\title{
THE TITAN REVERSED-FIELD-PINCH FUSION REACTOR S'TUDY
}

\author{
FINAL REPORT
}

1990

\section{Volume II: TITAN Plasma Engineering}

University of Californis, Los Angeles Departinent of Mechanical, Aerospace, and Nuclear Engineering and Institute of Plasma and Fusion Research Los Angeles, CA

General Atomics

San Diego, Ca
Los Alamos National Laboratory

Los Alamos, NM

Rensselaer Polytechnic Institute Department of Nuclear Engineering

Troy, NY

\section{MASTER}




\section{CONTRIBUINING AUTHORS}

\section{UNIVERSITY OF CALIFORNIA, LOS ANGELES}

Farrokh Najmabadi, Robert W. Conn, Nasr Ghoniem

James P. Blanchard

Steven P. Grotz

Radger C. Martin

Shahram Sharafat

Edward T. Cheng
Yuh-Yi Chu

Mohammad Z. Hasan

Anil K. Prinja(2)
Patrick I. H. Cooke ${ }^{(2)}$ Charlea E. Kessel George E. Orient Erik L. Vold

\section{GENERAL ATOMICS}

Kenneth R. Schultz

Richard L. Creedon

Charles G. Hoot

Clement P. C. Wong

LOS ALAMOS NATIONAL LABORATORY

Robert A. Krakowski

John R. Bartlit ${ }^{(3)}$

Charles G. Bathke

Ronald L. Miller

Ken A. Werley

\section{RENSSELAER POLYTECHNIC INSTITUTE}

Don Steiner

William P. Duggan

William P. Kelleher

\section{ARGONNE NATIONAL LABORATORY \\ Daj-Kai Sze}

\section{CANADIAN FUSION FUELS TECHNOLOGY PROJECT}

Paul J. Gierszewski

Otto K. Keuton

(1) Permanent address: Culham Laboratory, Abingion, Oxfordshire, United Kingdom.

(2) Present address: University of New Mexico, Albuquerque, New Mexico.

(3) TSTA Program. 
Contents

Volume I: EXECUTTVE SUMMARY

1. EXECUTTVE SUMMARY . . . . . . . . . . . . 1-1

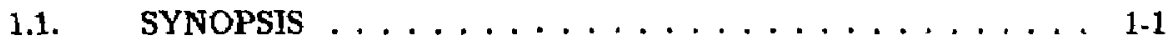

1.2. OVERVIEW OF TITAN PLASMA ENGINEERING $\ldots \ldots$. . . 18

1.3. OVERVIEW OF TITAN-I FUSION POWER CORE . . . . . 1-86

1.4. OVERVIEW OF TITAN-II FUSION POWER CORE $\ldots \ldots$. 1-133 RERERENCES . . . . . . . . . . . . . . . 1-176 
Volume II: TITAN PLASMA. ENGINEERING

2. REVERSED-FIELD PINCH AS A FUSION REACTOR . . . . . . 2-1

2.1. INTRODUCTION ............... 2-1

2.2. RFP THEORY $\ldots \ldots \ldots \ldots \ldots \ldots \ldots \ldots \ldots \ldots$

2.3. RFP EXPERIMENTS . . . . . . . . . . . 2-24

2.4. SUMMARY . . . . . . . . . . . 2-70 REFERENCES . . . . . . . . . . . . . . 2-71

3. PARAMETRIC SYSTEMS STUDIES. . . . . . . . . . . . 3-1

3.1. INTRODUCTION $\ldots \ldots \ldots \ldots \ldots \ldots \ldots \ldots \ldots \ldots$

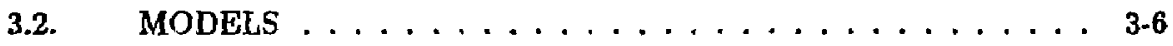

3.3. $\operatorname{costing} \ldots \ldots \ldots \ldots \ldots \ldots \ldots \ldots \ldots \ldots \ldots$

3.4. TITAN-I DESIGN POINT . . . . . . . . . . . 3-32

3.5. TITAN-II DESIGN POINT $\ldots \ldots \ldots \ldots \ldots \ldots \ldots$

3.6. SUMMARY AND CONCLUSIONS . . . . . . . . . . 3-57 REFERENCES . . . . . . . . . . . . . . . . 3-64

4. MAGNETICS ....................... . . . . . .

4.1. INTRODUCTION $\ldots \ldots \ldots \ldots \ldots \ldots \ldots \ldots \ldots, \ldots, 1$

4.2. INTEGRATED-BLANKET-COIL (IBC) CONCEPT . . . . 4-3

4.3. TOROIDAL-FIELD (TF) COILS . . . . . . . . . . . 4-4

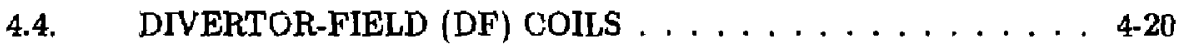

4.5. OHMIC-HEATING (OH) COILS $\ldots \ldots \ldots \ldots \ldots \ldots$. . . . . .

4.6. EQUILIBRIUM-FIELD (EF) COILS . . . . . . . . . 4-59

4.7. SUMMARY AND CONCLUSIONS . . . . . . . . 4-60 REFERENCES . . . . . . . . . . . . . . 4-63 
5. BURNING-PLASMA SIMULATIONS $\ldots \ldots \ldots \ldots \ldots \ldots \ldots$ 5-1

5.1. INTRODUCTION ............... 5 .1

5.2. EQULLIBRIUM AND STABILITY . . . . . . . 5.2

5.3. CORE-PLASMA SIMULATYONS . . . . . . . . . . 5-16

5.4. EDGE-PLASMA SIMULATIONS . . . . . . . . . 5-27

5.5. NEUTRAL TRANSPORT AND EROSION $\ldots \ldots \ldots \ldots$

5.6. FUELING . . . . . . . . . . . . . . 5-44

5.7. SUMMARY AND CONCLUSIONS . . . . . . . . 5-52

REFERENCES $\ldots \ldots \ldots \ldots \ldots \ldots \ldots \ldots \ldots \ldots \ldots$

6 PLASMA TRANSIENT OPERATIONS ............ 6-1

6.1. INTRODUCTION $\ldots \ldots \ldots \ldots \ldots \ldots \ldots \ldots \ldots$

6.2. RFP START-UP DATA BASE . . . . . . . 6 6-3

6.3. FORMATION OF THE TITAN PLASMA . . . . . . . 6-14

6.4. IGNITION REQUIREMENTS . . . . . . . . 6.19

6.5. CURRENT RAMP-UP TO IGNITION AND BURN . . . . . . 6-29

6.6. SHUTDOWN \& TERMINATION OF TITAN PLASMA . . . . $6-55$

6.7. SUMMARY AND CONCLUSIONS . . . . . . . 6-62 REFERENCES $\ldots \ldots \ldots \ldots \ldots \ldots \ldots \ldots \ldots \ldots \ldots \ldots$

7. CURRENT DRTVE. . . . . . . . . . . . . $7-1$

7.1. INTRODUCTION $\ldots \ldots \ldots \ldots \ldots \ldots \ldots \ldots \ldots$

7.2. CURRENT-DRIVE OPTIONS . . . . . . . 7 7-2

7.3. OSCILLATING-FIELD CURRENT-DRIVE MODEL . . . . . . 7-12

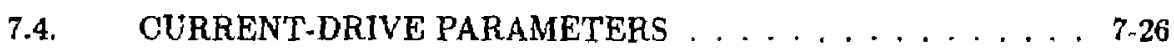

7.5. SUMMARY AND CONCLUSIONS $\ldots \ldots \ldots \ldots \ldots$

REFERENCES . . . . . . . . . . . . . . 7-40 
8. PHYSICS ISSUES FOR COMPACT RFP REACTORS $\ldots \ldots \ldots \ldots$. . . .

8.1. INTRODUCTION . . . . . . . . . . 8-1

8.2. CONFINEMENT . . . . . . . . . . . 8.8

8.3. CURRENT DRIVE $\ldots \ldots \ldots \ldots \ldots \ldots \ldots \ldots$. . . . . . .

8.4. IMPURITY CONTROL . . . . . . . . . . . 8-28

8.5. FORMATION AND START-UP $\ldots \ldots \ldots \ldots \ldots$

8.6. SUMMARY AND RECOMMENDATIONS . . . . . . 8.51

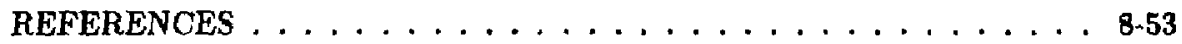


Volume III: TITAN-I FUSION POWER CORE

9. OVERVIEW OF TITAN-I DESIGN $\ldots \ldots \ldots \ldots \ldots$. . . . . . .

9.1. INTRODUCTION $\ldots \ldots \ldots \ldots \ldots \ldots \ldots \ldots$

9.2. CONFIGURATION $\ldots \ldots \ldots \ldots \ldots \ldots \ldots \ldots$

9.3. MATERIALS ................... 9-15

9.4. NEUTRONICS . . . . . . . . . . . . . 9-19

9.5. THERMAL AND STRUCTURAL DESIGN $\ldots \ldots \ldots \ldots \ldots$

9.6. MAGNEI ENGINEERING . . . . . . . . . . . . 9-28

9.7. POWER CYCLE . . . . . . . . . . . . . 9-32

9.8. DIVERTOR ENGINEERING . . . . . . . . . . . . 9.33

9.9. TRITIUM SYSTEMS $\ldots \ldots \ldots \ldots \ldots \ldots \ldots \ldots$

9.10. SAFETY DESIGN $\ldots \ldots \ldots \ldots \ldots \ldots \ldots \ldots$

9.11. WASTE DISPOSAL . . . . . . . . . . 9-46

9.12. MAINTENANCE . . . . . . . . . . . . . . . 9-48

9.13. SUMMARY AND KEY TECHNICAL ISSUES $\ldots \ldots \ldots \ldots$. . . $9-53$ REFERENCES . . . . . . . . . . . . . . . . 9-61

10. TITAN-I FUSION-POWER-CORE ENGINEERING $\ldots \ldots \ldots \ldots$ 10-1

10.1. INTRODUCTION $\ldots \ldots \ldots \ldots \ldots \ldots \ldots \ldots \ldots \ldots . \ldots \ldots$

10.2. MATERIAL SELECTION . . . . . . . . . . 10-1

10.3. NEUTRONICS . . . . . . . . . . . . . . 10-54

10.4. THERMAL AND STRUCTURAL DESIGN . . . . . . 10-66

10.5. MAGNET ENGINEERING . . . . . . . . . . 10-110

10.6. POWER-CYCLE ANALYSIS . . . . . . . . . 10-133

10.7. SUMMARY AND CONCLUSIONS $\ldots \ldots \ldots \ldots \ldots$ 10-142

REFERENCES . . . . . . . . . . . . . . . . . . . . 10-144 
11. TITAN-I DIVERTOR ENGINEERING . . . . . . . . . . 11-1

11.1. INTRODUCTION $\ldots \ldots \ldots \ldots \ldots \ldots \ldots \ldots \ldots$

11.2. MATERIALS ................ 11-3

11.3. TARGET FABRICATION . . . . . . . . . . 11-11

11.4. TARGET DESIGN . . . . . . . . . . 11-15

11.5. THERMAL AND STRUCTURAL ANALYSIS . . . . . . . 11-27

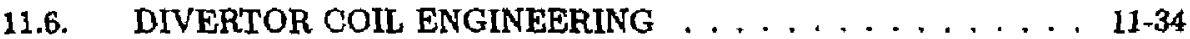

11.7. NEUTRONICS . . . . . . . . . . . . . 11-38

11.8. VACUUM SYSTEMS . . . . . . . . . . 11-39

11.9. SUMMARY AND CONCLUSIONS $\ldots \ldots \ldots \ldots \ldots \ldots \ldots$

REFERENCES . . . . . . . . . . . . . . . . . . 11-44

12. TITAN-I TRITIUM SYSTEMS $\ldots \ldots \ldots \ldots \ldots \ldots$ 12-1

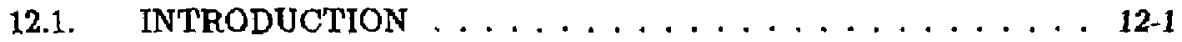

12.2. BLANKET TRITIUM SYSTEM $\ldots \ldots \ldots \ldots \ldots$. . . . . . . . .

12.3. FUEL-PROCESSING AND SAFETY SYSTEMS . . . . . . 12-10

12.4. SUMMARY AND CONCLUSIONS . . . . . . . . . 12-25

REFERENCES . . . . . . . . . . . . . . . . . . . . 12-29

13. TITAN-I SAFETY DESIGN AND RADIOACTIVE-WASTE DISPOSAL . 13-1.

13.1. INTRODUCTION . . . . . . . . . . . . 13-1

13.2. SAFETY-DESIGN GOALS $\ldots \ldots \ldots \ldots \ldots \ldots$

13.3. SAFETY-DESIGN FEATURES . . . . . . . . . . . . 13-5

13.4. LOSS-OF-FLOW \& LOSS-OF-COOLANT ACCIDENTS . . . . 13-14

13.5. LITHIUM-FIRE ACCIDENTS . . . . . . . . . . 13-32

13.6. PLASMA ACCIDENTS . . . . . . . . . . . . 13-37

13.7. RADIOACTIVE-WASTE NISPOSAL $\ldots \ldots \ldots \ldots \ldots$ 
13.8. SUMMARY AND CONCLUSIONS . . . . . . . . . 13-45 REFERENCES . . . . . . . . . . . . . . . 13-48

14. TITAN-I MAINTENANCE PROCEDURES $\ldots \ldots \ldots \ldots \ldots$ 14-1

14.1. INTRODUCTION . . . . . . . . . . . . 14-1

14.2. TITAN-I PLANT LAYOUT . . . . . . . . . . . . 14-2

14.3. MAINTENANCE PROCEDURES . . . . . . . . 14-6

14.4. FUSION POWER CORE PRETESTING $\ldots \ldots \ldots \ldots \ldots, \ldots, 14-12$

14.5. SUMMARY . . . . . . . . . . . . . . . 14-14

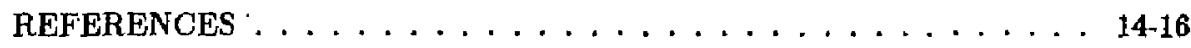

A. APPENDLX A ................ A

A.1. TABLE OF TITAN-I REACTOR PARAMETERS . . . . . . A-1

A.2. SYSTEMS CODE PARAMETERS OF TITAN-1 . . . . . . A A-24

A.3. COST SUMMARY OF TITAN-I REACTOR . . . . . . . A A-27

A.4. COST DATA BASE FOR TITAN-I REACTOR . . . . . . . A A-35 REFERENCES . . . . . . . . . . . . . . . . A A-43 
viii

Volume IV: TITAN-II FUSION POWER CORE

15. OVERVIEW OF TITAN-II DESIGN $\ldots \ldots \ldots \ldots \ldots \ldots \ldots$ 15-1

15.1. INTRODUCTION . . . . . . . . . . I5-1

15.2. CONFIGURATION $\ldots \ldots \ldots \ldots \ldots \ldots \ldots \ldots \ldots$

15.3. MATERIALS . . . . . . . . . . . . . 15-16

15.4. NEUTRONICS . . . . . . . . . . . . 15-23

15.5. THERMAL AND STRUCTURAL DESIGN . . . . . . 15-25

15.6. MAGNET ENGINEERING . . . . . . . . . . . . 15-29

15.7. POWER CYCLE . . . . . . . . . . . . . . 15-29

15.8. DIVERTOR ENGINEERING . . . . . . . . . . 15-30

15.9. TRITIUM SYSTEMS $\ldots \ldots \ldots \ldots \ldots \ldots \ldots \ldots$ 15-37

15.10. SAFETY DESIGN $\ldots \ldots \ldots \ldots \ldots \ldots \ldots \ldots \ldots \ldots$

15.11. WASTE DISPOSAL . . . . . . . . . . 15-45

15.12. MAINTENANCE. . . . . . . . . . . 15-48

15.13. SUMMARY AND KEY TECHNICAL ISSUES $\ldots \ldots \ldots \ldots \ldots 15-51$

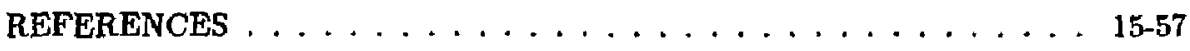

16. TITAN-II FUSION-POWER-CORE ENGINEERING . . . . . . . 16-1

16.1. INTRODUCTION . . . . . . . . . . . I6-1

15.2. MATERIAL SELECTION . . . . . . . . . . . 16-7

16.3. NEUTRONICS . . . . . . . . . . . . . . . . 16-95

16.4. THERMAL AND STRUCTURAL DESIGN . . . . . . . 16-104

16.5. POWER-CYCLE ANALYSIS . . . . . . . . . $16-127$

16.6. SUMMARY AND CONCLUSIONS . . . . . . . . . . 16-129

REFERENCES . . . . . . . . . . . . . . . 16-133 
17. TITAN-HI DIVERTOR ENGINEERING $\ldots \ldots \ldots \ldots \ldots \ldots \ldots$

17.1. INTRODUCTION $\ldots \ldots \ldots \ldots \ldots \ldots \ldots \ldots \ldots \ldots$

17.2. MATERIALS . . . . . . . . . . . . 17-4

17.3. TARGET FABRICATION . . . . . . . . . . 17-6

17.4. TARGET DESIGN . . . . . . . . . . . 17-10

17.5. THERMAL AND STRUCTURAL ANALYSES $\ldots \ldots \ldots \ldots 17-17$

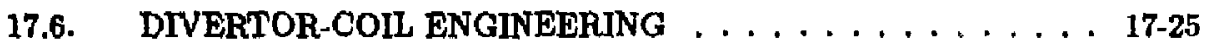

17.7. VACUUM SYSTEMS $\ldots \ldots \ldots \ldots \ldots \ldots \ldots \ldots \ldots$

17.8. SUMMARY AND CONCLUSTONS $\ldots \ldots \ldots \ldots \ldots \ldots$. . . . . . . REFERENCES . . . . . . . . . . . . . . . . . . . 17-29

18. TITAN-II TRITIUM SYSTEMS $\ldots \ldots \ldots \ldots \ldots \ldots \ldots$ 18-1

18.1. INTRODUCTION $\ldots \ldots \ldots \ldots \ldots \ldots \ldots \ldots \ldots$ 18-1

18.2. PERMEATION . . . . . . . . . . . . . . . 18-2

18.3. TRITIUM CONTROL . . . . . . . . . . . . . 18-4

18.4. BLANKET TRITIUM-RECOVERY SYSTEM . . . . . . . 18-10

18.5. PLASMA-EXHAUST-PURIFICATION SYSTEM . . . . . . . 18-23

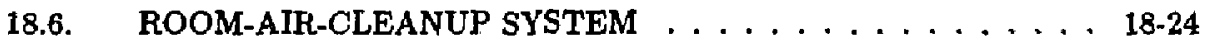

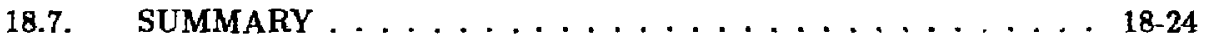

REFERENCES . . . . . . . . . . . . . . . . . 18-28

19. TITAN-II SAFETY DESIGN AND RADIOACTIVE-WASTE DISPOSAL 19-1 19.1. INTRODUCTION . . . . . . . . . . . . 19-1

19.2. SAFETY-DESIGN GOALS . . . . . . . . . . 19-1

19.3. SAFETY-DESIGN FEATURES . . . . . . . . . 19-3

19.4. LOSS-OF-FLOW \& LOSS-OF-COOLANT ACCIDENTS . . . . 19-11

19.5. FADIOACTIVE-WASTE DISPOSAL . . . . . . . . . 19-22 
19.6. SUMMARY AND CONCLUSIONS . . . . . . . . . . 19-29 REFERENCES . . . . . . . . . . . . . . . 19-30

20. TITAN-II MAINTENANCE PROCEDURES . . . . . . . . . 20-1

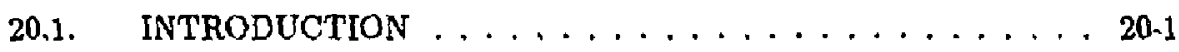
20.2. TITAN-II PLANT LAYOUT . . . . . . . . . . . 20-2 20.3. MAINTENANCE PROCEDURES $\ldots \ldots \ldots \ldots \ldots \ldots$

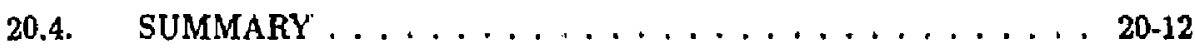
REFERENCES . . . . . . . . . . . . . . . . 20-14

B. APPENDIX B ...................

B.i. TABLE OF TITAN-1I REACTOR PARAMETERS . . . . . . B B-1

B.2. SYSTEMS CODE PARAMETERS OF TITAN-II . . . . . . B-22

B.3. GOST SUMMARY OF TITAN-II REACTOR . . . . . . . . B-25

B.4. COST DAMA BASE FOR TITAN-II REACTOR . . . . . . . B B-33 REFRRENCES . . . . . . . . . . . . . B B-41 


\section{ACKNOWLEDGEMENTS}

The TITAN design team wishes to acknowledge the consultations and advice given by the TITAN Physics Advisory Committe on reversed-field-pinch physics issues and by the TITAN Engineering Review Committee on engineering design and analysis. Useful diecuasions with Frank Clinard of Los Alamos National Laboratory on properties of ceramic insulators, with John Davis of McDonnel Douglas on refractory metals, with John Haines of McDo_rel Douglas on vacuum pumping issues, with Steve Piet of Idaho National Engineering Laboratory on safety aspects of the design, with Mark Tillack of University of California, Los Angeles on liquid-metal MHD issues, and with scientists of Fusion Engineering Design Center at Oak Ridge National Laboratory on design maintenance and integration are also acknowledged. The authors also wish to express their appreciation to Ms. Joan George for her effort in preparing this repoit.

The TITAN research program is supported by the U. S. Department of Energy, Office of Fusion Energy, at University of California, Los Angeies under grant DE-FG0386ER52126, at General Atomics under contract DE-AC03-84ER53158, at Rensselaer Polytechnic Institute under grant DE-FG02-85ER52118, and at Los Alamos National Laboratory which is operated by the University of California for the U. S. DOE under contract W-7405-ENG-36. 


\section{TITAN PHYSICS ADVISORY COMMITTEE}

Richard A. Nebel

Allan Newton

Stewart C. Prager

Michael J. Schaffer

Kurt F. Schoenberg

Teruo Tamano

Starnes Walker
Los Alamos National Laboratory

Culnam Laboratory

University of Wisconsin, Madison

General Atomics

Los Alamos Nationel Laboratory

General Atomics

Phillips Petroleum Company

\section{TITAN ENGINEERING REVIEW COMMITTEE}

Mohamed A. Abdou

Charles C. Baker

Dariei Cohn

James D. Gordon

Garl D. Henning

Michael J. Schaffer
University Of California, Los Angeles

Argonne National Laboratory

Massachusetts Institute Of Technology TRW

Lawrence Livermore National Laboratory

General Atomics 


\section{ABSTRACT}

The TITAN Reversed-Field-Pinch (RFP) fusion-reactor study is a multi-institutional research effort to determine the technical feasibility and key developmental issues for an RFP fusion reactor operating at high power dersity, and to determine the putential economic (cost of electricity), operational (maintenance and availability), safety and envirolumental features of high mass-power-density fusion systems. Mass power density $(M P D)$ is defined as the ratio of net electric output to the mass of the fusion potver core (FPC). The fusion power core includes the plasma chamber, first wall, blanket, shield, magnets, and related structure.

Two different detailed designs, TITAN-I and TITAN-II, have been produced to demonstrate the possibility of multiple engineering-design approaches to high-MPD reactors. TITAN-I is a self-cooled lithium design with a vanadium-alloy structure. TITAN-II is a self-cooled aqueous loop-in-pool design with $9 \mathrm{C}$ ferritic steel as the structural material. Bcth designs would use RFP plasmas operating with essentially the same parameters. Both conceptual reactors are based on the DT fuel cycle, have a net electric output of about $1000 \mathrm{MWe}$, are compact, and have a high MPD of $800 \mathrm{kWe}$ per tonne of FPC. The inherent physical characteristics of the RFP confinement concept make possible compact fusion reactorg with such a high mass power density. The TITAN designs would reet the $U$. S. criteria for the near-surface disposal of radioactive waste (Class $C, 10 C F T$. 61) and would achieve a high Level of Safety Assurance with respect to FPC damage by decay afterheat and radioactivity release caused by accidents. Very importantly, a "single-piece" FPC maintenance procedure has been worked out and appears feasible for both degigns.

Parametric system studies have been tused to find cost-optimized designs, to determine the parametric design unindow associated with each approach, and to assess the sensitivity of the designs to a wide range of physics and engineering requirements and assumptions. The design window for such compact RFP reactors would include machises with neutron wall loadings in the range of $10-20 \mathrm{MW} / \mathrm{m}^{2}$ with a shallow minimum-COE at about $18 \mathrm{MW} / \mathrm{m}^{2}$. Even though operation at the lower end of the this range of wall loading (10$12 \mathrm{MW} / \mathrm{m}^{2}$ ) is possible, and may be preferable, the TITAN study adopted the design point at the upper end $\left(18 \mathrm{MW} / \mathrm{m}^{2}\right)$ in order to quantify and assess the technical feasibility and physics limits for such high-MPD reactors. From this work, key physics and engineering issues central to achieving reactors with the features of TITAN-I and TITAN-II have emerged. 


\section{Contents}

2.1. INTRODUCTION $\ldots \ldots \ldots \ldots \ldots \ldots \ldots \ldots$ 2-1

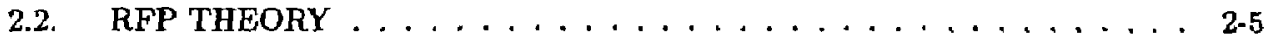

2.2.1. RFP Confinement Concept $\ldots \ldots \ldots \ldots \ldots \ldots \ldots$

2.2.2. Equilibrium . . . . . . . . . . . . 2.13

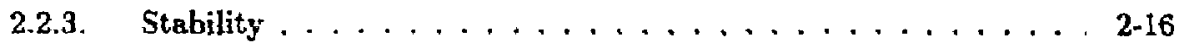

2.2.4. Relaxation and Sustainment . . . . . . . . . . 2-20

2.2.5. Transport and Confinement . . . . . . . . . . . 2-22

2.3. RFP EXPERIMENTS . . . . . . . . . . . . . . 2-24

2.3.1. Start-Up and Formation .............. 2-28

2.3.2. Typical Temperature and Density Responses . . . . . . . . 2-35

2.3.3. Density Control . . . . . . . . . . . . . 2-36

2.3.4. Plasma Fluctuations . . . . . . . . . . . 2-40

2.3.5. Scaling. . . . . . . . . . . . . . . 2-42

2.3.6. Highly Radiating Plasma . . . . . . . . . . . 2-57

2.3.7. Current Termination . . . . . . . . . . . . 2-61

2.3.8. Current Drive . . . . . . . . . . . . . . . 2-61

2.3.9. Operation with Resistive Shells . . . . . . . . . 2-65

2.4. SUMMARY . . . . . . . . . . . . . . . . . . 2-70

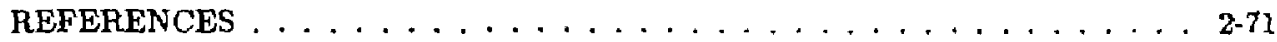




\section{REVERSED-FIELD PINCH AS A FUSION REACTOR}

\subsection{INTRODUCTION}

The TITAN research program is a multi-institutional [1] effort to determine the potential of the reversed-field-pinch (RFP) magnetic fusion concept as a compact, highpower-density, and "attractive" fusion energy system from economics (cost of electricity, COE), safety, environmental, and operational viewpoints.

In recent seactor studies, the compact resctor option [2-5] has been identified as one approach toward a more offordable and competitive fusion reactor. The main feature of a compact reactor is a fusion power core (FPC) with a mass power density in excess of 100 to $200 \mathrm{kWe} /$ tonne. Mass power density (MPD) is defined [2] as the ratio of the net electric power to the mase of the FPC, which includes the plasma chamber, first wall, blanket, shield, magnets, and related structure. The increase in MPD is achieved by increasing the plasme power density and neutron wall loading, by reducing the size and mass of the FPC through decreasing the blanket and shield thicknesses and using resistive magnet coils, as well as by increasing the blanket energy multiplication. A compact reactor, therefore, strives toward a system with an FPC comparable in mass and volume to the heat sources of alternative fission power plants, with MPDs ranging from 500 to $1000 \mathrm{kWe} /$ tonne and competitive cost of energy.

Other potential benefits for compact systems can be envisaged in addition to improved economics. The FPC cost in a compact reactor is a small portion of the plant cost and, therefore, the economics of the reactor will be less sensitive to changes in the unit cost of FPC components or the plasma performance. Moreover, since a high-MPD FPC is smaller and cheaper, a rapid development program at lower cost should be possible, changes in the FPC design will not introduce large cost penalties, and the economics of learning curves can be readily exploited throughout the plant life.

The RFP has inherent characteristics which allow it to operate at very high mass power densities. This potential is available because the main confining field in an RFP is the poloidal field, which is generated by the large toroidal current flowing in the plasma. This feature results in a low field at the external magnet coils, a high plasma beta, and a very high engineering beta (defined as the ratio of the plasma pressure to the square 
of the magnetic field strength at the coils) as compared to other confinement schemes. Furthermore, sufficiently low magnetic fields at the external coils permit the use of normal coils while joule losses remain a small fraction of the plant output. This option allows a thinner blanket and shield. In addition, the high current density in the plesme allows ohmic heating to ignition, eliminating the need for auxiliary heating equipment. Also, the RFP concept promises the possibility of efficient current-drive systems based on low-frequency oscillations of poloidal and torotdal fluxes and the theory of RFP relaxed states. The RFP confinement concept allows arbitrary aspect ratios, and the circular cross section of plasma eliminates the need for plasma shaping coils. Lastly, the higher plasma densities particulariy at the edge, together with operation with a highly radiative RFP plesma, significantly reduce the divertor heat flux and erosion problems.

These inherent characteristics of the RFP [6] allow it to meet, and actually far exceed, the economic threshold MPD value of $100 \mathrm{kWe} /$ tonne. As a result, the TITAN study also seeks to find potentially siguificant benefits and to illuminate main drawbacks of operating well above the MPD threshold of $100 \mathrm{kWe}$ / tonne. The program, therefore, has chosen a minimum cost, high-neutron wall loading of $18 \mathrm{MW} / \mathrm{m}^{2}$ as the reference case in order to quantify the issue of engineering practicality of operating at high MPDs. The TITAN study has also put strong emphasis on safety and environmental features in order to determine if high-power-density reactors can be designed with a high level of safety assuance and with low-ackivation material to qualify for Class- $\mathrm{C}$ wraste disposal.

Two differenit detailed designs, TITAN-I and TITAN-II, have been produced to demonstrate the possibility of multiple engineering design upproaches to high-MPD reactors. TITAN-I is a self-cooled lithium design with a vanadium-alloy structure. TITAN-II is a self-cooled aqueous "loop-in-pool" design with ferritic-steel alloy, 9C, as the structural material. Both designs use RFP plasmas operating, with essentially the same parameters.

The operating space of a compact RFP reactor has been exrmined using a comprehensive parametric systems model which includes the evolving state of knowledge of the physics of RFP confinement and embodies the TITAN-I and TITAN-II engineering approaches (Saction 3). Two key figures of merit, the cost of electricity (COE) and mass power density (MPD), are monitored by the parametric systems model and are displayed in Figure 2.1-1 as functions of the neutron wall loading. Figure 2.1-1 shows that the COE is relatively insensitive to wall loadings in the range of 10 to $20 \mathrm{MW} / \mathrm{m}^{2}$, with a shallow minimum at about $19 \mathrm{MW} / \mathrm{m}^{2}$. The MPD is found to increase monotonically with the wall load. For designs with a neutron wall load larger than about $10 \mathrm{MW} / \mathrm{m}^{2}$, the FPC is physically small enough such that single-piece FPC maintenance is feasible. These considerations point to a design window for compact $\mathrm{RFP}$ reactors with neutron wall 
loading in the range of 10 to $20 \mathrm{MW} / \mathrm{m}^{2}$. The TITAN-class RFP rezctors in this design window have an MPD in excess of $500 \mathrm{kWe} /$ tonne, and an FPC engineering power density in the range of 5 to $15 \mathrm{MWt} / \mathrm{m}^{3}$; these values represent improvements by factors of 10 to 30 compared with earlier fusion reactor designs. The FPC cost is a smaller portion of the tota' plant cost (typically about $12 \%$ ) compared with $25 \%$ to $30 \%$ for earlier RFP designs $[4,5]$. Therefore, the unit direct cost (UDC) is less sensitive to related physics and technology uncertainties.

Near-minimum-COE TITAN-I and TITAN-II design points, incorporating distinct blanket thermal-hydraulic options, materials choices, and neutronics performances have been identified in Figure 2.1-1. The major parameters of the TITAN reactors are summarized in Table 2.1-I. In order to permit a comparison, the TITAN reference design points have similar plasma parameters and wall loadings allowing for certain plasma engineering analyses to be common between the two designs.

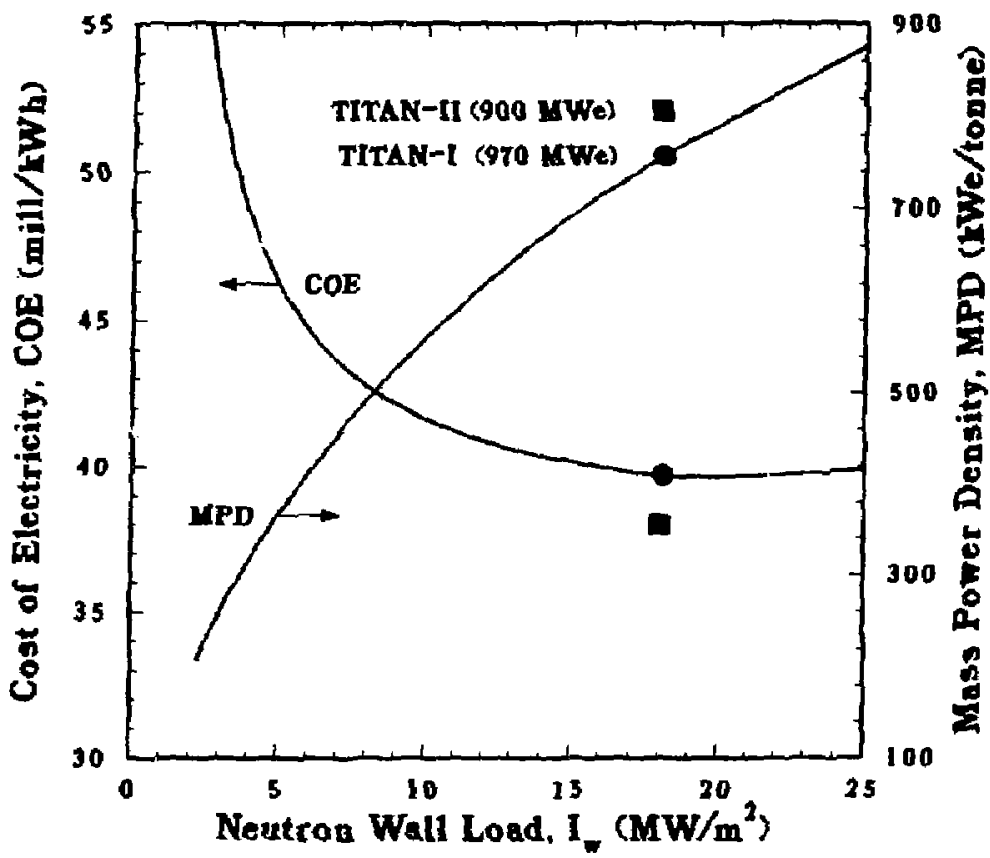

Figure 2.1-1. The COE (in 1986\$) and MPD as functions of neutron wall loading for the TITAN-class RFP reactors. TITAN-I (filled circle) and TITAN-II (filled squares) reference design points are also shown. 
Table 2.1-I.

OPERATING PARAMETERS OF TITAN FUSION POWER CORES

\begin{tabular}{|c|c|c|}
\hline & TITAN-I & TITAN-II \\
\hline Major radius (m) & 3.9 & 3.9 \\
\hline Minor plasma radius (m) & 0.60 & 0.60 \\
\hline First wall radius (m) & 0.66 & 0.66 \\
\hline Plasma current (MA) & 17.8 & 17.8 \\
\hline Torojdal field on plesme surface (T) & 0.36 & 0.36 \\
\hline Poloidal beta & 0.23 & 0.23 \\
\hline Neutron wall load $\left(\mathrm{MW} / \mathrm{m}^{2}\right)$ & 18 & 18 \\
\hline Radiation heat flux on first wall ( $\mathrm{MW} / \mathrm{m}^{2}$ ) & 4.6 & 4.6 \\
\hline Primary coolant & Liquid lithium & Aqueous solution \\
\hline Structural material & $V-3 T i-1 S i$ & Ferritic steel $9 . \mathrm{C}$ \\
\hline Breeder materis] & Liquid lithium & $\mathrm{LiNO}_{3}$ \\
\hline Neutron multiplier & none & $\mathrm{Be}$ \\
\hline Coolant inlet temperature $\left({ }^{\circ} \mathrm{C}\right)$ & 320 & 298 \\
\hline First-wall-coolant exit temperature $\left({ }^{\circ} \mathrm{C}\right)$ & 440 & 330 \\
\hline Blanket-coolant exit temperature $\left({ }^{\circ} \mathrm{C}\right)$ & 700 & 330 \\
\hline Coolant punping power (MW) & 48 & 49 \\
\hline Fusion power (MW) & 2301 & 2290 \\
\hline Total thermal power (MW) & 2935 & 3027 \\
\hline Net electric power ( $M W$ ) & 970 & 900 \\
\hline Gross efficiency & $44 \%$ & $35 \%$ \\
\hline Net efficiency & $33 \%$ & $30 \%$ \\
\hline Mass power density, MPD ( $k w_{e} /$ tonne) & 757 & 806 \\
\hline Cost of electricity, COE (mill/kWh) & 39.7 & 38.0 \\
\hline
\end{tabular}


In this section, the theoretical principles and understanding of the RFP confinement scheme are described, emphasizing those features that are relevant to a fusion reactor. The experimental data base is summarized in Section 2.3. More detailed descriptions of the theoretical and experimental aspects of RFP confinement concept are given in References $[6,9,10]$ and the references contained therein. A detailed description of the plasma engineering for TITAN reactors is given in Sections 4 through 7. Section 8 summarizes the major physics issues for realizing TITAN-class RFP reactors.

\subsection{RPP THEORY}

\subsubsection{RFP Confinement Concept}

The RFP, like the tolanak, belongs to a class of axisymmetric, toroidal confinement systems that uses both toroidal, $B_{\phi}$, and poloidal, $B_{\phi}$, magnetic fields to confine the plesma. Stability in the tokamak is provided by a strong toroidal field $\left(B_{\phi} \gg B_{\theta}\right.$ everywhere) such that the safety factor, $q=r_{p} B_{\phi} /\left(R_{T} B_{\theta}\right\rangle_{\text {, exceeds unity }}$ where $R_{T}$ and $r_{p}$ are, respectively, the major and minor radii of the plasma. In the RFP, on the other hand, strong magnetic shear produced by the radially varying (and decreasing) toroidal field stabilizes the plawn with $q<1$ at relatively modeat levels of $B_{\phi}$. Theoreticaily, an electrically conducting shell surrounding the plasma is required to stabilize the lons-wavelength MHD modes. In both the RFP and the tokamak, equilibrium may be provided by either an externally produced vertical field, a conducting toroidal shell, or a combination of both. Figure 2.2-1 compares the radial variation of the poloidal and toroidal fields and the safety factors for the tokamak and RFP.

The RFP magnetic topology is dominated by the poloidal field generated by the current flowing in the plasma. This feature has several reactor-relevant advantages. The poloidal field decreases inversely with the plasma radius outside the plasma. The toroijal field is also weak outside the plasma relative to the tokamak. The riagnetic field strength at the external conductors, therefore, is small, and a high engineering beta (defined as the ratio of the plasma pressure to the magnetic field pressure at the magneta) results; less-massive resistive coils with a low current density are possible. The RFP experiments operate at reactor-relevant values of total beta (5\% to $10 \%)$. Furthermore, by relying on the magnetic shear to stabilize the plasma, the RFP can support a large ratio of plasma current to toroidal field, and stability constraints on the aspect ratio, $R_{T} / r_{p}$, are removed; the choice of the aspect ratio, therefore, can be made solely on the basis of engineering constraints. High-current-density operation and strong ohmic heating to ignition are 

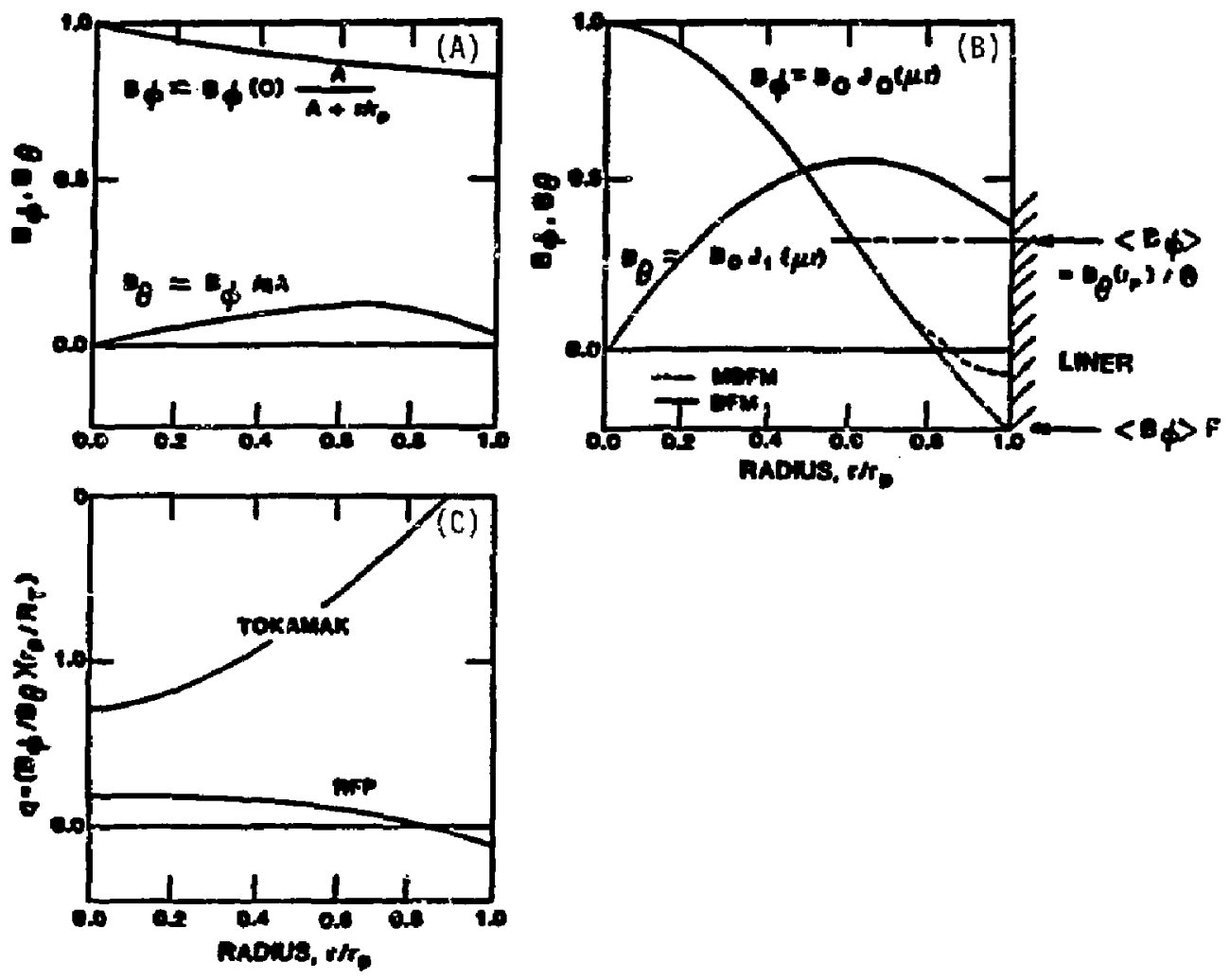

Figure 2.2-1. Magnetic-field distribution for tokamak ( $A$ ) and RFP (B) and the $q$ profiles for tokamak and RFP (C). 
also positive consequences of the shear-stabilized RFP. Lastly, the close coupling of the current and magnetic-field components within the RFP plasma also promises a unique and highly efficient current-drive technique.

The field configuration and toroidal-field reversal in the RFP are the result of the relaxation of the plasma to a near-minimum-energy state. A theory of relaxed states developed by Taylor [11] postulates that a pinch should relax to a magnetic-field configuration determined by minimizing the magnetic energy subject to constraints imposed on allowed motion or magnetic-field variation. Taylor then considered the relaxation of a plasma with small but finite resistivity in a flux-coneerving cylinder, subject to the invariance of the nagnetic helicity $K \equiv \int \mathbf{A} \cdot \mathbf{B} d V_{p}$, where $\mathbf{B} \equiv \nabla \times \mathbf{A}$ and the integration is over the plasma volume. The helicity is a measure of the linkage between the poloidal and toroidal magnetic flux. The relaxed, minimum-energy state was found to be force-free and described by $\nabla \times \mathbf{B}=\mu \mathrm{B}$, where $\mu=\mu_{0} \mathbf{j} \cdot \mathbf{B} / B^{2}$ is the ratio of local parallel carrent and field. For the minimum-energy Taylor state, the $\mu$ profile is uniform across the plasma. The solution to the equilibrium squation in cylindrical geometry for a spatially constant $\mu$ gives the Bessel-function model (BEM), with $B_{\phi} \propto J_{1}(\mu r)$ and $B_{\theta} \propto J_{0}(\mu r)$, where $J_{1}$ and $J_{0}$ are Bessel functions of the first kind. These relaxed states can be described solely in terms of two dimensionless quantities: the reversal parameter, $F$, and the pizch parameter, $\Theta$, where

$$
\begin{aligned}
F & \equiv \frac{B_{\phi}\left(r_{p}\right)}{\left\langle B_{\phi}\right\rangle}, \\
\Theta & \equiv \frac{B_{\theta}\left(r_{p}\right)}{\left\langle B_{\phi}\right\rangle} .
\end{aligned}
$$

The average toroidal field within the conducting shell, $\left\langle B_{\phi}\right\rangle$, is defined as

$$
\left\langle B_{\phi}\right\rangle=\frac{2}{r_{p}^{2}} \int_{0}^{r_{r}} B_{\phi}(r) r d r .
$$

The locus of relaxed states then form a curve in F- $\theta$ space, as is shown in Figure 2.2-2 (labeled as BFM). The same figure also shows the experimental data which lie to the right of the curve predicted by the BFM model. The experimental equilibria represented in Figure 2.2-2 differ from the Taylor model because plasma has finite pressure, $\mu$ is not uniform across the plasma, and a perfectly conducting wall is not used. These data represent "near-minimum-energy" states. The concept of a preferred locus of relaxed states in $F-\theta$ space, as originally postulated by Taylor, remains applicable, however.

Near the cool edge of practical RFPs, the $\mu$ profile deviates away from $\mu(r) \simeq$ constant condition of the minimum-energy assumption. At the highly resistive plasma edge, $\mu$ is 


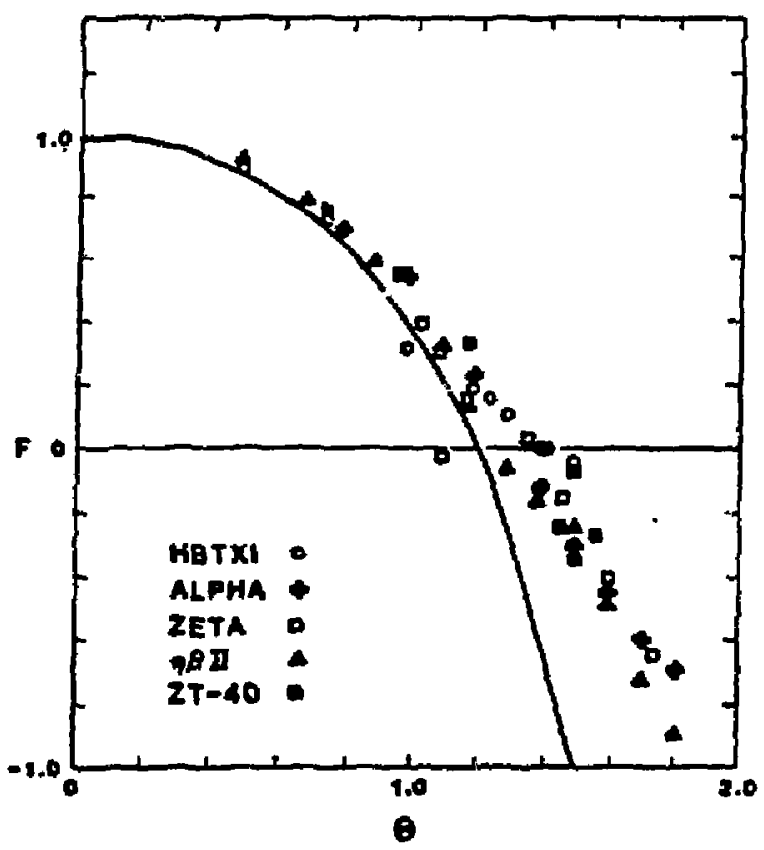

Figure 2.2-2. Locus of operating points on the $F \Theta$ diagram. The solid line (BFM) is the curve predicted by Taylor's theory [11] and the data points are from several RFP experiments.

assumed to dectease to zero in order to model the decreasing current expected in the edge region. These differences can be seen in Figure 2.2-3 [12] which compares the magnetic field profiles from experiments to the BFM model. The smaller magnitude of the toroidal fields observed in experiment reflects the fallofl of $\mu$ and $j_{\theta}$ in the cold plasma edge. Typically, the following expressions for $\mu(\mathbf{r})$ describes experimental results [13].

$$
\frac{\mu(r)}{\mu(0)}=1-\left(\frac{r}{r_{p}}\right)^{\alpha}
$$

where $\alpha \simeq 6$ to 8 and $\mu\left(0 j \equiv 2 \Theta_{0} / r_{p}=2 q(0) A / r_{p}\right.$ are fitting parameters used to noodel the experimental profiles with finite plasma beta. The impact of the cold plasma edge (smaller value of $\alpha$ ) on the $F-\Theta$ diagram is shown in Figure 2.2-4 along with experimental results form the ETA-BETA-II RFP experiment [12].

The theory of relaxed states as applied to the RIP concept has several important consequences. Firstly, the theory predicts that the relaxed states depend only on the 


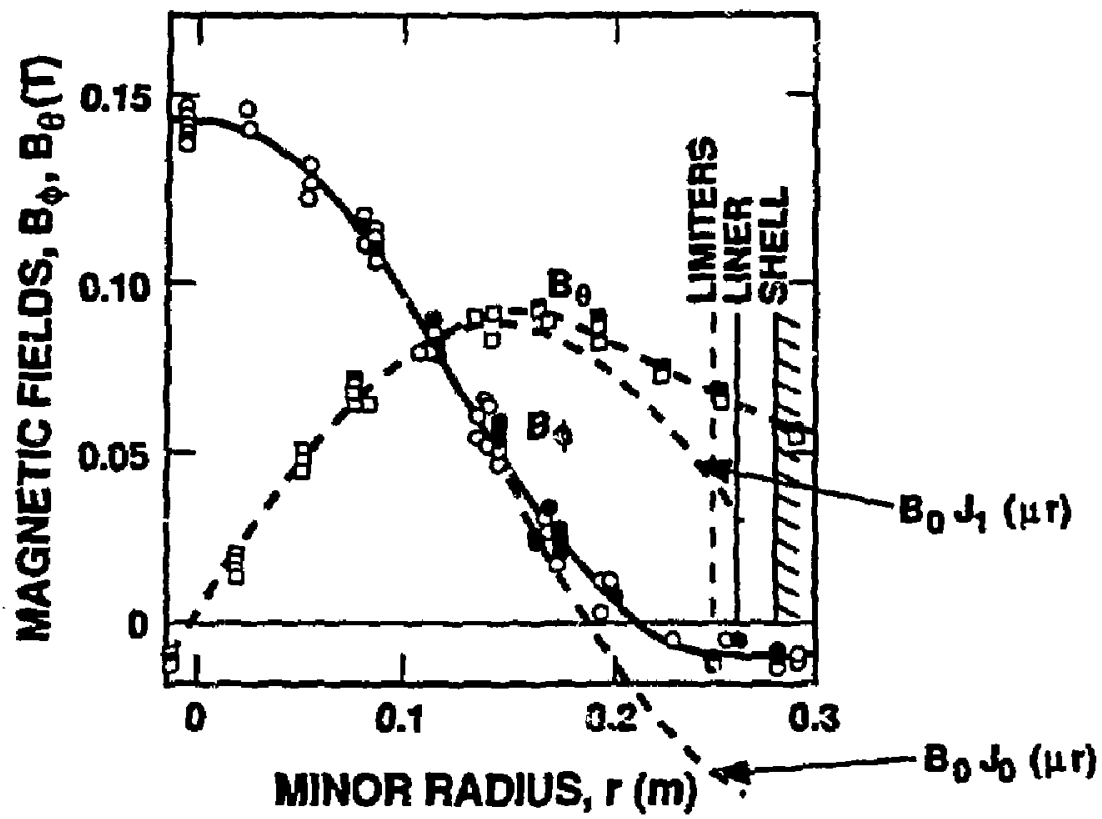

Figure 2.2-3. Magnetic-field profiles from the HBTX experiments [12] showing the derivation from the BFM for $\mu(r)$ profiles that are not consta. it across the plasma radius.

pinch parameter, $\Theta$, and these states are independent of initial conditions provided that the time scale is sufficiently long for the relaxation process to occur. Secondly, if the plasma current and toroidal flux are maintained constant in time (i.e., constant $\Theta$ ), then the relaxed-state equilibrium will be sustained. Experinentally, the RFP configuration shown in Figure 2.2-3 exists for times much larger than the resistive decay time of the field profiles. 1'his sustainment process involves continuous generation of the toroidal field within the plasma to compensate for the resistive decay of the toroidal field and to maintain the field profile; this process is often called the RFP "dynamo." The RFP dynamo converts poloidal flux or torisidal currents to poloidal currents of toroidal fux and is driven by localized plasma fluctuations of fluid velocity, $\delta u$, and magnetic field, $\delta B$, to give a non-zero time-averaged electric field, $\langle\delta u \times \delta B\rangle$.

The $F-\Theta$ relationship (Figure 2.2-2) reflects a strong coupling between the toroidal and poloidal fields within the plasina: the toroidal fietd is continuously regenerated by 


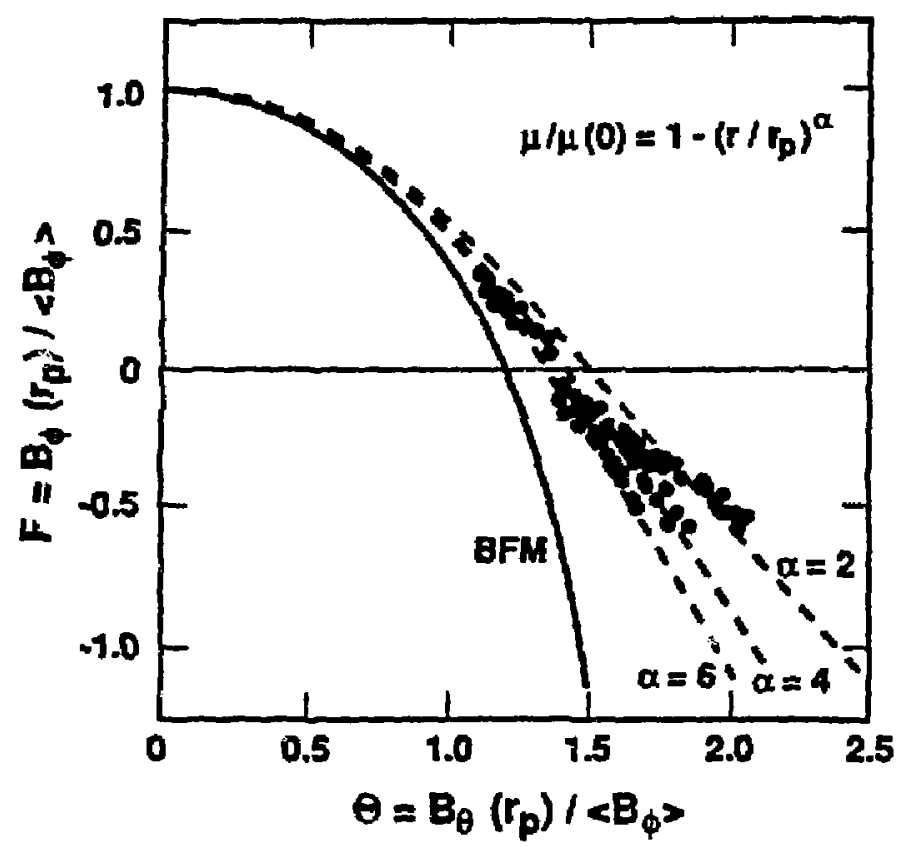

Figure 2.2-4. F- $\Theta$ diagram for Taylor's relaxed state and an equilibrium model with different values of the experiment, $\alpha$, describing the flatness of the $\mu$ profile (Equation 2.2-4). Circles indicate experimental configuration for the ETA-BETA-II experiment [12].

driving toroidal current with an external poloidal-field circuit. Indeed, such a relaxationassisted plasma current ramp has been demonstrated in RFP experiments and is envisioned for RFP reactors. Figure 2.2-5 illustrates a typical "matched-mode" RFP start. up [14]. After the vacuum toroidal field, $B_{\phi}$, reaches a maximum value, the plasma breakdown occurs, and current, $I_{q}$, is initiated, with $B_{\phi}$ at and beyond the plasma surface, $B_{\phi}\left(\tau \geq r_{p}\right)$, actually reversing direction while the average toroidal field, $\left\langle B_{\phi}\right\rangle$, and toroidal fux, $\Phi$, within the conducting shell remain positive. Upen reversal of the toroidal field and establishment of the quiescent near-mininum-energy RFP state, Figure 2.2-5 slows a dramatic decrease in the plasma resistive, $B_{p}$, which nevertheless remains large in comparison with the tokamak. Figure 2.2-6 $[15,16]$ illustrates explicitly the generation of toroidal flux within the plasma by the RFP dynamo, which assists and maintains the toroidal field during a slow current ramp-up on a time scale that is an order of magnitude greater than the resistive decay time for the toroidal field. 


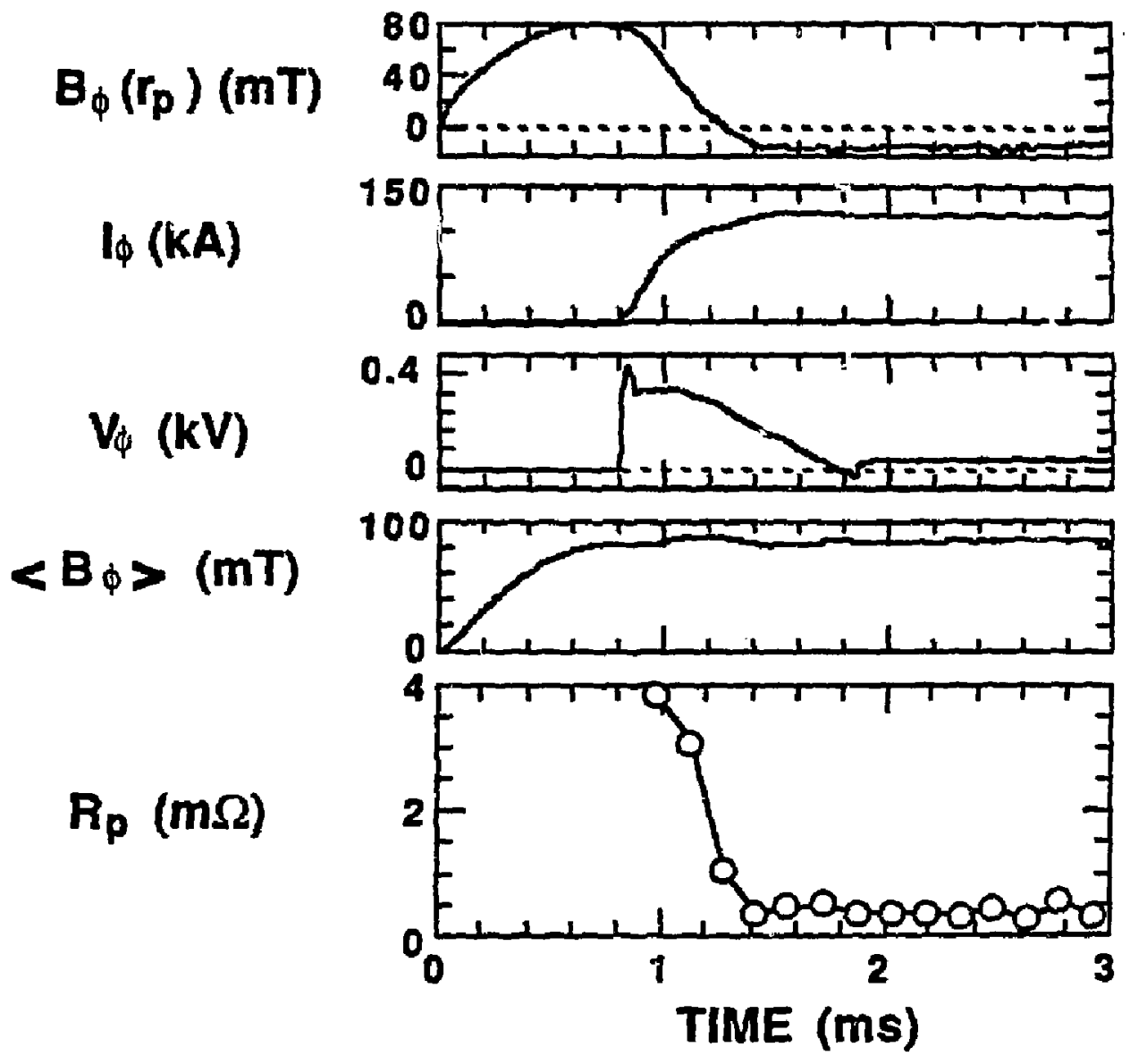

Figure 2.2-5. Typical "matched-mode" fornation and start-up of an RFP wherein the net transfer of toroidal flux across the conducting shell is zero. 

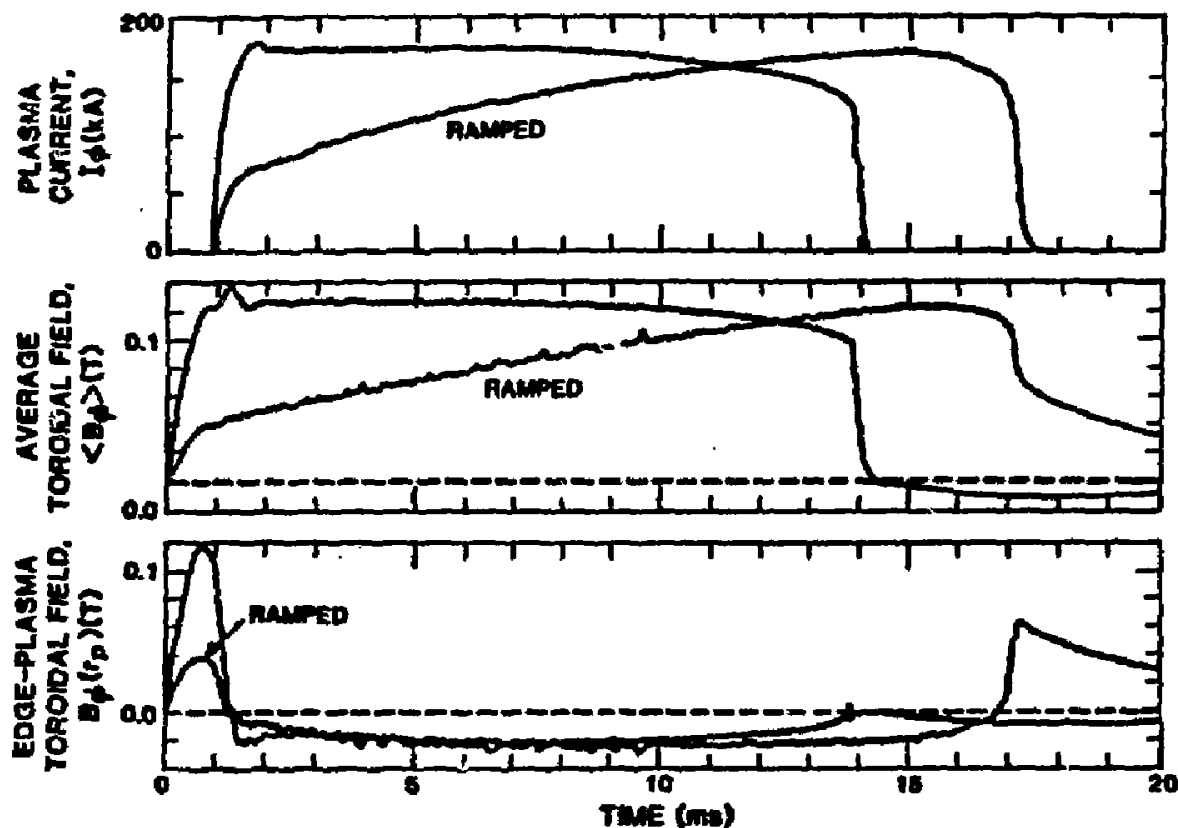

Figure 2.2-6. Dynamo-assisted current ramp-up wherein a majority of the toroidal flux, $\Phi=\pi r_{p}^{2}\left\langle B_{\phi}\right\rangle$, within the plasma is generated by fluid motion within the plasma, since $B_{\phi}\left(r_{p}\right)$ is fixed by the external circuit $[15,16]$.

The strong coupling of the poloidal and toroidal fields in RFPs also offers the possibility of a steady-state current-drive mechanism based on "magnetic helicity injection" [17] because the resistive decay of plasma currents can be viewed as a dissipation of magnetic helicity. Current drive through "electrostatic helicity injection" has been experimentally demonstrated in spheromaks [18], which are also relaxed-state systems like RPPs. Another heljcity injection technique is the oscillating-field current drive (OFCD) $[17,19]$. In this scheme, oscillating voltages are applied to the toroidal and poioidal circuits in the appropriate phase to drive a DC toroidal current in the plasma with the plasma, in effect, behaving as a nonlinear rectifier. The experiments on OFCD are not yet conclusive but are encouraging $[16,19-21\}$. Helicity injection as applied to TITAN is discussed in more detailed in Section 7, with Section 2.3.8 summarizing experimental results.

The general characteristics of a dynamo-sustained RFP, therefore, describe a system where $B_{\phi} \simeq B_{\theta}$ within the plasma, and fluctuation and transport are enhanced because 
of the dynamic sustainment of field and current profiles. Also, loop voltages and plasma resistance should be higher than in a comparable tokamak, and significant ohnic heating by the Iarger current densities in the low- $q$, shear-stabilized plasma is expected.

\subsubsection{Equilibrium}

As for any toroidal plasma, equilibrium in the RFP is described by the static idealMHD equations,

$$
\begin{aligned}
\nabla \boldsymbol{p} & =\mathbf{j} \times \mathbf{B}, \\
\nabla \times \mathbf{B} & =\mu_{0} \mathbf{j},
\end{aligned}
$$

where $p=2 k_{B}\left(T_{e}+T_{i}\right)$ is the plasma pressure, $k_{B}=1.602 \times 10^{-16} \mathrm{~J} / \mathrm{keV}$ is the Boltzmann constant, and $\mu_{0}=4 \pi \times 10^{-7} \mathrm{H} / \mathrm{m}$ is the permeability of free space. The analysis of equilibrium and stability in RFPs usually invokes the high-espect-ratio (straight cylinder) approximation. Such a model encompasses z-pinches $(q=0)$, $\theta$-pinches $(q \rightarrow \infty)$, large-aspect-ratio tokamaks $(q>1)$, and $\operatorname{RFPs}_{(}(q<1)$. The radial pressure balance in these systems is described by

$$
\frac{d}{d r}\left(p+\frac{B_{\theta}^{2}+B_{\phi}^{2}}{2 \mu_{o}}\right)+\frac{B_{\theta}^{2}}{\mu_{a} r}=0 .
$$

Information on the current and pressure profiles is required in order to find the equilibrium magnetic-field profiles. Because of the strong tendency for RFPs to relax towards a near-minimum-energy state, the field distributions obtained in modern experiments (Figure 2.2-3) are close to those given in Figure 2.2-1. The theory of relaxed states [11] prediets that $\mathbf{j}=\mu \mathrm{B}$, with $\mu \equiv \mu_{\circ} j_{\|} / B$ assumed to be spatially uniform across the plasma and leading to BFM field profiles (Figure 2.2-3). As noted previously, a constant $\mu$ profile implies a large parallel-current density in the outer region of cold, resistive plasma. A $\mu$ profile that is nearly constant over the bulk of the plasma and decreases in the outer region to match the practical condition that $j$ and $\mu\left(r_{p}\right) \simeq 0$ eliminates an unphysical feature of the BFM. Sample profiles used are as given by Equation 2.2-4 with $\alpha \simeq 6$ to 8 or a $\mu$ profile that is constant for $r<r_{r}$ (the radius of reversal surface) and decreases linearly to zero at $\tau_{p}$.

For any toroidal plasma system, including the RFP, equilibrium requires the compensation of the outward force from the plasma pressure and the plesma current (i.e., poloidal-field pressure). A perfectly conducting wall, a vertical field produced by the 
external circuits, or a combination of both is necessary to provide toroidal equilibrium. The required value of this vertical field is given by $[22,23]$

$$
B_{v}=\frac{\mu_{\theta} I_{\phi}}{4 \pi R_{T}}\left[\ln \left(\frac{8 R_{T}}{r_{p}}\right)+\beta_{\theta}+\frac{\ell_{i}}{2}-1.5\right],
$$

where

$$
\ell_{i}=\frac{1}{\pi r_{p}^{2} B_{\theta}^{2}\left(r_{p}\right)} \int_{0}^{\pi} \int_{0}^{r_{p}} B_{\theta}^{2} r d r d \theta
$$

is the normalized plasma internal inductance and $\beta_{\theta}$ is the poloidal beta $\beta_{\theta}=2 \mu_{\circ} p / B_{\partial}^{2}$. Typically, the vertical field required for equilibrium is small, and the ratio $B_{V} / B_{b}\left(r_{p}\right)$ is of the order of the inverse aspect ratio, $\epsilon=1 / A=r_{p} / R_{T}$.

A toroidal shift, $\delta$, develops between the center of a conducting shell of radius $r_{w}$ and the center of a circular plasma of radius $r_{p}$ in the process of compensating for the force acting on the plasme and achieving an equilibrium. A detailed anblysis $[22,23]$ gives the following expression for the equilibrium shift:

$$
\frac{\delta}{r_{p}}=\frac{\epsilon}{2 x^{2}}\left[-\ln (x)+\left(1-x^{2}\right)\left(\beta_{\theta}-\frac{\ell_{i}-1}{2}\right)\right],
$$

where $x \equiv r_{p} / r_{t w}$. Present-day RFP experiments use a conducting sheil to provide equilibrium (and stability), with vertical fields being applied, largely at insulating gaps provided in the shell, prior to or during the discharge. On longer time scales, these equilibriumproducing magnetic fields will diffuse into the shell, and externally produced vertical fields will be required to maintain a force equilibrium. Externally produced vertical fields will in fact be required for the next generation of RFP experiments [24], as are presently used on most modern tokamaks.

It follows from the ideal-MHD theory (Equation 2.2-5) that the current and the magnetic-field lines lie on constant-pressure surfaces. For axisymmetric, toroidal, currentcarrying plasma, the equilibrium, therefore, consists of toroidal flux surfaces that are nested about a magnetic axis. Each surface is generated by a large number of toroidal traverses of the helical magnetic-field lines. If the toroidal symmetry is violated (e.g., because of errors in the aligiment of magnet coils), the nested toroidal surfaces break up, resulting in a more complicated structure which includes helical magnetic islands [25]. The existence and interaction of these islands in the regions near the plasma edge impact plasma transport. Although both tokamaks and RFPs are susceptible to magneticisland formation, the number and location of the resonant flux surfaces, as indicated in 


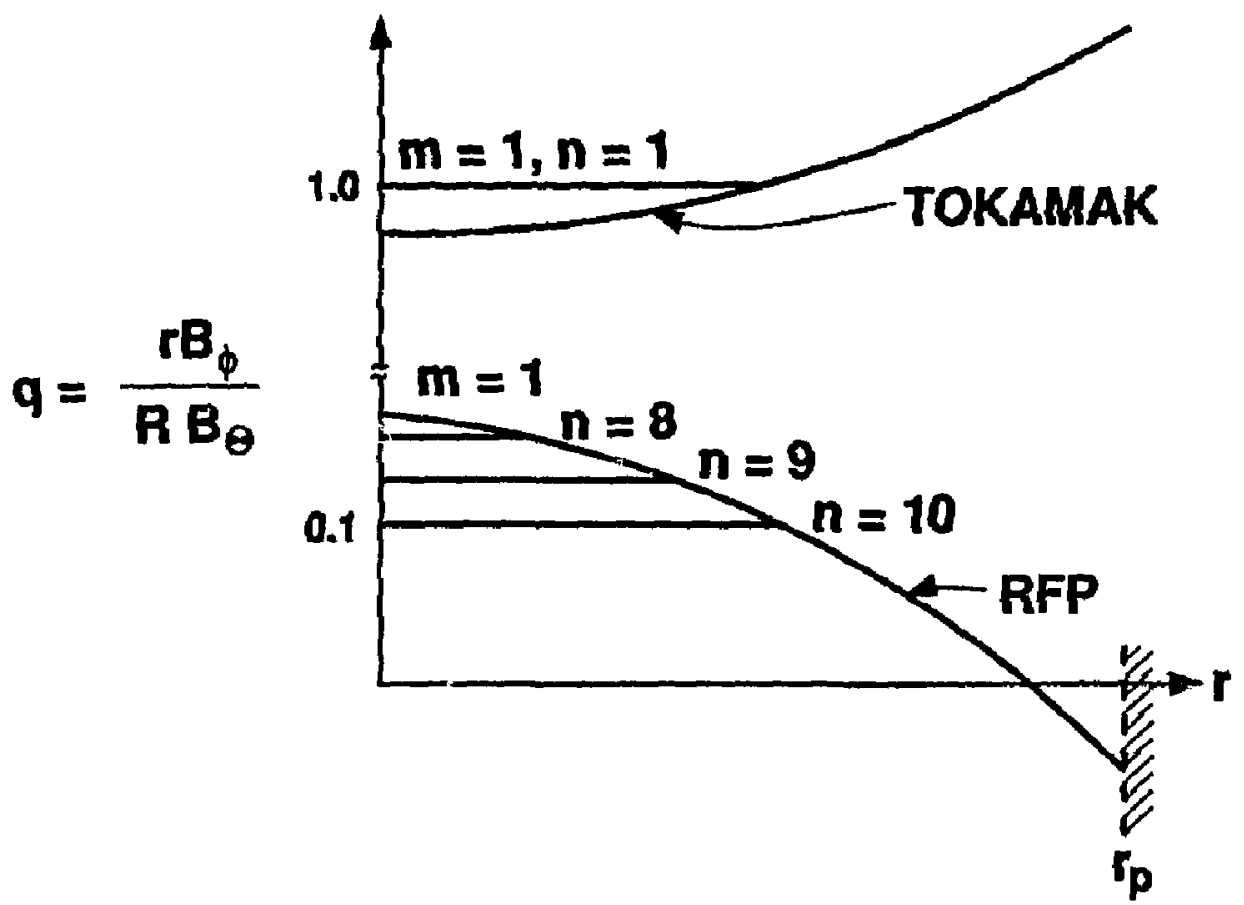

Figure 2.2-7. Comparison of magnetic-plasma configurations between RFPs and tokamaks, indicating the large number of toroidally resonant flux surfaces in the RFP compared with the tokamak.

Figure 2.2-7, are significantly different in the two concepts. Whereas the admission of a rational surface into the tokamak plasma nomlinearly leads to instability (disruptions), the inclusiou of a large number of helically interacting, rational surfaces in the RFP give rise to a large number of degrees of freedom for field-line reconnection and a mechanism (e.g., dynamo) for moving towards the Taylor minimum-energy state [11]. The large number of rational surfaces, however, makes field errors a greater concern in RFPs [24].

Since the plasma edge in RFPs plays an important role in plasma confinement and helicity dissipation [26-29], changes in this region through the equilibrium shift and /or field errors can be important in determining plasma performance. Figures $2.2-8$ and 2.2-9 illustrate, respectively, the reduction in loop voltage and the increase in energy confinement time as a result of better control of field errors and the equilibrium shift. 


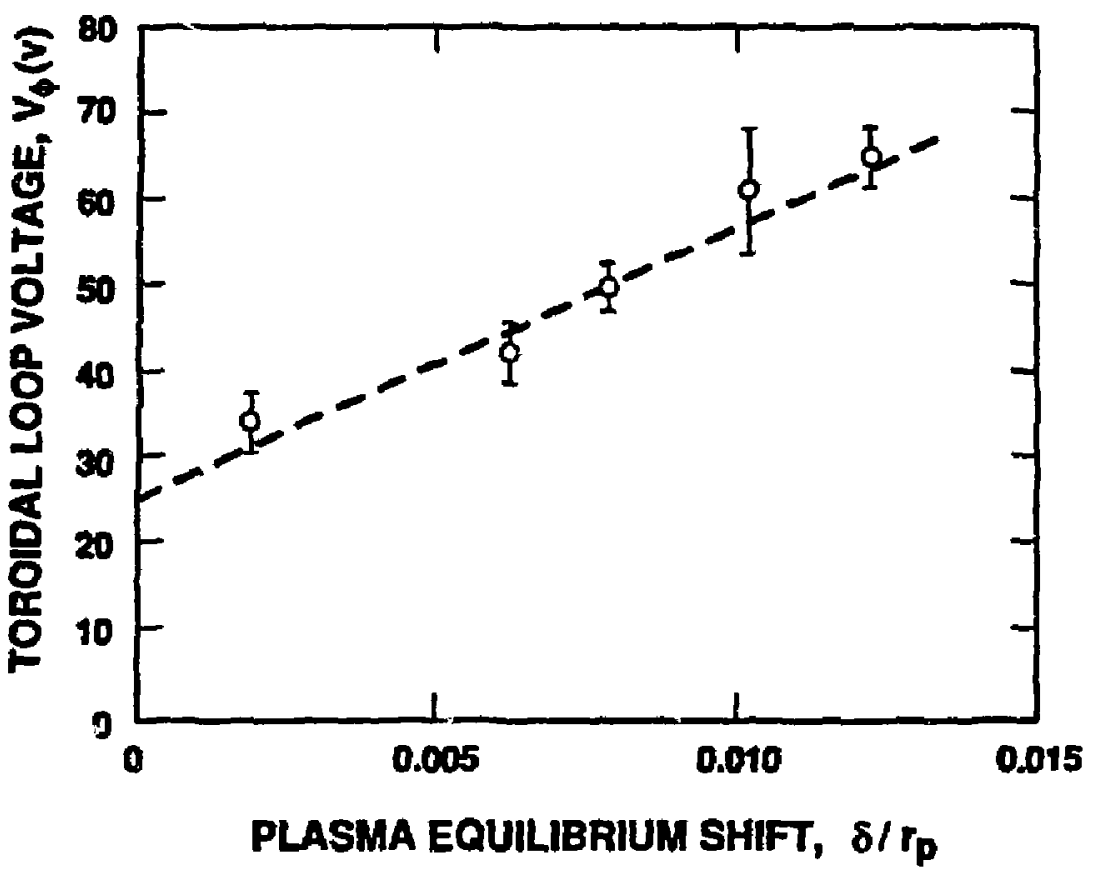

Figure 2.2-8. Impact of plasma equilibrium shift and wall contact on the toroidal loop voltage in the HBTX experiment. [27].

\subsubsection{Stability}

The equilibrium-pressure profile described by Equation 2.2-7 must be subjected to stability analysis using the energy principle [30] or normal-mode analysis. The Suydam criterion [31] gives the following necessary condition for stability against ideal-MHD pressure-driven modes for a straight cylinder:

$$
\frac{r}{4}\left(\frac{1}{q} \frac{d q}{d r}\right)^{2}+\frac{2 \mu_{o}}{B_{\phi}^{2}} \frac{d p}{d r}>0
$$

where $q(r)=(r / R)\left(B_{\phi} / B_{\theta}\right)$ is a relative measure of field-line pitch at minor-radial position $r$. This criterion simply states that the negative pressure gradient associated with the confinement of a hot plazma has a destabilizing effect and can be compensated for only by a sufficiently large radial variation in the field-line pitch $P(r)=\left(B_{\phi} / B_{\theta}\right) r=q R$ (i.e., large magnetic shears). 


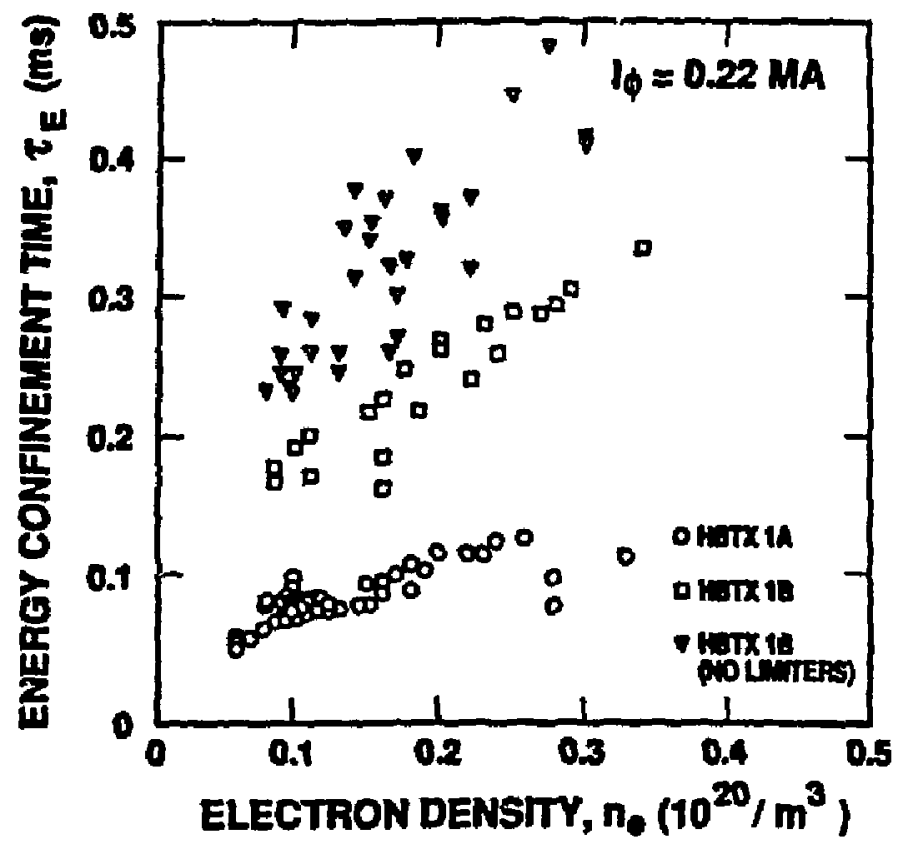

Figure 2.2-9. Impact of field errors and $D C$ vertical field on the energy confinement in the HBTX experiments [12]. Compared to HBTX1A, the field errors in HBTX1B have been reduced by a factor of $15[0.3 \%$ to $0.02 \%$, expressed as a percentage of error flux intersecting the wall relative to $B_{Q}\left(r_{\mathrm{p}}\right)$ l.

Inclusion of first-order toroidal effects inio the stability analysis leads to the Mercjer criterion [32]

$$
\frac{r}{4}\left(\frac{1}{q} \frac{d q}{d r}\right)^{2}+\frac{2 \mu_{a}}{B_{\phi}^{2}} \frac{d p}{d r}\left(1-q^{2}\right)>0 .
$$

The Mercier criterion indicates that if $q(r)>1$ everywhere, the system is ideally stable, even without the magnetic shear. This condition is the primary approach to stability used in the tokamak. Physically, the $q>1$ condition forces the wave length of the potentially unstable $m=1$ kink mode to exceed the major circumference of the torus, $2 \pi R_{T}$. Since $q<1$ in RFPs, sufficient shear must be present in order to satisfy the Mercier criterion. Moreover, a pitch minimum (i.e., $d q / d r=0$ ) within the plasma must be avoided.

Necessary conditions for stability against ideal-MHD current-driven modes have been derived by Robinson [33] on the basis of the energy principle [30]. The necessary condition 
cen be expressed as $\left|P\left(r_{w}\right)\right|<3 P(0)$, where $r_{w}$ is the location of the conducting wall. This condition can be approximately expressed as follows:

$$
\begin{aligned}
\left(\frac{r_{w}}{r_{p}}\right)^{2} & <3\left|\frac{B_{\phi}(0)}{B_{\phi}\left(r_{w}\right)}\right|, \\
\Phi & >0,
\end{aligned}
$$

where $\Phi$ is the total toroidal flux ins:de the conducting wall. These conditions do not include a vacuum boundary and require that both the amplitude and the region of the field reversal not be large.

In summary, the conducting wall should be close to the plasma to stabilize currentdriven modes. Furthermore, stability against current-driven modes also excludes a pitch minimum in the plasms. These conditions are usually well satisfied for experimental profiles and are also monitored and satisfied for the profiles calculated for the TITAN designs. The necessary conditions given by Equations 2.2-13 and 2.2-14 are in practice close to being sufficient. Based on ideal-MHD theory, RFP profiles are possible with plasma beta values as high as 0.3 . The Taylor theory predicts that all states described by the $F-\Theta$ diagran (Figure 2.2-2), including those with $F>0$, ure near-minimum-energy states and, therefore, are stable. Profiles without toroidal-field reversal, however, exhibit pressure-driven and current-driven modes. This conclusion is confirmed experimentally and an example is given in Figure 2.2-10 showing the plasma resistance in the H3TX1A experiment [34] as a function of the pinch parameter; as $\Theta$ increases, the resistance falls dramatically, particularly as $\theta$ exceeds the value where the field revernal occurs. For larger values of $\Theta$ and $|F|$, the plasma resistance increases again because of enhanced dynamo activity and the appearance of sawtooth-like oscillations in the plasma [35].

The ideal-MHD theory assumes a zero plasma resistivity. This assumption constrains magnetic-field lines to be "frozen" in the plasma, thereby limiting the class of potentially unstable modes. Resistive-MHD stability analysis has to be performed to provide a more realistic picture of the plasta behavior [33]. In general, the criteria for resistive stability are more stringent, and a closer fitting conducting wall and a lower value of $\beta$ generally result. A detailed analysis of current-driven resistive tearing modes has been made [36] and stable RFP configurations have been found with $\beta \simeq 0.2$. These configurations, however, have been found to be unstable to the so-called resistive g-modes (the " $\mathrm{g}$ " is used to emphasize the "gravity" analogy). Moreover, analyses show that resistive g-modes can become unstable for pressure gradients substantially smaller than those needed to drive ideal-MHD instabilities. These resistive g-modes are localized and may ultimately affect the confinement time. In fact, certain theoretical estimates of the confinement time 


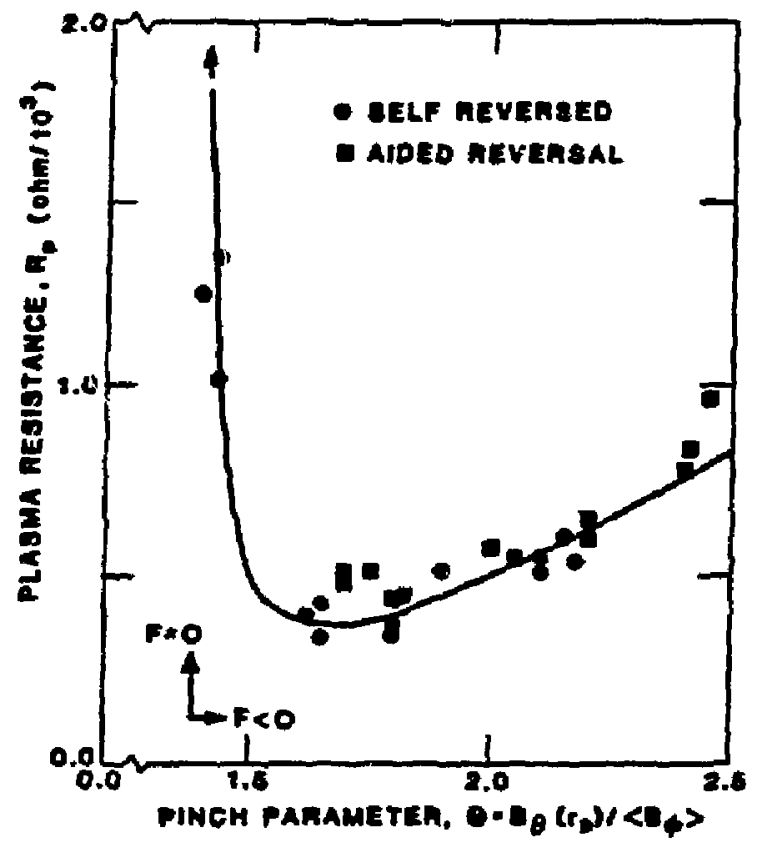

Figure 2.2-10. Plasma resistance in the HBTXIA experiment as a function of the pinch parameter, $\Theta[34]$.

have been proposed that are based on transport along stochastic field lines created by resistive g-nnode turbulence [37] and are discussed in Section 2.2.5.

The current-driven MHD instabilities have more recently been analyzed for an RFP in contact with a perfectly conducting wall [13], and even more recently for conditions where a vacuum annuius exists between the RFP and the conducting wall [38]. Figure 2.2-11 gives an example of a stability diagram $[13,38]$ for the fastest growing $m=1$ mode as a function of the $\mu$-profile shape factors, $\mu(0)$ and $\alpha$, as given in Equation 2.2-4. These analytic results show that a completely stable region exists for ideal and resistive currentdriven modes in an RFP over a range of $\Theta=\Theta_{0}$ and degrees of current-density peaking, and for a range of free-boundary conditions, $\delta_{v} \simeq\left(r_{w}-r_{p}\right) / r_{p}$. Generally, the external $m=1$ modes do not appear to be significant. These external modes occur for deep-reversal conditions that rarely arise for most experiments and are not of interest for reactors because of cost and technology demands (Section 8). A lower limit on $g(0) A=\theta_{0} \geq 2 / 3$ is identified which weakly depends on the profile exponent $\alpha$. This 


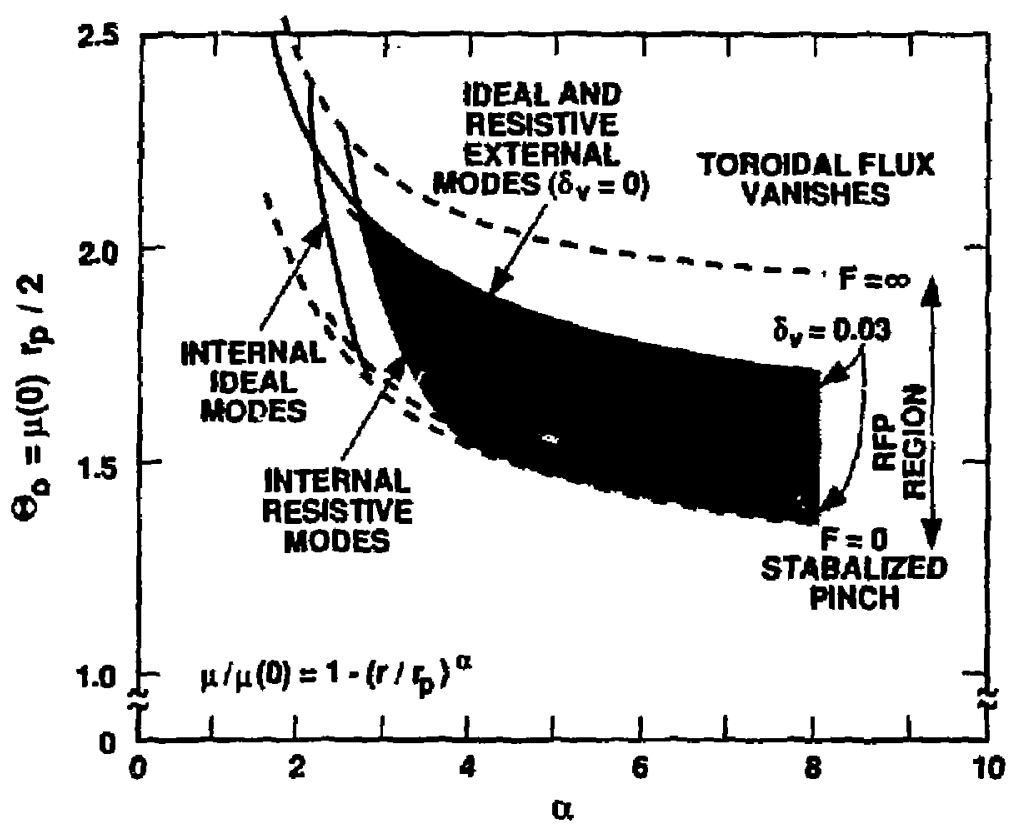

Figure 2.2-11. Stability diagram for $m=1$ internal (inside reversal layer) and external resistive current-driven modes for an RFP with a vacuum annulus equal to $\delta_{v}=0.0$ and 0.3 of the plasma radius $[13,38]$.

lower limit is associated with the internal $m=1$ resistive mode, and imposes an upper lizrit on the on-axis current density $[13,38]$ of $j_{\phi}(0) \leq 3 B_{\phi}(0) /\left(\mu_{o} r_{p}\right)$. As noted in Reference [13], resistive diffusion tends to peak the on-axis current, lowering $q(0)$ to below this critical limit and exciting internal current-driven instabilities, redistrifuting (i.e., fiattening) the current-density profile, and recovering the initial configuration that is stable to tearing modes. Hence, the field profiles in RFPs are a result of periodic relaxations that oppose processes related to resistive diffusion; such oscillations around near fields have been observed in a number of large- $\Theta$ RFP discharges $[39,40]$.

\subsubsection{Relaxation and Sustainment}

The RFP magnetic topology is maintained against diffusion by instability-driven luctuations and described by an evolving series of plasma-configurational relaxations. The theory of relaxed states has successfully predicted the behavior of RFPs in terms of the 
characteristics of the F- $\Theta$ diagram (Figure 2.2-2). The details of the process of relaxation to near-minimum-energy states are neither invoked nor required in this theory. These details are understood only in the broadest terms, but a complete description is ultimately required to explain and understand the sustainment, stability, and transport in RFPs.

Of particular interest is a more detailed account of RFP profile sustainment. Consider a cylindrically symmetric, resistive plasma with reversed-field profiles, and possibly with anisotropic resistivity. According to Ohm's law, a poloidal electric field, $E_{\theta}$, corresponding to the poloidal current at the reversal point should exist. To sustain this resistive electric field, Faraday's law requires a resistive decay of the toroidal flux inside the reversal radius. Reversed-field-pinch discharges, however, are maintained for times Far longer than the resistive diffusion time with $E_{\theta}=0$. A mechanism is necessary to drive the poloidal current at the plasma surface and cancel the resistive electric field at that point. This dynamo mechanism generates a magnetic field that opposes the resistive field diffusion.

Several mechanisms for toroidal-flux regeneration have been proposed, each offering a different explanation for the origin of the poloidal current at the toroidal-field reversal surface. For example, second-order effects of low-level MHD fluctuations resulting from nonlinear evolution of resistive-MHD modes can drive a poloidal current at the reversal surface that is sufficient to sustain the field reversal [41]. Alternatively, a plasma model with stochastic field lines has been proposed, wherein a global rather than a local Ohm's law applies. In this mode, the poloidal current at the reversel surface is driven by electromagnetic fields originating elsewhere in the plasma $[26,42-44]$.

In summary, quasi-stationary RFP equilibria are sustained through continuous relaxation and field generation. These time-averaged equilibria are stable to ideal and resistive-tearing modes because the relaxation process acts to maintain the stability. Relaxation and field-generation processes are driven continuously by a complex spectrum of resistive MHD modes and related nonlinear interactions [44], These processes also involve field-line reconnection and profile modification that can impact the cross-field transport. The plasma confinement and beta, therefore, are intimately related to and affected by the relaxation process [37]. A detailed explanation of the mechanisms that drive the relaxation and dynamo activities in RFPs, however, has yet to be devised. Lastly, resistive g-modes or pressure-driven interchange modes are always unstable for RFP field profiles. These modes, however, are highly localized, should contribute to transport, and in principle should become less a factor in hotter, reactor-grade plasmas with high magnetic Reynolds numbers $\left(S \equiv \tau_{n} / \tau_{A}\right.$, where $\tau_{n}$ is the ohmic time and $\tau_{A}$ is the Alfven time). 


\subsubsection{Transport and Confinement}

Theoretical models for the transport in RFPs based on micro-turbulence have been proposed [45]. A detailed transport model is not yet available for RFPs, however, and the precise scaling of the energy confinement is unknown. Empirical approaches can be used to evaluate present experimental results from ohmically heated RFPs and used to form a basis for the extrapolation of these results to reactor regimes. The details of the transport physics, therefore, are not considered here but instead, experimental observations are used to guide the formulation of a scaling theory. Pressure balance in the RFP (i.e., $p \propto \beta_{\theta} I_{\phi}^{2} / r_{p}^{2}$ ) can be expressed as follows:

$$
T=\frac{\mu_{0}}{16 \pi k_{B}}\left(\frac{I_{\phi}}{N}\right) \beta_{\theta} I_{\phi}
$$

where $\mathrm{N}=n \pi r_{p}^{2}$ is the plasma line density and $T$ is the density-weighted plasma temperature. Firstly, Equation 2.2-15 predicts that $T / I_{\phi} \propto I_{\phi} / N \simeq$ constant; this behavior is observed experimentally. Secondly, the plasme pressure under certain conditions is found to scale approximately as $I_{\phi}^{1.7} \rightarrow I_{\phi}^{2}$ over a wide range of conditions, indicating an approximately constant $\beta_{\theta}$. In fact, experimental evidence exists that RFPs operate near a beta limit; plasma energy transport and loss mechanisms in RFPs appear to self-adjust to lose energy at a rate that is sufficient to maintain $\beta_{\theta}$ nominally constant (Sections 2.3 .5 and 2.3.6). Equation 2.2-15 then suggests that the temperature varies linearly with the current for a fixed value of $I_{\phi} / N$, as has been reported for number of experiments.

The constant-beta assumption, however, remains an open question, since a strict linear relation between $T$ and $I_{\phi}$ is not observed under all RFP experimental conditions. Specifically, evidence is emerging from a number of RFP devices that the average plasma pressure may be scaling with current raised to a power less than two, indicating that under certain conditions $\beta_{\theta}$ may be decreasing somewhat with plasma curre.nt. Interpretation of scaling results from present devices is difficult because of less-than-ideal density control, impurity effects, $Z_{e f}$, and operation close to or within the electron runaway condition, $\xi=v_{D} / v_{T H e} \propto\left(I_{\phi} / N\right) T_{e}^{-1 / 2} \geq 0.1$.

Using the definition of energy confinement derived from the energy balance for a steady-state, ohmically heated discharge, and substituting from Equation 2.2-15 for $T$, the following expression for $\tau_{s}$ results:

$$
\tau_{E} \equiv \frac{3 n k_{B} T}{\eta j_{\phi}^{2}}=\frac{3 \mu_{0}}{16} \frac{\beta_{\theta} r_{P}^{2}}{\eta},
$$


where $j_{\phi} \equiv I_{\phi} /\left(\pi r_{p}^{2}\right)$ is the plasma current density and $\eta$ is the plasma electrical resistivity evaluated at the density-weighted average plasma temperature. The plasma resistance measured on a number of RFP experiments over wide range of parameters is observed to have classical temperature dependence, $\eta \propto T_{e}^{-3 / 2}$. Using the assumptions of constan: $\beta_{\theta}$ and linear defendence of $T$ on $I_{\phi}$, the energy confineinent time is found to scale with the plasma current according to $\tau_{s} \propto I_{\phi}^{3 / 2} r_{p}^{2}$.

Geometrical effects (i.e., spatial dependence of field-line pitch), impurities, and ihe anomalous resistance associated with the electric fields needed to drive the RFP dynamo must be considered in calculating the plasma resistance. The dynamo represents an added dissipation of the currents and, therefore, appears as a resistivity anomaly. The resistivity in RFP experiments over a wide range of conditions is close to the classical values, taking into account the geometrical and impurity effects. The dissipation associated with the dynamo effect, therefore, is generally small, and the ohmic power delivered to the plasma under optimal conditions as not expected to exceed the classical prediction by a significant amount. At low plasma densities (or high values of $I_{\phi} / N$ or streaming parameter, $\xi$ ) the resistance-anomaly factor is too high to be explained solely by geometrical or impurity effects.

Theories have been proposed to estimate the energy corfinement time associated with the electron parallel-field transport along stochastic magnetic-field lines caused by resistive-fluid turbulence. Turbulence caused by pressure-driven resistive-interchange modes has been considered [37] and gives a diffusion coefficient that scales as follows:

$$
D \propto \eta\left(\frac{M}{m}\right)^{1 / 2} \beta_{\theta}^{2},
$$

where $M$ and $m$ are ion and electron masses, respectively. Using this diffusion coefficient for ohmically heated discharges, the value of beta is found to be $\beta_{t} \equiv(\mathrm{m} / M)^{1 / 6}$ and is independent of machine parameters: the exponent of $1 / 6$ is a result of assuming a radial temperat ure profile that scales as $T \propto J_{0}(\mu r)$. This prediction combized with the pressure balance gives a linear temperatur-: current scaling, as reported in a number of experiments (Section 2.3.5).

Turbulence associated with resistive-tearing modes has been considered [44] and gives the following scaling:

$$
\begin{aligned}
\frac{1}{\beta_{\theta}} & \propto\left(\frac{I_{\phi}}{N}\right)^{1 / 3} I_{\phi}^{1 / 3} r_{p}^{1 / \theta} \\
T & \propto I_{\phi}^{2 / 3} T_{p}^{-1 / \theta} .
\end{aligned}
$$


Another theory based on current-driven drift-wave turbulence [46] gives the following scaling relationships:

$$
\begin{aligned}
\frac{1}{\beta_{\theta}} & \propto\left(\frac{I_{\phi}}{N}\right) I_{\phi}^{1 / 7} r_{p}^{-2 / 7}, \\
T & \propto I_{\phi}^{\theta / 7} r_{p}^{-2 / 7}, \\
r_{E} & \propto\left(\frac{I_{\phi}}{N}\right)^{-1} I_{\phi}^{8 / 7} r_{p}^{\theta / \tau} .
\end{aligned}
$$

This theory predicts a weak dependence of plasma beta on machine parameters and a nearly linear temperature-current dependence. A clear resolution of these various theoretical predictions must awajt experiments with a broader range of plasma and machine parameters (e.g., plasma current, current density, dimensions) and diagnostics (profiles, $\left.Z_{e f f}\right)$ under a level of density control that is sufficient to eliminate considerations and possible secondary effects of runaway electrons.

\subsection{RFP EYPERIMENTS}

An important achievement for the RFPs was the discovery in 1965 of a period of improved stability and reduced turbulence on the ZETA device [47]. The quiescent period observed in ZETA was preceded by a twibulent phase with large energy losses and strong plasma-wali interactions. Furthermore, self-reversal of the external toroidal field relative to the on-axis field was observed, but the importance of these observations was not fully appreciated at the time. To reduce energy losses and plasma-wall interactions, experimental RFPs during the 1970s used fast magnetic-field programing with typical rise-times of a few microseconds to force the reversal externally. These experiments required electrically insulated discharge tubes to accommodate the high voltages needed to generate fast-rising magnetic fields. Many important advances in RFP physics were made in these machines, although the reactor relevance of these high-voltage, pulsed devices was minimal.

With experience from fast-programing machines and a general theory of relaxed states [11], modern RFP experiments in the late 1970s and 1980s have returned to slowrising plasma current (0.1 to $1.0 \mathrm{~ms}$ ) and the facility for slow $B_{\phi}$ control to assist and optimize the self-reversal process and to minimize RFP formation losses. These machines use a metallic liner around thr: plasma, are equipped with improved vacuum systems, and operate with improved magnetic field geometry. The first of these modern machines 
was ETA-BETA-II at Padova [48-50], which began operation in 1979. Today, hightemperature plasmas are routinely produced in many intermediate-size machines, such as TPE-1R(M) at ETL, Sakura-Mura [51,52], ZT-40M at Los Alamos National Laboratory $[15,53,54]$, HBTX $1 A$ at Culham [55,56], and OHTE/RFP at General Atomics [57,58]. General parameters of these more recent RFP experiments are listed in Table 2.3-I. The design parameters of the TITAN reactor are also listed for comparison and Figure 2.3-1 gives a size comparison between existing, planned [24], and conceptual [59] RFP designs.

The plasma parameters obtained in these experiments have been improving steadily. Reactor-relevant values of $\beta$, in the range 0.1 to 0.2 are routinely achieved (total beta in RFPs is typically $50 \%$ of $\beta_{0}$ ). Electron temperatures in the range 0.4 to $0.6 \mathrm{keV}$, densities exceeding $10^{20} \mathrm{~m}^{-3}$, and energy confinement times approaching $1 \mathrm{~ms}$ are typical of these intermediate-size experiments. In addition, ion temperatures approaching $1 \mathrm{keV}$ heve been achieved under conditions where snomalous ion heating is observed (i.e., high values of $I_{\phi}(N)$. Data from a number of machines indicate a nearly linear temperaturecurrent scaling under some conditions, which suggests $T_{g} \propto T_{e}^{s / 2}$. Furthermore, both

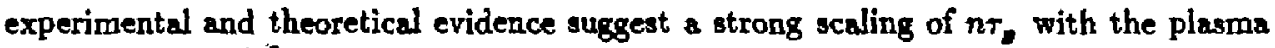
current $\left(n \tau_{n} \propto I_{\phi}^{5,2}\right)$. The scaling of plasma pressure with current suggests $p \propto I_{\phi}$, where $\nu \leq 2.0$; the equality indicates a constant- $\beta_{\theta}$ scaling, although evidence exists for a slow decrease in $\beta_{\theta}$ with plasma current.

This section presents the experimental dat from the key RFP experiments listed in Table 2.3-I with special emphasis on extrapolating the present data base to a reactorrelevant regime. The main scaling parameter is plasma current, $I_{\phi}$, with the larger RFPs ranging almost a factor of 10 in this variable, while the smaller devices vary $I_{\phi}$ by only a factor of 2 to 3. As concluded at a recent RFP workshop [60], the overall picture on RFP scaling remains somewhat uncertain, and prediction of performance "... is sukject to large errors." Specifically, on the basis of more than 4,000 ZT-40M discharges it was concluded [60] that the available information is "insufficient ... to formulate statistically defensible scaling laws, and that it may be more sensible to compare the data to predictions" at this point in the development of the RFP data base.

Reference [60] summarizes concisely the difficulties at this time in deriving statistically meaningful scaling laws for the present RFP data base; these difficulties are multifold and interconnected, and it is appropriate to list them here as follows:

- Each RFP device is improving performance with time (e.g., reduced field errors, better Bv control, etc.) and, therefore, remains in an optimization process; parameters, consequently, are changing and evolving. 
Table 2.3-I.

PARAMETERS OF MAJOR RFP DEVICES

\begin{tabular}{|c|c|c|c|c|c|c|c|}
\hline Deyice & $\begin{array}{c}\text { Major } \\
\text { Radius } \\
(\mathrm{m})\end{array}$ & $\begin{array}{c}\text { Minor } \\
\text { Radius } \\
\text { (m) }\end{array}$ & $\begin{array}{c}\text { Plasma } \\
\text { Current } \\
\text { (MA) }\end{array}$ & $\begin{array}{l}\text { Current } \\
\text { Density } \\
\left(\mathrm{MA} / \mathrm{m}^{2}\right)\end{array}$ & $\begin{array}{c}\text { Electron } \\
\text { Temperature } \\
\text { (keV) }\end{array}$ & $\begin{array}{c}\text { Average } \\
\text { Density } \\
\left(10^{20} \mathrm{~m}^{-3}\right)\end{array}$ & $\begin{array}{c}\text { Poloidal } \\
\text { Beta }\end{array}$ \\
\hline TPE-1RM ${ }^{(a)}$ & 0.50 & 0.09 & 0.13 & 5.1 & 0.60 & 0.3 & 0.1 \\
\hline ETA-BETA-II (b) & 0.65 & 0.125 & 0.15 & $\mathbf{3 . 0}$ & 0.08 & 1.0 & 0.1 \\
\hline $\operatorname{HBTX1A^{(c)}}$ & 0.80 & 0.26 & 0,32 & 1.5 & 0.10 & 0.2 & 0.05 \\
\hline OHTE/RFP(d) & 1.24 & 0,20 & 0.50 & 4.5 & $0.4-0.6$ & $0.5-3.0$ & $0.1 \cdot 0.2$ \\
\hline $\mathrm{ZT}-40 \mathrm{M}^{(e)}$ & 1.14 & 0.20 & 0.44 & 3.5 & $0.3-0.5$ & $0.4-0.9$ & $0.1-0.2$ \\
\hline $\mathrm{FFX}^{(f)}$ & 2.00 & 0.48 & 2.0 & 2.8 & $0.5-2.0$ & $0.3 \cdot 2.0$ & 0.10 \\
\hline CPRF/ZTH(v) & 2,40 & 0.40 & 4.0 & 8.0 & $0.5-5.0$ & $0.3-5.0$ & 0.10 \\
\hline FTF $/ \mathbf{R F P}^{(h)}$ & 1.80 & 0.30 & 10.4 & 37. & 10. - 20 . & $6.0-9.0$ & $0.1-0.2$ \\
\hline $\operatorname{TITAN}^{(i)}$ & 3.81 & 0.60 & 18.2 & 16. & 10. - 20. & 9.0 & 0.2 \\
\hline
\end{tabular}

(a) Existing experiment at ETL, Japan $[51,52]$.

(b) Existing experiment at Padova, Italy [48 - 50].

(c) Existing experiment at Culham, U. K. [55,56].

(d) Existing experiment at General Atomics, U. S. A. [57,58].

(e) Existing experiment at Los Alamos National Laboratory, U. S. A. [53,54].

(f) Planned experiment at Padova, Italy [24].

(g) Planned experiment at Los Alamos National Laboratory, U. S. A. [24].

(h) Conceptual neutron source, a Los Alamos National Laboratory study, U. S. A. [59].

(i) Conceptual reactor desjgn, a UCLA-led multi-institutional study, U. S A. 


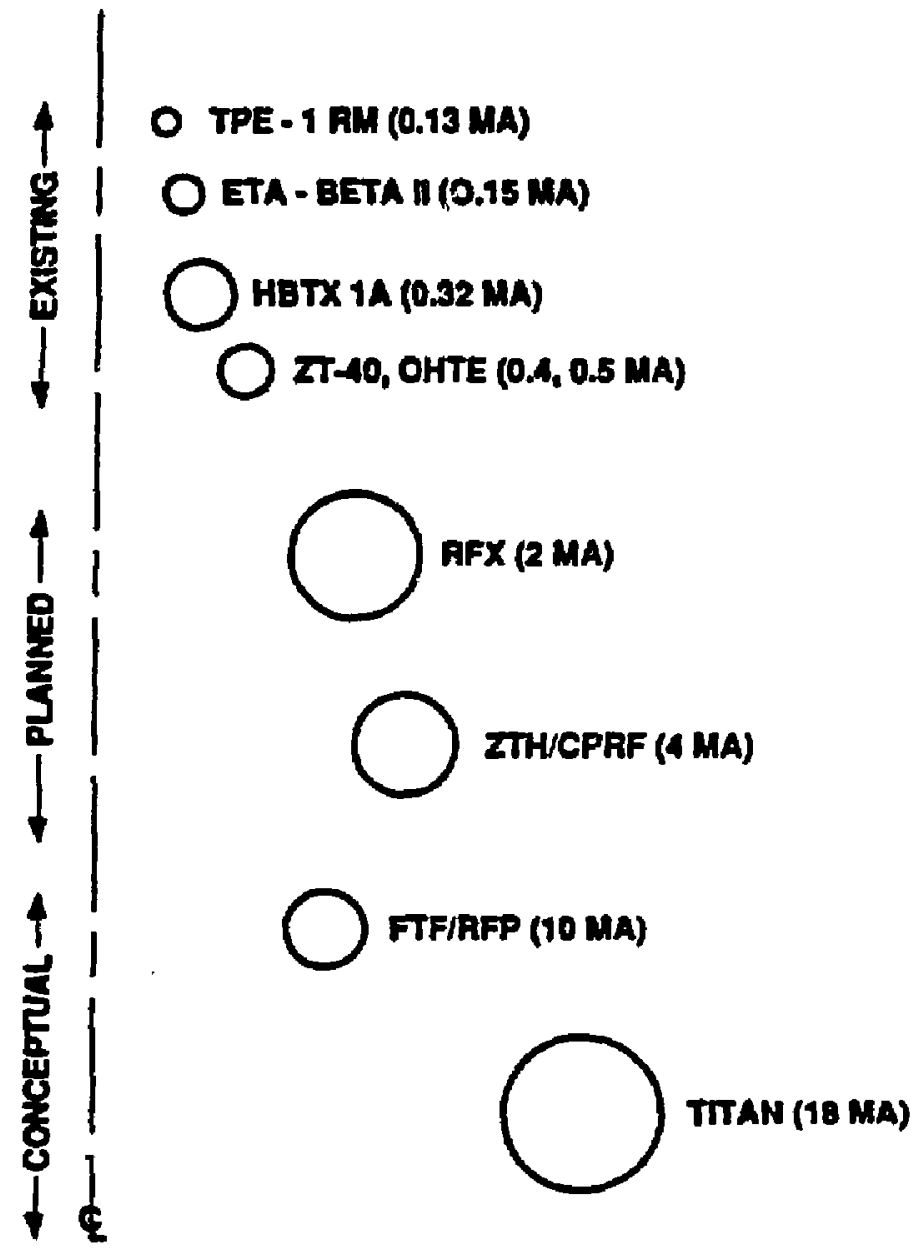

Figure 2.3-1. Comparison of plasma cross sections for present, planned, and conceptual RFP devices of Table 2.3-1. 
- Diagnostics generally could be improved in all RFPs (e.g., $Z_{\text {eff }}$, profiles, edgeplasma parameters, ion temperatures).

- Under some conditions, certain parameters presumed to be "independent" can be strongly correlated (e.g., $I_{\phi} / N$ or $\beta_{\theta}$ dependence on $I_{\phi}$ ).

- Most data bases are not sufficient in size or extent to permit statistically meaningful correlations to be made at this time.

- In addition to a constant improvement in machine parameters with time, sometimes within a given period machine performance is not reproducible, indicating unknown and/or uncontrollable parameter variations.

- Size scaling is highly limited for the existing RFPs with strong difference in field errors, diagnostics, construction, and other factors. It is, therefore, difficult to predict whether key confunement parameters are scaling with $I_{\phi}, I_{\phi}^{2} / N, j_{\phi}$, or combinations thereof.

Many of these uncertainties will dissolve with the advent of the next generation of highercurrent RFP experiments. The scaling of machine performance with $I_{\phi}$ and/or $I_{\phi} / N$ given in the following subsections, therefore, should be viewed as a comparison of sample data with the predictions of simplified theory, rather than the presentation of a fully corroborated RFP data base.

This section summarizes the experimental data base as applied to the physics assumed or extrapolated to describe the TITAN reactor. Emphasis is placed on the areas of atartup and formation, confinement, and transport. The status and experience in the areas of density control, highly radiating RFPs, current terminations, and RFP operation with resistive shells are also reviewed.

\subsubsection{Start-Up and Formation}

The time history of a typical RFP experimental discharge can be divided into three phases: (1) formation phase, (2) sustainment phase, and (3) termination phase. A representative time history of a RFP discharge is shown in Figures 2.2-5 and 2.2-6. The formation phase denotes the time from the start to the peak of the toroidal plasme current. The sequence of events during the formation phase begins by establishing a toroidal magnetic field inside the discharge chamber in the absence of the plasma. At the time of peak toroidal magnetic field, poloidal-field windings are activated to produce a flux 
change through the center of the tortus and, consequently, toroidal voltage around the discharge chamber. This voltage typically ionizes the gas in a few microseconds and the toroidal current is initiated in the resulting plasma. The tocoidal plasma current and the toroidal magnetic field within the plasma increase while the toroidal magnetic field at the wall decreases, keeping the average toroidal field (and the toroidal flux) in the chamber almost constant. Eventually the toroidal magnetic field at the wall changes sign and is crowbared in the reversed direction relative to the back-biased condition of the formation phase.

The duration of the RFP discharge is extended by using either a passive crowbar applied to the poloidal circuits, to give a decaying weveform, or an active (power) crowbar to produce a flat-top current waveform, as is illustrated in Figure 2.3-2 (upper trace). Figure 2.3-2 also gives the time dependence of plasma density and a soft-X-ray signal, which is messure of plasma temperature. It should be noted that the un-fueled RFP showa a density decrease or "pump-out," as does the tokamak. This pump-out can and must be controlled by wall conditioning in the short term and by gas-puffing, pellet injection, or both in the longer term.

Reversed-field-pinch discharges normally end abruptly and the plasma current decreases rapidly to zero. Accompanying this fast current "termination" is a positive pulse in the toroidal voltage at the liner, as is also shown on Figure 2.3-2. This current termination is in contrast to the negative spike in toroidal voltage that accompanies the current disruption in a tolamak, indicating a difference in the flow of magnetic energy to or from the plasme during the respective events. Furthermore, the RFP current termination can be influenced through the control of the density or toroidal magnetic (reversed) field [61], and in this sense also differs from a tokamak disruption; generally, the RFP terminates only when toroidal-field reversal is lost.

Three modes of operation are generally used for the RFP formation phase: selfreversal, matched, and aided-reversal. In the self-reversal mode, a conducting shell maintains and conserves the toroidal flux inside the chamber. The self-reversal mode of RFP formation is used on OHTE/RFP and often on HBTXIA. In the matched mode, the external circuits are programmed to conserve the toroidal flux inside the chamber by maintaining the poloidal electric field, $E_{A}$, near zero at the liner, thereby, simulating the action of a conducting shell. This mode is usually used on ZT-40M. In the aided-reversal mode, the external circuits supplement the plasma self-reversal effect, as typically used on ETA-BETA-II. Field control during the formation phase provides flexibility in varying the pinch parameter, $\Theta$, on which the configuration depends. The choice of the formation mode also affects the consumption of the poloidal flux during this phase of the RFP 

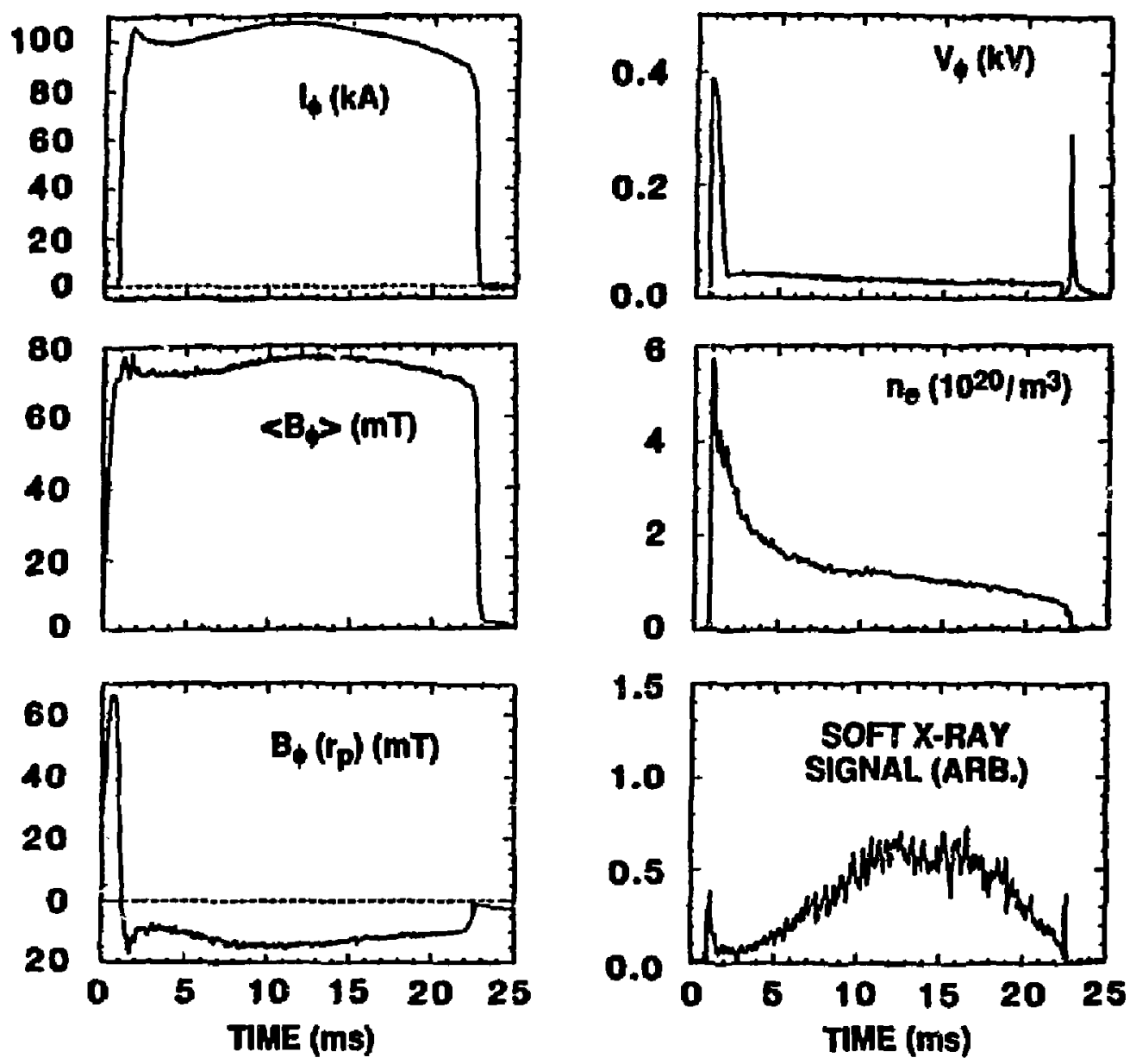

rigure 2.3-2. Typical waveforms for the toroidal current, $I_{\phi}$, average toroidal field, $\left\langle B_{\phi}\right\rangle$, toroidal field at the plasma edge, $B_{\phi}\left(r_{p}\right)$, loop voltage, $V_{\phi}$, plasma density, $n_{e}$, and soft-X-ray signals (measure of plasma temperature). 
discharge (Figure 2.3-3). The final plasma parameters, however, are not particuinily sensitive to the mode used for RFP formation.

The decrease in plasme resistance (Figure 2.2-10) or loop voltage (Figure 2.3-3) is typical of the attainment of the near-minimum-energy, reduced-turbulence RFP state. For larger values of $\Theta$ and deeper reversal, $V_{\phi}$ increases (Figure 2.3-3) because of the increased RFP-dynamo activity needed to maintain the larger toroidal fields within the plasma and the associated increase in the fluctuations and sawtooth-like oscillations. Start-up and formation modes, wherein the RFP is programmed to remain at the value of $\Theta$ where $V_{\phi}$ is minimum (minimum flux consumption), are desirable. This start-up mode has recently been achievea [62]. The ramp-scaling results shown in Figure 2.3-3, however, give a constant voltage, $V_{\phi} \simeq 32.5 \mathrm{~V}$, indicating that the plasma resistance, $R_{p}$, is decreasing only linearly with current, rather than as $R_{p} \propto T^{-3 / 2} \propto I_{\phi}^{-3 / 2}$.

Similar work on optimizing RFP formation and ramp-up has also been reported for HBTX1B [63], which now uses a matched-mode operation and is attempting to achieve constant- $\Theta$ operation. Figure 2.3-4 shows the value of planma resistance as anction of the current for different start-up methods. The plasma resistance values are inferred from $R_{p} J_{\phi}=V_{\phi}^{*}+\delta V_{\phi}$, wherein $V_{\phi}^{*}$ is associated with collisional conductivity and $\delta V_{\phi}$ describes an anomaly, possibly caused by field lines intersecting walls [26,64]. In HBTX1B, $\delta V_{\Phi} \sim 45$ to $55 \mathrm{~V}$, this voltage can be reduced by reducing field errors [63].

Another mode of RFP start-up has been demonstrated on the ZT-40M experiment. This mode is called ramped start-up because the plasma current is slowly ramped to a final value after an initial low-current RFP is formed. Figure 2.2-6(C) shows such a. ramped start-up. In a conventional start-up sequence, the peak current is nearly reached at the time the toroidal field at the wall reverses. This start-up mode is undesirable in a large experiment or a reactor because: (1) the RFP formation phase is power intensive until reversal is reached, and (2) large voltages are required. In a ramped start-up, however, the RFP configuration is set up in a relatively short time at a low current and low stored energy; the current is then slowly increased to the desired value while maintaining the RFP profiles. Ramped start-up operation has also recently been reported for the HBTXIB experiment [63].

The ramped start-up scenario relies on the plasma relaxation process. During the current ramp, the toroidal flux must be increased proportionally to the current to maintain the RFP profiles while holding $F$ and $\Theta$ constant. This process requires the generation of toroidal flux by the RFP dynamo, since the toroidal field at the wall is negative while the average toroidal flux within the conducting shell is positive. The plasma must generate an equal and opposite amount of negative and positive flux to satisfy Faraday's 

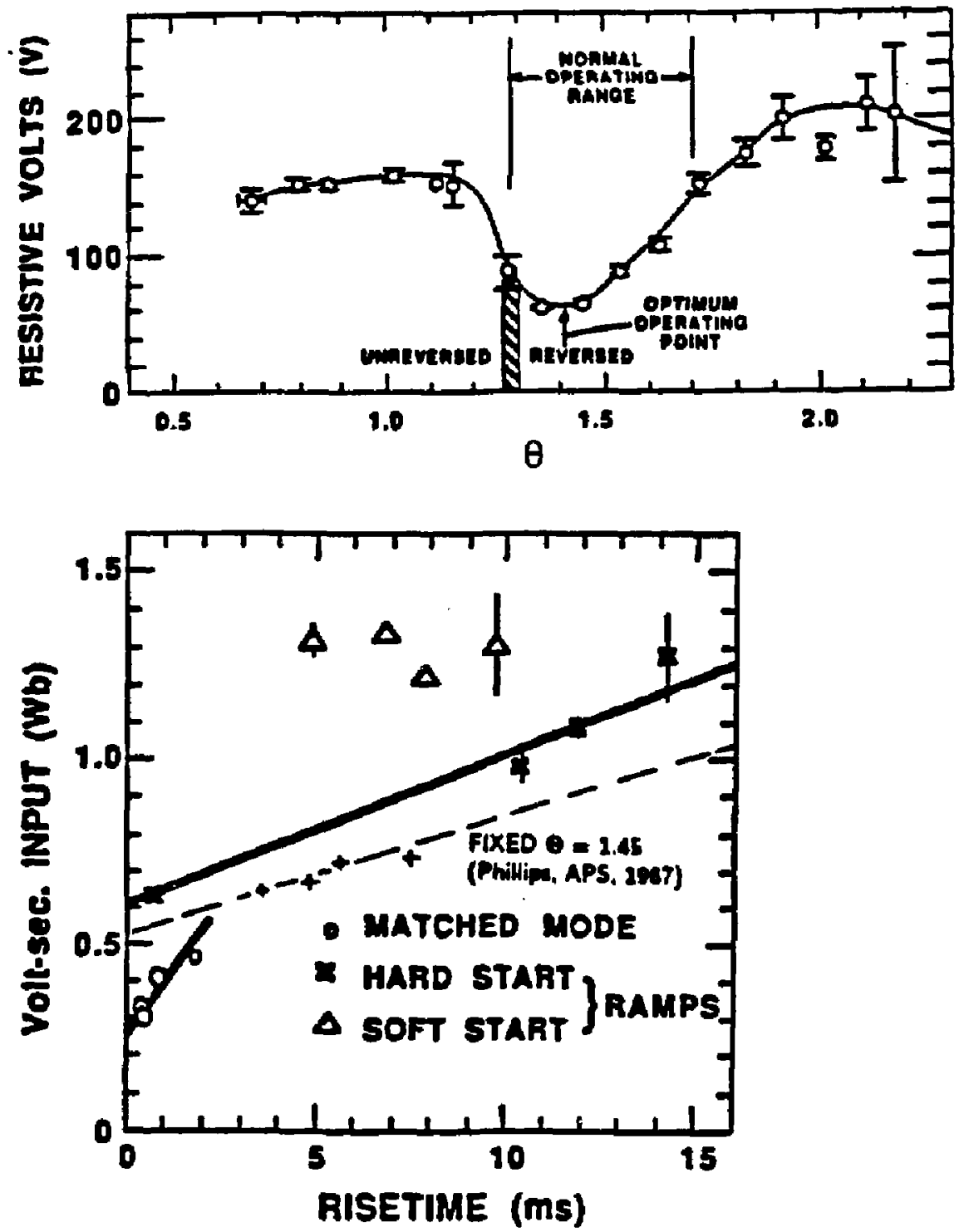

Figure 2.3-3. Dependence of loop voltage on pincli parameter, $\Theta$, and flux consumption on current rise-time for ZT-40 $[14,62]$. 


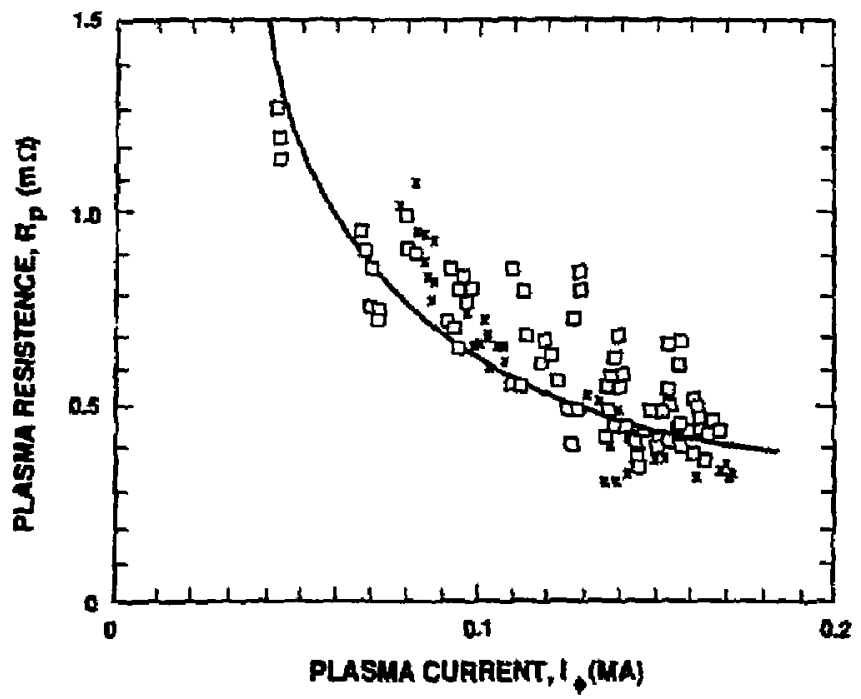

Figure 2.3-4. Plasma resistance as a function of the current in HBTX1B [63] for ramped (squares) and flat-top $(x)$ discharges.

law and then expel the negative flux from the plasma to generate a net positive flux increase. Indeed, the ramped discharges show that toroidal flux (Figure 2.2-6) continues to be generated, and negative flux is expelled from the plasma; this process occurs on a multi-millisecond time scale.

The TITAN reactor design relies on a rapid formation of a "seed" RFP followed by a slow plasma current ramp to the final value (Section 6). Special attention is given to the RFP formation phase. Experimental results point to an RFP formation "window" in the parameter space constrained by the volt-second requirement (i.e, poloidal-flux consumption), equilibrium and field-error constraints, plasma density, current-density, etc. For example [65], the dependence of RFP formation on density and initial bias field is shown in Figure 2.3-5 for ZT-40M and is extrapolated to define a formation window for the reactor. Other constraints imposed on the RFP formation include the relationsilip between the initial bias toroidal field $\left(B_{\infty \infty}\right)$ and the average toroidal field $\left(\left\langle B_{\phi}\right)\right)$ and the value of $I_{\phi} / N$ upon formation. Figure 2.3-6 gives the relationship between $B_{\phi \infty}$ and $\left\langle B_{\phi}\right\rangle$ derjved from $\mathrm{ZT}-40 \mathrm{M}$ [65]. The dependence of the radiation burn-through and reduced loop voltage on $I_{\phi} / N$ for the ETA-BETA-II experinent is shown in Figure 2.3-7. 

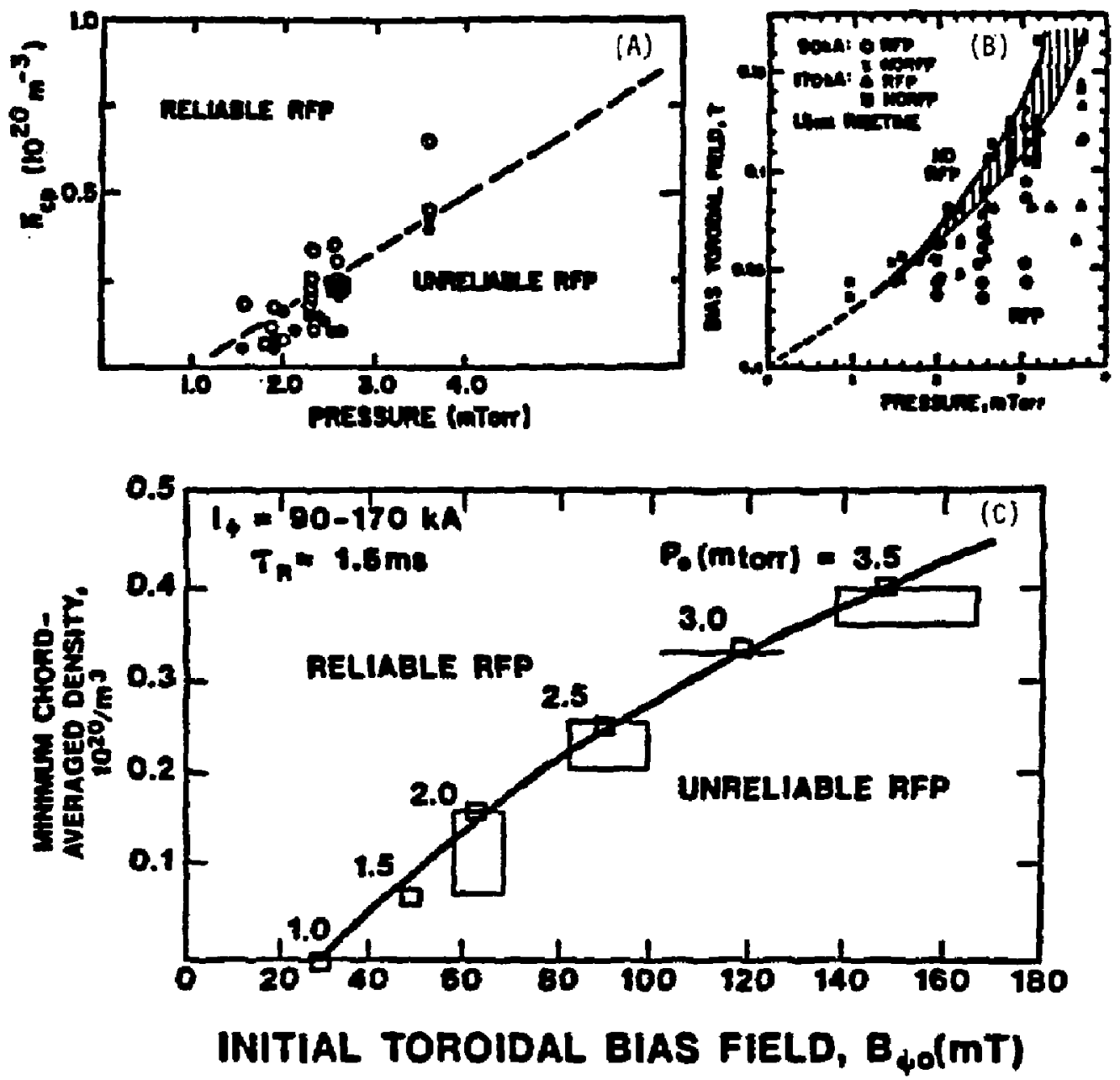

Figure 2.3-5. Typical RFP formation windows showing: (A) dependence on a critical plasma density, (B) magnitude of the original toroidal bias field, and (C) a combination of the two constraints. 


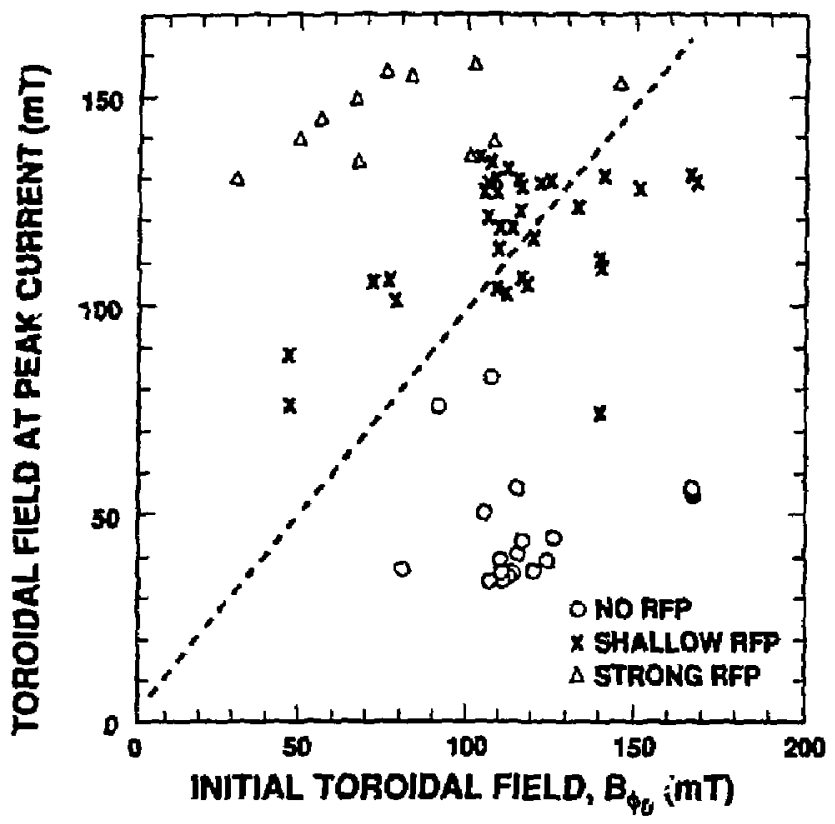

Figure 2.3-6. Relationship between $B_{\phi 0}$ and $\left(B_{\phi}\right)$ for a range of ZT-40M discharges [65] where robust RFP formation occurred, as well as no RFP formation as very shallow, spheromak-like RFP were formed.

\subsubsection{Typical Temperature and Density Responses}

The time variation of the temperature from several machines is shown in Figure 2.3.8. For discharges with a flat-top current waveform (Figure 2.3-8) the electron temperature, $T_{e}$, rises rapidly, reaching approximately 100 to $200 \mathrm{eV}$ near the time of peak current. Then $T_{e}$ increases more slowly as the density drops, ultimately reaching values in the range of 300 to $500 \mathrm{eV}$. At later times during the discharge, the temperature remains approximately constant or decreases slightly. This latter behavior is attributed to wall effects, possibly caused by field errors or inadequate equilibrium control. Similar belavior of the electron temperature is seen on smaller devices, as is illustrated in Figure 2.3.8 for ETA-BETA-1I, where the time scale is nuch shorter and $T_{e}$ rises continuously during the pulse. The data from ZT-40M in Figure 2.3-8 shows that the temperature increases with increasing plasma current. Similar beltavior is generally observed in all RFP experiments.

The ion temperature, $T_{i}$, is usually comparable to the electron temperature, but in

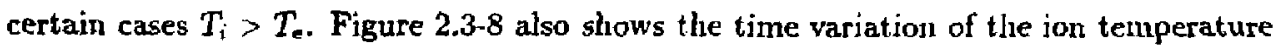




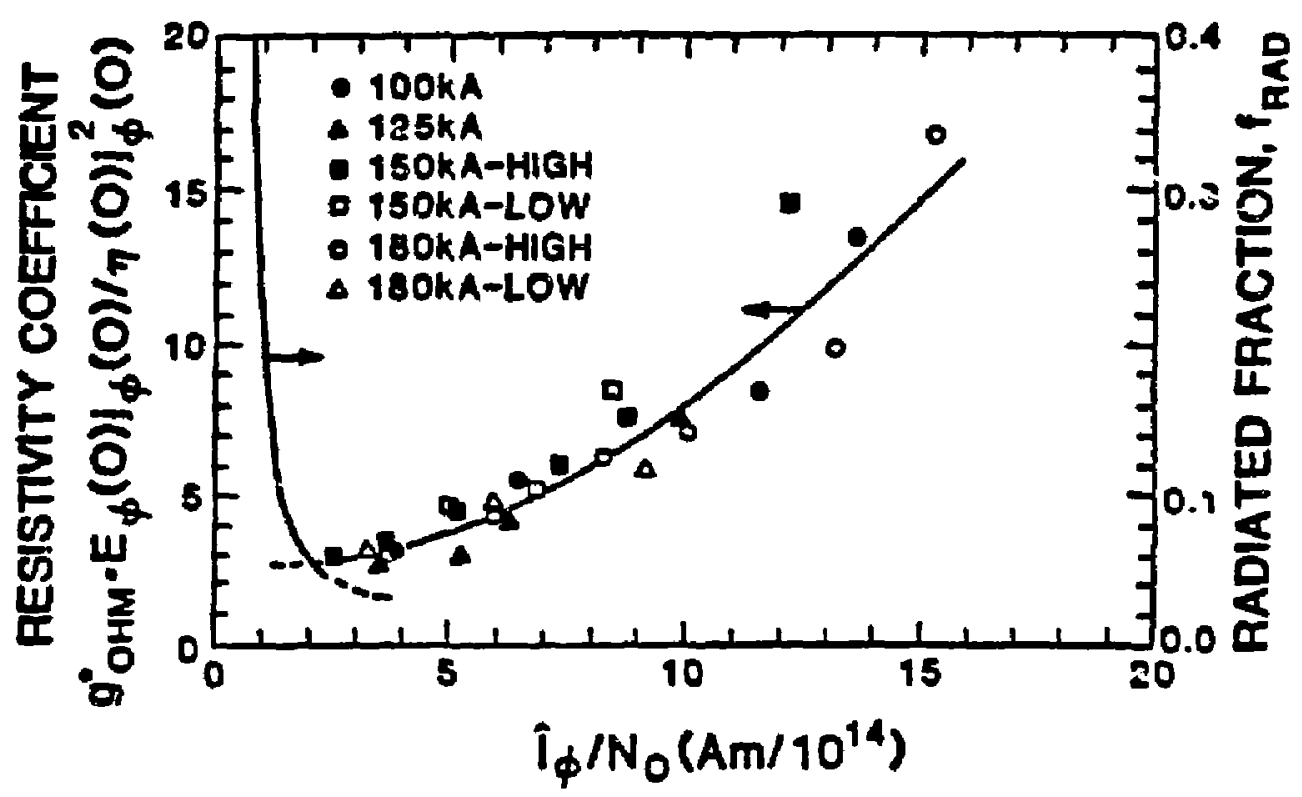

Figure 2.3-7. Dependence of burn-through and reduced-resistance constraints on $\hat{I}_{\phi} / N_{\circ}$ for the ETA-BETA-II RFP, where $\hat{I}_{\phi}$ is the peak current and $N_{o}=n_{o} \pi r_{p}^{2}$ is the initial filling pressure.

in TPE-1R(M). The electron-ion equilibration time is sufficiently long that the ions canu:ot be heated by collisions with electrons during the discharge, and some anomalous ion heating mechanism is apparent.

\subsubsection{Density Control}

Generally, RFP experiments operate without active refueling. The chamber is filled with gas prior to the discharge, and density is maintained by recycling with the chamber walls. Modern RFP discharges are of sufficient duration where active refueling by pellet injection has been used $(16,66-68)(0.5-$ to $2.0-1 \mathrm{~mm}$ radius pellets injected at speeds of 1 to $1.5 \mathrm{~km} / \mathrm{s}$ ). Figure $2.3-9$ shows the temporal variation of plasma density without pellet fueling. Typically, the electron density rises initially to a value corresponding to the filling density and then falls rapidly to $10 \%$ to $20 \%$ of the filling value during the formation phase. After this initial pump-out phase, the density decays more slowly. In 

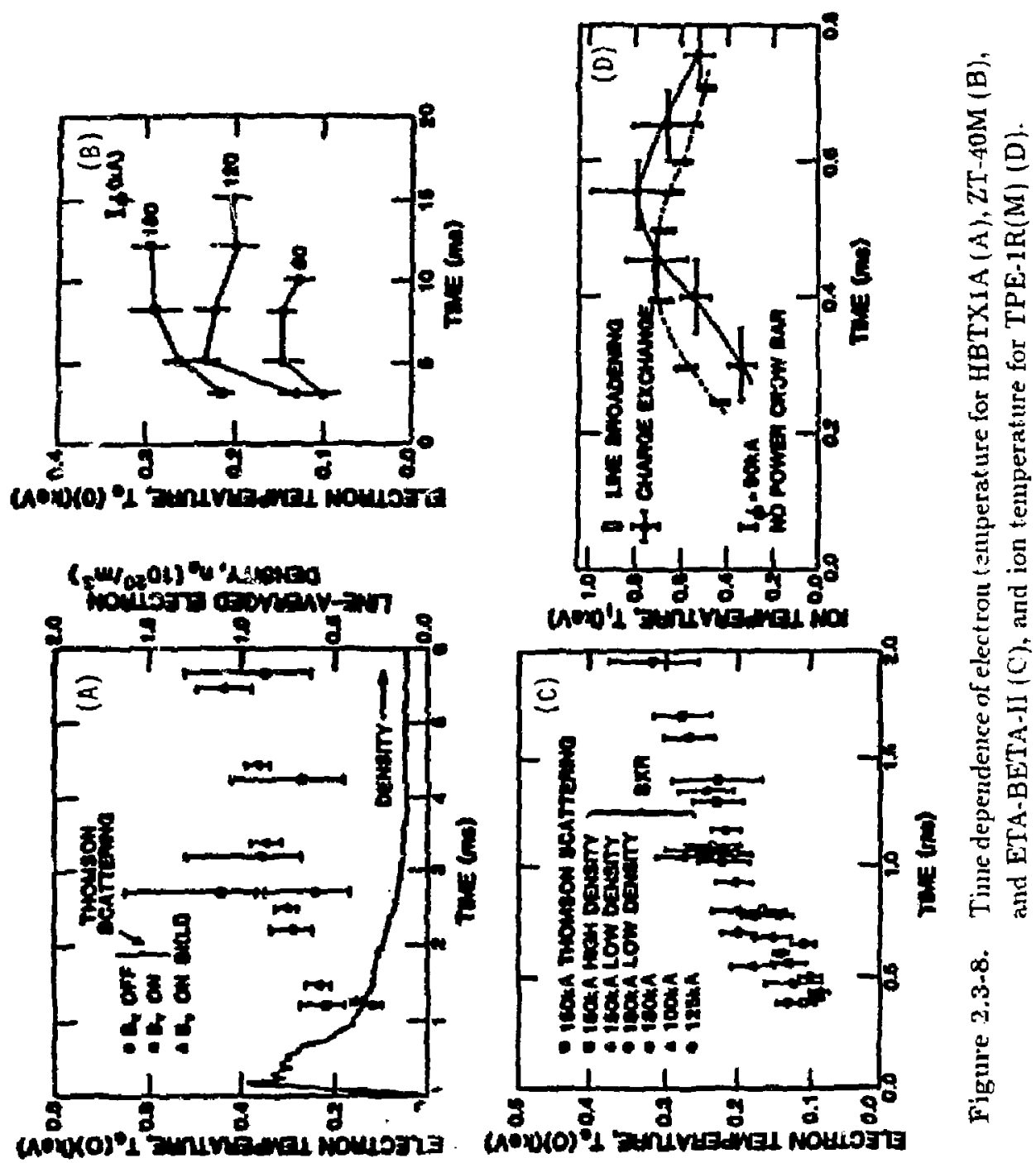
long-pulsed devices, the density tends to reach a steady-state value that shows little dependence on the initial filling density, but some dependence on the plasma current and on the wall conditioning is observed

The rate of intial pump-out depends strongly on the wall condition and is attributed to particles that leave the discharge and are not replaced sufficiently fast by wall recycling. This process can be affected by preconditioning the wall through pulsed discharge cleaning ( $P D C$ ). In fact, by operating the PDC cycle at varying filling pressures a varying amount of hydrogen is "preloaded" into the vacuum-vessel wall and subsequently changes the plasma density as this gas is "unloaded" by subsequent plasma-wall interactions. In the HBTXIB and ZT-40M experiments shown on Figure 2.3-9, the walls were loaded with hydrogen of denterium prior to the discharge initiation. The results show that the density decay can be reduced considerably, and the density is sustained approximately constant for up to $5 \mathrm{~ms}$ or longer without active refueling.

Ges-puffing through external fast-acting valves has been performed on both HBTXIA and ZT-40M. The gas valves inject a steady stream of gas for a preprogrammed portion of discharge. In these experiments, gas-puffing raised the density by at least a factor of two and also increased the ciuration of the current pulse. The electron temperature, however, decreased when the density was increased. Because gas-puffing often changes the equilibrium at a faster rate than can be controled by the feedback systems presently used on these experiments, additional gas-puffing experiments await better equilibrium control.

Figure 2.3-10 gives the density response to the injection of four hydrogen pellets in ZT-40M discharges with $I_{\phi}=120$ and $180 \mathrm{kA}$ [66]. A singie deuterium pellet has also been injected into the ETA-BETA-II experiment [68]. The strong poloidal currents flowing in the outer regions of the RFP cause substantial deflection of the peilet in the poloidal and toroidal directions because of the asymmetry of ablation driven by electrons streaming along field lines. On-axis density peaking was achieved, however, by off-axis injection to compensate for this ablation-driven deflection [67].

Pellet injection increases the density but the temperature of both electrons and ions decrease in a way that holds $\beta_{\theta}$ approximately constant. Typically, ion temperature is 200 to $250 \mathrm{eV}$ prior to the injection, decreases to 90 to $100 \mathrm{eV}$ after the injection, and then recovers to $\sim 200 \mathrm{eV}$ on a 1 to $2 \mathrm{~ms}$ time scale. For the $I_{\phi}=120-\mathrm{kA}$ discharge on ZT-40M, the edge-chordal density rises higher and more rapidly than the central-chordal density. The pellet deposition appears to be more central for the $I_{\phi}=180-\mathrm{kA}$ discharges. Because of the large size of the pellet and the shortness of the discharge in the singlepellet injection experiments on ETA-BETA-II [68], the pellet is not fully consumed and 

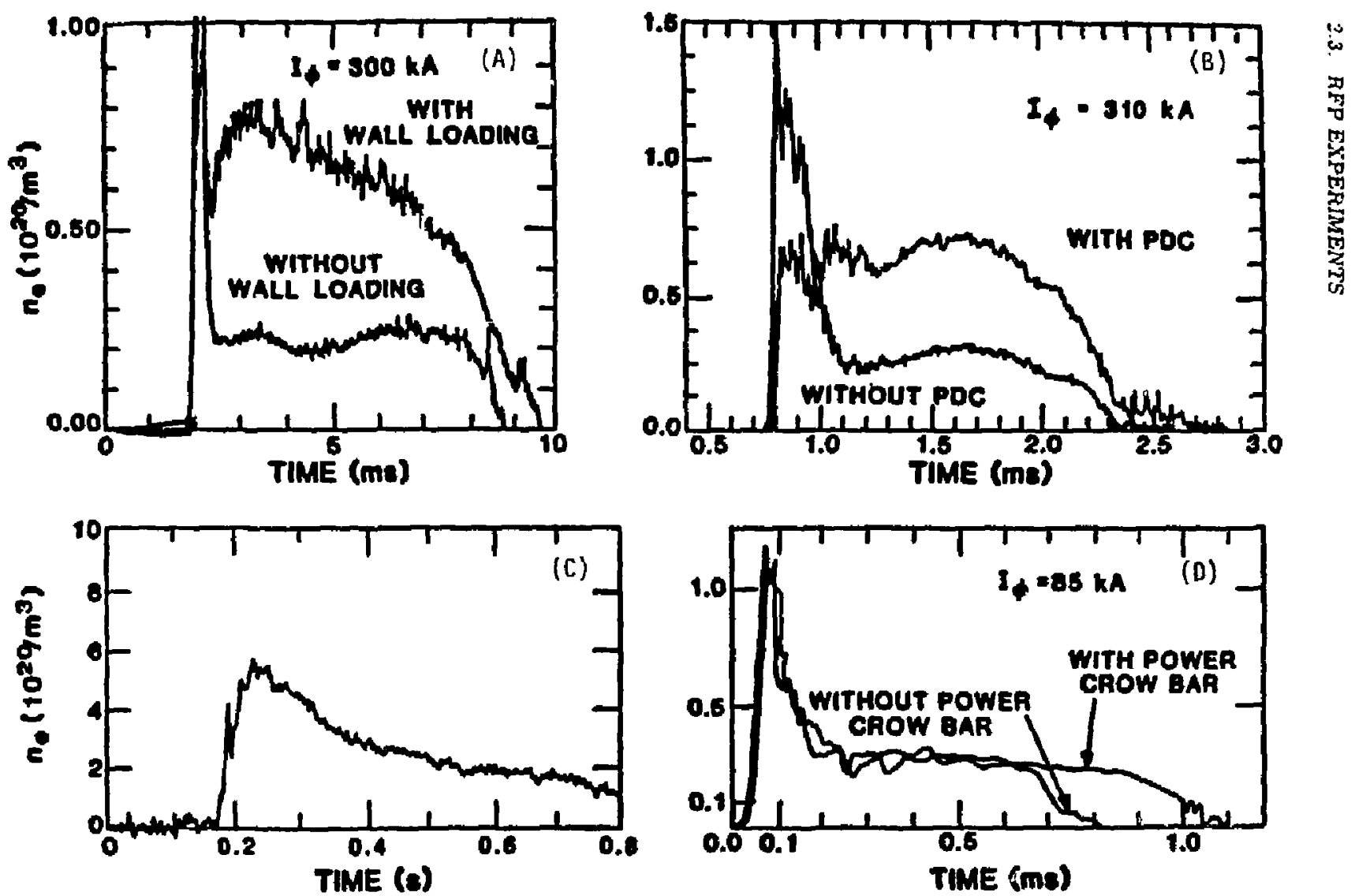

Figure 2.3-9. Time dependence of plasma density for HBTX1B (A), ZT-40M (B), ETA-BETA-II (C), and TPE-1R(M) (D). 


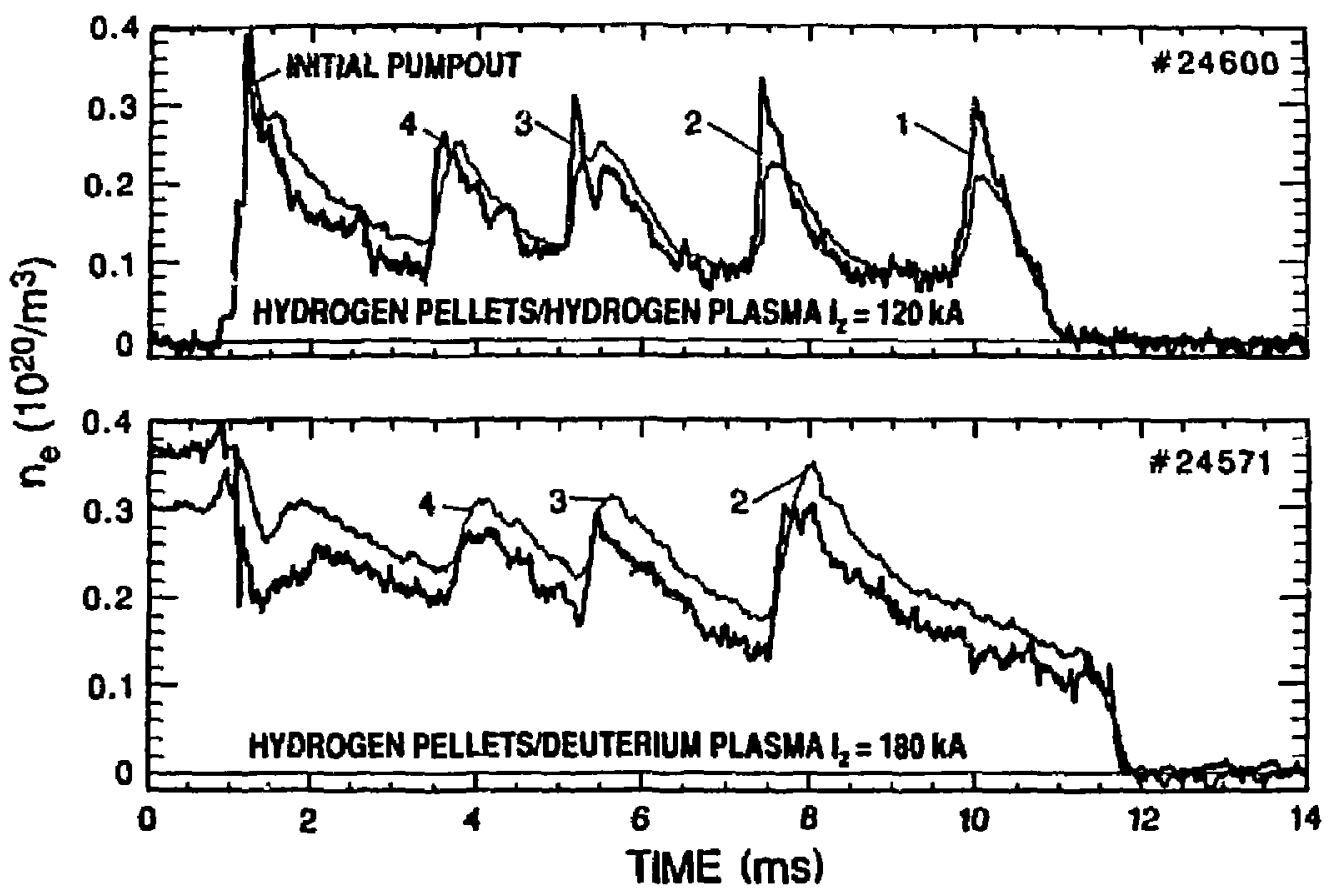

Figure 2.3-10. Density response in ZT-40M along a central chord ( $d$ urk line) and an edge-plasma chord to the injection of four hydrogen pellets into $I_{\phi}=120$ and 180-kA discharges.

the significant increase in density accompanying pellet injection is maintained throughout the discharge. In experiments on ETA-BETA-II, pellet injection is accompanied by a decrease in $T_{e}(0)$, similar to the ZT-40M experiments, but instead of constant beta, the ETA-BETA-II experiment indicates a slight increase in $\beta_{\theta}$.

\subsubsection{Plasna Fluctuations}

The RFP is intrinsically turbulent since, according to Figure 2.2-7, many unstable, helically coupled MHD modes simultaneously reside in the plasma. Fluctuations of MHD origin are thought to be important for toroidal-field generation and sustaimment. Fluctuations can also enhance cross-field transport because of the breakup of the magnetic surfaces and the resulting ergodic field-line behavior. Field and density fluctuations have been studied primarily on the HBTX and ETA-BETA-II experiments [69-73]. Measurements of nagnetic-field fluctuations in HBTX $1 \mathrm{~A}$, for exampie, indicate dominant global 
modes with $m=1$ and a broad spectrum in toroidal mode number centered around $n=-8$, corresponding to the aspect ratio of the device. The minus sign in the toroidal mode number indicates that these modes are resonant inside the reversal surface. Finescale activity with a short transverse correlation length, containing comparable power to the globsl modes, was also found in the core of the discharge. Modes with $m=0$ and small toroidal mode numbers were also observed. These dominant modes were observed throughout the discharge, including the formation phese where the amplitude was a factor 5 to 10 times higher. These oscillations, therefore, appear to a play a fundamental role in maintaining the RFP configuration.

More recent studies of magnetic fluctuations in HBTX1B [73] normal discharges $(\Theta=1.4, F=-0.1)$ indicate modes with $m<2$ and $|n|<6,10,1.3$, and 16 that are retonant inside the reversal surface. Magnitude of the magnetic-field fluctuations at edge-plasma is $\tilde{B} / B \simeq 0.01$ to 0.02 , with toroidal fluctuations dominating the poloidal component by a factor of 3 to 5 and the power being concentrated at frequencies below $150 \mathrm{kHz}$. Deep reversal discharges on HBTX1B give field-fluctuation amplitudes of $5 \%$ to $10 \%$, with the toroidal component being larger thwi: the poloidal fluctuations by only a factor of 2 to 3 .

In contrast to HBTX1A, coherent quasi-continuous $m=1$ activity in the center of the plasma was observed to dominate in low- $\Theta$ discharges in ZT-40M [75]. The $m=1$ modes were.consistent with the predictions of the resistive instability theory. Estimates of the width and separation of magaetic isiands resulting from the $m=1$ modes indicate that the field lines are stochastic inside the reversal surface, and probably throughout the plasme when the $m=0$ modes are taken into account. Qualitative estimates of the energy confineizent time based on stochastic field-line diffusion from the $m=1$ modes are similar in magnitude to those observed on HBTX1A.

Density fluctuations driven by pellet injection on ETA-BETA-II [68] were reported [74] to be $\bar{B} / B=0.01$ to 0.02 . These density fluctuations are poorly correlated with fluctuations of other quantities such as fields, $D_{a}$ sigarals, etc.

The level of magnetic-field fluctuations in RFPs is about $\tilde{B} / B \simeq 0.01$, which is approximately 10 or more times greater than the fluctuations typically observed in tokamaks. The magnetic-field fluctuation levels are observed to decrease with increasing plasma current or magnetic Reynolds number, $S \equiv \tau_{\Omega} / \tau_{A}$, where $\tau_{R}$ and $\tau_{A}$ are, respectively, the plasma resistive and Alfven times. This behavior is shown in Figure 2.3-11 for OHTE/RFP, where fluctuation levels appear to decrease as $S^{-1 / 2}$. Similar behavior is also reported for ETA-BETA-II [76]. 


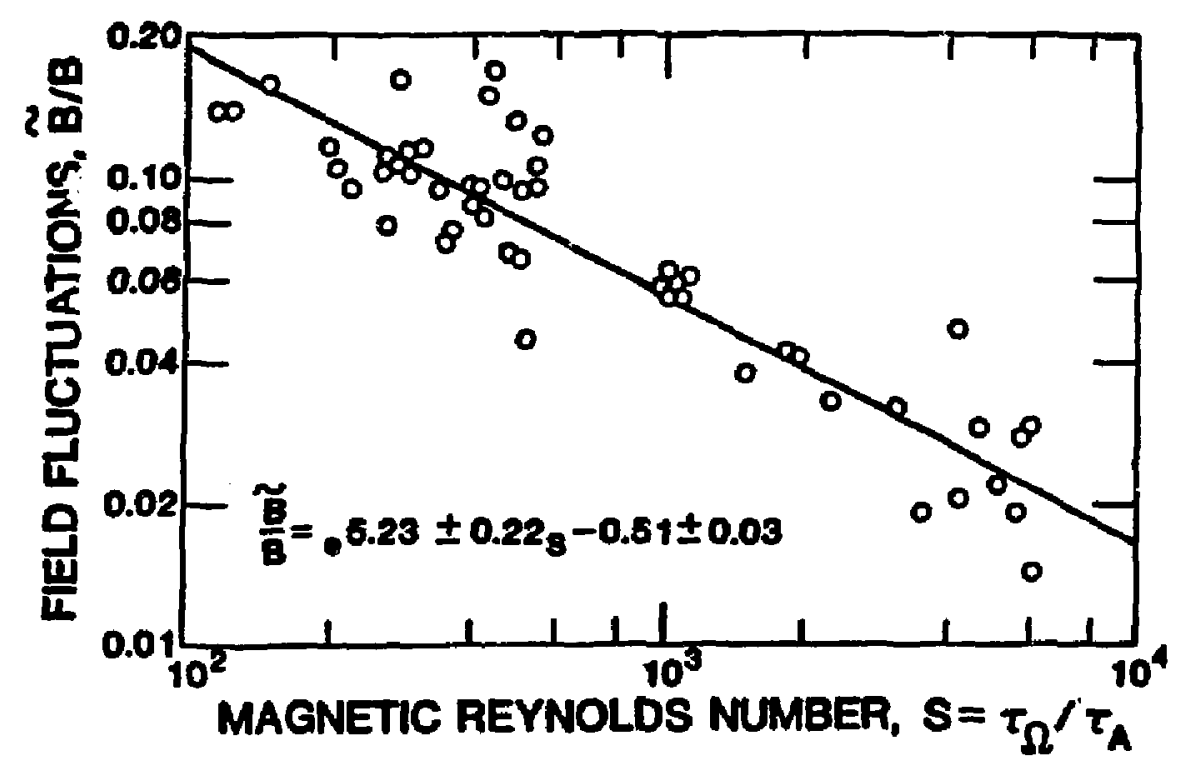

Figure 2.3-11. Magnetic-fluctuation amplitude as a function of magnetic Reynolds number for the OHTE/RFP device, where the plasma resistive and Alfven times are $\tau_{\Omega}$ and $\tau_{A}$, respectively.

The cross-field transport caused by stochastic field-line diffusion corresponds to the correlation of fluctuations with $S$, given in Figure 2.3-11. This correlation gives a favorable temperature-current scaling that is consistent with the observed linear dependence of electron temperature on plasma current. Since the RFP dynamo is thought to be closely associated with MHD-driven fluctuations related to the simultaneous admission of many instabilities into the plasma, some concern arises as to the ability of the dynamo to maintain the minimum-energy state if the RFP becomes too quiescent in the high- $S$ reactor regime.

\subsubsection{Scaling}

The experimental determination of the dependence of plasma resistance, density and temperature (pressure and beta), and confinement time as size and current is the main mealls by which present-day RFPs provide "scaling" to the next experiment [24], ignition devices [59], and the reactor. Since stability, transport, confinement, and heating are closely related in RFPs (or any ohnically heated device for that matter), the scaling 
of each of these phenomena is closely coupled and irterdependent. Recognizing this interdependence, the experimental status of RFP scaling is summarized here in a format that divides according to (1) plagme resistance, (2) density and temperature (pressure and beta), and (3) confinement time. In summarizing these reaults it should be emphasized that experimentally reported densities and temperatures generally refer to line-averaged and centerline values, respectively, with $n$ referring to the volume-averaged density and $T_{e, i}$ being the density-weighted volume average for the TITAN study.

It is recalled (Equation 2.2-15) that expressing the pressure balance in terras of a poloidal beta and setting $T_{\mathrm{e}}=T_{\mathrm{i}} \equiv T$ leads to

$$
T=\frac{\mu_{0}}{16 \pi k_{B}} \beta_{0} I_{\phi}\left(\frac{I_{\phi}}{N}\right),
$$

where $N \equiv n \pi r_{p}^{2}$ is the line density and the ratio $I_{\phi} / N=j_{\phi} / n$ is related to the electron streaming parameter, $\xi$

$$
\xi=\frac{v_{D}}{v_{T H e}}=\left(\frac{10^{\delta} m_{e}}{k_{\theta}^{3}}\right)^{1 / 2}\left(\frac{j_{\phi}}{n_{e}}\right) T_{*}^{-1 / 2},
$$

where $\xi \geq 0.1$ for the present experiment and $\xi \leq 0.004$ for the TITAN reactor designs.

Using the definition of energy confinement time in an ohmically heated plasma given by Equation 2.2-16 and defining the thermal diffusivity as $\chi_{ \pm} \equiv(3 / 16) r_{p}^{2} / \tau_{1}$ lead to

$$
\chi_{s}=g_{\text {OHM }} \frac{\eta}{\mu_{0} \beta_{0}},
$$

where $\eta \simeq 1.65 \times 10^{-9} Z_{\text {eff }} T_{e}^{-3 / 2} \ln \Lambda$ is the Spitzer resistivity. The composite profile factor, $g_{\text {ONM }}$, includes both density and temperature profiles as well as the average effects of the magnet-field "Acrew-up" factor which is larger for RFP.

Equation 2.3-1 suggests that if $\beta_{\theta}, I_{\phi} / N$, and $T(0) / T$ are constants, then $T(0) \propto I_{\phi}$. Furthermore, if $\eta$ scales classically with temperature and $g_{O H N}$ and $Z_{e / S}$ are constant, then $\eta \propto I_{\phi}^{-3 / 2}$. Unfortunately, the scaling data base described below contains some uncertainty and diffusiveness because of the variability of profiles (i.e., either $g_{\mathrm{OHM}}$ or $T(0) / T), Z_{\text {eff }}$, the contribution from locally nonclassical resistivities, and the relatively high value of $\xi$ and the potential for significant runaway electron populations, at least through the latter stages of a given discharge (i.e., $n$ decreasing because of pump-out). The general trends suggested by the purely classical scaling described in Equations 2.3-1 and 2.3-3, however, are generally substantiated by experiment and serve as the primary basis from which the TITAN physics performance is extrapolated. It should be noted that profiles and $Z_{\text {efs }}$ can vary with current, and this variation can significantly impact the measured dependence of $T$ or $\tau_{s}$ on $I_{\phi}$. 


\subsubsection{Resistance scaling}

The decrease in plasma resistance, $R_{p}$, with current for HBTX1B was shown in Figure 2.3-4. Figure 2.3-12 illustrates a similar behavior for OHTE/RFP indicating $R_{p} \propto I_{\phi}^{-\nu}$, where $\nu \simeq 1$ to 1.5. Figure 2.3-13 gives the dependence of $R_{p}$ on $T_{e}(0)$ for ZT- $40 \mathrm{M}$, with $R_{\mathrm{p}} \propto T_{e}^{-\nu}$ and $\nu \simeq 1$ to 1.5 being indicated. Noting the validity of the above-mentioned assumptions leading to the $T_{e} \propto I_{\phi}$ prediction, these data indicated a Spitzer-like temperature dependence of $R_{p}$. The $R_{p} \propto T_{e}^{-3 / 2}$ curve in Figure 2.3-13 correlates with a value of $[T(0) / T]^{3 / 2} Z_{\text {ef } f g_{\text {онM }}}=23.3$ which for $T(0) / T \simeq 2$ results in $Z_{\text {eff }} g_{O H M}=8.2$. The profile factor $g_{\text {OHM }}$ is expected to range from 2.9 to 5.1 , respectively, for the profile used for TITAN (Equation 2.2-4 with $\alpha=8$ ) and BFM. Hence, the data in Figure 2.3-13 match the Spitzer resistivity for $Z_{\text {eff }}$ values in the range of 1.6 to 2.8 .

Although this scaling of resistance with temperature, and indirectly with current, is favorable, the ZT-40M results for ramped discharge given in Figure 2.3-3 are less optimistic (i.e., $R_{p} I_{\phi} \approx 32.5$ ) and need further resolution. The sensitivity of $R_{p}$ to field errors (Figure 2.2-9) and equilibrium shifts (Figure 2.2-8), and general edge-plasma conditions are also noted. Generally, the scaling of plasma resistance and/or loop voltage represents the most near-term, premier issue for RFPs, closely followed by and related to confinement of hot plasma $\left(\beta_{\theta}\right)$ and transport $\left(\chi_{n}\right)$.

\subsubsection{Density and temperature $\left(\beta_{\theta}\right)$ scaling}

All major RFP experiments show an increase in confinement plasma pressure with increased current and a general tendency for $\beta_{\theta}$ (Equation 2.3-1) to be constant or possibly to decrease slightly with current. It should be noted that the experimental values of $\beta_{\theta}$ generally refer to the average density, but the on-axis electron temperature, $T_{e}(0)$, is used. Hence, decreases in this experimental $\beta_{\theta}$ with current can also reflect changes in $T(0) / T$, as well as $T_{q} / T_{i}$ which is assumed generally to be unity.

Figure 2.3-14 gives the results of a two-point electron-temperature measurement on ZT-40M, where $I_{\phi}$ was held fixed at $0.3 \mathrm{MA}$ and the filling pressure, $P_{o}$, was varied to give the desired variation in $n$ and $I_{\phi} / N$. These data illustrate the general trade-off of density with temperature at the most basic experimental level. Figure 2.3-15 gives the variation of central electron temperature, $T_{e}(0)$, and density, $n_{e}(0)$, with plasma current for the OHTE/RFP device. A similar data correlation from the TPE-1R(M) experiment is given in Figure 2.3-16. Figure 2.3-17 gives the variation of the on-axis tenperature 


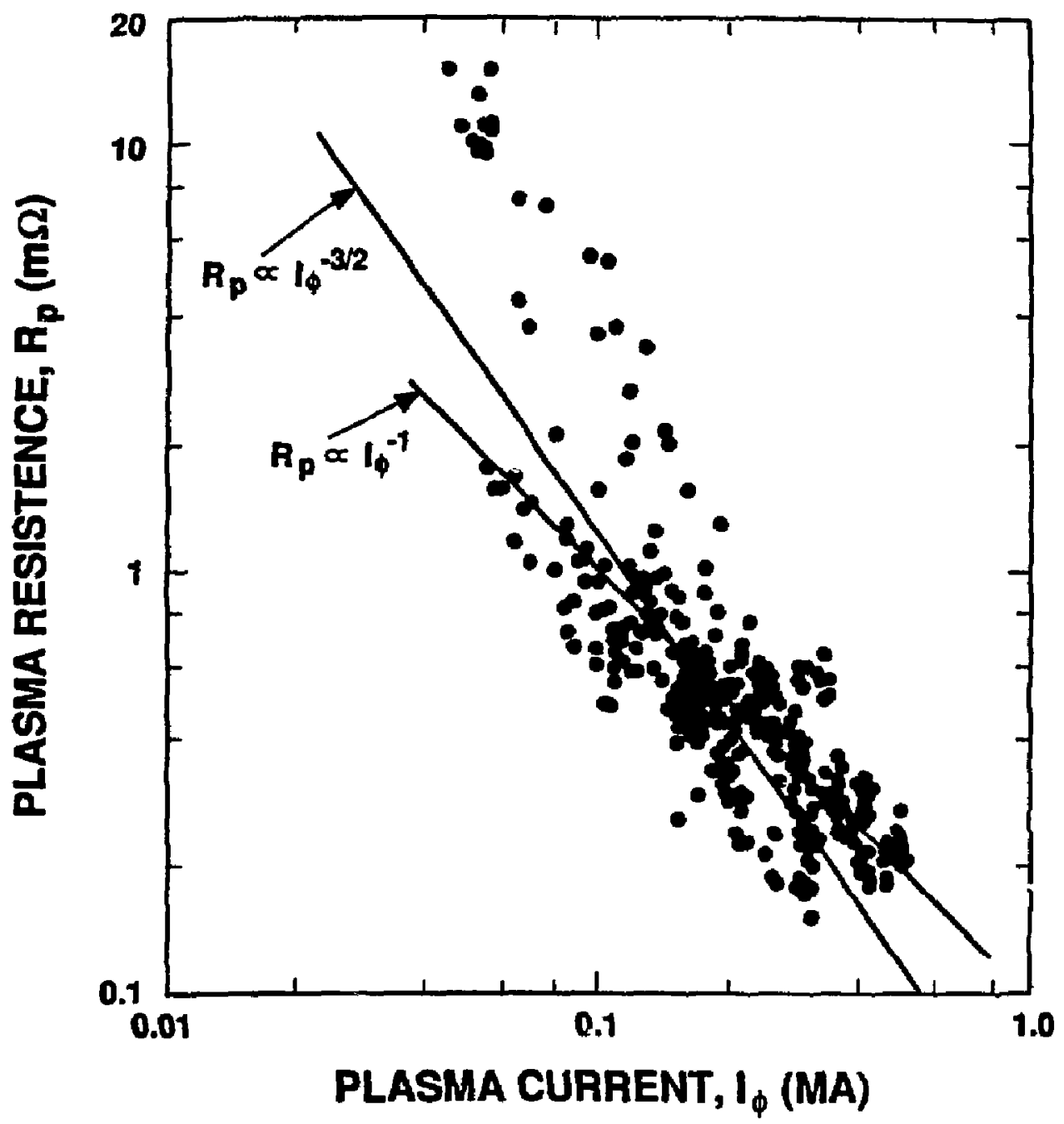

Figure 2.3-12. Dependence of plasma resistance on current for the OHTE/RFP experiment. 


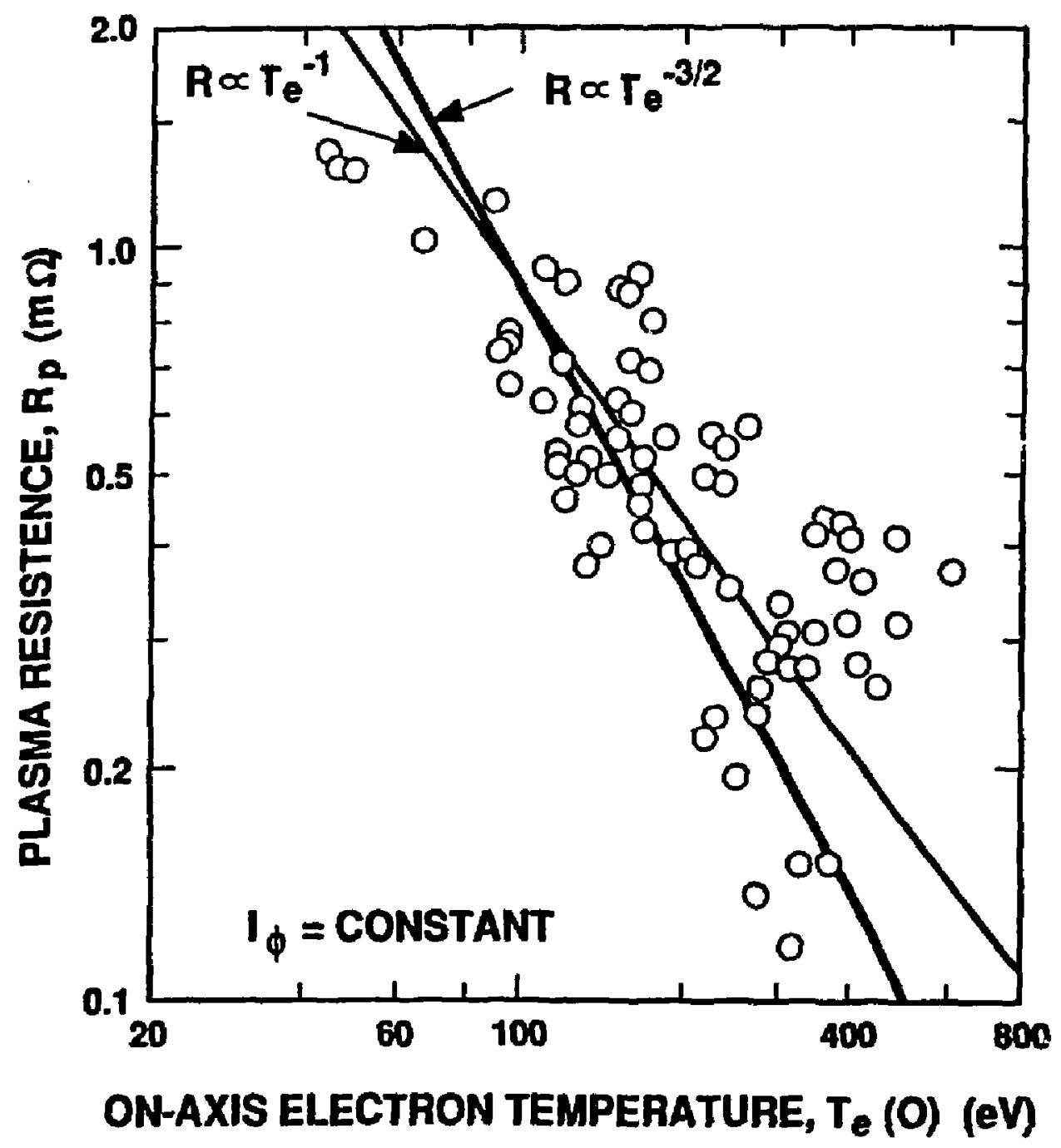

Figure 2.3-13. Dependence of plasma resistance on central electron temperature for ZT-40M experiment. 


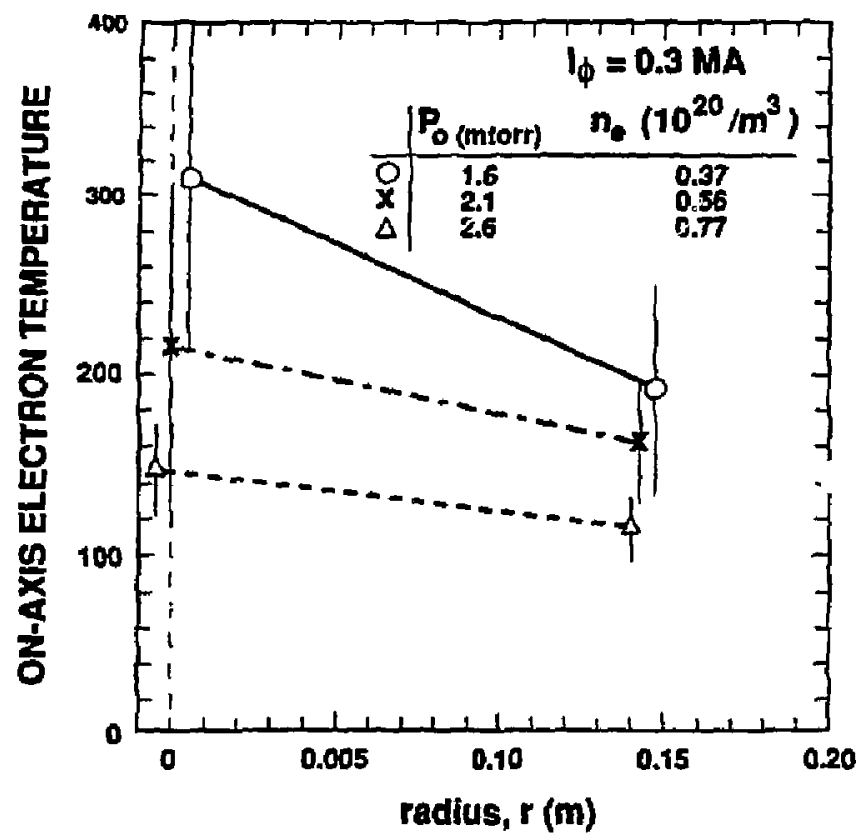

Figure 2.3-14. Two-point electron-temperature profiles for $I_{\phi}=0.3 \mathrm{MA}$ and three val. ues of $I_{2 i} N$ for the ZT-40M experiment.

and the product $n T_{e}(0)$ with plasma current for ZT-40M. The latter set of data gives a linear increase of $T_{e}(0)$ with $I_{\phi}$ and $n T_{e}(0) \propto I_{\phi}^{2}$, with the slope indicating a nominally constant value of poloidal beta of $\beta_{\theta} \simeq 0.05$.

The ZT-40M and OHTE/RFP pressure-scaling data are given in Figure 2.3-18. Correlation of this data with Equation 2.3-1 shows that poloidal beta values in the range of 0.05 to 0.30 are $p($ ssible. A correlation of selected data from a number of RFP experiments are shown on Figure 2.3-19, again substantiating the nominal constancy of $\beta_{\theta}$ in the range of 0.05 to 0.30 . The best-fit curve gives an "international" $\beta_{\theta}$ of 0.064 , nevertheless, a wide range of beta values are possible for a given machine, with the OHTE/RFP and TPE-1R(M) devices consistently reporting somewhat higher $\beta_{\theta}$ values. 

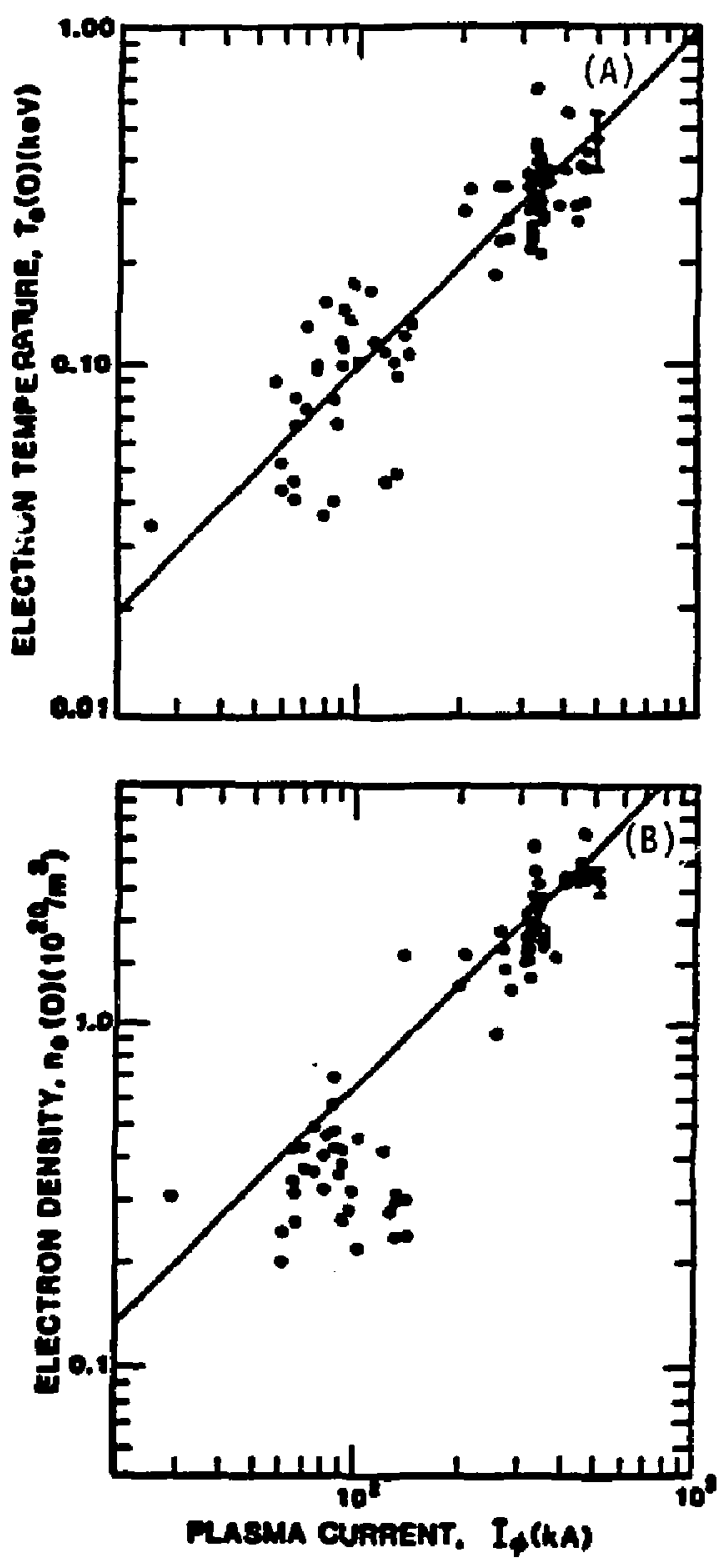

Figure 2.3-15. Variation of central electron temperature $(A)$ and central electron density (B) with the plasma current for the OHTE/RFP experinent. 


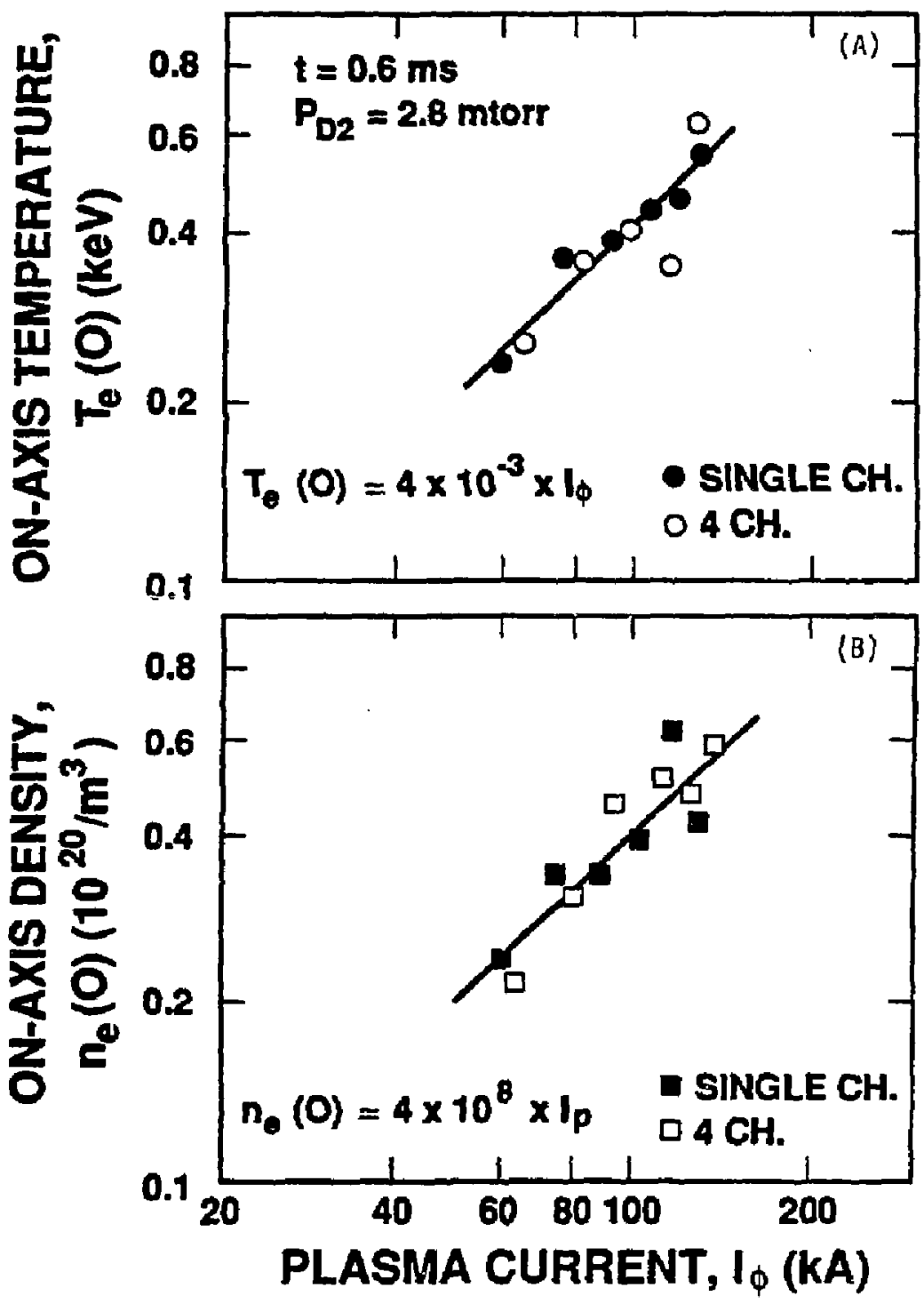

Figure 2.3-16. Variation of central election temperature (A) and central electron density (B) with the plasma current for the TPE-1R(M) experiment. 

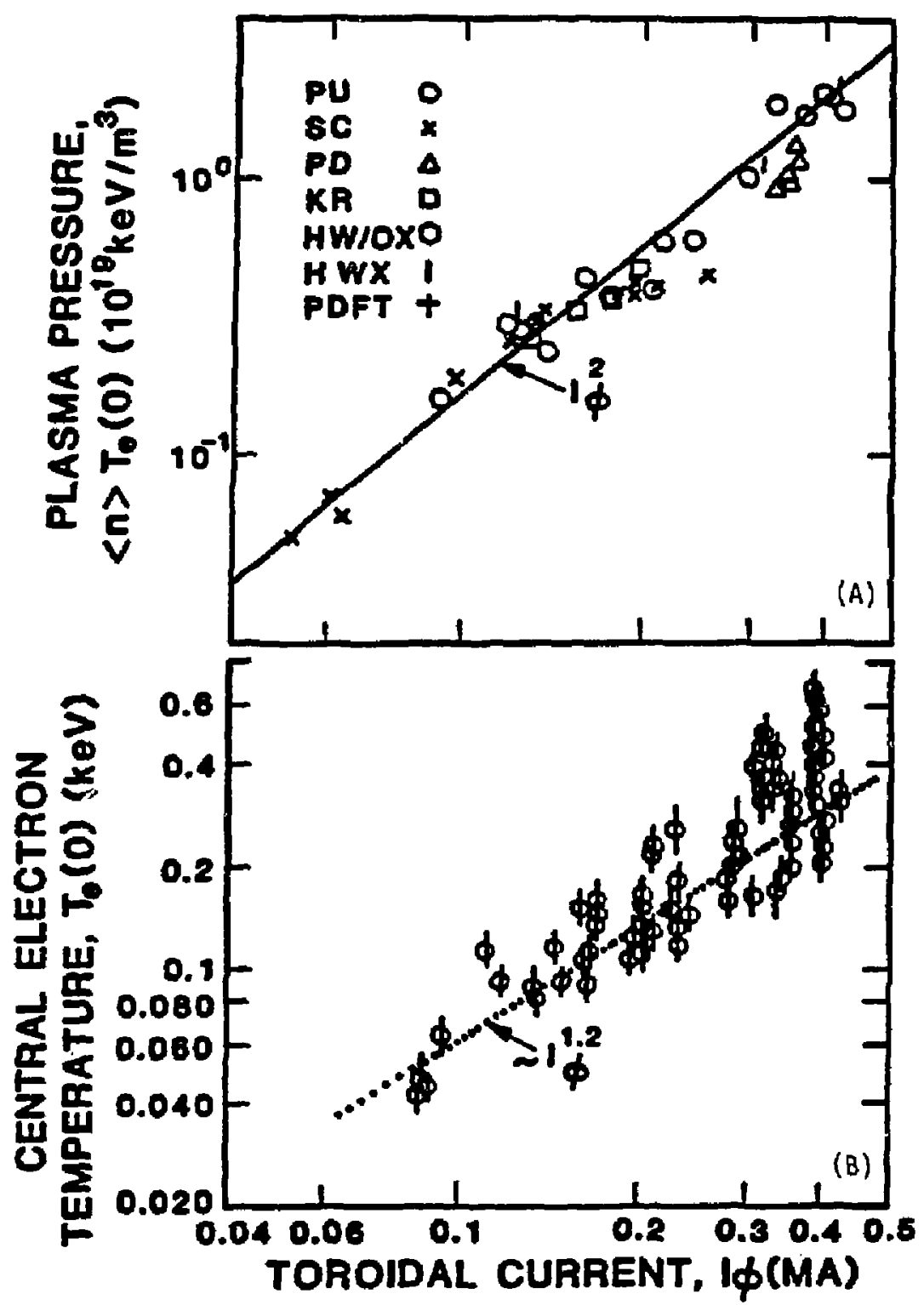

Figure 2.3-17. Variation of the product of central electron temperature with the average electron density current (A) and central electron temperature (B) with the plasma current for the ZT-40M experiment. 


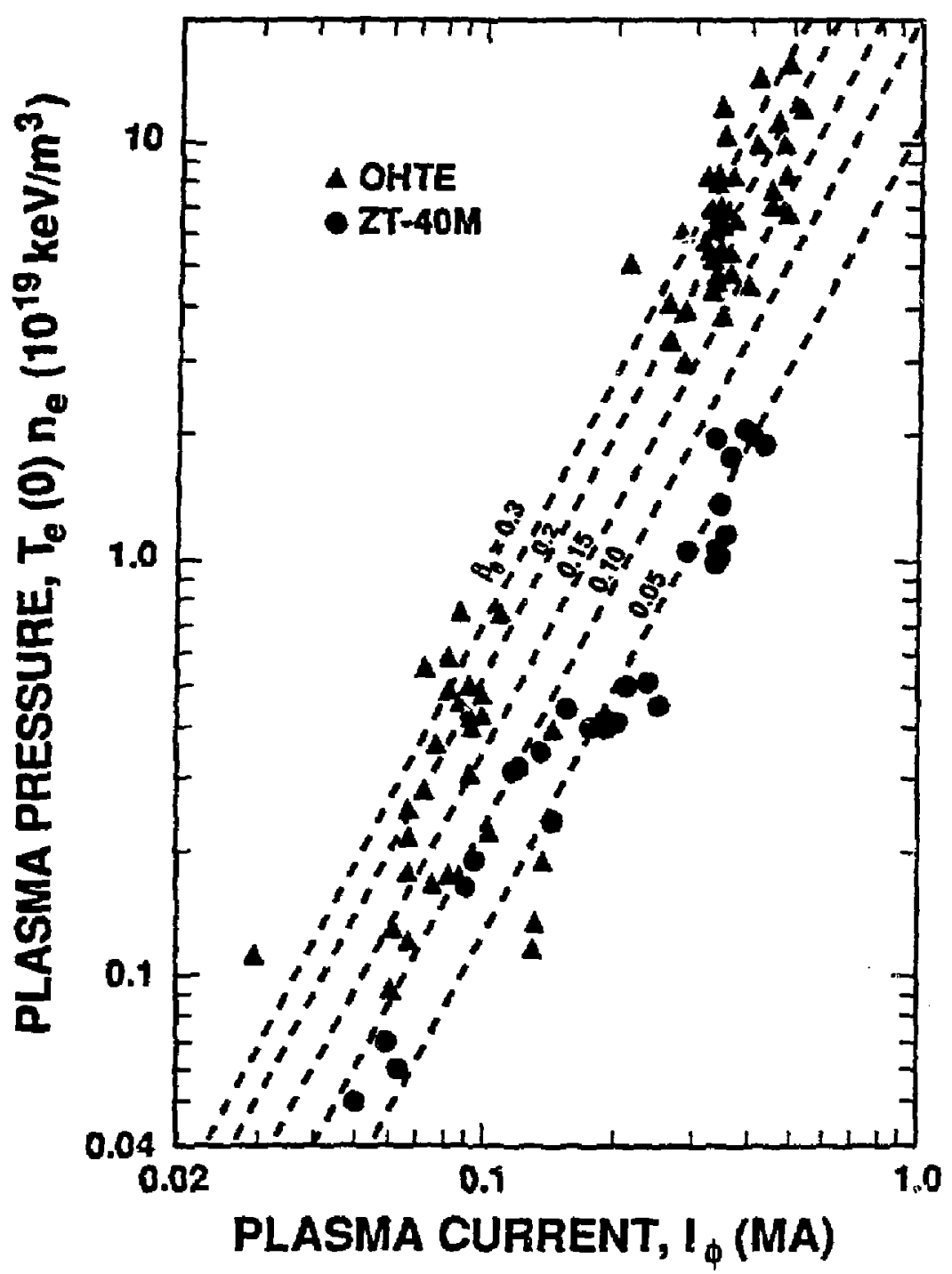

Figure 2.3-18. Corresation of ZT-40M and OHTE/FFP pressure-scaling data with Equation 2.3-1 for a range of $\beta_{\theta}$ and assuning $T_{-}(0) / T \simeq 2.0$. 


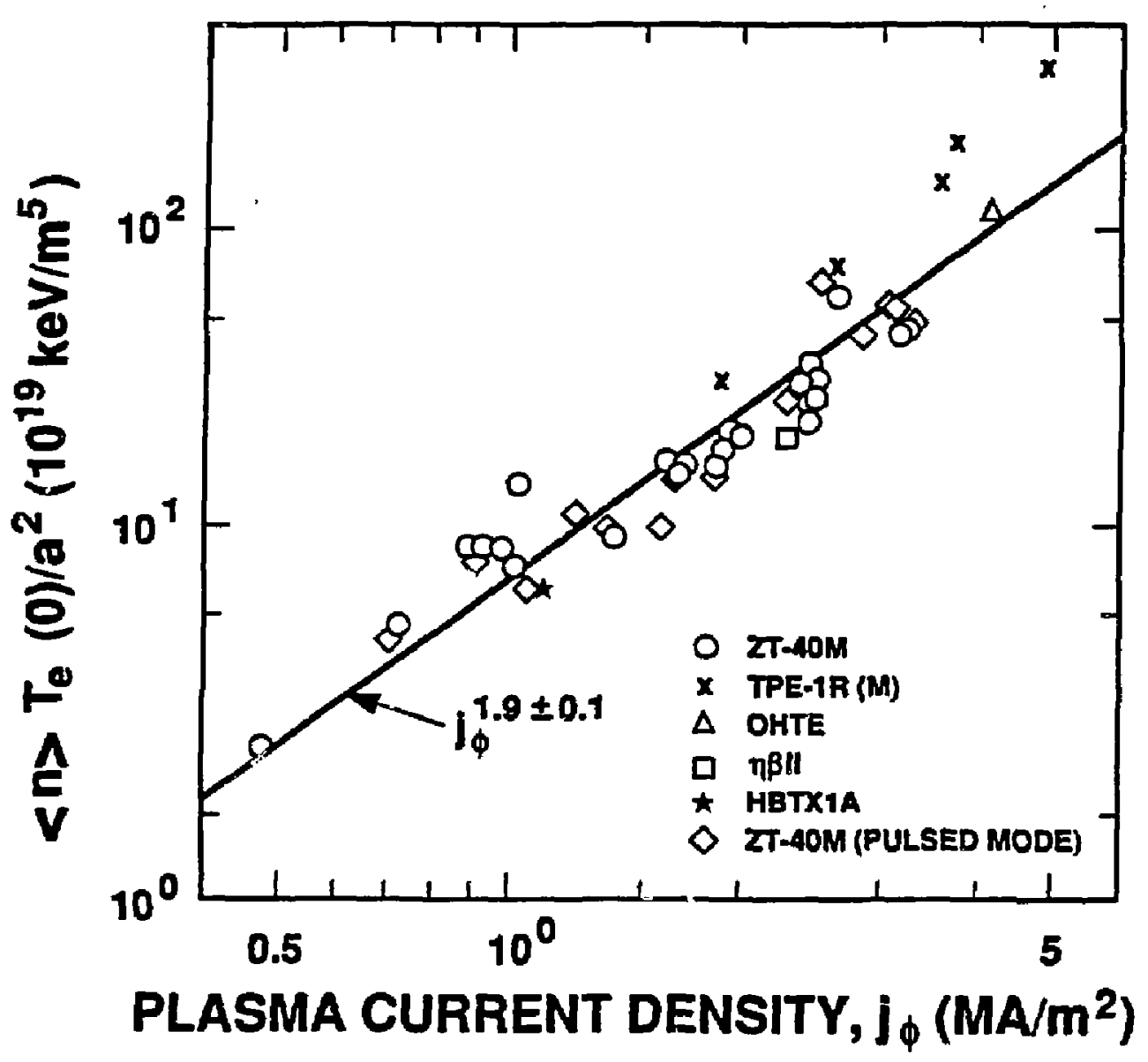

Figure 2.3-19. Correlation of plasma pressure with current and geometry for a number of RFP experiments. The "best-fit" curve gives $\beta_{\theta} \simeq 0.064$ under the assumption that $T_{e}(0) / T \simeq 2.0$. 


\subsubsection{Transport scaling}

As noted previously, only a heuristic approach to the scaling of $\tau_{3}$ and $n \tau_{3}$ is possible until plasma profites and transport are intrinsically and raproducibly measured. Combining Equations 2.3-1 and 2.3-3 and using the previously stated expression for Spitzer resistivity with $\ln \Lambda \simeq 15$ gives the followic

$$
\begin{aligned}
\tau_{m} & =1.86 \times 10^{13} \frac{\tau_{p}^{2} \beta_{\theta}^{5 / 2}}{g_{\text {ofr }} Z_{\text {eff }}}\left(\frac{I_{\phi}}{N}\right)^{3 / 2} I_{\phi}^{3 / 2}, \\
n \tau_{\text {a }} & =5.9 \times 10^{12} \frac{\beta_{\theta}^{b / 2}}{g_{\text {ors }} Z_{\text {eff }}}\left(\frac{I_{\phi}}{N}\right)^{1 / 2} I_{\phi}^{5 / 2} .
\end{aligned}
$$

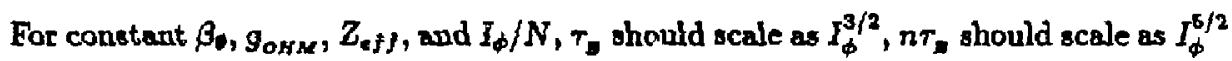
and, according to Equation 2.2-1, $T \propto I_{\phi}$. Figure 2.3-20 gives an early data correlation used to formulate and calibrate the following scaling in previous RFP reactor studies [8] as well as the present one

$$
\tau_{z}=C_{\nu} I_{\phi}^{\nu} r_{p}^{2} f\left(\beta_{\theta}\right)
$$

where the coefficient, $C_{v}$, and exponent, $\nu$, are fitting parameters to the extrapolation shown in Figure 2.3-20 and are given in Table 3.2-III.

More recent and extensive measurements on ZT-40M [77] show that the invarialility of $I_{\phi} / N$ is not sufficient to assume this parameter constant, and that $\tau_{0} \propto\left(I^{2} / N\right)^{3 / 2}$ gives a better correlation with recent data. Figure 2.3-21 gives this correlation with ZT-4OM data. The deviation from the ideal slope of $\sim 3 / 2$ on this figure could be explained by increasing values of $Z_{\text {eff }}\left(\tau_{,} \propto 1 / Z_{\text {eff }}\right)$ as the plasma current and heat loads on invacuum components are increased. Unfortunately, a measurement of $Z_{\text {efs }}$ is not yet available; the curves on Figure 2.3-22 are derived from Equation 2.3-4. Figure 2.3-22 correlates these ZT-40M data according to $\chi_{p} \equiv(3 / 16) \tau_{p}^{2} / \tau_{z}$ and extiepolates to the TITAN reactor requirement.

It should be noted that recent scaling experiments on the 2T-40M device [77] have generated considerably more data points than reported on Figures 2.3-21 and 2.3-22. Many of these additional discharges have been performed at lower currents (70 to $100 \mathrm{kA}$ ) and in this regiou show improved performance (e.g., increased $\beta_{\theta}$ and $\tau_{s}$ ). These newer, low-current data skew the distributions when combined with higher-current data, which do not show improvement relative to past experiments on ZT-40M. The result is an 


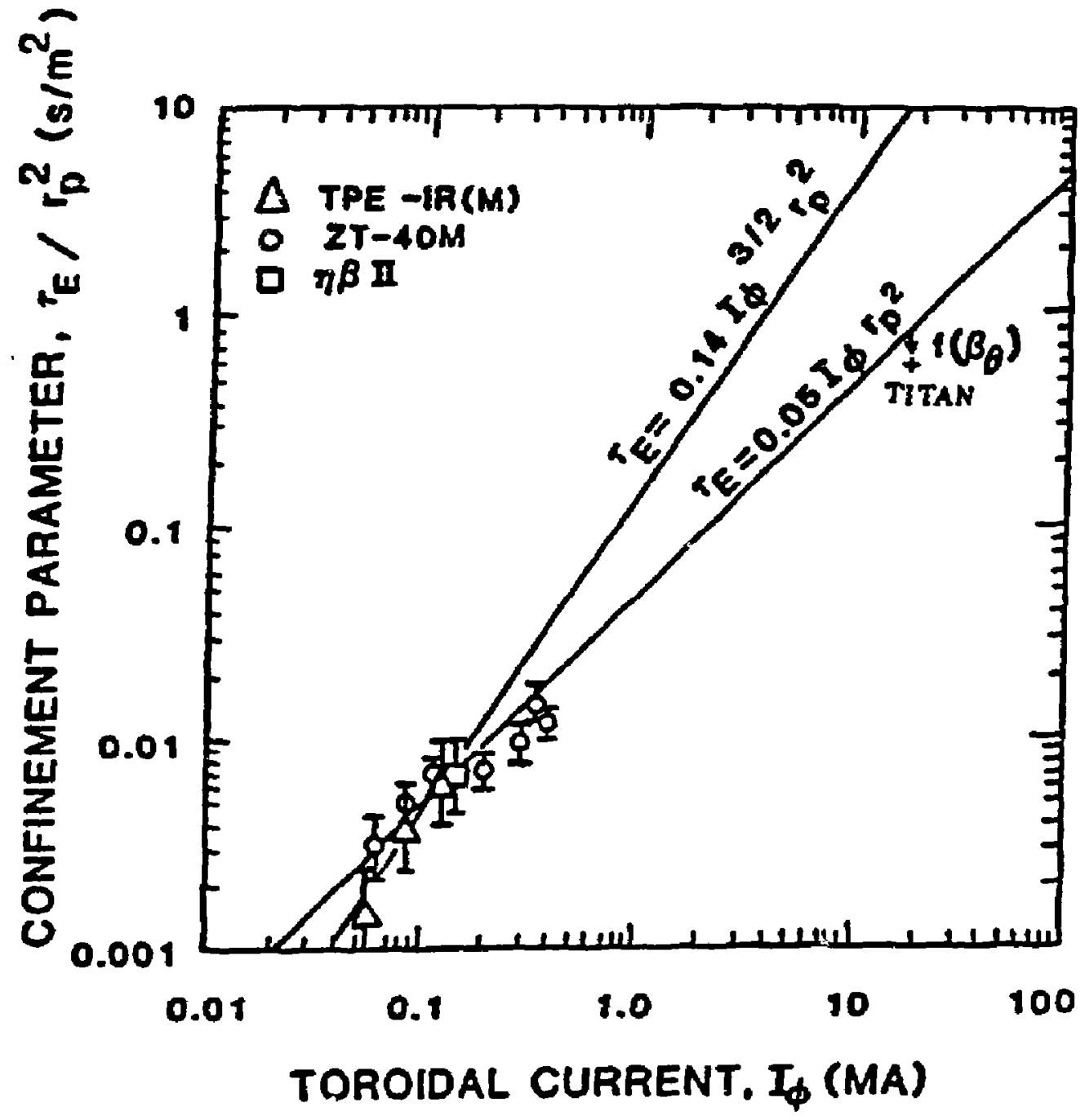

Figure 2.3-20. Variation of the confinement parameter with plasma current with data from several experiments. These early data formed the basis of scaling relationships used in early studies of the RFP reactor [8]. 


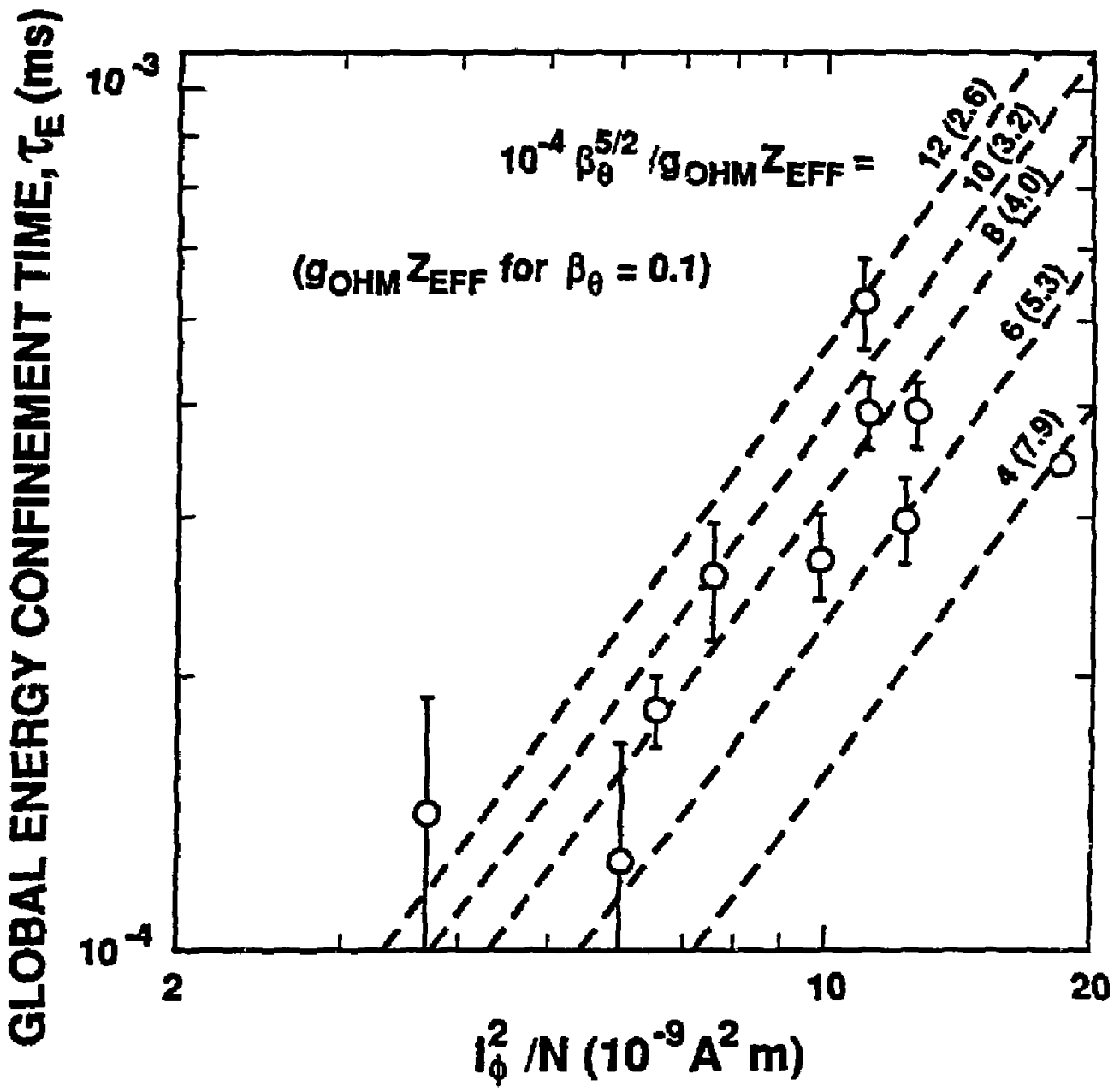

Figure 2.3-21. Correlation of global energy confinement time in ZT-40M with the parameter $\left(I_{\phi}^{2} / N\right\}^{3 / 2}$ in order to accommodate variability in the parameter $I_{\phi} / N$. The lines of constant $\beta_{\theta}^{5 / 2} / g_{O H M} Z_{e f f}$ are generated by Equation 2.3-4. The values of $g_{O H A} Z_{e j /}$ are given in parenthesis fot $\beta_{\theta}=0.1$. 


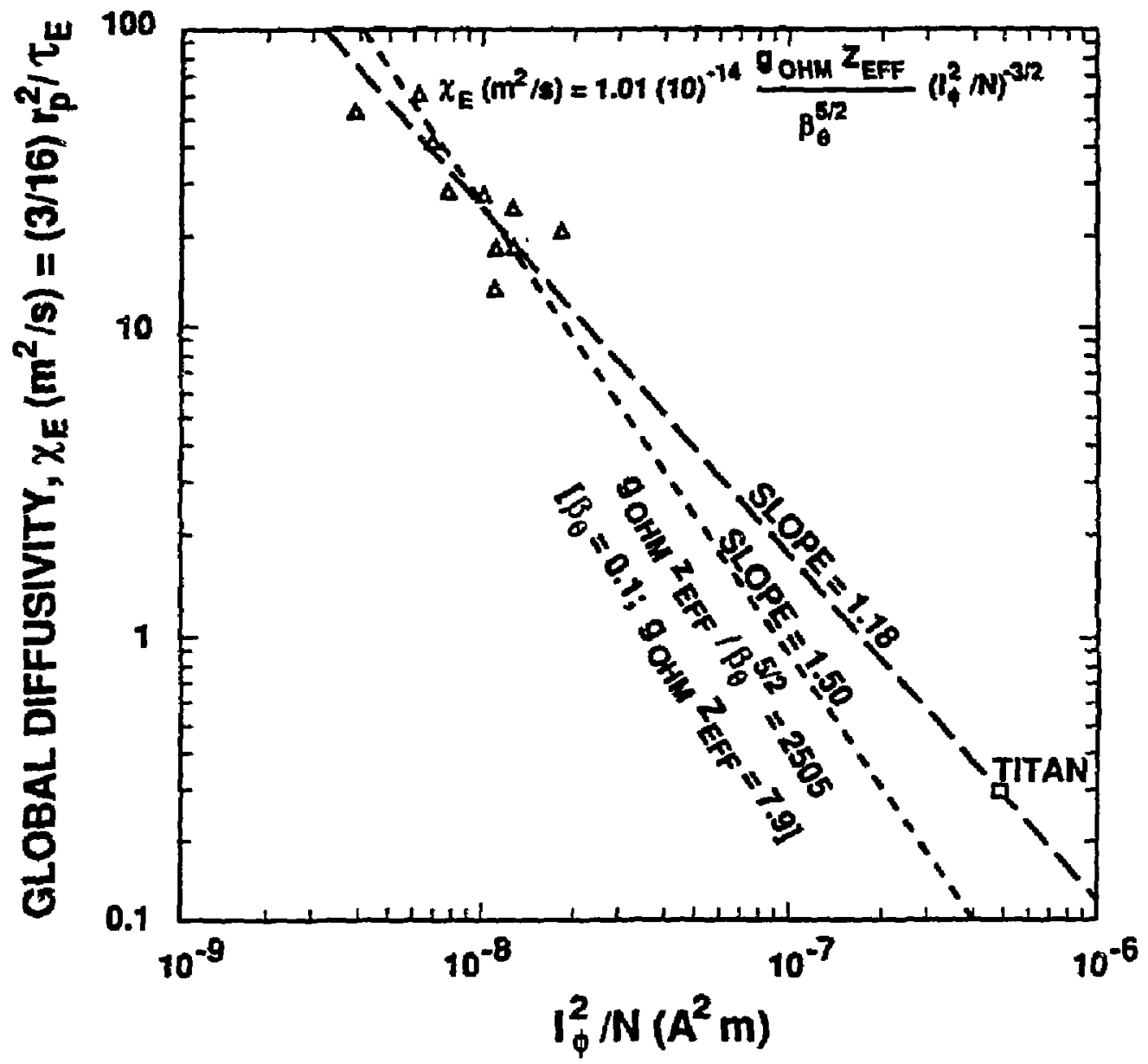

Figure 2.3-22. Correlation of global diffusivity for ZT-40M $[77]$ and an extrapolation to the TITAN reactor conditions using Equation 2.3-4. 
overall current scaling that is less favorable than indicated on Figures 2.3-21 and 2.3-22. Data from higher-current discharges with better density control, and with improved and extended diagnostics of $Z_{\text {aff }}$ and profiles are required. It is likely that these issues will not be fully resolved until data from the higher-current RFP device [24] become available.

Equation 2.3-5 shows that a correlation of transport with plasma current using a Lawson-like parameter, $n \tau_{n}$, should reduce the impact of uncontrolled variations in $I_{\phi} / N$. Figure 2.3-23 given such a plot for both ZT-40M and OHTE/RFP experiments. The line drawn on this figure has the slope of $5 / 2$ which, taking a nominal value of $I_{\phi} / N \simeq 8 \times 10^{-14} \mathrm{~A} / \mathrm{m}$, hoo an intercept that predicts $g_{\text {oHrs }} Z_{\text {eff }} \simeq 1650 \beta^{5 / 2}$. Hence, for $\beta_{0} \simeq 0.1, g_{\text {oNs }} Z_{\text {eff }} \simeq 5.2$. Both the ZT-40M and OHTE/RFP data show a derivation from the ideal $n r_{2} \propto I_{+}^{5 / 2}$ sialing the higher currents, again possibly refiecting increases in heat losd and $Z_{\text {aff }}$ and/or changing (flattening) profiles for the higher-current discharges.

In summary, experimental results a few years ago $[78,79]$ suggested that the scaling of temperature with current might be described by the postulate of a constant $\beta_{\theta}$ and $n T_{a} \propto I_{\phi}^{2}$, with the slope of $T_{a}$ versus $I_{\phi}$ being determined by $I_{\phi} / N$. More recent data correlations from both ZT-40M and HBTX1A indicate that the scaling of $n T_{e}(0)$ with current could under certain circumstances be less than quadratic, with a lower limit of $n T_{*}(0) \propto I_{\phi}^{1.7}$ being suggested by some deta analyses $[77,80]$. Preliminary indications that $\beta_{0}$ may be decreasing somewhat with current awaits a better experimental resolution. As for TITAN, parametric results on the impact of reduced $\beta$, on the reactor performance are given in Sections 3 and 8 .

Also of great interest is the scaling of transport with plasma size, a variable which to date is limited in range. In many respects the main issue for the compact, small-FPC approach to commercial fusion is the degree to which size $\left(r_{p}\right)$ and current can be traded to give the smallest plesma exhibiting bulk-like plasma properties and acceptable firstwall and divertor heat loads. Central to this goal is the achievement of highly radiating, but well-confined plasmas.

\subsubsection{Highly Radiating Plasma}

The observed scaling of plasma pressure with the toroidal current, $n T_{e}(0) \propto I_{\phi}^{2}$, is suggestive of operation near a beta limit. Under this condition, the intrinsic transport would adjust by MHD activity, radiation, or other mechanigm to lose energy at a sufficient rate to maintain $\beta_{\mathcal{E}}$ constant. To test this hypaihesis, experiments were performed on 


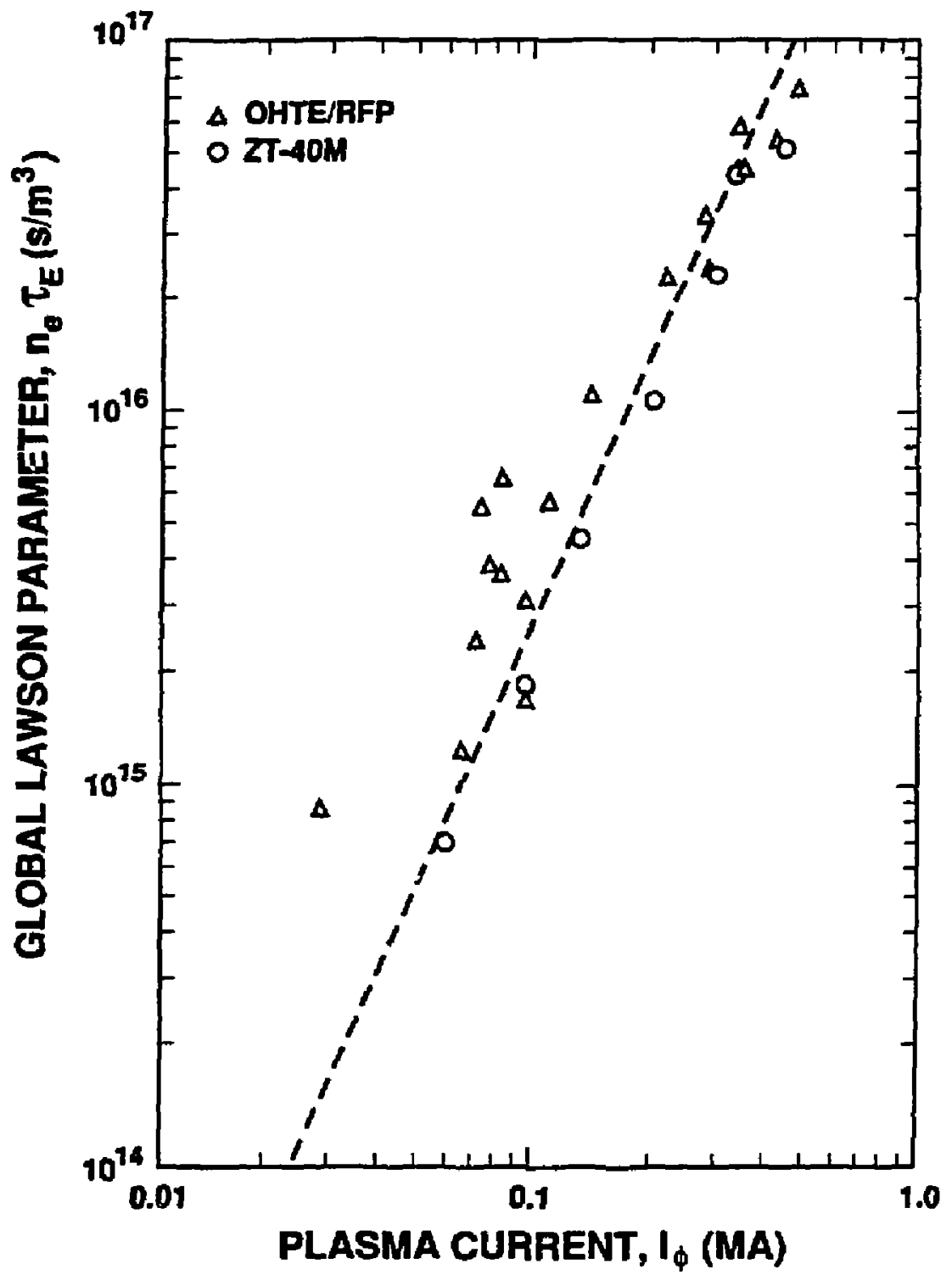

Figure 2.3-23. Correlation of a Lawson-like global energy confinement parameter, $n \tau_{R}$, with plasma current for both OHTE/RFP and ZT-40M devices. 
ZT-40M by adding trace quantities of krypton impurity [81] to enhance the radiative losses. Krypton was chosen to maximize the ratio of radiated power to the ohmic-heating power. It was found that as the impurity was injected, the rate of radiation lose, $P_{\text {RAD }}$, was incressed, but simultuneously, the ohmic power, POHM, only slightly increased. Most importantly, $\beta_{0}$ remained constant. This observation suggests that as radiation losses are increased, the non-radiative or "intrinsic" transport losses, $P_{N R}$, decrease to maintain the beta constant.

A simplified zero-dimensional power-balance model for a steady-state plasma gives $P_{O H M}=P_{R A D}+P_{N R}$. Using the definitions of the global energy confinement time, $\tau_{\text {n }}$, and a non-radiative (intrinsic) energy-confinement time, $\tau_{E}^{N R}$, the following expression results:

$$
\tau_{E}^{N R}=\tau_{I}\left(1-\frac{P_{R A D}}{P_{R A D}+P_{N R}}\right)^{-1}
$$

For the assumed constant-beta scating and self-similar profiles of dentity and electron temperature before and after krypton injection, the values of the total energy loss $\left(P_{R A D}+P_{N R}\right)$ and $\tau_{s}$ remain unchanged. Equation 2.3-7 indicates that as the radiative losses increase, the non-radiative losses decrease (or $\tau_{E}^{N R}$ increases) to maintain the energy content of the plasms and hold $\beta_{\theta}$ constant. The data from the krypton impurity experiments are plotted in Figure 2.3-24, and close agreement with predictions of Equation 2.3-7 is indicated.

A second experimental test was made of the beta-limit hypothesis. The energy confinement scaling given by Equation 2.3-4 predicts that the total energy confinement scales inversely with $Z_{\text {eff. }}$ By injectiug krypton impurities, the plesma resiatance was varied and the total confinement time was measured. These experimental data shown on Figure 2.3-24 indicate that $\tau_{s}$ scales as $Z_{e f f}$, as suggested by Equation 2.3-4.

It is important to point out that, while suggestive of a beta-limit hypothesis, these results are not conclusive. Furthermore, it appears that far more power is being supplied to the discharge than is needed to maint in the plasma at a given beta limit [81]. Therefore, these experiments are not expected to exhibit an underlying transport which is not affected by the beta-limit hypothesis. Beta-limited plasma operation with a high radiation fraction, $f_{R A D} \equiv P_{R A D} /\left(P_{O H M}+P_{\alpha}\right)$, is crucial to the compact high-power-density devices in order to meet realistic constraints on heat and particle loads on the first wall and divertor plates. 

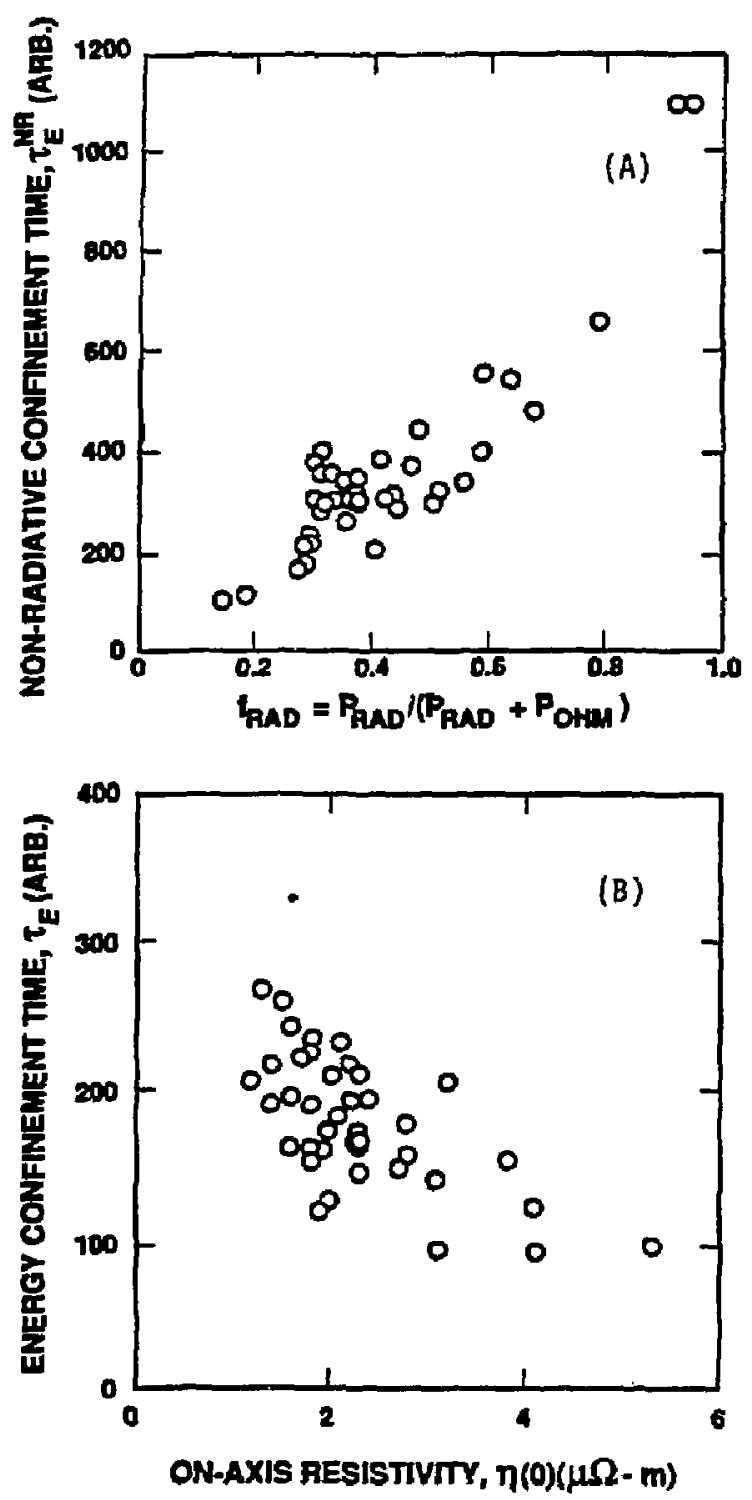

Figure 2.3-24. Scaling of the non-radiative energy confinement time with the fractional radiative power loss $(A)$ and dependence of $\tau_{E}$ on the effective plasma resistivity $(B)$ in the krypton-seeded ZT-40M discharges [81]. 


\subsubsection{Current Termination}

Operating RFP experimental dischargets usually end with a current-termination phase, wherein the plasma current is rapidly reduced to approximately zero (Figure 2.3-2). The current termination occurs in a few tenths of a microsecond in small devices and up to a few hundred microseconds in the larger machines. Current terminations are accompanied by a positive voltage spike and large density and magnetic-field fluctuations. A number of variables, such as plasma radius, density, toroidal-field reversal, magnetic-field errors, and inpurities appear to affect the termination. A complete and satiofactory explanation of RFP current terminations is not yet available. Because of large atored magnetic energy in the plasma, currant termination at TITAN reactors at full plasma current must be avoided in favor of controlled ramp-down of the plasma current.

Fvidence auggests that the onset of termination may be related to a loss of density and confinement, possibly leading to a streaming parameter, $\xi$, that exceeds a critical value for runaway electrons. Studies on $2 \mathrm{~T}-40 \mathrm{M}$ indicate $[80]$ that termination occurs when $I_{\phi} / N$ approaches $\sim 1$ to $2 \times 10^{-13} \mathrm{~A} / \mathrm{m}$, which is consistent with the occurrence of a critical drift threshold when $\xi$ approuches unity.

Rapid current termination can have severe consequences in large, high-current experiments or reactors because of the large voltage spikes and the localized heating of the walls. A method of controlled "run-down" has been teated experimentally on HBTX1B $[61,63,82]$ in which the toroidal-field-coil circuit is controlled so that the pinch parameter, $\theta$, is maintained at a given value as the current is decreased. The field reversal in this case is maintained until the current reaches a relatively low level. Maintaining reversal in this way is found to delay termination, and the current can be reduced to between $10 \%$ and $20 \%$ of the maximum value before the termination occurs. Controlled ramp-downs of this kind forestall the loss of toroidal-field reversal as long as possible and will be required of the reactor.

\subsubsection{Current Drive}

Because of the relatively small values for $L_{p} / h_{p}$ in the compact RFP reactor, inductive pulse lengths in excess of 100 to $200 \mathrm{~s}$ are not possible, and some form of current drive is required. Although the use of radio-frequency (RF) fast-wave current-drive scheme has not been fully explored for the RFP, the high plasma density and currents relative to those for the tolamak indicate problems with the efficiency of RF current-drive schemes (Section 7). On the other hand, because of the relaxation processes in RFPs, there is no 
need to drive the current at the plasma center and some of the issues related to wave penetration may be negated.

Bootstrap current, given by $j_{s c}=-\left(\epsilon^{1 / 2} / B_{\theta}\right)(\partial p / \partial r)[83]$, is also expected to be low (if such current exists at ail in RFPs) since $\beta_{\theta}$ and $\epsilon=r_{p} / R_{T}$ are small. For example, if the pressure profile is given by $p / p_{o}=1-\left(r / r_{p}\right)^{\nu}$, then the contribution of bootstrap current to the overall current density is given by

$$
\frac{j_{\theta c}}{j_{\phi}}=\beta_{\theta} \epsilon^{1 / 2} \frac{\nu^{2}}{4(\nu+2)},
$$

which for $\nu \simeq 2, \beta_{\theta} \simeq 0.2$, and $\epsilon \simeq 1 / 6$ (TITAN plasma conditions) gives $j_{g o} / j_{\phi} \simeq 0.02$.

The close coupling of poloidal and toroidal currents and magnetic fields that determine the near-minimum-energy states of the RFP offers the possibility of a currentdrive method based on "magnetic helicity injection" [17]. Current drive through "electrostatic helicity injection" has been experimentally demonstrated in spheromaks [18], which are also relaxed-state systems !jke RFPs. Another helicity-injection technique is the oscillating-field current drive (OFCD) $[17,19]$. In this scheme, audio-frequency oscillating voltages are applied to the toroidal and poloidal circuits in the appropriate phase $(\delta=\pi / 2)$ to drive a DC toroidal current in the plasma with the plesms, in effect, behaving as a nonlinear rectifier. As originally proposed [17]; OFCD is based on the premise that maintenance of the RFP configuration simply requires the supply of magnetic helicity at a rate equal to its dissipation. The helicity balance is given by $[17,84]$

$$
\frac{d K}{d t}=2 \Phi V_{\phi}-2 \int \mathbf{E} \cdot \mathbf{B} d V_{\mathbf{P}},
$$

where the integral gives the rate of helicity dissipation throughout the plasma volume and the remaining product of toroidal flux and voltage gives the rate of helicity injection or ejection through the plasma surface. Helicity is effectively injected into the plasma if $\Phi$ and $V_{\phi}$ are sinuscidal and are oscillated in phase with each other (e.g., $V_{\theta}=-\dot{\Phi}$ and $V_{\phi}$ are in quadrature), even though the time-averaged electric fields are zero. Hence, with the $F-\Theta$ diagram providing the required connection between $V_{\theta}$ and $V_{\phi}$, a noninwasive and potentially efficient means to drive currents in high-density thermonuclear plasma is possible. Current drive by helicity injection has also been proposed for tokamaks [85 - 87], provided that a similar, globally nondisruptive mechanism for profile relaxation exists.

The effects of $\mathrm{AC}$ magnetic-field oscillations on ZT-40M were first studied using a zero-dirnensional simulation, assuming that the plasma evolved through a series of quasirelaxed equilibrium states. The OFCD was predicted for these experiments, provided that 
the plasma relaxation tirue was shorter than the modulation period and that such field modulations did not adversely affect plasms performance. Subsequent field-oscillation experiments on 2T-40M [19] shoped relaxation times of $\sim 0.1$ ms without deleterious impacts, provided toroidal-field reversal was maintained. Based on these RFP relaxation experiments a full test of OFCD on ZT-40M was conducted $[16,21,88]$.

The test of OFCD on the relatively cold, $T_{e}(0) \simeq 200 \mathrm{eV}$, resistive ZT-40M plasma was anticipated to be difficult because of the strong field oscillations and high frequencies required to observe an effect. The main OFCD discharge variables that have to be optimized relative to RFP physics and machine limitations are $V_{\phi}, V_{\theta}, \delta, \bar{\theta}$, and $\omega$, where $\bar{\Theta}(F)$ is the pinch parameter about which the oscillations would occur at frequency $\omega$. This optimization procedure is described in References [16] and [21]. Operational constraints on $\omega$ lie in the range 5 to $500 \mathrm{kHz}$, although consideration of the shell electrical penetration time forced an upper imit of $5 \mathrm{kHz}$, and a lower limit of $2 \mathrm{kHz}$ was set by temporal characteristics of sawtooth oscillations [40]. Ideally, $\bar{\Theta}$ is selected as the point of minimum plasma resistance $(\bar{\Theta} \simeq 1.45$, Figure 2.3-3). Maximum OFCD power, however, required $V_{\theta}$ to be maximized which forced deeper reversal, larger $\bar{\theta}$ (more negative $F$ ), increased plasma resistance (Figure 2.3-3), and required more power. Lastly, as previously noted, the maximum OFCD effect is expected for $\delta \simeq \pi / 2$.

High-power ( $\sim 40 \mathrm{MVA}$ ) OFCD tests on $I_{\phi}=180$ to $200 \mathrm{kA}$ discharges were first performed and the results are given in Figure 2.3-25. The real rms power input amounts to $\sim 20 \mathrm{MW}$ compared to $7 \mathrm{MW}$ for the DC power input. "Anti-OFCD" conditions, wherein helicity is drawn out from the plasma at an accelerated rate, could be obtained if $\delta=-\pi / 2$. These high-power OFCD tests showed an increasing plasma resistance, total radiation power, and metal line radiation which, together, indicate a strong plasma-wall interaction. A slight decrease in $I_{\phi}$ was seen because of increased plasma resistance related to increased impurity content. Calculations indicated that the adverse effect of the plasma-wall interaction was comparable to any expected OFCD current enhancement.

The low-power OFCD tests ( $\sim 7 \mathrm{MVA}, I_{\phi} \simeq 60$ to $70 \mathrm{kA}$ ), also shown in Figure 2.3-25, were conducted to reduce the extent of the increase in plasma resistance caused by the plasma-wall interaction. These ramped discharges, however, were at lower temperature and, hence, higher plasma resistance. In this case, no substantial increase in plasma resistance was observed between the OFCD and DC discharge. With optimal phasing $(\delta \simeq \pi / 2)$, an approximately $5 \%$ increase in poloidal fux was observed when OFCD was applied. Futhermore, the amplitude and phase of the on-axis oscillation of the toroidal field compared to that in the edge-plasma region were what would be expected if the mean magnetic fields remained in a quasi-relaxed state, as described by a modified BFM $[17,89]$. 


\section{Low Power}

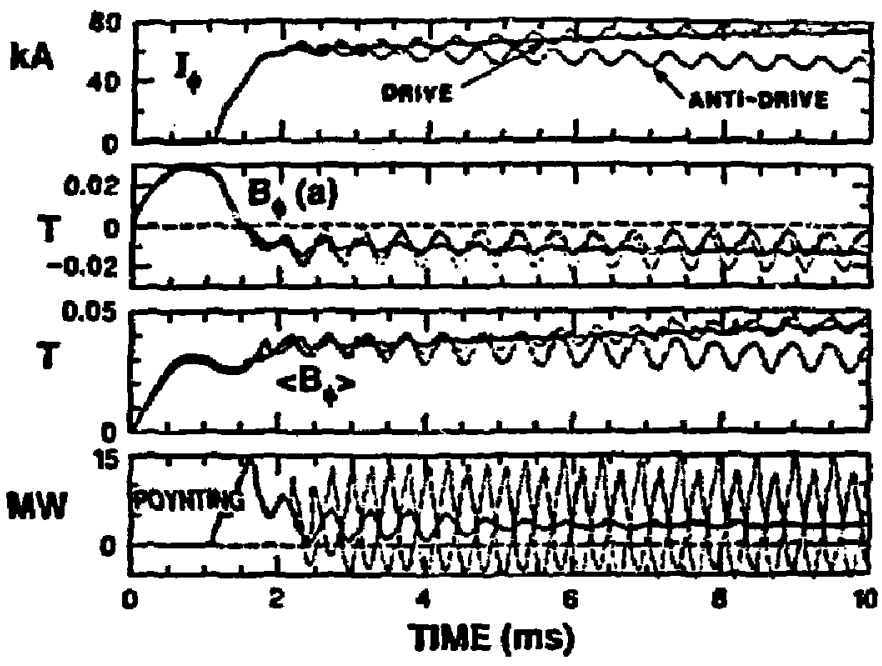

\section{High Power}

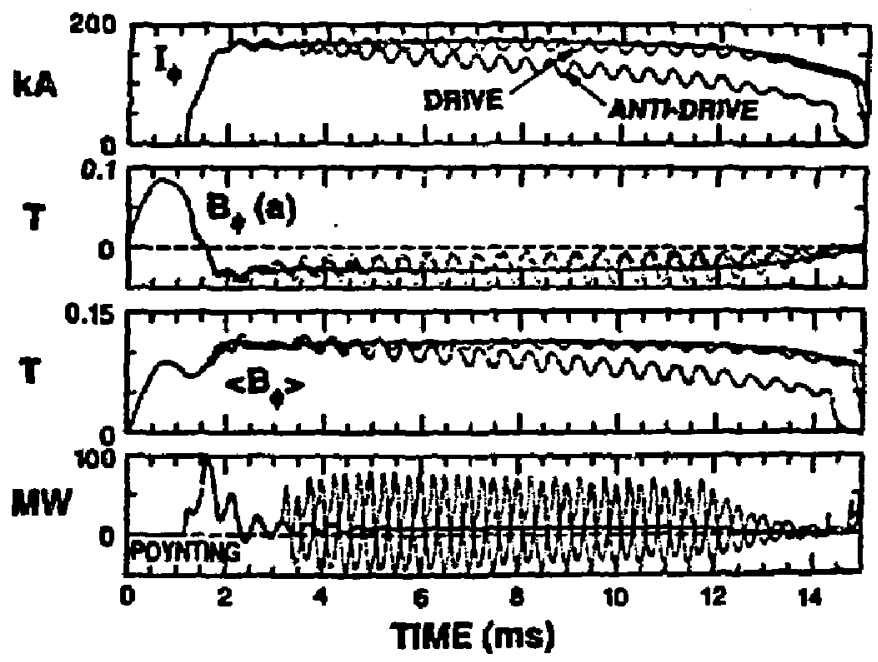

Figure 2.3-25. The OFCD results from ZT-40M $[16,21]$ for low-power $(\sim 7 \mathrm{MVA}$, $I_{\phi}=60$ to $\left.70 \mathrm{kA}\right)$ and high-power $\left(\sim 40 \mathrm{MVA}, I_{\phi}=180\right.$ to $\left.200 \mathrm{kA}\right)$ tests. 
While a clear demonstration of substantial current drive by OFCD must await RFPs operating with hotter plasmas and reduced wall interaction [24], the strong dependence of the plasma response on 6 and the spatial and temporal behavior of the mean magnetic fields are in generkl agreement with magnetic helicity models and simulations.

\subsection{P. Operation with Resistive Shells}

Since the confinement in an ohmically heated discharge is related to the plasma resistance $\left(x_{1}=g_{\text {orm. }} \eta / \mu_{0} \beta_{0}\right)$, the key to increased $\tau_{n}$ and reduced $\chi_{0}$ is the reduction in plasma resistivity through increased plasma temperature and reduced wall interactions. Improved plasma performance is expacied if the magnetic-helicity dissipation occurring in the edge-plasma region can be significantly reduced. For a number of present-day RFP experiments, a better control of the plasma position through an externally applied vertical field has led to inproved plasma parameters and extended discharge durations. Increased external concrol on the plasma, however, requires thinner stabilizing shells.

Historically, RFP experiments have closely surrounded the plasme with thick conducting shells to stabilize against kink instabilities $[9,33,38]$. The czucial trade-off between the desire and/or need for active control of the plasma position and the plasma-wall interaction on the one hand, and the control of instabilities to a level that also minimizes the plasma-wall interaction on the other (both being diectly but inversely dependent upon the thickness of the stabilizing sheij represents a crucial issue for the next major RFP experiments [24] as well as for reactors.

Table 2.3-II and Figure 2.3-26 show that the claracteristic time for vertical-field or shell diffusion in most present day KFPs is greater than or comparable to typical discharge times. Those experiments that apply an external vertical field, $B_{V}$, do so in a preprogrammed mode; active feedback of $B_{V}$ is yet to be done in any RFP experiment. The impact of this preprogramed vertical field on the plasma performance can be significant $[12,15,51,52]$. The results from the most recent experiments on OHTE [90] are given in Figure 2.3-26, which shows the time dependence of the plasma current and radical displacement for a number of vertical-field conditions. The plasma-position control indicated on this figure was made possible by decreasing the shell time constant from 25 to $1.5 \mathrm{~ms}$ [91]. There was no change in the gross MHD modes whan operating with the more resistive shell, although linear MHD theory predicts RFP plasmas surrounded by a resistive shell should be unstable [33]. A toroidally localized kink mode was observed [92]. This mode perhaps resulted from nonlinear coupling of internal kink modes and seemed to grow initially and then self-heal. If in fact a relatively resistive shell can 
Table 2.3-II.

VERTICAL-FIFLD TIME CONSTRAINTS FOR A NUMBER OF RFP EXPERIMENTS

\begin{tabular}{lccccc}
\hline \multicolumn{1}{c}{ Device } & $R_{T}(\mathrm{~m})$ & $r_{p}(\mathrm{~m})$ & $I_{\phi}(\mathrm{kA})$ & $\tau_{v}(\mathrm{~ms})^{(a)}$ & $\tau_{D}(\mathrm{~ms})^{(a)}$ \\
\hline Reversatron & 0.50 & 0.08 & 25. & 0.004 & 0.28 \\
TPE-1RM15 & 0.50 & 0.09 & 130. & 100. & 7. \\
ETA-BETA-II & 0.65 & 0.125 & 150. & 8. & 2. \\
HBTX1B & 0.80 & 0.26 & 320. & $80 . .^{(f)}$ & 10. \\
ZT-40M & 1.15 & 0.20 & 400. & 65. & 38. \\
OHTE & 1.24 & 0.20 & 500. & $1.5^{(b)}$ & 14. \\
CPRF/ETH(c) & 2.40 & 0.40 & $4,000$. & $\sim 50 .(d)$ & $\sim 800$. \\
RFX ${ }^{(e)}$ & 2.00 & 0.48 & $2,000$. & 300. & $\sim 250$. \\
TITAN & 3.80 & 0.60 & $18,000$. & 10. & $\infty$ \\
& & & & & \\
\hline
\end{tabular}

(a) Characteristic resistive shell time for vertical-field diffusion, $\tau_{v}=\mu_{0} r_{0} \delta_{v} /\left(2 \eta_{0}\right)$, where $r_{0}$ is the shell minor radius and $\delta_{s}$ is the shell thickness.

(b) Decreased from an original value of $25 \mathrm{~ms}[91,92]$.

(c) Planned experiments.

(d) Original design was $250 \mathrm{~ms}$.

(e) Maximum discharge time.

(f) Plans exist to install a $\tau_{v}=2$-ms shell in early 1988. 

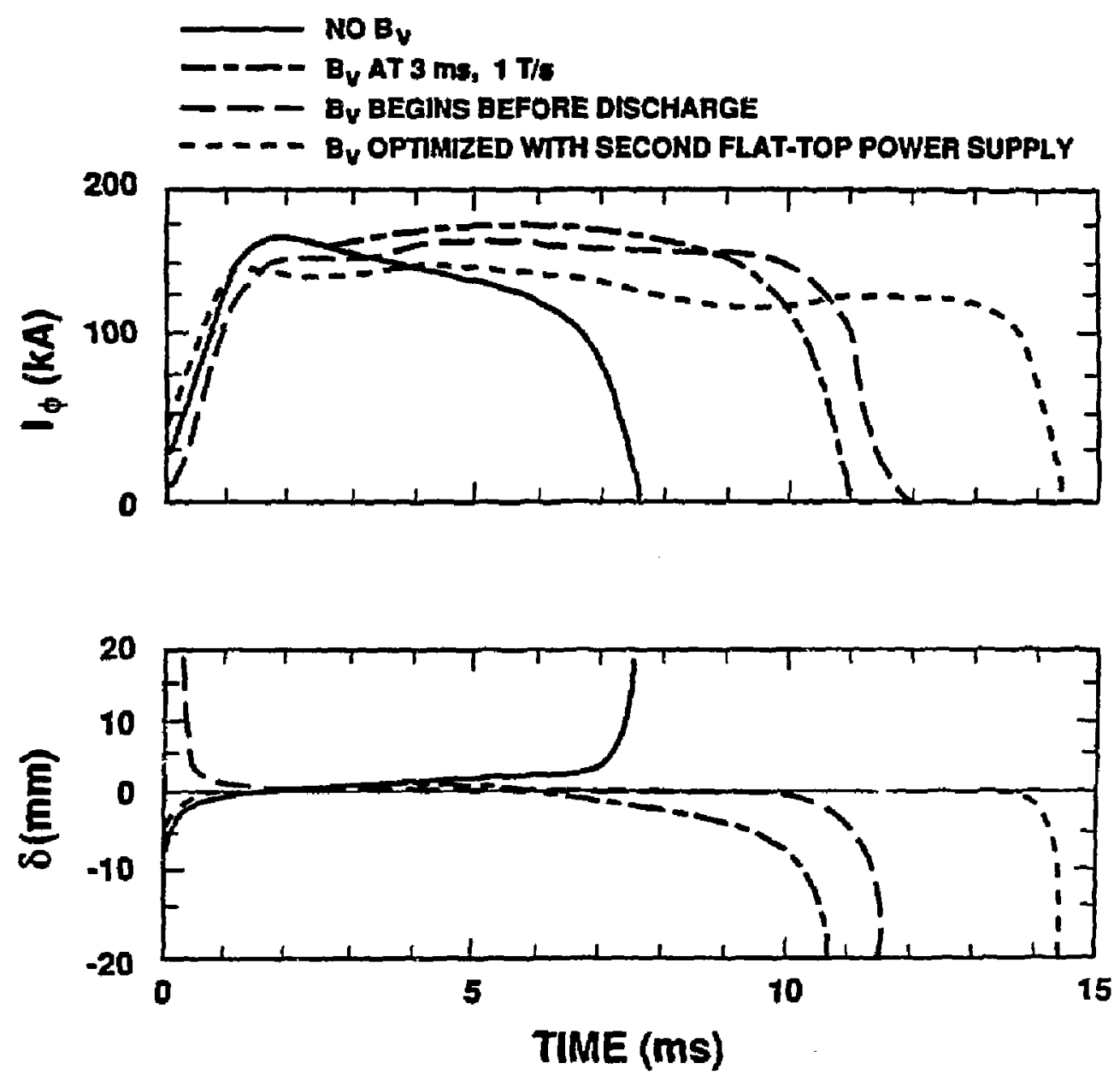

Figure 2.3-26. Impact of vertical field on OHTE discharges carried out in resistive shells with $\tau_{v}=1.5 \mathrm{~ms}[90]$. 
provide a boundary that is adequately stabilizing, major design simplification and optimization for both the next generation RFPs and reactors may be possible. Field-error constraints become less important. Also, active control of plasma position should reduce the plasma-wall interaction, edge-plasma dissipation, and the loop voltage, and should thereby increase the energy confinement time [92]. This trend towards $\tau_{D}>\tau_{v}$ and the ability to exert more external control on the plasma is reflected in the RFX and ZTH experiments (Figure 2.3-27), and is a major step towards reactor-relevant operation of RFPs.

The Reversatron experiment [93] is pursuing the limits of RFP operation with virtually no conducting shell (Table 2.3-II). This device is somewhat small in size and is subject to large field errors, but nevertheless the Reversatron has the flexibility to operate as an RFP or as a high-aspect-ratio spheromak (i.e., $F \rightarrow 0$ ). Shell-less discharges in both RFP and spheromak configurations are terminated by global MHD modes with toroidal mode numbers $n=1$ and 2. Depending on the vertical-field index, $n^{\prime}$, this shellless RFP is found to be unstable to the $n=1$ tilt or shift modes. The growth time scales for the "rigid" (vertical) shift and tilt modes, respectively, were shown to scale as follows [94]:

$$
\begin{aligned}
& \left(\frac{1}{\gamma}\right)_{S F T}=\left(\frac{2 A^{2}}{\left|n^{\prime}\right| \Lambda-1}\right) \tau_{v} \simeq A^{2} \tau_{\nu}, \\
& \left(\frac{1}{\gamma}\right)_{T L T}=\left(\frac{4}{\pi}\right)^{2}\left(\frac{A^{2}}{\Lambda\left|1-n^{\prime}\right|}-1\right) \tau_{v},
\end{aligned}
$$

where $A=\ln (8 A)+\ell_{i} / 2+\beta_{\theta}-3 / 2, n^{\prime}=-\left(R_{T} / B_{V_{0}}\right)\left(\partial B_{V} / \partial R\right)$, and $A=R_{T} / r_{p}$ is the plasma aspect ratio, with $|n| \Lambda / 2 \simeq 1$. On the basis of this simplified theory, the OHTE discharges given on Figure 2.3-26 have not reached a point where these instabilities should be seen $\left(\tau_{v}=1.5 \mathrm{~ms}, A=6.3\right.$, or $\left.1 / \gamma \simeq 60 \mathrm{~ms}\right)$.

In summary, considerable experimental and theoretical work is required to resolve the role of the close-fitting, stabilizing shell that ideal-MHD theory has historically demanded for the RFP. The longer-pulsed, higher-current, higher-power-density RFPs of the future, however, must operate with active control of the plasma position to achieve a better control of the edge-plasma conditions and better confinement. The first steps in this direction are being taken by the new OHTE and Reversatron experiments, but considerably more remains to be done in this crucial area. 


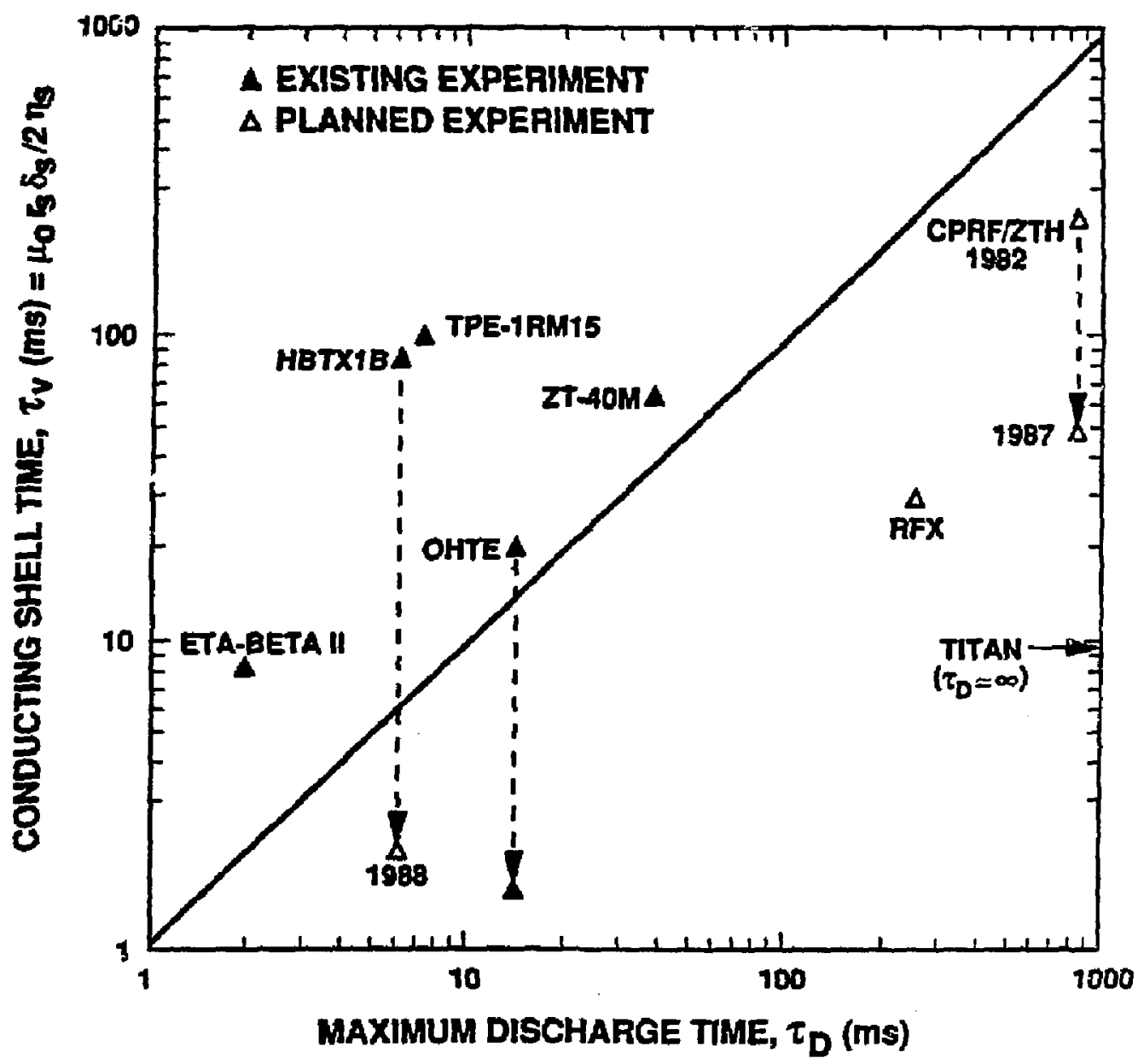

Figure 2.3-27. Relationship between shell and discharge times for a range of existing and planned RFP experiments. 


\subsection{SUMMARY}

The principles of the RFP confinement concept were discussed and the experimental data base was briefly reviewed. This data base is not as extensive as that of tokamaks and, therefore, requires a larger extrapolation to reactor-relevant regimes. Modern RFP experiments such as those of Table 2.3-I, however, have demonstrated the robuttness of the RFP dynamo and an emerging commonality of the basic physical processes operative in RFPs.

The key physics requirements and uncertainties for a RFP reactor include heating, transport, plasno-wall interaction, current drive, and impurity contral and particle exhaust with pumped limiters or magnetic divertors. The largest uncertainties in the existing RFP data base remain in the confinement physics and, in particular, in the mechnism, magnitude, and the regional extent of cross-field transport in the near-minimumenergy-state RFP configuration. Experimento with higher currents (and possibly higher current densities) and variable plasma size are needed to distinguish between possible scaling laws.

The modern RFP experiments are physically small, but operate close to ohmic-power and current densities expected for the reactor. These experiments, therefore; can be strongly influenced by plasma-wall interaction. The increased particle and heat load on the firet wall and impurity-control system, and the need to control field errore and the plasma-wall interaction in general, also represent major challenges for the next-atep multi-mega-ampere experiments [24]. Data from these next-generation experiments are expected in the early 1990s. These data are of the utmost importance in resolving key physics requirements and uncertainties for RFP reactors. Furthermore, these next-step experiments can provide valuable technc'ugical insight for devining a development path towards economically and technologically attractive RFP fusion reactors with strong safety and environmental attributes. 


\section{REFERENCES}

[1] F. Najmabadi, N. M. Ghoniem, R. W. Gonn, et al., "The TITAN Reversed-Field Pinch Reactor Study, Scoping Phase Report," joint report of University of California Los Angeles, General Atomics, Los Alamos National laboratory, and Rensselaer Polytechnic Institute, UCLA-PPG-1100 (1987).

[2] R. W. Conn (chairman and Ed.), "Magnetic Fusion Advisory Committee Panel X Report on High Power Density Fusion Systems," (May 8, 1985).

[3] J. Sheffield, R. A. Dory, S. M. Cohn, J. G. Delene, L. F. Parsley, et al., "Cost Assessment of a Generic Magnetic Fusion Reactor," Oak Ridge National Laboratory report ORNL/TM-9311 (1986) 103.

[4] R. A. Krakowski, R. L. Miller, and R. L. Hagenson, "The Need and Prospect for Improved Fusion Reactors," J. Fusion Energy 5 (1986) 213.

[5] R. A. Krakowski, R. L. Hagenson, N. M. Schnurr, C. Copenhaver, C. G. Bathke, R. L. Miller, and M. J. Embrechts, "Compact Reversed-Field Pinch Reactors (CRFPR)," Ntclear Eng. Design/Fusion 4 (1986) 75.

[6] H. A. Bodin, R. A. Krakowski, and O. Ortolani, "The Reversed-Field Pinch: from Experiment to Reactor," Fusion Technol. 10 (1986) 307.

[7] R. L. Hagenson, R. A. Kiakowski, C. G. Bathke, R. L. Miller, M. J. Embrechts, et al., "Compact Reversed-Field Pinch Reactors (CRFPR): Preliminary Engineering Considerations," Los Alamos National Laboratory report LA-10200-MS (1984).

[8] C. Copenhaver, R. A. Krakowski, N. M. Schnurr, R. L. Miller, C. G. Bathke, et al., "Compact Reversed-Fie'd Pinch Reactors (CRFPR)," Los Alamos National Laboratory report LA-10500-MS (1985).

[9] H. A. B. Bodin and A. A. Newton, "Review Paper: Reversed-Field Pinch Research," Nucl. Fusion 20 (1980) 1255.

[10] D. A. Baker and W. E. Quinn, "The Reversed-Field Pinch" FUSION I, Purt A, Edward Teller (Ed.), Academic Press, NY (1981) p. 438.

[11] J. B. Taylor, "Relaxation of Toroidal Plasma and Generation of Reversed Magnetic Field," Phys. Lett. 33 (1974) 1139. 
[12] H. A. B. Bodin, "Reversed-Field Pinch: Status and Trends," Plasma Phys. and Cont. Fusion 28 (1987) 1297.

[13] V. Antoni, D. Merlin, S. Ortolani, and R. Paccagnella, "MHD Stability Analysis of Force-Free Reversed-Field Pinch Configurations," Nucl. Fusion 20 (1987) 1711.

[14] J. A. Phillips, D. A. Baker, R. F. Gribble, and C. Munson, "Start-up of ReversedField Pinches and Current Ramping Using Dynamo Action," submitted to Nucl. Fusion (1987).

[15] R. S. Massey, R. G. Watt, P. G. Weber, G. A. Wurden, D. A. Baker, C. J. Buchenauer, et al., "Status of the ZT-40M RFP Experimental Program," Fusion Technol. 8 (1985) 1571.

[16] G. A. Wurden, K. F. Schoenberg, M. M. Pickrell, and the RFP Experimental Team, "RFP Experiments: Results From ZT-40M and ZT-P," in Proc. Int. School of Plasma Phys. Course and Workshop on Physics of Mirrors, Reversed-Fielc: Pinches, and Compact Tori, Varenna, Italy (September 1987).

[17] M. K. Bevir and J. W. Gray, "Relaxation, Flux Consumption and Quasi Steady State Pinches," Proc. RFP Theory Workshop, Los Alamos National Laboratory report LA-8944-C (January 1982) 176.

[18] C. W. Barnes, J. C. Fernandez, I. Henins, H. W. Hoida, T. R. Jarboe, S. O. Knox, G. J. Marklin, and K. F. McKenna, "Experimental Determination of the Conservation of Magnetic Helicity from the Balance Between Source and Spheromak," Phys. Fluids 29 (1986) 3415.

[19] K. F. Schoenberg, C. J. Buchenauer, R. S. Messey, J. G. Melton, R. W. Moses, R. A. Nebel, and J. A. Phillips, " $F-\Theta$ Pumping and Field Modulation Experiments on a Reversed Field Pinch Discharge," Phys. Fluids 27 (1984) 548.

[20] K. F. Schoenberg, C. P. Munson, D. A. Baiker, R. F. Gribble, R. W. Moses, P. G. Weber, R. A. Nebel, and R. F. Scardovelli, "Oscillating-Field Current Drive Experiments on the ZT-40M RFP," Bthl. Am. Phys. Soc. 31 (1986) 1547.

[21] K. F. Schoenberg, J. C. Ingraham, C. P. Munson, P. G. Weber, D. A. Baker, R. F. Gribble, et al., "Oscillating-Field Current Drive Experiment in ZT-40M Revereed Field Pinch," submitted to Phys. Fluids (1988).

[22] V. D. Shafranov, "Plasma Equilibrium in a Magnetic Field," Reviews of Plasma Physics 2, Consultant Bureau, New York (1966) 103. 
[23] V.S. Mukhovatov and V. D. Shafranov, "Plasma Equilibrium in a Tokamak," Nucl. Fusion 11 (1971) 605.

[24] D. B. Thomson (Ed.), Proc. Int. Workshop on Engineering Design of Next Step Reversed Field Pinch Devices, Los Alamos National Laboratory (July 13-17, 1987).

[25] B. B. Kadmontsev, "Disruptive Instabilities in Tokamaks," Sov. J. Plasma Phys. 1 (1975) 389.

[26] T. R. Jarboe and B. Alper, "A Model for Loop Voltage of a Reversed-Field Pinch," Phys. Fluids 30 (1987) 1177.

[27] H. Y. W. Tsui, "Helicity Transport and Anomalous Resistance in Reversed-Field Pinch," in Proc, 14th Ezumean Conf. on Cont. Fusion and Plasma Phys. Madrid, Spain (June 22-26, 1987).

[28] A. R. Jacobsen and R. W. Moses, "Non-local DC Electrical Conductivity of a Lorentz Plasma in a Stochastic Magnetic Field," Phys. Rev. A 29 (1984) 3335.

[29] R. W. Moses, K. F. Schoenberg, and D. A. Baker, "Empirical Modelling and Dependence of RFP Loop Voltage on Edge Plasma Conditions," private communication, Los Alamos National Laboratory (December 1987).

[30] I. B. Bernstein, E. A. Frieman, M. D. Kruskal, and R. M. Kulsrud, "An Ene:gy Principle for Hydromagnetic Stability Problems," Proc. Royal Soc. 244 (1958) 17.

[31] B. R. Suydam, "Stability of Linear Pinch," Proc. 2nd United Nations Int. Conf. on the Peaceful Uses of Atomic Energy, Geneve, Switzerland (September 1958), United Nations, Geneva 31 (1958) 157.

[32] C. Mercier, "A Necessary Criterion for the Hydrodynamic Stability for an Axisymmetric Plasma," Nucl. Fusion 1 (1960) 47.

[33] D. C. Robinson, "High- $\beta$ Diffuse Pinch Configuraticns," Plasma Phys. 13 (1971) 439.

[34] I. H. Hutchinson, "Plasma Resistance Studies in HBTX1A Reversed-Field Pinch," in Proc. 11th European Conf. on Cont. Fusion and Plasma Phys., Aachen, F, R. G. (September 1984).

[35] R. G. Watt and E. M. Little, "RFP Energy Evolution and Containment in the Presence of Giant Sawteeth," Phys. Fluids 27 (1984) 784. 
[36] D. C. Robinson, "Tearing-Mode-Stable Diffuse-Pinch Configurations," Nucl. Fusion 18 (1978) 939.

[37] J. W. Connor and J. B. Taylor, "Resistive Fluid Turbulence and Energy Confinement," Phys. Fluids 27 (1984) 2676.

[38] A. D. Merlin, S. Ortolani, and R. Paccagnella, "Free Boundary MHE Stability Gonfigurations," in Proc. 14th European Conf. on Cont. Fusion and Plasma Phys., Madrid, Spain (June 22-26, 1987).

[39] S. Ortolani, in Proc. Int. School of Plasma Phys. Course on Mirror-based and FieldReversed Approaches to Magnetic Fusion, Varenne, Italy' (1983).

[40] R. G. Watt and R. A. Nebel, "Sawteeth, Magnetic Disturbances, and Magaetic Flux Regeneration in the Reversed-Field Pinch," Phys. Fluids 26 (1983) 1168.

[41] D. D. Schnack, E. J. Caramana, and D. C. Barnes, "Simulation of Current Ramp Operation in the Reversed- Field Pinch," Bull. Am. Phys. Soc, 30 (1985) 1399.

[42] M. G. Rusbridge, "A Model of Field Reversal in the Diffuse Pinch," Flasma Phys. 18 (1977) 499.

[43] M. G. Rusbridge, "Fjeld-Line Reconnection and Density Fluctuations in ZETA," Plasma Phys. 22 (1980) 331.

[44] Z. G. An et al., "Role of Multiple Helicity Non-Linear Interaction of Tearing Modes in Dynamo and Anomalous Thermal Transport in Reversed Field Pinch," Proc. 10th Int. Conf. Plasma Phys. and Cont. Nucl. Fusion Res., London, U. K. (September 1984), IAEA, Vienna, 2 (1985) 231.

[45] N. T. Gladd and N. A. Krall, JAYCOR report J530-84-221 (1984).

[46] S. Briguglio, F. Romanelli, and G. Vlad, "Current Driven Drift Waves as a Possible Mecharism for Dynamo Effect and Transport in Reversed Field Pinches," Nucl. Fussion 26 (1986) 1293.

[47] D. C. Robinson and R. E. King, "Factors Influencing the Period of Improved Stability in ZETA," Proc. Ind Int. Conf. on Plasma Phys. and Cont. Nucl. Fusion Res., Novoribirsk, U. S. S. R. (Aug. 1968), IAEA, Vienne, 1 (1969) 263.

[48] A. Buffa et al., "First Results from the ETA-BETA-II RFP Experiment," Prac. 9th European Conf. on Cont. Fusian and Plasma Phys., Oxford, U. K. (September 1979), Culham Laboratory (1979) 544. 
[49] V. Antoni et al, "Studies on High- Density RFP Plasmas in the ETA-BETA-II Experiment," Proc. 9th Int. Conf. on Plasma Phys. and Cont. Nucl. Fiusion Res., Baltimore, U. S. A. (September 1982), IAEA, Viemna, i (1983) 619.

[50] V. Antoni et al., "Reversed Field Pinch Plasma with Current Flat-Top in ETA-BETA-II," Proc. 10th Int. Conf. on Plasma Phys. and Cont. Nucl. Fresion Res., London, U. K. (September 1984), IAEA, Vienna, 2 (1985) 487.

[51] K. Ogewa, Y. Maejima, T. Shimada, Y. Hirano, P. G. Carolan, C. W. Gowers, A. Nagata, H. Ashida, T. Amano, and Y. Kondoh, "Experimental and Computational Studies of Reversed-Field Pinch on TPE-1R(M)," Pnoc. 9th Int. Canf. on Plasma Phys, and Cont. Nucl. Fusion Res., Baltimore, U. S. A. (September 1982) IAEA, Vienna, 1 (1983) 575.

[52] Y. Hirano, T. Shimada, Y. Maejima, and K. Ogawa, "Improved Stability Period in High-Current-Density Operation of Reversed-Field Pinch of ETL-TPE-1R(M)," Nucl. Fusion 22 (1982) 1613.

[53] D. A. Baker, M. D. Bausman, C. J. Buchenauer, L. C. Burkhardt, G. Chandler, and J. N. DiMarco, "Performance of the ZT-40M Reversed-Fi-ld Pinch with an Inconel Liner," Proc. 9th Int. Conf. on Plasma Phys. and Cont. Nucl. Fusion Reg,, Baltimore, U. S. A. (September 1982) LAEA, Vienna, 1 (1983) 587.

[54] D. A. Baker, C. J. Buchenauer, L. C. Firfthardt, E. J. Caramana, J. N. DiMarco, J. N. Downing, et al., "Experimental and Theoretical Studies of the ZT-40M Reversed-Field Pinch," in Proc. 10th Int. Conf. on Plasma Phys. and Cont. Nucl. Fusion Res.: London, U. K. (September 1984) IAEA, Vienna (1985).

[55] H. A. B. Bodin et al., Proc. 9th Int. Conf. on Plasma Phys. and Cont. Nucl. Fusion Res., Baltimore, U. S. A. (September 1982), IAEA, Vienna, 8 (1983) 641.

[56] P. Carolan et al., "New Results from HBTX1A Reversed Field Pinch," Proc. 10th Int. Conf. on Plosma Phys. and Cont. Nucl. Fusion Res. London, U. K. (September 1984), LAEA, Vienna, 2 (1985) 449.

[57] T. Tamano et al., "Pinch Experiments in OHTE," Proc. 9th Int. Conf. on Plasma Phys. and Cont. Nucl. Fusion Res., Baltimore, U. S. A. (September 1982), IAEA, Vienna, 1 (1983) 609.

[58] T. Tamano, W. D. Bard, T. N. Carlstrom, C. Chu, B. Curwe, R. K. Fisher, et al., "High Current, High Beta Toroidal Pinch Experiment in OHTE," in Proc. 1Oth Int. 
Conf. on Plasma Phys. and Cont. Nucl. Fusion Res., London, U. K. (September 1984) IAEA, Vienna (1985).

[59] C. G. Bathke, R. A. Krakowski, R. A. Krakowski, and R. L. Miller, "A DT Neutron Source Based on the Reversed Field Pinch," Proc. 12th IEEE Symp. ro Fision Eng., Monterey, CA (October 1987) 829.

[60] G. Maiesani (Chair.), Trilatemal Workshop to Promote International Cooperaion on RFP Research, Padova, Italy (September 1987) Instituto Ges ionizzati del C.N.R. report IGI 87/03 (1987).

[61] A. A. Newton and P. G. Noonan, "Controlled Termination of Reversed-Field-Pinch Discharges," Nucl. Instr. and Methods A245 (1986) 167.

[62] J. A. Phillips, "Improved Start-up of Reversed-Field Pinches Using Current Ramping with Toroidal Flux Generation," Bull. Am. Phys. Soc. 32 (1987) 1785.

[63] P. G. Noonen, C. G. Gimblett, and A. A. Noonan, "Plasma Biahavior During Programmed Current Decay and Ramping in the Reversed-Field Pinch," in Proc. 14th European Conf. on Cont. Fusion and Plasma Phys., Madrid, Spain (June 1987).

[64] B. Alper and H. Y. W. Tsui, "Effect of a Moveable Tile Limiter on the Loop Voltage in HBTX1B RFP," in Proc. 14th European Conf. on Cont. Fusion and Plasma Phys., Madrid, Spain (June 1987).

[65] J. A. Phillips, L. C. Burkhardt, A. Haberstich, R. B. Howrell, J. C. Ingraham, E. M. Little, K. S. Thomas, and R. G. Watt, "ZT-40M Current Ris time Study," Los Alamos National Laboratory repart LA-9717-MS (1983).

[66] R. G. Watt, G. A. Wurden, P. G. Weber, K. Buechl, and E. K. Spanos, "Pellet Injection on the ZT-40M Reversed-Field Pinch," Rev. Sci. Instr. 58 (1987) 1401.

[67] G. A. Wurden, P. G. Weber, R. G. Watt, C. P. Munson, T. E. Cayton, and K. Buchl, "Refueling and Density Control in the ZT-40M Reversed-Field Pinch." in Proc. Int. School of Plasma Phys, Course and Workshop on Physics of Mirrors, Reversed-Field Pinches, and Compact Tori, Varenna, Italy (September 1987).

[68] V. Antoni, E. Baseggio, L. Carraro, L. Flora, L. Gabellieri, and L. Giudicotti, " $D_{2}$ Pellet Injection in ETA-BETA-II Plasma," in Proc, 14th European Conf. on Cont. Fusion and Plasma Phys., Madrid, Spain (June 1987). 
[69] 1. H. Hutchinson et al., "The Structure of Magnetic Fluctuations in HBTX1A Reversed-Field Pinch," Nucl. Fustion 14 (1984) 59.

[70] V. Antoni and S. Ortolani, "Characteristic of the Magnetic-Field Fluctuations in the ETA-BETA-II Reversed Field Finch Experiment," Plasma Phys. 25 (1983) 799.

[71] D. Brotherton-Ratcliffe, C. G. Gimblett, and 1. H. Hutchinson, "Analysis of Internal Magnetic Fluctuations in HBTX1A Reversed Field Pinch," Oulham Laboratory report CLM-P742 (1985).

[72] R. J. LaHaye et al., "Measurements of Magnetic-Field Fluctuations in the OHTE Toroidal Pinch," Phys. Fluids 27 (1984) 2576.

[73] H. Y. W. Tsui, J. Cunnane, and D. E. Evans, "Magnetic Island Fluctuations in the HBTX1B Reversed-Field Pinch," in Proc. 14th Eturopean Conf. on Cont. Fusion and Plasma Phys., Madrid, Spain (June 1987).

[74] V. Antoni, P. DeSimone, P. Innocente, P. Martin, S. Martini, S. Ortclani, et al, "Fluctuation Studies on the ETA-BETA-II Experiment," in Proc. 14th European Conf. on Cont. Fusion and Fiasma Phys., Madrid, Spain (June 1987).

[75] G. A. Wurden, "Soft X-ray Array Results on ZT-40M Reversed Field Pinch," Phys. Fluids 27 (1984) 551.

[76] V. Antoni and S. Ortolani, "Magnetic Field Fluctuation Studies on ETA-BETA-II," Proc. of the rorkshop on Mirror-Based and Field-Reversed Approaches to Magnetic Fusion, Varenna (September 1983), Monobypia Franchi, Perugia, 2 (1984) 97.

[77] G. Wurden, private cummunication, Los Alamos National Laboratory (December 1987).

[78] B. Alper et al,, "RFP Confinement Studies in ETA-BETA-II," Proc. 12th European Conf. on Cont. Fusion and Plasma Phys., Budapest, Hungary (September 1985), European Phys. Soc. 1 (1985) 578.

[79] P. G. Weber et al., "Results from the Los Alamos RFP Experiments," Proc. 12th European Conf. on Cont. Fusion and Plasma Phys., Budapest, Hungary (September 1985), European Phys. Soc. 1 (1985) 570.

[80] T. Cayton, private communication, Los Alamos National Laboratory (1987). 
[81] M. M. Pickrell, J. A. Phillips, C. J. Buckenauer, T. Cayton, J. N. Downing, A. Haberstich, et al., "Evidence for a Poloidal Beta Limit on ZT-40M," Bull. Am. Phys. Soc. 29 (1984) 1403.

[82] A. R. Jacobsen and M. G. Rusbridge, "Observed Mechanism of Termination in ZT-40M Reversed-Field Pinch," Los Alamos National Laboratory report LA-9589MS (1982).

[83] R. Gross, Fusion Energy, Wiley and Sons, NY (1984) 118.

[84] K. F. Schoenberg, R. F. Gribble, and D. A. Baker, "Oscillating Field Current Drive for Reversed Field Pinch Discharges," J. Appl. Phys. 56 (1984) 2519.

[85] J. T. Hogan, Bull. Am. Phys. Soc. 31 (1986) 1548.

[86] J. M. Finn and T. M Antonsen, Jr., "Anomalous Current Penetration and Oscillating Current Drive in Tokarnaks," Phys. Fluids 30 (1987) 2450.

[87] M. Ono, G. J. Greene, D. Dajrrow, C. Forest, H. Park, and T. H. Stix, "Steady-State Tokamak Discharge via Helicity Injection," Phys. Rev. Lett. 50 (1987) 2165.

[88] W. A. Reass, R. F. Gribble, and C. F. Hammer, "Design Construction and Electrical Test Results of Dual Phase Controlled Multi-Megawatt Oscillators for OscillatingField Current Drive on ZT-40M," Proc. IEEE 11th Symp. Fusion Eng. (1985) 1214.

[89] K. F. Schoenberg, G. A. Wurden, P. G. Weber, J. C. Ingraham, C. P. Munson, et al., Proc. 11th Int. Conf. on Plasma Phys. and Cont. Nuci. Fusion Res., Kyoto, Japan (November 1986) IAEA, Vienne, 2 (1987) 423.

[90] S. Ortolani, V. Antoni, S. Martini, R. LaHaye, M. Schaffer, T. Tameno, and P. Taylor, "Reversed-Field Pinch Operation with a Thin Shell," in Proc. 14th European Conf. on Cont. Fusion and Plasma Phys., Madrid, Spain (June 1987).

[91] R. R. Goforth, T. N. Carlstran, C. Chu, B. Curwen, D. Grauman, P. S. C. Lee, et at., "Reversed-Field Pinch Experiment with a Resistive Shell," Nucl. Fusion 26 (1986) 414.

[92] T. Tamano, W. D. Bard, C. Chu, T. N. Carstram, R. R. Goforth, Y. Kondoh, et al., "Characteristics of Reversed-Field Pinch Plasmas with a Resistive Shell," in Proc. 11th Int. Conf. on P'asma Phys. and Cont. Nucl. Fusion Res., Kyoto, Japan (November 1986). 
[93] P. Schmid and S. Robertson, "Design of an Air-Core Reversed-Field Pinch," IEEE Trans. Plasma Sci. (June 1986).

[94] S. Robertson and P. Schmid, "Instability of a Reversed Field Pinch Without a Conducting Shell," private communication, University of Colorado, Boulder (1987). 


\title{
3. PARAMETRIC SYSTEMS STUDIES AND DESIGN-POINT SELECTION
}

\author{
Ronald L. Miller
}

Robert A. Krakowski 


\section{Contents}

3.i. INTRODUCTION $\ldots \ldots \ldots \ldots \ldots \ldots \ldots \ldots \ldots \ldots \ldots \ldots$

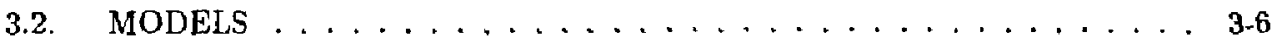

3.2.1. Plasna Physics Morel ... . . . . . . . . . . 3-6

3.2.2. Reactor Engineering Madel . . . . . . . . . . . 3-16

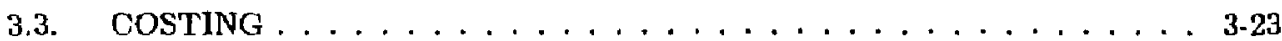

3.4. TITAN-I DESIGN FOINT $\ldots \ldots \ldots \ldots \ldots \ldots \ldots . \ldots \ldots$. . . . . . . . . .

3.4.1. Design Point Selection . . . . . . . . . . . . . . 3-32

3.4.2. Trade-off and Sensitivity Studies . . . . . . . . . . 3-43

3.5. TITAN-II DESIGN POINT . . . . . . . . . . . . 3-53

3.5.1. Designt-Point Selection . . . . . . . . . . . . 3-53

3.5.2. Trade-of and Sensitivity Studies $\ldots \ldots \ldots \ldots$ 3-57

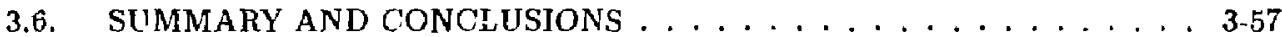

REFERENCES . . . . . . . . . . . . . . . . . . . 3-64 


\section{PARAMETRIC SYSTEMS STUDIES AND DESIGN-POINT SELECTION}

\subsection{INTRODUCTION}

The objective of the systems analysis activity is the systematic study and determination of plant operating parameters through economic analysis and optimization of the power station, emphasizing the performance of the fusion power core. The fusion power core (FPC) includes the plasma chamber, first wall, blanket, shield, coils, and associated structure. The reference design points are chosen to meet overall design goals of the TITAN study such as minimal cost of electricity (COE) and high mass power density (MPD). In addition to the reference design points, trade-off and sensitivity studies were performed to establish and characterize the "design window" for attractive RFP reactor operation.

A parametric systems analysis (PSA) conputer code is used for this study. This code was originally developed for use in the Los Alamos compact reversed-field pinch reactor (CRFPR) studies [1-3], and the cost data base was updated in the course of the Los Alamos modular stellarator reactor, MSR [4] and spherical torus reactor, AT'R/ST [5] studies. Further updates of the cost data base made in the course of the TITAN study are discussed in Section 3.3. Code models include steady-state surveys designed to assess sensitivities and trade-offs related to various TITAN operating configurations and assumptions. These models are benchmarked and colibrated against more detailed plasma physics and magnetics models to provide a framework for the overall design process (Figure 3.1-1).

The PSA code algorithm used in the CRFPR study (Figure 6.1 of $R$ _ierence [3]) has been modified to treat the equilibrium-field (EF) and ohmic-heating $(\mathrm{OH})$ coil sets separately, allowing the consideration of superconducting EF coils. As indicated on Figure 3.1-2, the PSA code identifies optimal reactor parameters for a specified net electric power, $P_{E}$, using a set of nested search loops centered on a convergence operation for the engineering Q-value, $Q_{E}=1 / \epsilon$, where $\epsilon$ is the recirculating power fraction. This innermost iteration procedure searches for the value of $Q_{E}$ that yields the specified $P_{E}$ for a given total coil thickness, $\delta_{c}$, as the split between the toroidal-field (TF) coil and $\mathrm{OH}$ coil geometry is varied. 


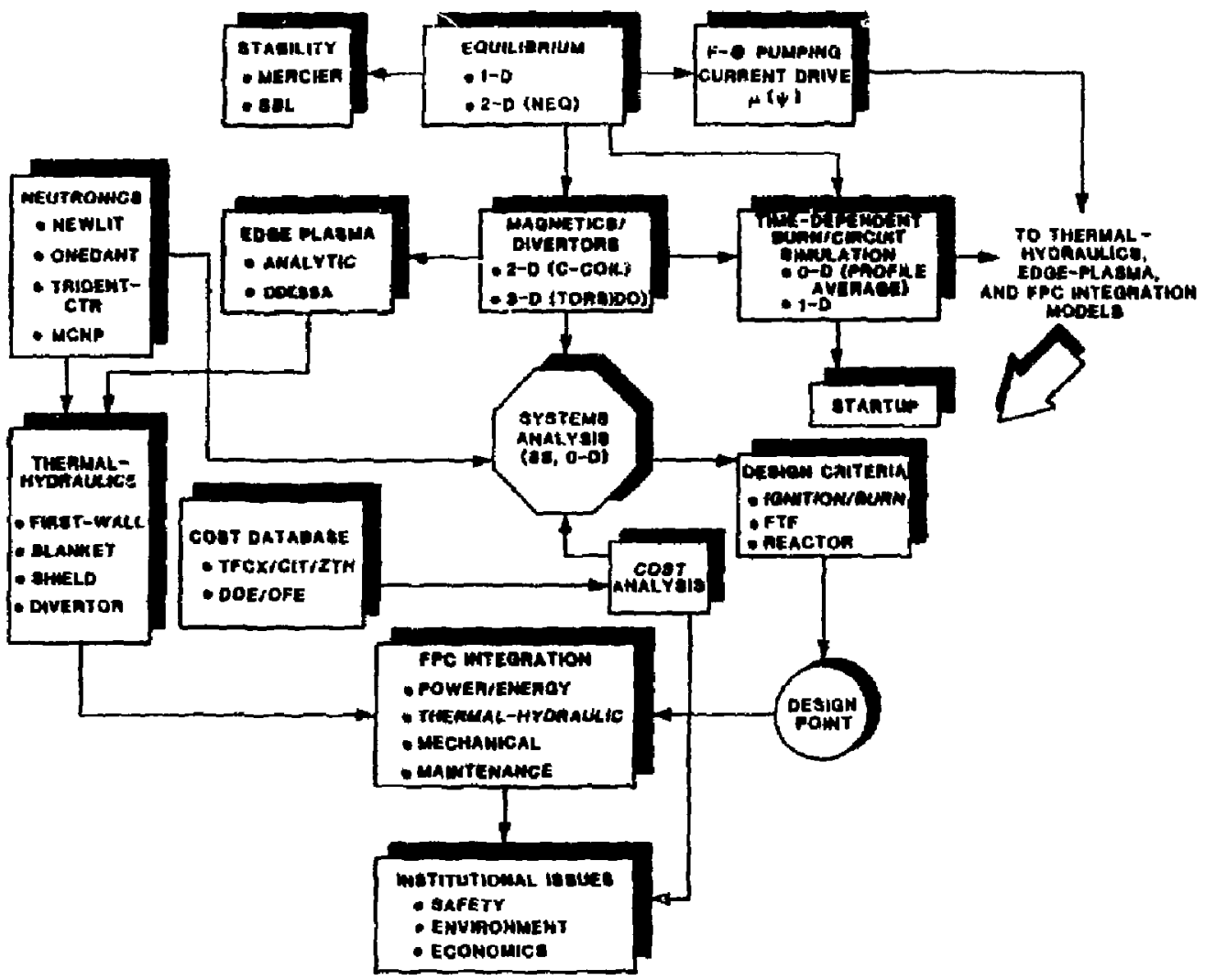

Figure 3.1-1. TITAN study approach organized by key plasma and nucleas engineering activities and interfaces. Some of the computer codes used in the TITAN study are NEQ [6,7], NEWLIT [8], ONEDANT [9], TRIDENT-CTR [10], MCNP [11], CGOIL [2], TORSIDO [4], and ODESSA [12]. 


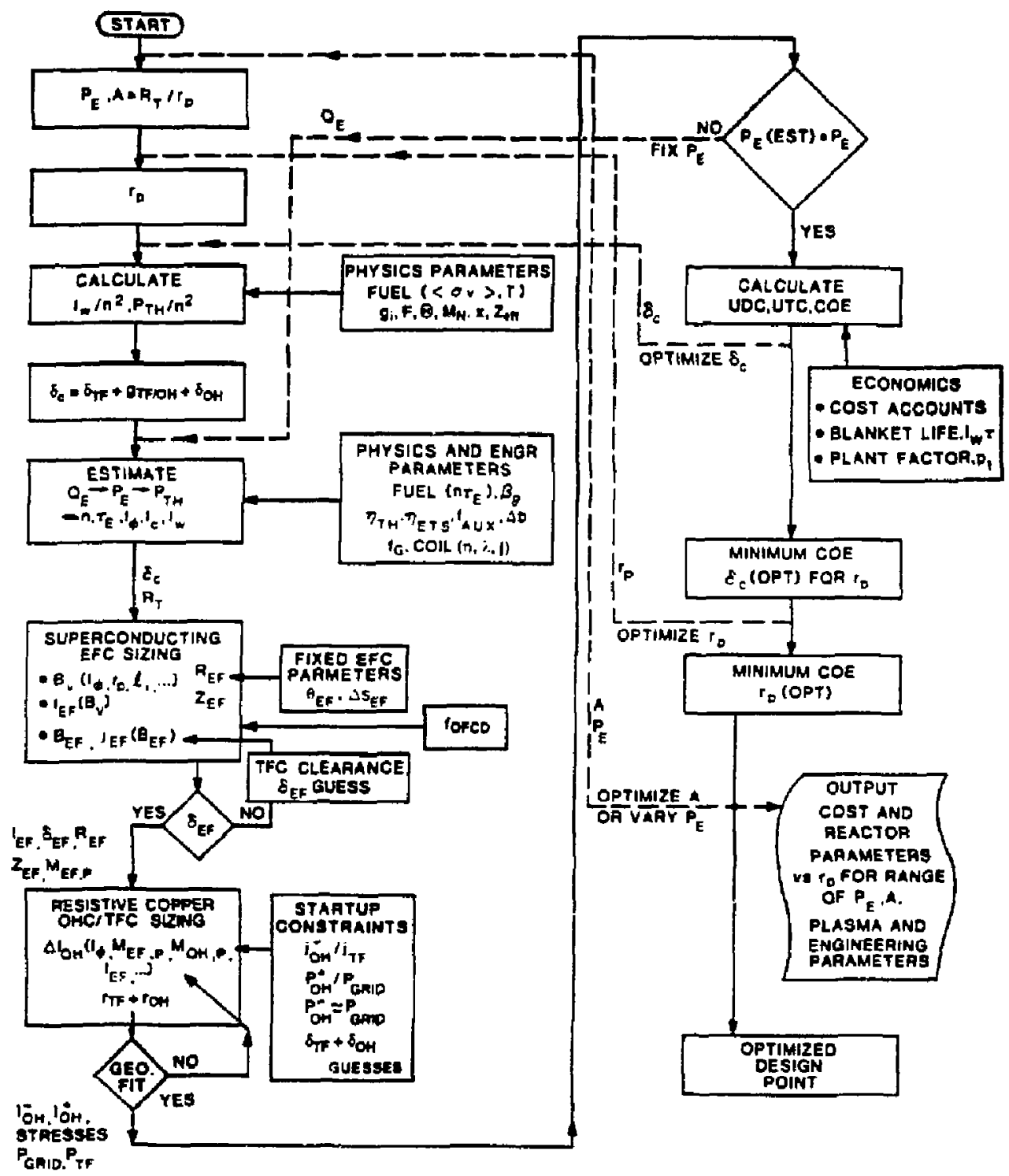

Figure 3.1-2. Parametric systems analysis (PSA) procedure used for the TITAN study, as adapted from CRFPR study methods [1-3]. 
First, for a fixed geometry (including plasma major and ninor radii and aspect tatio), the inner loop finds the total TF coil and $\mathrm{OH}$ coil thickness, $\delta_{c}$, which produces a minimum-COE design for the specified $P_{E}$. This procedure is repeated for different values of plasma minor radius; $r_{p}$, to determine a higher-order (lower) COE ninimum. Generally, $r_{p}$ is used as a display variable, with the respective ninimum-COE design corresponding to a particular value of $P_{E}$ and plasma aspect ratio, $A \equiv R_{T} / r_{p}\left(R_{T}\right.$ is the plasma major radius). The outermost loop then varies $A$ in search of an even lower minimum-COE system although, within realistic bounds, the optimum in $A$ is found to be relatively flat for the RFP. These fully cost-optimized design points are then examined as a function of $P_{E}$ and various physics, engineering, and econonic parameters and options. The results of this analysis identify preliminary design points which serve as the starting points for engineering analysis. As this subsequent analysis characterizes the global performance of various subsystems, the optional parameters of the PSA code are updated to provide an evolving, self-consistent picture of the TITAN design points.

The PSA model geometry is illustrated in Figure 3.1-3. A moderate aspect-ratio plasma is surrounded by a first-wall with radius, $r_{w}$. A conventional design fits a blanket and shield annulus around the first wall followed by a resistive copper-alloy TF coil set and a dominant resistive $\mathrm{OH}$-coil set. An EF-coil set could be either superconducting or normal conducting. The former option (used for TITAN) requires additional local radiation shielding. The integrated blanket coil (IBC) option [13] is used in TITAN-I (Section 3.4) which consolidates the TF-coil set with the blanket.

The PSA code initialization section, executed prior to the $\left(A, P_{E}\right)$ search loops, establishes the plasma physics conditions. A plasma density-weighted, volume-averaged temperature, $T\left(T_{e} \simeq T_{i}\right)$, and corresponding Lawson parameter, $n_{i} T_{E}$, are selected. The radial profiles of plasma temperature, density, and $\mu(r) \equiv \mu_{\mathrm{o}} \mathbf{j} \cdot \mathbf{B} / B^{2}$ are obtained from empirical fits to the results of 1-D RFPBURN plasma simulations (Section 5.3). The ratio of electron to ion density, $n_{\mathrm{e}} / \boldsymbol{n}_{\mathrm{i}}$, is calculated from an input value of $Z_{e f f}$.

Other input values include the poloidal beta, $\beta_{\theta} \simeq 2 \mu_{0} k_{B}\left(n_{t}+n_{i}\right) T / \bar{B}_{\theta}^{2}\left(r_{p}\right)$, the reversal parameter, $F=\bar{B}_{\phi}\left(r_{p}\right) /\left\langle B_{\phi}\right\rangle$, and a representative value of toroidal plasma current, $I_{\phi}$. Here $\mu_{o}$ is the permeability of free space, $k_{B}=1.602 \times 10^{-1 \theta} \mathrm{J} / \mathrm{keV}$ is the Boltzmanu constant, $\bar{B}_{\theta}(r), \bar{B}_{\phi}(r)$ denote, respectively, the flux-surface-averaged poloidal and toroidal magnetic field, and $\left(B_{\phi}\right\rangle$ is the volume-averaged toroidal fielci. From these input values, a self-consistent value of the pinch parameter, $\Theta=\bar{B}_{\theta}\left(r_{p}\right) /\left(B_{\phi}\right)$, and the profile enhancement (of fusion power, Bremsstrahlung, and olmmic lieating) factors, $g_{j}$, are computed using a numerical equilibrium solver (Section 5.2) and the assumed plasma density, temperature, and $\mu$ profiles. 
The magnitude of the plasma current, $I_{\phi}$, is adjusted within the $\left(A, P_{E}\right)$ search loops, as required. For a fixed value of $n_{i} \tau_{E}$, the ion density is adjusted to yield the required fusion power, $P_{F}$, and neutron wall load, $I_{w}$. For each particular geometric configuration, the cost-optimized confinement time, $\tau_{E}(\mathrm{OPT})$, is determined at the minimumCOE design point. These values of $\tau_{E}(\mathrm{OPT})$ are then compared with $\tau_{E}(\mathrm{PHYS}) \propto I_{\phi}^{\nu}$ (Section 3.2) to assess the accessibility of the tentative design point.

Section 3.2 summarizes the physics and engineering models used in the PSA code. Section 3.3 describes the costing algorithms. The TITAN-I and TITAN-II design points and sensitivities studies are presented in Sections 3.4 and 3.5, respectively. A summary of the results of the systems-studies activity is given in Section 3.6.

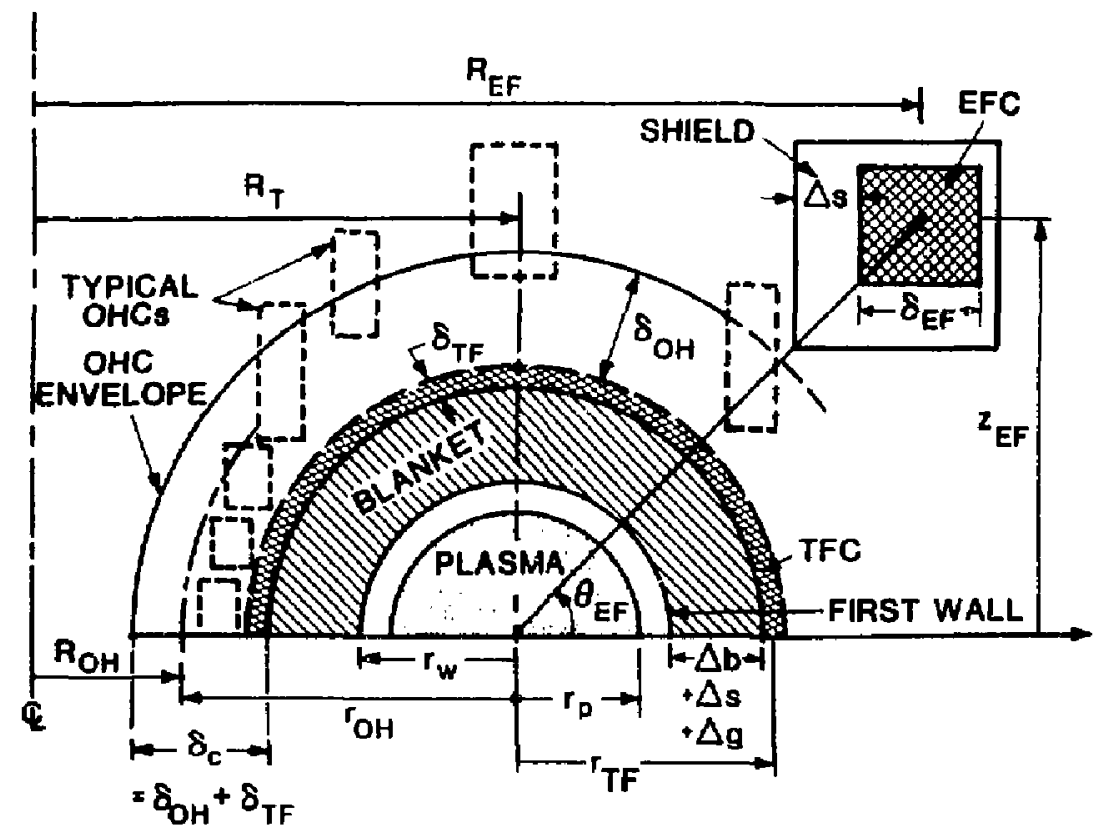

Figure 3.1-3. The FPC model used to optimize and perform sensitivity studies of TITAN reactors. The computational model loops through all FPC and plasma dimensions in search of minimum-COE designs. The IBC option [13] combines the TF-coil set with the blanket in TITAN-I. 


\subsection{MODELS}

\subsubsection{Plașma Physics Model}

An RFP plasma is confined by a combination of a poloital field, $B_{\theta}$, generated by a toroidal current, $I_{\phi}$, flowing in the plasna, and a toroidal field, $B_{\phi}$, produced partly by currents flowing in the plasma and partly by extermal coils. The distinguishing features of the RFP are: (a) $B_{\theta} \simeq\left|B_{\phi}\right|$ within the plasma, and (b) the toroidal field is reversed and mucl smaller in magnitude in the outer region with respect to the value on the axis. A cundamental property of the RFP is that the field configuration and the generation of the reversed toroidal field is a natural consequence of the relaxation of the plasma to a near-minimum-energy state. This relaxation process results in a close coupling of poloidal and toroidal circuits through the plasma and allows the use of oscillatingfield current drive (OFCD) for steady-state sustainment. The RFP edge safety factor, $q=r_{\mathrm{p}} B_{\phi} / R_{T} B_{\theta}$, is less than unity, creating the possibility of large plasma current density, strong ohmic heating, and low magnetic fields at coils. Typical values of poloidal beta, $\beta_{\theta}$, equal or exceed 0.20 , allowing high plasma DT-fusion power densities $\left(>70 \mathrm{MW} / \mathrm{m}^{3}\right.$ ). Energy confinement is assumed to scale as $\tau_{E} \propto I_{\phi}^{\nu} r_{p}^{2}$, where $\nu \simeq 1.0$. These inherent characterisitics of the RFP confinement concept lead to a high-power-density, steadystate plasma configuration with a relatively weak external toroidal field and promise an improved commercial reactor.

The TITAN systems model begins with a steady-state, point-plasma model, corrected for profile effects. It is convenient to define a plasma-filling froction, $x \equiv r_{p} / r_{w}$, for the circularized plasma in a toroidal chamber, where $r_{w}$ is the minor radius of the first wall and $r_{p}$ is the plasma-core radius (i.e., to the separatrix). The parameter $x$ is chosen to anticipute the first-wall geometry and scrape-off layer thickness $\left(r_{w}-r_{p}\right)$ in relating the volumetric fusion power to the average 14.1-MeV neutron first-wall load.

The average plasma DT-fusion (17.58 MeV/fusion) power density, $P_{F} / V_{p}$, is given by

$$
P_{F} / V_{P}\left(\mathrm{~W} / \mathrm{m}^{3}\right)=2.186 \times 10^{-12} g_{D \tau} n_{D} n_{T}\langle\sigma v\rangle_{D \tau},
$$

where $n_{D, T}$ is the average DT fuel-ion density $\left(n_{D, T}=f_{D, T} n_{i}\right), n_{i}$ is the total ion density, and $g_{D r}$ is the fusion-power profile correction factor. The values for the DT fusion reactivity, $\langle\sigma v\rangle_{D T}$, are based on the recent Los Alamos experimental measurements and temperature-dependent fitting function [14] in the range $0<T<20 \mathrm{keV}$, with typical results summarized in Table $3.2-1$. 
Table 3.2-I.

MAXWELLIAN-AVERAGED DT FUSION REACTIVITIES [14]

\begin{tabular}{ccc}
$\begin{array}{c}\text { Temperature, } T \\
(\mathrm{keV})\end{array}$ & $\begin{array}{c}\text { Reactivity, }\langle\sigma v\rangle \\
\left(\mathrm{m}^{3} / \mathrm{s}\right)\end{array}$ & $\begin{array}{c}\langle\sigma v\rangle / T^{2} \\
\left(\mathrm{~m}^{3} / \mathrm{s}-\mathrm{keV}^{2}\right)\end{array}$ \\
\hline 0.5 & $5.58 \times 10^{-29}$ & $2.23 \times 10^{-28}$ \\
1.0 & $6.71 \times 10^{-27}$ & $6.71 \times 10^{-27}$ \\
5.0 & $1.33 \times 10^{-23}$ & $5.30 \times 10^{-25}$ \\
10.0 & $1.12 \times 10^{-22}$ & $1.12 \times 10^{-24}$ \\
13.5 & $2.21 \times 10^{-22}$ & $1.215 \times 10^{-24}(a)$ \\
15.0 & $2.71 \times 10^{-22}$ & $1.20 \times 10^{-24}$ \\
20.0 & $4.29 \times 10^{-22}$ & $1.07 \times 10^{-24}$ \\
$25.0^{(b)}$ & $5.95 \times 10^{-22}$ & $9.52 \times 10^{-25}$ \\
& & \\
\hline
\end{tabular}

(a) Maximum value.

(b) Out of the nominal range of the fitting function used.

Differences in physics assumptions between the TITAN sturiy and earlier CRFPR studies [2,3] are summarized in Table 3.2-Il. For purposes of the TITAN study, a lower value of poloidal beta, $\beta_{\theta}$, and fiatter radial profiles of plasma temperature and density have been assumed (the flatter profiles are a result of 1-D RFPBURN-code plasma simulations, described in Section 5.3). These profiles are displayed in Figure 3.2-1. The $g$ values reported in Table 3.2-II measure the peaked profile enhancement of fusion power $\left(g_{\mathrm{DT}}\right)$, ohmic heating ( $\left.g_{\mathrm{OH}}\right)$, and Bremsstrahlung $\left(g_{B R}\right)$ relative to the values obtained from flat profiles.

Generally, the volume-averaged power densities are given by

$$
P_{j} \equiv\left\langle F_{j}(r)\right\rangle=\left\langle f_{j}[B(r), j(r), n(r), T(r)]\right\rangle,
$$

where the subscript $j$ denotes fusion, radiation, or olunic-lieating power density. The systems model calculates volume-average power densities, $P_{j}$, using average parameters; 
Table 3.2-II.

\section{COMPARISON OF PHYSICS PARAMETERS OF RFP REACTOR STUDIES}

\begin{tabular}{|c|c|c|}
\hline Paraneter & CRFPR $[2,3]$ & TITAN \\
\hline$\mu(r) / \mu(0)$ & $\begin{array}{ll}1 & \left(r<r^{*}\right) \\
\frac{r_{p}-r^{*}}{r_{p}-r^{*}} & \left(r^{*}<r<r_{p}\right)\end{array}$ & $(\text { Section } 5.3)^{(\alpha)}$ \\
\hline$T(r) / T(0)$ & $J_{0}(\mu r)$ & $(\text { Section } 5.3)^{(a)}$ \\
\hline$n(r) / n(0)$ & $J_{0}(\mu r)$ & $(\text { Section } 5.3)^{(a)}$ \\
\hline Ion temperature, $T_{i}(\mathrm{keV})$ & 10. & 10. \\
\hline Electron temperature, $T_{e}(\mathrm{keV})$ & 10. & 9.5 \\
\hline Poloidal beta, $\beta_{\theta}$ & 0.20 & $0.20(0.22)^{\{b\}}$ \\
\hline Pinch parameter, $\Theta$ & 1.55 & 1.556 \\
\hline Reversal parameler, $F$ & -0.12 & -0.10 \\
\hline Fusion reactivity enhancement, $g_{D} T$ & 2.23 & 1.403 \\
\hline Ohnic-heating endsancement, gоHM & 5.08 & 2.924 \\
\hline Bremsstrahlung enhancement, $g_{B A}$ & 1.52 & 1.172 \\
\hline Lawson parameter, $n \tau t,\left(\mathrm{~s} / \mathrm{m}^{3}\right)^{(\mathrm{c})}$ & $1.60 \times 10^{20}$ & $1.92 \times 10^{20}$ \\
\hline Effective plasma charge, $Z_{e f f}{ }^{(d)}$ & $\sim 1.0$ & $1.69^{(d)}$ \\
\hline
\end{tabular}

(a) Profiles of plasma parameters from 1-D simulations of Section 5.3.

(b) Includes coniribution of fusion-product alpha particles $\left(E_{\alpha} \simeq 60 \mathrm{keV}\right)$ and impurities $\left(T_{X e}=T_{i}=10 \mathrm{keV}\right)$.

(c) At $T \simeq 10 \mathrm{keV}$.

(d) $Z_{\text {eff }} \equiv \sum n_{j} Z_{j}^{2} / \sum n_{j} Z_{j}$, where $j$ denotes the ion species.

Defining $f_{j}=n_{j} / n_{i}$, typically $f_{D, T}=f_{T}=f_{D}=0.48484, f_{a}=0.0030$, and $f_{X e}=0.00033$ with $Z_{X e}=45$ for the partially stripped $X e$ ir.spurity ions. 


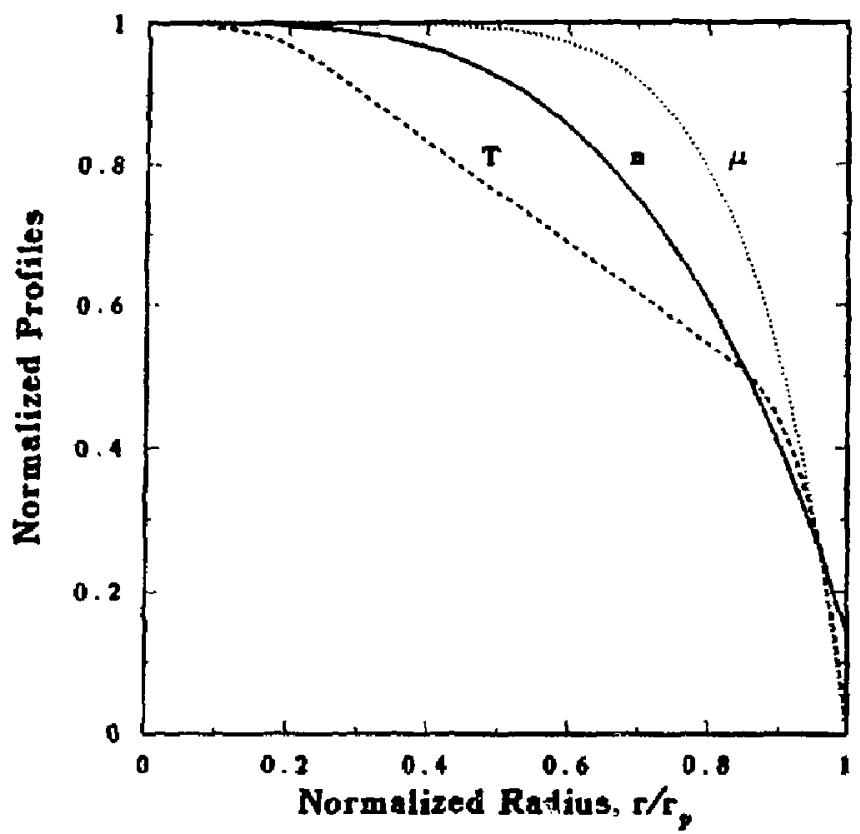

Figure 3.2-1. Profiles of core-plasma normalized density, $n$, temperature, $T$, and $\mu=\mu_{\mathrm{o}} \mathbf{j} \cdot \mathbf{B} / B^{2}$ as obtained from 1-D simulations (Section 5.3).

all profile information is contained in the profle-enhancement factors $g_{j}$, where

$$
P_{j}=g_{j} f_{j}(\langle B\rangle,\langle j\rangle,\langle n\rangle,\langle T\rangle) .
$$

Henceforth, these quantities are volume-averaged and ( ) is dropped except otherwise specified so that the notation can be simplified.

The average current density, plasma density, and temperature used in Equation 3.2-1 are defined as follows:

$$
\begin{aligned}
j_{\phi} & \equiv \frac{I_{\phi}}{A_{p}}, \\
n & \equiv \frac{2 \pi}{A_{\mathrm{p}}} \int_{0}^{r_{p}} n(r) r d r, \\
T & \equiv \frac{2 \pi}{n A_{p}} \int_{0}^{s_{\mathrm{p}}} T(r) n(r) r d r,
\end{aligned}
$$


where $J_{\phi}$ is the toroids! plasma current and $A_{p}=\pi r_{p}^{2}$ is the plasma minor cross-sectional area. The profile factors are then defined as

$$
g_{j} \equiv \frac{2 \pi}{P_{j} A_{p}} \int_{0}^{r_{p}} P_{j}(r) r d r
$$

The olmuc-heating profile correction factor, for example, becomes

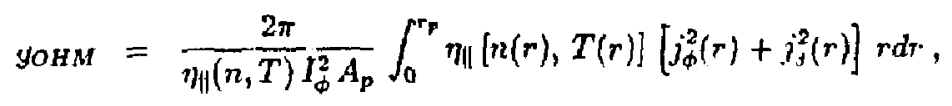

where $\eta_{\|}$is the classical Spitzer resistivity $\left(\propto Z_{e f} T_{e}^{-3 / 2}\right.$ ).

The profiles of fields and current densities in the plasma are found using the ideal MHD equations,

$$
\begin{aligned}
\nabla \times \mathbf{B} & =\mu_{o} \mathbf{j}=\mu_{o}\left(\mathbf{j}_{\|}+\mathbf{j}_{\perp}\right), \\
\mathbf{j} \times \mathbf{B} & =\nabla p,
\end{aligned}
$$

where $\mathbf{j}_{\|}$and $\mathbf{j}_{\perp}$ are, respectively, the components of the current density parallel and perpendicular to the magnetic field. It is the natural tendency for the RFF configuration to relax to a force-free Woltjer-Taylor minimum-energy state $[15,16]$. These relaxed states are characterized by the reversal naraneter, $F$, the pinch parameter, $\theta$, and the parameter $\mu(r)$, where

$$
\begin{aligned}
F & \equiv \frac{\bar{B}_{\phi}\left(r_{p}\right)}{\left\langle B_{\phi}\right)}, \\
\theta & \equiv \frac{\hat{B}_{\theta}\left(r_{p}\right)}{\left\langle B_{\phi}\right)}, \\
\mu(r) & \equiv \frac{\mu_{o} \mathbf{j}(r) \cdot B(r)}{B^{2}},
\end{aligned}
$$

with $\bar{B}_{\phi}$ and $\bar{B}_{\theta}$ being flux-surface-averaged quantities. Typically, $\mu(r)$ is constant within the central plasma and decreases to zero across the cold plasma edge, where the highly resistive edge plasma cannot support large current densities.

The parallel and perpendic.:'ir component of the current density can be found, respectively, from the definition oi $\mu(r)$ (Equation 3.2-13) and the ideal MHD pressure-balance Equation 3.2-10 to be

$$
\begin{aligned}
& \mathbf{j}_{\|}=\frac{(\mathbf{j} \cdot \mathbf{B}) \mathbf{B}}{B^{2}}=\mu(\tau) \mathbf{B}, \\
& \mathbf{j}_{\perp}=\frac{\nabla p \times \mathbf{B}}{B^{2}} .
\end{aligned}
$$


Substituting for the components of the current density in Equation 3.2-9 and expanding the resultant equation in the cylindrical coordinates yields the following working equations:

$$
\begin{aligned}
-\frac{\partial B_{\phi}}{\partial r} & =\mu_{\circ} j_{\theta}=\mu B_{\vartheta}+\mu_{o} \frac{\partial p}{\partial r} \frac{B_{\phi}}{B^{2}}, \\
\frac{1}{r} \frac{\partial}{\partial r}\left(r B_{\theta}\right) & =\mu_{o} j_{\phi}=\mu B_{\phi}-\mu_{o} \frac{\partial p}{\partial r} \frac{B_{\theta}}{B^{2}} .
\end{aligned}
$$

These equations are derived based on a large aspect-ratio approximation. For a given $\mu$ and pressure profiles, these equations yield the components of the magnetic field. Usually values of polojdal beta, $\beta_{\theta}$, toroidal plasma current, $I_{\phi}$, and either reversal or pinch parameters are specified as the boundary conditions. The plasma-surface-averaged poloidal magnetic field, $\bar{B}_{\theta}\left(\tau_{p}\right)$, is approximated by

$$
\bar{B}_{\theta}\left(r_{p}\right)=\frac{\mu_{o} I_{\phi}}{2 \pi r_{p}}
$$

Except for special cases, such as the Bessel-function profiles, these equations must be integrated numerically (Section 5.2).

The plasma self-inductance, $L_{p}$, can be approximated by

$$
L_{p}(H)=\mu_{o} R_{T}\left[\ln \left(\frac{8 R_{T}}{r_{p}}\right)+\frac{l_{i}}{2}-2.0\right],
$$

where $L_{i} \equiv\left\langle B_{\theta}^{2}\right\rangle / \bar{B}_{\theta}^{2}\left(r_{p}\right)$ is the plasma internal inductance per unit length normalized to $\iota_{0} \equiv 4 \pi \times 10^{-7} \mathrm{H} / \mathrm{m}$. The vertical field, $B_{V}$, required to maintain the plasma toroida] equilibrium becomes $[17]$

$$
B_{V}(T)=-\frac{\mu_{\mathrm{o}} I_{\phi}}{4 \pi R_{T}}\left[\ln \left(\frac{8 R_{T}}{r_{\mathrm{p}}}\right)+\beta_{\theta}+\frac{l_{i}}{2}-\frac{3}{2}\right],
$$

which must be provided by the EF-coil pair (Section 4.6).

As implemented in the PSA code, the plasma inductance is expressed as a sum of an external inductance, $L_{p_{i} e x}$, and an internal inductance, $L_{p, \text { in }}$ (i.e., $L_{p}=L_{p_{1} \text { in }}+L_{p, e x}$ ). The external inductance is taken to be that for a wire with the same dimensions as the plasnia [18]:

$$
L_{\mathrm{p}, \mathrm{ex}}=\mu_{o} R_{T}\left[\operatorname{in}\left(\frac{8 R_{T}}{r_{p}}\right)-2.0\right]
$$


The plasma internal inductance, as derived from results of the 1-D equilibrium calculation, is given by

$$
L_{p, \text { in }}=\frac{1}{I_{\phi}^{2}}\left[2 \pi R_{T}\left(W_{B}+W_{\phi}\right)-\frac{\Phi^{2}}{2 L_{\mathrm{o}}}\right],
$$

where the internal magnetic energies, $W_{\theta}$ and $W_{\phi}$, the toroidal magnetic flux, $\Phi$, and the vacuum toroidal inductance, $L_{0}$, are given by

$$
\begin{aligned}
W_{\theta, \phi} & \equiv \frac{\pi}{\mu_{o}} \int_{0}^{r_{p}} B_{\theta, \phi}^{2}(r) r d r, \\
\Phi & \equiv 2 \pi \int_{0}^{r_{p}} B_{\phi}(r) r d r, \\
L_{o} & \equiv \frac{\mu_{o} r_{p}^{2}}{2 R_{T}} .
\end{aligned}
$$

Neglecting corrections for $Z_{e f f}>1$, the plasma pressure balance can be written in the approximate form

$$
I_{\phi}=2 \pi r_{p}\left(\frac{2 p}{\beta_{\theta} \mu_{o}}\right)^{1 / 2}
$$

where the average plasma pressure is $p \simeq 2 n k_{B} T$ and $k_{B}$ is the Boltzmann constant. The plasna line density is $N=n \pi r_{p}^{2}$ and the streaming parameter $\xi \equiv v_{D} / v_{T H_{e}} \propto I_{\phi} / N \sqrt{T_{e}}$, where $v_{T H_{e}} \equiv\left(2 k_{B} T_{e} / m_{\epsilon}\right)^{1 / 2}$.

The PSA code searches for minimum-COE design points satisfying the steady-state burn condition, while balancing the plasma ohnic and fusion-product alpha power inputs against combined radiation and transport losses, such that

$$
P_{O H M}+P_{\alpha}=P_{R A D}-P_{T R},
$$

at a profile-corrected Lawson parameter, $n \pi \tau_{E}$, that is consistent with the stipulated plasma density and temperature profiles and the average plasma operating temperature, $T$ [19]. The prompt alpha-particle loss to the first wall is expected to be negligible for typical RFP-reactor plasma currents of 15 to $20 \mathrm{MA}$.

The plasma ohmic power, $P_{O H M}$, is given by

$$
P_{O H M}=g_{O H M} I_{\phi}^{2} R_{p}=g_{O H M} \eta_{\|} j_{\phi}^{2} V_{p},
$$

where $R_{\mathrm{p}}$ is the plasma resistance and $V_{p}$ is the plasma volume. The TITAN reactors operate with a highly radiating plasma. A fraction $f_{R A D} \equiv P_{R A D} /\left(P_{O H M}+P_{\mathrm{a}}\right) \simeq 0.95$ 
Table 3+2-III.

RFP ELECTRON ENERGY CONFINEMENT

SCAIING PARAMETERS(a)

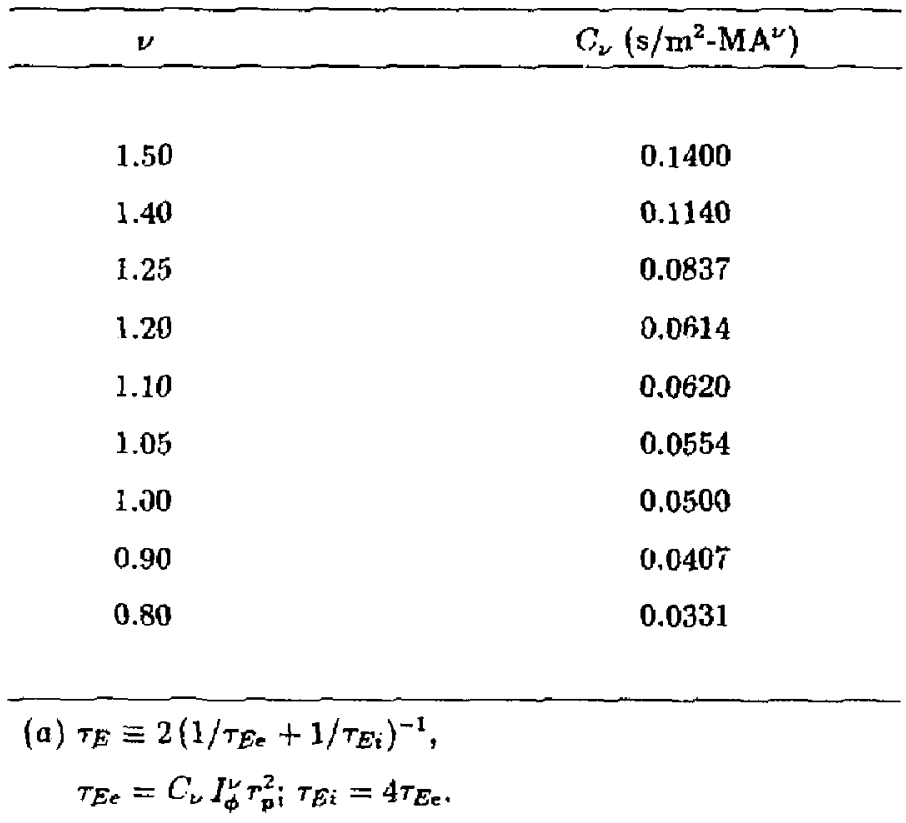

of plasma power is radiated uniformly to the first wall to recluce the heat flux on the divertor-plate surfaces to a manageable level. Sinultaneously, the plasma temperature and density in front of the divertor plate are reduced such that the sputtering of first-wall and divertor-plate structural surfaces is acceptable (Section 5 ).

The RFP electron energy confinement time, $\tau_{E e}$, is assumed to scale from values. obtained in present-day experiments (Section 2.3.5) according to

$$
r_{E e}(P H Y S)=C_{\nu} I_{\phi}^{\nu} r_{p}^{2} f\left(\beta_{\theta}\right),
$$

with typical values of the current scaling exponent, $v$, and the corresponding mumerical coefficient, $C_{\nu}$, summarized in Table 3.2-III and Figure 2.3-20. The function $f\left(\beta_{\theta}\right)$ nodels the soft beta linit and is assumed to have a value of one for beta values below the beta limit and going to zero when the maximum beta value is exceeded. 
The electrons lose energy by radiation and conduction (convection), while the ions lose energy only by conduction (convection). The total loss from the plasma, $P$, is

$$
P=\frac{1.5 n_{i} k_{E} T_{i}}{\tau_{E i}}+\frac{1.5 n_{e} k_{B} T_{e}}{\tau_{E e}},
$$

where it is assumed that $\tau_{B i} \simeq \tau_{c i} \simeq 4 \tau_{E e}$ is the ion energy confinement time and $\tau_{B_{e}}$ is the electron energy loss time given by Equation 3.2-29 above, which also includes the radiation losses (Section 5). For $n=n_{i} \simeq n_{e}$ and $T=T_{i} \simeq T_{e}$, and defining the plasma internal energy as $W_{p}=3 n k_{B} T$, the global plasma-energy loss rate can be rewritten as

$$
P \equiv \frac{W_{\mathrm{p}}}{\tau_{E}}=\frac{3 n k_{B} T}{2}\left(\frac{1}{\tau_{E i}}+\frac{1}{\tau_{E e}}\right) \text {. }
$$

Therefore, the global energy confinement tine, $\tau_{E}$, is given by

$$
\tau_{E}=2\left(\frac{1}{\tau_{E i}}+\frac{1}{\tau_{E e}}\right)^{-1} \text {. }
$$

In the linit $\tau_{E i} \rightarrow \infty, \tau_{E}=2 \tau_{E_{e}}$, and for $\tau_{E i}=\tau_{E e}, \tau_{E}=\tau_{E e}$. A typical result for the TITAN design points is $\tau_{E} \simeq 0.2 \mathrm{~s}$.

The global energy confinement time of a minimum-COE design point, $\tau_{E}(O P T)$, at $P_{E} \simeq 1,000 \mathrm{MWe}$ as determined from the PSA code is typically consistent with $\nu \simeq 1.0$. Smaller output reactors with smaller values of $r_{p}$ typically require $\nu>1.0$ (i.e., better intrinsic energy confinement). The effective global diffusivity is defined for parabolic temperature and flat density profiles such that $\chi_{E}=(3 / 16) r_{p}^{2} / \tau_{E}$. The confinement time parameter space for the TITAN-I reference desigtl is fllustrated in Figure 3.2-2. A viable design requires $\tau_{E}(E C O N)<\tau_{E}($ PHYS), so that only a sufficiently high value of $\nu$ is of interest.

In steady state, the fusion alplia-particle confinement time, $\tau_{p a x}$ is defined by

$$
\dot{r}_{\alpha}=0.25 g_{D T} n_{i}^{2}\langle\sigma v\rangle_{D T}-\frac{n_{Q}}{\tau_{F \alpha}}=0 \text {, }
$$

The inpurity control and particle exhaust system should be desigied to have adequate pumping efficiency to temove the alpha ash. The fuel-ion fractional burnup, $f_{B}$, is given by

$$
f_{B}=\left(1+\frac{2}{g_{D T} n_{i}\langle\sigma v\rangle_{D T} T_{p i}}\right)^{-1} .
$$

A typical result for a TITAN design point is $f_{B} \simeq 0.06$.

The PSA code couples the above-described physics model with engineering and models described in the following subsections. Elaboration of the physics basis and related isstues of the TITAN study is found in Sections 2 and 4 through 8. 


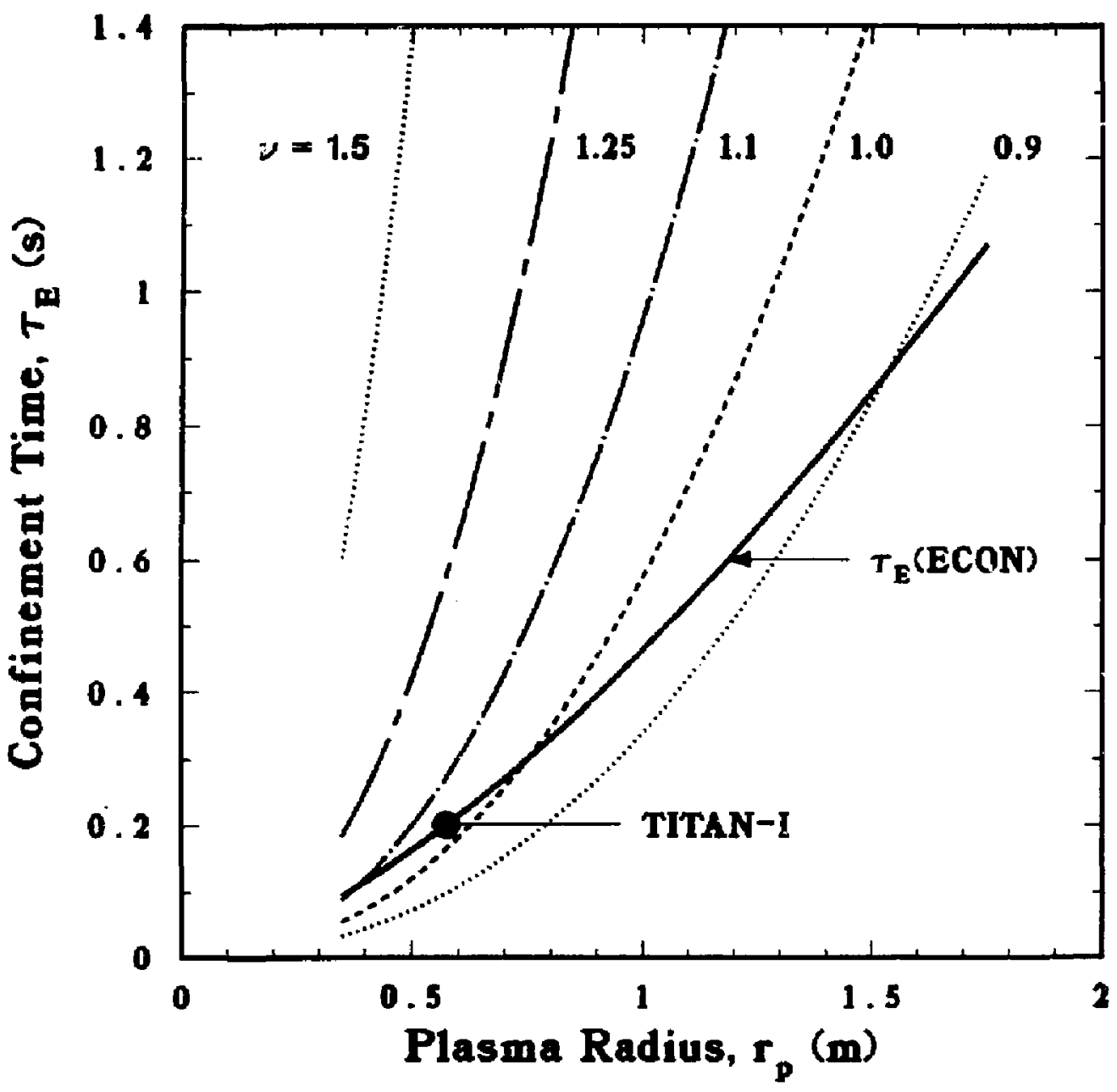

Figure 3.2-2. Comparison of the dependence of the global energy confinement time on plasma minor radius for various values of the plasma-current scaling exponent, $\nu$. The economic confinement time, $\tau_{E}(E C O N)$, for TITANclass reactors is also shown as a function of $r_{p}$. The TITAN-I reference design point is denoted by the filled circle. 


\subsubsection{Reactor Engineering Model}

Given a stipulated target for the net electric-power output, ' , the thermal-power output, $P_{T H}$, is determined for a noninal value of the thermal conversion efficiency, $\eta T$; such that $P_{E}=\pi_{T H}(1-\epsilon) P_{T H}$, where $\epsilon=1 / Q_{E}$ is the recirculating power fraction. The gross electric-power output is $P_{E T}=\eta_{T H} P_{T H}$. A fraction $f_{A U X}$ of $P_{E T}$ is allocated for auxiliary functions, such that $P_{A U X}=f_{A U X} P_{E T}$. A fraction $f_{\text {pump }}$ of $P_{E T}$ is allocated for primary-coolant punping power, such that $P_{\text {pump }}=f_{\text {pump }} P_{E T}$. Of this latter contribution to the recirculating power, it is assumed that $0.85 P_{\text {pump }}$ is recoverable as useful thertual power in the primary loop. The plasma ohmic-leating power, $P_{\text {OHM }}$, joule dissipation in the respective resistive coil sets, $P_{T F}^{n}$ and $P_{D F}^{n}$, and current-drive power, $P_{C D}$ (including $\left.P_{O H M}\right)$ complete the components of recirculating power for the TITAN. Power dissipation attributable to OFCD in the various coil sets and in the first wall, blanket, and shield is calibrated by separate calculations (Section 7). If the EF coil is taken to be superconducting, $P_{E F}^{\Omega}=0$. Additionally; following the start-up transient, the $\mathrm{OH}$-coil current would be slowly ramped to zero, such that for purposes of the average steady-state power balance, $P_{O H}^{\mathbb{R}}=0$.

The engineering $Q$-value figure of merit, $Q_{E}$, can be written as

$$
Q_{E}=\frac{1}{\epsilon}=\eta_{X H} \frac{M_{N} P_{N}+P_{a}+P_{O H A}+0.85 P_{\text {pump }}+P_{I B C}^{\Omega}}{P_{A U X}+P_{\text {pump }}+P_{T F}^{\Omega}+P_{D F}^{\Omega}+P_{C D}},
$$

where $M_{N}$ is the blanket neutron energy multiplication ratio and is found fron neutronics calculations to be 1.2 for the TITAN-I design (Section 10.3) and 1.36 for the TITAN-II design (Section 16.3). For the TITAN-I design with the IBC option [13], the dissipated power, $P P_{I B C}$, in the resistive TF and divertor IBCs is also included in the useful thermal power, $P_{T H}$.

The average 14.1-MeV neutron first-wall load, $I_{w}$, is given by

$$
I_{w}=\frac{14.06}{17.58} \frac{P_{F} x}{4 \pi^{2} A r_{\mathrm{p}}^{2}},
$$

where $P_{F}$ is the DT fusion power and $x \equiv r_{p} / r_{w}$ is the plasma filling fraction. A nominally constant scrape-off layer thickness is presently assuned such that $r_{w}=r_{p}+0.06 \mathrm{~m}$ for TITAN designs. The ratio of the first-wall radius to the plasma radius is $r_{w} / r_{p} \simeq 1.1$ at the TITAN reference design points. The dependence of $I_{w}$ on $r_{p}$ for TITAN-1 is illustrated in Figure 3.2-3. For these reactors, the various components of $Q_{E}$ included in Equation 3.2-35 adjust among themselves so that $Q_{E}$ itself is quite insensitive to FPC size, as is also illustrated in Figure 3.2-3. 


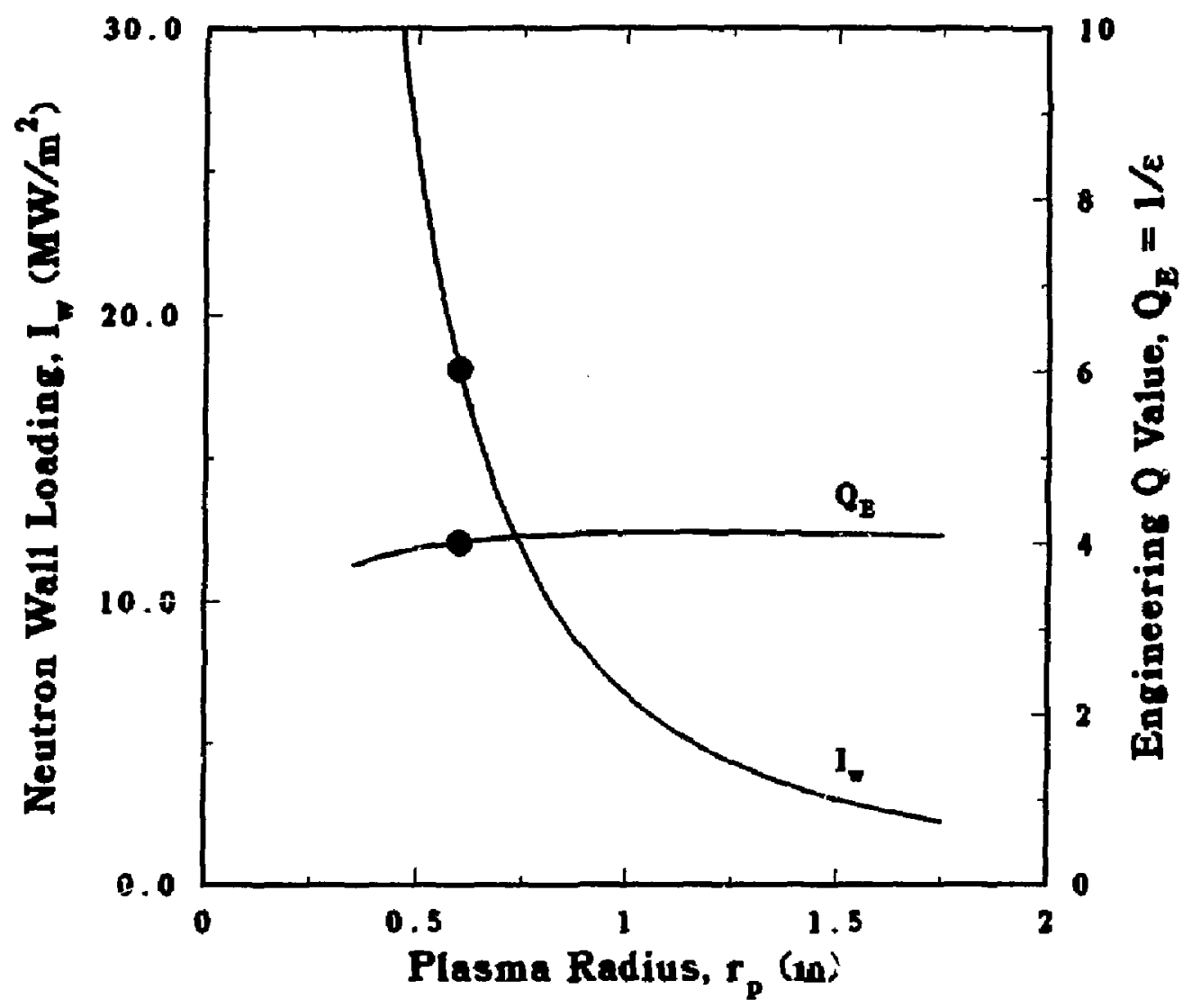

Figure 3.2-3. Dependence of the neutron wall loading ard the engineering Q-value on plasma minor radius for the TITAN-I-class reactors. The TITAN-I reference design point is denoted by the filled circle. 
The resistivity of the copper coil is taken to be $\rho_{\mathrm{Cu}}=2.0 \times 10^{-8} \Omega \mathrm{m}$ at the coil operating temperature $\sim 20^{\circ} \mathrm{C}$. Typically, the effective resistivity is increased by $1 / \lambda_{6}$, where $\lambda_{c} \simeq \mathbf{0 . 7}$ is the assumed conductor filling fraction, pending a more detailed design of the coil internals. The mass density of the copper coils is consistent with a composition of $10 \%$ coolant, $10 \%$ structure, and $10 \%$ insulator (all by volvune). A similar assumption applies to the superconducting EF coils resulting in a effective coil mass density of $p_{\mathrm{c}} \simeq 7300 \mathrm{~kg} / \mathrm{m}^{3}$. For superconducting EF coils, the maximum coil current density is given by $[20]$

$$
j_{m}\left(\mathrm{MA} / \mathrm{m}^{2}\right)=\frac{96-B_{\psi_{c}}}{1+\left(B_{\theta_{c}} / 12\right)^{1.5}}
$$

where $B_{\theta_{c}}$ is the Jocal magnetic-field strength, in units of tesla, calculated at the EF-coil surface.

The OH-coil set is sized by applying the start-up flux-swing condition,

$$
L_{p} I_{\phi}\left(1+f_{R E S}\right)=M_{E F, p} I_{E F}+M_{O H, p} \Delta I_{O H}
$$

where $M_{E F, p}$ is the mutual inductance between the EF-coil set and the plasma, $M_{\cap H_{1}}$ is the mutual inductance between the $O H$-coil set and the plasma, $\Delta I_{O H} \equiv I_{O H}^{+}-I_{O H}^{-}$ is the cliange (swing) during the start-up rise time, $\tau_{R}$, of the $\mathrm{OH}$-coil current from its initial (back-bias) value $I_{O H}^{-}$to its value at full plasma current, $I_{O H}^{+}$. After the OFCD system is fully operational, the $\mathrm{OH}$ current is slowly ramped down to zero. The factor

$$
f_{R E S} \simeq 2 \operatorname{goFM} \frac{R_{p}}{L_{p}} \tau_{R}
$$

provides for resistive flux dissipation in the plasma during the start-up transient.. The parameter $f_{G} \equiv I_{O H}^{+} /\left|I_{O H}^{-}\right|$is used to characterize the symmetry of the bipolar current swing and $f_{G}=1$ achieves a symmetric $O H$-coil current swing. Smaller values of $f_{G}$ represent deeper initial back-bias current (i.e., larger $I_{O H}^{-}$) and result in higher values of OH-coil stress ai back bias.

Both resistive and inductive factors contribute to the power supply requirements and cost of the separate $\mathrm{OH}$ and EF-coil sets according to

$$
P_{c}=I_{c}^{2} R_{c}\left(1+\frac{L_{c} / R_{c}}{\tau_{\boldsymbol{R}}} \frac{\Delta I_{c}}{I_{c}}\right),
$$

where subscript $c$ dessotes either OH or EF coils. The two-stage ramp-uj, of the TITAN RFP draws power from the grid only during the slow second stage ( $\sim 8$ to $15 \mathrm{~s}$ ) which follows the discharge of the stored magnetic energy in the $\mathrm{OH}$-coil set during the first-stage, 


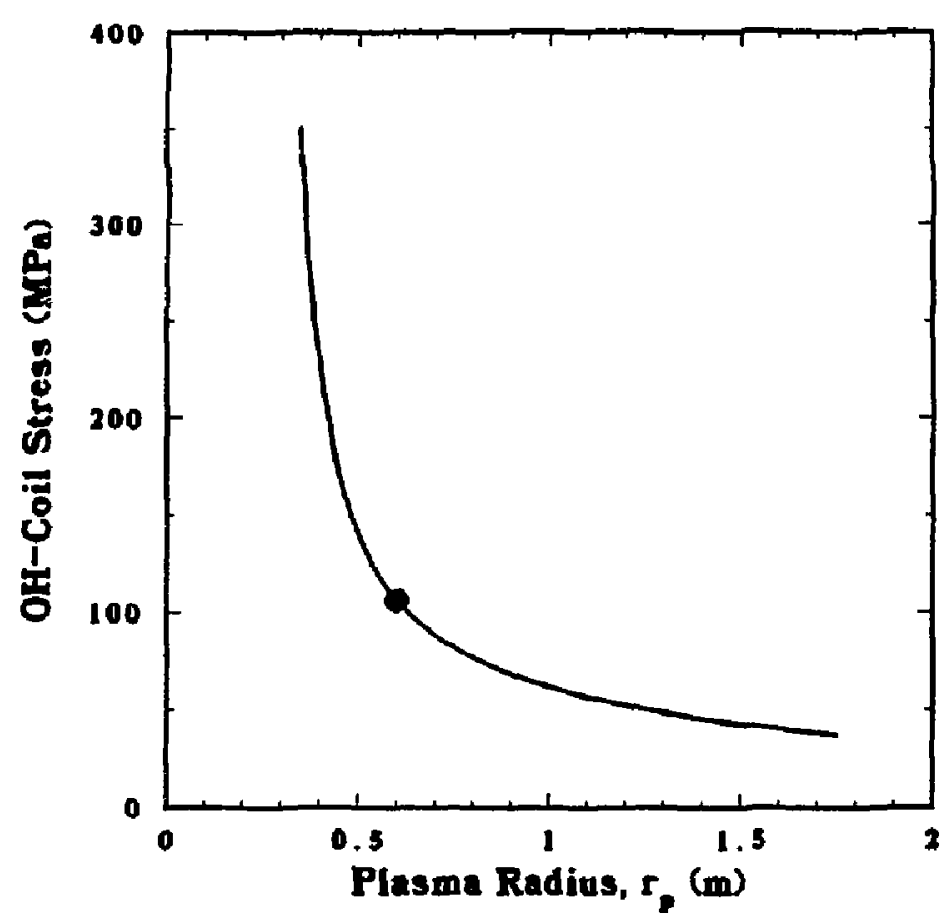

Figure 3.2-4. Dependence of back-bias OH-coil von Mises stress at $f_{G} \equiv I_{\mathrm{OH}}^{-} / I_{\mathrm{O}_{H}}^{+}=1.0$ on plasma minor radius. The TITAN-I reference design is denoted by a filled circle. The stress for $f_{G}=0.2$ approaches the nominal design limit of $200 \mathrm{MPa}$.

fast-ramp phase (1 to $2 \mathrm{~s})$. Therefore, the PSA code approximates $\Delta I_{O H} \simeq\left(I_{O H}^{+}+3\right) \mathrm{MA}$ and $\Delta I_{E F} \simeq\left(I_{E F}^{+}-13\right) \mathrm{MA}$ for purposes of Equation 3.2-40. A more detailed start-up simulation is considered in Section 6.2.

The OH-coil stresses are estimated approximately using a von Mises stress [21] given by

$$
\sigma_{\mathrm{OH}}=\left(\sigma_{r}^{2}+\sigma_{\theta}^{2}-2 \nu_{O H} \sigma_{r} \sigma_{\theta}\right)^{1 / 2},
$$

where $\sigma_{r}$ and $\sigma_{\theta}$ are the respective radial and loop stress components and $\nu_{O H} \simeq 0.3$ is the effectjve OH-coil Poisson ratio. A maximum upper limit to $\mathrm{OH}$-coil stress evaluated at the back-bias condition is taken to be $\sigma_{O H} \leq 200 \mathrm{MPa}$. The dependence of $\sigma_{O H}$ on $r_{p}$ for TITAN-I is illustrated in Figure 3.2-4. 
TITAN reactors operate at steary state using an OFCD system to maintain the plasma current (Section 7). Factors calibrating the AC joule dissipation in the first wall, blanket, shield, and coils have been calculated separately and incorporated into the PSA model. The efficiency of the OFCD power supply is monitored by means of a circuit Qvalue, $Q_{P S} \simeq 100$. The $P_{C D}$ term in Equation $3.2-35$ represents the sum of these losses.

Drained blanket, shield, and coil masses are calculated using homogenized densities. The FPC mass, $M_{F P C}$, is used to compute mass utilization $M_{F P C} / P_{T H}$ (tonne/MWt) and mass power density, MPD ( $k W_{e} /$ tonne), figures of merit (Reference [22] and $A \mu-$ pendix $C$ of Reference [5]). These figures of merit have been found to be useful, but not rigorous, predictors of cost trends. Minimum-COE TITAN design points occur for $M P D \simeq 800 \mathrm{kWe} /$ tonne

The small physical size and mass of high MPD reactors makes a single-piece maintenance procedure feasible. In this scheme, the entire reactor torus is replaced as a single unit. Single-piece maintenance is expected to have a strong infuence on the plant availability. System redundancy, steady-state operation, ease of reactor torus replacement, and development of reliable components should permit the nominal overall plant availability of $p_{f} \simeq 76 \%$ for the TITAN designs. Steady-state operation should also considerably improve reliability for the application of economically optimum engineering safety factors. The plant availability is reduced from $100 \%$ Lscause of the outage tine for unscheduled maintenance, $t_{\mathrm{u}}$, and scheduled maintenance, $t_{a}$ (days per year),

$$
p_{f}=1-\frac{t_{u s}+t_{s}}{305} .
$$

The scheduled outage time, $t_{n}$, is the maximum of the outage time for the maintenance of the balance of plani, $t_{s 0}$, and the maintenance of the FPC, $t_{\theta}^{F P C}=\tau_{\theta} / t$, where $\tau_{s}$ is the reactor-torus replacement time (in days) and $t=\left(I_{w} \tau\right) /\left(I_{w} p_{f}\right)$ is the operational lifetime of the FPC (in years) which is limited by the fluence lifetime of the first wall, $I_{\omega} \tau$.

To achieve the target availability, the unscheduled outage time, $t_{u}$, is set at 60 days per year, the scheduled maintenance of the balance of plant, $t_{s o}$ is set at 28 days per year, it is assumed that scheduled maintenance of the FPC requires $\tau_{s}=28$ days per reactortorus replacement, and a typical first-wall fluence lifetime of $I_{w} r=15 \mathrm{MWy} / \mathrm{m}^{2}$ is used. For a plaut availability of $76 \%$, the operational lifetime is one year at $l_{w} \simeq 20 \mathrm{MW} / \mathrm{m}^{2}$. For lower wall loads, the lifetime of the FPC is longer than one year and the FPC: can be replaced during the scheduled maintenance time of the balance of plant. Then, the scheduled outage time, $t_{*}=t_{s o}=28$ days per year and the plant availability remains at. $76 \%$ and independent of $I_{w}$. 


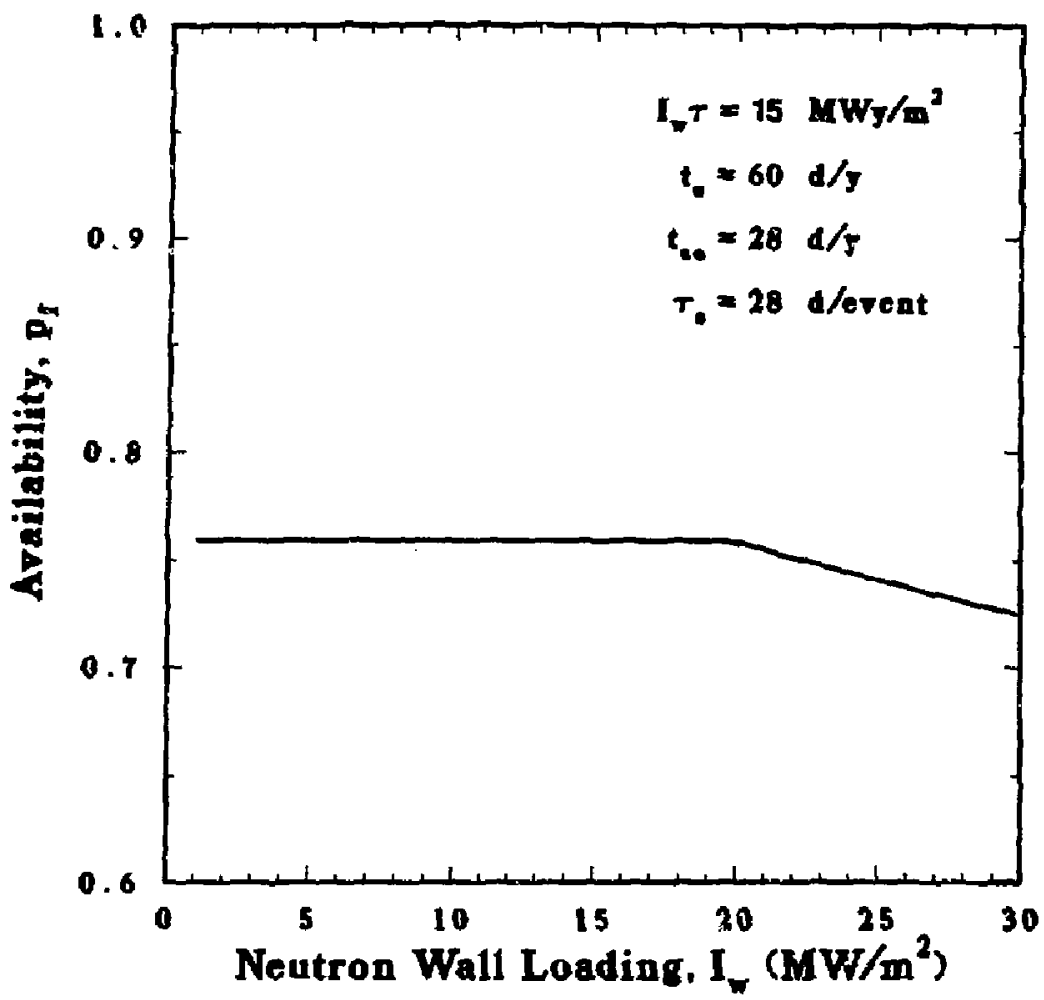

Figure 3.2-5. TITAN plent availability, $p_{f}$, as a function of neutron first-wall load, $I_{w}$, for the indicated nominal parameters. At very high $I_{w}$, the FPC change-out rate becomes large such that $p_{f}$ is penaljzed.

For wall loads higher than $20 \mathrm{MW} / \mathrm{m}^{2}$, the operational lifetime of the $\mathrm{FPC}$ is less than one year and the scheduled outage time is $t_{s}=t_{0}^{P P C}=\tau_{0} / t$, which results in a decrease in the plant availability factor for high $I_{w}$. This dependence of $p_{f}$ on $I_{w}$ is expressed by

$$
p_{f}= \begin{cases}\frac{365-t_{u}-t_{\rho o}}{365}=0.76 & \text { for } \frac{I_{w}}{\left(I_{w} \tau\right)}<\frac{1}{0.76} y^{-1} \\ \frac{365-t_{u}}{365+\left(I_{w} \tau_{o}\right) /\left(I_{w} \tau\right)} & \text { for } \frac{I_{w}}{\left(I_{w} \tau\right)}>\frac{1}{0.76} y^{-1}\end{cases}
$$

and is illustrated in Figure 3.2-5. For wall loads such that $I_{w} \leq\left(I_{w} \tau\right) / p_{f}$, the annual change-out of the FPC is assumed such that $p_{f} \simeq 0.76$. As $I_{w}$ exceeds $I_{w} \tau / p_{f}$, the FPC 


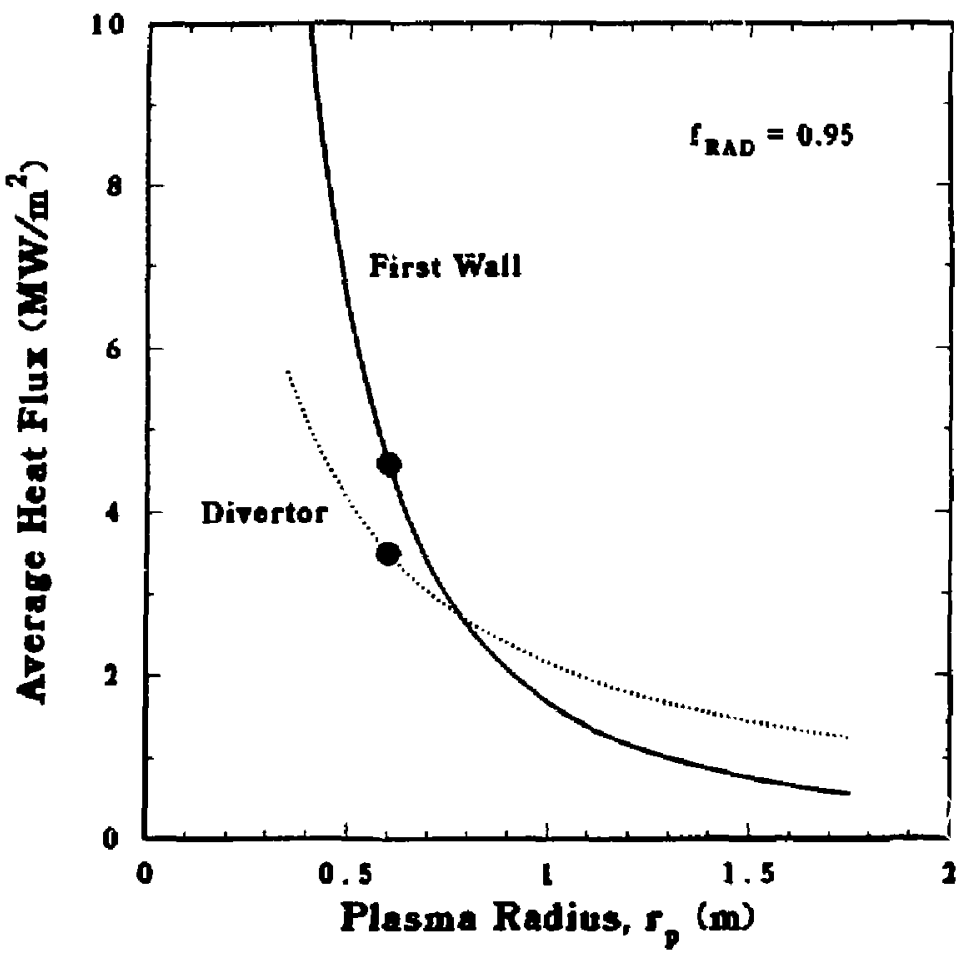

Figure 3.2-6. Dependence of first-wall and divertor-plate surface heat loads on plasma minor radius for a radiation fraction of $f_{R A D}=0.95$. The TITAN-1 reference design point is denoted by a filled circle.

change-out rate is accelerated and $p_{f}$ is penalized as is shown. It is seen that at very high wall loads, the periodic first-wall and blanket. replacement becomes an important. operational feature.

A high-power-density RFP reactor must operate with a highly radiating plasma to distribute the plasma energy uniformly on the first wall and reduce the heat flux on the divertor plates to a manageable level. The TITAN plasma radiates a considerable fraction, $f_{R A D} \simeq 0.95$, of the combined plasma power, $P_{\alpha}+P_{O H M}$ ( $70 \%$ radiation from the core and additional $25 \%$ from the scrape-off layer). Assuming three divertors with area $A_{D X V} \simeq 7 \mathrm{~m}^{2}$ (Section 11), the average surface heat load for TITAN-I design is illustrated in Figure 3.2-6. 


\subsection{COSTING}

The estimated cost of electricity (COE) is the most important evaluation tool to optimize and compare with alternative energy sources. The estimated COE is the object function of the PSA code. Both constant-1986 and then-current-1992 dollar analyses are used to evaluate the TITAN economic parameters for $\mathrm{n} n$ assumed six years construction tine. The general equation for bus-bar energy cost is given by

$$
C O E=\frac{C_{A C}+\left(C_{O Q_{C} M}+C_{S C R}+\Xi_{F}\right)(1+y)^{Y}}{8760 P_{E} p_{f}},
$$

where

COE Cost of electricity in constant or then-current dollars (mill $/ \mathrm{kWh}$ ),

$C_{A C}$ Annual capital cost charge, equals the "overnight" total capital cost (TCC) multiplied by the fixed charge rate (FCR),

$C_{i} \quad$ Cost of account $i$,

$C_{O \& M}$ Annual operations and maintenance (O\&M) cost, $C_{40}+C_{41}+\ldots+C_{47}$,

$C_{S O R}$ Annual scheduled component replacement (SCR) cost, $C_{50}+C_{51}$,

$C_{F} \quad$ Annual fuel costs, $C_{02}$ and $C_{03}$,

$y \quad$ Annual escalation rate,

$Y \quad$ Construction period (year),

$P_{E} \quad$ Net plant electric-power output (MWe),

$p_{f} \quad$ Plant availability factor,

TDC Total direct cost $=\sum_{i=20}^{26} C_{i}$,

$C_{I D C} \quad$ Interest during construction, $C_{97}=f_{I D C}$ TDC,

$C_{E D C}$ Escalation during construction $C_{98}=f_{E D C} \mathrm{TDC}$,

TCC Total capital cost $=\sum_{i=20}^{98} C_{i}$.

The detailed methodoiogy for calculating the TITAN time-related cost factors is described in References [20] and [23] and will not be repeated here. This description cliffers from the U.S. fusion-reactor-community stantarcls [2:-26] used in the period 1980-1985 because of a slightly-different "s-shaped" spending profile assumed, but represents the pending standard for U. S. fusion-reactor studies for the forseeable future [2T]. Fixed charge rate (FCR) values are summarized in Table 3.3-I. The reference TITAN case assumed an annual inflation/escalation rate, $y=0.06 / y$, to give, under the standlard assumptions [23] an annual utility cost of money $(\mathrm{CO}) \mathrm{M})$ of $x=0.09 / \mathrm{y}$, a constantdollar-mode levelized annual fixed clarge rate (LAFriR) of $0.08 / y$, and a corresponding 
then-current-dollar-mode LAFCR of $0.136 / y$. Factors used to obtain interest-duringconstruction (IDC) and escalation-during-construction (EDC) costs are summarized in Table 3.3-II. The TITAN study uses the upper set of factors, consistent with $y=0.06 / y$ and $x=0.09 / y$. The lower set, assuming $y=0.05 / y$ and $x=0.10 / y$, is characteristic of the older U.S. fusion-community standard [23-25]. Differences between the standards are not large.

The direct cost account entries, $C_{i}$, are obtained by applying relevant (instalied) unitcost estimates (e.g., $\left.\$ / \mathrm{W}, \$ / \mathrm{kg}, \$ / \mathrm{m}^{3}\right)$, where known, to the calculated usage of these items in the conceptual design, such that $C_{i}(\$)=u_{i}(\$ /$ unit $) X_{i}$ (unit). A learning-curve or

\section{Table 3.3-I.}

EFFECTIVE COST OF MONEY (COM) AND LEVELIZED ANNUAL FIXED CHARGE RATE (LAFCR) ${ }^{(a)}$

\begin{tabular}{ccc}
$\begin{array}{c}\text { Escalation Kate, } y \\
(\% / y)\end{array}$ & $\begin{array}{c}\text { COM, } x \\
(\% / y)\end{array}$ & $\begin{array}{c}\text { LAFCR } \\
(\% / y)\end{array}$ \\
\hline 0 & 4.2 & $8.0^{(b)}(10.0)^{(c, d)}$ \\
2 & 5.8 & 9.7 \\
4 & 7.4 & 11.6 \\
5 & $\mathrm{NA}$ & $\mathrm{NA}(15.0)^{(d)}$ \\
$6^{(b, e)}$ & $9.0^{(b, e)}$ & $13.6^{(e)}(16.5)^{(e)}$ \\
8 & 10.6 & 15.8 \\
10 & 12.2 & 18.1
\end{tabular}

(a) Reference [23].

(b) TITAN reference case for constant-dollar mode.

(c) Reference [20j.

(d) Reference [24].

(e) TITAN reference case for then-current-dollar mode. 
Table 3.3-II.

TIME-RELATED COST FACTORS ${ }^{(\circ)}$ AS FUNCTIONS OF CONSTRUCTION LEAD TIME

\begin{tabular}{cccc}
$\begin{array}{c}\text { Lead Time, } y \\
(\mathrm{y})\end{array}$ & $\begin{array}{c}\text { Capitalization Factors } \\
f_{\text {cap }}^{\prime} \text { (current-dollar) }\end{array}$ & $\begin{array}{r}\text { Interest Fact } \\
\text { (constant-dollar) }\end{array}$ & $f_{I D C}^{\prime}$ \\
\hline & For $y=0.06 / y$ and $x=0.09 / \mathbf{y}^{(b)}$ & \\
1 & & & \\
2 & 0.0788 & 0.0177 & 0.0556 \\
3 & 0.1558 & 0.0287 & 0.0908 \\
4 & 0.2386 & 0.0399 & 0.1274 \\
5 & 0.3273 & 0.0513 & 0.1652 \\
$6^{(c)}$ & 0.4224 & 0.0529 & 0.2042 \\
7 & 0.5244 & 0.0747 & 0.2444 \\
8 & 0.6338 & 0.0866 & 0.2858 \\
9 & 0.7511 & 0.0986 & 0.3284 \\
10 & 0.8768 & 0.1109 & 0.3723 \\
& 1.0117 & 0.1233 & 0.4176
\end{tabular}

For $y=0.05 / y$ and $x=0.10 / y$ :

$\begin{array}{rlll}1 & 0.0812 & 0.0297 & 0.0617 \\ 2 & 0.1558 & 0.0484 & 0.1011 \\ 3 & 0.2359 & 0.0676 & 0.1424 \\ 4 & 0.3217 & 0.0873 & 0.1853 \\ 5 & 0.4135 & 0.1076 & 0.2299 \\ 6 & 0.5120^{(d)} & 0.1282^{(d)} & 0.2761^{(d)} \\ 7 & 0.6174 & 0.1494 & 0.3240 \\ 8 & 0.7303 & 0.1711 & 0.3738 \\ 9 & 0.8512 & 0.1933 & 0.4254 \\ 10 & 0.9808 & 0.2161 & 0.4790\end{array}$

(a) $f_{j} \equiv 1+f_{j}^{\prime}$.

(b) cf. Table D.I, p. 237, Reference [20].

(c) TITAN reference case for $Y=6 \mathrm{y}$ construction time.

(d) cf. Reference [24]:

$f_{C A P}^{\prime} \equiv\left(F_{I D C}+F_{E D C}\right)=(0.316+U .190)=0.506, f_{c a p 0}^{\prime}=0.129, f_{I D C}^{\prime}=0.316$. 
mass-production credit is taken for a "tenth-of-a-kind" conmercial reactor installation, consistent with U. S. fusion-reactor-design-commumity practice, Often, the cost data base consist $\mathrm{s}$ of cost-scaling relationships of the form

$$
C_{j}(\$)=c_{j}\left(X_{j}\right)^{\mathfrak{e}_{j}}
$$

where $X$ can be either a descriptive variable (e.g., power, mass, volume) or a scaled variable, $X_{k} / X_{R E F}$, related to a reference value, $X_{R E F}$ of $X_{j}$, and $e_{j}$ is an appropriate scaling exponent (usually $0<e_{j}<1$ ). Equation 3.3-2 can be rewritten in the form

$$
c_{j}(\$)=\frac{c_{j}\left(X_{j}\right)^{1-e_{j}}\left(X_{j}\right)^{e_{j}}}{\left(X_{j}\right)^{1-e_{j}}}=\left[\frac{c_{j}}{\left(X_{j}\right)^{1-e_{j}}}\right] X_{i}
$$

which allows the definition of a (variable) unit cust

$$
u_{j}=\left[\frac{c_{j}}{\left(X_{j}\right)^{1-e_{j}}}\right]
$$

which is a dependent function of the descriptive variable, $X_{j}$, itself. While the cust accountiug scheme allows for detailed cost breakdowns (to four levels), only a relatively sparse, but comprehensive, subset of items are estinnated reported explicitly. The cost scaling exponents, $e_{j}$, used in the TITAN study are typically consistent with those of the U. S. nuclear-fission-reactor experience [28] and represent the inclusis $: n$ of qualitycontrol costs associated with nuclear-grade ("N-stamped") components. Potential cost savings derived from the substitution of conventional (non-nuclear) components under the condition of demonstrable inherent safety (Sections 13 and 19) are significant $[29,30]$ but controversial [31]. These savings (up to $25 \%$ on selected items) have not been included in the TITAN data base. 'The essential elements of the TITAN cost data base $\{2,5\}$ are sumunarized in Table 3.3-III. Costs which date from sources using a 1980-dollar reporting base are scaled to a 1986 reporting base using the multiplicative factor 1.348 [5].

For purposes of costing in the PSA code, the reactor building is divided into a variablevolume reactor cell, housing the FPC and vacuum tank, and a fixed-volume region, housing the primary heat-transport loops. The volume of the latter portion is estimated to be $1.55 \times 10^{5} \mathrm{mi}^{3}$ and is similar to that of the STARFIRE [32] design after escalatjng costs. The reactor room is modeled by a rectilinear enclosure extencling horizontally $9 \mathrm{~nm}$ beyond the FPC with a height approximately three times that of the FPC, such that $V_{R B}\left(1 \mathrm{~m}^{3}\right)=16\left(R_{\mathrm{T}}+r_{\mathrm{s}}+9\right)^{2}\left(6 r_{s}\right)+1.55 \times 10^{5}$. The basic building structure (Account 21.2.1) is priced at $300 \mathrm{~m}^{3}$, a value between that of STARFIRE [32] and MARS [33] designs. To this value, $2 \mathrm{MS}$ is added for building services (Account 21.2.2), 
Table 3.3-III.

SUMMARY OF TITAN COST DATA BASE ${ }^{(a, b)}$

Acct. No.

Account Title

Cost $\left(M \$, 1980^{(c)}\right)$

20. Land and Land Rights

3.3

21. Structures and Site Facilities

21.1 Site improvements and facilities

11.28

$21.2 \quad$ Reactor building

21.3 Turbine building

$3.0 \times 10^{-4} V_{R B}+39.5$

21.4 Cooling structures

33.5

21.5 Power supply and energy storage bldg.

$7.135\left(P_{E T} / 1000\right)^{0.3}$

21.6

Miscellaneons buildings

9.16

21.7

Ventilation stack

76.5

1.81

22. Reactor Plant Equipment (RPE)

22.1 Réactor equipment

22.1.1.1 Breeding material:

Liquid metal (LM): $\mathrm{PbLi}^{(d, e)}$

$$
\mathrm{Li}^{(\alpha, f)}
$$

Water solution: $\mathrm{LiNO}_{3}{ }^{(d, g)}$

22.1.1.2 Blanket and first-wall structure

22.1.1.3 Be multiplier ${ }^{(g)}$

22.1.2 Shield:

$\checkmark$ alloy $(f)$

Ferritic stee $\}^{(s\}}$

22.1.3 Magnet coils:

Normal couducting $\quad 0.065 M_{\mathrm{c}}$

Superconducting $\quad 0.130 M_{c}$

22.1.4 Supplemental (RF) heating systems ${ }^{(h)}$

$1.65 P_{\mathrm{RF}}$

Primary structure and support

(see Acct, no. 26.1)

(see Acct. no. 26.1)

$\left(7.83 f_{\mathrm{B}_{L i}}+2.46\right) \times 10^{-3} \mathrm{M}$

$0.0533 M_{B L}$

$0.3338 M_{B e}$

$0.1855 M_{\text {SHD }}$

$0.015 T M_{S H D}$

22.1 .5

Reactor vacuum system

$0.1125 V_{S T R}$

$0.015 M_{V A C}^{(f)}+2.5(\mathrm{~kg} / \mathrm{d})$ 


\section{Table 3.3-III (Cont'd)}

Acct. No. Account Title

Cost $\left(\mathrm{M} \$, 1980^{(c)}\right)$

22.1.7 Power supply (switciting, energy storage):

Normal-conducting coils

Superconducting coils

$\operatorname{IBC}^{(f)}$

OFCD

Other

TF IBC busbars $(f)$

DF IBC busbars $(f)$

22.1.8 Impurity control system

22.1.9 Direct energy conversion $(h)$

22.1.10 ECRH breakdown system

22.2 Main heat-transfer system

22.2.1 Primary coolant:

$$
\begin{aligned}
& \mathrm{Lj}^{(f)} \\
& \mathrm{H}_{2} \mathrm{O}^{(g)}
\end{aligned}
$$

22.2.2 Internediate-coolant system

22.2.3 Secondary-coolant. system

22.3 Auxiliary cooling systems

22.4 Radioactive-waste treatment

22.5 Fuel handling and storage

22.5.1 Pellet injectors

22.5.2 Furel processing system

22.5.3 Fuel storage

22.5.4 Atmospheric tritium recovery

22.5 .5
Water-cletritiation system:

TITAN-1

TITAN-II
$18.55 \$ / \mathrm{kVA}$

$296.7 \$ /(\mathrm{kVA})^{0.8}$

37.09 \$ $\mathrm{YA}$

$37.09 \$ / \mathrm{kVA}$

1.0

3.034

1.625

$0.66 A_{\text {DIV }}$

0.0

1.589
$u_{j}=0.2013\left[P_{T H}\left(1-f_{w}\right)\right]^{-0.2(i)}$

$X_{j}=P_{T H}\left(1-f_{\omega}\right)$

$u_{j}=0.1030\left[P_{T H}\left(1-f_{w}\right)\right]^{-0.2(i)}$

$X_{j}=P_{T H}\left(1-f_{w}\right)$

$u_{j}=0.1030 P_{\mathrm{TH}}^{-0.2(i)}$

$X_{j}=P_{T H}$

$\tau_{i}=0.1030\left(P_{T H} f_{w}\right)^{-0.2(j, e)}$

$X_{j}=P_{T H} f_{w}$

$6.7 \times 10^{-4} P_{T H}$

$1.2 \times 10^{-3} P_{T H}$

$3.709 \mathrm{M} \$$ each $\times 2(J, g, i)$

$0.5(\mathrm{~g} / \mathrm{day})^{0.7}$

3.709

$0.2\left(\mathrm{~m}^{3} / \mathrm{h}\right)^{0.6}$

5

140 
Table 3,3-III (Cont'd)

\begin{tabular}{|c|c|c|}
\hline Acct. No. & Account Title & Cost $\left(M \$, 1980^{(c)}\right)$ \\
\hline 22,6 & Other reactor plant equipment & $1.09 \times 10^{-3} P_{T H}$ \\
\hline 22.7 & Instrumentation and control & 23.41 \\
\hline 23. & Turbine Plant Equipment & \\
\hline 23.1 & Turbine generators & $59.9\left(P_{E T} / 1000\right)^{0.7}$ \\
\hline 23.2 & Main steam system & $4.80\left(P_{T H} / 2860\right)$ \\
\hline 23.3 & Heat rejection systems & $0.0632\left(P_{T H}-P_{E T}\right)^{0.8}$ \\
\hline 23.4 & Condensing system & $13.8\left(P_{E T} / 1000\right)^{0.9}$ \\
\hline 23.5 & Feed heating system & $7.55\left(P_{T H} / 2860\right)$ \\
\hline 23.6 & Other turbine plant equipment & $40.9\left(P_{E T} / 1000\right)^{0.6}$ \\
\hline $23 . \bar{T}$ & Instrumentation and control & $7.8\left(P_{E T} / 1000\right)^{0.3}$ \\
\hline 24. & Electric Plant Equipment & \\
\hline 24.1 & Switchgear & $8.6\left(P_{E T} / 1000\right)$ \\
\hline 24.2 & Station service equipment & $14.2\left(P_{E T} / 1000\right)$ \\
\hline 24.3 & Switcliboards & $5.4\left(P_{E T} / 1000\right)$ \\
\hline 24.4 & Protective equipment & 2.11 \\
\hline 24.5 & Electrical structures and wiring containers & $11.12+6.28\left(P_{E T} / 1440\right)$ \\
\hline 24.6 & Power and control wiring & $23.0+13.0\left(P_{E T} / 1440\right)$ \\
\hline 24.7 & Electrical lighting & 8.2 \\
\hline 25. & Miscellaneous Plant Equipment & \\
\hline 25.1 & Transportation and lifting equipment & 15.68 \\
\hline 25.2 & Air and water service systems & 12.35 \\
\hline 25.3 & Communications equipment & 6.22 \\
\hline 25.4 & Furnishings and fixtures & 1.20 \\
\hline 26. & Special Materials & \\
\hline \multirow[t]{2}{*}{26.1} & Reactor LM coolant/breeder ${ }^{(d)}$ & \\
\hline & $\begin{array}{l}\operatorname{Pbli}^{(e)} \\
\mathrm{Li}^{(f)}\end{array}$ & $\begin{array}{l}\left(7.83 f_{a_{L i}}+2.46\right) \times 10^{-3} M_{L M} \\
\left(1169 f^{6} L i+58.0\right) \times 10^{-3} M_{L M}\end{array}$ \\
\hline 26.4 & Other & 0.25 \\
\hline 26.5 & Reactor-building cover gas (Argon) ) $^{(f)}$ & 0.13 \\
\hline
\end{tabular}


Table 3.3-III (Cont'd)

\begin{tabular}{ll}
\hline Acct. No. & Account Title \\
90. & Total Direct Cost (TDC) \\
01. & Construction Services and Equipment (10\% of TDC) \\
92. & Hone-Office Engineering and Services (10\% of TDC) \\
93. & Field-Office Engineering and Services $(10 \%$ of TDC \\
94. & Owner's Cost (5\% of TDC) \\
65. & Process Contingency (5\% of TDC) ${ }^{(h)}$ \\
96. & Project Contingency (10\% of TDC) \\
97. Interest during Construction (IDC) \\
98. $\quad$ Escalation during Construction (EDC) \\
99. $\quad$ Total Capital Cost (TCC)
\end{tabular}

(a) Gross electric power, $P_{E T}$, net electric power, $P_{E}$, and total thermal power, $P_{T H}$, are given in $M W$. Volumetric $V\left(\mathrm{~m}^{3}\right)$ or corresponding mass $M$ (tonne) unit costs for the fusion power core (FPC) and related items are given as follows:

Reactor building, $V_{R B}=4\left(R_{T}+r_{s}+9\right)^{2}\left(6 r_{s}\right)+1.55 \times 10^{5}\left(\mathrm{~m}^{3}\right)$,

Blanket structure ( $5 \%), M_{B L}$ (tomne), Shield, $M_{S H D}$ (tonne),

Magnet coils, $M_{C}$ (tonne), Structure, $V_{S T R}\left(\mathrm{~m}^{3}\right)$,

Vacturm tank, $M_{V A C}=(0.07)(7.8) 2 \pi\left[\left(R_{T}+r_{s}+3\right)^{2}+4 r_{s}\left(R+r_{s}+3\right)\right]$ (tonne),

Divertor-plate surface area, $A_{D I V}\left(\mathrm{~m}^{2}\right)$.

(b) Refer to Appendices A and B for detailed TITAN design cost summaries.

(c) 1980 costs are multiplied by 1.348 to yield 1986 costs.

(d) Liquid metal, $M_{L M}$ (tonne): ${ }^{6} L i$ enriched, $0.075<f_{6_{L i}}<0.90$.

(e) Applicable to CRFPR.

(f) Applicable to TITAN-I.

(g) Applicable to TITAN-II.

(h) Not applicable to TITAN.

(i) cf. Equation 3.3-4. 
$40 \mathrm{M} \$$ for containment structures (Account 21.2.3), and 10.1 $\mathrm{M} \$$ for architectural costs (Account 21.2.4). The TITAN FPC is represented by most of the Reactor Equipment (Account 21.1) items. Supplemental-RF-heating system (Account 22.1.4) and directenergy-conversion system (Account 22.1.9) are not applicable to the TITAN design. On a unit-cost basis, superconducting-coil power supplies are considerably more expensive than resistive-coil power supplies. Power supplies for IBC and associated electrical bussing are more expensive than power supplies for conventional multi-turn resistive coils.

The main heat-transfer system for TITAN-I includes a liquid-metal primary loop serving the blanke!, divertor, and shield. Allowances are made for a fraction, $f_{w}$, of thermal power to be delivered to a pressurized-water loop. The cost of the liquid-metal loop (Account 22.2.1) calibrated by the dual-media (PbLi and water) MARS design [33] with a reduction of $80 \%$ of the dominant piping costs of that design to reflect the shorter pipe runs in the TITAN case. This model results in an $\sim 50 \mathrm{M} \$$ increase in cost over the pressurized-water main heat-transfer system in STARFIRE [32]. In the TITAN-I case, where $\mathrm{Li}$ is the sole primary coolant, $f_{w}$ is zero; in the TITAN-II case, $f_{w}$ is one. Tine liquid-metal inventory in the system consists of $95 \%$ of the blanket volume, corrected by a factor of 1.09 to account for the FPC ducts connecting the blanket through the magnet sets to the main manifolds. To this variable volume is added a fixed increment $\left(\sim 500 \mathrm{~m}^{3}\right)$ for the primary-loop inventory, a value assumed to be relatively constant over the parameter range of interest. The cost of the liquid metal in the primary loop is reported under Special Material (Account 26), insofar as it is salvageabie and rewsable. The unit cost of the liquid metal (PbLi or $\mathrm{Li}$ ) is a linearly increasing function of the ${ }^{6} \mathrm{Li}$ enrichment, $f_{L_{i}}$, as shown on Figure XIV.3-1 of Reference [34].

The reactor-torus replacement-cost estimate applies a factor of two to the direct cost of these components to allow for the lrandling and replacement of the spent reactor torus. For an assumed first-wall fluence life, $I_{\mathrm{r}} \mathrm{T}=15 \mathrm{MWy} / \mathrm{m}^{2}$ at a cost-optinized neutron wall loading of $I_{w} \simeq 20 \mathrm{MW} / \mathrm{m}^{2}$ and a plant factor $p_{f} \simeq 0.76$, routine FPC replacement accurs annually. Account 50 represents $\sim 9 \%$ of the basecase COE for TrTAN-I and $\sim 3 \%$ for TITAN-II, and is distinct from the nominal annual operations and maintenanre (O\&M) charge (Accounts $40-47,51$ ), conservatively estimated $[24,25]$ to be $2 \%$ of the direct. cost. This schen: 3 costs the first reactor, first wall, and blanket twice, and credit is not taken for any reuse of reactor-torus components (i.e., TITAN-II TF coils or shield). 


\subsection{TITAN-I DESIGN POINT}

\subsubsection{Design Point Selection}

The Los Alamos CRFPR framework studies $[2,3,35,36]$ focused on a design with a first-wall neutron wall load of $I_{w} \simeq 20 \mathrm{MW} / \mathrm{m}^{2}$, and high-coverage, poloidal pumped limiters. This design uses lithium lead, $\mathrm{Pb}_{83} \mathrm{Li}_{17}$, as the coolant and the breeder, ferriticsteel (HT-9) as the structural material, and a thin $(0.10 \mathrm{~m})$ steel shield. The magnet system includes closely coupled, copper-alloy TF and poloidal-field (PF) coils. The PF coils include both $O H$ and EF coils. The CRFPR design operates at steady state using OFCD $[37,38]$. A single-piece FPC maintenance procedure was envisioned. The heaviest components are the PF-coil set at $\mathbf{8 0 0}$ tonne and the reactor torus (first wall, blanket, shield, and TF coils) at 300 tonne.

During the TITAN study, a wide range of FPC configurations were considered and two main engineering design options emerged for detail design: (a) TITAN-I, a self-cooled liquid-lithium design with vanadium alloy (V-3Ti-1Si) as the structural material; and (b) TITAN-II, a "loop-in-pool" configuration with an aqueous solution with dissolved $\mathrm{LiNO}_{3}$ salt as the coolant and brecder, and ferritic steel alloy 9-C [39] as the structural material. The TITAN-II FPC is submerged in pool of water to achieve a high level of safety assurance. The TITAN-I design incorporates the IBC concept [13], wherein the toroidal magnetic field is produced by poloidal currents conducted in the liquid-lithium coolant, with joule losses being recovered directly in the thermal cycle. The TITAN-II design uses copper-alloy TF and divertor coils. The TITAN designs operate at steady state using $O F C D$, feature high-recycling toroidal-field divertors for impurity control and particle exhaust [40], and operate at an aggressive first-wall neutron load of $18 \mathrm{MW} / \mathrm{m}^{2}$. Because of the small physical size and mass, the TITAN FPCs are made of a few factoryfabticated pieces that are assembled on-site into a single torus, tested to full operational condition, and installed as a single unit in the reactor vault.

In order to eliminate steady-state power consumption in the resistive EF coil ( $53.5 \mathrm{MW}$ for the CRFPR design [2,3]), the TITAN designs use superconducting (SC) EF coils. This option also allows a more open FPC geometry, which requires higher currents in the EF coils because of the poorer coupling of PF coils to the plasma. The $\mathrm{OH}$ and TF coils in TITAN, however, are normal conducting (NC) to retain a compact reactor torus, with the OH-coil set being used and sized for start-up conditions only. Both OH and TF coils (or a subset of their windings) may also serve OFCD functions, depending on the electrical design of the intervening first wall, blanket, and shield. 
The systems model described in References [2-3] was expanded for the TITAN study to include a self-consistent treatment of separate $\mathrm{OH}$ and EF coils (Figure 3.1-3). The scaling of poloidally symmetric toroidal-field divestors and OFCD was also included in the revised optimization algorithm. The basic computational algorithm remains essentially as described in Reference [3]. As for any model of this nature, the best choices are made on the basis of separate and detailed analyses of various subsystems such as neutronics, plasma equilibrium, OFCD, divertor, and thermal-hydraulic calculations. Some important trade-offs like construction time versus size and complexity, mean-time-torepair versus mean-time-to-failure as a functic: of power density and size, and elasticity of nuclear and size economies of scale for key components remain inadequately resulved and in need of future work. Table 3.4-I lists key design variables that were either fixed or varied in the re-optimization of the RFP reactor for the TITAN study.

A typical PSA code output curve is shown on Figure 3.4-1, which displays COE as a function of plasma minor radius, $r_{p}$, for fixed aspect ratio, $A=6.5$, and net pcwer output $P_{E}=970 \mathrm{MWe}$. The higher dashed curve illustrates the COE based on thencurrent dollars, and the solid curve illustrates the constant-dollar-mode, which is reported exclusively in the remainder of this section. The minimum-COE design point is denoted by an open circle on the solid curve. It should be noted that the choice of costing mode does not alter the value of plasma radius producing the minimum-COE design point. The minima in the curves result from the trade-off between higher capital cost for larger FPCs as $r_{p}$ increases and the reduced plant availability factor, $p_{f}$, for small values of $r_{p}$ as neutron first-wall load, $I_{w}$, increases for fixed $P_{E}$.

A feature of Figure 3.4-1 is the shallowness of the COE versus $r_{p}$ (and hence, $I_{w}$ ) minimum, although the compressed COE scale should be noted. Nevertheless, increasing $I_{\mathrm{t}}$ from 5 to $10 \mathrm{MW} / \mathrm{m}^{2}$ and then to the near-minimum-COE design at $I_{\omega} \simeq 18 \mathrm{MW} / \mathrm{m}^{2}$ results only in a $3 \%$ and $11 \%$ reduction in COE, respectively. Other developmental and operational incentives (i.e., single-picce maintenance) not included in the present costing model may justify the higher- $I_{w}$, high-MPD design points that reside closer to the COE minimum.

The varjation of the cost of neat minimum-COE design points with plasma aspect ratio, $A=R_{T} / r_{p}$ is weak in the range exan'sed ( $A=4$ to 8 ), as illustrated in Figure 3.4-2. Establishing a maxinum grid power of $P_{G R I D} \simeq 300 \mathrm{MWe}$ delivered to the $\mathrm{OH}$ coil in the back-bias condition during start-up, and maintaining the peak (inboard) von Mises stress in the $\mathrm{OH}$ coil at $\sigma_{O H} \leq 200 \mathrm{MPa}$ set a limit of $A \geq 5.5$ to 6: a TITAN baseline value of $A=6.5$ was selected to allow for added start-up flux and provides sufficient symmetrization of the TF for an efficient magnetic-divertor design (Section 4.4). 
Table 3.4-I.

FIXED AND VARIABLE PARAMETERS FOR TITAN REACTOR OPTIMIZATION AND SENSITIVITY STUDIES

\begin{tabular}{|c|c|c|}
\hline Parameter & TITAN-I & TITAN-II \\
\hline \multicolumn{3}{|l|}{ Varied Paranneters ${ }^{(\alpha)}$} \\
\hline Plasma aspect ratio & 6.5 & 6.5 \\
\hline Plasma minor radius ( $\mathrm{m}$ ) & 0.60 & 0.60 \\
\hline Poloidal beta & 0.20 & 0.20 \\
\hline Plasma average temperature (keV) & $\sim 10$ & $\sim 10$ \\
\hline Lawson parameter, $n \tau_{F}\left(10^{20} \mathrm{~s} / \mathrm{m}^{3}\right)^{(b)}$ & 1.92 & 1.92 \\
\hline Reversal parameter, $F$ & -0.10 & -0.10 \\
\hline Pinch parameter, $\Theta^{(\mathrm{c})}$ & 1.56 & 1.56 \\
\hline TF- and divertor-coil options & Cit of IBC & $\mathrm{Cu}$ \\
\hline EF-coil option & $\mathrm{SC}$ or $\mathrm{NC}$ & $\mathrm{SC}$ or $\mathrm{NC}$ \\
\hline EF-coil shield standoff (m) & $0.5(\mathrm{SC}), 0.0(\mathrm{NG})$ & $0.5(\mathrm{SC}), 0.0(\mathrm{NC})$ \\
\hline FPC radiation lifetime, $I_{w} \tau\left(\mathrm{MWy} / \mathrm{m}^{2}\right)$ & 15 & 15 \\
\hline Plant factor, $p_{f}^{(d)}$ & $\leq 0.76$ & $\leq 0.76$ \\
\hline
\end{tabular}

\section{Fixed Parameters}

Primary coolant

Structural material

Blanket energy multiplication ratio, $M_{N}$

First-wall, blanket, \& shield standoff (m)

$\mathrm{SC}$ current density, $j_{c}\left(\mathrm{MA} / \mathrm{m}^{2}\right)$

Cu current density, $j_{c}\left(\mathrm{MA} / \mathrm{m}^{2}\right)^{(e)}$

$\begin{array}{cc}\text { Liquid lithium } & \text { Aqueous solution } \\ \text { Vanadium } & \text { Ferritic steel (9-C) } \\ 0.44 & 0.35 \\ 0.77 & 0.50\end{array}$

Equation 3.2-37

$\leq 50$
Equation 3.2-37

$\leq 50$

(a) Nominal design values are given.

(b) Consistent with plasma temperature. Also $n=\sum n_{j} f_{j}$ with $f_{D}=f_{T}=0.484, f_{\alpha}=0.03, f_{X e}=0.003 ; Z_{\text {eff }}=1.69$.

(c) Consistent with reversal parameter, plasma current, polojdal beta, etc.

(d) Calculated from Equation 3.2-43 and consistent witl. FPC lifetine, 28 days FPG scheduled maintenance, and $60 \mathrm{~d} / \mathrm{y}$ of unscheduled maintenance.

(e) Trade-ofis between cost of the power supply and cost of copper usually set. $j_{c}$ below this limit. Typically $j_{c}=10$ to $15 \mathrm{MA} / \mathrm{m}^{2}$. 


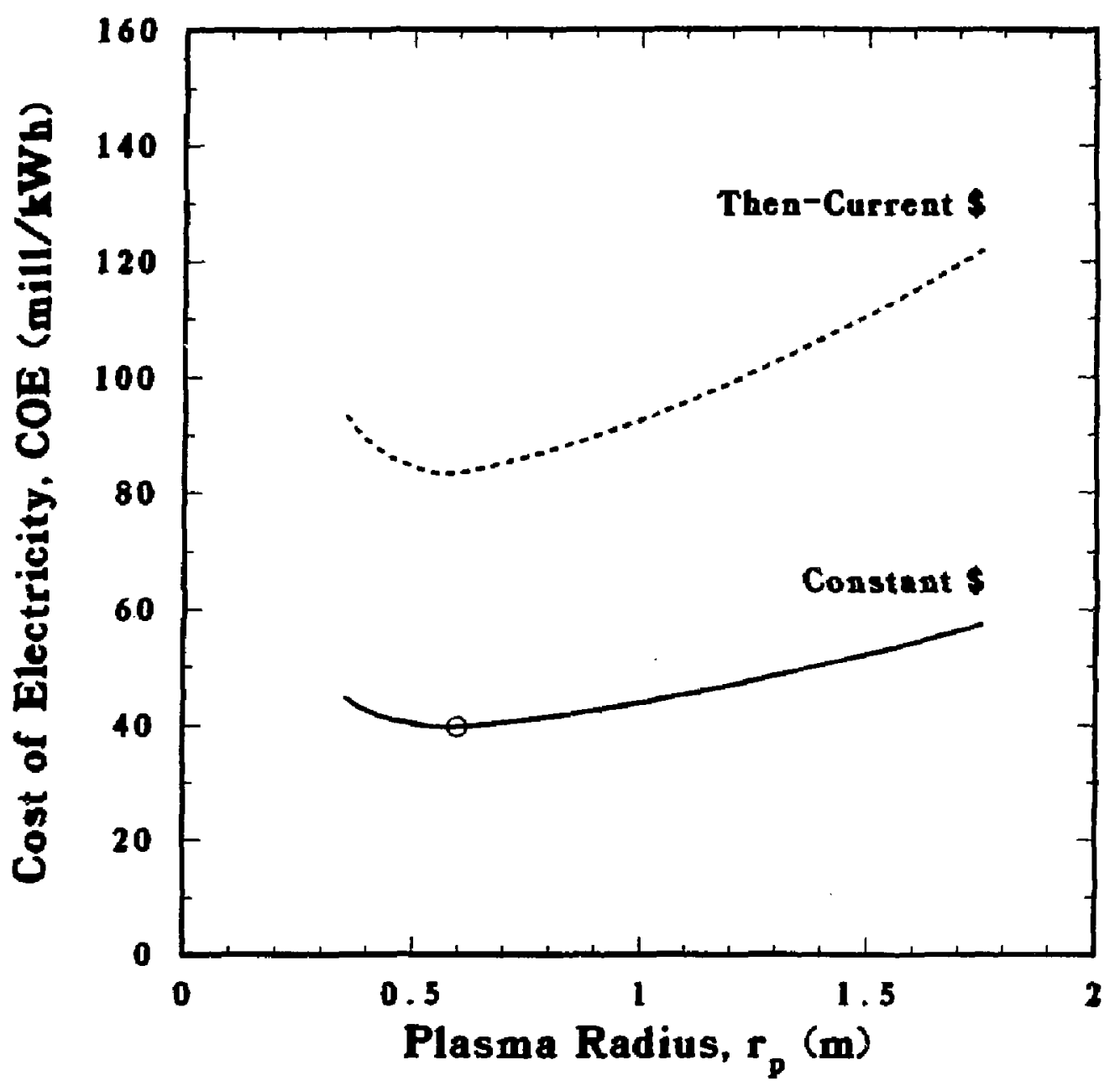

Figure 3.4-1. Dependence of COE on plasma minor radius for constant-dollar and thencurrent-dollar costing modes analyses. The minimum-COE operating point is denoted by an open circle. The net power output is fixed at $j_{E}=970 \mathrm{MWe}$ and the plasma aspect ratio is $A=6.5$. 


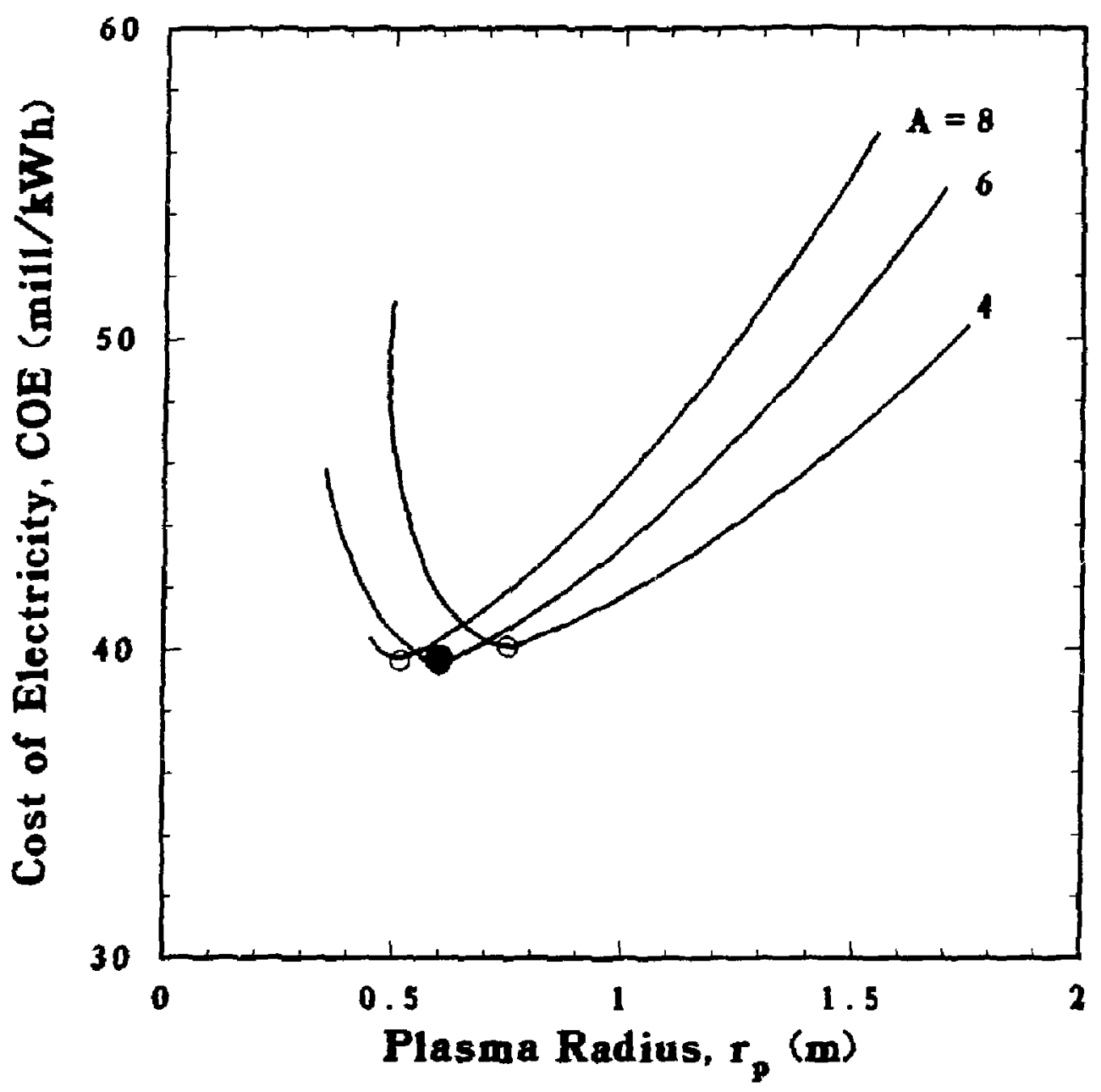

Figure 3.4-2. Dependence of COE on plasma aspect ratio, $A \equiv R_{T} / r_{p}$. MinimumCOE design points for each value of $A$ are denoted by open circles. The near-minimum-COE TITAN-I reference design point at $A=6.5$ and $r_{p}=0.60 \mathrm{~m}$ is denoted by a filled circle. 


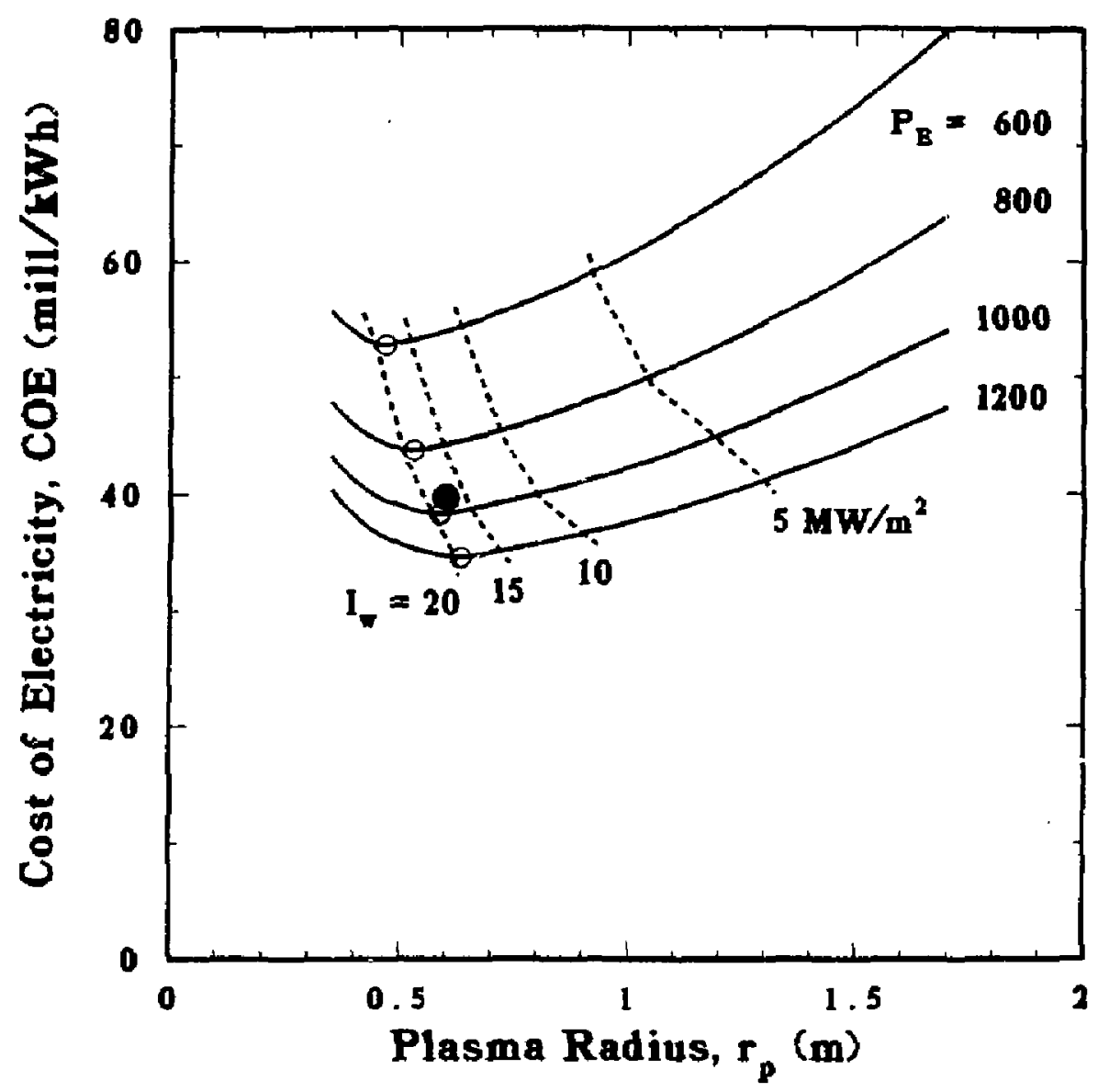

Figure 3.4-3. Dependence of COE on plasma minor radius for a fixed plasma aspect ratio, $A=6.5$, for various indicated values of net power output, $P_{E}$. The crowding of the curves at higher values of $P_{E}$ is indicative of the nuclear economies of scale built into the cost data base ( $c f$. Table 3.3III). For each value of $P_{E}$, the minimum-COOE design point is denoted by an open circle. The dashed lines are contours of constant neutron wall loading, $I_{w}$. The near-ninimum-COE TITAN-I reference design point at $P_{E}=970 \mathrm{MWe}, I_{w}=18.1 \mathrm{MW} / \mathrm{m}^{2}$, and $r_{p}=0.60 \mathrm{~m}$ is indicated by the filled circle. 
The dependence of $\mathrm{COE}$ on $P_{E}$ given on Figure 3.4-3 is typical of nuclear economies of scale, resuiring in smaller marginal cost benefits as $P_{E}$ increases. The TITAN-1 design window is also elaborated in Figure 3.4-3, which displays contours of constant nelutron first-wall load, $I_{w}$, together with contours of net power output $P_{E}$ for the indicated fixed parameters. Above values of $I_{w} \simeq 20 \mathrm{MW} / \mathrm{m}^{2}$ for the nominal first-wall and blanket fluence lifetime, $I_{w} \tau=15 \mathrm{MWy} / \mathrm{m}^{2}$, faster than annual change-out of the FPC results in plant availability, $p_{f}$, reduced below $76 \%$ and higher values of COE. Only a modest cost penalty is incurred for operation at lower values of $l_{w}\left(\right.$ i.e., down to $\left.10 \mathrm{MW} / \mathrm{m}^{2}\right)$. Below this value, however, the applicability of a single-piece FPC maintenance approach, which is important to the justification of the nominal value $p_{f} \simeq 0.76$, becomes questionable. It should be noted that the shallowness of these cost minima is a resulk of the FPC in TITAN already repesenting a small percentage of the total direct cost.

The TITAN PSA nodel was used to characterize several tentative designs before settling on a reference TITAN-1 case diuring an iterative process involving input from the subsystem design activities of the study. Several of these prelininary designs are summarized in Table 3.4-11. Case A is a conventional copper-coil configuration conceptually similar to the CRFPR $[2,3]$. Case B incorporates the IBC option for both the TF and divertor-field (DF) coils functions, but retains a copper EF-coil pair. Higher recirculating power results in significantly higher neutron first-wall load, $I_{w}$, and $C O E$ for the same value of net power output, $P_{E}$. Case $C$, the TITAN reference case, retains the IBC: option but uses a superconducting EF coil pair to reduce the steady-state recirculating power, offsetting the increased capital cost of the superconducting EF coils to give COE performance nearer that of Case $\mathrm{A}$.

The PSA geometric model (Figure 3.1-3) assumes an annular-shell representation for the $\mathrm{OH}$-coil set. After appropriate magnetics discretization (Section 4.5), a tentative TITAN I FPC model results. This model is subsequently refined by more detailed engineering analysis (Section 10), and appropriate adjustments are made to a re-calibrated PSA model.

The average neutron wall load for the TITAN-I reference design is $I_{w} \simeq 18.1 \mathrm{MW} / \mathrm{m}^{2}$. The NEWLIT code [8] was used to map the poloidal variation of $I_{w}$. The volumetric neutron source distribution assumed is jllustrated in Figure 3.4-4. The peaking is consistent with the $g_{D T} n_{i}^{2}(r)\langle\sigma v\rangle_{D T}\left[T_{i}(\cdot)\right]$ source distribution of Equation 3.2-1. The outward Shafranov shift of an RFP plasma at the nominal aspect ratio $A=6.5$ is liegligible. For purposes of NEWLIT modeling, the TITAN-I first-wall tube bank is represented by an inscribed polygon with 16 sides. This approximation results in a geometric correction such that $I_{w}=18.1=\Gamma_{a v}(16 / \pi) \sin (\pi / 16)$. The minimum wall load modeled by NEWLIT is 
Table 3.4-II.

SUMMARY DF TITAN-I REACTOR DESIGNS ${ }^{(a, b)}$

\begin{tabular}{|c|c|c|c|}
\hline Parameter & Case A & Case B & Ref. Case (e) \\
\hline EF-cojl optjon & Copper & Copper & $S C^{(d)}$ \\
\hline DF-c i' option & Copper & $\mathrm{IBC}(e)$ & $\mathrm{IBC}^{(e)}$ \\
\hline TF-coil option & Copper & IBC(e) & $\mathrm{IBC} \mathrm{G}^{(e)}$ \\
\hline \multicolumn{4}{|l|}{ Plasma Parameters } \\
\hline Plasma current, $I_{\phi}(M A)$ & 18.24 & 18.23 & 17.82 \\
\hline Plasma ion density, $n_{i}\left(10^{20} \mathrm{~m}^{-3}\right)$ & 8.75 & 9.36 & 8.93 \\
\hline Plasma electron density, $n_{e}\left(10^{20} \mathrm{~m}^{-3}\right)$ & 9.14 & 9.78 & 9.33 \\
\hline Poloidel field at plasma surface, $B_{\theta}\left(r_{p}\right)(\mathrm{T})$ & 5.88 & 6.08 & 5.94 \\
\hline Thermal diffusivity, $\chi_{E}\left(\mathrm{~m}^{2} / \mathrm{s}\right)$ & 0.308 & 0.330 & 0.315 \\
\hline Fusion power density, $P_{F} / V_{p}\left(\mathrm{MW} / \mathrm{m}^{3}\right)$ & 79.7 & 91.1 & 83.0 \\
\hline Plasma ohmic dissipation, $P_{O H M}(\mathrm{MW})$ & 28.0 & 29.8 & 28.5 \\
\hline \multicolumn{4}{|l|}{ Poloidal-Field Quantities } \\
\hline OH-coil thickness, $\delta_{O H}(\mathrm{~m})$ & 0.23 & 0.27 & 0.27 \\
\hline A verage minor radius of coil, roH (m) & 1.57 & 1.57 & 1.56 \\
\hline$O H$-coil field, $B_{\theta_{c}}(\mathrm{~T})$ & 2.24 & 2.33 & 2.28 \\
\hline OH-coil current density, for $\left(\mathrm{MA} / \mathrm{m}^{2}\right)^{(f)}$ & 15.8 & 13.7 & 13.1 \\
\hline Mass of OH-coil set, $M_{O H}$ (tonne) & 290. & 343. & 343. \\
\hline EF-coil current density, $j_{E F}\left(\mathrm{MA} / \mathrm{m}^{2}\right)$ & 6.6 & 6.1 & $19.2^{(d)}$ \\
\hline Mass of EF-coil set, $\boldsymbol{M}_{E F}$ (tonne) & 521. & 573 & 305. \\
\hline Poloidal-field stored energy, $W_{M \theta}(G J)$ & 1.8 & 1.8 & 5.2 \\
\hline OH-coil dissipation during back-bias (MW) & 211. & 132. & 121. \\
\hline \multicolumn{4}{|l|}{ Toroidal-Field Quantities } \\
\hline TF-coil thickness, $\delta_{T F}(\mathrm{~m})$ & 0.03 & $0.28^{(e)}$ & $0.28^{(e)}$ \\
\hline A verage ninor radius of coil, rTF (m) & 1.45 & 0.68 & 0.68 \\
\hline Mass of TF-coil set, $M_{T F}$ (tonne) & 31. & 41. & 41. \\
\hline Reversed stoidal field, $-B_{\phi R}(\mathbf{T})$ & 0.378 & 0.391 & 0.382 \\
\hline Toroidal-field stored energy, $W_{A_{\phi}}(G J)$ & 0.73 & 0.17 & 0.16 \\
\hline TF-coil current density, jTF $\left(\mathrm{MA} / \mathrm{m}^{2}\right)$ & 15.8 & 1.68 & 1.64 \\
\hline Ohmic dissipation during burn, $P_{T F}^{\Omega}$ (MW) & 30.2 & 28.9 & $2 \pi .6$ \\
\hline Mass of DF-coil set, $M_{D F}$ (tonne) & 2.0 & 0.57 & 0.55 \\
\hline Ohmic dissipation in divertor, $P_{D F}^{\Omega}(M W)$ & 12. & 145. & 142 . \\
\hline
\end{tabular}


Table 3.4-II (Cont'd)

\begin{tabular}{|c|c|c|c|}
\hline Parameter & Case $\mathbf{A}$ & Case B & Ref. Case (c) $^{(1)}$ \\
\hline \multicolumn{4}{|l|}{ Engineering Summary } \\
\hline Neutron first-wall loading, $I_{w}\left(\mathrm{MW} / \mathrm{m}^{2}\right)$ & 17.4 & 19.9 & 18.1 \\
\hline Engineering $Q$-value, $Q_{E}=1 / \epsilon$ & 6.20 & 3.20 & 4.02 \\
\hline Fusion power, $P_{F}(M W)$ & 2,207 . & 2,526 . & 2,301 . \\
\hline Total thermal power, $P_{T H}(\mathrm{MW})$ & 2,630 . & 3,207 . & 2,935 . \\
\hline Net electrical power output, $P_{E}(\mathrm{MWe})$ & 970. & 970. & 970. \\
\hline Fusion-power-core minor radius, $r_{s}(m)$ & 1.69 & 1.70 & 1.70 \\
\hline \multicolumn{4}{|l|}{ Masses (tonne) } \\
\hline - First wall and blanket & 41. & 41. & 41. \\
\hline . OH-coil "hot shield" & 267. & 267. & 267. \\
\hline - EF-coil shield & 0 . & $\mathbf{0 .}$ & 325. \\
\hline - Total coil set & 843. & 917. & 648. \\
\hline - Total fusion power core $e^{(g)}$ & $1,152$. & 1,225 & $1,282$. \\
\hline FPC power density, $P_{T H} / V_{F P C}\left(\mathrm{MWt} / \mathrm{m}^{3}\right)$ & 12.0 & 14.4 & 13.2 \\
\hline Mass power density, MPD (kWe/tonne $)^{(g)}$ & 842. & 792. & 757. \\
\hline \multicolumn{4}{|l|}{ Cost Summary } \\
\hline Cost of electricity, COE (mill/kWh) $)^{(h)}$ & 37.6 & 42.3 & 39.7 \\
\hline Unit direct cost, UDC $(\$ / \mathrm{kWe})$ & 1,449 . & 1,627 . & 1,531 . \\
\hline Total cost, TC (M\$) & 2,267 . & 2,545 . & 2,396 . \\
\hline FPC unit cost $(\$ / \mathrm{kg})$ & 147. & 144. & 146. \\
\hline \multicolumn{4}{|l|}{ Fractions of total direct cost (TDC): } \\
\hline - Reactor plant equipment, RPE /TDC & 0.42 & 0.45 & 0.43 \\
\hline - Fusion-power-core cost, FPC/TDC $(\alpha)$ & 0.12 & 0.11 & 0.13 \\
\hline
\end{tabular}

(a) All designs are for baseline parameters given in Table 3.4-1: $A=6.5, R_{\mathrm{T}}=3.9 \mathrm{~m}, r_{\mathrm{p}}=0.60 \mathrm{~m}, V_{p}=27.7 \mathrm{~m}^{3}, r_{w}=0.66 \mathrm{~m}$.

(b) $M_{N}=1.20$, $T_{T H}=0.44$, and water detritiation system cost $\simeq 5 \mathrm{MS}$.

(c) Iterated basis for detailed elaboration and analysis.

(d) Superconducting coils.

(e) Integrated blanket coil (IBC).

(f) Symmetric bipolar swing $\left(f_{G} \equiv I_{O H}^{+} / I_{O H}^{+}=1\right)$, $I_{\mathrm{O} H}^{+}$subsequently decays to zero upon initiation of OFCD.

( $g$ ) Includes first wall, blanket, shield, and coils, but not FPC support structures.

(h) Costs reported in constant 1986-dollars, assuming 6 years construction time. 


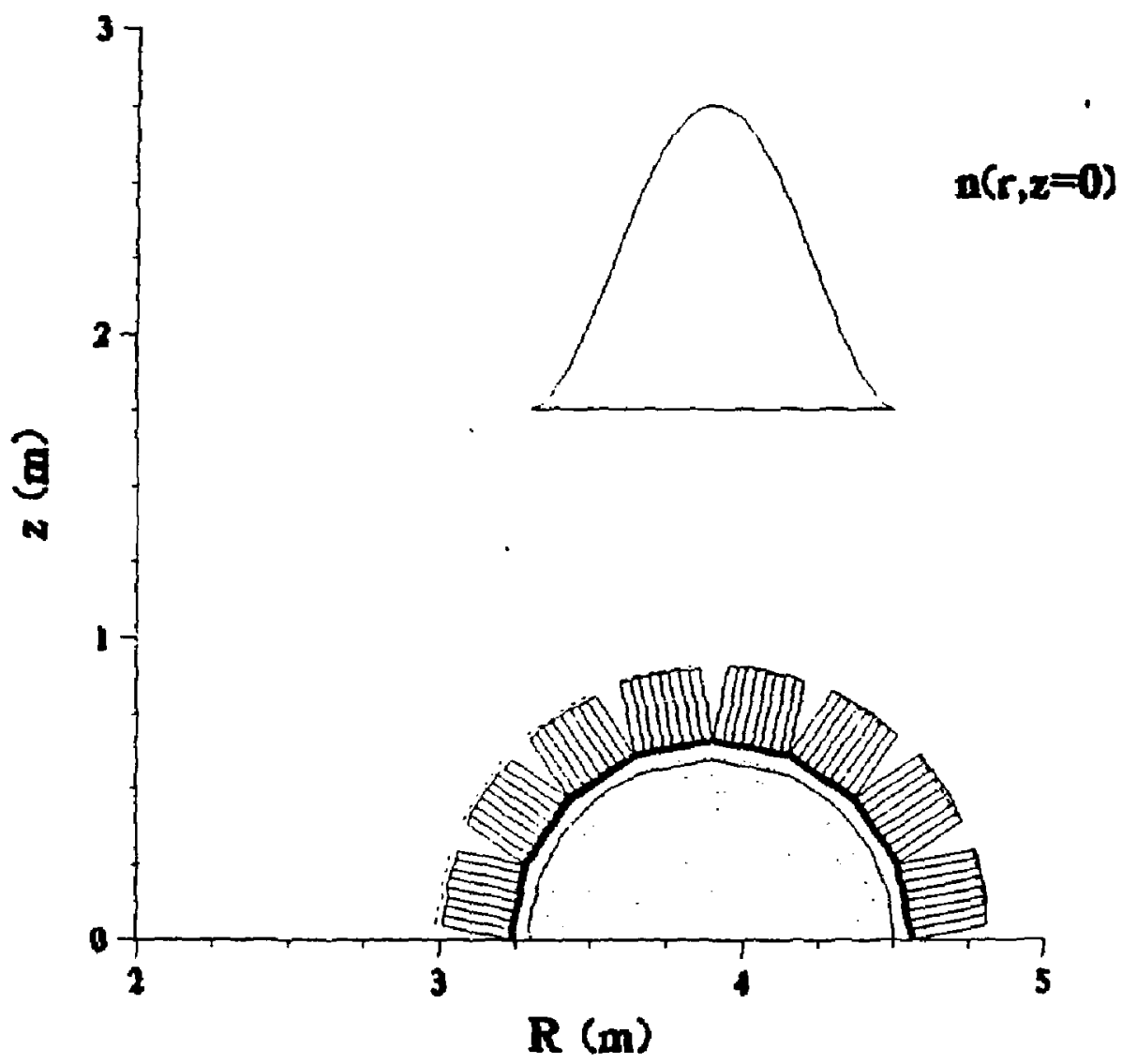

Figure 3.4-4. Un-collided fusion neutrons source distribution used in the modeling of the poloidal variation of the neutron wail loading for the TITAN-I reference design point (with $r_{p}=0.60 \mathrm{~m}, r_{w}=0.66 \mathrm{~m}$, and $R_{T}=3.9 \mathrm{~m}$ ) using the NEWLIT code [8]. The first wall is represented by an inscribed polygon with 16 sides. 


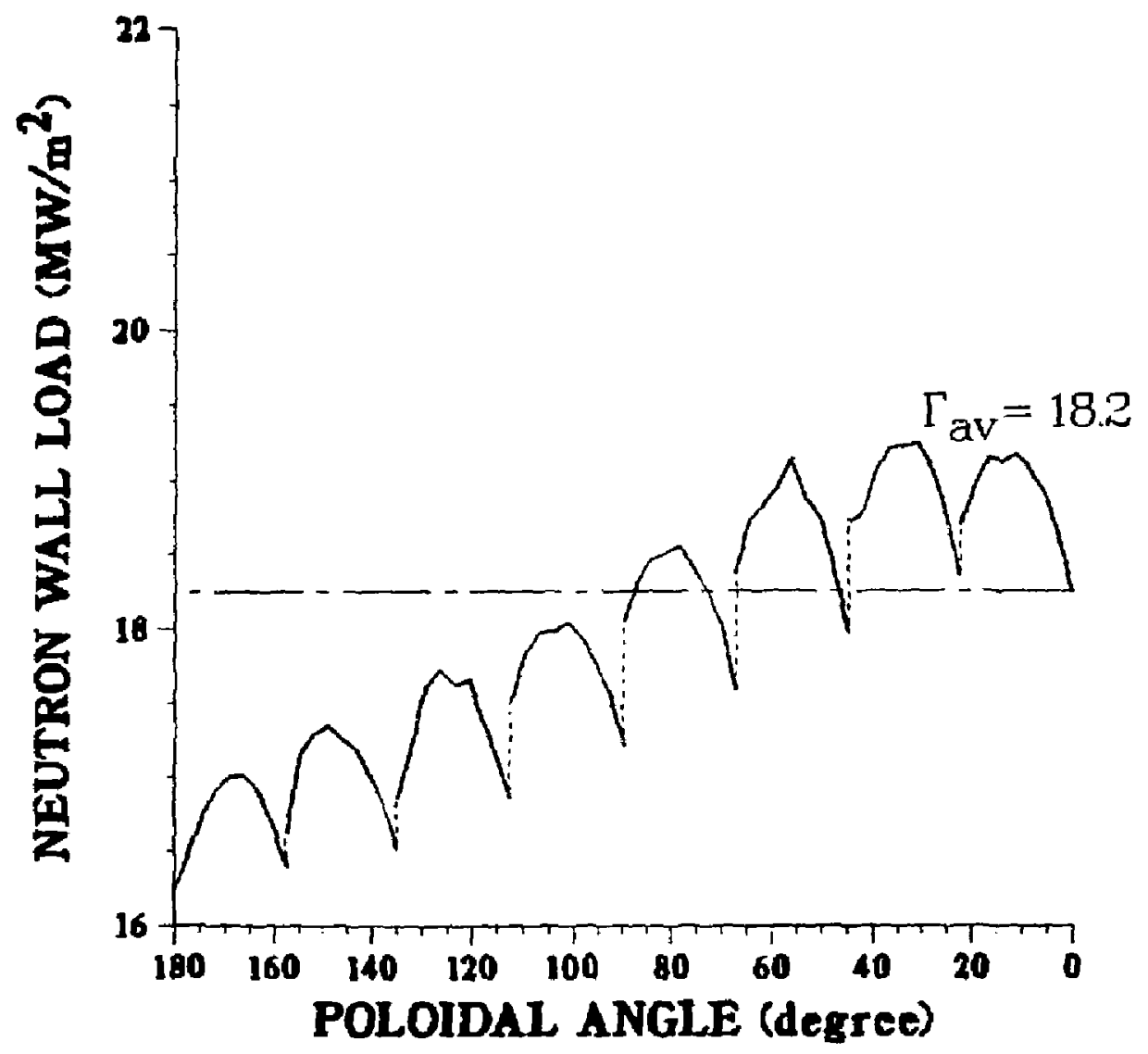

Figure 3.4-5. Poloidal variation of the neutron wall load, $I_{w}$, for the TITAN-1 reference design point. The inboard and outboard first-wal] locations in the equatorial plane occur, respectively, at the poloidal angles $\theta=180$ and 0 degrees. The fusion power is $P_{F}=2300 \mathrm{MW}$. The first wall is represented in the NEWLIT code [8] by an inscribed polygon with 16 sides. 
16.24 MW $/ \mathrm{m}^{2}$ on the inboard side at the first wall in the equatorial plane, and the maximum wall load is $19.22 \mathrm{MW} / \mathrm{m}^{2}$ at the outboard equatorial plane. The fine structure in the profile shown in Figure 3.4-5 is an artifact of the NEWLIT polygonal approximation and of negligible importance. The general poloidal variation in $I_{w}$ is sufficiently small for $A=6.5$. The surface heat-flux distribution resulting from the distributed racliated power, $P_{R}(\theta)=f_{A A D}\left(P_{a}+P_{O H M}\right)$, is qualitatively similar to $P_{n}(\theta) \propto I_{w}(\theta)$. Therefore, local peaking of the surface heat load on the first-wall coolant tubes is also small. Peaking in a low-aspect-ratio device is potentially more severe, resulting in a constraint on accessible values of $I_{w}$.

\subsubsection{Trade-off and Sensitivity Studies}

In addition to the TITAN design points emerging from the PSA code, trade-off and sensitivity studies were performed to establish and characterize the "design window" of attractive RFP reactor operation. Several of the key fixed parameters are re-examined. with a view toward establishing the sensitivity of the reference design to changes in these parameters.

The reference TITAN-I plasma operating temperature is $T \simeq 10 \mathrm{keV}$. Table 3.4 -III summarizes the sensitivities of key reactor paraneters such as $\mathrm{COE}$, engineering $\mathrm{Q}$-value $Q_{E}=1 / \epsilon$, and first-wall neutron load $I_{w}$ on $T$ in the range 10 to $20 \mathrm{keV}$. The variation of these global parameters is fairly small on this temperature range although a slight peaking nearer to $T \simeq 15 \mathrm{keV}$ may be indicated, consistent with the peaking of $\langle\sigma v\rangle_{D T} / T^{2}$ at $T \simeq 13.5 \mathrm{keV}$. Separate studies suggest operation at the higher tenperatures to maximize the efficiency of OFCD (Section 7). An overriding consideration suggesting the lower values of $T$, however, was the establishment of a high- $f_{\text {RAD }}$ core plasma with edgeplasma conditions consistent with efficient magnetic-divertor operation (Section 5). For fixed values of $\beta_{\theta}$ and magnetic field, temperature trades off directly with density for constant pressure, but the plasma fusion-power density and neutron wall load, $I_{w}$, are nearly constant.

Consistent with the best operation of present-day RFP experiunents, the noninal TITAN poloidal-beta value is $\beta_{\theta}=0.20$; this value includes only thermalized fusionproduct alpha-particle and $X$ e impurities at $T_{X e}=10 \mathrm{keV}$. The energetic alpha-particles add about 0.02 to this beta value. The response of the TITAN-I design to lower values of $\beta_{\theta}$ is shown in Figure 3.4-6. The COE increases as $\beta_{\theta}$ is decreased which is caused primarily by the need to establish and drive more plasma current, as reflected in increased 
OFCD power consumption, increased coil mass, and reduced MPD. Values of $\beta_{\theta}$ nuch below $\sim 0.1$ would result in large increase in COE.

Using the experimental scaling of the confinement time, $\tau_{E e} \propto I_{\phi}^{\nu} r_{p}^{2} f\left(\beta_{\theta}\right)$, the impact of the plasma curreut scaling exponent, $\nu$, on achieving the ninimum-COE TITAN-I design is illustrated in Figure 3.4-7. For each respective constant $\nu$ curve, the condition $\tau_{E}(\mathrm{ECON}) \leq \tau_{E}(\mathrm{PHYS}) \equiv 2\left(1 / \tau_{E e}+1 / \tau_{E i}\right)^{-1}$ with $\tau_{E i} \simeq 4 \tau_{E e}$ is met to the right (i.e., higher $\left.r_{p}\right)$. The accessibility to minimum-COE designs depends on the value of $\nu$. In addition, for $\nu$ values much below $\sim 0.8$, the demands on the $O H$-coil system during the ohmic-heating transient to ignition and burn can be serious. Also, it should be noted that the flexibility of operation of the TITAN-I device at lower than nominal power (as for load following or checkout) requires better intrinsic plasma confinement (i.e., higher v).

Table 3.4-III,

\section{DEPENDENCE OF KEY TITAN-I PARAMETERS ON ION TEMPERATURE ${ }^{(a)}$}

\begin{tabular}{lccc} 
Ion temperature, $T_{i}(\mathrm{keV})$ & $10^{(b)}$ & 15 & 20 \\
\hline Lawson parameter, $n T_{E}\left(10^{20} \mathrm{~s} / \mathrm{mi}^{3}\right)$ & 1.92 & 1.30 & 1.10 \\
Electron temperature, $T_{e}(\mathrm{keV})$ & 9.5 & 14. & 19. \\
Plasma current, $I_{\phi}(\mathrm{MA})$ & 17.82 & 17.87 & 18.60 \\
Fusion power density, $P_{F} / V_{\mathrm{p}}\left(\mathrm{MW} / \mathrm{m}^{3}\right)$ & $\mathbf{8 3 . 0}$ & 82.3 & 82.6 \\
Plasma ion density, $n_{i}\left(10^{20} \mathrm{~m}^{-3}\right)$ & 8.93 & 5.99 & 1.87 \\
Neutron wall load, $I_{w}\left(\mathrm{MW} / \mathrm{m}^{2}\right)$ & 18.11 & 17.96 & 18.01 \\
Engineering Q-value, $Q_{E}=1 / \epsilon$ & 4.02 & 4.16 & 4.11 \\
Cost of electricity, $\mathrm{COE}(\mathrm{mill} / \mathrm{kWh})$ & 39.7 & 39.5 & 39.8
\end{tabular}

(a) Fixed geometric parameters: $A \equiv R_{\mathrm{T}} / r_{\mathrm{p}}=6.5$ and $r_{\mathrm{p}}=0.60 \mathrm{~m}$.

(b) Reference TITAN-I case. 


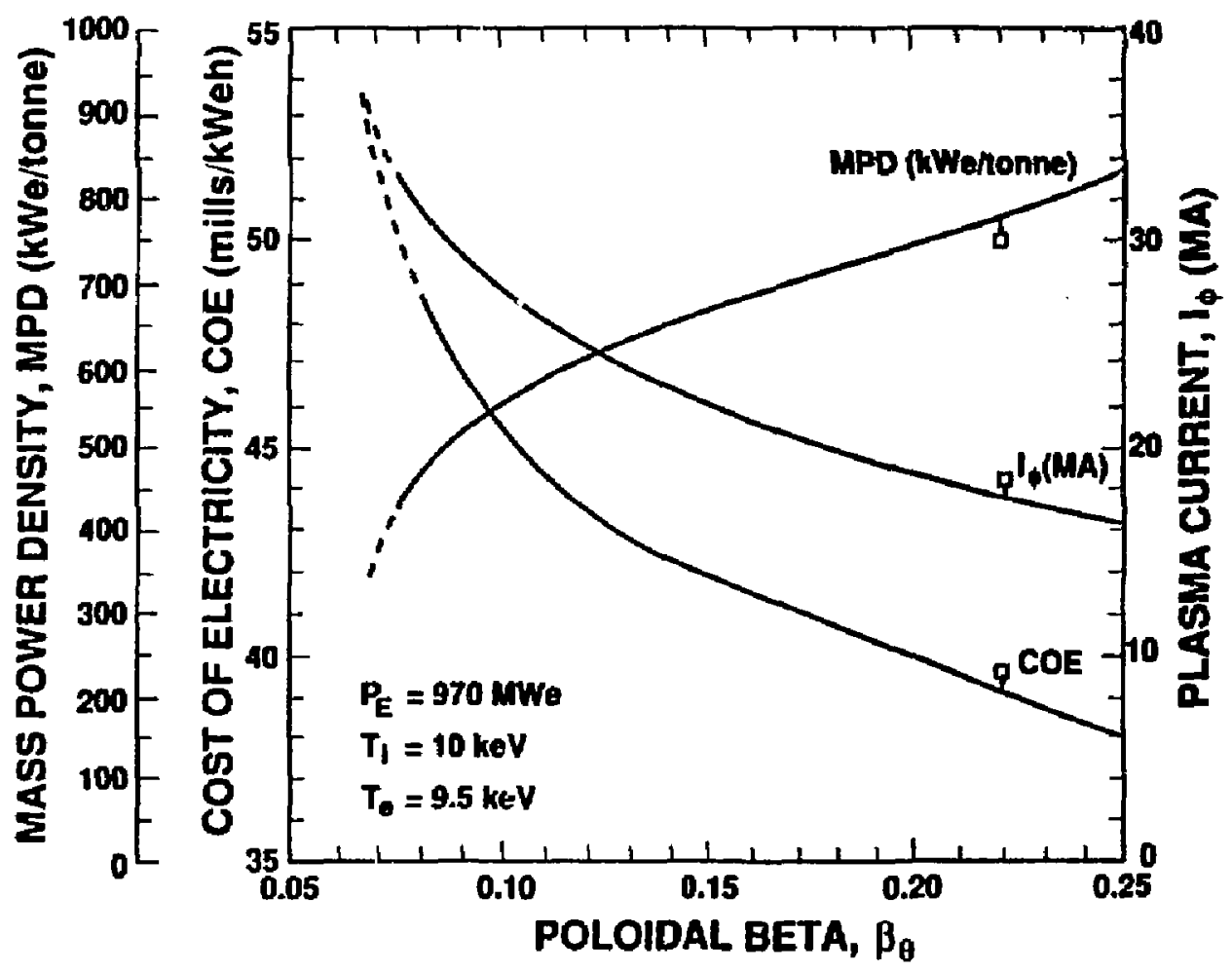

Figure 3.4-6. Dependence of minimum-COE TITAN-I design values on poloidal beta; the near-minimum-COE TITAN-I reference design values are also shown. 


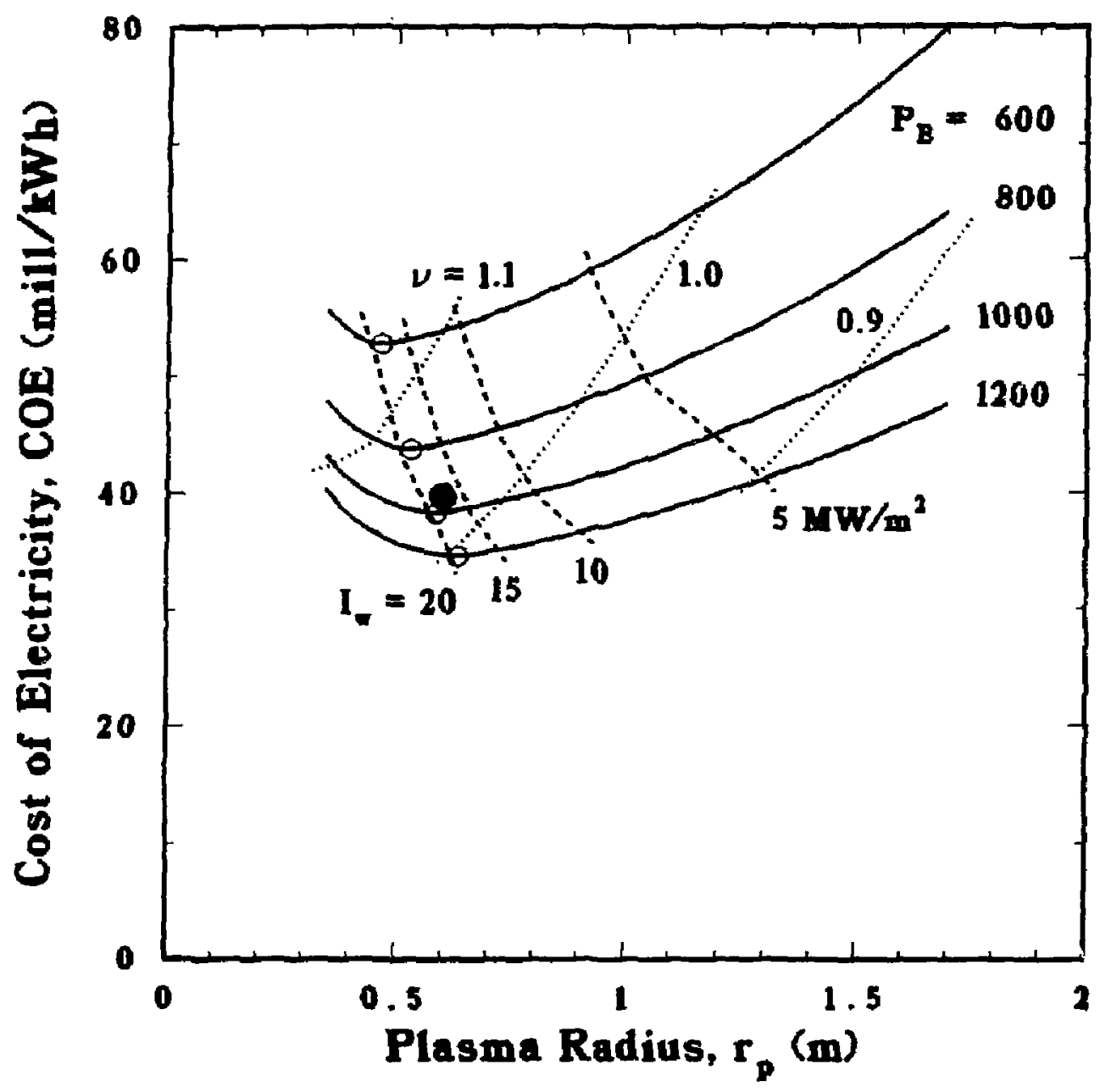

Figure 3.4-7. Parametric trade-off of TITAN-I cost with level of confinement required $\left\{\tau_{E_{e}}=C_{v} I_{\phi}^{\nu} \tau_{p}^{2} f\left(\beta_{\theta}\right\}_{,} \tau_{E_{i}}=4 \tau_{E_{\mathrm{e}}}\right)$, expressed as the magnitude of exponent $\nu$ required for $\tau_{E}(E C O N) \leq \tau_{E}(\mathrm{PHYS})=2\left(1 / \tau_{E i}+1 / \tau_{E_{e}}\right)^{-1}$. Accessible design points are to the right of curves of constant $\nu$. Minimum.COE design points with $\tau_{E}(\mathrm{OPT})=\tau_{E}(E C O N)$ for fixed net power output $P_{E}$ are indicated by open circles. The near-minimum-COE TITAN-I reference design at $P_{E}=970 \mathrm{MWe}$ and $I_{r p}=18.1 \mathrm{MW} / \mathrm{m}^{2}$ is denoted by a filled circle. 
The magnitude of the reversal parameter, $F \equiv \bar{B}_{\phi}\left(r_{p}\right) /\left\langle B_{\phi}\right\rangle$, drives the magnitude of the toroidal field at the coil, and the power reguired by the magnetic divertor system, $P_{D F}$, to locally cancel that field. The dependence of key TITAN-I parameters on $F$ is illustrated in Figure 3.4-8. For deeper reversals compared with the reference value $F=-0.1$, the divertor power, $P_{D F}$, grows linearly and reduces $Q_{E}=1 / \epsilon$. To maintain the nominal net power output of $P_{E}=970 \mathrm{MWe}$, the plasma current rises slowly and $I_{w} \propto I_{\phi}^{4}$ increases until $I_{w} \simeq 20 \mathrm{MW} / \mathrm{m}^{2}$, whereupon the plant availability, $p_{f}$, begins to decrease below 0.76 ( $c f$. Figure 3.2-2). Because the dissipated power in the divertor and toroidal-field IBC sets is recovered in the thermal cycle, this power can actually displace some primary fusion power, resulting in somewhat lower values of $l_{w}$ near $F=-0.3$. The current-drive efficiency, $I_{\phi} / P_{C D}$, also decreases as $F$ becomes more negative, further lowering $Q_{E}$, although it should be mentioned that a self-consistent modeling of the OFCD system was not done at these deeper values of $F$. The nominal value of $F$ in this study was not raised above -0.10 so as to provide a reasonable margin for the OFCD oscillations in $F$ without loss of reversal altogether. Further, more exact (coupled) work in this area is warranted.

Figures 3.4-9 and 3.4-10 show the impact on COE of parametrically increasiug the resulting poloidal-flux consumption as the ramp-up rise time, $\tau_{R}$, is increased, with the main cost drivers being the OH-coil power supply (power-grid-driven reatifiers at $25 \$ / \mathrm{kVA}$ ) and the increased coil mass; the increased power, current, and von Mises stress in the OHI coil, $\sigma_{O H}$, are also shown for both symmetric $\left(f_{G}=1\right)$ and asymuetric $\left(f_{G}=0.2\right)$ current swings. To provide for the increased poloidal-flux consumption as $\tau_{A}$ is increased, a deeper back-bias current must be provided (i.e., higher $I_{O H}^{-}$). For the symmetric case, $I_{O H} \equiv I_{O H}^{-}=I_{O H}^{+}$. The resistive flux consunption in the plasma during start-up scales as $f_{R E S} L_{p} I_{\phi}$ and is directly proportional to $\tau_{R}$ as given by Equation 3.2-39.

The dependence of $\mathrm{OH}$-coil back-bias stress, $\sigma_{\mathrm{OH}}$, and $\mathrm{OH}$-coil power-supply requirement is illustrated in Figure 3.4-11. For the superconducting EF-coil option selected for TITAN, a symmetric OH-coil current swing $\left(f_{G}=1\right)$ is preferred from the viewpoint of minimizing $\sigma_{\mathrm{OH}}$ and $P_{\mathrm{OH}}$ (cf. Equation 3.2-40). The cost of the copper OH-coil power supply (25\$/kVA) does not result in a significant cost impact for the values of $f_{G}$ examined. A model that included a thicker value of $\delta_{O H}$ (as $f_{G}$ decreases to prevent $\sigma_{O H}$ from increasing) would provide additional cost incentive for the choice of $f_{G} \simeq 1$ for TITAN. A copper EF-coil option ( $c f$. CRFPR) leads to a preference for $f_{G} \simeq 0.2$.

The dependence of TITAN-I operating parameters on the assumed efficiency of the OFCD power supply, monitored by a circuit $Q$-value, $Q_{P S}$, is illustrated in Figure 3.4-12. For a range of values $Q_{P S}>75$, performance is relatively insensitive to $Q_{P S}$. Below 

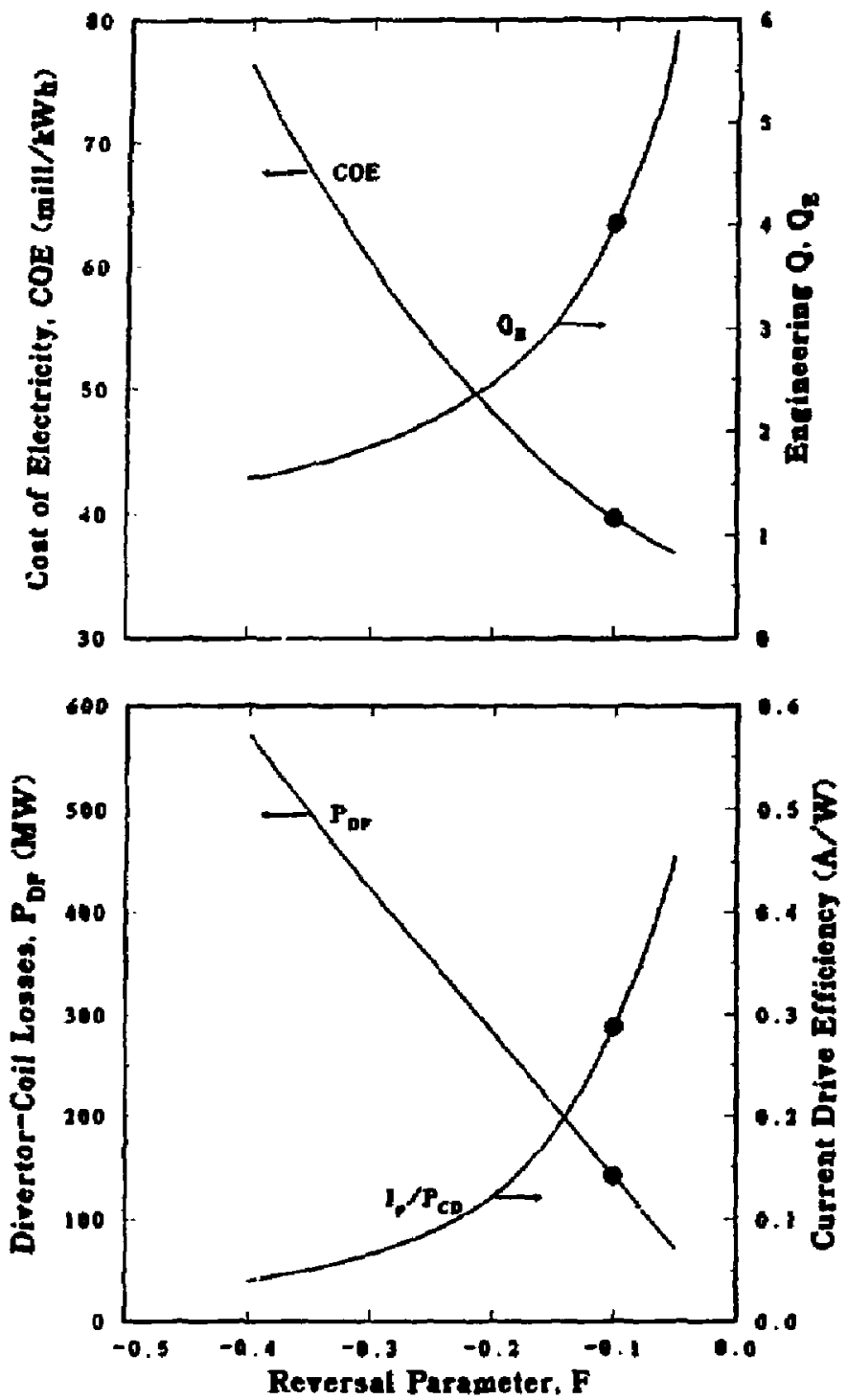

Figure 3.4-8. Impact of the reversal parameter on the overall TITAN-I design and cost. The TITAN-I reference design at $F=-0.1$ is denoted by a filled circle. 

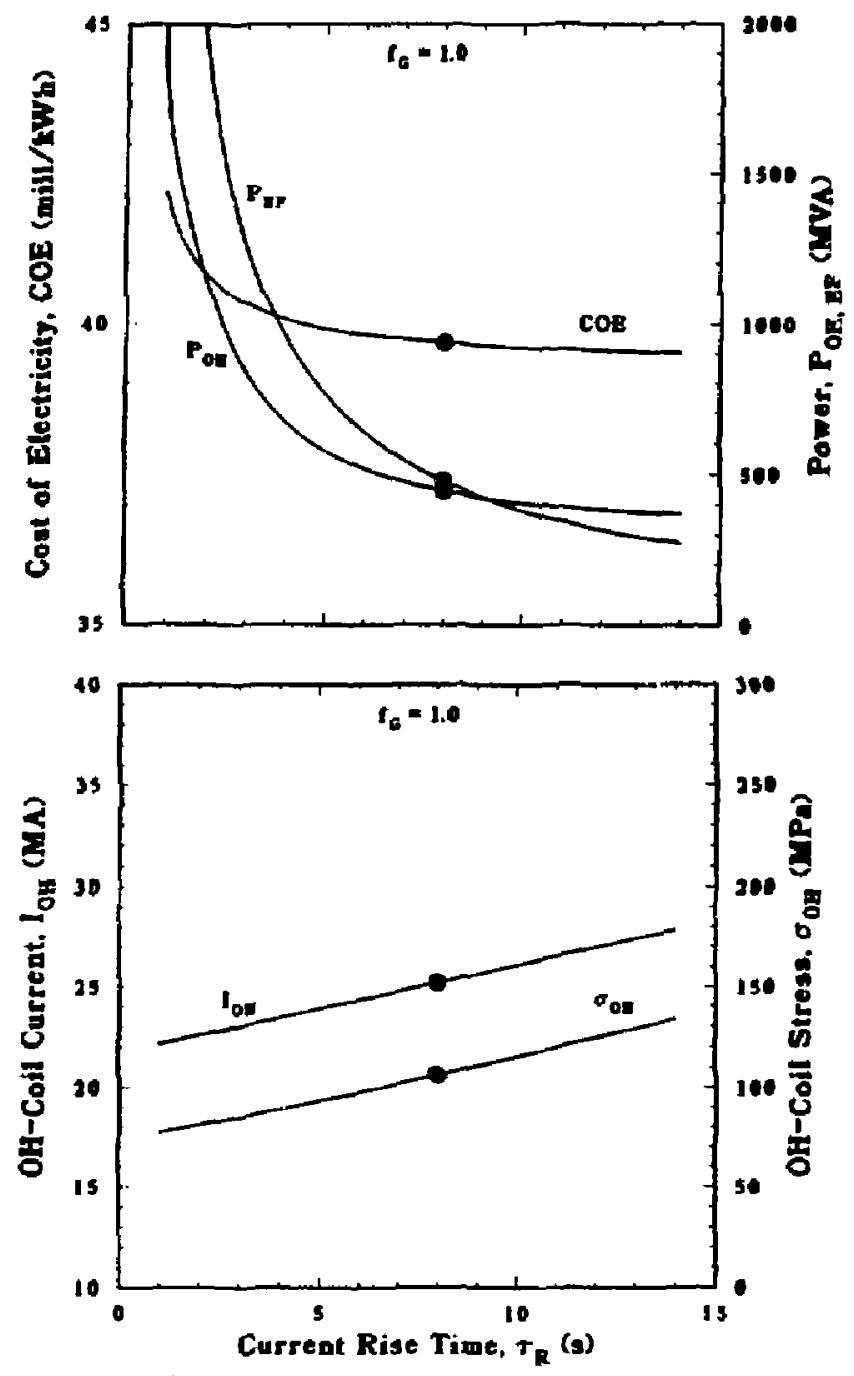

Figure 3.4-8. Dependence of COE and main design parameters (PF-coil puwer, OHcoil current and stress) on resistive poloidal flux consumption for the TITAN-I design expressed in terms of nominal current rise time for a symmetric back-biased flux swing $\left(f_{G}=1.0\right)$. The TITAN-I reference design is denoted by a filled citcle at $\tau_{R}=8 \mathrm{~s}$. 

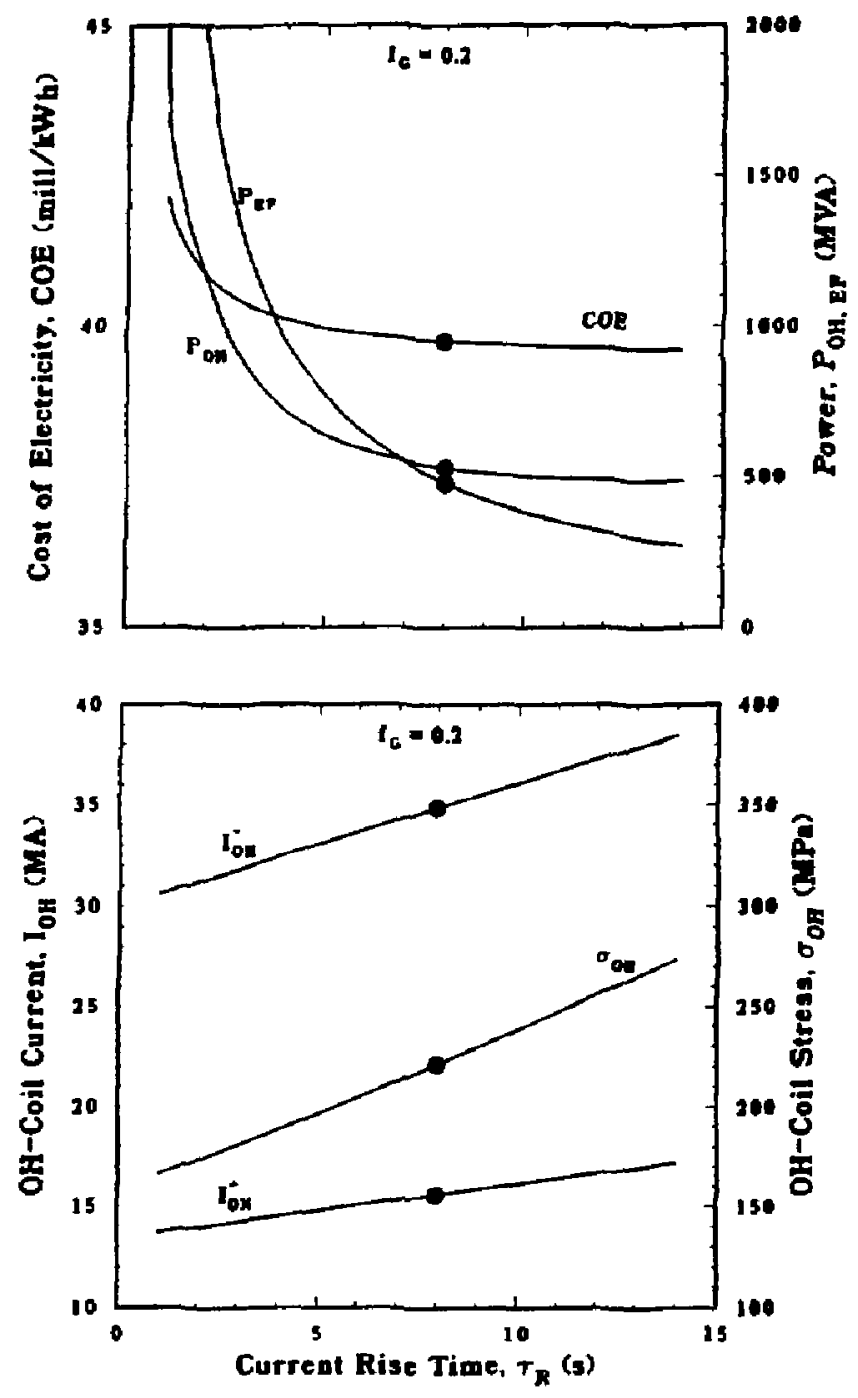

Figure 3.4-10. Dependence of COE and main design parameters (PF-coil power, $\mathrm{OH}$ coil current and stress) on resistive poloidal flux consumption for the TITAN-I design expressed in terms of nominal current rise time for an asymmetric back-biased flux swing $\left(f_{G}=0.2\right.$ ). The TITAN-I reference design is denoted by a filled circle at $\tau_{R}=8 \mathrm{~s}$. 
$Q_{P S} \simeq 50$, however, the OFCD system becomes inefficient and the recirculating power fraction, $\epsilon=1 / Q_{E}$, begins to incresse significantly. As a result, higher values of neutron wall load, $I_{w}$, is needed to maintain $P_{E}=970 \mathrm{MWe}$ and higher values of COE as $p_{s}$ dropa below 0.76 ( $c f$. Figure 3.2-5).

Generally, the TITAN-I design point is fairly robust and insensitive to perturbation in values of key parameters, whether physics or engineering. Most such choices are firily conservative and are grounded in experimental performance (Section 3.3) or engineering practice. Incorporation of the IBC option for the TITAN-I divertor coils introduces a significant recirculating porrer contribution, which is offset by the negligible joule losses of the superconducting EF-coil set, in contrast te the all-copper-coil GRFPR. The impact of using expensive $(250 \mathrm{~s} / \mathrm{kg}) \mathrm{V}$-alloy structures in the TITAlN-I FPC for safety and environmental reasons is mitigated by the small physical size and impact of the FPC on total cost.

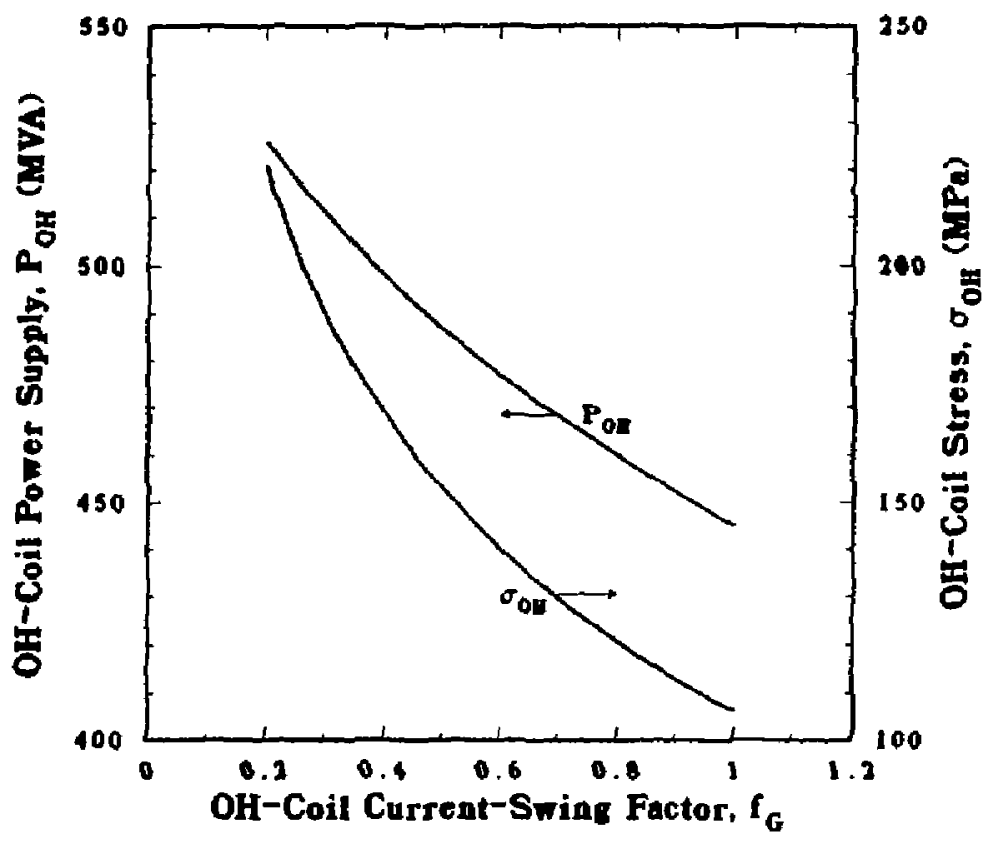

Figure 3.4-11. Dependence of OH-coil hack-bias stress and OH-coil powersupply requirement on the $O H$-coil current-swing symmetry factor, $f_{G} \equiv I_{O H}^{+} / I_{O H}^{-}$, for TITAN-I. 

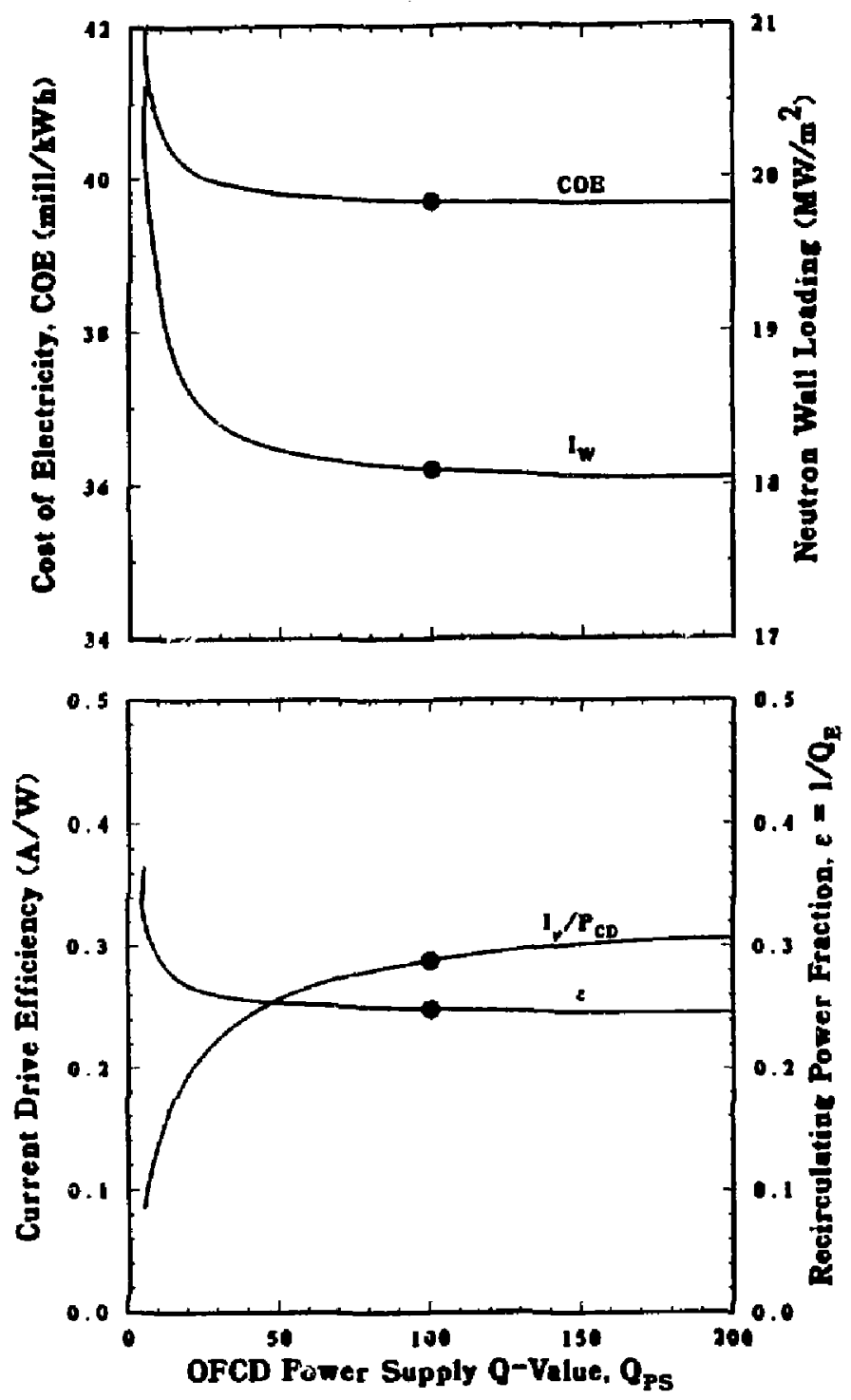

Figure 3.4-12. Impact of OFCD power supply Q-value on TITAN-I operating paranleters. The TITAN-I reference case is denoted by a filled circle at $Q_{P S}=100$. 


\subsection{TITAN-II DESIGN POINT}

\subsubsection{Design-Point Selection}

The TITAN-II FPC design is an aqueous loop-in-pool concept with a dissolved Li salt $\left(\mathrm{LiNO}_{3}\right)$ as the breeder, a $\mathrm{Be}$ neutron multiplier, and a reduced activation, highstrength ferritic steel 9-C [39] as the structural material. The complete FPC and the primary coolant system are submerged in a pool of low-temperature, low-pressure water to achieve passive safety. The TITAN-II pool configuration, therefore, provides an aiternative approach to inlzerently safe operation of an RFP reactor, but does not require a significantly different plasma performance or FPC configuration. The TITAN-I IBC configuration is replaced by a more conventional blanket, hot-shield, TF-coil configuration in TITAN-II.

The full coil optimization procedure of the PSA code (Section 3.1) applies to the TITAN-II FPC configuration. The overall coil thickness, $\delta_{c}$, including the copper TF-coil thickness, $\delta_{T F}$, followed by a gap thickness, $g_{T F / O F}$, and the homogenized $O H$-coil-set thickness, $\delta_{O H}$. The overall coil thickness is varied to obtain a minimum-COE design point, trading the cost of the recirculating power (joule dissipation) in the TF-coil set. with the direct cost of the FPC. The dissipated power in the TF-coil set, PTF, decreases monotonically as the $T F$-coil thickness, $\delta_{T F}$, is ailowed to increase and the current density, $j T F$, decreases for relatively fixed values of $I_{T F}$ and $B_{\phi}=\mu_{0} I_{T F} i\left(2 \pi R_{T}\right)$.

The TITAN-II plasma aspect ratio, $A$, is retained at 6.5 to facilitate comparison with TITAN-i. As a function of minor plasma radius, $r_{p}$, the minimum-COE design point occurs at $r_{p}=0.575 \mathrm{~m}$ and corresponding ueutron wall $\mathrm{lcad}, I_{w}=19.58 \mathrm{MW} / \mathrm{m}^{2}$, for a fixed net power output, $P_{\varepsilon}=900 \mathrm{MWe}$. It was decided to nove slightly of of the minimum-COE design point to $r_{p}=0.60 \mathrm{~m}$ (the same value as used for TITAN-I, which is itself slightly off of its own minimum-COE design point) in order to obtain nearly equal values of $I_{w} \simeq 18 \mathrm{MW} / \mathrm{m}^{2}$ and corresponding surface heat fluxes for both TITAN-I and TITAN-II. It is not possible to simultaneously achieve equal values of net power output, $P_{E}$ (970 MWe for TITAN-I and $900 \mathrm{MWe}$ for TITAN-II), while fixing the FPC: geometry because of the differences in power flows between the two TITAN reactor embodinents. The dependence of COE on coil thickness is illustrated on Figure 3.5-1 for both the minimum-COE $\left(r_{p}=0.575 \mathrm{~m}\right)$ and near-minimum-COE TITAN-11 reference case $\left(r_{p}=0.60 \mathrm{~m}\right)$. The PSA code searches to find the total coil thickness, $\delta_{c}$, and partition, $\delta_{T F} / \delta_{O H}$, that minimizes the overall COE. For each value of $r_{p}$, there exists a siuilar, but shifted, curve of COE versus $\delta_{c}$. The greatly expanded COE scale of Figure 3.5-1 exaggerates the sharpness of the minima of these curves. 
Table 3.5-I.

SUMMARY OF TITAN-II REACTOR DESIGNS ${ }^{(a, b)}$

\begin{tabular}{|c|c|c|}
\hline Parameter & With IHX(c) & Without IHX ${ }^{(c)}$ \\
\hline EF-coil option & $S C^{(d)}$ & $S C^{[d]}$ \\
\hline DF-coil option & Copper & Copper \\
\hline TF-coil option & Copper & Copper \\
\hline \multicolumn{3}{|l|}{ Plasma Parameters } \\
\hline Plasina current, $I_{\phi}(M A)$ & 17.80 & 17.80 \\
\hline Plastra ion density, $n_{i}\left(10^{20} \mathrm{~m}^{-3}\right)$ & 8.91 & 8.91 \\
\hline Plasma electron density, $n_{e}\left(10^{20} \mathrm{~m}^{-3}\right)$ & 9.31 & 9.31 \\
\hline Poloidal field at plasma surface, $B_{\theta}\left(r_{p}\right)(T)$ & 5.93 & 5.93 \\
\hline Thernal diffusivity, $\chi_{E}\left(\mathrm{~m}^{2} / \mathrm{s}\right)$ & 0.314 & 0.314 \\
\hline Fusion power density, $P_{F} / V_{p}\left(\mathrm{MW} / \mathrm{m}^{3}\right)$ & 82,6 & 82.6 \\
\hline Plasma ohmic dissjpation, $P_{O H M}(\mathrm{MW})$ & 28.5 & 28.5 \\
\hline
\end{tabular}

\section{Poloidal-Field Quantities}

OH-coil thickness, $\delta_{\mathrm{OH}}(\mathrm{m})$

Average minor radius of coil, $\mathrm{rOH}_{\mathrm{O}}(\mathrm{m})$

OH-coil field, $B_{\theta c}(T)$

$\begin{array}{ll}0.38 & 0.37 \\ 1.37 & 1.37 \\ 2.60 & 2.61 \\ 9.1 & 9.2\end{array}$

$O H$-coil current density, jOH $\left(\mathrm{MA}_{\mathrm{A}} / \mathrm{m}^{2}\right)^{(e)}$

420.

Mass of OH-coil set, $M_{O H}$ (tonne)

$20 . E^{(d)}$

414.

EF-coil current density, $j_{E F}\left(\mathrm{MA} / \mathrm{m}^{2}\right)$

247.

$20.5^{(d)}$

Mass of EF-coil set, $M_{E F}$ (toune)

Poloidal-field stored energy, $W_{M \theta}$ (GJ)

4.1

247.

OH-coil dissipation during back-bias (MW)

106.

4.1

$10 \%$.

\section{Toroidal-Field Quantities}

TF-coil thickness, $\delta_{T F}(\mathrm{~m})$

0.047

0.046

Average minor radius of coil $r_{T F}(\mathrm{~m})$

1.11

1.11

Mass of TF-coil set, $M_{T F}$ (tome)

41.

41.

Reversed toroidal field, $-B_{\phi N}(\mathrm{~T})$

Toroidal-field stored energy, $W_{M \phi}$ (GJ)

0.381

0.381

0.43

0.43

$\mathrm{TF}$-coil current density, jTF $\left(\mathrm{MA} / \mathrm{m}^{2}\right)$

9.1

9.1

Olunic dissipation during burn, $P_{T F}^{\Omega}$ (MW)

13.4

13.6

Mass of DF-coil set, $M_{D F}$ (tome)

2.0

2.0

Ohmic dissipation in divertor, $P_{D F}^{\cap}$ (MW)

12.

12. 
Table 3.5-I (Cont'd)

\begin{tabular}{|c|c|c|}
\hline Parameter & With IHX ${ }^{(c)}$ & Witlout IHX(c) \\
\hline \multicolumn{3}{|l|}{ Engineering Summary } \\
\hline Neutron first-wall loading, $I_{\omega}\left(\mathrm{MW} / \mathrm{m}^{2}\right)$ & 18.0 & 18.0 \\
\hline Engineering $Q$-value, $Q_{E}=1 / \epsilon$ & 7.22 & 7.20 \\
\hline Fusion power, $P_{F}(M W)$ & 2,289 . & 2,290 . \\
\hline Total thermal power, $P_{T^{\prime} H}(\mathrm{MW})$ & 2,985 . & 2,986 . \\
\hline Net electrical power output, $P_{E}$ (MWe) & 900. & 900 . \\
\hline Fusion-power-core minor radius, $r_{\mathrm{a}}(\mathrm{m})$ & 1.56 & 1.5 \\
\hline \multicolumn{3}{|l|}{ Masses (tonne) } \\
\hline . First wall and blanket & 21. & 21. \\
\hline . OH-coil "hot shield" & 106. & 106. \\
\hline - EF-coil shield & 287. & 286. \\
\hline - Total coil set & 711. & 704. \\
\hline - Total fusion power $\operatorname{core}^{(f)}$ & 1,125 & 1,117 . \\
\hline FPC power delisity, $P_{T H} / V_{F P C}\left(\mathrm{MWt} / \mathrm{m}^{3}\right)$ & 16.0 & 16.1 \\
\hline Mass power density, MPD (kWe/tonne) ${ }^{(f)}$ & 800. & 806. \\
\hline \multicolumn{3}{|l|}{ Cost Summary } \\
\hline Cost of electricity, COE (mill $/ \mathrm{kWh})^{(s)}$ & 40.2 & 38.0 \\
\hline Unit direct cost, UDC $(\$ / \mathbf{k W e})$ & 1,635 . & 1,543 \\
\hline Total cost, TC (M\$) & 2,347 . & 2,488 . \\
\hline FPC unit cost $(\$ / \mathrm{kg})$ & 118. & 119. \\
\hline \multicolumn{3}{|l|}{ Fractions of total direct cost (TDC): } \\
\hline - Reactor plant equipment, RPE/TDC & 0.51 & 0.48 \\
\hline - Fusion-power-core cost, FPC/TDC $(f)$ & 0.09 & 0.10 \\
\hline
\end{tabular}

(a) All designs are for baseline parameters given in Table 3.4-I:

$A=6.5, R_{T}=3.9 \mathrm{~m}, r_{p}=0.60 \mathrm{~m}, V_{p}=27.7 \mathrm{~m}^{3}, r_{w}=0.66 \mathrm{~m}$.

(b) $M_{N}=1.36$, $\eta_{T H}=0.35$, and water detritiation system cost $\simeq 140 \mathrm{MIS}$.

(c) Intermediate lheat exchanger (IHX) loop

(d) Superconducting coils.

(e) Symmettic bipolar swing ( $f_{G} \equiv I_{O H}^{+} / I_{O H}^{-}=1$ ), $I_{O f}^{+}$subsequently decays to zero upon initiation of OFCD.

(f) Includes first wall, blanket, shield, and coils, but not FPC support structures.

(g) Costs reported in constant 1986-dollars, assuming 6 years construction time. 
Two TITAN-II options are summarized on Table 3.5-I, the first including an intermedjate heat exchange (IHX) loop and the second without it. Because of the significant cost savings and because of no significant adverse consequences on the area of tritium removal and safety (Section 16, 18, and 19), the second option was sejected as the TITAN-II reference design. The deletion of the IHX system increnses the relative impact of the FPC on the tatal capital cost, resulting in a small reduction in the optimal coil thickness and changes in other parameters leading to a slightly different near-minimum-COE configuration.

Plasma parameters for TITAN-II are quite close to those of TITAN-I so some of the plasme engineering effort has not been duplicated. Magnetic-divertor performance (Section 4) and OFCD results (Section 7) are modified somewhat by the influence of a differeat coil set.

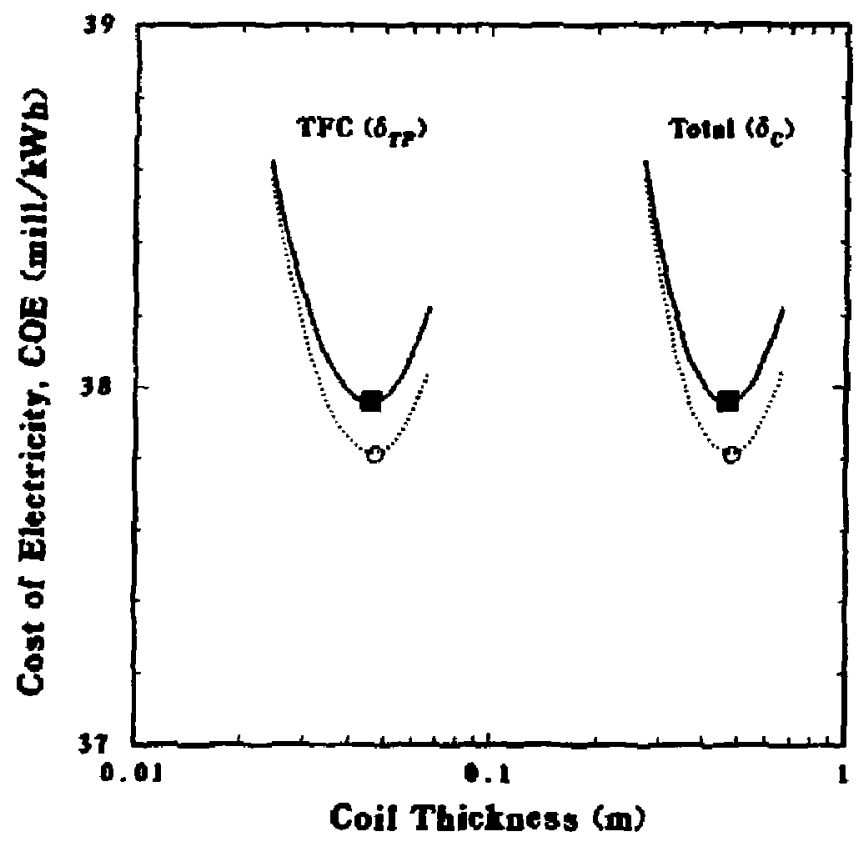

Figure 3.5-1. Dependence of COE on coil thickness at a fixed net power output, $P_{E}=900 \mathrm{MWe}$. The minimum-COE case $\left\langle r_{p}=0.575 \mathrm{~m}\right.$ and $\left.I_{w}=19.58 \mathrm{MW} / \mathrm{m}^{2}\right)$ is denoted by an open circle. The TITAN-II nearminimum-CrE reference case $\left(r_{p}=0.60 \mathrm{~m}\right.$ and $\left.I_{w}=18.0 \mathrm{MW} / \mathrm{m}^{2}\right)$ is shown as filled square. 


\subsubsection{Trade-off and Sensitivity Studies}

Because the TITAN-I and TITAN-II embodiments share sinilar plasma configurations, it is not necessary to repeat the temperature, poloidal beta, and reversal parameter variations considered in Section 3.4.2. The rationale of the cloice of a superconducting EF-coil set for TITAN-1 remains valid for TITAN-II altlough it is significantly weakened by the reduced lost power in the TITAN-II copper-alloy divertor coil set $(\sim 12 \mathrm{MW})$ relative to the divertor IBC selected' $r$ TITAN-l whicl consumes $\left(1-\eta_{T H}\right) \times 145=81 \mathrm{MW}$.

Several representative TITAN-II design options are sumnarized in Table 3.5-II. Case A includes a copper EF-coil set similar to the TITAN-1 Case A of Table 3.4-II. The large water-detritiation-system cost of TITAN-II $(\sim 140 \mathrm{M} \$)$ results in a $\sim 2 \mathrm{mill} / \mathrm{kWh}$ COE penalty, offsetting the cost savings of lower FPC blanket and shield costs (9-C versus $V$ alloy). Case $B$ incorporates a superconducting EF-coil set and an exaggerated gap between TF and OH coil sets, $g_{T F / O H}=0.31 \mathrm{~m}$. For this case, the TITAN-II OHcoil set is nearly coincident with that of TITAN-I, substituting a water-filled gap for part of the thicker blanket and shield annulus of TITAN-I. A penalty associated with this option is less-than-ideal coupling of the $\mathrm{CH}$-coil set to the plasma and higher COE relative to the reference case, so this option was de-emphasized. Case C includes a nominal gap, $g_{T F / O H}=0.05 \mathrm{~m}$ and an asymmetric $\mathrm{OH}$-coil current swing characterized by $f_{G} \equiv I_{O H}^{+} / I_{O H}^{-}=0.2$. The TITAN-II reference case, with the lowest COE projection of these four options, has a symmetric OH-coil swing $\left(f_{G}=1.0\right)$.

\subsection{SUMMARY AND CONCLUSIONS}

The operating space of a compact RFP reactor has been examined using a comprehensive parametric systems model which includes the evolving state of knowledge of the physics of RFP confinement and embodies the TITAN-I and TITAN-II engineering approaches. Two key figures of merit, the cost of electricity (COE) and mass power density (MPD), are monitored by the parametric systems model, both of which are displayed in Figure 3.6-1 as functions of the neutron wall loading. Figure 3.6-1 slows that the COE is relatively insensitive to wall loadings in the range of 10 to $20 \mathrm{MW} / \mathrm{m}^{2}$ with a sliallow nimimum at about $19 \mathrm{MW} / \mathrm{m}^{2}$. Mass power density is found to increase monotonically with the wall load. For desigus with a neutron wall load larger than about $10 \mathrm{MW} / \mathrm{m}^{2}$, the FPC is physically small enough such that single-piece FPC maintenance is feasible. These considerations point to a design window for compact RFP reactors with loading in the range of 10 to $20 \mathrm{MW} / \mathrm{m}^{2}$. The TITAN-class RFP reactors in this design window 
Table 3.5-II.

SUMMARY OF TITAN-II REACTOR DESIGNS ${ }^{(a, b)}$

\begin{tabular}{|c|c|c|c|c|}
\hline Parameter & Case $A^{(c)}$ & Case $\mathrm{B}^{(\mathrm{d})}$ & Case $\mathrm{C}^{(c, e)}$ & Ref. Case ${ }^{(f)}$ \\
\hline EF-coil option & Copper & $\mathrm{SC}^{(g)}$ & $\mathbf{S C}(g)$ & $\mathrm{SC}^{(g)}$ \\
\hline DF-coil option & Copper & Copper & Copper & Copper \\
\hline TF-coil option & Copper & Copper & Copper & Copper \\
\hline \multicolumn{5}{|l|}{ Plasma Parameters } \\
\hline Plasma current, $I_{\phi}(M A)$ & 17.89 & 17.83 & 17.82 & 17.80 \\
\hline Plasma ion density, $n_{i}\left(10^{20} \mathrm{~m}^{-3}\right)$ & 9,00 & 8.94 & 8.93 & 8.91 \\
\hline Plasma electron density, $n_{e}\left(10^{20} \mathrm{~m}^{-3}\right)$ & 9.40 & 9.34 & 9.33 & 9.31 \\
\hline Poloidal field at plasma surface, $B_{\mathcal{O}}\left(r_{p}\right)(\mathrm{T})$ & 5.96 & 5.94 & 5.94 & 5.93 \\
\hline Thennal diffusivity, $\chi E\left(\mathrm{~m}^{2} / \mathrm{s}\right\}$ & 0.317 & 0.315 & 0.315 & 0.314 \\
\hline Fusion power deusity, $\boldsymbol{P}_{\boldsymbol{F}} / V_{\mathrm{p}}\left(\mathrm{MW} / \mathbf{m}^{3}\right)$ & 84.3 & 83.2 & 83.0 & 82.6 \\
\hline Plasma olunic dissipation, PoHm (MW) & 28.7 & 28.6 & 28.5 & 28.5 \\
\hline \multicolumn{5}{|l|}{ Poloidal-Field Quantities } \\
\hline OH-coil thickness, $\delta_{O H}(m)$ & 0.22 & 0.31 & 0.44 & 0.37 \\
\hline A verage minor radius of coil, roH (m) & 1.27 & 1.59 & 1.39 & 1.37 \\
\hline OII-coil field, $B_{\theta c}(\mathrm{~T})$ & 2.82 & 2.25 & 2.56 & 2.61 \\
\hline OH-coil current density, joH $\left(\mathrm{MA} / \mathrm{m}^{2}\right)$ & $16.1^{(f)}$ & $11.4^{(f)}$ & $10.8^{(e)}$ & $9.2^{(f)}$ \\
\hline Mass of $O H$-coil set, $M_{O H}$ (tonue) & 226. & 403. & 500. & 414. \\
\hline EF-coil current density, $j E F\left(\mathrm{MA} / \mathrm{m}^{2}\right)$ & 4.6 & $20.2^{(\theta)}$ & $20.4^{(s)}$ & $20.6^{(s)}$ \\
\hline Mass of EF-coil set, $M_{E F}$ (tonne) & 674. & 259. & 251. & 247. \\
\hline Poloidal-field stored energy, $W_{M \theta}(G J)$ & 1.4 & 4.4 & 4.2 & 4.1 \\
\hline OH-coil dissipation during back-bias (MW) & 181. & 153. & 176 & 107. \\
\hline \multicolumn{5}{|l|}{ Toroidal-Field Quantities } \\
\hline TF-coil thickuess, $\delta_{T F}(\mathrm{nn})$ & 0.026 & 0.037 & 0.039 & 0.046 \\
\hline Average nunor radius of coil, $r_{T F}(\mathrm{~m})$ & 1.10 & 1.10 & 1.10 & 1.11 \\
\hline Mass of TF-coil set, $M_{T F}$ (tonue) & 23. & 33. & 35. & 41. \\
\hline Reversed-toroidal field, $-\boldsymbol{B}_{\phi R}(\mathrm{~T})$ & 0.383 & 0.382 & 0.382 & 0.381 \\
\hline Toroidal-field stored energy, $W_{M d \phi}$ (GJ) & 0.43 & 0.43 & 0.43 & 0.43 \\
\hline TF-coil current density, $j_{2} F\left(\mathrm{MA} / \mathrm{m}^{2}\right)$ & 16.1 & 11.4 & 10.8 & 9.2 \\
\hline Olmuic dissipation during burn, $p_{T F}^{n}(\mathrm{MW})$ & 23.6 & 16.8 & 15.9 & 13.6 \\
\hline Mass of DF-coil set, $M_{D F}$ (tonne) & 2.0 & 2.0 & 2.0 & 2.0 \\
\hline Olunic dissipation in divertor, $P_{D F}(M W)$ & 12. & 12. & 12. & 12. \\
\hline
\end{tabular}


Table 3.5-II (Cont'd)

\begin{tabular}{|c|c|c|c|c|}
\hline Parameter & Case $A^{(c)}$ & Case $B^{(d)}$ & Case $C^{(c, e)}$ & Ref. Case $(f)$ \\
\hline \multicolumn{5}{|l|}{ Engineering Summary } \\
\hline Neutron first-wall loading, $I_{w}\left(\mathrm{MW} / \mathrm{m}^{2}\right)$ & 18.4 & 18.1 & 18.1 & 18.0 \\
\hline Eagineering $Q$-value, $Q_{E}=1 / \epsilon$ & 6.41 & 6.92 & 7.00 & 7.20 \\
\hline Fusion power, $P_{F}(\mathrm{MW})$ & $2,336$. & $2,305$. & 2,300 & 2,290 \\
\hline Total thertnal power, $P_{T H}(\mathrm{MW})$ & 3,047 & 3,006 & 3,000 & $2,986$. \\
\hline Net etrical power output, $P_{E}(\mathrm{MWe})$ & 900. & 900. & 900. & 900. \\
\hline Fusion-power-core minor radius, $r_{e}(\mathrm{~m})$ & 1.38 & 1.74 & 1.70 & 1.55 \\
\hline \multicolumn{5}{|l|}{ Masses (tonne) } \\
\hline - First wall and blanket & 21. & 21. & 21. & 21. \\
\hline . OH-coil "lnot shield" & 91. & 91. & 106. & 106. \\
\hline - EF-coil shield & 0. & 295. & 289. & 286. \\
\hline - Total coil set & 926. & 697. & 787. & 704. \\
\hline - Total fusion power core ${ }^{(h)}$ & 1,053 & 1,119 & 1,204 & $1,11 \pi$ \\
\hline FPC power density, $P_{T H} / V_{F P C}\left(\mathrm{MWt} / \mathrm{m}^{3}\right)$ & 20.8 & 12.9 & 15.0 & 16.1 \\
\hline Mass power density, $M P D(\mathrm{kWe} / \text { tonne })^{(h)}$ & 854. & 804. & 747. & 806. \\
\hline \multicolumn{5}{|l|}{ Cost Summary } \\
\hline Cost of electricity, COE (mill/kWh) $)^{(i)}$ & 39.8 & 38.4 & 38.5 & 38.0 \\
\hline Unit direct cost, UDC $(3 / k$ We $)$ & 1,617 . & $1,561$. & 1,563 & $1,543$. \\
\hline Total cost, TC (M\$) & 2,608 . & $2,267$. & 2,269 & 2,239 \\
\hline FPC unit cost $(\$ / \mathbf{k g})$ & 118. & 119. & 116. & 119. \\
\hline \multicolumn{5}{|l|}{ Fractions of total direct cost (TDC): } \\
\hline - Reactor plant equipment, RPE/TDC & 0.50 & 0.48 & 0.49 & 0.48 \\
\hline - Fusion-power-core cost, FPC/TDC ${ }^{(h)}$ & 0.09 & 0.10 & 0.10 & 0.10 \\
\hline
\end{tabular}

(a) All designs are for baseline parameters given in Table 3.4-I:

$A=6.5, R_{T}=3.9 \mathrm{~m}, r_{p}=0.60 \mathrm{~m}, V_{p}=27.7 \mathrm{~m}^{3}, r_{w}=0.66 \mathrm{~m}$.

(b) $M_{N}=1.36,7 r H=0.35$, and water detritiation system cost $\simeq 5 \mathrm{MS}$.

(c) Gap between TF coil and OH coil, $G_{T F / O H}=0.05 \mathrm{~m}$.

(d) Gap between $\mathrm{TF}$ coil and $\mathrm{OH}$ coil, $G_{T F / O H}=0.31 \mathrm{~m}$.

(e) Asymmetric bipolar swing $\left(f_{G} \equiv I_{O H}^{+} / I_{O H}^{-}=0.2\right)$,

$I_{O H}^{+}$subsequently decays to zero upon initiation of OFCD.

(f) Symmetric bipolar swing $\left(f_{G} \equiv I_{O H}^{+} / I_{O H}^{-}=1\right)$.

(g) Superconducting coils.

(h) Includes first wall, blanket, shield, and coils, but not FPC support structures.

(i) Costs reported in constant 1986-dollars, assuming 6 years construction time. 


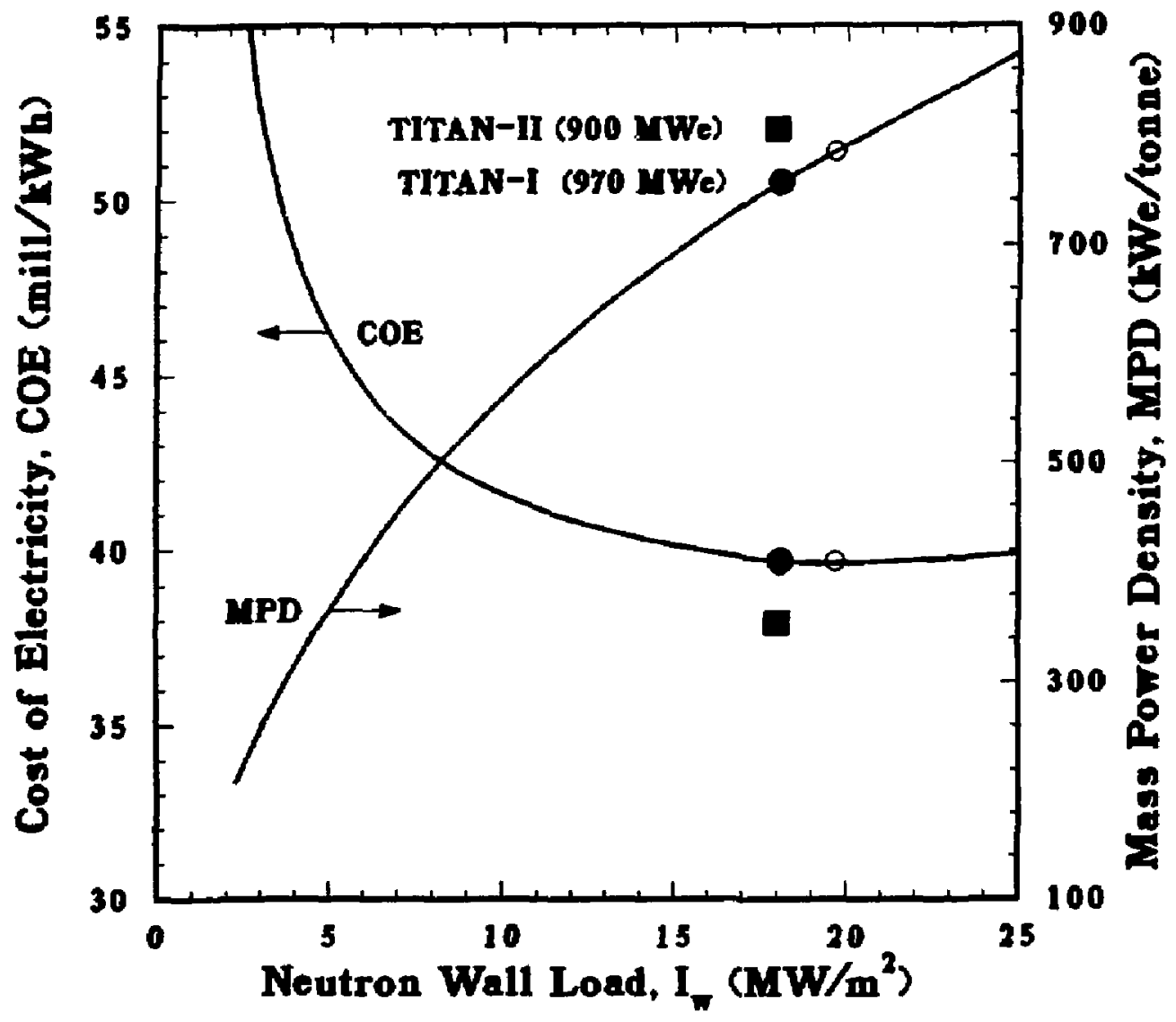

Figure 3.6-1. The COE and mass power density as functions of neutron wall load for the TITAN RFP reactor. Minimum-COE TITAN-I design point is indicated as an open circle. The near-minimum-COE reference design points for TITAN-I (filled circle) and TITAN-II (filled square) are also shown. 
have a MPD in excess of $500 \mathrm{KWe} /$ tonne, and a FPC engineering power density in the range 5 to $15 \mathrm{MWt} / \mathrm{m}^{3}$, representing inprovements by factors of 10 to 30 compared witl earlier fusion reactor designs. The FPC is a smaller portion of the total plant cost (typically about $12 \%$ ) conpared with $25 \%$ to $30 \%$ for earlier RFP designs [41,42]. Therefore, the unit direct cost, UDC $(\$ / \mathrm{kWe})$, is less sensitive to related physics and technology uncertainties.

The reference TITAN design points have been identified at a high neutton wall londing of $I_{w} \simeq 18 \mathrm{MW} / \mathrm{m}^{2}$ with $A=6.5, r_{p}=0.60 \mathrm{~m}$, and net power outputs of $970 \mathrm{MWe}$ (TITAN-I) and $900 \mathrm{MWe}$ (TITAN-II). The TITAN reference designs are summarized in Table 3.6-I. Previously reported TITAN-I results $[43,44]$ are updated and superceded. Detailed subsystem design for TITAN-I and TITAN-II FPCs are reported throughout this report. The parameters of the TITAN-I and TITAN-Il reference design points, based on detailed subsystem design, are included in Appendices $A$ and $B$, respectively, following the DOE/OFE standard reporting format. The Appendices also include detailed cost tables and paranetric systems code predictions of subsystem parameters for comparison with DOE/OFE tables. 
Table 3.6-I.

SUMMARY OF REFERENCE DESIGNS OF TITAN REACTORS ${ }^{(\star)}$

\begin{tabular}{|c|c|c|}
\hline Paranneter & TITAN-I ${ }^{(b)}$ & TI'TAN-II (c) \\
\hline EF-coil option & $s C^{(d)}$ & $S C^{(d)}$ \\
\hline DF-coil option & $\operatorname{IBC}(e)$ & Copper \\
\hline TF-coil option & $\mathrm{IBC}^{(\mathrm{e})}$ & Copper \\
\hline
\end{tabular}

\section{Plasma Parameters}

Plaswa current, $I_{\phi}(M A)$

17.82

17.80

Plasma ion density, $n_{i}\left(10^{20} \mathrm{~m}^{-3}\right)$

8.93

8.91

Plasma electron density, $n_{\varepsilon}\left(10^{20} \mathrm{~m}^{-3}\right)$

$9.33 \quad 9.31$

Poloidal field at plasma surface, $B_{\theta}\left(r_{p}\right)(\mathrm{T})$

5.94

5.93

Thermal diffusivity, $\chi_{E}\left(\mathrm{~m}^{2} / \mathrm{s}\right)$

0.315

0.314

Fusion power density, $P_{F} / V_{p}\left(M W / \mathrm{m}^{3}\right)$

83.0

82.6

Plasma ohnic dissipation, POHM (MW)

28.5

28.5

\section{Poloidal-Field Quantities}

OH-coil thickness, $\delta_{\mathrm{OH}}(\mathrm{m})$

$$
0.27
$$

$0.3 \overline{7}$

A.verage minor radius of coil, $r_{O H}(\mathrm{~m})$

1.56

1.37

OH-coil field, $B_{\theta_{c}}(\mathrm{~T})$

2.28

2.61

OH-coil current density, joH (MA/ $\left./ \mathrm{m}^{2}\right)^{(f)}$

13.1

9.2

Mass of $O H$-coil set, $M_{O H}$ (tonne)

343.

414.

EF-coil current density, $j E F\left(\mathrm{MA} / \mathrm{m}^{2}\right.$ )

$19.2^{(d)}$

$20.6^{(d)}$

Mass of EF-coil set, $M_{E F}$ (tonne)

305.

247.

Poloidal-field stored energy, $W_{\text {MI }}$ (GJ)

OH-coil dissipatjon during back-bias (MW)

\section{2}

4.1

121.

107.

\section{Toroidal-Field Quantities}

TF-coil thickness, $\delta_{T F}(\mathrm{~m})$

$0.28^{(e)}$

0.046

Average minor radius of coil, $r_{T F}(\mathrm{~m})$

0.68

1.11

Mass of TF-coil set, $M_{T F}$ (tome)

41.

41.

Reversed-toroidal field, $-B_{\phi R}(T)$

0.38

0.381

Toroidal-fiejd stored energy, $W_{M S_{\phi}}$ (GJ)

TF-coil current density, jTF $\left(\mathrm{MA} / \mathrm{m}^{2}\right)$

0.16

0.43

Olunic dissipation during burn, $P_{T F}^{n}(\mathrm{MW})$

1.54

9.2

Mass of $D F$-coil set, $M_{D F}$ (tonne)

27.6

13.6

Olunic dissipation in divertor, $P_{D F}^{\Omega}$ (MW)

0.55

2.0

142.

12. 
Table 3.6-I (Cont'd)

\begin{tabular}{|c|c|c|}
\hline Parameter & TITAN-I $^{(b)}$ & TITAN-II ${ }^{(c)}$ \\
\hline \multicolumn{3}{|l|}{ Engineering Summary } \\
\hline Neutron first-wall loading, $I_{w}\left(\mathrm{MW} / \mathrm{m}^{2}\right)$ & 18.1 & 18.0 \\
\hline Engineering Q-value, $Q_{E}=1 / \epsilon$ & 4.02 & 7.20 \\
\hline Fusion power, $P_{F}$ (MW) & 2,301 . & 2,290 . \\
\hline Total thernnal power, $P_{T H}(\mathrm{MW})$ & 2,935 . & 2,986 . \\
\hline Net electrical power output, $P_{E}$ (MWe) & 970. & 900. \\
\hline Fusion-power-core nunor radius, $r_{s}(\mathrm{~m})$ & 1.70 & 1.55 \\
\hline \multicolumn{3}{|l|}{ Masses (tonne) } \\
\hline - First wall and blanket & 41. & 21. \\
\hline - OH-coil "hot shield" & 267. & 106. \\
\hline - EF-coil shield & 325. & 286. \\
\hline - Total coil set & 648. & 704. \\
\hline Total fusion power core $e^{(g)}$ & 1,282 & 1,117 . \\
\hline FPC power density, $P_{T H} / V_{F P S}\left(\mathrm{MWt} / \mathrm{m}^{3}\right)$ & 13.2 & 16.1 \\
\hline Mass power density, MPD (kWe/tonne $)^{(\theta)}$ & 757. & 806. \\
\hline \multicolumn{3}{|l|}{ Cost Summary } \\
\hline Cost of electricity, COE (mill/kWh) ${ }^{(h)}$ & 39.7 & 38.0 \\
\hline Unit direct cost, UDC ( $\$ / \mathbf{k W e})$ & $1, \mathbf{5 3 1}$ & $1,543$. \\
\hline Total cost, TC (M\$) & 2,396 . & 2,239 . \\
\hline FPC unit cost $(\$ / \mathrm{kg})$ & 146. & 119. \\
\hline \multicolumn{3}{|l|}{ Fractions of total direct cost (TDC): } \\
\hline - Reactor plant equipinent, RPE/TDC & 0.43 & 0.48 \\
\hline - Fusion-power-core cost, FPC/TDC & 0.13 & 0.10 \\
\hline
\end{tabular}

(a) All designs are for baseline parameters given in Table 3.4-I: $A=6.5, R_{T}=3.9 \mathrm{~m}, r_{p}=0.60 \mathrm{~m}, V_{p}=27.7 \mathrm{~m}^{3}, r_{w}=0.66 \mathrm{~m}$.

(b) $M_{N}=1.20, \eta_{T H}=0.44$, and water detritiation system cost $\simeq 5 \mathrm{M}$.

(c) $M_{N}=1.36, \eta_{T H}=0.35$, and water detritiation system cost $\simeq 140 \mathrm{M} \$$.

(d) Superconducting coils.

(e) Integrated blanket coil (IBC).

(f) Symmetric bipolar swing $\left(f_{G} \equiv I_{O H}^{+} / I_{O H}^{-}=1\right)$, $I_{O H}^{+}$subsequently decays to zero upon initiation of OFCD.

(g) Includes first wall, blanket, shield, and coils, but not FPC support structures.

(h) Costs reported in constant 1986-dollars, assuming 6 years construction time. 


\section{REFERENCES}

[1] R, L. Hagenson and R. A. Krakowski, "Compact Reversed-Field Pinch Reactors (CRFPR): Sensitivity Study and Designt-Point Determination," Los Alanos National Laboratory report LA-9389-MS (July 1982).

[2] R. L. Hagenson, R. A. Krakowski, C. G. Bathke, R. L. Miller, M. J. Enubrechts, et al., "Compact Reversed-Field Pinch Reactors (CRFPR): Preliminary Eugineering Considerations," Los Alamos National Laboratory report LA-10200-MS (August 1984).

[3] C. Copenhaver, R. A. Krakowski, N. M. Schnurr, R. L. Miller, C. G. Bathke, R. L. Hagenson! et al., "Compact Reversed-Field Pinch Reactors (CRFPR): FusionPower-Core Integration Study," Los Alamos National Laboratory report LA-10500MS (August 1985).

[4] R. L. Miller, G. G. Bathke, R. A. Krakowski, F. M. Heck, L. Green, J. S. Karbowski, et al., "The Modular Stellarator Reactor: A Fusion Power Plant," Los Alamos National Laboratory report LA-9737-MS (July 1983).

[5] R. L. Miller, R. A. Krakowski, C. G. Bathke, C. Copenhaver, N. M. Sclunurr, A. G. Engelhardt, T. J. Seed, and R. M. Zubrin, "Advanced Tokanıak Reactors Based on the Spherical Torus (ATR/ST)," Los Alamos National Laboratory report LA-10740-MS (June 1986).

[6] J. D. Callen and R. A. Dory, "Magnetohydrodynamic Equilibria in Sharply Curved Axisymmetric Devices," Phys. Fluids 15 (1972) 1523.

[7] D. J. Strickler, J. B. Miller, K. E. Rotlie, and Y-K. M. Peng, "Equilibrium Modeling of the TFCX Poloidal Field Coil System," Oak Ridge National Laboratory report ORNL/FEDC-83/10 (April 1984).

[8] H. M. Attaya and M. E. Sawan, "NEWLIT-A General Code for Neutron Wall Loading Distribution in Torojdal Reactors," Fusion Tectnol. 8 (1985) 609.

[9] R. D. O'Dell, F. W. Brinkley, Jr., and D. R. Marr, "User's Manual for ONEDANT: A Code Package for One-Dimensional Diffusion-Accelerated Neutral-Particle Tratsport," Los Alamos National Laboratory report LA-9184-M (February 1982).

[10] T. J. Seed, “TRIDENT-CTR User's Manual," Los Alamos Scientific Laboratory report LA-7835-M (May 1979). 
[11] J. F. Briesmeister, Ed., "MCNP. A General Monte Carlo Code for Neutron and Photon Transport, Version 3A," Los Alamos National Laboratory report LA-7396M, Rev. 2 (September 1986).

[12] A. K. Prinja and R. W. Conn, "An Axially Averaged Radial Transport Model of Tokamak Edge Plasma," J. Nucl. Mater. 128-129 (1984) 135.

[13] D. Steiner, R. C. Block, and B. K. Malaviya, "The Integrated-Blanket-Coil Concept Applied to the Poloidal Field and Blanket Systems of a Tokamak Reactor," Fusion Technol. 7 (1985) 66.

[14] N. Jarmie, R. E. Brown, and R. A. Hardenkopf, "Fusion-Energy Reaction ${ }^{2} H(t, \alpha) n$ from $E_{\mathrm{t}}=12.5$ to $117 \mathrm{keV}$, Phys. Rev. $C 29$ (1984) 2031; also relevant Erratum in Phys. Rev. C 33 (1986) 385.

[15] L. Woltjer, "A Theorem on Force-Free Magnetic Fie]ds," Proc. National Academy of Sciences 44 (1958) 489.

[16] J. B. Taylor, "Relaxation of Toroidal Plasma and Generation of Reversed Magnetic Field," Phys. Rev. Lett. 33 (1974) 1139.

[17] V. S. Mukhovatov and V. D. Shafranov, "Plasmua Equilibrium in a Tokamak," Nucl. Fision 11 (1971) 605.

[18] W. R. Smyth, Static and Dynamic Electricity, McGraw-Hill Book Company, NY (1939).

[19] J. Kessner and R. W. Conn, "Space-Dependent Effects on the Lawson and Ignition Conditions and Thermal Equilibria in Tokamaks," Nuc!. Fusion 16 (1976) 397.

[20] J. Sheffield, R. A. Dory, S. M. Cohn, J. G. Delene, L. Parsly, D. E. T. F. Ashiby, and W. T. Reiersen, "Cost Assessment of a Generic Magnetic Fusion Reactor," Fusion Techrol. 9 (1986) 199.

[21] S. Timoshenko and J. N. Goodier, Theory of Elasticity, 2nd edition, McGraw-Hill Book Company, Inc., New York (1951).

[22] R. W. Conn (Chairman), "Panel X Report to the USDOE Magnetic Fusion Advisory Committee," University of California Los Angeles (May 8, 1985).

[23] W. R. Hamilton, D. C. Keeton, and S. L. Thomson, "Cost Accounting System for Fusion Studies," Oak Ridge National Laboratory report ORNL/FEDC-85/7 (December 1985). 
[24] S. C. Schulte, T. L. Wilke, and J. R. Young, "Fusion Reactor Design StudiesStandard Accounts for Cost Estimates," Battelle Pacific Northwest Laboratory report PNL-2648 (May 1978).

[25] S. C. Schulte, W. E. Bickford, C. E. Willingham, S. K. Ghose, and M. G. Walker, "Fusion Reactor Design Studies - Standard Unit Costs and Cost Scaling Rules," Pacific Northwest Laboratory report PNL-2987 (Septemlber 1979).

[26] D. L. Phung, "A Method for Estimating Escalatiou' and Interest During Construction (EDC and IDC)," in Proc. 2nd Miami Conf. on Allernative Energy Sources, Miami, FL (December 10-13, 1979).

[27] S. L. Thomson, "Reference Cost Datá for Fusion," Oak Rijge National Laboratory report ORNL/FEDC-86-8.

[28] J. G. Delene, H. I. Bowers, and M. L. Meyers, "Nuclear Energy Clost Data Base," U. S. Department of Energy report DOE/NE-0041/3 (June 1985).

[29] J. D. Lee (Ed.), "MINIMARS Conceptual Design: Final Report," Lawrence Livermore Natiunal Laboratory repnrt UCID-20773 (Septemljer 1986).

[30] I. Maya, K. R. Schultz, R. F. Bourque, E. T. Cheng, R, L. Creedon, J. H. Norman, et al., "Lisertial Confinement Fusion Reaction Chamber and Power Conversion System Study," GA Technologies report GA-A 7842 (October 1985).

[31] J. P. Holdren (Chairman), "Summary of the Report of the Senior Committee on Environment, Safety, and Ecor omic Aspects of Magnetic Fusion Energy," Lawrence Livermore National Laboratory report UCRL-53766-Summary (September 1987).

[32] C. C. Baker, M. A. Abdou, R. ' 2. Arons, A. E. Bolon, C. D. Boley, J. N. Brooks, et al. "STARFIRE - A Commercial Tokanak Fusion Power Plant Study," Argonne National Laborato y report ANL/FPP-80-1 (September 1980).

[33] B. G. Logan, C. D. Henning, G. A. Carlson, R. W. Werner, D. E. Baldwin, W. L. Barr, et al., "MARS Mirror Advanced Reactor Study Final Report," Lawrence Livermors National Laboratory report UCRL-53480 (July 1984).

[34] B. Badger, I. N. Sviatoslavsky, S. W. Van Sciver, G. L. Kulcinski, C. A. Emmert, D. T. Anderson, et al., "UWTOR-M: A Conceptual Modular Stellarator Power Reactor," University of Wisconsin report UWFDM-550 (October 1982). 
[35] R. A. Krakowski, R. L. Hagenson, N. M. Schnurr, C. Copenhaver, C. G. Bathke, and R. L. Miller, "Fusion-Power-Core Integration Study for the Compact Reversed-Field Pinch Reactor (CRFPR): A Follow-On Study," Nucl. Eng. and Design 4 (1986) 75; also Los Alamos National Laboratory report LA-UR-85-3614.

[36] R. A. Krakowski, R. L. Miller, C. G. Bathke, R. L. Hagenson, C. Copenhaver, and K. A. Werley, "Compact Reversed-Field Pincl Reactors (CRFPR)," Proc of IAEA Technical Committee Merting and Workshop on Fusion Renctor Design and Technology, Yalta, USSR (May 29 - June 6, 1986) 1 (1986) 331; also Los Alamos National Laboratory report LA-UR-86-1229.

[37] M. K. Bevir and J. W. Gray, "Relaxation, Flux Consumption and Quasi Steacly State Pinches," Proc. RFP Theory Workshop, Los Alamos Natioual Laboratory April 28May 2, 1980, Los Alamos National Laboratory report LA-8944-C (January 1982) 176.

[38] K. F. Schoenberg, R. F. Gribble, and D. A. Baker, "Oscillating Field Current Drive for Reversed Field Pinch Discharges," J. Appl. Phys. 56 (1984) 2519.

[39] D. S. Geiles, N. M. Ghoniem, and R. W. Powell, "Low Activation Ferritic Alloys Patent Description," University of California Los Angeles report UICILA/ENG-87-9 PPG-1049 (1987).

[40] C. G. Bathke and R. A. Krakowski, "A Comparison Study of Toroidal-Field and Bundle Divertors for a Compact Reversed-Field Pincl Reaclor," Fusion Technol. 8 (1985) 1616 .

[41] R. A. Krakowski, R. I. Miller, and J. G. Deiene, "Directions for Inuproved Fusion Reactors," Proc. IAEA Technical Committee and Workshop on Fusion Reactor Design and Technology, Yalta, USSR (May 26-June $\widetilde{\cup}, 1986$ ) 1 (1986) 265; also Los Alamos National Laboratory report LA-UR-86-1386.

[42] R. A. Krakowski, R. L. Miller, and R. L. Hagenson, "Prospects for Improved Fusion Reactors," in Proc. sth European Nuclear Conference (ENC-86), (June 1-6, 1986); also Los Alamos National Laboratory report LA-UR-86-641.

[43] R. L. Miller, R. A. Krakowski, C. G. Batlıke, K. A. Werley, and R. L. Hagenson, "Fusion Reactor Options and Alternatives for the RFP," Fusion Technol. 10 (1986) 1159. 
[44] R. L. Miller, "The TITAN Reversed-Field Pinch Reactor: Design-Point Determuination and Parametric Studies," in Proc. 12th IEEE Symp. Fusion Engineering, Monterey, CA (October 12-16, 1987). 


\section{MAGNETICS}

Charles G. Bathke William P. Duggan Farrokh Najmabadi
William P. Kelleher

Don Steiner 


\section{Contents}

4.1. INTRODUCTION $\ldots \ldots \ldots \ldots \ldots \ldots \ldots \ldots \ldots \ldots \ldots$ 4.1

4.2. INTEGRATED-BLANKET-COIL (IBC) CONCEPT $\ldots \ldots \ldots \ldots$. 4-3

4.3. TOROIDAL-FIELD (TF) COILS $\ldots \ldots \ldots \ldots \ldots \ldots \ldots .4 .4$

4.3.1. Models and Constraints . . . . . . . . . . . 4-4

4.3.2. TITAN-I TF-Coil Design . . . . . . . . . . . . . . 4-10

4.3.3. TITAN-II TF-Coil Design . . . . . . . . . . . . . . 4-15

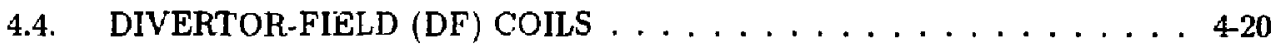

4.4.I. Models and Constraints $\ldots \ldots \ldots \ldots \ldots \ldots \ldots .4420$

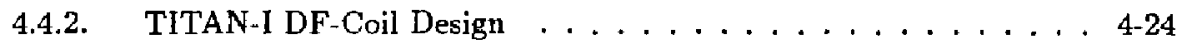

4.4.3. TITAN-II DF-Coil Design . . . . . . . . . . . . . 4-31

4.5. OHMIC-HEATING (OH) COILS $\ldots \ldots \ldots \ldots \ldots \ldots \ldots \ldots$

4.5.1. Models and Constraints $\ldots \ldots \ldots \ldots \ldots \ldots \ldots 4.32$

4.5.2. OH-Coil Configurations $\ldots \ldots \ldots \ldots \ldots \ldots \ldots \ldots$ 4.40

4.6. EQULIBRIUM-FIELD (EF) COILS $\ldots \ldots \ldots \ldots \ldots \ldots \ldots$. 4-59

4.6.1. Models and Constraints $\ldots \ldots \ldots \ldots \ldots \ldots \ldots$ 4.59

4.6.2. EF-Coil Design . . . . . . . . . . . . . . . 4-60

4.7. SUMMARY AND CONCLUSIONS $\ldots \ldots \ldots \ldots \ldots \ldots \ldots .4 .60$

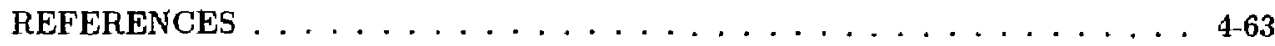




\section{MAGNETICS}

\subsection{INTRODUCTION}

The design points for the TITAN reversed-field-pinch (AFP) reactors (Table 3.6-1) were generated with the parametric systems model described in Section 3.2. The parametric systems analysis (PSA) model uses a simplified description of the magnet. configuration illustrated in Figure 3.1-3 to estimate the magnet performance as characterized by the coil positions, masses, and ohmic losses summarized in Table 4.1-I. To advance the reactor design from the PSA level, the magnet design must be considered in more detail using the models described below. The coil parameters listed in Table 4.1-I provide design direction.

The magnet configuration consists of the following sets of coils: toroidal-field (TF), divertor-field (DF), and poloidal-field (PF) coils. The TITAN reactors operate at steady state using the oscillating-field current-drive (OFCD) system, which is described in Section 7 . Rather than using a separate coil set to sustain the toroidal plasma current of $I_{\phi} \simeq 18 \mathrm{MA}$, the TF, DF, and PF coils are oscillated about their steady-state currents and used as the OFCD driver coils. In addition, the TF coils provide the initial bias field, $B_{\phi \infty}$, on which the initjal RFP configuration is formed. These coils subsequently maintain the external reversed field, $B_{\phi R}$, with a minimum ripple, $\Delta B_{R} / B_{\theta}$, at the plasma edge.

Two TITAN fusion power core (FPG) designs have been evaluated: (1) TITAN-I, a liquid-lithium, self-cooled design with vanadium-alloy (V-3Ti-1Si) structural material, and (2) TITAN-II, an aqueous-solution, self-cooled design with dissolved $\mathrm{LiNO}_{3}$ salt as the breeder, and ferritic-steel alloy (9-C) as the structural material. The TITAN-II FPC is submerged in a pool of cold water to achieve passive safety. Two TF-coil design approaches were examined. The TITAN-I desjgn uses the integrated-blanket-coil (IBC) concept [1], wherein poloidal currents are driven in the primary lithium coolant to produce the toroidal magnetic field (Section 4.2). The TITAN-II FPC design uses normalconducting, Cu-alloy TF coils encasing the blanket, which is sinilar to the CRFPR design [2]. The TF-coil models, constraints, and designs are described in Section 4.3.

A magnetic divertor is used in conjunction with a highly radiating plasma to reduce peak heat loads on the divertor plate and also limit the sputtering rates to reasonable levels. The TITAN divertor nulls the minority toroital field to minimize effects on the 
Table 4.1-1.

SUMMARY OF PARAME'TRIC SYSTEMS CODE ESTIMATES OF KEY PARAMETERS USED IN TITAN MAGNETICS DESIGNS ${ }^{(a)}$

\section{Parameter}

EF-coil option

DF-coil option

TF-coil option
TITAN-I

SC

$\mathrm{IBC}^{(b)}$

$\mathrm{IBC}^{(b)}$

17.82

3.90

0.60

0.66

$-0.382$

5.94

Poloidal field at plasma surface, $B_{\theta}\left(r_{P}\right)(T)$

Poloidal-Field Quantities

OH-coil thickness, $\delta_{O H}(\mathrm{~m})$

0.27

1.56

Average minor radius of OH coil, $\mathrm{rOH}_{\mathrm{H}}(\mathrm{m})$

Mass of $\mathrm{OH}$-coil set, $M_{O H}$ (tonne)

OH-coil current density, joH (MA/m $\left.\mathrm{m}^{2}\right)$

OH-coil dissipation during back-bias (MW)

Mass of EF-coil set, $M_{E F}$ (tonne)

EF-coil current density, $j_{E F}\left(\mathrm{MA} / \mathrm{m}^{2}\right)$
343.

13.1

121.

305.

19.2
TITAN-II

SC

Copper

Copper

Toroidal-Field Quantities

$T F$-coil thickness, $\delta_{T F}(\mathrm{~m})$

0.28

0.046

Average ruinor radius of coil, $r_{T F}(\mathrm{~m})$

0.68

1.11

Mass of TF-coil set, $M_{T F}$ (tonne)

41.

41.

TF-coil current density, $j_{\mathrm{TF}}\left(\mathrm{MA} / \mathrm{m}^{2}\right)$

1.64

9.2

TF-coil dissipation, $P_{T F}^{n}$ (MW)

27.6

13.6

Mass of DF-coil set, $M_{D F}$ (tonne)

0.55

2.0

DF-coil dissipation, $P_{D F}^{n}(M W)$

142.

(a) Section 3.6, Table 3.6-1.

(b) Integrated blanket coil (IBC) [1]. 
plasma and to minimize the DF-coil currents and ohmic losses. The DF coils also are designed to minimize toroidal-field ripple and are described in Section 4.4.

The PF-coil set performs an equilibrium and an ohmic-heating (start-up) function. The equilibrium function requires that a vertical field of appropriate magnitude, $B_{V}$, and index, $\partial\left(\ln B_{V}\right) / \partial(\ln R)$, corresponding to the values of the plasina current and beta $[3-5]$ be imposed over the plasma cross section. The ohmic-heating function provides the poloidal-flux swing required to establish the steady-state plasma current, which is then subsequently sustained by OFCD. Since the ohtnic-heating function is required only during start-up and the equilibrium function is required continuously, the PF-coil set is naturally, but not necessarily, separated into two sets of coils: equilibrium-field (EF) and ohmic-heating $(\mathrm{OH})$ coils. This separation has also helped eliminate the need for on-site energy storage during the start-up procedure (Section 6). The OH- and EF-coil designs are described in Sections 4.5 and 4.6, respectively. A summary of the TITAN-I and TITAN-II inagnet designs and conclusions are presented in Section 4.7.

\subsection{INTEGRATED-BLANKET-COIL (IBC) CONCEPT}

The integrated-blanket-coil (IBC) concept combines the blanket functions of tritium breeding and high-temperature energy recovery (of both fusion and ohmic heating) with the coil function of magnetic field production in a single component. The IBC resembles a conventional blanket sector, but the coolant also serves as the electrical conductor. The overall design is simplified by eliminating the coil shielding requirement and also the additional, but separate, coolant lines for the magnet coils found in conventional magnet designs. The IBC also improves reactor performance by increasing the tritjum-breeding ratio (TBR) and energy recovery through a greater overall volume for capturing neutrons productively. Toroidal-field ripple can also be reduced.

Previous studies $[1,6]$ indicate that adopting the IBC approach offers the following benefits:

- The IBC dual functions of coil and blanket permit a closer placement of the coil to the plasma without sacrificing neutronics performance, which potentially increases the mass power density of the FPC.

- Moving the coils closer to the plasma improves magnetic coupling as measured by inductance, thereby reducing current requirements in PF and DF coils. 
- For compact reactors especially, the IBC simplifies design and maintenance because fewer coolant lines and the associated connections are required.

Several combinations of materials for the coolant (conductor) and the structure are possible for the IBC. The TITAN study has adopted an IBC based on liquid lithium and the vanadium alloy, V-3Ti-1Si. Liquid lithium is unique in combining cooling and tritium-breeding capability with high electrical conductivity relative to mont blanket materials. Vanadium alloys are chemically compatible with liquid lithium and provide a suitable match of materials for use in the IBC.

One concern with the IBC concept is the large electrical resistivity of lithium relative to copper. If the concept is to be economically attractive, the joule losses should be comparable to those with a conventional resistive magnet system. However, even when a $70 \%$ fill fraction is assumed for a wound coil, the resistivity of the IBC is about 13 times larger than that of a room-temperature copper coil. Part of this difference is negated by capturing the ohmic heat in the blanket/coil, which serves as the main energy recovery component; therefore, about $\mathbf{4 0 \%}$ of the resistive losses in the IBC reappear as electrical power, albeit added costs associated with added thermal-conversion capacity is incurred.

The IBC concept requires that the electrical and therraal hydraulic systems be physically connected, leading to relatively low-voltage, high-current (few-turn) coils. The coil leads carry large currents and require careful design to minimize the error fields and the power consumption therein. The design of the low-voltage, high-current power supplies is the most critical engineering issue for the IBC. The magnet engineering aspects of the TITAN IBCs are discussed in Section 10.5.

\subsection{TOROIDAL-FIELD (TF) COILS}

\subsubsection{Models and Constraints}

The major goal for the TF-coil resign of RFPs is the achievement of minimal toroidalfield ripple. Toroidal-field ripple produces magnetic islands within the edge-plasma re gion. Particles and energy flow freely within this island structure, and plasma confinement is thereby degraded according to the island size. To ensure that confinement is not adversely affected by the TF ripple, the radial extent of the islands is required to be smaller than the radial distance between the reversal surface, $r_{r}$, and the plasma surface, $r_{p}$; this region is perceived to be primarily responsible for confinement in an RFP [7]. 
An estimate of the magnetic-island size produced by TF ripple is given in terms of the radial thickness of an island [8]:

$$
\Delta r=4\left[\frac{r \Delta B_{R}}{n B_{\theta}(d q / d r)}\right]^{1 / 2},
$$

where $r$ is the minor radius of the resonant surface, $\Delta B_{R}$ is the amplitude of the radial magnetic-field perturbation, $n$ is the toroidal mode number of the perturbation, $B_{\theta}$ is the poloidal field at the resonant surface, and the derivative of the safety factor $(d q / d r)$ is evaluated at the resonant surface. The safety factor at a given minor radius, $r$, is given by

$$
q(r)=\frac{r B_{\phi}(r)}{R_{T} B_{\theta}(r)},
$$

where $R_{T}$ is the plasma major toroidal radius and $B_{\theta}$ and $B_{\phi}$ are the poloidal and toroidal magnetic fields, respectively. In the case of TF ripple, the toroidal mode number of the perturbation is equal to the nutnber of TF coils, $N_{X F}$. The resonant surface occurs, by definition, at the minor radius where the field lines and the magnetic-field perturbation have the same periodicity in the direction of the perturbation. Then the primary resonaut surface for $T F$ ripple occurs at the minor radius where $q(r)=N_{T F}^{-1}$, which in the linit of $N_{T F} \gg 1$ is approximated as $r_{r}$, the radius of the reversal surface where $B_{\phi}\left(r_{r}\right)=q\left(r_{r}\right)=0$.

A circularized, 1-D ideal-MHD model described in Section 5.2 is used to determine $r_{r}$ and $d q / d r$. The MHD model is based on the following specification of the current density parallel and perpendicular to the magnetic field:

$$
\begin{aligned}
\nabla \times \mathbf{B} & =\mu_{\mathbf{0}} \mathbf{j}=\mu_{0}\left(\mathbf{j}_{\|}+\mathbf{j}_{\perp}\right), \\
\mathbf{j}_{H} & =\mu \mathbf{B}, \\
\mathbf{j}_{\perp} & =\frac{\nabla p \times \mathbf{B}}{B^{2}},
\end{aligned}
$$

where Equation 4.3-3 is Ampere's law, Equation 4.3-4 gives the prescription for supporting a plasma pressure, $p$, in equilibrium, and Equation 4.3-5 invokes Taylor's hypothesis of the ninimum energy state [9]. The MHD model requires as input the $\mu$ profile and the pressure profile. The pressure profile is derived from the assumed $\mu$ profile and from fits to density and temperature profiles calculated with the 1-D plasma simulations reported in Section 5.3. Given the poloidal beta, the plasma current, and either reversal or pinch parameters, the MHD model then determines the magnetic fields internal to the plasma, 
Table 4.3-I.

\section{PARAMETERS USED TO DETERMINE LOCATION OF REVERSAL SURFACE AND DERIVATIVE OF SAFETY FACTOR}

Plasma current, $I_{\phi}$ (MA)

17.82

Plasma major toroidal radius, $R_{r}(\mathrm{~m})$

Plasma minor radius, $r_{p}(m)$

0.6

Poloidal beta, $\beta_{\theta}$

0.22

Reversal parameter, $F$

$-0.1$

Pinch parameter, $\Theta$

Reversal surface minor radius, $n_{r}(m)$

Reversal region thickness, $r_{p}-r_{r}(\mathrm{~mm})$

50.65

Edge-plasma safety factor, $q\left(r_{p}\right)$

$-0.0099$

Safety factor derivative at reversal surface, $q^{\prime}\left(r_{r}\right)\left(m^{-1}\right)$

$-0.308 r$

as shown in Figure 4.3-1, from which the $q$ profile is derived. From the $q$ profile, $r_{r}$ and $d q / d r$ are obtained and reported in Table 4,3-I. In addition, the $B_{\theta}$ profile indicatea that the poloidal field at the resonant surface can be approximated conservatively by the poloidal field at the plasma edge. This approximation for $B_{6}$ permits the decoupling of design changes between TITAN-I and TITAN-II and the MAD calculation for $r_{r}$ and $d q / d r$, which are relatively insensitive to the changes in $I_{\phi}$.

The TF-coil designs for most RFP reactors [2] and experiments [10] strive for island widths $\Delta r \leq r_{p}-r_{r}$ which are achieved with ripples, $\Delta B_{R}$, of a few $\mathrm{mT}$ produced by $N_{T F} \geq 25$ TF coils. The ripple for the TITAN TF-coil configurations is derived from 2-D field-line tracings at the plasma surface, where $\Delta B_{R}$ is slightly larger than at the resonant surface; the 3-D vacuum-field magnetics code, TORSIDO [11], is used with only the TF-coils simulated for this purpose.

In addition, the field errors produced by the gap in the IBC needed to permit the ingress and egress of the first-wall coolant channel and by the current leads to the single- 
4.3. TOROIDALFIELD (TF) COILS
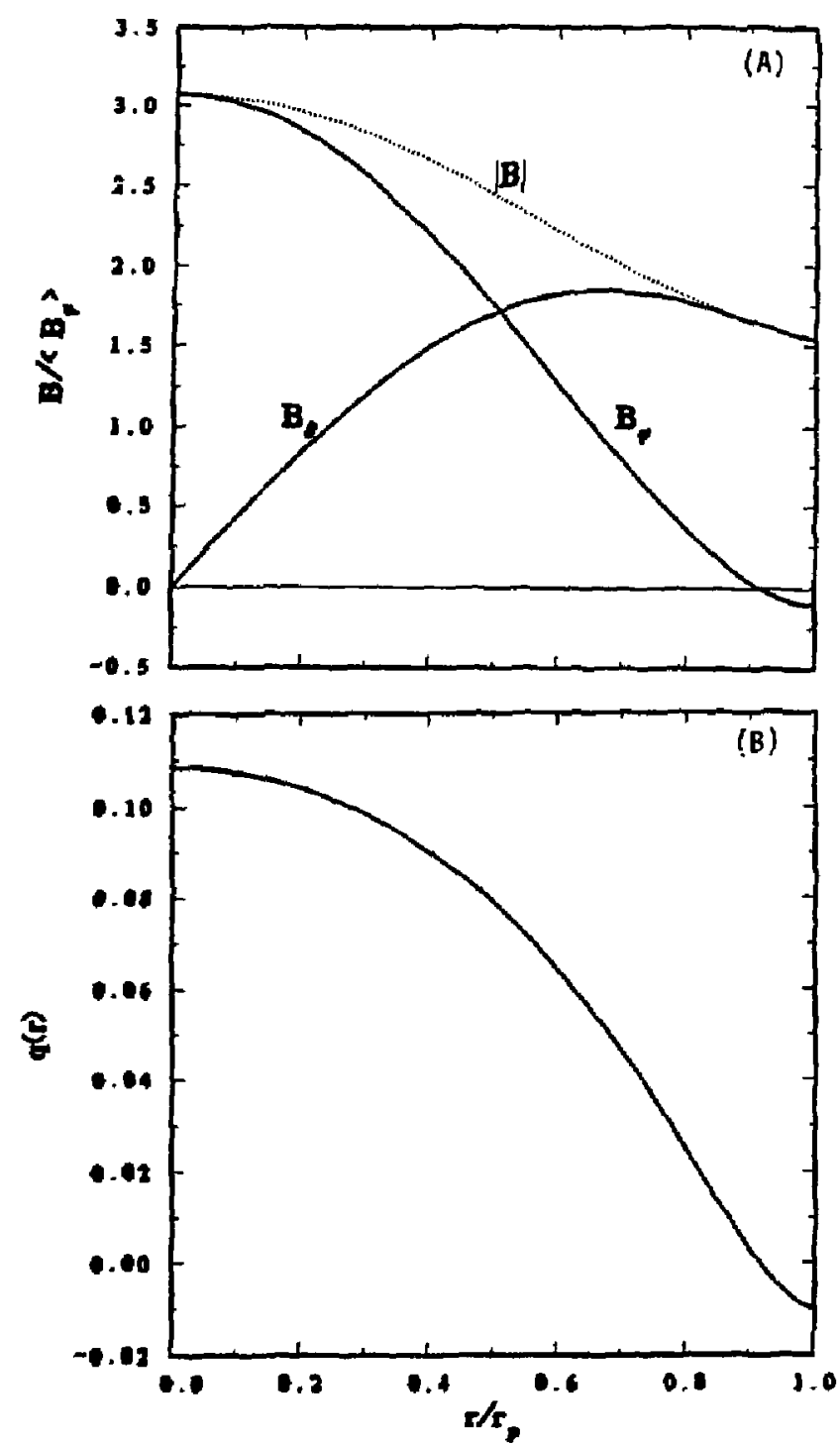

Figure 4.3-1. The radial profiles of (A) the magnetic field and (B) the safety factor, $q=(r / R)\left(B_{\phi} / B_{\phi}\right)$ from the 1-D MHD equilibrium model. These profiles are used to determine the location of the reversal surface, $r_{r}$, and $(d q / d r)_{\text {r. }}$ for magnetic-island width estimates. 


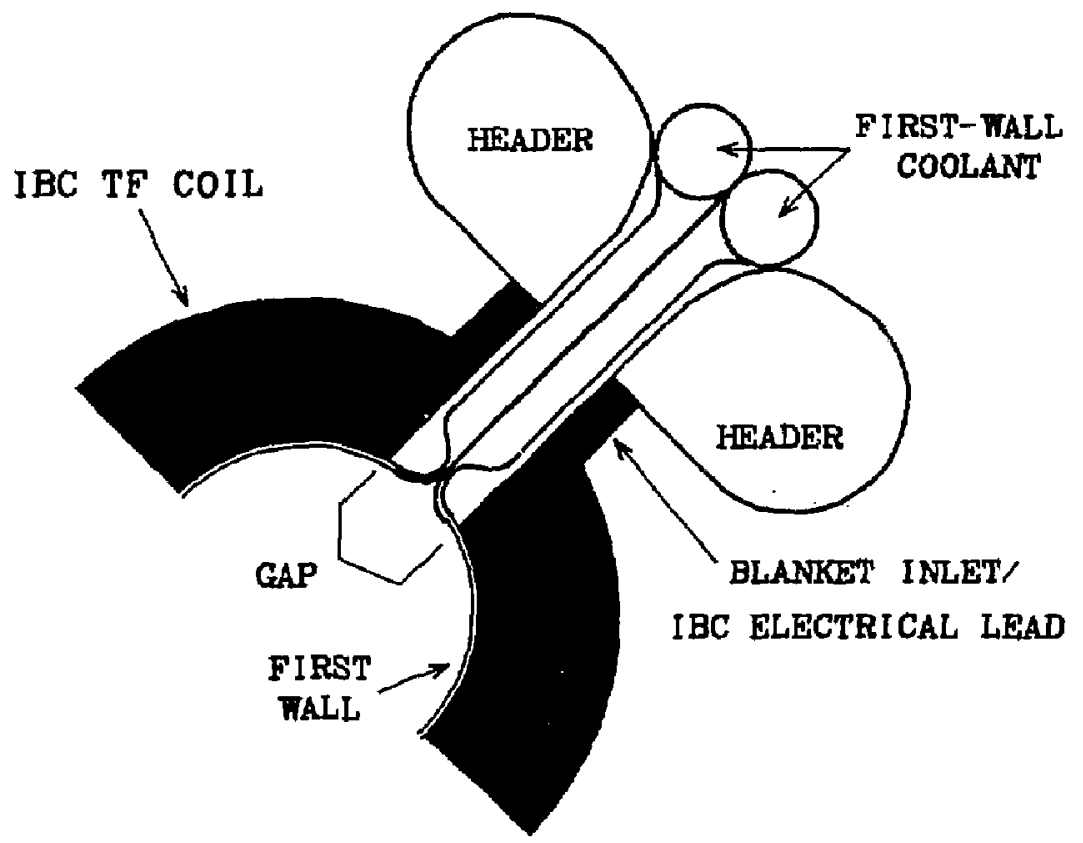

Figure 4.3-2. Schematic view of the IBC cross section, showing the electrical leads and the gap that are potential sources of field errors.

turn TF IBC, as shown in Fjgure 4.3-2, must also be minimized. The field-error constraint adopted is that the field errors be less than $\Delta B_{R}$ derived from the constraint imposed on magnetic-island widths. The IBC field errors are calculated with the $3-\mathrm{D}$ magnetics code EFFI [12].

Additional constraints, summarized in Table 4.3-II, on the magnitude of the toroidal field and the geometry shown in Figure 4.3-3 are imposed on the TF-coil design. The coil geometry for TITAN-I is largely determined by thermal-hydraulic considerations (Section 10); the TF-coil design is analyzed only to verify compliance with the ripple and field-error constraints. For TITAN-II the coil geometry is mainly predetermined, with $N_{\text {TF }}$ being the only degree of freedom. The TF-coil inner core radius is determined by blanket neutronics requirements. The blanket-coolant heariers pass vertically through the space between TF coils, as is shown in Figure 4.3-3. Provision for these headers constrains the TF-coil "transparency" factor, which is defined as

$$
f_{T F}=1-\frac{\ell_{T F} N_{T F}}{2 \pi R_{T F}}
$$




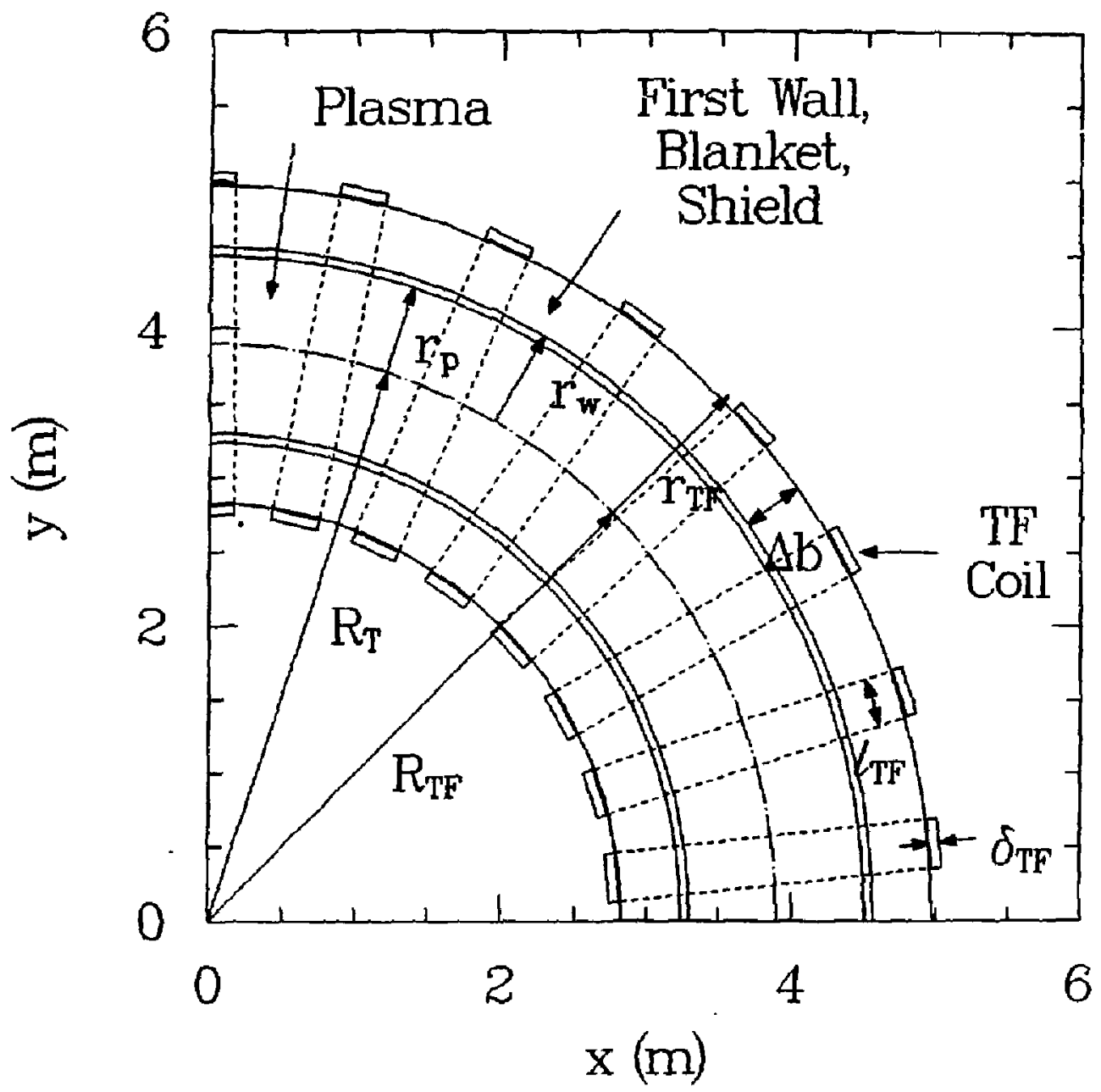

Figure 4.3-3. A schematic diagram of a conventional, Cu-alloy TF-coil design, indicating the notation used herein. 
Table 4.3-II.

MAGNETIC FIELD AND GEOMETRY CONSTRAINTS IMPOSEL ON TF-COIL DESIGN

\begin{tabular}{|c|c|c|}
\hline Parameter & TITAN-I & TITAN-II \\
\hline Reversed toroidal field, $B_{\phi R}(\mathrm{~T})$ & -0.36 & -0.38 \\
\hline Edge poloidal field, $B_{\theta}\left(r_{p}\right)(\mathrm{T})$ & 5.60 & 5.91 \\
\hline Plasma-to-TF-coil clearance $(\mathrm{m})$ & 0.08 & 0.4825 \\
\hline TF-coil transparency factor, $f_{T F}$ & 0.0 & 0.6 \\
\hline Conductor fill fraction, $\lambda$ & $\mathrm{TBD}^{(\boldsymbol{c})}$ & 0.7 \\
\hline TF-coil dissipated power (MW) & $\mathrm{TBD}^{(\boldsymbol{a})}$ & 16. \\
\hline TF-cojl major radius, $R_{T F}(\mathrm{~m})$ & 3.9 & $\mathrm{TBD}^{(a)}$ \\
\hline TF-coil minor radius, $r_{T F}(\mathrm{~m})$ & 0.82 & $\operatorname{TBD}^{(\alpha)}$ \\
\hline TF-coil radial thickness, $\delta_{T F}(\mathrm{~m})$ & 0.28 & $\operatorname{TBD}^{(a)}$ \\
\hline
\end{tabular}

(a) These values are determined after TF-coil design is completed rather than serving as design constraints.

where $\ell_{T F}$ is the toroidal width of the TF-coil cross section and $R_{T F}$ is the inajor radius of the coil, which may differ from $R_{T}$. The TF-coil dissipation is determined by cost optimization with the PSA model (Section 3.2). An additional constraint for TITAN-II is that $N_{T F}$ be an integer multiple of the number of diveriors to maintain symmetry of the divertors and ease of assembly and maintenance.

\subsubsection{TITAN-I TF-Coil Design}

For the interim TITAN-I TF IBC with a blanket thickness of $0.45 \mathrm{~m}$, the amplitude of the ripple field was found to be a few $\mu \mathrm{T}$, which in turn results in a magnetic-island width $\Delta r \leq 0.1 \mathrm{~mm}$ for $N_{T F} \sim 10^{3}$ and is more than two orders of magnitude below the jesign constraint. Such a small ripple and, hence, island width is a result of the TF IBC being 
an excellent approximation to a toroidally uniform conducting shell. The deviation from a shell is only a funchin of the diameter of the coolant tubes used in a TF IBC design and the spacing betweer the tubes. A close packing of the tubes is achit: ed by varying the tube cross section from a circle in the outboard equatorial plane to an ellipse which preserves the radial build in the inboard equatorial plane (Section 10). Since tlie final TF IBC design closely packs six adial rows of tubes in a radial build of $0.28 \mathrm{~m}$ as $\mathrm{G}_{\text {p }}$ posed to six rows in 0.45-m radial build for the interim design, the final design is expected to have even smaller ripple and island widiths than the interim design. Consequently, TF ripple was judged noi to be an issue for the TITAN-I TF-coil design given in Figure 4.3-4 and described in Table 4.3-III.

The current-element models of the TF IBCs, shown in Figure 4.3-5, were developed as input to EFFI [12] to calculate the error fields produced by the combination of gap and leads as shown in Figure 4.3-2. The current-element models are for the following ideal cases: no gaps or leads, for a gap of variable poloidal width but without lead, and a gap of variable width with leads forming a closed poloidal locp. Furthernore, the poloidal-current elements are modeled as 24 toroidally discrete coils of poloidally uniform cross section, as is shown in Figure 4.3-6, because of EFFI modeling limitations. Points $A$ through $F$ in Figure 4.3-5 mar: the locations at which the fields are evaluated for the various cases. Points $A$ and $E$ me located at the plasma edge in the inboard and outboard midplan.e; $B$ and $D$ arc located at the top and bottom plasna edges at the plasma major radius; $C$ is located on the plasma minor anis; and $F$ is located on the plasma edge at the gap center.

Possible error fields are: (1) perturbations to the toroidal field, (2) stray radial fields, and (3) stray vertical fields. Table 4.3-IV summarizes the results of the EFFI estimates of the toroidal-field strength for the various gap sizes. The cases with gaps out no leads produce significantly different toroidal fields compered to the calculated toroidal fields for the poloidally continuous cases of the "ideal loop" and the gap with leads. For example, at the plasma minor axis, point $\mathrm{C}$, the ideal loop has a toroidal field of $0.364 \mathrm{~T}$; the $54^{\circ}$ gap las $0.323 \mathrm{~T}$; and the $54^{\circ}$ gap with leads has $0.362 \mathrm{~T}$. Although the values are not exactly equal for the loop and gap with leads cases, they are sufficiently close to regard the differences as resulting from imperfections in the model geometry. These results indicate that the field topology within the plasma chamber is unaffected by the slape of the poloidal loup as long as the loop is closed, as is predicted by Ampere's law.

Tine same cu-rent-element models were used to evaluate the stray radial and vertical fields resulting from the gap and leads. The radial field in the "ideal loop" case is on the order of a few $\mu \mathrm{T}$. As shown in Table $4.3 * \mathrm{~V}$, the cases with gap and leads have fields of 


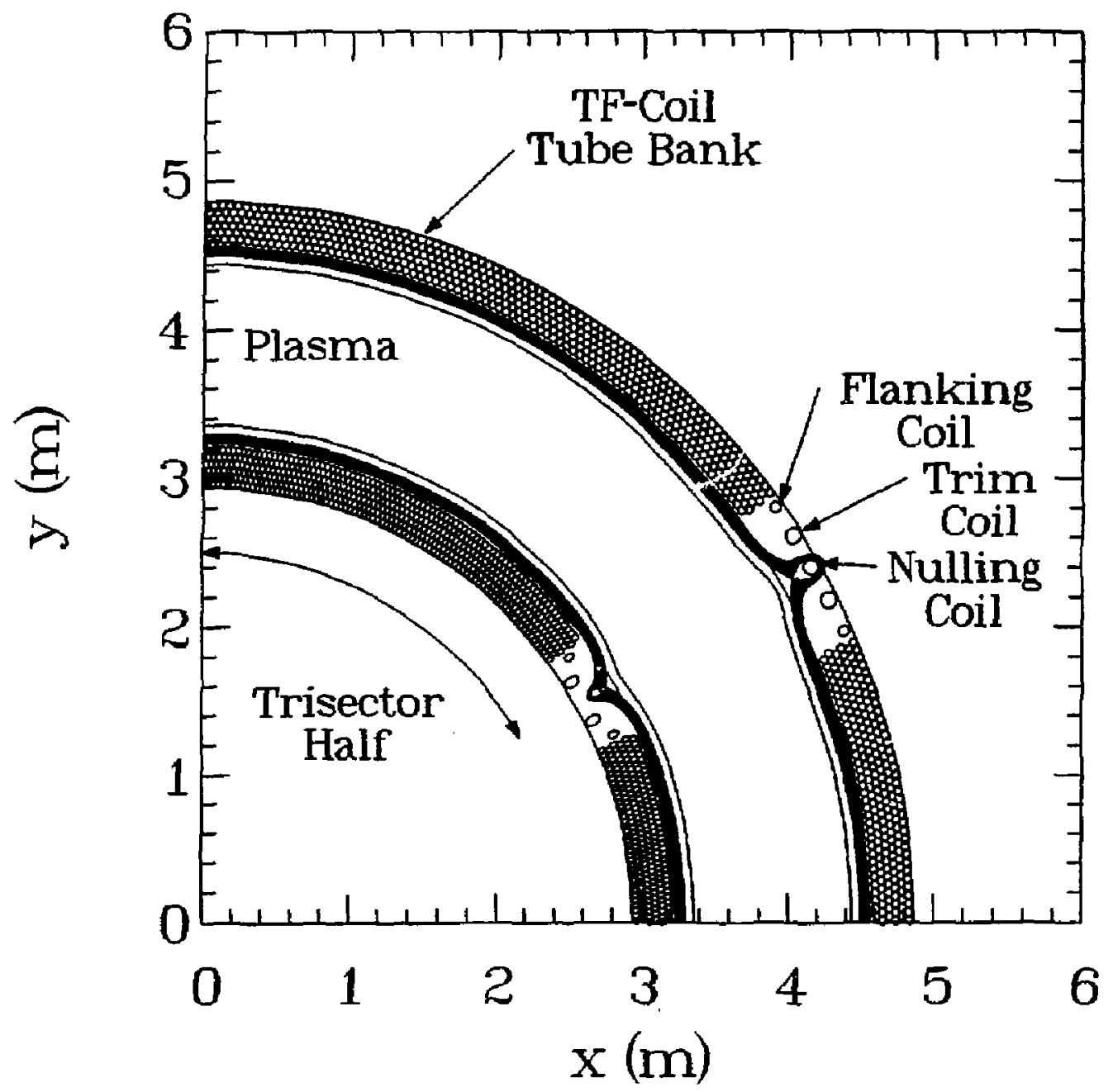

Figure 4.3-4. Equatorial-plane view of TF-IBC design with divertor. Also shown are field-line tracings at inboard and outboard minor radii of $r=0.5494$, $0.5995,0.6005,0.6010,0.6030,0.6060,0.6090,0.6120,0.6180,0.6240$ $0.6300,0.6360,0.6420,0.6480,0.6540$, and $0.6600 \mathrm{~m}$. 
Table 4.3-III.

PARAMETERS OF TITAN-I TOROIDAL-FIELD COILS

Current per trisector (MA)

Reversed toroidal field, $B_{\phi R}(\mathrm{~T})$

Number of tubes per trisector

Average current per tube (kA)

Tube inner diameter $(\mathrm{mm})^{(a)}$

Tube wall thickness (mm) $)^{(a)}$

Tube inner area $\left(\mathrm{m}^{2}\right)$

Average current density $\left(\mathrm{MA} / \mathrm{m}^{2}\right)$

Resistivity, $\eta(\mu \Omega-\mathrm{m})$

Total power, $P_{T F}^{\Omega}(\mathrm{MW})$

Blanket coverage

Tube data for row number:

Poloidal radius, $r_{\theta_{c}}(\mathrm{~m})$

Number per trisector

Current, $I_{B_{c}}(\mathrm{kA})$

Current density $\left\langle j_{\theta_{c}}\right)\left(\mathrm{MA} / \mathrm{m}^{2}\right)$ $\begin{array}{llllll}1 & 2 & 3 & 4 & 5 & 6\end{array}$

$\begin{array}{llllll}0.706 & 0.752 & 0.797 & 0.843 & 0.888 & 0.934\end{array}$

$\begin{array}{llllll}162 & 163 & 162 & 163 & 162 & 163\end{array}$

$\begin{array}{llllll}2.22 & 2.19 & 2.15 & 2.11 & 2.08 & 2.04\end{array}$

$\begin{array}{llllll}1.54 & 1.54 & 1.53 & 1.52 & 1.52 & 1.51\end{array}$

(a) Values evaluated at outboard equatorial plane and vary poloidally. 


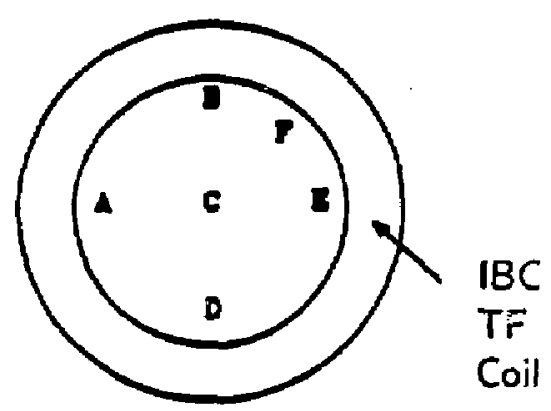

MAGNETICS

(a) Ideal Loop

(b) Gap, No Leads

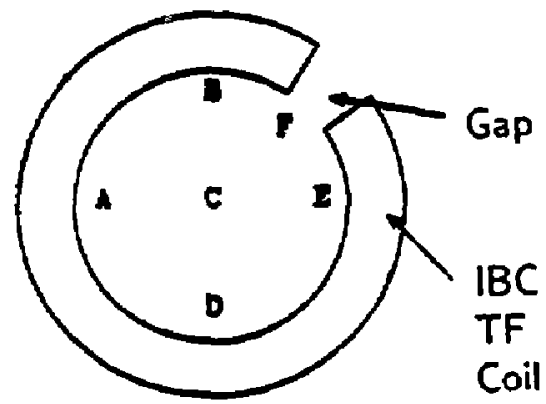

(c) Gap with Leads

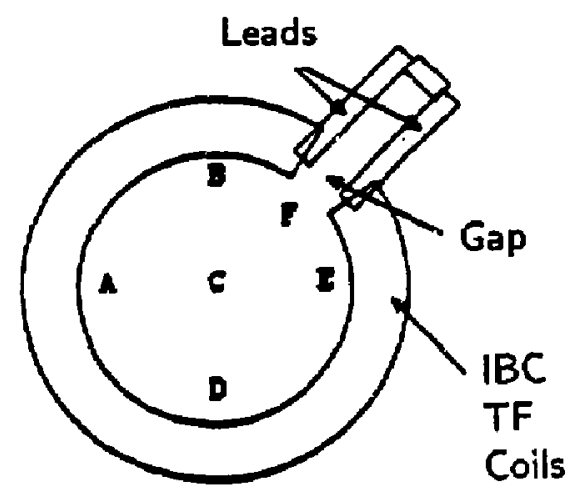

Figure 4.3-5. A schematic view of the poloidal cross section of current-element models inputted to EFFI to evaluate field errors for the TF IBCs. Also showu are the locations where field errors are calculated. 


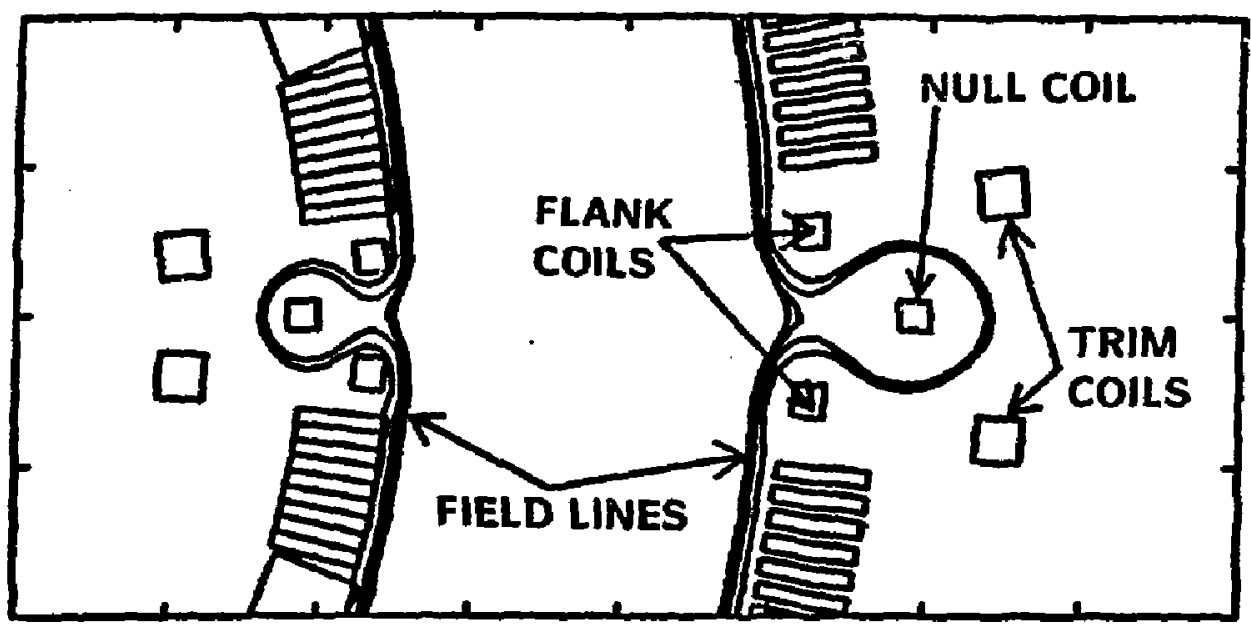

Figure 4.3-6. A schematic view of the toroidal cross section of the current-element models inputted to EFFI to evaluate field errors for the TF IBCs, Also shown are divertor coils.

similar magnitude. The stray vertical fields, also listed in Table 4.3-V, are even smaller ( $\sim 1$ to $2 \mu \mathrm{T}$ ). The TF-ripple constraint calculated for TITAN allows a spatial radialfield variation of a few $\mathrm{mT}$. In this context, the $\mu \mathrm{T}$ fields generated by the leads are insignificant. This conclusion is especially true when compared to the poloidal fields of several tesla generated by the plasma and PF coils.

\subsubsection{TITAN-II TF-Coil Design}

As discussed in Section 4.3.1, the TITAN-II geometry is specified to the point that the only degree of freedom in designing the TF coils is $N_{T F}$. The total TF-coil cross-sectional area is independent of $N_{T F}$ because the magnetic field and the power dissipation are held constant near values suggested by the PSA as being nearly minimum COE. The toroidal width, $\ell_{T F}$, of a single TF coil varies inversely with $N_{T F}$ and the radial tluckness, $\delta_{T F}$, of a TF coil is independent of $N_{T F}$, because the TF-coil transparency factor, $f_{T F}$ is also constant. Because $\ell_{T F}$ is varying, the TF-coil major and minor radii (respectively, $R_{T F}$ and $T_{T F}$ ) must change with $N_{T F}$ to provide a constant plasma-to-TF-coil clearance in 
Table 4.3-TV.

TOROIDAL FIELD CALCULATED FOR VARIOUS CURRENT-ELEMENT MODELS OF TITAN-I TOROIDAL-FIELD IBC(a)

\begin{tabular}{lccccccc}
\hline & \multicolumn{6}{c}{ Toroidal Field (T) at Location (a) } \\
\cline { 2 - 7 } \multicolumn{1}{c}{ Case } & $\mathrm{A}$ & $\mathrm{B}$ & $\mathrm{C}$ & $\mathrm{D}$ & $\mathrm{E}$ & $\mathrm{F}$ \\
\hline \multirow{2}{*}{$\begin{array}{l}\text { Ideal loop } \\
10^{\circ} \mathrm{gap}\end{array}$} & 0.424 & 0.385 & 0.364 & 0.386 & 0.362 & 0.364 \\
$22^{\circ} \mathrm{gap}$ & 0.420 & 0.378 & 0.364 & 0.382 & 0.355 & 0.327 \\
$54^{\circ}$ gap & 0.415 & 0.369 & 0.348 & 0.378 & 0.346 & 0.289 \\
$10^{\circ}$ gap and leads & 0.423 & 0.382 & 0.362 & 0.385 & 0.358 & 0.361 \\
$22^{\circ}$ gap and leads & 0.422 & 0.379 & 0.362 & 0.384 & 0.356 & 0.361 \\
$54^{\circ}$ gap and leads & 0.422 & 0.374 & 0.362 & 0.384 & 0.349 & 0.353
\end{tabular}

(a) For the current-element models and gaps shown in Figure 4.3-5.

toroidal geometry. This clearance is measured in the coil midplane on the outboard side of the equatorial plane and along a ray from the plesma major axis which passes through a corner of the TF-coil cross section on the coil inner surface on the inboard side of the equatorial plane, resulting in a slight inward shift of the TF coil relative to the plasms minor axis.

The results of the ripple calculations over a range of $N_{T F}$ values are presented in Table 4.3-VI and Figure 4.3-7. The amplitude of the ripple field, $\Delta B_{R}$, decreases with incressing $N_{T F}$ so that the magnetic island width, $\Delta r$, falls off faster than the explicit prediction of Equation 4.3-1 that $\Delta r \propto N_{r F}^{-1}$; this behavior is shown in Figure 4.3-7. The TITAN-II TF-coil design described in Table 4.3-VII and shown in Figure 4.3-3 meets the magnetic-island constraint with only a small safety margin. The safety margin could be increased if so desired by increasing the plasma-to-TF-coil clearance and the TF-coil minor radius as illustrated by results in Figure $4.3-7$ for a $65-\mathrm{mm}$ larger value of $r_{T F}$. 
Table 4.3-V.

STRAY FIELD CALCULATED FOR VARIOUS CURRENT-ELEMENT MODELS OF TITAN-I TOROIDAL-FIELD IBC(a)

\begin{tabular}{|c|c|c|c|c|c|c|}
\hline \multirow[b]{2}{*}{ Case } & \multicolumn{6}{|c|}{ Stray Field $(\mu \mathrm{T})$ at Location (a) } \\
\hline & A & B & $\mathrm{C}$ & D & $\mathbf{E}$ & $F$ \\
\hline
\end{tabular}

Stray Radial Field

$\begin{array}{lllllll}\text { Ideal loop } & 9.701 & 5.899 & 6.605 & 5.899 & 0.832 & 2.524 \\ 16^{\circ} \text { gap } & 9.621 & 5.957 & 6.672 & 5.971 & 1.056 & 2.715 \\ 22^{\circ} \text { gap } & 9.523 & 6.026 & 6.754 & 6.057 & 1.330 & 2.940 \\ 54^{\circ} \text { gap } & 9.280 & 6.208 & 6.964 & 6.274 & 2.020 & 3.501 \\ 10^{\circ} \text { gap and leads } & 8.365 & 4.041 & 4.953 & 4.743 & 0.954 & 0.573 \\ 22^{\circ} \text { gap and leads } & 7.303 & 4.110 & 3.733 & 3.547 & 2.523 & 0.334 \\ 54^{\circ} \text { gap and leads } & 8.237 & 4.299 & 5.277 & 5.235 & 0.241 & 1.028\end{array}$

Stray Vertical Field

$\begin{array}{lllllll}\text { Ideal Loop } & 0 . & 2.005 & 0 . & 2.005 & 0 . & 1.820 \\ 10^{\circ} \text { gap } & 0.169 & 2.088 & 0.071 & 1.957 & 0.093 & 1.771 \\ 22^{\circ} \text { gap } & 0.369 & 2.181 & 0.151 & 1.904 & 0.208 & 1.706 \\ 54^{\circ} \text { gap } & 0.864 & 2.327 & 0.288 & 1.803 & 0.562 & 1.443 \\ 10^{\circ} \text { gap and leads } & 0.787 & 0.366 & 1.336 & 2.932 & 1.718 & 0.054 \\ 22^{\circ} \text { gap and leads } & 1.115 & 0.874 & 1.467 & 3.159 & 1.585 & 0.451 \\ 54^{\circ} \text { gap and leads } & 0.579 & 0.947 & 0.789 & 2.399 & 2.662 & 0.812\end{array}$

(a) For the current-element models and gaps shown in Figure 4.3-5. 
Table 4.3-VI.

RESULTS OF TITAN-II RIPPLE CALCULATIONS

\begin{tabular}{cccccccc}
\hline$N_{T F}$ & $\begin{array}{c}\Delta B_{R} \\
(\mathrm{mT})\end{array}$ & $\begin{array}{c}\Delta r \\
(\mathrm{~mm})\end{array}$ & $\begin{array}{c}\left|r_{0}-r_{S}\right| \\
(\mathrm{mm})\end{array}$ & $\begin{array}{c}\ell_{T F} \\
(\mathrm{~mm})\end{array}$ & $\begin{array}{c}\Delta_{T F} \\
(\mathrm{~mm})\end{array}$ & $\begin{array}{c}R_{T F} \\
(\mathrm{~m})\end{array}$ & $\begin{array}{c}r_{T F} \\
(\mathrm{~m})\end{array}$ \\
\hline & & & & & & & \\
24 & 27.72 & 74.65 & 28.69 & 408.3 & 70.5 & 3.896 & 1.121 \\
27 & 19.72 & 59.36 & 18.34 & 363.1 & 70.4 & 3.897 & 1.121 \\
30 & 14.14 & 47.69 & 11.96 & 326.7 & 70.4 & 3.898 & 1.120 \\
33 & 10.16 & 38.55 & 7.90 & 297.1 & 70.4 & 3.898 & 1.120 \\
36 & 7.37 & 31.43 & 5.30 & 272.2 & 70.3 & 3.898 & 1.119 \\
39 & 5.28 & 25.56 & 3.53 & 251.3 & 70.3 & 3.899 & 1.119 \\
& & & & & & &
\end{tabular}

Cost penalties associated with an increase in coil mass, obviously, result from exercising this option.

The magnetic-island width predicted by Equation 4.3-1 is not valid because the resonant surface (where $q(r)=N_{T F}^{-1}$ ) does not lie between $r_{r}$ and $r_{p}$. Three-dimensional field-line tracings in the plasma edge region with the TF and PF coils and plasma simulated under the same conditions of non-resonance of the $T F$ ripple [13] indicate that the Inagnetic island width or flux surface broadening is the same as the difference, $\left|r_{O}-r_{I}\right|$, in the radial amplitudes of outboard and inboard field-line tracings in two dimensions with only the TF coils simulated. The island widths in this case are $\sim 0.2$ of the constraint, as is indicated in Table 4.3-VI.

The sensitivity of the TF-coil thickness to the ohmic dissipation constraint is explored in Figure 4.3-8 for the parameters listed in Table 4.3-VI. The design point occurs at the knee of the dissipation versus thickness curve indicating a cost balance between power dissipation and coil mass. Changing the TF-coil thickness has little effect on island widths for a given $N_{T F}$, but a variation in thickness would change $f_{T F}$. A self-consistent changing of the ohmic-dissipation constraint which affects the TF-coil thickness would then change $N_{T F}$. Changes to $N_{T F}$ are compounded by the requirement that $N_{T F}$ be an integer multiple of the number of divertors. 

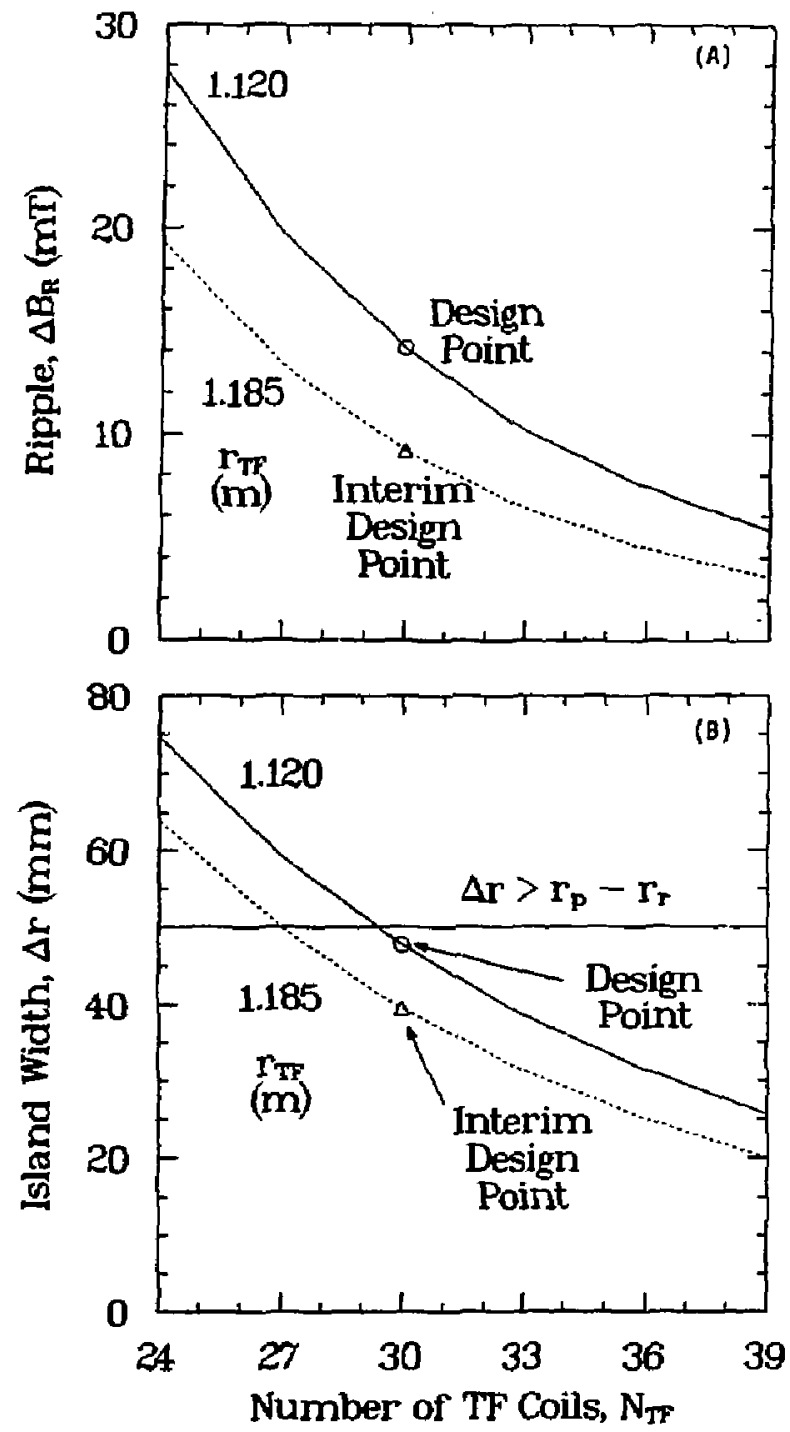

Figure 4.3-7. Amplitude of the ripple magnetic field (A) and the corresponding magnetic-island widths (B) as functions of the number of TF coils, $N_{\text {TF }}$. Also shown are results for an interim design point illustrating the effect of changing the plasma-to-TF-coil clearance by $65 \mathrm{~mm}$. 
Table 4.3-VII.

\section{PARAMETERS OF TITAN-II TOROIDAL-FIELD COILS}

Number of TF coils, $N_{T F}$

Major radius, $R_{T F}(\mathrm{~m})$

Minor radius, $r_{T F}(\mathrm{~m})$

Radial thickness, $\sigma_{X F}(\mathrm{~mm})$

Toroidal thickness, $\ell_{T F}$ (mm)

Reversed toroidal field, $B_{\phi R}(\mathrm{~T})$

Current per coil, $I_{T F} / N_{T F}(\mathbf{k A}\}$

Current density, $j_{T F}\left(\mathrm{MA} / \mathrm{m}^{2}\right.$ )

Steady-state peak coil field, $B_{\phi c}(\mathrm{~T})$

TF-coil mass, $M_{T F}$ (tonne)

Total ohmic power, $P_{T F}^{R}$ (MW)
30.

70.4

326.7

0.38

247.0

10.7

0.69

$35.5^{(a)}$

16.

(a) Assuming a coil density of 7.3 tonne $/ \mathrm{m}^{2}$.

\subsection{DIVERTOR-FIELD (DF) COILS}

\subsubsection{Models and Constraints}

The divertor design approach adopted by the TITAN study builds directly on the results of References $[13-16]$. The divertor nulls the minority toroidal field to minimize perturbations to the confining Inagnetic field in the plasma and to minimize the DF-coil currents required to produce a null. The TF null a line poloidally encircling the plasma cross section upon which the toroidal field is zero. The plasma surface is taken to be the separatrix, which is the boundary differentiating open (leaving the plasma chamber) and closed (contained within the plasma surface) field lines. The separatrix surface also contains the null. The null is produced by a mulling coil, as is shown in Figure 4.3-4. The nulling coils (one per divertor location) form a solenoid within the TF-coil solenoid; the 


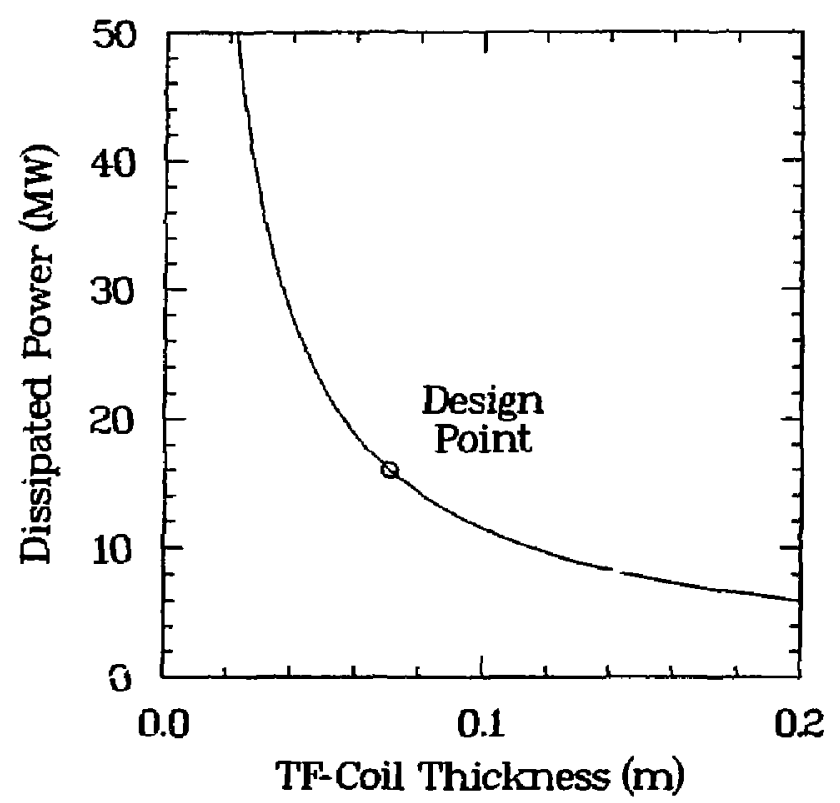

Figure 4.3-8. Sensitivity of the TF-coil ohmic dissipation to changes in coil thickness for the parameters of Table 4.3-VII.

nulling-coil solenoid nearly cancels the toroidal field over the volume of the inner solenoid. To localize the effect of the nulling coil, two flanking coils are positioned symmetrically atout the nulling coil, as is shown in Figure 4.3-4. The flanking coils carry a combinet current equal to that in the nulling coil, but of opposite sign. In the case of the IBC divertor, the divertor assembly dispiaces a portion of the IBC TF-coil tube bank. A pair of trim coils is required to conduct a current equal to that of the displaced tube bark in order to control the TF ripple.

Particles and energy diffuse across the separatrix, creating a plasma exhaust that flows along the open field lines to the divertor or diffuses across field lines to the first wall. The open field lines form a plume that encircles the nulling coil upon entering the divertor region. A nestralizer or collector plate is positioned to intercept the plasma exhaust confined to the field-line plume.

Two divertor configurations are possible depending on the location of the collector plate: a closed divertor and an open divertor. The collector plate in the closed divertor 
is positioned to intercept the plume at the furthest possible location from the plasma in order to inluibit neutral particles from entering the plasma because of the remoteness of the point of interaction between the plate and the plasma exhaust. In the open divertor approach, the plate is positioned near the null, requiring several neutral mean free paths (four are assumed sufficient here) between the plate and the separatrix to prevent the neutral particles from entering the plasma.

The open divertor has a decided advantage over a closed divertor in an RFF because adjacent field lines are decompressed near the null and compressed at the outermost part of the plume relative to the spacing of adjacent field lines far away from a divertor. This decompression of field lines causes a substantial decrease in the heat and particle fluxes incident upon the plate in the open divertor relative to the closed divertor. Furthermore, a poloidal asymmetry in the field-line density exists in the plume which is negligible at the null and increases monotonically to a maximum at the outermost point of the plume. This asymunetry enhances the differences in peak heat and particle fluxes between the two approaches with the open divertor showing less poloidal asymmetry. Consequently, the open divertor approach was adopted for the TITAN divertor designs.

The divertor magnetic topology is deternined with the 3-D vacuum magnetics code TORSIDO [11], but only 2-D field lines confined to the equatorial plane are traced, with ouly the TF and DF coils being simulated. The more economical 2-D field-line tracings were found to reproduce the 3-D field-line tracings in the portion of the scrapeoff layer (SOL, the open field-line region between the separatrix and first wall) of interest for an open divertor. A flux-surface expansion factor that measures the expansion or compression of adjacent field ines relative to the spacing at the divertor midplane is also calculated along a field line. The calculation of the flux-surface expansion factor appropriately assumes that the flux in the equatorial plane is constant along a field line; this factor is then simpiy the ratio of the magnitude of the field at the divertor midplane (toroidal and radial components only) to the magnitude of the local field.

A 3-D field-line length, $L$, is also calculated by

$$
L=\int \frac{\left(B_{V}^{2}+B_{\phi}^{2}+B_{R}^{2}\right)^{1 / 2}}{\left(B_{\phi}^{2}+B_{R}^{2}\right)^{1 / 2}} d l,
$$

where $d l$ is the integration step size in the equatoxial plane for the 2-D field-line tracings. The vertical field in the equatorial plane is $B_{V}$ and is produced by EF coils (positioned as in the TITAN-I design described in Section 4.6.1) and by the plasme, which is simulated with a hoop current at a major radjus of $R_{T}^{+}=3.908 \mathrm{~m}$ to account for the Shafranov slift [10]. The magnetic fields $B_{\phi}$ and $B_{R}$ are the toroidal and radial fields produced by 
the TF and DF coils. Field-line lengths from the divertor midplane to the null, $L_{N}$, and to the plate, $L_{p}$, are calculated specifically for use in the edge-plasma modeling discussed in Section 5.4. The field-line coordinates for field-line tracings from the divertor midplane to a point past the divertor at minor radii of $600.5,601.0,603.0,606.0,609.0,612.0,618.0$, $624.0,630.0,636.0,642.0,048.0,654.0$ and $660.0 \mathrm{~mm}$ in the SOL are tabulated for use in the divertor-plate design (Section 11.4). In addition, the magnetic field components, the flux-surface expansion factors, and field-line lengths corresponding to the above field-line coordinates are included in the divertor tabulations.

The divertor magnetics connection * edge-plasma modeling and target design was exercised iteratively to establish divertor performance criteria. The TITAN-I and TITAN-II target designs have different critical heat-flux limits set by the choice of coolant, structural material, and configuration. The critical heat-flux limits translate into magnetics requirements that the TITAN-I divertor design produce a peak flux-surface expansion factor of $\sim 2$ at a plate location $22.1 \mathrm{~mm}$ (four neutral mean free paths) from the null. The TITAN-II divertor design produces an expansion factor of $\sim 3$. Only three divertors are used in either design. Production of a higher flux-surface expansion factor requires a larger nulling-coil minor radius resulting in a larger wamic dissipation in the divertor coils: Reactor economics, which in turn is driven by the thermal-energy conversion efficiency, $\eta_{T H}$, restricts the divertor ohmic dissipation to $P_{D F}^{\mathrm{n}} \simeq 100 \mathrm{MW}$ for TITAM-I and to $P_{D F} \simeq 10 \mathrm{MW}$ for TITAN-II. The ohmic dissipation is affected by the angular spread between flanking and nulling coils which, in turn, affects the ripple produced by the divertor. The divertor ripple is large, but this ripple is also characterized by a high mode number (Equation 4.3-1). A 3-D analysis of the divertor ripple in the CRFPR [13] at parameters similar to TITAN indicates that a $4^{\circ}$ spacing between the flanking and nulling coils produces magnetic islands smaller than those produced by the TF coils. Consequently, a $4^{\circ}$ spacing was adopted as a guideline in the TITAN design to meet the ripple constraint. In addition, symmetrizing the edge-plasma region $\left(r_{+} \leq r \leq r_{p}\right)$ from inboard to outboard was found to play an important role in reducing divertor-introduced island widths and is imposed as an additional goal.

The location of the divertor coils consistent with the above constraints is determined by the following algorithm. The uulling coil is located close to the plasma on the inboard side to minimize the divertor-coil current and to obtain acceptable flux-surface expansion factors. The outboard locations of all divertor coils are determined by requiring an inboard/outboard symmetry of the edge-plasma region. The divertor coils also are constrained to remain inside the blanket envelope in order not to displace shielding. The flanking coils are positioned to mininize TF ripple. The trin coils recuired in the 


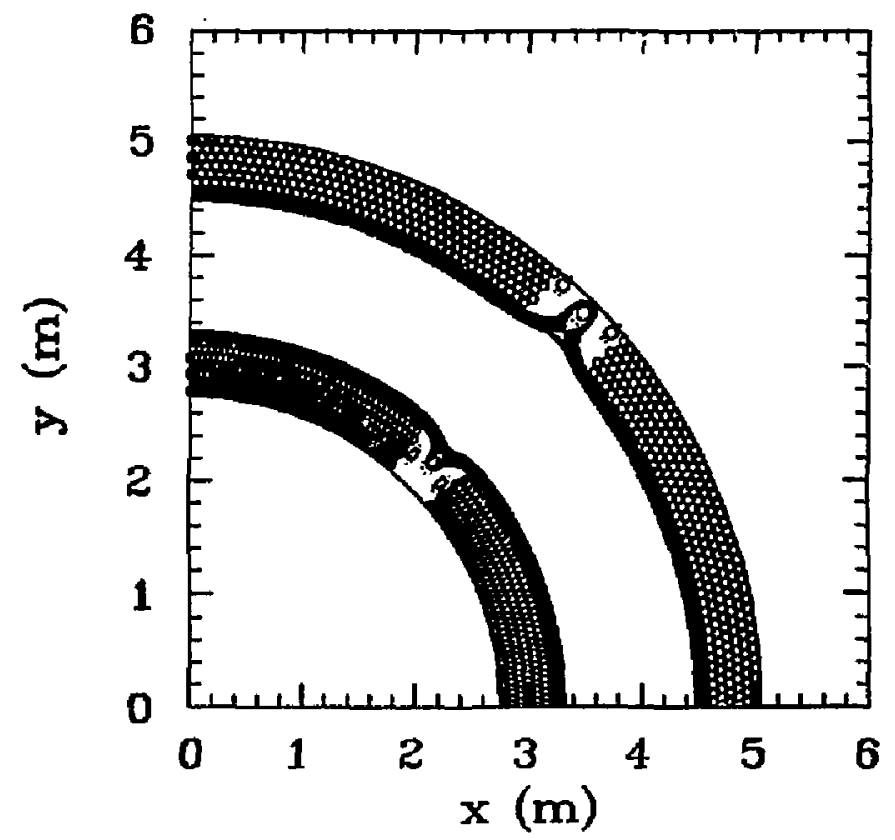

Figure 4.4-1. The divertor configuration for Case I of the nulling-coil radial-position sensitivity study summarized in Table 4.4-I.

TIT AN-I IBC design are positioned radially as far as possible from the nulling coil within the blanket envelope to minimize the divertor currents; the toroidal position minimizes TF ripple when operated without the divertor as in the RFP formation phase.

\subsubsection{TITAN-I DF-Coil Design}

The higher resistivity of $\mathrm{Li}(0.35 \mu \Omega \mathrm{m})$ relative to $\mathrm{Cu}(0.02 \mu \Omega \mathrm{m})$ results in a large ohmic dissipation for divertor IBCs. Because of spatial restrictions imposed by the target design, the DF-coil cross-sectional area cannot be increased sufficiently to achieve a tolerable ohmic dissipation. Management of the IBC divertor ohmic dissipation was investigated first with a study of the impact of radial variations of the nulling coil, as reported in Tabie 4.4-I. The DF- and TF-coil configurations corresponding to the three cases in Table 4.4-I are shown in Figures 4.4-1 and 4.4-2 with the flanking and trim coils combined. The DF coils are shown with two cross sections. The ohmic dissipation for the smaller cross section was evaluated with a $\mathrm{Cu}$-like resistivity. The second cross section 
Table 4.4-I.

TITAN-I DIVERTOR COILS SENSITIVTTY STUDY

\begin{tabular}{c} 
Parameter \\
\hline
\end{tabular}

Nulling coil:

Current (MA)

Current density $\left(\mathrm{MA} / \mathrm{m}^{2}\right)^{(a)}$

Major radius (m)

Minor radius (m)

Cross-sectional radius (mm)

Radius enlargement factor

$$
-0.331
$$$$
-0.705
$$

$30.0(37.0)$

$30.0(37.8)$

$30.0(38.4)$

3.9847

0.9327

4.0152

1.0392

59.3

1.9

86.49

2.1

134.69

1.7

Flanking coil:

Current (MA)

Current density $\left(\mathrm{MA} / \mathrm{m}^{2}\right)^{(a)}$

Major radius (m)

Minor radjus (m)

Cross-sectional radius (mun)
0.263

$30.0(37.5)$

3.983

0.9960

52.83
0.45

$30.0(37.7)$

4.0

1.025

69.10

Ohmic losses $(M W)$ :

$$
\begin{array}{ll}
\mathrm{Cu} & (\eta=0.020 \mu \Omega-\mathrm{m}) \\
\mathrm{Li} & (\eta=0.347 \mu \Omega-\mathrm{m})
\end{array}
$$

22.3

44.8

107.4

117.8

199.2

611.3

Inboard null minor radius ( $\mathrm{m}$ )

Shielding thickness (mm)

0.725

0.728

0.729

Flux surface expansion factor (at $r=0.6005 \mathrm{~m}$ ):

$\begin{array}{llll}\text { Inboard } & 3.41 & 3.89 & 4.85 \\ \text { Outboard } & 5.59 & 6.19 & 5.87\end{array}$

Divertor connection lengths (m) (at $r=0.6005 \mathrm{~m}$ ):

Inboard

42.9

46.0

46.7

Outboard

71.6

73.2

75.0

Average

57.2

59.6

60.8

(a) Values in the outboard equatorial-plane cross section. Poloidally averaged values are listed in parenthesis. 

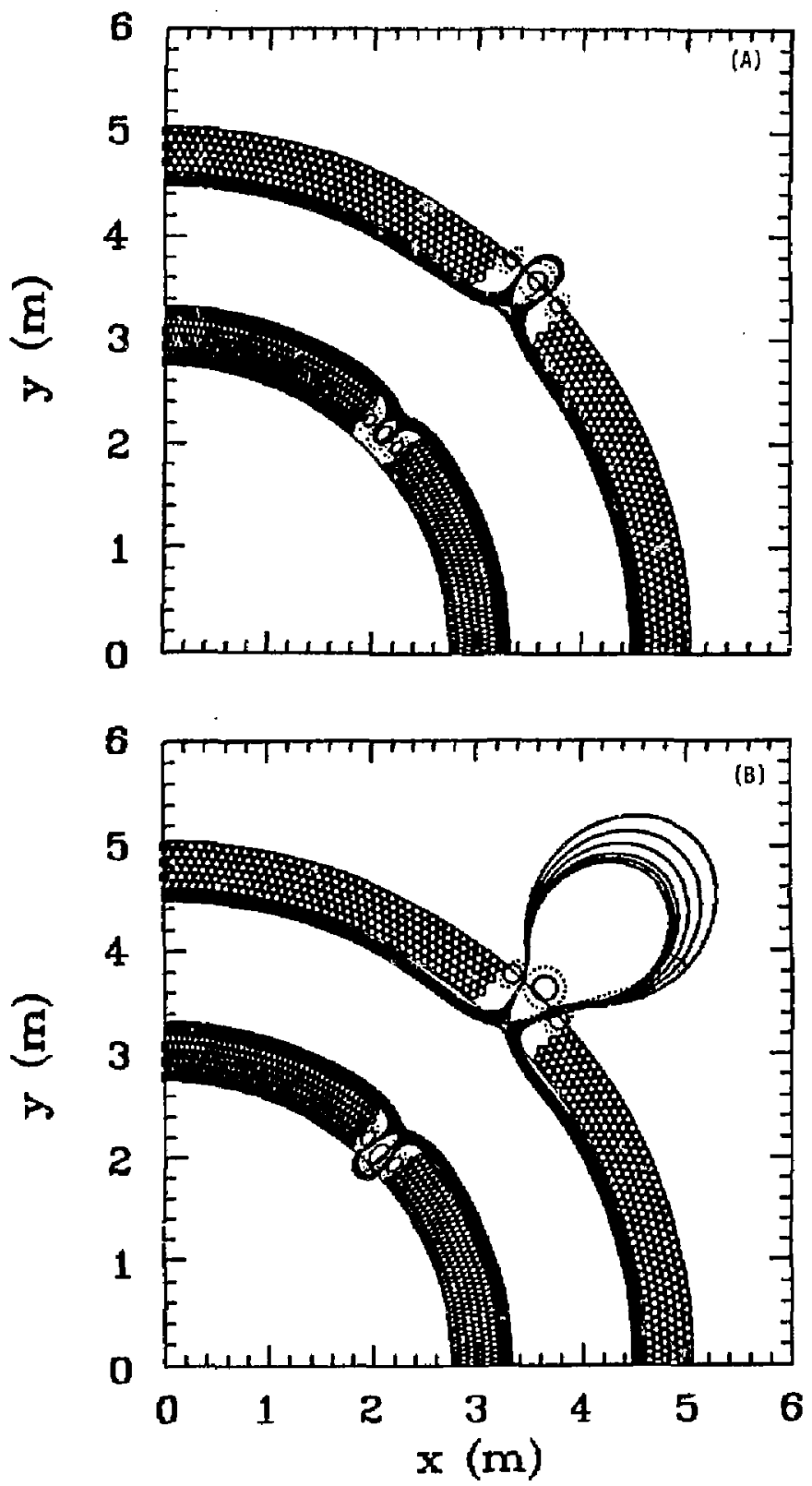

Figure 4.4-2. The divertor configuration for Case II (A) and Case III (B) of the nullingcoil radial-position sensitivity study summarized in Table 4.4-I. 
represents the largest cross section possible in the design. The increase in cross-sectional area can be gauged from the radius enlargement factor listed in Table 4.3-I. This factor is applied uniformly to the nulling coils, but only radially to the flanking coils whjeh creates elliptical cross sections at both inboard and outboard positions. The enlargement factor is bounded by the null at the inboard equatorial plane or the flanking coils. With the enlarged cross sections, only Case III has room between the nulling coil and the null for a collector plate with a shape that reduces the peak heat flux to an acceptable level $\left(\leq 100 \mathrm{MW} / \mathrm{m}^{2}\right.$ ). However, the ohmic dissipation evaluated for a Li-like resistivity in the larger cross section for Case III is unacceptable. The space between the null and the smaller cross sections is tabulated in Table 4.4-I as an available shielding thickness. Based on the shielding thicknesses and ohmic losses reported in Table 4.4-I, a copper DF-coil solution is possible with $\sim 4^{\circ}$ spacing between the flanking and nulling coils. However, the IBC option was adopted for both the TF and DF coils.

The resulting divertor design is shown in Figure 4.3-4 and described in Table 4.4-II. The angular spread between the flanking and nulling coils had to be increased to $5.7^{\circ}$ to achieve a peak flux-surface expansion factor of $\sim 2$ at the collector plate and an ohmic dissipation of $\sim 120 \mathrm{MW}$. An expanded view of the divertor region is shown in Figure 4.4-3, which illustrates the density of the field-line tracings in the SOL. Along each field line shown in Figures 4.3-4 and 4.4-3, the magnetic field parallel to a field line in the equatorial plane (consisting of a radial component, $B_{R}$, and a toroidat component, $B_{\phi}$ ), the magnetic field perpendicular to the equatorial plane (equivalently, the vertical field, $\left.B_{v}\right)$, and the flux-surface expansion factor are tabulated for input to the target-design task (Section 11.4). Results for a few representative lines are shown in Figures 4.4-4 and 4.4-5. The magnetic fields and the expansion factor are uniform far from the divertor (i.e., $L=0$ ), with the inboard fields being larger than the outboard fields because of toroidal effects. The separatrix is bracketed tightly by the field-line tracings at $r=0.5995$ and $0.6005 \mathrm{~m}$, so that the dotted lines in Figures 4.4-4 and 4.4-5 are superimposed upon the solid lines in the plasma chamber until these lines separate in the vicinity of the null where the toroidal field is a minimum and the expansion factor is a maximum. As the field lines proceed around the back of the nulling coil and actually intercept the coil on the irboard side, the parallel field reaches its maximum value at the same lo ation where the perpendicular field and expansion factor are a minimum. The inverse minor-radius dependence of the perpendicular field and the inverse major-radius dependence of the parallel field are demonstrated between the results at the separatrix $(r=0.6005 \mathrm{~m})$ and at the first wall ( $r=0.6600 \mathrm{~m}$ ). Although flux-surface expansion factors much larger than two occur along the field line adjacent to the separatrix, the collector plate cannot be positioned at those locations because of the required four neutral-mean-free-path buffer 
Table 4.4-II.

\section{DF-COIL PARAMETERS FOR TITAN-I AND -II}

Number per trisector

Toroidal angle $\left({ }^{\circ}\right)$

Major radius ( $m$ )

Minor radius ( $m$ )

Cursent per coil (kA)

Current density (c) (MA/m ${ }^{2}$ )

Average current density $\left(M A / m^{2}\right)$

Resistivity, $\eta(\mu \Omega m)$

Power pef coil $(M W)$

Conductor volume $\left(10^{-3} \mathrm{~m}^{3}\right)$

Coil volume $\left(10^{-2} \mathrm{~m}^{3}\right)$

Total average current density $\left(M A / m^{2}\right)$

Conductor filling fraction, $\lambda$

Steady-state dissipated power( $M W$ )

Inboard/outboard/average values at $r=0.6005 \mathrm{~m}$

Watershed-to-null distance, $L_{N}(m)$

Watershed-to-plate distance, $L_{P}(m)(f)$

Peak flux-surface expansion factor $(f)$

$\begin{array}{ccl} & \text { TitaN-1(d) } & \\ & & \\ \text { Nulling } & \text { Flanking } & \text { Trim } \\ 1 & 2 & 2 \\ 0 & 5.717 & 2.935 \\ 3.946 & 3.945 & 3.9 \\ 0.855 & 0.860 & 0.900 \\ 164.00 & 82.00 & 131.07 \\ 20^{(d)} & 20^{(d)} & 15^{(d)} \\ 27.532^{(e)} & 29.095^{(e)} & 20.835^{(e)} \\ 0.35313 & 0.35313 & 0.35313 \\ 11.754 & 6.238 & 7.382 \\ 3.2001 & 1.5229 & 3.5574 \\ 3.6206 & 1.8189 & 4.0147 \\ & 25.1 & \\ & 0.874 & \\ & 116.980 & \end{array}$

$53.9 / 91.4 / 72.7$

$55.2 / 94.0 / 74.6$

$2.27 / 4.23 / 3.25$

\begin{tabular}{clcc}
$\begin{array}{c}\text { TitaN-!!(t) } \\
\text { (internesiate) }\end{array}$ & \multicolumn{2}{c}{$\begin{array}{c}\text { TITAN-II(b) } \\
\text { (Final) }\end{array}$} \\
Nulling & Flanking & Nulling & Flanking \\
1 & 2 & 1 & 2 \\
0 & 5.729 & 0 & 4.011 \\
3.962 & 3.945 & 3.963 & 3.940 \\
0.846 & 0.850 & 0.879 & 0.030 \\
162.50 & 81.25 & 280.00 & 140.00 \\
25 & 25 & 50 & 25 \\
25 & 25 & 50 & 25 \\
0.020 & 0.020 & 0.020 & 0.020 \\
0.617 & 0.310 & 2.209 & 0.522 \\
2.419 & 1.215 & 2.165 & 2.044 \\
3.456 & 1.736 & 3.093 & 2.920 \\
& 25 & \multicolumn{2}{c}{35.7} \\
0.7 & \multicolumn{2}{c}{0.7} \\
3.711 & \multicolumn{2}{c}{9.757}
\end{tabular}

$50.7 / 86.0 / 68.3$ $52.1 / 88.0 / 70.0$ $2.47 / 4.25 / 3.36$
$53.5 / 88.3 / 70.9$ $55.9 / 91.6 / 73.8$ $3.07 / 5.14 / 4.11$

(-) Design plasma-surface field values: $B_{\phi R}=0.36 T$ and $B_{\theta}\left(r_{p}\right)=5.6 T$.

(b) Design plasma-sulface field values: $B_{\phi R}=0.38 T$ and $B_{\theta}\left(r_{p}\right)=5.91 T$.

(c) Outboard equatorial-plane value.

(d) Current density obtained by averaging current over conductor and strueture.

(e) Includes effect of variable coil cross section and constant structural cross-sectional area associated with 2.5-mm-thick walls.

(f) Values are measured at $22.1 \mathrm{~mm}$ from the null. 

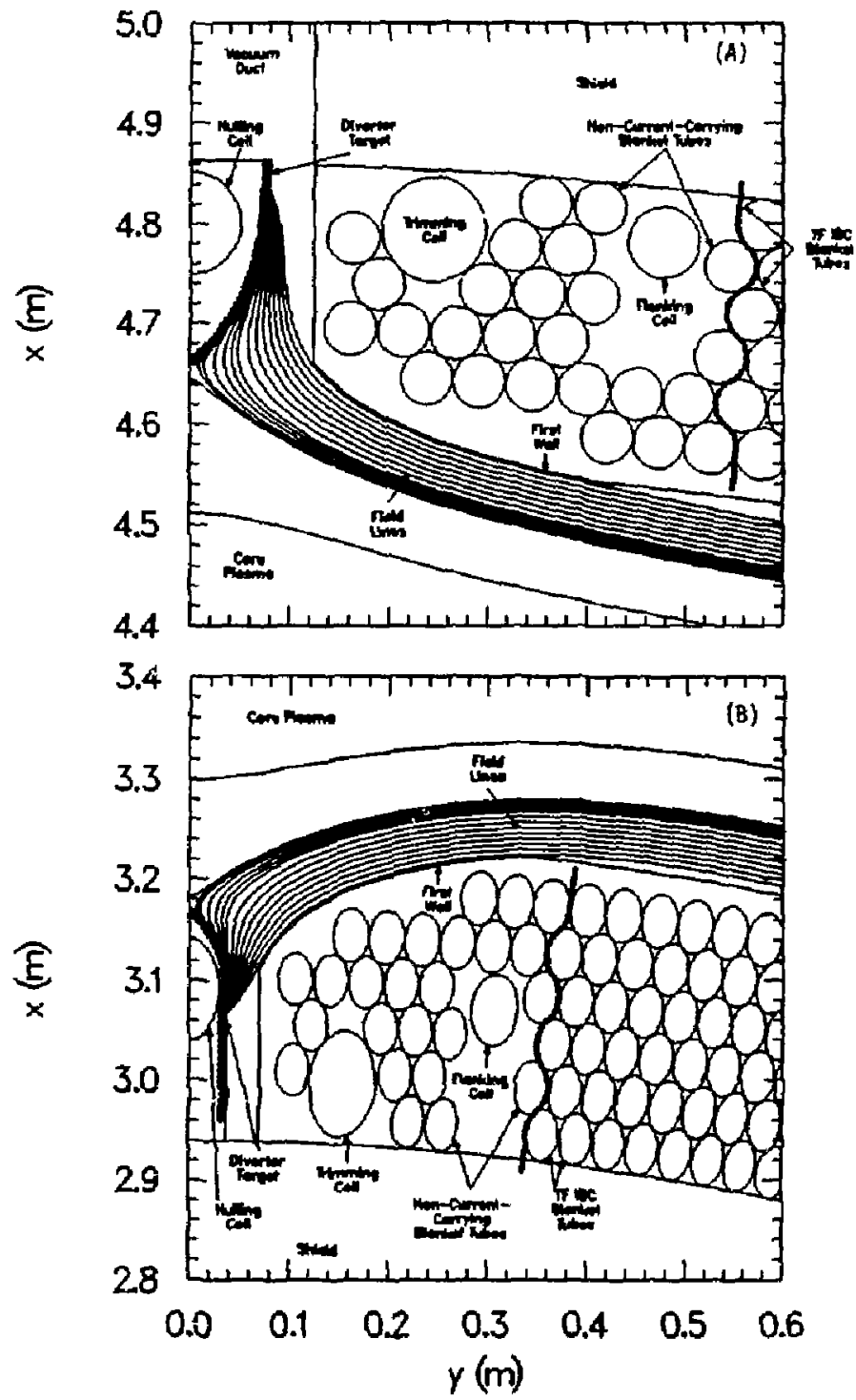

Figure 4.4-3. An expanded view of the TITAN-I divertor configuration outboard (A) and inboard (B) shown in Figure 4.3-4 and described in Table 4.4-II. The field lines are at $r=0.5449,0.5995,0.6005,0.6010,0.6030,0.6060$, $0.6090,0.6120,0.6180,0.6240,0.6300,0.6360,0.6420,0.6480,0.6540$, and $0.6600 \mathrm{~m}$. 

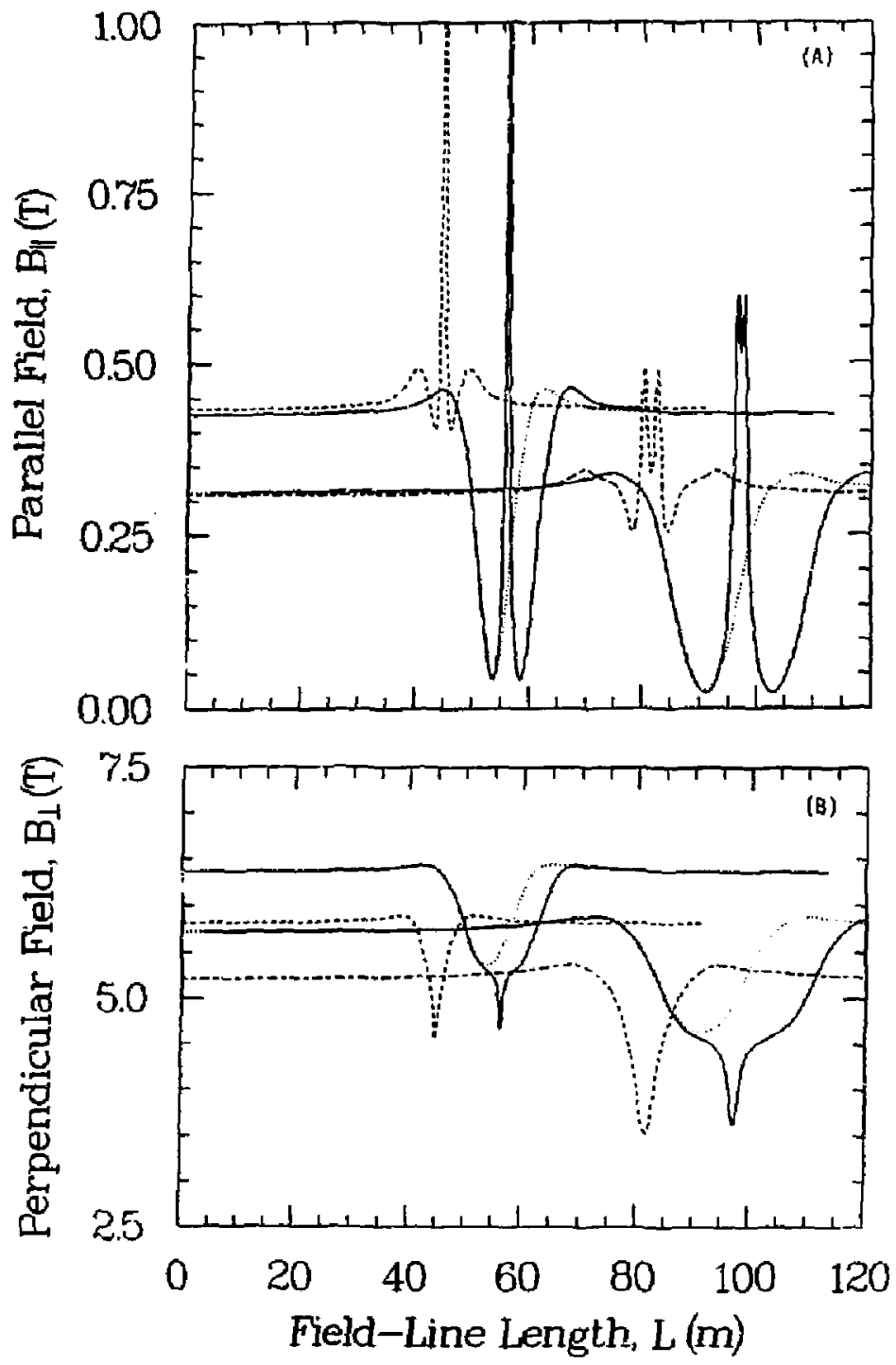

Figure 4.4-4. Parallel (A) and perpendicular (B) magnetic-field strength along the field line with length measured from the divertor midplane for TITAN-1. The fields correspond to field lines at $r=0.5995$ (dotted line), 0.6005 (solid line), and $0.6600 \mathrm{~m}$ (dashed line) both inboard (higher initial field values) and outboard. 


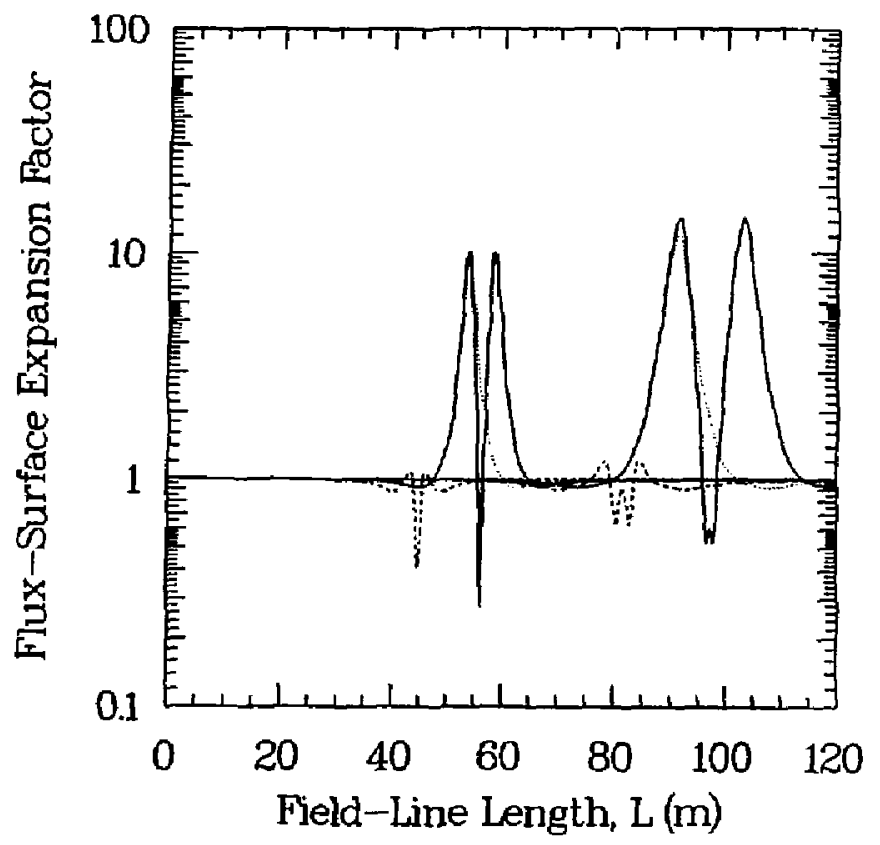

Figure 4.4-5. Flux-surface expansion factor along the field line with the length measured from the divertor midplane for TITAN-I. The expansion factors correspond to field lines at $r=0.5995$ (dotted line), 0.6005 (solid line), and $0.6600 \mathrm{~m}$ (dashed line) both inboard (curves with spikes between 40 and $70 \mathrm{~m}$ ) and outboard.

zone between the divertor plate and the null. This buffer zone is needed to attenuate the neutral flux before they can enter the plasma.

\subsubsection{TITAN-II DF-Coil Design}

Since TITAN-I and TITAN-II have similar blanket thicknesses $(\sim 0.28 \mathrm{~m})$, the divertor design for TITAN-II was initiated with a simple conversion of the TITAN-I design into the intermediate TITAN-II divertor design presented in Table 4.4-II. The similarity of these two designs in all respects except the conductor (i.e., Li versus $\mathrm{Cu}$ alloy) permits a precise documentation of the impact of the two conductors on overall performance. The difference in resistivity accounts for a factor of 0.06 reduction in the olnmic dissipation from TITAN-I to the intermediate TITAN-II design. A factor of 0.7 from TITAN-I to 
the intermediate TITAN-II is attributable to the IBC being a single-turn coil, in which the olmic losses in the two meters of leads cannot be ignored, as is the case for multituru Cu-alloy coils. Another factor of 0.6 results from the requirement that the DF coils include a trim coil for the IBC design.

This intermediate TITAN-II design, however, requires a target design with a critical leat-flux linit which in turn requires a higher $(\sim 3)$ flux-surface expansion factor. The divertor design which meets the various constraints is shown in Figure 4.4-6 and described in Table 4.4-II. The design constraints were met by returning to the $4^{\circ}$ spread between the nulling and flanking coils required to ensure acceptable magnetic islands. In addition, the nulling-coil minor radius and current were increased to achieve an expansion factor of $\sim 3$ within the constraint of an ohmic dissipation of $\leq 10 \mathrm{MW}$. A maxinum of 20 to $50 \mathrm{num}$ of shielding is possible inboard; this shield thickness increases to $>100 \mathrm{~mm}$ outboard, as is shown in the expanded view of the divertor region in Figure 4.4-7. A maximum of $120 \mathrm{~mm}$ of shielding is possible for the flanking coils. Shielding is not necessary, however, to protect the insulators (Section 10.2), but shielding is desirable for purposes of energy recovery.

Plots of the parallel and perpendicular magnetic fields and the flux-surface expansion factor along the field line are shown in Figures 4.4-8 and 4.4-9. These three plots are similar to the TITAN-I plots of Figures 4.4-4 and 4.4-5 with the exception of the effect of TF ripple. The field-ljne tracings begin in the divertor midplane, which is midway between TF coils where the toroidal field is a minimum. Because the opening between TF coils is larger at the outboard than at the inboard position, the ripple is larger for the outboard field lines. Furthermore, a ripple is observed in the perpendicular or poloidal field because the radial excursions of the toroidal field lines result in minor-radius variations, which in turn affect the magnitude of the poloidal field. The ripple of both components of the magnetic field in the SOL could result in a weak mirror trapping of particles and enhance radia! diffusion to the first wall. For the purposes of edge-plasma modeling, however, field ripple is neglected.

\subsection{OHMIC-HEATING (OH) COILS}

\subsubsection{Models and Constraints}

The single-turn back-bias and forward-bias OH-coil currents (respectively, $I_{O H}^{-}$and $\left.I_{O H}^{+}\right)$are determined by the inductive-flux conservation, and ignoring the resistive losses:

$$
L_{p} I_{\phi}=M_{E F, p} I_{E F}+M_{O H, p}\left(I_{O H}^{+}-I_{\bar{O} H}^{-}\right) \text {, }
$$




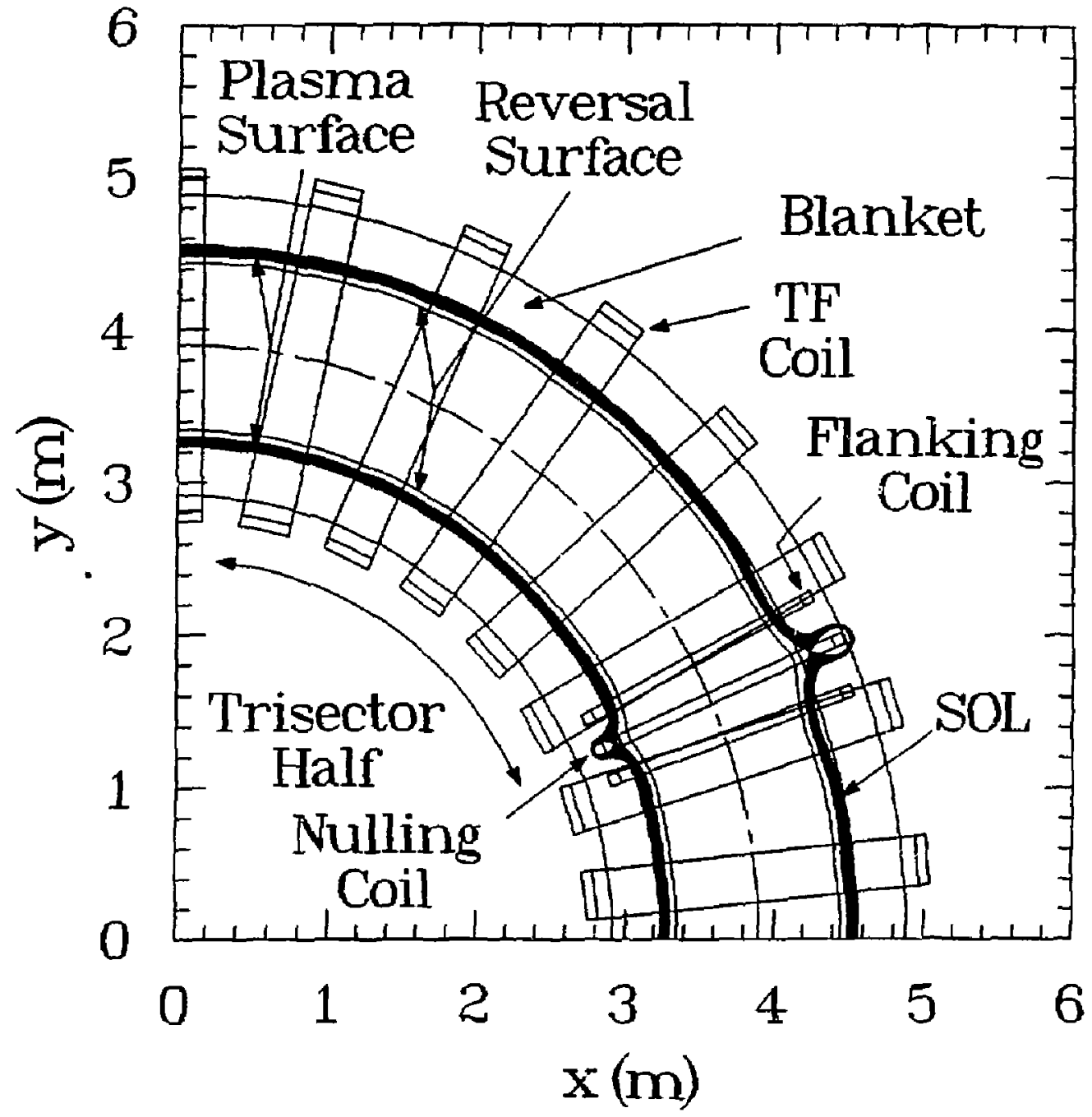

Figure 4.4-6. Equatorial-plane view of TITAN-II clivertor design. Also shcwn are fieldline tracings at inboard and outboard minor radii of $r=0.5494,0.5995$, $0.6005,0.6010,0.6030,0.6060,0.6090,0.6120,0.6180,0.6240,0.6300$, $0.6360,0.6420,0.6480,0.6540$, and $0.6600 \mathrm{~m}$. 

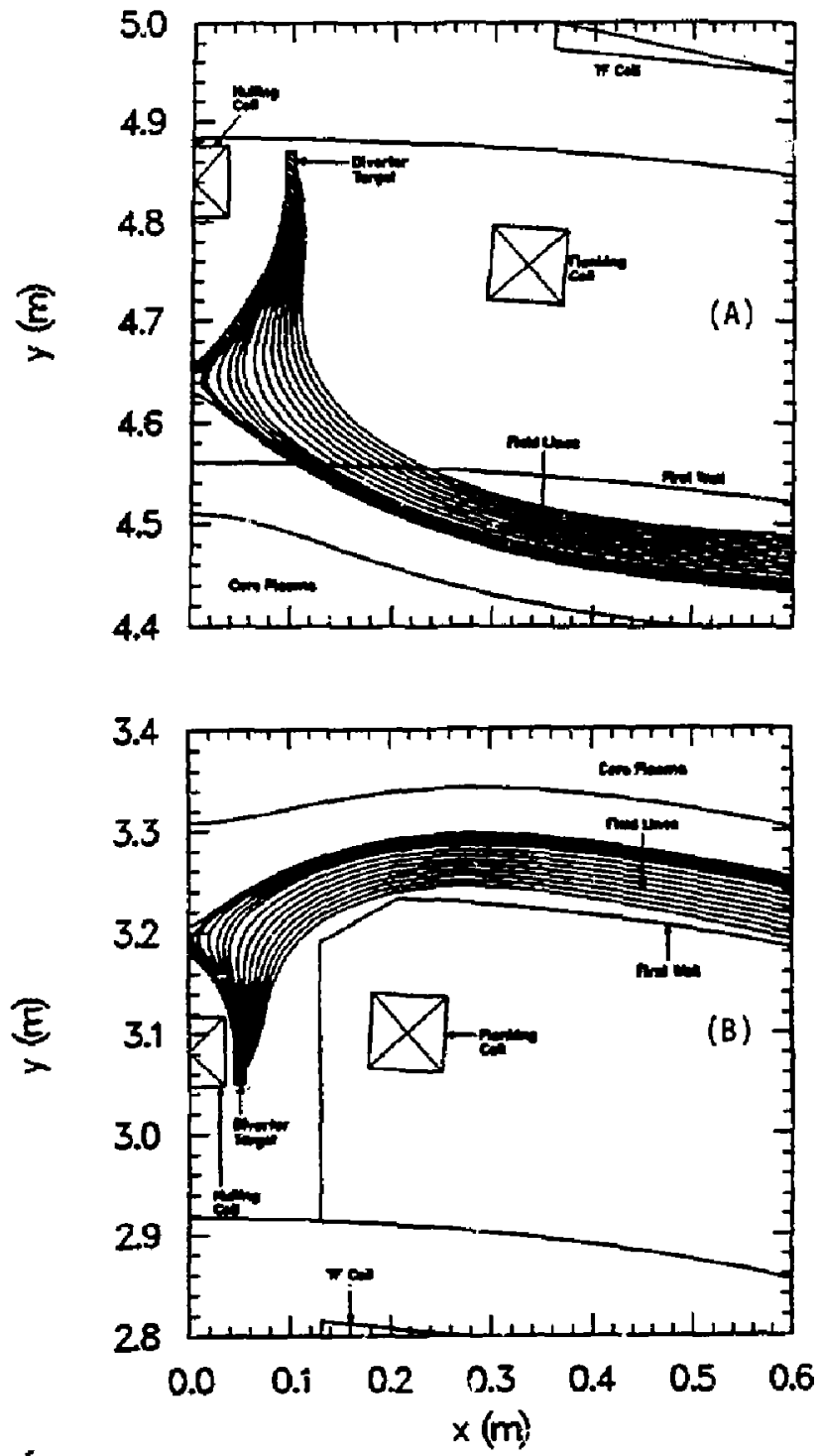

Figure 4.4-7. An expanded view of the TITAN-II divertor configuration outboard (A) and inboard (B) shown in Figure 4.4-6 and described in Table 4.4-II. The field lines are at $r=0.5 .194,0.5995,0.6005,0.6010,0.6030,0.6060$, $0.6090,0.6120,0.6180,0.6240,0.6300,0.6360,0.6420,0.6480,0.6450$, and $0.6600 \mathrm{~m}$. 

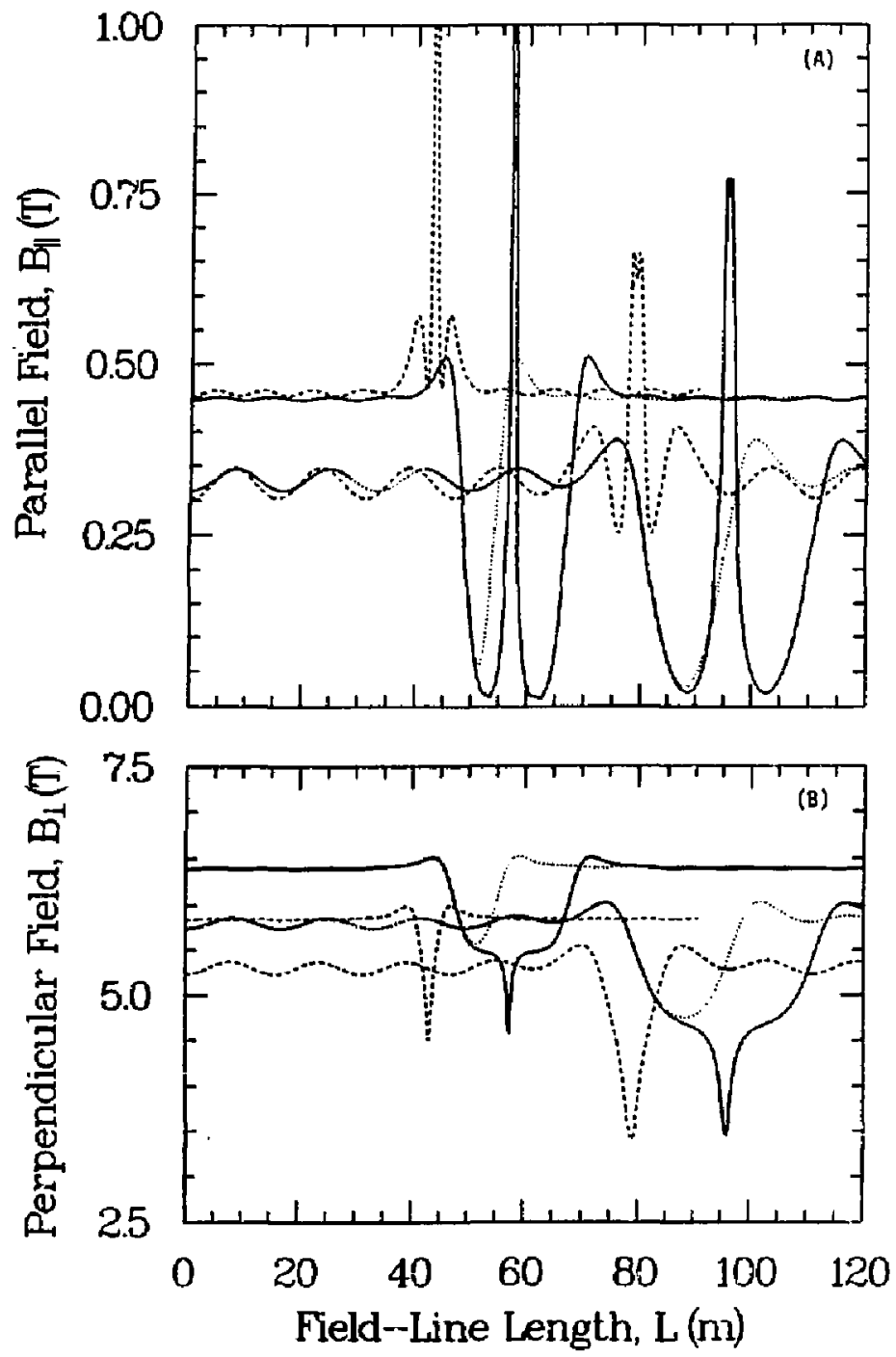

Figure 4.4-8. Parallel (A) and perpendicular (B) magnetic-field strength along the field line with length measured from the divertor midplane for TITAN-II. The fields correspond to field lines at $r=0.5995$ (dotted line), 0.6005 (solid line), and $0.6600 \mathrm{~m}$ (dashed line) both inboard (ligher initial field vaiues) and outboard. 


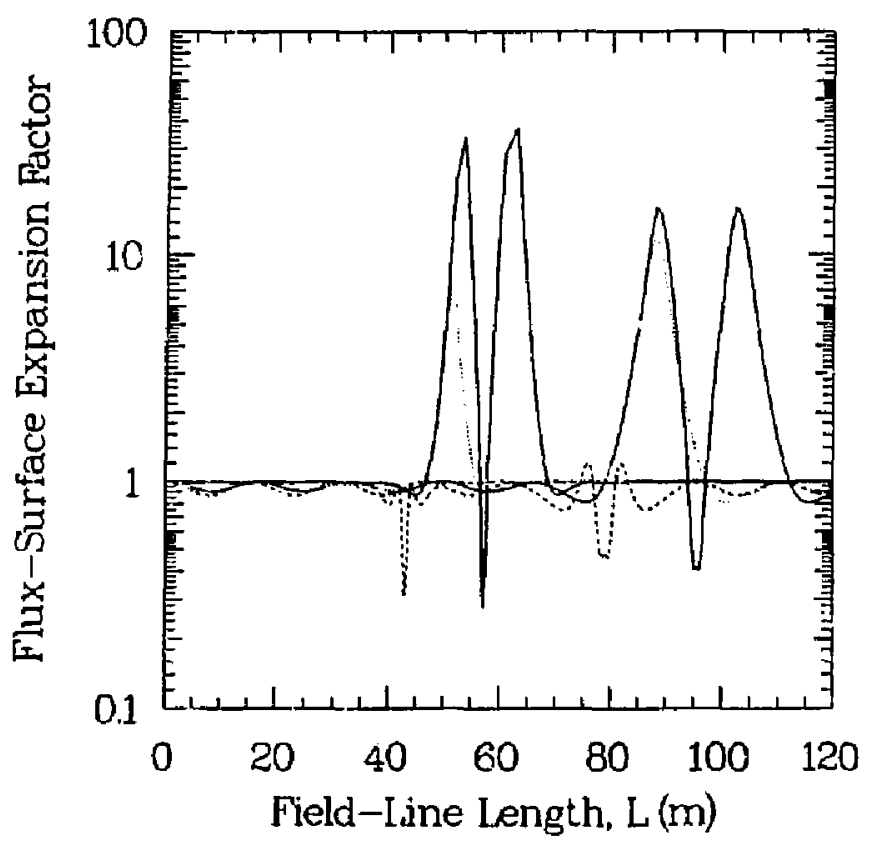

Figure 4.4-9. Flux-surface expansion factor along the seid line with the length measured from the divertor midplane for TITAN.Il. The expansion factors correspond to field lines at $r=0.5995$ (totted line), 0.6'J05 (solid line), and $0.6600 \mathrm{~m}$ (dashed line) brith inboard (curves with spikes between 40 and $70 \mathrm{~m}$ ) and outboard.

where $I_{\phi}$ and $I_{E F}$ are the steady-state plasmia and EF-coil currents, respectively, $M_{i, j}$ is the mutual inductance between the $i^{\text {th }}$ and $j^{\text {th }}$ circuit elements, and $L_{p}$ is the plasme self-inductance. The parameter $f_{G} \equiv I_{O H}^{+} / \mid\left(I_{O H}^{-} \mid\right.$is used to characterize the symumetry of the bipolar $\mathrm{OH}$-current swing. For \& symmetric bipolar swing, $f_{G}=1$ while smaller values of $f_{G}$ represent deeper initial Dack-bias current (i.e., larger $I_{O H}^{-}$). The required OH-coil back-bias volt seconds is

$$
M_{O H, p} J_{j H}=\frac{L_{p} I_{\phi}-M_{E F_{, P}} I_{E F}}{1+f_{G}}
$$

The scoping analysis of the various OH-coil configurations for TITAN-I (Section 4.5.2) uses the code, CCOIL $[2,17]$. The locations of the OIf coils are determined using CCOIL by first specifying an arc upon which the coils are to be arrayed; this arc is defined as a 
segment of an ellipse that is symmetric about the equatorial plane. The coeficients for a Fourier series representation of the current distribution on the arc that excludes flux from the entire plasma cross section are then determined. Assuming equal-current coils to facilitate series electrical connection of the coils, the current distribution is integrated along the arc to yield the $\mathrm{OH}$-coil current-center locations. This integration process used to determine the OH-coil locations introduces an error of $\geq 20 \mathrm{mT}$ in the stray vertical field within the plasma. A manual adjustment of the $\mathrm{OH}$-coil locations is then required to achieve the desired level of stray vertical field. A modified version of CCOIL was used to analyze the vertical-stack configuration discussed in Section 4.5.2. The locations of the vertical-stack $\mathrm{OH}$ coils are taken to be uniformly spaced within a stack. The coils within a stack are of equal current. The current distribution between stacks is determined by requiring the coil set to exluibit an on-axis field null in order to facilitate breakdown and formation of the initial RFP (Section 6.2).

The computer code, CCOIL, estimates the mutual inductances used in Equation 4.5-1 from the following formula for two coaxial hoops [18]:

$$
M_{i, j}=\frac{2 \mu_{\mathrm{o}}\left(r_{i} r_{j}\right)^{1 / 2}}{k}\left[\left(1-\frac{k^{2}}{2}\right) K(k)-E(k)\right],
$$

where

$$
k=\frac{4 r_{i} r_{j}}{\left(r_{i}+r_{j}\right)^{2}+\Delta_{z}^{2}} .
$$

The radii of the $i^{\text {th }}$ and $j^{\text {th }}$ hoops are $r_{i}$ and $r_{j}, \Delta_{2}$ is the distance between the two parallel coil planes, and $K(k)$ and $E(k)$ are the complete elliptic integrals of the first and second kinds, respectively. Each coil in a set is simulated by 100 hoops to ensure a high degree of accuracy, especially in computing coil self-inductances.

When the calculation of the single-turn mutual inductance involves a coil set, a sumination is perforned over each hoop in each coil in the set; for example,

$$
M_{O H, j}=\sum_{i=1}^{n_{O H}} M_{i, j}
$$

where $n_{O H}=100 N_{O H}$ is the number of loops used to simulate the number of $O H$ coils, $N_{O H}$. The single-turn self-inductances of the coil sets are determined by application of the formula for mutual inductance with both summations over the same coil set as follows:

$$
L_{k}=\sum_{i}^{n_{k}} \sum_{j}^{n_{k}} M_{i_{j} j}
$$


where all of the filaments are equi-spaced and carry equal currents. The singuhr element $M_{i, i}$ is replaced with the following expression for the self-inductance of a wire of infinite minor radius [18]:

$$
M_{i, i}=\mu_{0} R_{i}\left[\ln \left(\frac{8 R_{i}}{\Delta}\right)-1.75\right]
$$

where $R_{i}$ is the major radius of the hoop used to simulate a coil and $\Delta$ is the separation between the flaments and assumed to be $0.01 \mathrm{~m}$.

For the final TITAN-I OH-coil design as well as that of TITAN-II, a new computer code is developed which allows a higher degree of flexibility in coil design. In these designs, the current densities in all coils are kept uniform and currents in Oll coils are allowed to vary such that the number of turns in each coil remains an integer. The coil currents and locations are determined by matching the magnetic flux prodiaced by the OH-coil, $\psi$, on the outermost flux surface to $\psi_{o}=M_{O H, p} I_{O H}^{-}$, as prescribed by Equation 4.5-2. For TIPAN designs, the outermost flux surface is a circle with muluor raclius of $r_{p}=0.6 \mathrm{~m}$. This magnetic-flux matching is performed by a numerical optimizer routine whicl minimizes the functional

$$
F=\sum_{s=1}^{N_{s}}\left[1-\frac{\psi^{\prime}\left(\mathrm{r}_{\mathrm{s}}\right)}{\psi_{\mathrm{o}}\left(\mathrm{r}_{\mathrm{s}}\right)}\right]^{2}
$$

whe $N_{i} N_{S}$ points on the niatching flux surface are cliosen, each located at position $\mathbf{r}_{\mathrm{g}}$. Because of the up-down symmetry of the TITAN designs, only points on the upper half of the flux surface (above equatorial plane) are considered. The number of points $N_{S}$ is usually set at a prine number to ensure that resonant multi-poles would not result in a false matching. For initial calculations, $N_{S}=23$ is used and the final design is checked with $N_{S}=53$ points.

The components of the magnetic field and the vector potential are found by direct. integration of the Ampere's law over the coil cross section. Using a cylindrical coordinates system $(R Z \theta)$ with $Z$ axis lying on the axis of the torus and noting the toroida) symmetry, only one component of the vector potential is non-zero. For a circular coil with a rectangular cross section and the coil axis lying on the $Z$ axis, the resulting vector potential at point $(R, Z)$ is

$$
A_{\theta}(R, Z)=\frac{\mu_{o} J}{2 \pi} \int_{o}^{\pi} \cos \theta d \theta \int_{Z_{i}-Z}^{Z_{o}-Z} d Z^{\prime} \int_{R_{\mathrm{i}}}^{K_{0}} \frac{R^{\prime} d R^{\prime}}{\left(Z^{\prime 2}+R^{2}+R^{\prime 2}-2 R R^{\prime} \cos \theta\right)^{1 / 2}}
$$

where $R_{i}$ and $R_{0}$ are inner and outer coil radii and $Z_{i}$ and $Z_{0}$ are the heights of the botton and top of the coil, respectively. The nagnetic flux, $\psi$, can be directly calculated 
from

$$
\psi \equiv \boldsymbol{R} \boldsymbol{A}_{\theta}
$$

and the components of the magnetic field are given by

$$
\begin{aligned}
& B_{R}=-\frac{\partial A_{\theta}}{\partial Z}, \\
& B_{Z}=\frac{A_{\theta}}{R}+\frac{\partial A_{\theta}}{\partial R} .
\end{aligned}
$$

Equation 4.5-9 is substituted in the above equations, resulti: : in similar 3-D integrals for $B_{n}$ and $B_{Z}$.

Approximating each coil by a set of filaments is equivalent to carrying out the $\theta$ integration in Equation 4.5-9 analytically and using "mid-point" rules for $R^{\prime}$ and $Z^{\prime}$ integrals. As a result, the filamentary approximation requires a large number of filaments and will be inaccurate inside or near the coil beceuse of the denominator of the integrand in Equaticn 4.5-9. For final TITAN-I design as well the TITAN-HI OH-coil design, a different technique is used. The $R^{\prime}$ and $Z^{\prime}$ integrals in Equation 4.5-9, and similar equations for $B_{R}$ and $B_{Z}$, are solved analytically and the integral over $\theta$ is evaluated numerically using an adaptive quadrature routine [19]. Similarly, the expression for mutual inductance of two coaxial circular coils with rectangular cross sections and the self-inductance of such coils can be writlen as 6-D integrals over the coils cross section. These 6-D integrals can be reduced analytically to $2-\mathrm{D}$ integrals which are then evaluated numerically using adaptive quadratures [19].

Both the CCOIL computer code and the final TITAN OH-coil designs simulate the plasma as a single-hoop current that is positioned in the equatorial plisie at a major radius, $R_{T}^{*}$, which includes a Shafranov shift $[3]$;

$$
R_{r}^{*}=R_{T}+\frac{r_{w}^{2}}{2 R_{T}}\left[\left(\beta_{\theta}+\frac{l_{i}}{2}-\frac{1}{2}\right)\left(1-x^{2}\right)-\ln x\right],
$$

where $R_{T}$ is the torus unajor radius, $r_{w}$ is the first-wall minor radius, and $x=r_{p} / r_{w}$ is the ratio of plasma and first-wall minor radii. This is a good approximation for circular plasmas with high aspect ratios.

The plasma self-inductance is expressed as a sum of an external inductance, $L_{p, e x}$, and an internal inductance, $L_{p, i n}$, (i.e., $L_{p}=L_{p, i n}+L_{p, e x}$ ). The external inductance is taken to be that for a wire with the same dinensions as the plasma [18]

$$
L_{p, e x}=\mu_{o} R_{T}\left[\ln \left(\frac{8 R_{T}}{r_{p}}\right)-2\right] .
$$


The internal inductance is derived from a 1-D equilibrium calculation (Section 5.2) and is given by

$$
L_{\mathbf{p}, \text { in }}=\left[2 \pi R_{T}\left(W_{\theta}+W_{\phi}\right)-\frac{\phi^{2}}{2 L_{o}}\right] I_{\phi}^{2},
$$

where

$$
\begin{aligned}
W_{i} & \equiv \frac{\pi}{\mu_{0}} \int_{0}^{r_{p}} B_{i}^{2}(r) r d r \\
\phi & \equiv 2 \pi \int_{0}^{r_{p}} B_{\phi}(r) r d r \\
L_{0} & \equiv \frac{\mu_{0} r_{p}^{2}}{2 R_{T}}
\end{aligned}
$$

In addjtion to providing the adequate volt-seconds, the $\mathrm{OH}$-coil design is constrained by the maximum level of the stray vertical field during breakdown (Section 6.2). This constraint specifies the stray vertical field produced by the $\mathrm{OH}$-coil set as a fraction of the initial toroidal field, $B_{\phi \sigma}$. In principle, the initial toroidal field, $B_{\phi o}$, can be increased to ensure compliance with the stray-vertical-field constraint. Any increase in $B_{\phi o}$, however, would result in increases in the $\mathrm{OH}$-coil flux consumption as well as increases in the formation energy and power. A single order of magnitude increase in the value of $B_{\phi_{0}}$ would result in a flux consumption during formation $\geq 80 \mathrm{~V} \cdot \mathrm{s}$, a formation energy $\geq 200 \mathrm{MJ}$, and a formation power $\geq 1 \mathrm{GW}$ (Section 6.2). Consequently, a maximum value of $2.45 \mathrm{mT}$ for the stray vertical field is adopted. A secondary constraint is that the $O H$-coil set exhibit a field null within the plasma chamber. A field null provides a closed field line upon which to initiate a current cliannel. Finally, the PF-coil parameters listed in Table 4.1-I provide design guidance for the OH coils.

\subsubsection{OH-Coil Configurations}

The desire to optimize the $\mathrm{OH}$-coil performance, as measured by the electrical coupling between the $\mathrm{OH}$ coil and the plasma, conflicts with design-integration requirements for access to the TF coils, blanket, and vacuum chamber. Other nagnet engineering issues such as stresses, cooling requirements, and the magnet support struct ure should also be considered. Consequently, a search was conducted for an $\mathrm{OH}$-coil configuration with both good access and performance properties. The OH-coil configurations surveyed are a "close-fitting" configuration (shown in Figure 4.5-1), and a "vertical stack" configuration and a range of "pill box" configurations (shown in Figures 4.5-2 and 4.5-3). 


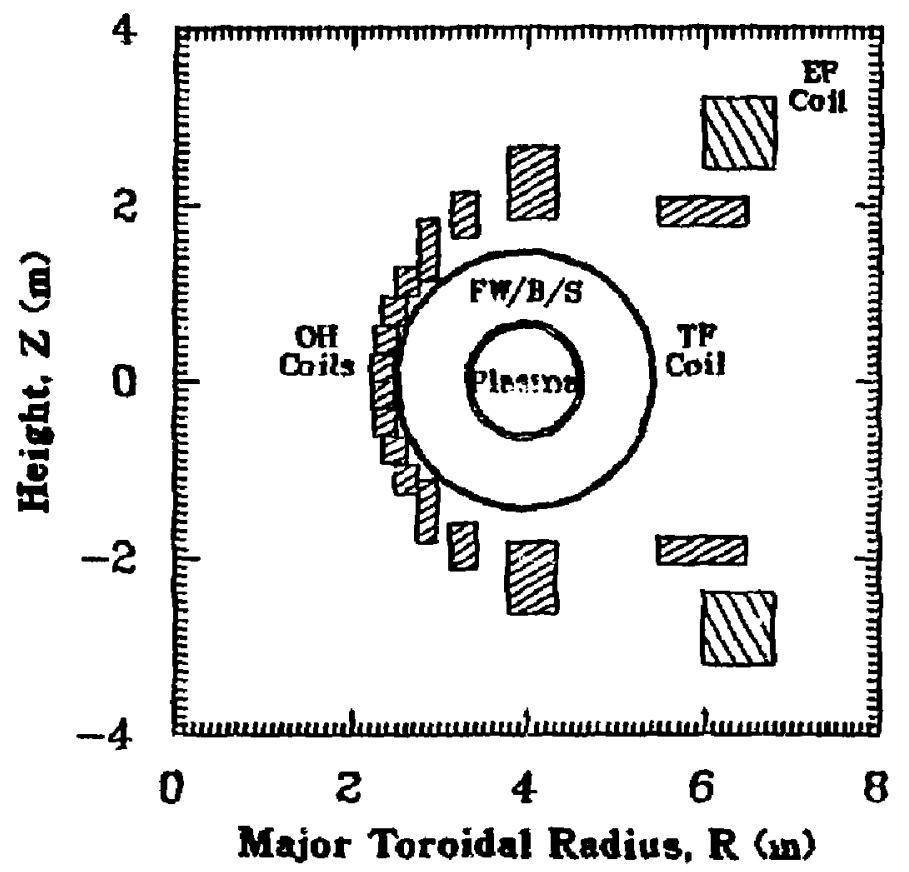

Figure 4.5-1. A "close-fitting" OH-coil configuration that maximizes the electrical coupling between the plasma and $\mathrm{OH}$ coils used in $\mathrm{OH}$-coil configurational analysis.

The close-fitting configuration yields the best electrical coupling to the plasma, as is indicated by the mutual inductances between the $\mathrm{OH}$ coils and the plasma (Table 4.5-I); $\mathrm{OH}$-coil transparency is sacrificed, however. The OH-coil transparency reported in Table 4.5-11 is defined as the fraction of the equatorial-plane cross-sectional area of the torus bounded by the TF coils that is vertically unobstructed by $\mathrm{OH}$ coils. The transparency is maximized by the vertical-stack configuration. The vertical-stack configuration, however, violates by nearly two orders of magnitude the liberal stray-vertical-field constraint of $\leq 8 \mathrm{mT}$ operative during this configurational study. Con oliance with a stray-vertical-field constraint of a few $\mathrm{mT}$ requires the placement of $\mathrm{OH}$ coils over the torus. A compronise configuration between the close-fitting and vertical-stack configuratjons is the pill-box configuration. By varying the height of the pill-box configuration, the inductive coupling to the plasma approaches $93 \%$ of that for the close-fitting configuration, but the $\mathrm{OH}$-coil transparency decreases below that of the close-fitting configuration. 

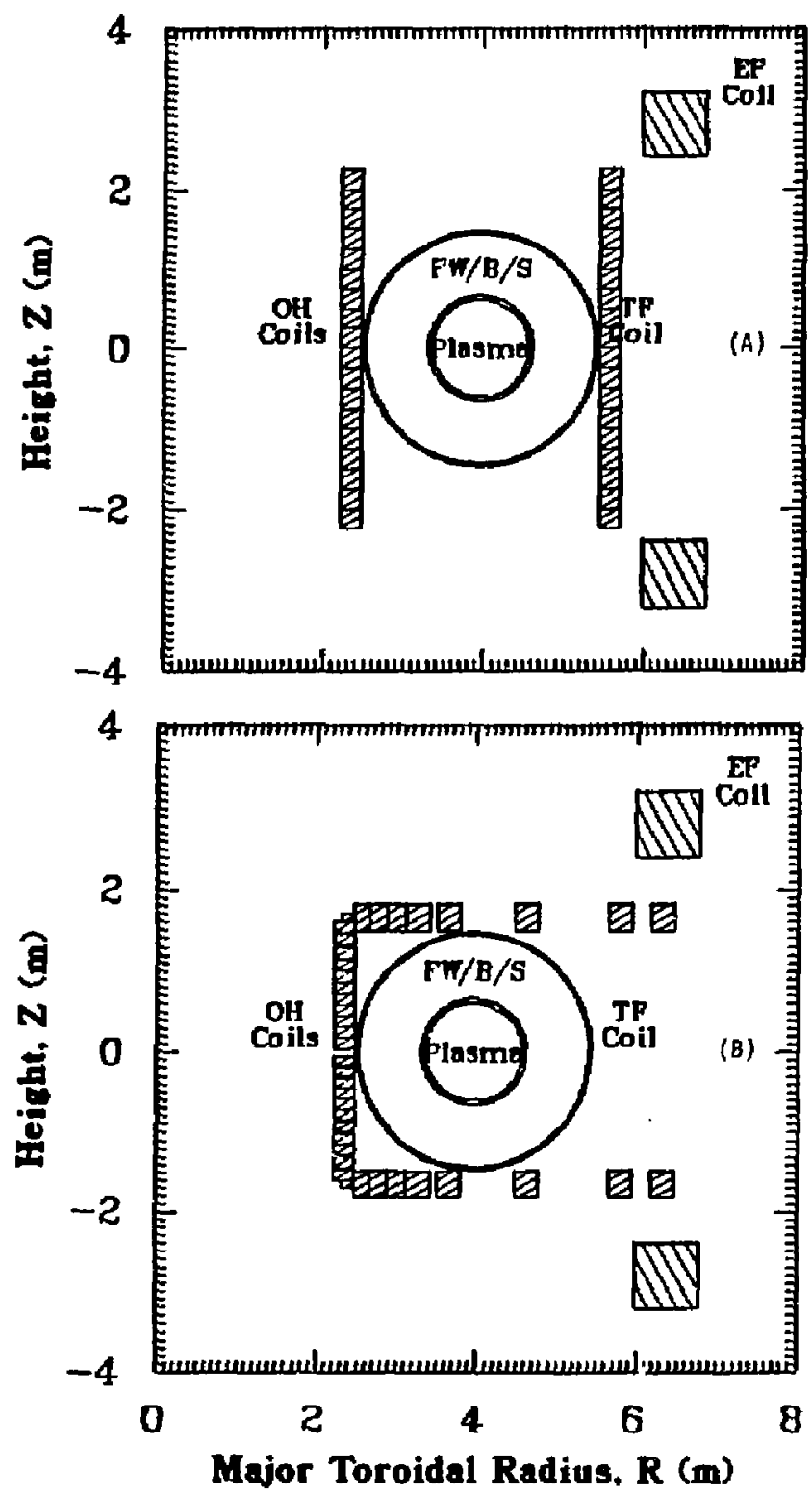

Figure 4.5-2. A "vertical-stack" configuration that maximizes the OH-coil transparency (A) and a "pill-box" configuration with a low vertical extent (B) used in $\mathrm{OH}$-coil configurational analysis. 

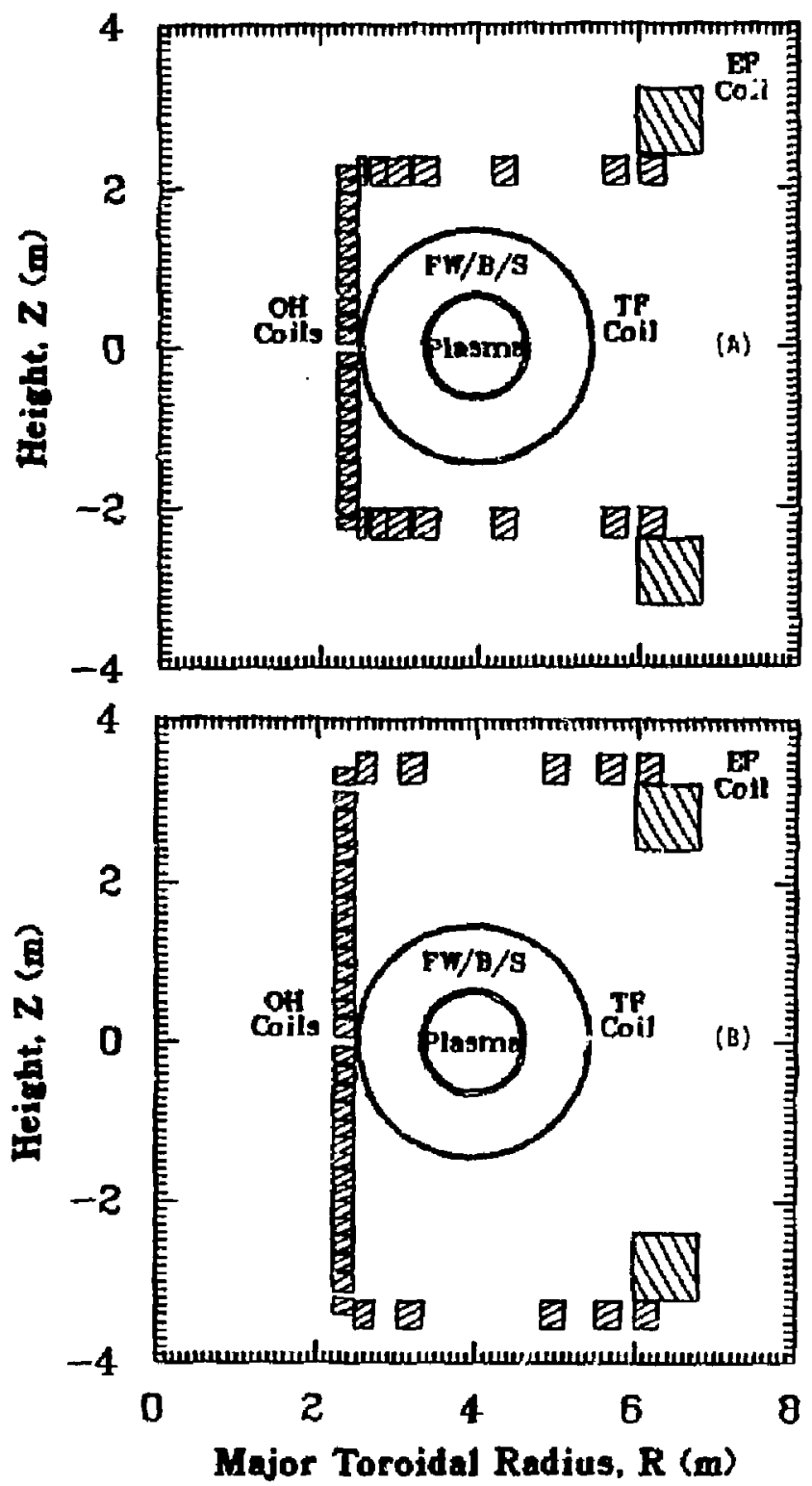

Figure 4.5-3. "Pill-box" configurations with a medium vertical extent (A) and a ligh vertical extent (B) used in OH-coil configurational analysis. 
Table 4.5-I.

CIRCUIT PARAMETERS FOR

TITAN-I OH-COIL CONFIGURATIONAL ANALYSIS

\begin{tabular}{|c|c|c|c|c|c|}
\hline & \multirow{2}{*}{$\begin{array}{c}\text { Close } \\
\text { Fitting }\end{array}$} & \multirow{2}{*}{$\begin{array}{c}\text { Vertical } \\
\text { Stack }\end{array}$} & \multicolumn{3}{|c|}{ Piit Box } \\
\hline & & & Low & Medium & High \\
\hline \multicolumn{6}{|c|}{ Self inductances $(\mu \mathrm{H})$} \\
\hline$\cdot L_{\mathrm{p}}$ & 13.5 & 13.5 & 13.5 & 13.5 & 13.5 \\
\hline$\cdot L_{E F}$ & 29.1 & 29.1 & 29.1 & 29.1 & 29.1 \\
\hline$L_{\text {OH }}$ & 4.31 & 3.32 & 3.46 & 2.98 & 2.41 \\
\hline \multicolumn{6}{|c|}{ Mutual inductances $(\mu \mathrm{H})$} \\
\hline - $M_{O H, P}$ & 3.60 & 3.02 & 3.34 & 2.82 & 2.24 \\
\hline$M_{O H, E F}$ & 3.01 & 2.12 & 2.54 & 2.52 & 2.44 \\
\hline$\cdot M_{E F, p}$ & 3.72 & 3.72 & 3.72 & 3.72 & 3.72 \\
\hline \multicolumn{6}{|c|}{ Current levels (MA) } \\
\hline$\cdot I_{\phi}$ & 21.7 & 21.7 & 21.7 & 21.7 & 21.7 \\
\hline$\cdot I_{E F}$ & 23.2 & 23.2 & 23.2 & 23.2 & 23.2 \\
\hline$\cdot \Delta I_{O H}$ & 56.9 & 67.9 & 61.5 & 72.8 & 91.5 \\
\hline \multicolumn{6}{|c|}{ Magnetic fluxes ( $W b$ ) } \\
\hline - Plasma & 291.5 & 291.5 & 291.5 & 291.5 & 291.5 \\
\hline - EF conil & 86.4 & 86.4 & 86.4 & 86.4 & 86.4 \\
\hline . OH coil & 205.1 & 205.1 & 205.1 & 205.1 & 205.1 \\
\hline
\end{tabular}


Table 4.5-II.

RESULTS OF TITAN-I OH-COIL CONFIGURATIONAL ANALYSIS

\begin{tabular}{|c|c|c|c|c|c|}
\hline & \multirow{2}{*}{$\begin{array}{c}\text { Close } \\
\text { Fitting }\end{array}$} & \multirow{2}{*}{$\begin{array}{c}\text { Vertical } \\
\text { Stack }\end{array}$} & \multicolumn{3}{|c|}{ Pill Box } \\
\hline & & & Low & Medium & High \\
\hline OH-coil back-bias current (MA) & 28.5 & 34.0 & 30.7 & 36.4 & 45.7 \\
\hline OH-coil volume $\left(\mathrm{m}^{3}\right)$ & 66.1 & 60.5 & 49.4 & 47.9 & 52.2 \\
\hline OH-coil mass (tonne) & 482.5 & 441.7 & 360.6 & 349.7 & 381.1 \\
\hline OH-coil joule losses (MW) & 214.7 & 319.0 & 261.7 & 324.6 & 411.6 \\
\hline OH-coil von Mises stress ( $\mathrm{MPa}$ ) & 198.6 & 113.2 & 135.9 & 124.0 & 127.8 \\
\hline OH-coil peak field (T) & 7.5 & 5.7 & 6.1 & 5.8 & 5.9 \\
\hline OH-coil current density $\left(\mathrm{MA} / \mathrm{m}^{2}\right)^{(a)}$ & 22.9 & 24.4 & 48.0 & 29.7 & 21.1 \\
\hline OH-coil stray vertical field $(\mathrm{mT})$ & 7.8 & 658.4 & 8.0 & 6.8 & 0.3 \\
\hline $\mathrm{OH}$-coil transparency $(\%)$ & 61.7 & 100. & 55.6 & 65.9 & 73.8 \\
\hline
\end{tabular}

(a) Maximum values.

The failure of the low-profile pill-box configuration to outperform the close-fitting configuration led to the adoption of the close-fitting configuration as the interin TITAN-I $\mathrm{OH}$-coil design. The close-fitting TITAN-I OH-coil design is shown in Figure 4.5-4 and described in Table 4.5-III. The presence of a field null at the back bias is shown in Figure 4.5-5. Compliance with the 2.45-mT stray-vertical-field constraint was monitored only in the equatorial plane, as is shown in Figure 4.5-6. As a result, compliance witb the stray-vertical-field constraint does not occur in a small region of the plasma cross section, as is shown in Figure 4.5-5; compliance can be obtained, however, witll only small adjustments to the coil locations.

The final TITAN-I OH-coil design strives to combine the good features of the closefitting and pill-box configurations as shown in Figure 4.5-7. The final design also incorporates the magnet engineering issues of the stresses, cooling, and magnet support structure. The vertical stack of the pill-box configuration is retained which allows the 


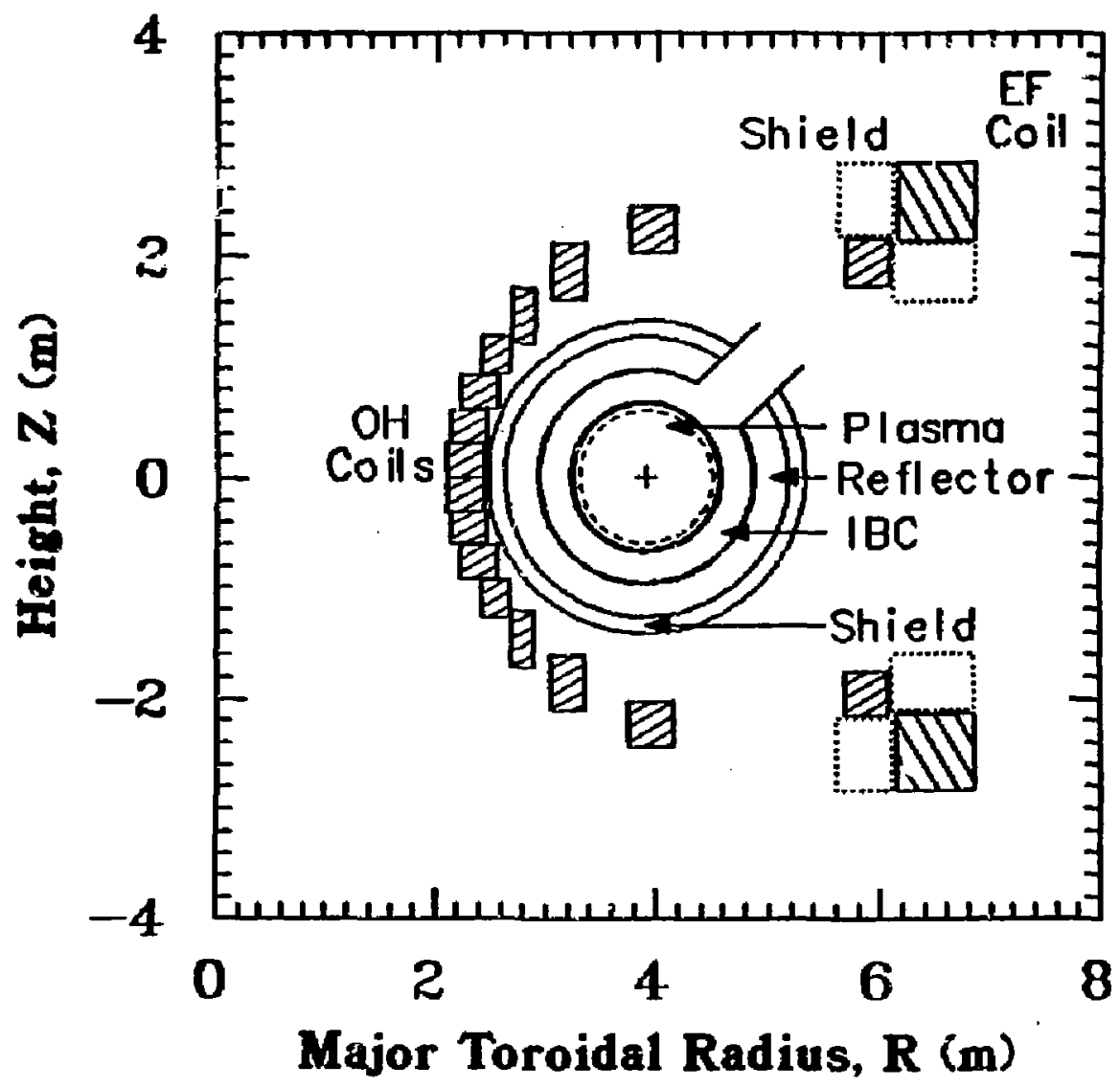

Figure 4.5-4. A cross-sectional view of the "close-fitting" OH-coil set for the intermediate TITAN-I design. The locations of the TF IBCs, the reflector, the shield, and the plasma are shown in addition to the EF coils and the $\mathrm{OH}$ coils. 
Table 4.5-III.

PARAMETERS OF INTERIM TITAN-I POLOIDAL-FIELD COLLS

\begin{tabular}{cccccccc} 
& $R$ & $\pm z$ & $\Delta R$ & $\Delta z$ & $I^{(a)}$ & $j^{(a, b)}$ & Mass $^{(c)}$ \\
Function & $(\mathrm{m})$ & $(\mathrm{m})$ & $(\mathrm{m})$ & $(\mathrm{m})$ & $(\mathrm{MA})$ & $\left(\mathrm{MA} / \mathrm{m}^{2}\right)$ & (tonne) \\
\hline
\end{tabular}

$\begin{array}{crrrrrrr}\text { Trim } & 5.7700 & 1.2000 & 0.2000 & 0.3000 & 0 . & 0 . & 15.9 \\ \text { EF } & 6.4959 & 2.4873 & 0.6973 & 0.6973 & -9.6189 & 19.78 & 144.9 \\ \text { OH-1 } & 5.8699 & 1.9473 & 0.4000 & 0.4000 & 1.4556 & 9.10 & 43.1 \\ \text { OH-2 } & 3.9472 & 2.2299 & 0.4100 & 0.4100 & 1.4556 & 8.66 & 30.4 \\ \text { OH-3 } & 3.1958 & 1.8533 & 0.3000 & 0.5000 & 1.4556 & 9.70 & 22.0 \\ \text { OH-4 } & 2.7905 & 1.4625 & 0.2000 & 0.5000 & 1.4556 & 14.56 & 12.8 \\ \text { OH-5 } & 2.5503 & 1.1031 & 0.2500 & 0.3300 & 1.4556 & 17.64 & 9.7 \\ \text { OH-6 } & 2.4028 & 0.7705 & 0.3300 & 0.3000 & 1.4556 & 14.70 & 10.9 \\ \text { OH-7 } & 2.3163 & 0.4557 & 0.3200 & 0.3000 & 1.4556 & 15.16 & 10.2 \\ \text { OH-8 } & 2.2759 & 0.1508 & 0.3300 & 0.3000 & 1.4556 & 14.70 & 10.3\end{array}$

(a) Mean steady-state values for the EF coils and back-bias values for the OII coils for a symmetric bipolar swing.

(b) Averaged over the entire coil cross section.

(c) A density of 7.3 tonne $/ \mathrm{m}^{3}$ is assumed. 

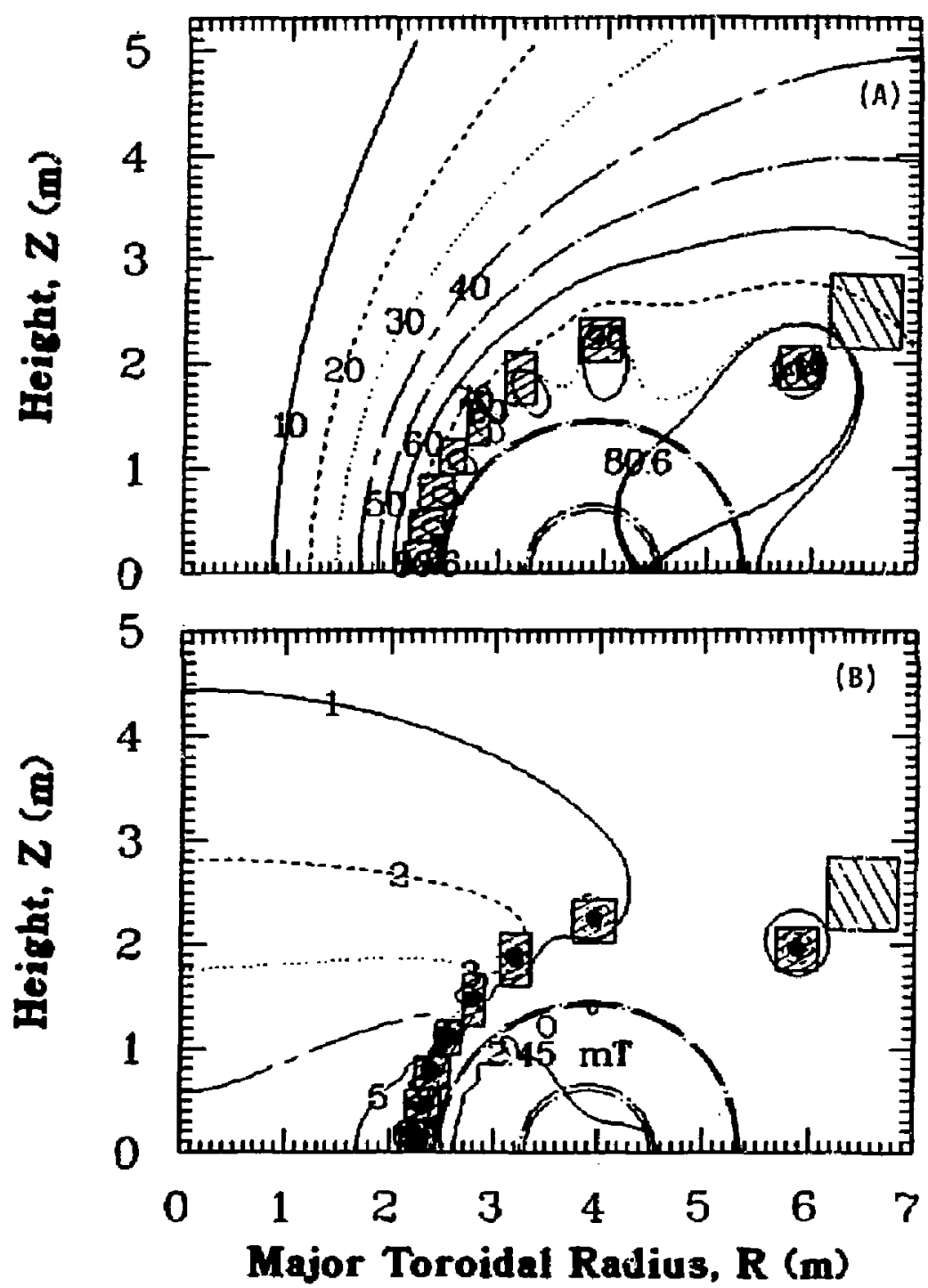

Figure 4.5-5. Contour plots of flux (A) and magnetic-field strength (B) for the intermediate TITAN-I OH-coil design. The flux contours, labeled in weber, demonstrate the presence of a field null within the plasma chamber. The magnetic-field contours are labeled in tesla, except the $2,45-\mathrm{m} T$ contour which corresponds to the stray-vertical-field constraint. 


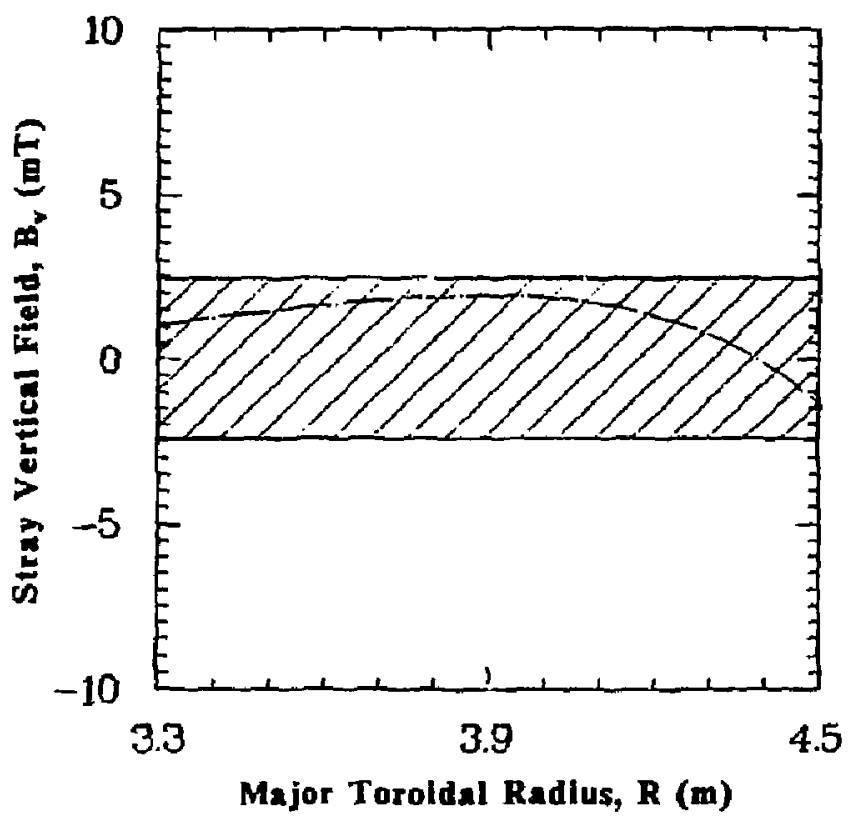

Figure 4.5-6. The stray vertical field for the intermediate TITAN-1 OH-coil design in the equatorial plane. The hatched region repi ssents the stray-vertical field constraint.

Lorentz forces from the uppe: coil set to be transmitted and canceled by the equal and opposite forces on the lower coil set. The coil OH-1 in Figure 4.5-7 is placed further away from the EF-coil set in order to reduce the forces on this coil during start-up transients. Coils $\mathrm{OH}-2$ through $\mathrm{OH}-4$ are arranged in a coil assembly which includes the support structure against bending moments produced by magnetic forces. During the maintenarce operations, only the upper $\mathrm{OH}$-coil assembly is removed; the vertical stack is installed permanently in the reactor vault. Detailed designs of the internal of the $\mathrm{OH}$ coils as well as the support structure are given in Section 10.5.

The final TITAN.I design is shown in Figure 4.5-7 and described in Tables 4.5-IV through 4.5-VI and compared with the parameters of the interim design. As expected, the intermediate design couphes better to the plasma than the final design, which results in a $20 \%$ larger OH-coil current swing. But, the final design has the same coil mass and volume as the interim design, and lower peak joule heating and stresses. The stray vertical field produced by the final design is less than $0.5 \mathrm{mT}$ in the entire plasma chamber. 


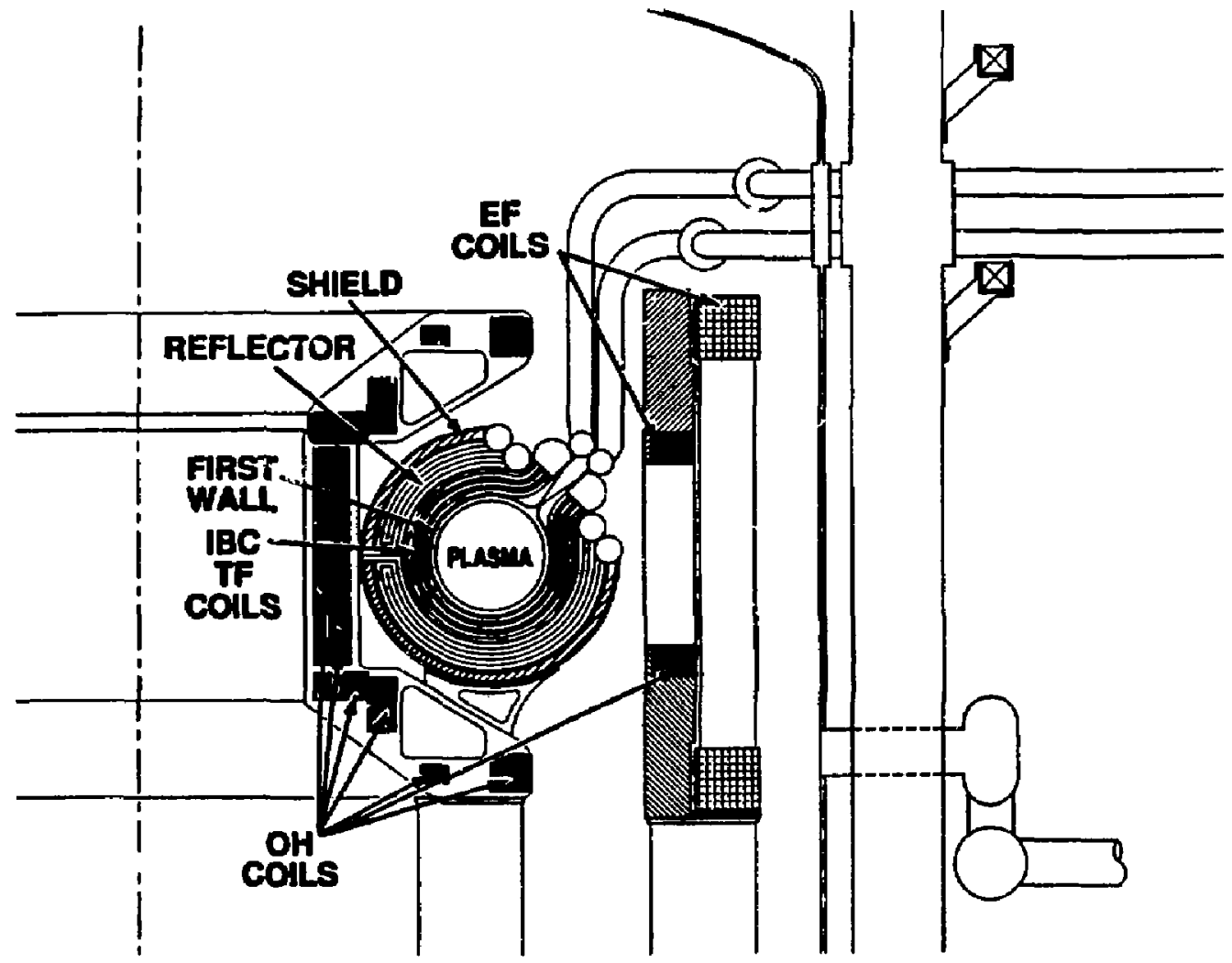

Figure 4.5-7. A cross-sectional view of the final TITAN-I OH-coil design. Also shown are the locations of the plasma, first wall, TF IBCs, reflector, shield, and EF coils. 
The compliance of the final TITAN-I design with the constraints of a field null and the magniiude of the stray vertical field are demonstrated in Figure 4.5-8.

The TITAN-II OH-coil design shown in Figure 4.5-9 and described in Tables 4.5-VII through 4.5-IX is sinular to the TITAN-I OH-coil design. The PF coils are closer to the plasma in TITAN-II, however, because of a thinner first wall, blanket, and shield ( $\sim 0.45 \mathrm{~m}$ as opposed to $\sim 0.75 \mathrm{~m}$ for TITAN-I) and the OH-coil mass is larger in TITAN-II as dictated by the parametric systems model of Section 3.2. The TITAN-II $\mathrm{OH}$-coil design has the prerequisite field null and the stray vertical field complies with the 2.45- $\mathrm{mT}$ constraint, as is shown in Figure 4.5-9.

Table 4.5-TV.

PARAMETERS OF FINAL TITAN-I POLOIDAL-FIELD COILS

\begin{tabular}{|c|c|c|c|c|c|c|c|c|}
\hline Coil & $\begin{array}{c}R \\
(\mathrm{~m})\end{array}$ & $\begin{array}{l} \pm z \\
(\mathrm{~m})\end{array}$ & $\begin{array}{l}\Delta R \\
(\mathrm{~m})\end{array}$ & $\begin{array}{l}\Delta z \\
(\mathrm{~m})\end{array}$ & $\begin{array}{c}I^{(a)} \\
(\mathrm{MA})\end{array}$ & $\begin{array}{c}i^{(a),(b)} \\
\left(\mathrm{MA} / \mathrm{m}^{2}\right)\end{array}$ & $\begin{array}{l}\text { Mass }^{(c)} \\
\text { (tonne) }\end{array}$ & Turns \\
\hline Trim & 5.7700 & 1.2000 & 0.2000 & 0.3000 & 0. & 0. & 15.9 & 10 \\
\hline $\mathrm{EF}$ & 6.4959 & 2.4873 & 0.6973 & 0.6973 & -9.6189 & 19.78 & 144.9 & 44 \\
\hline OH-1 & 6.0000 & 1.1963 & 0.2000 & 0.3000 & 0.7500 & 12.50 & 16.5 & 10 \\
\hline $\mathrm{OH}-2$ & 4.1073 & 2.4050 & 0.4000 & 0.4200 & 2.1000 & 12.50 & 31.7 & 28 \\
\hline $\mathrm{OH}-3$ & 3.2919 & 2.4000 & 0.3000 & 0.2000 & 0.7500 & 12.50 & 9.1 & 10 \\
\hline OH! -4 & 2.7125 & 1.6538 & 0.3000 & 0.6000 & 2.2500 & 12.50 & 22.4 & 30 \\
\hline $\mathrm{OH}-5$ & 2.2500 & 1.4307 & 0.6000 & 0.3000 & 2.2500 & 12.50 & 18.6 & 30 \\
\hline OH-6 & 2.1500 & 0.8100 & 0.4000 & 0.7800 & 3.9000 & 12.50 & 30.8 & 52 \\
\hline OH-7 & 2.1500 & 0.2050 & 0.4000 & 0.3900 & 1.9500 & 12.50 & 15.4 & 26 \\
\hline
\end{tabular}

(a) Mean steady-state values for the EF coils and back-bias values for the $\mathrm{OH}$ coils for a symmetric bipolar swing.

(b) Averaged over the entire coil cross section.

(c) A density of 7.3 tonne $/ \mathrm{m}^{3}$ is assumed. 

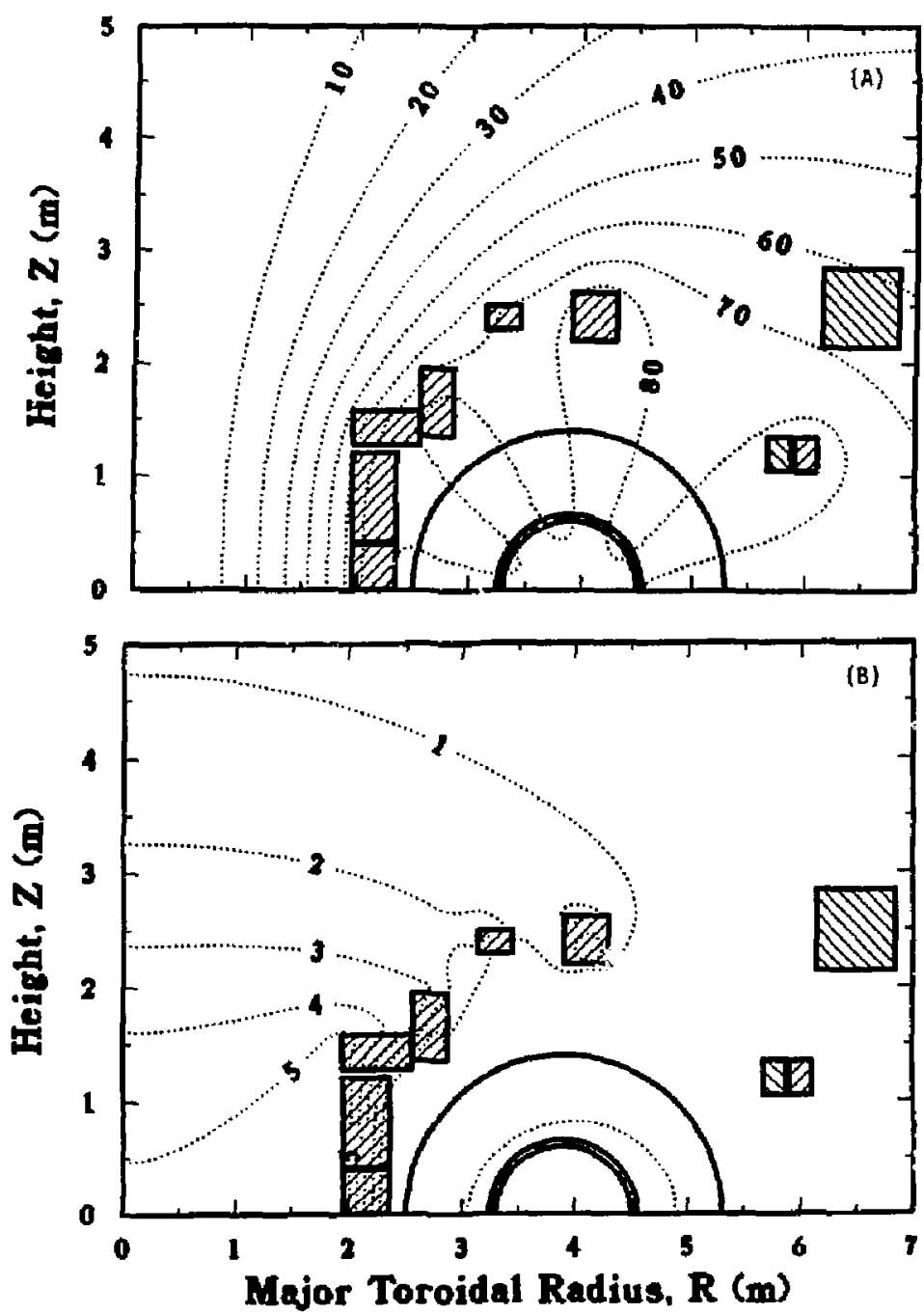

Figure 4.5-8. Contour plots of flux (A) and magnetic-field strength (B) for the final TITAN-I OH-coil design. The flux contours, labeled in weber, demonstrate the presence of a field null within the plasma chamber. The magnetic-field contours are labeled in tesla, except the 2.45-mT contour around the plasma chamber which corresponds to the stray-vertical-field constraint. 

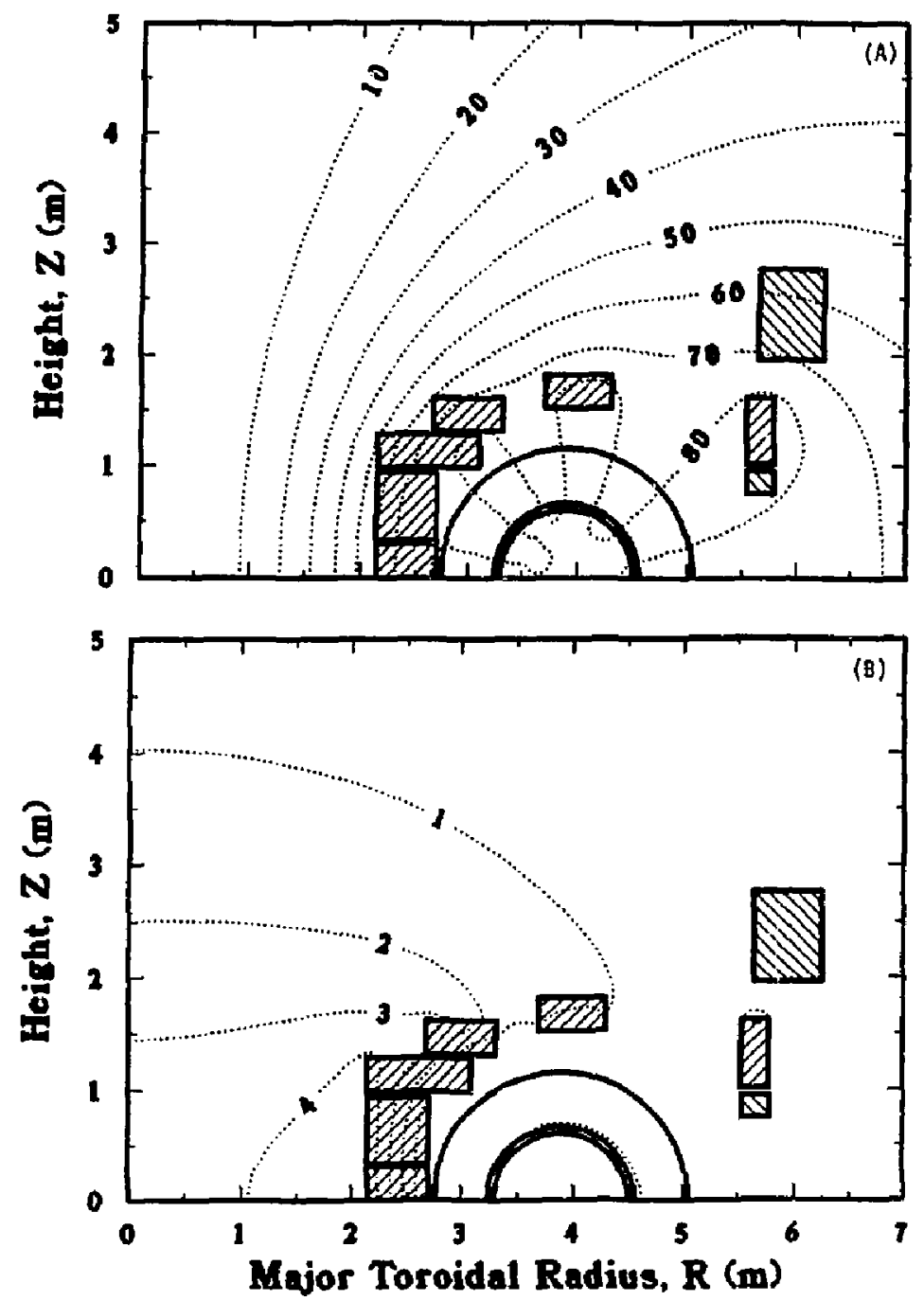

Figure 4.5-8. Contour plots of flux (A) and magnetic-field strength (B) for the TITAN-1I OH-coil design. The flux contours, labeled in weber, demonstrate the presence of a field null within the plasma chamber. The magnetic-field contours are labeled in tesla, except the $2.45-\mathrm{mT}$ contour around the plasma chamber which corresponds to the stray-vertical-field constraint. 
Table 4.5-V.

CIRCUIT PARAMETERS FOR TITAN-I PF-COIL DESIGNS ${ }^{(\alpha)}$

\begin{tabular}{|c|c|c|}
\hline & Interim & Final \\
\hline \multicolumn{3}{|c|}{ Self inductances $(\mu \mathrm{H})$} \\
\hline$\cdot L_{p}$ & 13.29 & 13.29 \\
\hline - $L_{O H}$ & 3.39 & 2.74 \\
\hline - $L_{E F}$ & 14.80 & 15.02 \\
\hline - $L_{\text {Trim }}$ & & 18.36 \\
\hline \multicolumn{3}{|c|}{ Mutual inductances $(\mu \mathrm{H})$} \\
\hline - MOH,p & 3.47 & 2.87 \\
\hline . $M_{O H, E F}$ & 3.08 & 2.26 \\
\hline - MOH,Trim & & 2.99 \\
\hline$M_{E F, p}$ & 3.88 & 3.86 \\
\hline$M_{E F, T \text { rim }}$ & & 8.69 \\
\hline$M_{\text {Trim,p }}$ & & 5.60 \\
\hline \multicolumn{3}{|c|}{ Current levels (MA-turn) } \\
\hline$I_{\phi}$ & 17.75 & 17.75 \\
\hline$I_{E F}$ & 19.24 & 19.24 \\
\hline$\Delta I_{O H}$ & 46.58 & 55.80 \\
\hline \multicolumn{3}{|c|}{ Magnetic fluxes (Wb) } \\
\hline - Plasma & 236.0 & 236.0 \\
\hline - EF roil & 74.5 & 74.5 \\
\hline . OH coil & 161.5 & 161.5 \\
\hline
\end{tabular}

(a) Equivalent single-turn inductance values are given. 
Table 4.5-VI.

\section{PARAMETERS OF TITAN-I PF-COIL DESIGNS}

\begin{tabular}{|c|c|c|}
\hline & Interim & Final \\
\hline EF-coil current (MA-turn) $)^{(a)}$ & 19.2 & 19.2 \\
\hline EF-coil volume $\left(m^{3}\right)$ & 39.7 & 39.7 \\
\hline EF-coil mass (tonne) & 289.7 & 289.7 \\
\hline EF-coil peak field $(T)^{(a)}$ & 6.4 & 6.4 \\
\hline EF-coil current density $\left(\mathrm{MA} / \mathrm{m}^{2}\right)^{(a)}$ & 19.8 & 19.8 \\
\hline Vertical field index, $n$ & 0.16 & 0.16 \\
\hline OH-coil current (MA-turn) $)^{(b)}$ & 23.3 & 27.9 \\
\hline OH-coil volume & 40.9 & 39.5 \\
\hline OH-coil mass (tonne) & 298.8 & 288.4 \\
\hline OH-coil joule losses $(\mathrm{MW})^{(6)}$ & 161.0 & 103. \\
\hline OH-coil von Mises stress $(\mathrm{MPa})^{(b)}$ & 100.9 & 89. \\
\hline OH-coil peak field $(\mathrm{T})^{(b)}$ & 5.9 & 5.7 \\
\hline OH-coil current density $\left(\mathrm{MA} / \mathrm{m}^{2}\right)^{(b)}$ & 8.6 to 17.6 & 12.5 \\
\hline OH-coil stray vertical field $\left(\mathrm{m}^{\mathrm{T}} \mathrm{T}\right)^{(b)}$ & $1.92^{(e)}$ & $0.43^{(c)}$ \\
\hline OH-coil transparency (\%) & 67.2 & 63. \\
\hline
\end{tabular}
(a) Mean steady-state values.
(b) Back-bias values for a symmetric bipolar swing.
(c) Satisfies the stray-vertical-field constraint $(<2.45 \mathrm{mT}$, Section 6.2). 
Table 4.5-VII.

PARAMETERS OF TITAN-II POLOIDAL-FIELD COILS

\begin{tabular}{|c|c|c|c|c|c|c|c|c|}
\hline Coil & $\begin{array}{c}R \\
(\mathbf{m})\end{array}$ & $\begin{array}{l} \pm z \\
(\mathrm{~m})\end{array}$ & $\begin{array}{l}\Delta R \\
(\mathrm{n})\end{array}$ & $\begin{array}{l}\Delta z \\
(\mathrm{~m})\end{array}$ & $\begin{array}{c}I^{(a)} \\
(\mathrm{MA}) \\
\end{array}$ & $\begin{array}{c}j^{(a),(b)} \\
\left(\mathrm{MA} / \mathrm{m}^{2}\right) \\
\end{array}$ & $\begin{array}{l}\text { Mass }(e) \\
\text { (tonne) } \\
\end{array}$ & Turns \\
\hline Trim & 5.6600 & 0.8500 & 0.2500 & 0.2000 & 0. & i. & 12.98 & 10 \\
\hline EF & 5.9356 & 2.3608 & 0.6000 & 0.8000 & -9.3021 & 19.38 & 130.68 & 22 \\
\hline OH-1 & 5.6598 & 1.3134 & 0.2500 & 0.6000 & 1.1730 & 7.82 & 38.94 & 20 \\
\hline $\mathrm{OH}-2$ & 3.9978 & 1.6684 & 0.6000 & 0.3000 & 1.4076 & 7.82 & 33.01 & 24 \\
\hline OH-3 & 2.9896 & 1.4600 & 0.6500 & 0.3000 & 1.5249 & 7.82 & 26.74 & 26 \\
\hline $\mathrm{OH}-4$ & 2.6200 & 1.1300 & 0.9500 & 0.3000 & 2.2287 & 7.82 & 34.25 & 38 \\
\hline $\mathrm{OH}-5$ & 2.4200 & 0.6320 & 0.5500 & 0.6000 & 2.5806 & 7.82 & 36.63 & 44 \\
\hline $\mathrm{OH}-6$ & 2.4200 & 0.1500 & 0.5500 & 0.3000 & 1.2903 & 7.82 & 18.31 & 22 \\
\hline
\end{tabular}

(a) Mean steady-state values for the EF coils and back-bias values for the $\mathrm{OH}$ coils for a symmetric bipolar swing.

(b) Averaged over the entire coil cross section.

(c) A density of 7.3 tonne $/ \mathrm{m}^{3}$ is assumed. 
Table 4.5-VIII.

CIRCUIT PARAMETERS FOR TITAN-II PF-COIL DESIGNS ${ }^{(a)}$

Self inductances $(\mu \mathrm{H})$

- $L_{\mathrm{p}}$

13.23

- $L_{O H}$

$3.6 \mathrm{E}$.

- $L_{E F}$

13.35

- $L_{\text {trim }}$

19.35

Mutual inductances $(\mu \mathrm{H})$

- MOH,p

3.92

- MOH,EF

3.04

- MOH,Trim

4.03

- $M_{E F, p}$

4.12

- $M_{E F, T \text { rim }}$

8.22

- MTrim,p

6.15

Current levels (MA-turn)

- $I_{\phi}$

17.82

- $I_{E F}$

18.60

- $\Delta I_{O H}$

40.82

Magnetic fluxes (Wb)

- Plasma

236.9

- EF coil

77.1

- $\mathrm{OH}$ coil

159.8

(a) Equivalent single-turn inductance values are given. 
Table 4.5-IX.

PARAMETERS OF TITAN-II PF-COIL DESIGNS

EF-coil current (MA-turn) ${ }^{(a)}$

EF-coil volume $\left(\mathrm{m}^{3}\right)$

EF-coil mass (tonne)

EF-coil peak field $(\mathrm{T})^{\{a\}}$

EF-coil curtent density $\left(\mathrm{MA} / \mathrm{m}^{2}\right)^{(\mathrm{a})}$

Vertical field index, $n$

OH-coil current (MA-turn) $)^{(b)}$

$\mathrm{OH}$-coil volume

$\mathrm{OH}$-coil mass (tonne)

OH-coil joule losses (MW) ${ }^{(b)}$

OH-coil won Mises stress $\left(M P_{a}\right)^{(b)}$

OH-coil peak field $(\mathrm{T})^{(b)}$

GH-coil current density $\left(\mathrm{MA} / \mathrm{m}^{2}\right)^{(b)}$

OH-coil stray vertical field $(\mathrm{mT})^{(6)}$
18.6

35.8

261.4

7.2

19.4

0.40

20.4

51.5

375.8

52.5

33.4

4.7

7.8

$2.30^{(c)}$

(a) Mean steady-state values.

(b) Back-bias values for a symmetric bipolar swing.

(c) Satisfies the stray-vertical-field constraint $(<2.45 \mathrm{mT}$, Section 6.2$)$. 


\subsection{EQUILIBRIUM-FIELD (EF) COILS}

\subsubsection{Modele and Constraints}

Since the EF coils are continuously active, the recirculating power can be minimized by using superconducting EF coils. Superconducting EF coils, however, require $\sim 1.5 \mathrm{~m}$ of blanket and shielding between the coilh and plasma compared to $\leq 0.4$ to $0.8 \mathrm{~m}$ for normal-conducting EF coils; hence, more current is needed to produce the same field resulting in an increase in the stored energy and a more massive and expensive coil set. The trade-off between normal-conjieting and superconducting EF coils was examined and found to weigh slightly in fayor of superconducting EF coils (Section 3.4). Consequently, the use of superconducting EF coils is adopted for the TITAN resctor study. An additional constraint is imposed of using only a single pair of EF coils positioned not to interfere with vertical or horizontal movement of the first wall, blenket, shield, and TF. coil assembiy during maintenance procedures. This EF-coil set would be a life-of-plant item.

The steady-state EF-coil currents are determined by equating the on-axi: vacuum field produced by the EF coil to the vertical field required for toroidal equilibrium. The required vertical field, $B_{V}$, is given by $[3,4]$

$$
B_{V}=\frac{\mu_{0} I_{\phi}}{4 \pi R_{T}}\left[\ln \left(\frac{8 R_{T}}{r_{P}}\right)+\frac{l_{i}}{2}+\beta_{\vartheta}-1.5\right],
$$

where $R_{T}$ and $r_{p}$ are the plesma major and minor radii, reapectively, $I_{\phi}$ is the steadystate plasma current, $\beta_{0}$ is the poloidal beta, and $l_{i}$ is the internal inductance per unit length of plasma. Typically, $l_{i} \simeq 1$ for RFP field and current profiles. The position of the EF coils is determined sucin that the value of the decay index,

$$
n \equiv \frac{\partial\left(\ln B_{V}\right)}{\partial(\ln R)} \simeq \frac{R_{T}}{r_{p}} \frac{B_{V}\left(R_{T}-r_{p}\right)-B_{V}\left(R_{T}+r_{p}\right)}{B_{V}\left(R_{T}-r_{p}\right)+B_{V}\left(R_{T}+r_{p}\right)},
$$

remains in the range $0 \leq n \leq 1.5$ [5]. Having a circular plasma cross section further constrains the index [4] to $0<n \leq 0.65$, which js the criterion used herein.

During the plasma suart-up, an additional EF-trim coil is required to ensure the plesma remains in equilibrium, as is discussed in Section 6.3. Furthermore, the reactive power associated with maintaining the plasma in equilibrium during oscillating-field current-drive (OFCD) is substantially reduced if the applied voltage is held fixed on the superconducting EF coil and the OFCD transient is followed with the EF-trim coil, as is 
described in Section 7.4. The current requirements of the trim coil are small ( $~ 1 M A)$, permitting a normal-conducting $\mathrm{Cu}$-alloy trim coil. Furthermore, the equilibrium feld and the decay index are relatively insensitive to the position of the trim coils. Consequently, the trim-coil positions are determined primarily by the requirements for vertical access.

\subsubsection{EF-Coil Design}

The TITAN-I EF- and trim-coil desigr is shown in Figure 4.5-7 and described in Tables 4.5-IV through 4.5-VI. The steady-state flux contours shown in Figure 4.6-1 indicate that the liquid-Li flow paths in the TITAN-I blanket and shield flow parallel to the dominant poloidal field. An inverse minor-radius dependency of the poloidal-field magnitude was assumed to determine the MHD pressure drops associated with the liquid-li flow paths described in Section 10; this dependency is consistent with the steady-state magnetic-field-strength plot in Figure 4.6-1.

The TITAN-II EF. and trim-coil design is shown in Figure 4.5-9 and described in Tables 4.5-VII through 4.5-LX. The TITAN-II design is similar to the TITAN-I but the coils are closer to the plasma. Consequently, the EF-coil current for TITAN-II is smaller than for TITAN-I (18.6 compared with 19.2 MA for TITAN-I) and the inductive coupling of the TITAN-II EF coils to the plasma is better (77.1 Wb compared with $74.5 \mathrm{~Wb}$ for TITAN-I).

\subsection{SUMMARY AND CONCLUSIONS}

Two distinct coil designs were developed for the TITAN reactor to demonstrate that a range of designs are capable of efficient, high-mass-power-density operation. A comparison between the TITAN-I and TITAN-II coil designs made solely on the basis of magnetics performance is not appropriate, because other subsystems play an equally important role in determining the overall reactor performance as measured by the COE or the plant capital cost (Section 3.4). Both TITAN-I and TITAN-II coil designs are characterized by operation at low fields ( $\leq 6 \mathrm{~T}$ for copper-alloy $\mathrm{OH}$ coils and $<8 \mathrm{~T}$ for superconducting EF coils) and stresses ( $\leq 100 \mathrm{MPa}$ ). The only major development area envisioned is the demonstration of divertors in RFPs, which should begin with the next generation of RFP experiments [10]. The design constraints imposed generally were met by a wide margin in a range of design options, with the exception of the vertical-stack $\mathrm{OH}$ coil configuration. This design margin facilitates the integration of the coil sets into the 

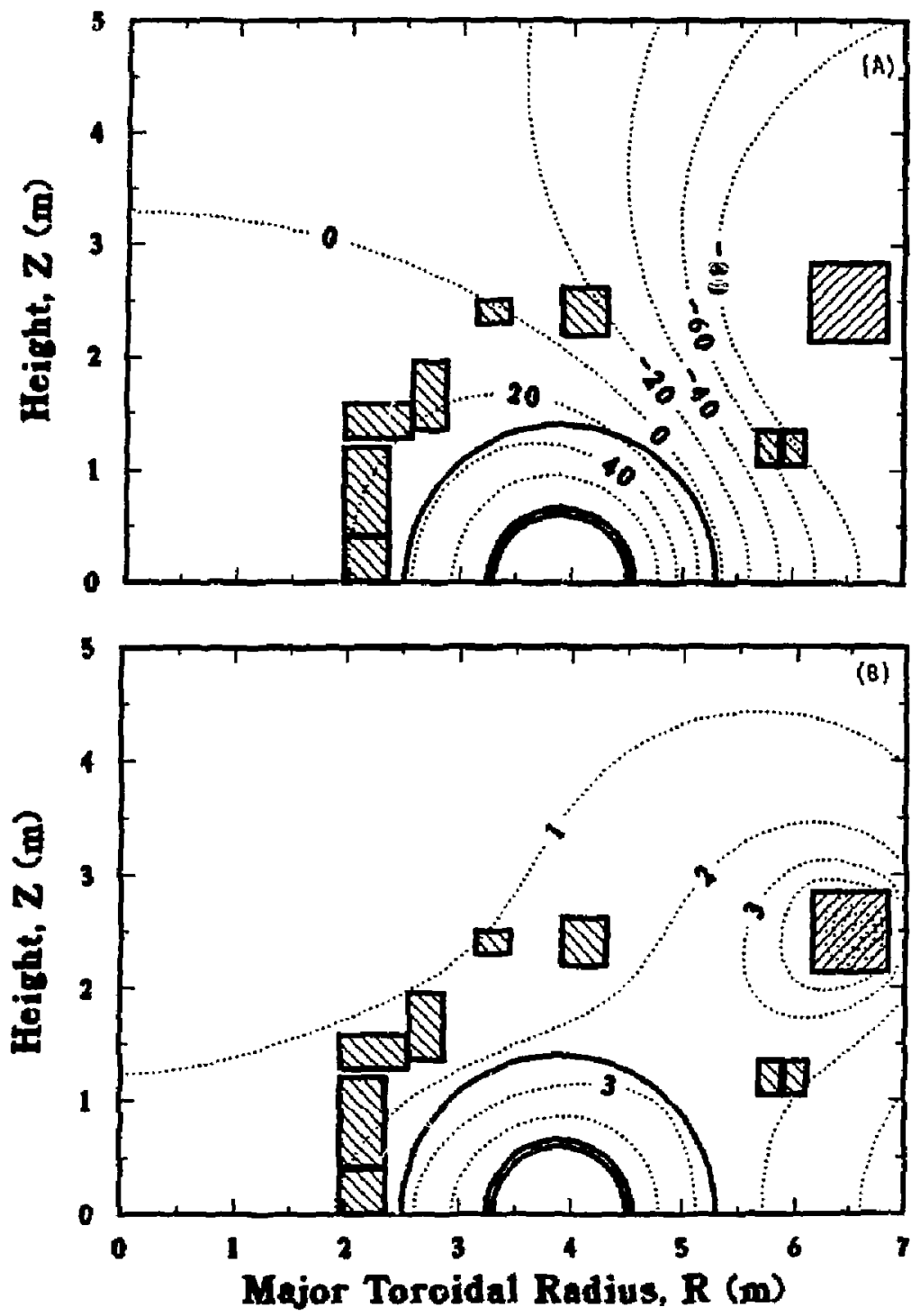

Figure 4.6-1. Contour plots of flux (A) and magnetic-field strength (B) for the EF coils and plasma at steady-state current for TITAN-I. The flux contours are labeled in weber and the magnetic-field contours are labeled in tesla. 
overall reactor torus design. Additionally, non-magnetic considerations such as safety (e.g., He-cooled $\mathrm{OH}$ coils), economics (e.g., TF IBCs that recover ohmic losses), and maintenance (e.g., non-interfering EF-coil locations) were included as major constraints of the overall design. 


\section{REFERENCES}

[1] D. Steiner, R. C. Block, and B. K. Malaviya, "The Integrated- Blanket- Coil Concept Applied to the Poloidal Field and Blanket Systems of a Tokamak Reactor," Fusion Technol. 7 (1985) 66.

[2] R. L. Hagenson, R. A. Krakowski, C. G. Bathke, R. L. Miller, M. J. Embrechts, et al., "Compact Reversed-Field Pinch Reactors (CRFPR): Prelininary Engineering Considerations," Los Alamos National Laboratory report LA-10200-MS (August 1984).

[3] V. D. Shafranov, "Plasma Equilibrium in a Magnetic Field," Revieves of Plasma Physics, Vol. 2, Consultant Bureau, New York (1966) p. 103.

[4] J. M. Greene, J. L. Johnson, and K. E. Weimer, "Tokamak Equilibrium," Phys. Fluids 14 (1971) 671.

[5] V. S. Mukhovatov and V. D. Shafranov, "Plasma Equilibrium in a Tokaunak," Nucl. Fusion 11 (1971) 605.

[6] D. Steiner, M. J. Embrechts, R. Mohanti, and W. Kelleher, "The Integrated-BlanketCoil Concept Applied to the Spherical Torus," Proc. 11th Symp. on Fusion Eng., Austin, TX (November 1985) 530.

[7] H. A. Bodin, R. A. Krakowski, and O. Ortolani, "The Reversed-Fjeld Pincli: from Experiment to Reactor," Fusion Technol. 10 (1986) 307.

[8] R. L. Spencer, "Magnetic Islands and Stochastic Field Lines in the RFP," Proc. RFP Theory Workshop, Los Alamos, NM (April 29 - May 2, 1980), Los Alamos National Laboratory report LA-8944-C (January 1982) 129.

[9] J. B. Taylor, "Relaxation of Toroidal Plasma and Generation of Reversed Magnetic Field," Phys. Lett. 33 (1974) 1139.

[10] P. Thullen and K. Schoenberg (Eds.), "ZT-H Reversed-Field Pinch Experinent Technical Proposal," Los Alamos National Laboratory report LA-UR-84-2602 (1984) 26.

[11] R. L. Miller, C. G. Bathke, R. A. Krakowski, F. M. Heck, L. Green, J. S. Karbowski, et al., "The Modular Stellarator Reactor: A Fusion Power Plant," Los Alamos National Laboratory report LA-9737-MS (July 1983). 
[12] S. J. Sackett, "EFFI - A Code for Calculating the Electronagnetic Field, Force, and Inductance in Coil Systems of Arbitrary Geometry - User's Manual," Lawrence Livermore National Laboratory report UCID-17621 (1977).

[13] C. G. Bathke and R. A. Krakowski, "A Comparison Study of Toroidal-Field and Bundle Divertors for a Compact Reversed-Field Pinch Reactor," Ftusion Technol. 8 (1985) 1616.

[14] C. G. Bathke, R. L. Miller, and R. A. Krakowski, "Magnetic Divertor Design for the Compact Reversed Field Pinch Reactor," Proc. 13th Symp. on Fusion Tech., Varese, Italy (September 1984) 1259.

[15] G. Copenhaver, R. A. Krakowski, N. M. Schunurr, R. L. Miller, C. G. Bathke, R. L. Hagenson, et al., "Compact Reversed-Field Pincl Reactors (CRFPR): FusionPower-Crie Integratiou Study," Los Alamos National Laboratory report LA-10500$\mathrm{M}$ ( A ugust 1985).

[16] K. A. Werley, C. G. Batlike, and R. A. Krakowski, "Edge Plasmas and Plasma/Wall Interactions in an Ignition-Class Reversed Field Pinch," in Proc, 12th IEEE Symp on Fusion Eng., Monterey, CA (October 1987).

[17] R. W. Moses, Jr. and J. K. Ballou, "Indurtive Shielding for Pulsed Energy Storage Magnets," IEEE Trans. an Magnetics MAG-11 (1975) 493.

[18] W. R. Smyth, Static and Dynamic Electricity, McGraw-Hill Book Company, NY (1939).

[19] F. Najmabadi, "Computing Magnetic Field, Vector Potential, and Inductance of Circular Coils with Rectangular Cross Section," University of California Los Angeles report UCLA-PPG-1255 (1989). 


\title{
5. BURNING-PLASMA SIMULATIONS
}

\author{
Farrokh Najmabadi Charles G. Bathke \\ Patrick I. H. Cooke \\ William P. Kelleher Ronald L. Miller \\ Erik L. Vold \\ Ken A. Werley
}




\section{Contents}

5.1. INTRODUCTION $\ldots \ldots \ldots \ldots \ldots \ldots \ldots \ldots \ldots \ldots$ 5-1

3.2. EQUILIBRIUM AND STABILITY $\ldots \ldots \ldots \ldots \ldots \ldots \ldots$

5.3. CORE PLASMA SIMULATIONS . . . . . . . . . . 5-16

5.3.1. Model . . . . . . . . . . . . . . . . . 5.18

5.3.2. Impurity Radiation $\ldots \ldots \ldots \ldots \ldots \ldots \ldots \ldots \ldots$

5.3.3. R=ference Design . . . . . . . . . . . . . . . . 5-24

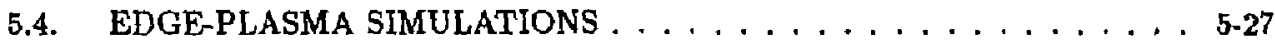

5.4.1. Edge-Plasma Model . . . . . . . . . . . . 5-28

5.4.2. Edge-Plasma Parameters . . . . . . . . . . 5-31

5.4.3. Sensitivity Study . . . . . . . . . . . . . 5-33

5.5. NEUTRAL TRANSPORT AND EROSION . . . . . . . . 5-36

5.5.1. Neutral Transport Calculations . . . . . . . . . . 5-36

5.5.2. First Wall Erosion $\ldots \ldots \ldots \ldots \ldots \ldots \ldots \ldots \ldots$. $\ldots \ldots 2$

5.5.3. Divertor Plate Erosion . . . . . . . . . . . . . . 5-42

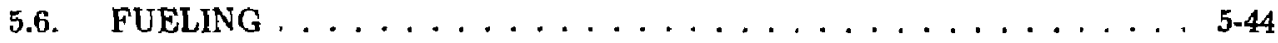

5.7. SUMMARY AND CONCLUSIONS $\ldots \ldots \ldots \ldots \ldots \ldots \ldots$.52 $\ldots \ldots \ldots$ REFERENCES . . . . . . . . . . . . . . . . 5-55 


\section{BURNING-PLASMA SIMULATIONS}

\subsection{INTRODUCTION}

Extensive studies of the TITAN burning plasma have been performed in order to analyze the plasma behavior and performance (e.g., equilibrium, stability, and transport), and to study key plasma-support systems (e.g., current drive, fueling, impurity control, and particle exhaust). As a whole, these analyses provided detailed descriptions of the burning plasma for all engineering activities and design efforts. The results also provided information to the system analysis activity for improving parametric systems models that were then used to generate new cost-optir ized design points for further conceptual design and analysis.

The TITAN burning-plasma simulations incorporate the latest understanding and models developed for reversed-field pinches (RFPs), as is summarized in Section 2; in several cases, new and improved models had to be developed for the TITAN study. Because of the relative lack of theoretical and experimental data bases for RFPs, the sensitivity of the design point to various physics assumptions has also been investigated (Section 3.4.2). A detailed description of the necessary R\&D areas for compact RFP reactors has also been produced and is reported in Section 8 .

The TITAN burning-plasma simulations include analyses of the equilibrium and stability of the TITAN plasma (Section 5.2). These equilibrium analyses were performed using a large-aspect-ratio approximation. Two-dimensional equilibrium analysis, however, was also performed to substantiate the accuracy of such an approximation for the TITAN effort. The TITAN plasma operates with a highly radiative core plasma, deliberately doped with xenon impurities, to reduce the heat flux on the divertor plates. Therefore, one-dimensional transport simulations of the TITAN core plasma were performed to study the behavior of highly radiative core plasmas and are reported in Section 5.3.

The characteristies of the edge plasma have been of major importance in driving the TITAN design and extensive simulations of the edge plasma have been undertaken during the study. The main objectives of this work are to predict the plasma conditions at the first wall and divertor target, to provide specifications for the engineering design of these components, and to estimate the requirements for particle removal. To obtain a self-consistent view of the behavior oi the plasma and neutral particles, this analysis has 
been coupled to the modeling of the core plasma (Section 5.3) and the neutral particle transport (Section 5.5). An overview of fueling requirements for the TITAN designs is reported in Section 5.6, and the summary and conclusions of the activities are given in Section 5.7 .

Although two separate fusion-power-core designs were studied for the TITAN reactor, lithium cooled for TITAN-I and an aqueous solution of a lithinm salt as the coolant and breeder for TITAN.II, both designs had the same plasma parameters. Therefore, most of the burning-plasma simulation effort wes not duplicated and, although there are certain references specifically to the TITAN-I design, only minor modifications would be required for TITAN-II.

\subsection{EQUILIBRIUM AND STABILITY}

The analysis of equilibrium and stability in RFPs usually invokes the large-aspectratio (straight cylinder) approximation. Such a model encompasses z-pinches $(q=0)$, $\theta$-pinches $(q \rightarrow \infty)$, large-aspect-ratio tokamaks $(q>1)$, and $\operatorname{RFPs}(q<1)$. The safety factor, $q(r) \equiv r B_{\phi} / R B_{\theta}$, where $B_{\phi}$ and $B_{\theta}$ are, respectively, toroidal and poloidal field, and $r$ and $R$ are measured, respectively, in directions of plasma minor $\left(r_{p}\right)$ and major $\left(R_{T}\right)$ radii. The radial pressure balance in these systems is described by:

$$
\frac{d}{d r}\left(p+\frac{B_{\phi}^{2}+B_{\theta}^{2}}{2 \mu_{0}}\right)+\frac{B_{\theta}^{2}}{\mu_{0} r}=0,
$$

where $p$ is the plasma pressure and $\mu_{0}$ is the permeability of free space. The equilibrium profiles are then subjected to stability analysis via the energy principle [1] or normal-mode technique.

For a toroidal system, equilibrium also requires the compensation of the outward force from the plasma pressure and the plasma current (poloidal-field pressure). Either a perfectly conducting wall, a vertical field produced by the external circuits, or a combination of both, is necessary for equilibrium. The required value of this vertical field is given by Shafranov [2],

$$
B_{V}=\frac{\mu_{0} I_{\phi}}{4 \pi R_{T}}\left[\ln \left(\frac{8 R_{T}}{r_{p}}\right)+\beta_{\theta}+\frac{l_{i}}{2}-1.5\right],
$$

where $l_{i}$ is the plasma internal inductance per unit length.

In principle, some information on the current and pressure profiles is required to find the equilibrium magnetic-field profiles. Because of the strong tendency for RFPs to relax, 
the field distributions obtained in modern experiments are near-minimum-energy states. The theory of relaxed states predicts the condition,

$$
\nabla \times \mathbf{B}=\mu \mathbf{B},
$$

with $\mu=\mu_{0} J_{\|} / B$ spatially uniform across the plasma, leading to Bessel-function model (BFM) field profiles. However, a constant $\mu$ (or constant $J_{\|} / B$ ) implies large parallel current dersity near the wall in a region of cold, resistive plasma. A $\mu$ profile that is nearly constant over the bulk of the plasma and decreases in the outer region to match the practical $\mu\left(r_{p}\right)=0$ condition eliminates the unphysical features of the BFM. Such $\mu$ profiles are observed in RFP experiments.

Combining Equations $5.2-1$ and $5.2-2$ results in

$$
\begin{aligned}
-\frac{d B_{\phi}}{d r} & =\mu(r) B_{\theta}(r)+\mu_{0} \frac{d p}{d r} \frac{B_{\phi}}{B^{2}}=\mu_{0} J_{\theta}(r), \\
-\frac{1}{r} \frac{d}{d r}\left(r B_{\theta}\right) & =\mu(r) B_{\phi}(r)-\mu_{0} \frac{d p}{d r} \frac{B_{\theta}}{B^{2}}=\mu_{0} J_{\phi}(r) .
\end{aligned}
$$

These equations are solved to obtain profiles of magnetic field and current density in the plasma. Values of plasma internal inductances, required vertical field, and ohmic losses in the plasma can then be computed. This information is used for analyses of steady-state burn point, magnetics, and the transient operation (Section 6).

We denote normalized profiles by "hats," i.e., $\mu(r) \equiv \mu(0) \hat{\mu}(r), p(r) \equiv p(0) \hat{p}(r)$, etc., with $\hat{\mu}(0)=\hat{p}(0)=\ldots=1$. Then, defining $x \equiv r / r_{p}$, Equations $5.2-4$ and $5.2-5$ can be written as

$$
\begin{aligned}
-\frac{d B_{\phi}}{d x} & =r_{p} \mu(0) \hat{\mu}(x) B_{\theta}(x)+\mu_{0} p(0) \frac{d \hat{p}}{d x} \frac{B_{\phi}}{B^{2}}, \\
\frac{d B_{\theta}}{d x}+\frac{B_{\theta}}{x} & =r_{p} \mu(0) \hat{\mu}(x) B_{\phi}(x)-\mu_{0} p(0) \frac{d \hat{p}}{d x} \frac{B_{\theta}}{B^{2}},
\end{aligned}
$$

with the following boundery conditions

$$
\begin{aligned}
& B_{\theta}(x=0)=0, \\
& B_{\theta}(x=1)=\frac{\mu_{0} I_{\phi}}{2 r_{p}}, \\
& \lim _{x \rightarrow 0} \frac{d B_{\theta}}{d x}=\lim _{x \rightarrow 0} \frac{B_{\theta}}{x}=\frac{1}{2} r_{p} \mu(0) B_{\phi}(0), \\
& \lim _{x \rightarrow 0} \frac{d B_{\phi}}{d x}=0 .
\end{aligned}
$$


The above equations are solved for given $\mu$ and $p$ normalized profiles, poloidal beta $\beta_{\theta} \equiv 2 \mu(0)\langle p\rangle / B_{\theta}^{2}\left(r_{p}\right)$ [or $\left.p(0)\right]$, and either the reversal parameter, $F$, or the pinch parameter, $\theta$;

$$
\begin{aligned}
F & \equiv \frac{B_{\phi}\left(r_{p}\right)}{\left\langle B_{\phi}\right)}, \\
\Theta & \equiv \frac{B_{\theta}\left(r_{p}\right)}{\left\langle B_{\phi}\right\rangle},
\end{aligned}
$$

where the average toroidal field within the conducting shell, $\left\langle B_{\phi}\right\rangle$, is defined as

$$
\left\langle B_{\phi}\right\rangle=\frac{2}{r_{p}^{2}} \int_{0}^{r} B_{\phi}(r) r d r .
$$

Because of the mixed nature of the boundary conditions (Equations 5.2-8 through 5.2-11), the equilibrium equations are solved by iterating over parameters $B_{\phi}(0)$ and $\mu(0)$ until values of $B_{\theta}\left(r_{p}\right)$ and $F$ or $\Theta$ match the desired values. Previously, the equilibrium equations were solved for magnetic field values normalized to $B_{\phi}(0)\left[\vec{B}_{\phi} \equiv B_{\phi} / B_{\phi}(0)\right.$ and $\left.\tilde{B}_{q} \equiv B_{\theta} / B_{\phi}(0)\right]$ :

$$
\begin{aligned}
\frac{d \bar{B}_{\phi}}{d x} & =-2 \Theta_{0} \hat{\mu}(x) \bar{B}_{\theta}(x)-\frac{\beta_{\phi 0}}{2} \frac{d \hat{p}}{d x} \frac{\bar{B}_{\phi}}{\bar{B}^{2}}, \\
\frac{d \bar{B}_{\theta}}{d x}+\frac{\bar{B}_{\theta}}{x} & =+2 \Theta_{0} \hat{\mu}(x) \bar{B}_{\phi}(x)-\frac{\beta_{\phi 0}}{2} \frac{d \hat{p}}{d x} \frac{\bar{B}_{\theta}}{\bar{B}^{2}},
\end{aligned}
$$

with boundary condutions, $\bar{B}_{\theta}(0)=0, d \bar{B}_{\theta} / d x(0)=\Theta_{0}, \bar{B}_{\phi}(0)=1$, and $d \bar{B}_{\phi} / d x(0)=0$. Parameters $\Theta_{0}$ and $\beta_{\phi 0}$ are defined as

$$
\begin{aligned}
& \theta_{0}=\frac{1}{2} \mu(0) r_{p}, \\
& \beta_{\phi 0}=\frac{2 \mu_{0} p(0)}{B_{\phi}^{2}(0)} .
\end{aligned}
$$

Again, the equilibrium equations are solved by iterating over parameters $\Theta_{0}$ and $\beta_{\phi 0}$ until values of $B_{\theta}\left(r_{p}\right)$ and $F$ or $\Theta$ match the desired values. Good starting estimates for the iteration process are $\beta_{\phi 0}=0.5 \beta_{\theta}$ and $\Theta_{0}=\theta$ or $\Theta_{0}=1.5-F$ (depending on whether $\Theta$ of $F$ value is specified).

An alternative has been developed for the TITAN study that is more robust numerically. Normalizing the magnetic field to the value of poloidal field at the plasma edge, 
$\tilde{B}_{\phi} \equiv B_{\phi} / B_{\theta}\left(r_{p}\right)$ and $\tilde{B}_{\theta} \equiv B_{\theta} / B_{\theta}\left(r_{p}\right)$, the equilibrium equations can be written as

$$
\begin{aligned}
\frac{d \bar{B}_{\phi}}{d x} & =-2 \Theta_{0} \hat{\mu}(x) \bar{B}_{\theta}(x)-\frac{\beta_{\theta}}{2 g_{p}} \frac{d \hat{p}}{d x} \frac{\bar{B}_{\phi}}{\bar{B}^{2}}, \\
\frac{d \bar{B}_{\theta}}{d x}+\frac{\bar{B}_{\theta}}{x} & =+2 \Theta_{0} \hat{\mu}(x) \bar{B}_{\phi}(x)-\frac{\beta_{\theta}}{2 g_{p}} \frac{d \hat{p}}{d x} \frac{\bar{B}_{\theta}}{\tilde{B}^{2}},
\end{aligned}
$$

with the boundary conditions, $\bar{B}_{\theta}(0)=0, d \bar{B}_{\theta} / d x(0)=\Theta_{0} \bar{B}_{\phi}(0)$, and $d \bar{B}_{\phi} / d x(0)=0$. Parameters $\Theta_{o}$ and $\beta_{\phi 0}$ are defined as before (Equations 5.2-17 and 5.2-18) and $g_{p}$ is the pressure profle factor,

$$
g_{p} \equiv \frac{\langle p\rangle}{p(0)}
$$

As before, these equilibrium equations are solved by iterating over parameters $\Theta_{0}$ and $\bar{B}_{\phi}(0)$ until values of $\bar{B}_{\vartheta}\left(r_{p}\right)=1$ and $F$ or $\Theta$ match the desired values. Good starting estimates for the iteration process are $\tilde{B}_{\phi}(0)=\sqrt{2 / g_{\mathrm{p}}}$ (corresponding to $\beta_{\phi 0}=0.5 \beta_{0}$ ) and $\Theta_{0}=\Theta$ or $\Theta_{0}=1.5-F$ (depending on whether $\Theta$ of $F$ value is specified). The above equations are solved by standard predictor/corrector meihod over a uniform mesh for $x$ since magnetic field profiles are then integrated to find the plasma internal inductance as is described below. The iteration on $\Theta_{0}$ and $\dot{B}_{\phi}(0)$ are performed using NewtonRaphson method. It is found that using an 80 -point mesh will yield accuracies of $<10^{-6}$ in computed values of plasma inductances and ohmic dissipation in the plasma (error in the field and current density profiles are considerably smaller). The Newton-Raphson iteration procedure is also found to converge after 3 to 5 iterations.

It is important to note that Equations 5.2-19 and 5.2-20 show that the profiles of magnetjc field and current density only depend on the profiles of $\mu$ and pressure, the poloidal beta, and $F$ or $\Theta$ values. These profiles are independent of the plasma current and for plasma minor radius. This observation is also true for plasma internal inductances and plamme-resistance composite-profile factor, $g_{O A M}$. The plasma internal inductance per unit length, $l_{i, P} \equiv L_{i, p} /\left(2 \pi R_{T}\right)$, can be written as

$$
l_{i, P}=l_{i, p}+l_{i, t}=\frac{2}{I_{\phi}^{2}} W_{p}+\frac{2}{I_{\phi}^{2}}\left(W_{t}-\frac{\Phi^{2}}{4 \pi R_{T} L_{0}}\right)
$$

where $l_{i, p}$ and $l_{i, t}$ are, respectively, poioidal and toroidal plasma internal inductances, and

$$
W_{i}=\frac{\pi}{\mu_{0}} \int_{0}^{r} B_{i}^{2} r d r, \quad(i=p \text { or } t)
$$




$$
\begin{aligned}
\Phi & =2 \pi \int_{0}^{r_{p}} B_{\phi} r d r, \\
L_{0} & =\frac{\mu_{0} r_{p}^{2}}{2 R_{T}} .
\end{aligned}
$$

Using the normalized field profiles, $\bar{B}_{\phi}$ and $\bar{B}_{\theta}$, the internal inductances per unit length can be written as

$$
\begin{aligned}
& l_{i, p}=\frac{\mu_{0}}{2 \pi} \int_{0}^{1} \tilde{B}_{\theta}^{2}(x) x d x \\
& l_{i, \ell}=\frac{\mu_{0}}{2 \pi}\left[\int_{0}^{1} \tilde{B}_{\phi}^{2}(x) x d x-2\left(\int_{0}^{1} \tilde{B}_{\phi}(x) x d x\right)^{2}\right] .
\end{aligned}
$$

The equilibrium profiles are also used to compute the plasma-resistance compositeprofile factor, $g_{\text {oHs }}$, which is defined through

$$
P_{\text {oHM }}=g_{\text {oHM }}\left\langle\eta_{\|}\right\rangle\left\langle J_{\|}\right\rangle^{2}
$$

where $P_{\text {orm }}$ is the ohmic heating in the plasma and $\left\langle\eta_{\|}\right)$is classical resistivity of the plasma which is computed based on the average electron temperature, $T_{e}$,

$$
\begin{aligned}
\left\langle\eta_{\|}\right\rangle & =0.51 \frac{4 \sqrt{2 \pi}}{3} \frac{m_{e}^{1 / 2}}{k^{3 / 2}}\left(\frac{e}{4 \pi \epsilon_{0}}\right)^{2} \frac{Z_{e f f}}{N\left(Z_{e f f}\right)} \lambda_{e} i T_{e}^{-3 / 2}, \\
N\left(Z_{e f f}\right) & \simeq 1+0.718\left(\frac{Z_{e f f}-1}{Z_{e f f}}\right)^{2},
\end{aligned}
$$

where $m_{e}$ is the electron mass, $k$ is the Boltznann constant, $\epsilon_{0}$ is the vacuum permittivity, $Z_{\text {eff }}$ is the effective plasma charge, and $\lambda_{e i}$ is the Coulomb logarithm. Substitisting in Equation 5.2-28 for $P_{\text {oH }}$ from

$$
P_{\text {OHM }}=\frac{2}{r_{\mathrm{p}}^{2}} \int_{0}^{r_{p}} \eta_{\|}(r) J_{\|}^{2}(r) r d r,
$$

and noting $J_{\|}=\mu(r) B(r) / \mu_{0}$, the plasma-resistance composite-profile factor, which includes the effect of the temperature profile as well as the field-line screw-up factor, is

$$
g_{\text {OHM }}=2 \Theta_{0}^{2} g_{T}^{3 / 2} \int_{0}^{1} \frac{\hat{\mu}^{2}(x)}{\hat{T}^{3 / 2}(x)} \tilde{B}^{2}(x) x d x
$$

Note that values of $l_{i, p}, l_{i, c}$, and $g_{O H M}$ are all independent of plasma current and size and only depend on $\mu$ and $p$ profiles, $\beta_{\theta}$, and $F$ or $\Theta$ values. 
For the analysis of the TITAN plasma, two distinct sets of $\mu$ and $p$ profiles have been used. For start-up and transient calculation, a standard set of profiles,

$$
\begin{aligned}
& \mu(r)=\mu(0)\left[1-\left(r / r_{p}\right)^{8}\right], \\
& n(r)=n(0)\left[1-\left(r / r_{p}\right)^{2.5}\right], \\
& T(r)=T(0)\left[1-\left(r / r_{p}\right)^{4}\right],
\end{aligned}
$$

are used. At steady-state full-power operation, the TITAN plasma is deliberately doped with a trace amount of $\mathrm{Xe}$ impurity to enhance core-plasma radiation and to reduce the heat load on the divertor target plates. One-dimensional transport analysis (Section 5.3) has been performed and the following plasma profiles were obtained:

$$
\begin{aligned}
& \mu(r)=2.843\left[1-0.44\left(r / r_{p}\right)^{6}-0.56\left(r / r_{p}\right)^{6}\right], \\
& T_{e}(r)= \begin{cases}14.40-46.94\left(r / r_{p}\right)^{2.8504} & \left(0 .<r / r_{p}<0.25\right) \\
16.07-10.29\left(r / r_{p}\right) & \left(0.25<r / r_{p}<0.833\right) \\
8.111-7.886\left(r / r_{p}\right)^{14.026} & \left(0.833<r / r_{p}<1\right)\end{cases} \\
& n(r)= \\
&
\end{aligned}
$$

These profiles have been used in the burning-plasma simulations.

Figures 5.2-1 through 5.2-4 show, respectively, the normalized profiles of plasma parameters $(n, T, P$, and $\mu$ ), magnetic field, current density, and safety factor, $q$, for both sets of profiles. Table 5.2-I compares the values of plasma profile factors and plasma internal inductances for both profiles. These equilibrium profiles and parameters are computed for $\beta_{\theta}=0.2$ and $F=-0.1$. Values of magnetic-field strength and current density are also given for $r_{p}=0.6 \mathrm{~m}$ and $I_{\phi}=17.8 \mathrm{MA}$.

The above cylindrical equilibrium calculations have been used extensively for simulation of the TITAN plasma. The accuracy of this model has been checked against equilibrium calculations performed by the computer code NEQ [3]. Figure 5.2-5 illustrates the flux surfaces of the TITAN reactors. The equilibrium parameters computed by NEQ are in good agreement with those reported in Table 5.2-1.

The equilibrium presoure profile described by Equation 5.2-1 must be subjected to stability analysis using the energy principle [1] or normal-mode anslysis. Necessary conditions for stability against ideal-MHD current-driven modes have been derived by Robinson [4] on the basis of the energy principle [1]. The necessary condition can be expressed 

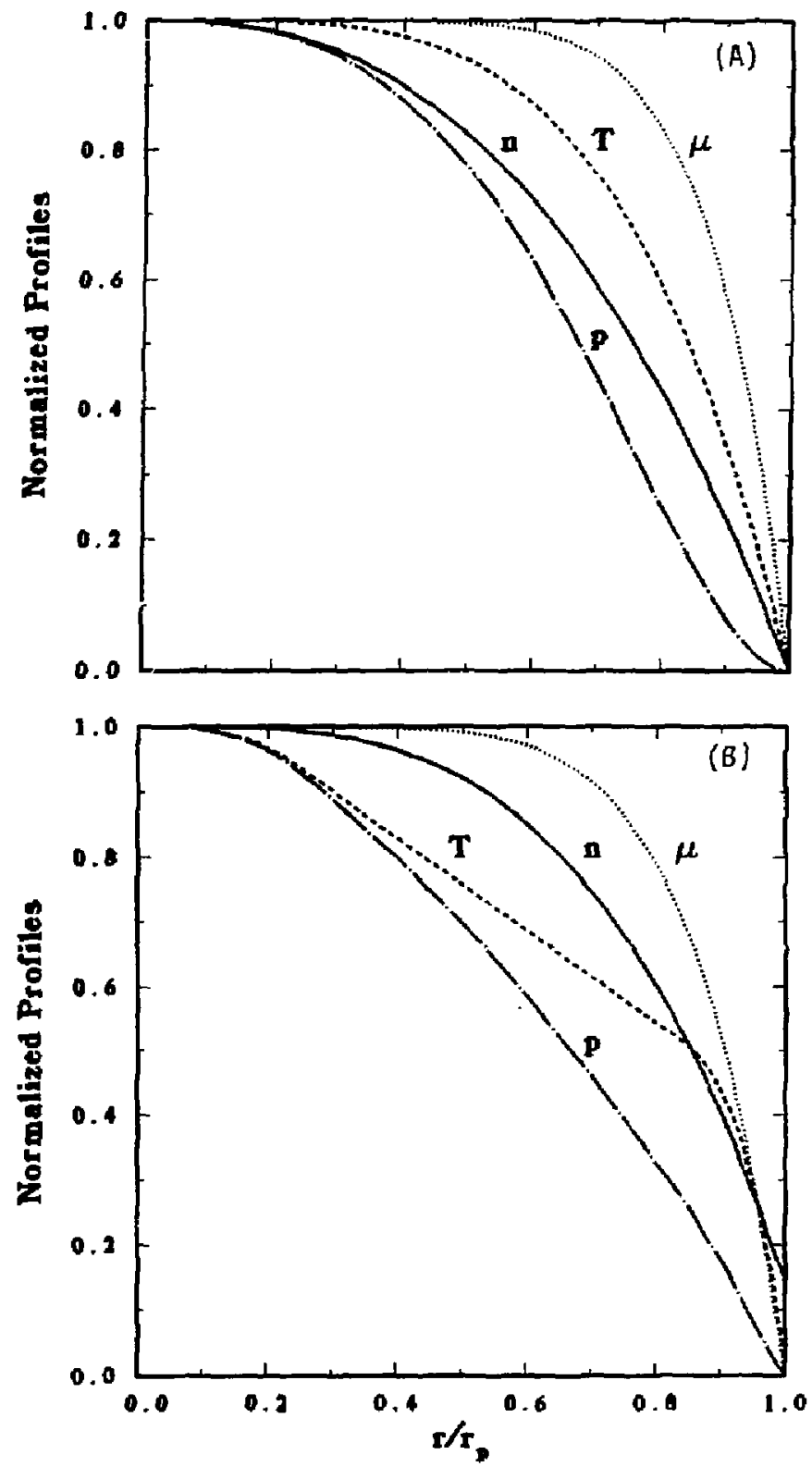

Figure 5.2-1. Plasme density $(n)$, temperature $(T)$, pressure (p), and $\mu$ profiles for power $(A)$ and radiative profiles $(B)$. 

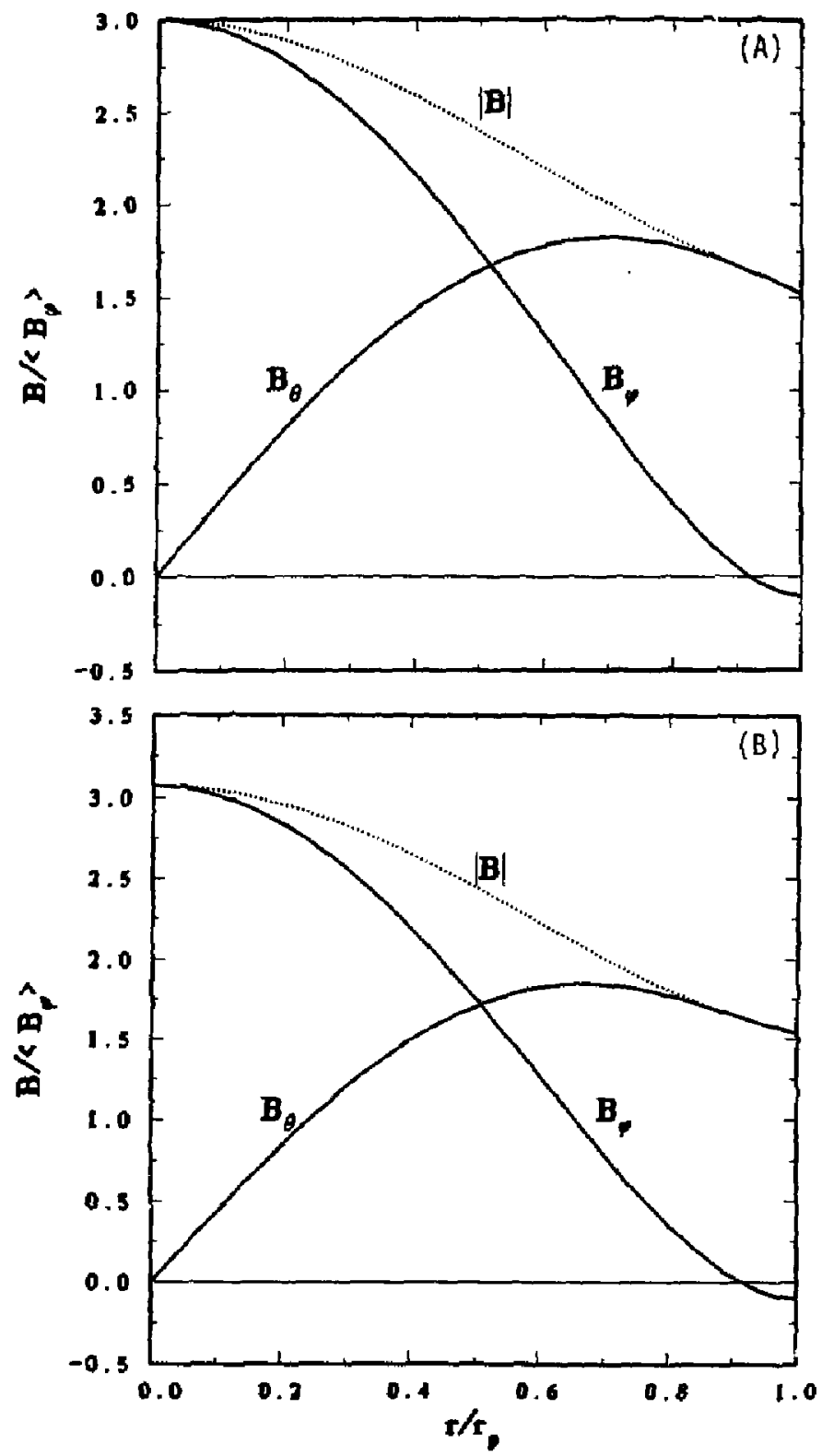

Figure 5.2-2. Equilibrium profiles of magnetic field for power (A) and radiative profiles (B). 

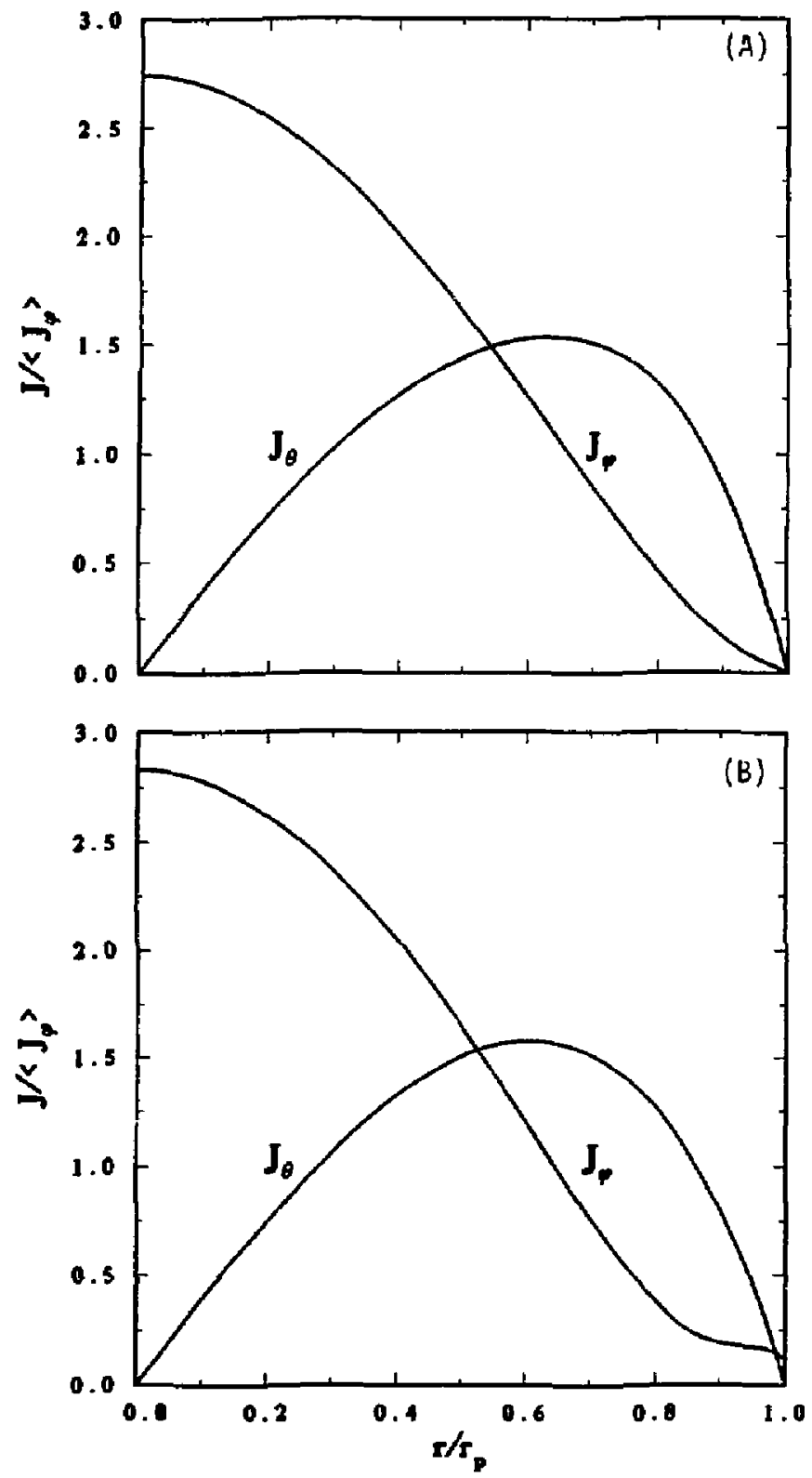

Figure 5.2-3. Equilibrium profiles of plasma current density for power $(A)$ and radiative profiles (B). 

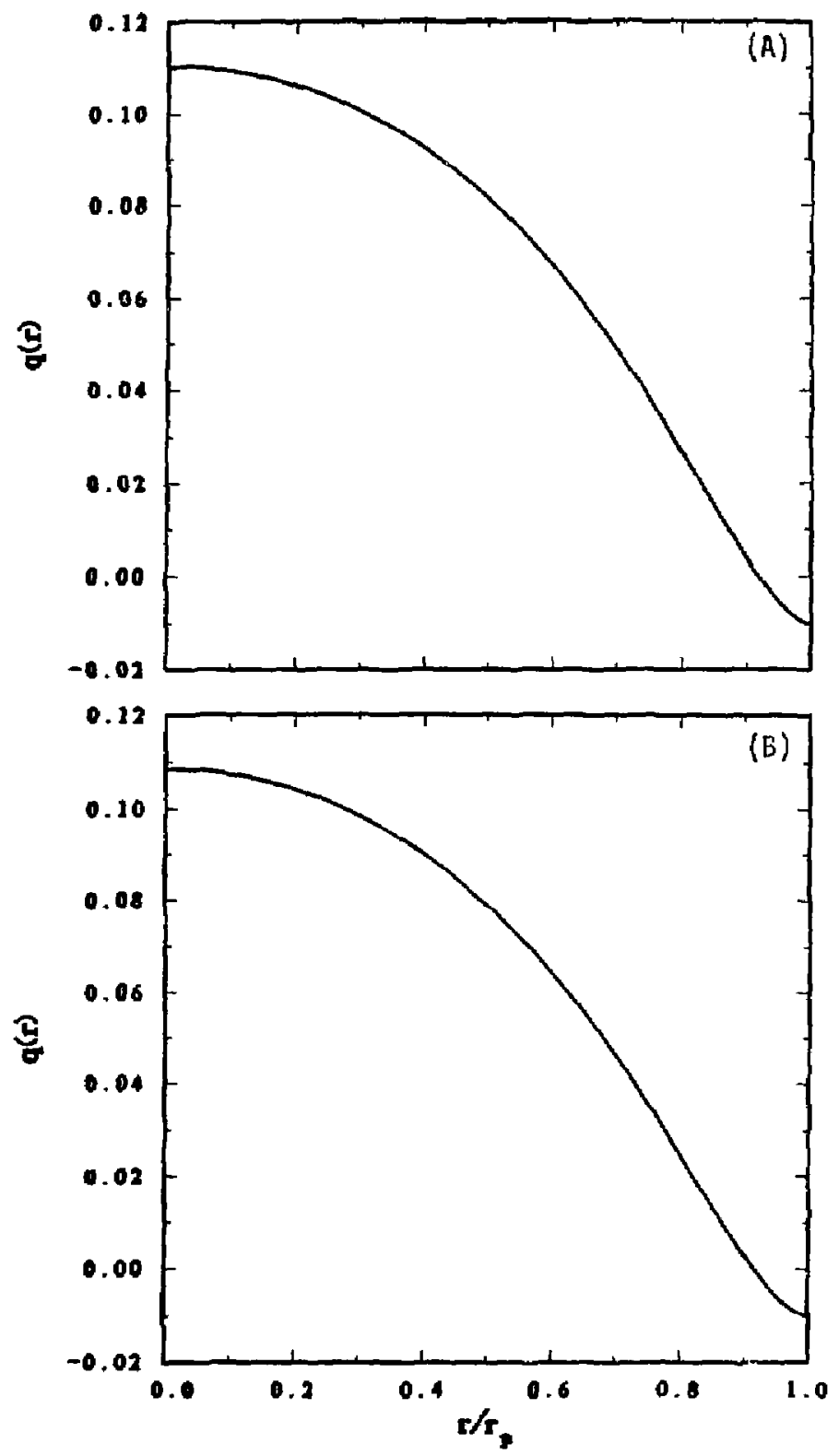

Figure 5.2-4. Equilibrium profiles of the safety factor for power (A) and radiative profilea (B). 
Table 5.2-I.

EQUILIBRIUM PAFAMETERS OF TITAN PLASMA

\begin{tabular}{|c|c|c|}
\hline Parameter & Power Profiles(a) & Radiative Profiles ${ }^{(6)}$ \\
\hline Poloidal beta, $\beta_{\theta}$ & a.2 & 0.2 \\
\hline Reversal parameter, $F$ & -0.1 & -0.1 \\
\hline Pinch parameter, $\theta$ & 1.522 & 1.538 \\
\hline \multicolumn{3}{|l|}{ Safety factor, $q$} \\
\hline On axis & 0.110 & 0.109 \\
\hline At planma edire & -0.011 & -0.010 \\
\hline \multicolumn{3}{|l|}{ Field-line pitch, $P$} \\
\hline On axis & 0.430 & 0.423 \\
\hline At plasion edge & -0.039 & -0.039 \\
\hline \multicolumn{3}{|c|}{ Plsisma internal inductance per unit length $(\mathrm{H} / \mathrm{m})$} \\
\hline Poloidal, $l_{i, p}$ & $1.136 \times 10^{-7}$ & $1.140 \times 10^{-7}$ \\
\hline Toroidal, $l_{i, t}$ & $3.724 \times 10^{-8}$ & $3.824 \times 10^{-8}$ \\
\hline Total, $l_{i, P}$ & $1.508 \times 10^{-7}$ & $1.522 \times 10^{-7}$ \\
\hline Plasma-resistance profile factor, $g_{O H M}$ & 3.419 & 2.918 \\
\hline Poloidal field at plasma edge, $B_{\theta}\left(r_{p}\right)(\mathrm{T})^{(e)}$ & 5.93 & 5.93 \\
\hline \multicolumn{3}{|l|}{ Toroidal field(e) } \\
\hline On axis, $B_{\phi}(0)(\mathrm{T})$ & 11.69 & 11.85 \\
\hline At plasma edge, $B_{\phi}\left(r_{p}\right)(T)$ & -0.390 & -0.386 \\
\hline Average, $\left(B_{\phi}\right\rangle(T)$ & 3.90 & 3.86 \\
\hline \multicolumn{3}{|l|}{ Toroidal current density ${ }^{(e)}$} \\
\hline On axis, $J_{\phi}(0)\left(\mathrm{MA} / \mathrm{m}^{2}\right)$ & 43.2 & 44.6 \\
\hline At plasma edge, $J_{\phi}\left(r_{p}\right)\left(\mathrm{MA} / \mathrm{m}^{2}\right)$ & 0. & 1.90 \\
\hline Average, $\left(J_{\phi}\right\rangle\left(\mathrm{MA} / \mathrm{m}^{2}\right)$ & 15.7 & 15.7 \\
\hline
\end{tabular}

(a) Profiles of plasma $n, T$, and $\mu$ are given in Equations 5.2-33 through 5.2-35.

(b) Profiles of plasma $\pi, T$, and $\mu$ are given in Equations 5.2-36 through 5.2-38.

(c) Values for $r_{p}=0.6 \mathrm{~m}$ and $I_{\phi}=17.8 \mathrm{MA}$. 


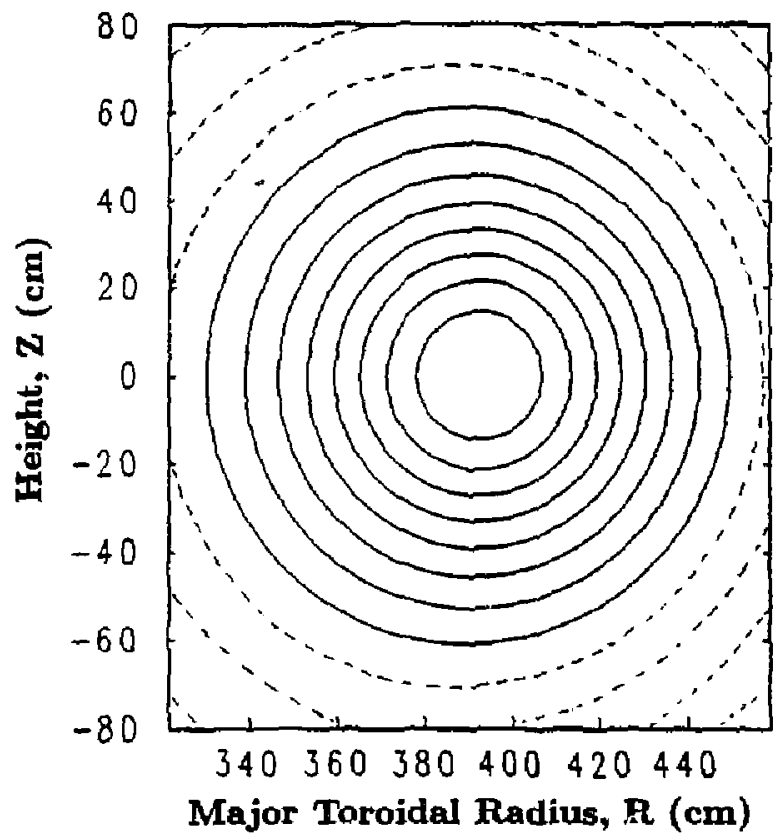

Figure 5.2-5. Equilibrium flux surface for the TITAN plasma computed by NEQ \{3\}.

as $\left|P\left(r_{w}\right)\right|<3 P(0)$, where $P \equiv q R$ is the field-line pitch and $r_{w}$ is the location of the conducting wall. This condition can be approximately expressed as follows:

$$
\begin{aligned}
\left(\frac{r_{w}}{r_{p}}\right)^{2} & <3\left|\frac{B_{\phi}(0)}{B_{\phi}\left(r_{w}\right)}\right|, \\
\Phi & >0,
\end{aligned}
$$

where $\Phi$ is the total toroidal flux inside the conducting wall. These conditions do not include a vacuum boundary and require that both the amplitude and the region of the field reversal not be large.

In summary, the conducting wall should be close to the plasma to stabilize currentdriven modes. Furthermore, stability against current-driven modes also excludes a pitch minimum in the plasma. These conditions are usually well satisfied for experimental profiles and are also monitored and satisfied for the profiles calculated for the TITAN designs. The necessary conditions given by Equations 5.2-39 and 5.2-40 are in practice close to being sufficient. Based on ideal-MHD theory, RFP profiles are possible with plasme beta values as high as 0.3 . 
The ideal-MHD theory assumes a zero plasma resistivity. This assumption constrains magnetic field lines to be "frozen" in the plasma, thereby limiting the class of potentially unstable modes. Resistive-MHD stability analysis has to be performed to provide a more realistic picture of the plasma behavior [4]. In general, the criteria for resistive stability are more stringent, and a closer fitting conducting wall and a lower value of $\beta$ generally result. A detailed analysis of current-driven resistive tearing modes has been made [5] and stable RFP configurations have been found with $\beta \simeq 0.2$. These configurations, however, have been found to be unstable to the so-called resistive g-modes (the " $\mathrm{g}$ " is used to emphasize the "gravity" analogy). Moreover, analyses show that resistive g-modes can become unstable for pressure gradients substantially smaller than those needed to drive ideal-MHD instabilities. These resistive g-modes are localized and may uliimately affect the confinement time. In fact, certain theoretical estimates of the confinement time have been proposed that are based on transport along stochastic field lines created by resistive g-mode turbulence [6] and are discussed in Section 2.

The current-driven MHD instabilities have more recently been analyzed for an RFP in contact with a perfectly conducting wall [7], and even more recently for conditions where a vacuum annulus exists between the RFP and the conducting wall [8]. Figure 5.2-6 gives an example of a stability diagram for the fastest growing $m=1$ mode as a function of the $\mu$-profile shape factors, $\mu(0)$ and $\alpha$, as given in Equation 5.2-4. These analytic results show that a completely stable region exists for ideal and resistive current-driven modes in an RFP over a range of $\Theta \simeq \Theta_{0}$ and degrees of current-density peaking, and for a range of free-boundary conditions, $\delta_{v} \simeq\left(r_{w}-r_{p}\right) / r_{p}$. Generally, the external $m=1$ modes do not appear to be significant. These external modes occur for deep-reversal conditions that rarely arise for most experiments and are not of interest for reactors because of cost and technology demands (Section 8). A lower limit on $q(0) A=\Theta_{0} \geq 2 / 3$ is identified which weakly depends on the profile exponent $\alpha$. This lower limit is associated with the internal $m=1$ resistive mode, and imposes an upper limit on the on-axis current density $[7,8]$ of $J_{\phi}(0) \leq 3 B_{\phi}(0) /\left(\mu_{o} r_{p}\right)$. As noted in Reference [7], resistive diffusion tends to peak the on-axis current, lowering $q(0)$ to below this critical limit and exciting internal current-driven instabilities, redistributing (i.e., flattening) the current-density profile, and recovering the initial configuration that is stable to tearing modes. Hence, the field profiles in RFPs are a result of periodic relaxations that oppose processes related to resistive diffusion; such oscillations around near fields have been observed in a number of large- $\Theta$ RFP discharges $[9,10]$.

Given the above considerations, detailed stability analyses of TITAN profiles were not performed. Rather, a poloidal beta of $\beta_{\theta}=0.23$ (corresponding to fuel ion beta of 


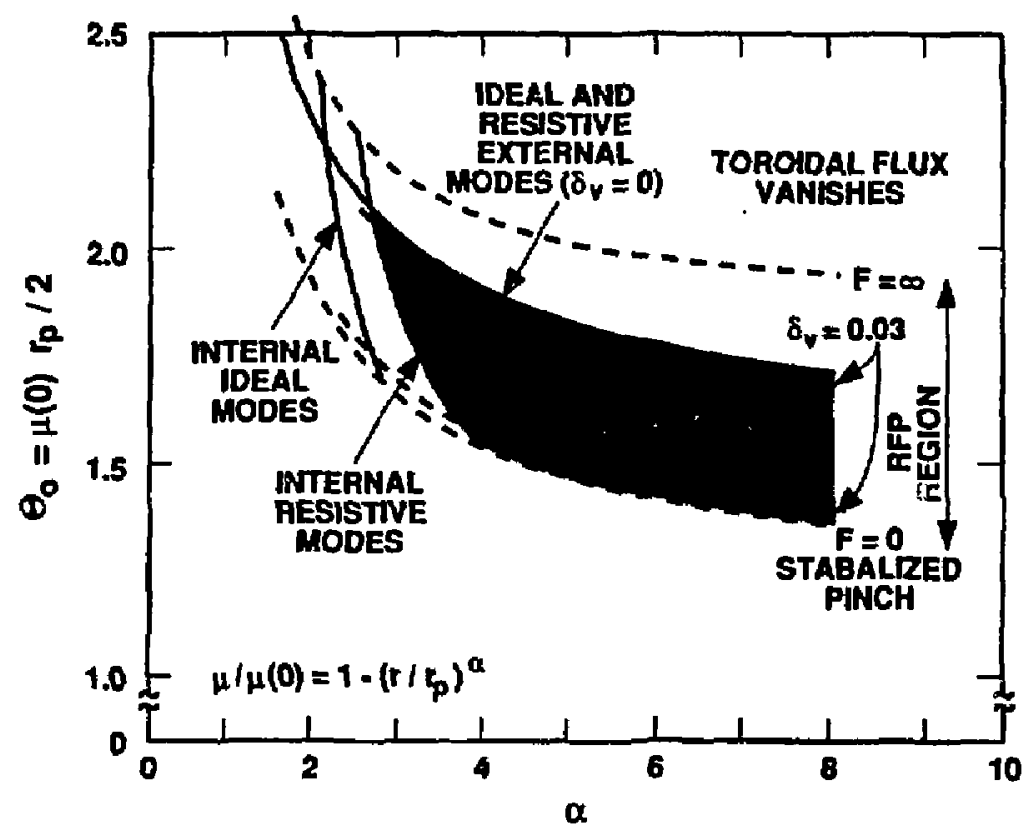

Figure 5.2-6. Stability diagram for $m=1$ internal (inside reversal layer) and external resistive current-driven modes for an MFP with a vacuum annulus equal to $\delta_{v}=0.0$ and 0.3 of the plasma radius $[7,8]$.

0.2) was assumed. The sensitivity of the reference design to achievable stable value of poloidal beta was also studied and reported in Section 3.4.2.

Finally, the existence and role of a close-fitting conducting shell that surrounds the RFP strongly impacts all physics and eagineering aspects of the design. The data base for RFP operation with resistive shells is summarized in Section 2.3.9. However, the need for and characteristics of a conducting shell with electrical breaks and its impact on RFP formation and start-up, confinement, current drive, and impurity control represent an important but inadequately mapped issue for the RFP. Because of the lack of data base, the TITAN study has circumnavigated this issue numerous times by assuming that the first wall and blanket would act as the conducting shell. 


\subsection{CORE-PLASMA SIMULATIONS}

A 1-D RFP plasma burn code, RFPBURN $[11,12]$, has been used to study transport and other 1-D effects in the bulk plasma of the TITAN reactors. While a 0-D plasma and circuits code has been used as the main tool to examine TITAN reactor transients (Section 6), RFPBURN supplements the 0-D model by examining 1-D aspects of local transport assumptions, impurity radiation with beta limits, pellet refueling, dynamo, and current drive. A brief summary of RFPBURN models is given in Section 5.3.1.

Experimental observation of the scaling of RFP plasma pressure with the toroidal current, $n T_{\varepsilon}(0) \propto I_{\phi}^{2}$, is suggestive of operation near a beta limit. Under this condition, the intrinsic transport would adjust by MHD activity, radiation, or other mechanism to lose energy at a rate sufficient to maintain $\beta_{\theta}$ constant. To test this hypothesis, experiments were performed on ZT-40M by adding trace quantities of krypton impurity [13] to enhance the radiative losses. Krypton was chosen to maximize the ratio of radiated power to the ohmic heating power. It was found that as the impurity was injected, the rate of radiation loss, $P_{R A D}$, was increased but, simultaneously, the ohmic power, $P_{\text {OHM }}$, only slightly increased. Most importantly, $\beta_{\theta}$ remained constant. This observation suggests that as radiation losses are increased, the non-radiative or "intrinsic" transport losses, $P_{N R}$, decrease to maintain the beta constant.

A simplified zero-dimensional power-balance model for a steady-state plasma gives $P_{O H M}=P_{R A D}+P_{N R}$. Using the definitions of the global energy-confinemenc time, $\tau_{n}$, and a non-radiative (intrinsic) energy-confinement time, $\tau_{E}^{N R}$, the following expression results:

$$
\tau_{E}^{N A}=\tau_{s}\left(1-\frac{P_{R A D}}{P_{R A D}+P_{N R}}\right)^{-1}
$$

For the assumed constant-beta scaling and self-similar profiles of density and electron temperature before and after krypton injection, the values of the total energy loss $\left(P_{R A D}+P_{N R}\right)$ and $\tau_{a}$ remain unchanged. Equation 5.3-1 indicates that as the radiative losses increase, the non-radiative losses decrease (or $\tau_{E}^{N R}$ increases) to maintain the energy content of the plasma and hold $\beta_{\varepsilon}$ constant. The data from the krypton impurity experiments are plotted in Figure 5.3-1, and close agreement with predictions of Equation 5.3-1 is indicated.

This characteristic of the RFP is in marked contrast with other confinement schemes such as the tokamak, where increasing the impurity content would increase the total 


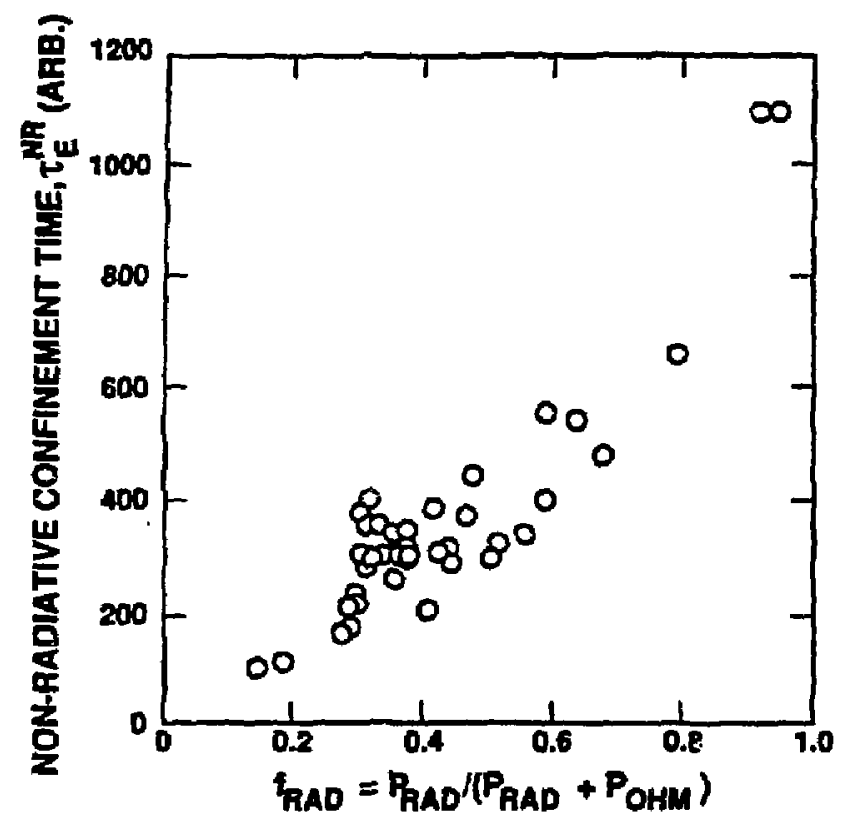

Figure 5.3-1. Scaling of the non-radiative energy-confinement time with the fractional radiative-power loss in the ZT-40M experiments [13].

energy-lass rate and degrade the plasma pressure. Enhanced radiation from a (high) beta-limited plasma is important becuuse it permits first wall designs to receive a higher average (but more uniform) heat flux and to minimize the divertor (or limiter) power losds, thereby optimizing the overall design for the maximum power density while maintaining realistic engineering constraints on all systems.

The RFPBURN code has been used to examine the possibility of seeding the TITAN plasma with impurities in order to enhance the ratio of radiative-to-transport losses and to reduce peak heat loads on the divertor target plates. This analysis has led to the TITAN reference design which is seeded with Xe impurities (Section 5.3.2). The plasma profiles obtained from this 1-D analysis are then used in the $0-D$ analysis. Additionally, RFPBURN results are coupled to the edge-plasma models to provide selif-consistent core/edge-plasma boundary conditions (Section 5.4). 


\subsubsection{Model}

Table 5.3-I summarizes the key features of the RFPBURN code. The 1-D RFPBURN model includes a dynamo electromotive-force term which permits a steady-state normalized profile where $\mu \equiv \mu_{a} \mathbf{j} \cdot \mathbf{B} / B^{2}$. The shape of the desired $\mu$ profile is determined prior to the application of the 1-D transport model by using the equilibrium solver routine from the Hewett-Freiberg linear resistive-stability code [14] to match the $F$ and $\Theta$ values of the 0-D design point. The equilibrium inputs are the $\mu$ profile and a normalized pressure profile of the following form:

$$
\begin{aligned}
& \mu(r)=\mu(0)\left[1+a_{2}\left(r / r_{p}\right)^{2}+a_{4}\left(r / r_{p}\right)^{4}+a_{6}\left(r / r_{p}\right)^{8}+a_{g}\left(r / r_{p}\right)^{8}\right], \\
& p(r)=\frac{0.05 B^{2}(0)}{\mu_{0}}\left[1-0.9973\left(r / r_{p}\right)^{2}\right] .
\end{aligned}
$$

The $\mu$ profile is constrained to be a positive monotonic function decreasing to zero at the edge. A parabolic normalized pressure profile was assumed with the on axis and edge values obtained from transport calculations that self-consistently coupled the core and edge plasma.

In order to examine some of the properties of a beta-limited and radiation-dominated reactor-grado plasma, a radiation model with coronal equilibrium and a constant impurity density is assumed. In order to incorporate a soft $\beta$ limit in a 1-D formulation, the transport coefficients were assumed with the following form:

$$
\begin{aligned}
K_{e} & =K_{\perp e}^{\mathrm{cl}} f\left(\beta_{\theta}\right), \\
K_{i} & =K_{\perp i}^{\mathrm{cl}}+K_{e} / 4, \\
D & =D^{c l}\left[1+0.1 f\left(\beta_{\theta}\right)\right],
\end{aligned}
$$

where $K_{\perp}^{c l}$ and $D^{a l}$ are classical values of the cross-field thermal conductivity and particle diffusivity, and the following function, $f\left(\beta_{\theta}\right)$, is used to model the observed soft $\beta$ limit:

$$
\begin{aligned}
f\left(\beta_{\theta}\right) & =\left\{\begin{array}{ll}
1 & \beta_{\theta} \leq \beta_{\theta c} \\
g(r) \exp \left[\left(\beta_{\theta} / \beta_{\theta c}\right)^{15}-1\right] & \beta_{\theta}>\beta_{\theta c}
\end{array},\right. \\
g(r) & = \begin{cases}1 & r>0.84 r_{p} \text { or } \beta(0) \leq 0.11 \\
1+10\left[1-\left(r / 0.84 r_{p}\right)^{2}\right] & \beta(0)>0.11\end{cases}
\end{aligned}
$$

Here, the exponential factor represents the poloidal beta limit. The parabolic factor, $g(r)$, represents an enhanced internal transport that excludes highly peaked temperature profiles caused by highly localized radiation losses. This enhanced internal transport is 
consistent with the flat denoity and temperature profiles observed on 2T-40M [15], which could result either from internal large-scale $m=1$ current-driven modes, or from "local" $\beta$-limit effects. The model uses boundary conditions at $r_{p}$ that include the toroidal magnetic field and the toroidal loop voltage as well as extrapolation endpoint conditions for the density and temperatures.

Table 5.3-1.

\section{KEY FEATURES OF THE 1-D RFP TRANSPORT CODE, RFPBURN}

- The code follows the time-dependent, cylindrical plasma evolution of ion and alphaparticle densities, ion and electron temperatures, and poloidal and axial (toroidal) magnetic fields.

- Physics constraints include Ohm's law, radial pressure balance, and local quasineutrality.

- Physical terms include conduction, diffusion, convection, resiative disipation, fusion reactions, Bremsatrahlung radiation, impurity radiations besed on a coronal equilibrium model, a volumetric ion source and/or a pellet injection model, and finally, a simple dynamo model that conserves helicity and allows the RFP configuration to be maintained at steady state.

- Boundary conditions consist of six regularity conditions at the origin $(r=0)$ and six wall conditions that include the toroidal magnetic field, the toroidal voltage, and either extrapolation endpoint or pedestal conditions on the densities and temperatures.

- Giobal plasma particle and energy balance is followed and used to ensure particle and energy conservation. 


\subsubsection{Impurity Radiation}

The interim TITAN design parameters, as described in Table 5.3-II, were used in this analysis. The computational results are summarized in Table 5.3-III. The required impurity fraction and the resultant $Z_{\text {eff }}$ as functions of the core radiation fraction are shown in Figure 5.3-2.

A minimum core-plasma radiation fraction, $f_{R A D}^{c}=0.12$, is computed for an $\sim 4 \%$ alphe-particle esh with no other impurities. While low- $Z$ impurities such as carbon can radiate the necessary power, a high impurity level $\left(f_{j}=0.04\right)$ is required. For a given plasma beta and plasma current, a large impurity level changes the shape of the plasma profiles and thus the fusion (alpha-particle) power, $P_{a}$, as shown in Table 5.3-III. Small increases in $I_{\phi}$, however, result in iarge increases in $P_{\alpha}$ and, therefore, design powers can be recovered easily with moderate (5\%) increases in $I_{\phi}$. The fusion power can also be increased by trading off density with temperature. Such optimizations can best be done at the 0-D level of analysis. The high value of $Z_{e f f}=2$ for the carbon impurity would double the current-drive power requirements.

High- $Z$ impurities, such as xenon, require a much smaller impurity concentration and give lower values of $Z_{\text {eff }}\left[e . g ., Z_{\text {eff }}(0)=1.3\right.$ for Xe and the $Z_{\text {eff }}$ decreases with radius]. High- $Z$ impurities, therefore, are favored for enhancing the plasma radiation fraction. In addition to determining that high- $Z$ impurities can radiate the same power as low- $Z$ impurities with a lower $Z_{\text {eff }}$ (and hence, $P_{O H M}$ ), an additional study evaluated the optimal plasma temperature for minimizing the ohmic dissipation, POHM. Results are presented in Table 5.3-IV for a plasma with a fixed $\beta_{\theta}, I_{\phi}$, and $f_{R A D}^{c}$. Plasmas with large radiation fractions and a significant xenon-impurity density $\left(10^{17}-10^{18} \mathrm{~m}^{-3}\right)$, actually have higher dissipation at higher plasma temperatures because $Z_{\text {efs }}$ increases more quickly than $T_{\varepsilon}^{-3 / 2}$ decreases. Minimizing $P_{O H M}$ is crucial for reducing current drive requirements (Section 7 ). The optimum operating temperature for a highly radiative plasma, therefore, is the minimum required to produce the required fusion powim $18 \mathrm{~s}$ $10 \mathrm{keV}$ ) while minimizing OFCD power requirements and cost.

The profiles of plasma parameters for these 1-D simulations are uncertain since local transport is not known. But the highly localized radiative losses in the outer regions of the plasma are not expected to change the plasma profiles dramatically if a local beta limit or some other source of rapid transport in the plasma core exists. It is noted that if the transport in the plasma core is too low, then high radiation losses at the plasma edge could result in a temperature collapse of the entire plasma column as the cold, radiating edge propagates inward. 
Table 5.3-II.

REACTOR CONDITIONS USED FOR THE IMPURITY SEEDING ANALYSIS

$\begin{array}{lc}\text { Plasma minor radius, } r_{p}(\mathrm{~m}) & 0.71 \\ \text { Plasma major toroidal radius, } R_{T}(\mathrm{~m}) & 3.93 \\ \text { Toroidal current, } I_{\phi}(\mathrm{MA}) & 18.0 \\ \text { Aversge ion density, } n_{\mathrm{i}}\left(\mathrm{m}^{-3}\right) & 8.67 \times 10^{20} \\ \text { Critical poloidal beta, } \beta_{\theta c} & 0.19\end{array}$

Table 5.3-III.

STEADY-STATE RADIATION RESULTS OF THE 1-D MODEL

\begin{tabular}{lcccc}
\hline & None & $\mathrm{C}$ & $\mathrm{Xe}$ & $\mathrm{U}$ \\
\hline$Z_{j}$ & & & & \\
$f_{j}$ & - & 12. & 54. & 92. \\
$Z_{e j f}$ & 0.0 & $4 \times 10^{-2}$ & $1 \times 10^{-4}$ & $3 \times 10^{-5}$ \\
$\tau_{E}^{N R}(\mathrm{~s})$ & 1.04 & 2.0 & 1.3 & 1.15 \\
$\tau_{E}(\mathrm{~s})$ & 0.64 & 7.9 & 1.2 & 1.8 \\
$P_{a}(\mathrm{MW})$ & 0.25 & 0.53 & 0.35 & 0.35 \\
$f_{\mathrm{AAD}}$ & 336. & 233. & 306. & 321. \\
& 0.12 & 0.76 & 0.71 & 0.81 \\
\hline
\end{tabular}



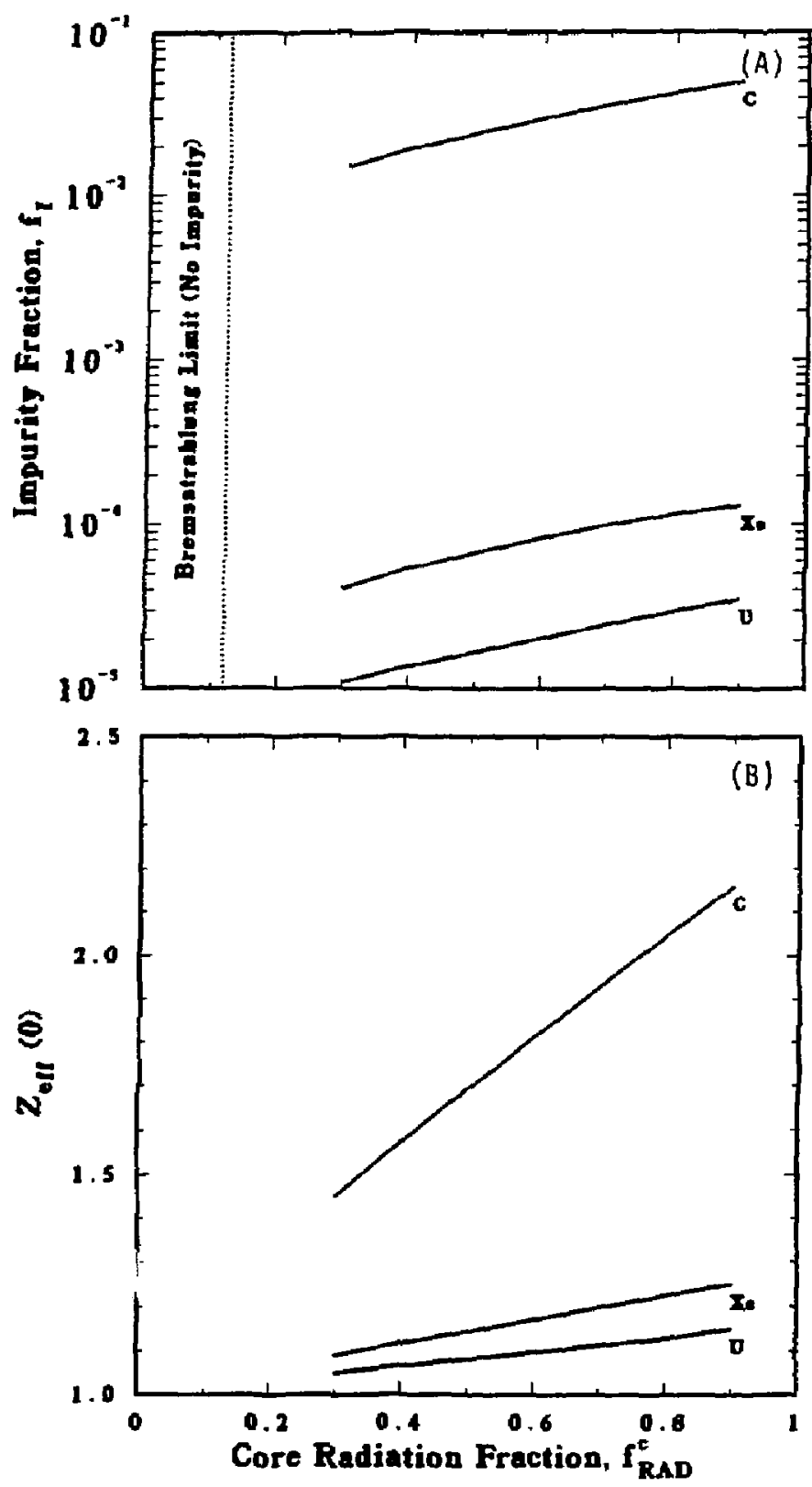

Figure 5.3-2. The required impurity fraction (A) and the resultant $Z_{\mathrm{e} / f}(\mathrm{~B})$ as functions of the core radiation fraction, $f_{\text {RAD }}^{c}$, for different impurities. 
Table 5.3-IV.

IMPACT OF Xe IMPURITIES ON OHMIC DISSIPATION ${ }^{(a)}$

\begin{tabular}{lcccccc}
\hline$n_{X e}\left(\mathrm{~m}^{3}\right)$ & $f_{R M O}^{c}$ & $T_{i}(\mathrm{keV})$ & $T_{e}(\mathrm{keV})$ & $Z_{\text {eff }}$ & $g_{\text {OHM }}$ & $P_{\text {OKM }}(\mathrm{MW})$ \\
\hline $2.9 \times 10^{17}$ & 0.77 & 10.6 & 9.6 & 1.67 & 4.88 & 46.6 \\
0. & 0.11 & 10.0 & 10.0 & 1.0 & - & 23.4 \\
$5.6 \times 10^{17}$ & 0.73 & 23.9 & 15.5 & 4.28 & 12.38 & 62.6 \\
0. & 0.11 & 20.0 & 20.0 & 1.0 & - & 8.0 \\
\hline
\end{tabular}

(a) $\beta_{*}=0.203, I_{\phi}=17.9 \mathrm{MA}$.

Listed below are the major unknowns associated with assuming $\beta$ limits for RFP reactors. Even though a few of these issues may be partially addressed by $1-D$ analysis, the majority of the unresolved issues will require experimental demonstration at reactor conditions and, therefore, will remain unknown for some time.

- $\beta$-limited confinement scaling in the reactor plasma regime,

- Limit on the local $\beta$,

- The level and mechanism of the intrinsic transport,

- The collapse of the temperature profile due to impurity injection,

- The interaction of competing profile effects (e.g., refueling: alpha-particle transport, $T_{i}: T_{\varepsilon}: n$ split),

- The impact of the impurities on the edge plasma and the wall (e.g., sputtering, wall buildup, vacuum, and tritium systems), and

- The effects of the impurities on the start-up transient. 
Based on both experiment (Section 2.3) and theory (Section 2.2), it appears feasible that RFP reactors can exhibit a soft $\beta$ limit. Such a $\beta$ limit was assumed in choosing ZT-H experimental parameters [16]. If such $\beta$ limits exist, it may be possible to adjust $f_{\text {RAD }}$ to any level between 0.12 and 0.95 , with only a minor increase $(10 \%$ to $30 \%)$ in plasma resistance by injecting high- $Z$ impurities into the plasma core. Only small variations in the impurity fraction are required to vary $f_{R A D}^{c}$ significantly. In practice, the maximum operating value for $f_{R A D}^{c}$ will be determined by the level of intrinsic transport. If the intrinsic transport mechanisms are classical, then the $f_{\text {RAD }}^{c}$ upper limit could be higher than 0.99. Finally, it is noted that the impurity fraction of Xe required for $f_{R A D}^{c}=1$ is two orders of magnitude smaller than that required for $f_{R A D}^{c}=1$ in the divertor chamber.

\subsubsection{Reference Design}

Based on the 1-D analysis, the TITAN reference design is sected with trace amount of Xe impurities $\left(n_{X e}=2.9 \times 10^{17} \mathrm{~m}^{-3}\right)$, resulting in a core radiation frection, $f_{\hat{R}_{1 D}}=0.695$ and an overall $Z_{e f f}=1.68$. The computed equilibrium density and temperat.ure profiles are shown in Figure 5.3-3. Analytical fits to these equilibrium profiles are:

$$
\begin{aligned}
& \mu(r)=2.843\left[1-0.44\left(\frac{r}{r_{p}}\right)^{6}-0.56\left(\frac{r}{r_{p}}\right)^{8}\right], \\
& T_{e}(r)= \begin{cases}14.40-46.94\left(\frac{r}{r_{p}}\right)^{2.8504} \quad\left(0 . \quad<\frac{r}{r_{p}}<0.25\right) \\
16.07-10.29\left(\frac{r}{r_{p}}\right) & \left(0.25<\frac{r}{r_{p}}<0.833\right), \\
8.111-7.886\left(\frac{r}{r_{p}}\right)^{14.025} \quad\left(0.833<\frac{r}{r_{p}}<1\right) & 1.23 \times 10^{21}\left[1-0.8577\left(\frac{r}{r_{p}}\right)^{3.44}\right] .\end{cases}
\end{aligned}
$$

The above analytical fits are plotted in Figure 5.2-1(B). The results of the 1-D code are compared in Table 5.3-V with the 0-D code results, and good agreement is found when the 0-D model uses density and temperature profiles that are similar to the 1-D results. 

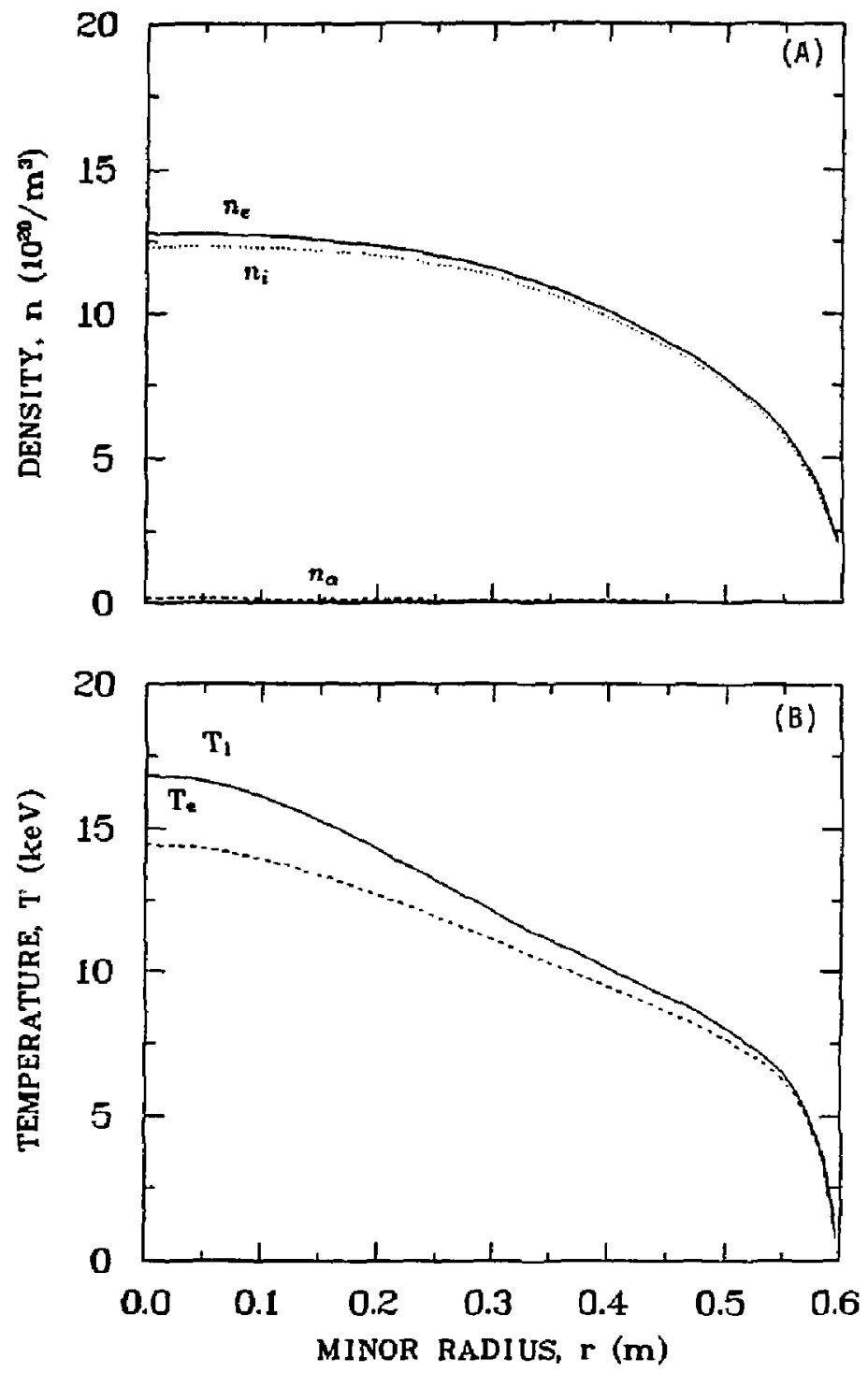

Figure 5,3-3. Profiles of plasma density (A) and electron temperatura (B) from RFP. BURN. 
Table 5.3-V.

\section{COMPARISON OF O-D AND 1-D PLASMA SIMULATION Of TITAN DESIGN POINT}

\begin{tabular}{|c|c|c|}
\hline Parameter & $0-\mathrm{D}$ & 1-D \\
\hline Minor plasma radius, $r_{p}(\mathrm{~m})$ & 0.6 & 0.6 \\
\hline Major toroidal radius, $R_{T}(\mathrm{~m})$ & 3.9 & 3.9 \\
\hline Average ion density, $n_{i}\left(10^{20} \mathrm{~m}^{-3}\right)$ & 9.93 & 8.90 \\
\hline Density peaking, $n_{i o} / n_{i}$ & 1.46 & 1.46 \\
\hline Alpha-particle fraction, $n_{\alpha} / n_{i}{ }^{(a)}$ & 0.030 & 0.005 \\
\hline Ion temperature, $T_{i}(\mathrm{keV})$ & 10.0 & 10.8 \\
\hline Ion temperature peaking, $T_{i o} / T_{i}$ & 1.46 & 1.56 \\
\hline Electron temperature, $T_{e}(\mathrm{keV})$ & 9.5 & 9.9 \\
\hline Electron temperature peaking, $T_{e o} / T_{e}$ & 1.46 & 1.46 \\
\hline Global particle-confinement time, $\tau_{p}(s)^{(b)}$ & 0.86 & 3.6 \\
\hline Global energy-confinement time, $\tau_{E}(\mathrm{~s})$ & $0.215^{(c)}$ & 0.20 \\
\hline Lawson parameter, $n_{i} \tau_{E}\left(10^{20} \mathrm{~s} / \mathrm{m}^{3}\right)$ & 1.92 & 1.78 \\
\hline Pcividal beta, $\beta_{\theta}^{(d)}$ & $0.22^{(e)}$ & 0.207 \\
\hline$Z_{\text {eff }}$ & 1.39 & 1.68 \\
\hline Core plasma-radiation fraction, $f_{\text {rad }}^{c}$ & $\mathrm{~N} / \mathrm{A}$ & 0.695 \\
\hline Toroidal plasma current, $I_{\phi}$ (MA) & 17.8 & 18.0 \\
\hline Ohmic power, PОНM $_{\text {OHW) }}$ & 28.5 & 28.1 \\
\hline Fusion power in plasma, $P_{F}(\mathrm{MW})$ & 2,301 & 2,636 \\
\hline \multicolumn{3}{|l|}{ Profile enhancement 5 ctors: } \\
\hline DT reactivity, $g_{D T}$ & 1.40 & 1.32 \\
\hline Ohmic dissipation, $g_{\text {oHM }}$ & 2.92 & 2.72 \\
\hline Bremsstrahlung, $g_{B \pi}$ & 1.17 & 1.19 \\
\hline
\end{tabular}

(a) Steady-state $n_{\alpha}$ depends upon $\tau_{p \alpha}$ assumption.

(b) $\tau_{p} \simeq 4 \tau_{E}$ for $0-D$ and $\tau_{p} \simeq 4 \tau_{E} /\left(1-f_{R A D}^{c}\right)$ for 1-D.

(c) Based on the empirical scaling $\tau_{E e}=C_{\nu} I_{\phi}^{\nu} T_{p}^{2}$ with $\nu \simeq 1$ and $C_{\nu} \simeq 0.05$ based on a fit to experimental data (Figure 2.3-20).

(d) At steady state, 3 to 3 times this $n \tau_{E}$ value is required for ignition.

(e) Including enerzetic alpha-particle pressure. 


\subsection{EDGE-PLASMA SIMULATIONS}

The characteristics of the edge plasma have been of major importance in the TITAN design and extensive simulations of the edge plasma were undertaken. The main objectives of these simulutions were to predict the plasma conditions at the first wall and divertor target and provide specifications for the engineering design of these components, and to estimate the requirements for particle removal. To obtain a self-consistent view of the behavior of the plasma and neutral particles, this analysis has been coupled to the modeling of the core plasma (Section 5.3) and the neutral particle transport (Section 5.5).

The design of in-vessel components (divertor plate, limiter, first wall) is a critical issue for all reactors and poses a particularly severe constraint for high-power-density reactors, sucb as TITAN. The key problem is to dispose of the steady-state plasme power (alpha-particle and ohmic) while maintaining acceptable heat fluxes and exosion rates on all components. In the scoping phase of the TITAN study [17], a simulation of the edge plasma without impurity radiation indicated plasma temperatures in the range $50-100 \mathrm{eV}$ at the first wall, with very high heat fluxes on the divertor target. Attempts to reduce these loadings, by creating a region of localized raciation in the divertor, failed, as described more fully later, because of difficulties in preveiting uncontrolled contamination of the core plasma by the injected impurities. The final proposed solution is to produce balanced radiation from the core plasma, the edge or scrape-off layer (SOL) plasma, and from the divertor plasma. The plasma is deliberately doped with a trace amount of a high- $Z$ xenon impurities to create strong radiative cooling and spreading the heat load uniformly over the largest possible area (first wall). This high-radiation regime of operation, which appears to be an essential ingredient for a high-power-density reactor, may be more easily achieved in RFPs than in tokamaks because experimental evidence suggests that RFPs operate with a soft $\beta$ limit. These issues are described in deiail in Section 5.3.

Although two separate fusion-power-core designs were studied for the TITAN reactor, lithium-cooled for TITAN-I and an aqueous solution of a lithium salt as the coolant and breeder for TITAN-II, both designs had the arme plasma parameters. Therefore, edgeplasme analysis was not duplicated and, although there are certain references specifically to the TITAN-I design, only minor modifications would be required for TITAN-II. A cross section of the outboard mid-plane of the TITAN-I divertor region is given in Figure 5.4-1, which shows the location of coils, divertor target, field lines, and the vacuum duct. This design represents the result of extensive iterations between edge-plasma and neutral transport analyses, magnetics design, and engineering design of the divertor target plate. 


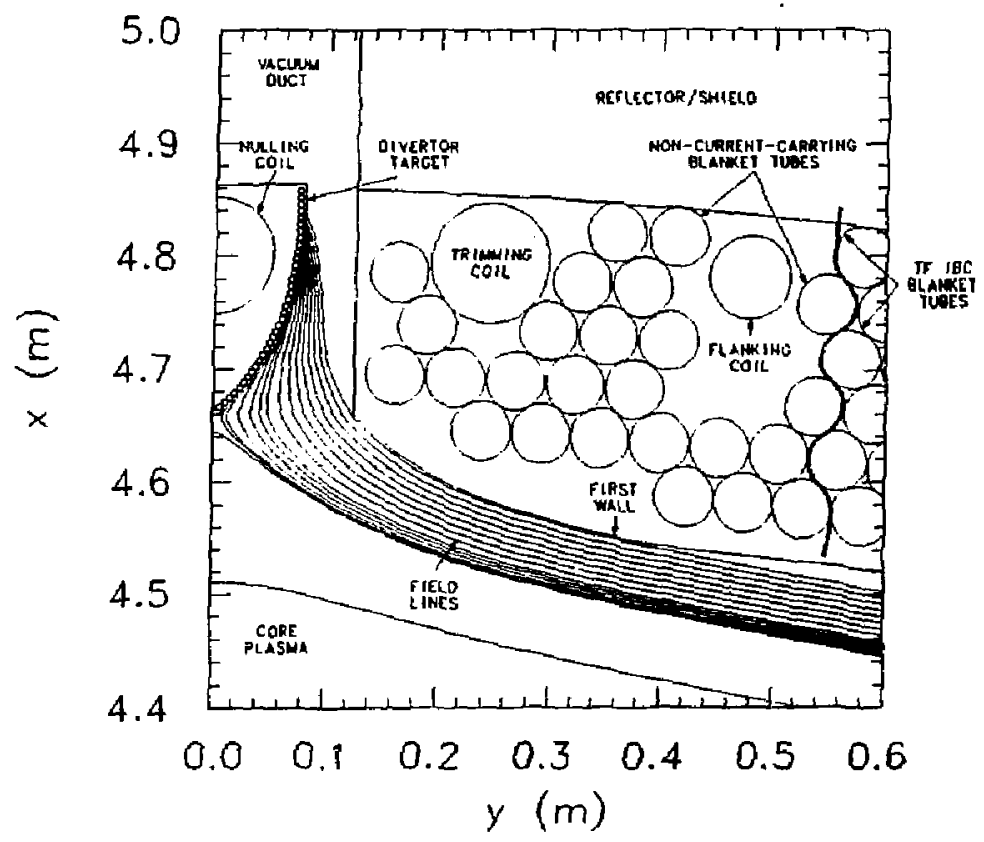

Figure 5.4-1. Outboard equatorial plane view of divertor section for TITAN-I.

\subsubsection{Edge-Plasma Medel}

The edge-plasma analysis was carried out using the recently developed quasi-2-D transport code ODESSA $[18,19]$. The code retains the computational advantinges of a 1-D radial transport code but incorporates important parallel physics. The localjred nature of recycling at the divertor target allows two essential axial domains of interest, namely, the upstream or SOL zone and the downstream or recycling zone in the divertor (Figure 5.4-1) to be identified. By introducing suitable axial averaging operators, a set of coupled 1-D time-dependent equations for the plasma parameters in the SOL and divertor zones is obtained that incorporates explicit radial variation and essential paralleI-transport processes, as well as the sheath condition. The 1-D nature of the code is a major design virtue computationally because extensive parametric stadies are possible and necessary to achieve a converged design. A detailed description of the model and numerical approach is given in References [18] and [19]. Recent modifications to the model [20] are the addition of a self-consistent neutral-atom recycling model in the divertor chamber and a high- $Z$ (xenon) impurity radiation model for radiative cooling at sub-200 $\mathrm{eV}$ temperatures. 
The neutral density model is a simplified but reasonably accurate time-dependent model that assumes a radially flat neutral profile. However, the magnitude evolves selfconsistently in time with changing plasma conditions in the divertor through temperaturedependent reaction rates and a prescribed pumping rate, which depends on such factors as geometry and pipe conductances. Prescribing the pumping time is believed to be more physical than the customery practice of fixing the recycling coefficient [21]. The recycling coefficient is then computed self-consistently from the relative amounts of ionization and leakage when the plasma reaches steady state. The flat profile assumption is justified on the grounds that charge-exchange-induced diffusion, which strongly dominates ionization at the low divertor temperatures encountered $\left(T_{e} \sim 5 \mathrm{eV}\right)$, smoaths spatial gradients of neutral density. This behavior is also seen in Monte Carlo simulations of neutral transport in the divertor.

In the absence of reliable data for the radiative cooling rate of high- $Z$ elements at low plasma temperatures, a simple model is used to estimate the impurity radiation. Post has tabulated date for the radiative cooling rate in coronal equilibrium of many impurities in relatively high-temperature plasmas [22]. For xenon, these data are available only for temperatures above $80 \mathrm{eV}$ and, therefore, have been extrapolated here for lower temperatures. Consideration of the electronic structure of xenon ions at these plasma temperatures suggests that, with reducing temperature, the cooling rate may increase somewhat befote falling and finally drops to a very low value for temperatures below a few $\mathrm{eV}$. To avoid introducing unwarranted complexity into the model, the cooling rate is assumed to remain constant with falting temperature, before falling to zero at the lowest temperatures. The model used for the radiative cooling rate as a function of plasma temperature, $L(T)$, of xenon impurities is

$$
L(T)=L_{0}\left\{1-\exp \left\{-\left\{T / T_{0}\right)^{n}\right\}\right\}
$$

where $L_{0}$ is a constant (equal to $5 \times 10^{-12} \mathrm{eV} \mathrm{m}^{3} / \mathrm{s}$ ), $T_{0}$ is taken as $10 \mathrm{eV}$, and a value of 2 was used for the exponent $n$. The rate of decay was varjed to give a reasonable physical model that was not constant to zero temperature on the one hand, and that did not trigger thermal instabilities on the other hand. It is recognized that this model for the magnitude of the cooling rate is rather uncertain, not only because of the extrapolation to lower temperatures, but also because it is not clear that the assumption of coronal equilibrium itself is valid.

The impurity density profile was assumed radially uniform in the core and edge, and justified on the grounds that with an anticipated edge fueling of xenon, a flat profile was more reasonable than a constant fraction of the electron density. However, in the 
divertor, recycling of the impurities is likely to drive the impurity concentration to some fraction of the electron density. This fraction was made consistent with the upstream concentration.

Transport coefficients used were similar to those utilied for 2-D simulations of the NET/INTOR tokamak designs [21]. There are few reports of the transport coefficients in the edge of RFP plasmas but, for large burning plasmas, it might be expected that they would be comparable to the values in tokamaks. The radial particle-diffusion coefficient was taken as $1 \mathrm{~m}^{2} / \mathrm{s}$ with a thermal diffusivity of $4 \mathrm{~m}^{2} / \mathrm{s}$. Boundary conditions at the first wall and divertor target were also standard $[21,23]$. Electron and ion temperature radial scale lengths at the first wall of $10 \mathrm{~cm}$ were assumed, with a zero density gradient. This latter assumption is necessary to prevent the loss of particles to the first wall, which will act as a perfect reflector in the steady-state plasma operation. A sheath boundary condition was imposed to model energy transmission at the divertor target.

An important difference from other edge-plasma simulations, however, was the treatment of the core/edge interface. In standard 2-D fluid simulations of edge plasmas $[21,23\}$, either fluxes of particles and heat from the core are prescribed at the separatrix, or the plasma density and temperatures are specified there. The core is thus assumed to be in steady state and not to respond to the dynamics of the SOL and divertor. This is a computational necessity since the iterative coupling of $1 \frac{1}{2} . \mathrm{D}$ core-transport and 2-D edge-transport codes would be prohibitively expensive. However, ODESSA is not bound by the same computational restrictions and, thus, extensive iterations with the core transport code described in Section 5.3 were performed to ensure self-consistency at the core/edge boundary of heat and particle fluxes, and of plasma density and temperatures.

The simplified neutral model in ODESSA was introduced to avoid having to complement the plasma evolution with detailed neutral-source distributions from Monte Carlo calculations at every time step. Although the model is adequate for the description of recycling in ODESSA, it does not provide estimates of leakage of neutral atoms and molecules acrose the separatrix back to the main plasma, or relative fluxes of $D, T$, and He that are pumped. It also cannot address the question of He enrichment at the pumps. For these reasons, the plasma computations were supplemented with 3-D Monte Carlo neutral-transport simulations in the relevant TITAN divertor geometry, using the DEGAS code [24] (Section 5.5). 


\subsubsection{Edge-Plasma Parameters}

Detniled field-line tracing calculations (Section 4.4) show the field-line connection length, $\mathrm{L}_{\|}$(defined as the distance along the field line from the "watershed"point between divertors to the target), to be sensitive to the distance into the SOL, especially in the vicinity of the separatrix. However, since the resolution on transport scales is at best an island width, a suitable average over the SOL width was taken for the ODESSA simulations, at a value $L_{\|}=69 \mathrm{~m}$.

After considerable iteration between core- and edge-plasma models, as well as thermal and mechanical design of the divertor and nagnetica analysis, the converged and optimum design consists of three divertors and an SOL width of $6 \mathrm{~cm}$. The choice of the number of divertors was a compromise between conflicting desires. As the number of divertors increases, the total area of the divertor targets increases, leading to a lower heat flux (as shown in Section 5.4.3), but the ohmic losses in the lithium divertor coils also increase. The choice of SOL width was also a trade-off; a small SOL thickness is desirable for reasons of plasma MHD stability, but it leads to higher plesme temperatures at the first wall and a consequent increase in erosion.

In the final converged stage between core- and edge-plasma codes, stable profiles of plasma density, temperature, and flow velocity in the SOL and divertor were obtained for $\sim 150 \mathrm{MW}$ of power flowing into the SOL at a particle throughput of $\sim 10^{22} \mathrm{~s}^{-1}$. In the global-plasme power balance, $\sim 70 \%$ of the steady-state heating power was radiated in the core plasma, $\sim 23 \%$ in the SOL, $4 \%$ in the recyclinr; zone, and $3 \%$ was deposited on the target through the plasma sheath.

Figure 5.4-2 depicts the radial upstream and downstream ion and electron temperature and density profiles. High separatrix density $\left(\sim 2 \times 10^{20} \mathrm{~m}^{-3}\right)$ and temperatures $\left(\mathrm{T}_{\mathrm{t}} \sim 220 \mathrm{eV}, \mathrm{T}_{1} \sim 380 \mathrm{eV}\right)$ are achieved, with the latter decaying strongly to $\sim 2 \mathrm{eV}$ at the first wall. This is largely due to the intense radiative cooling and strong rethermalization between ions and electrons near the first wall. Near the separatrix, however, strong parallel-electron conduction results in a depressed electron-temperature profile at that location compared with that of the ions. The density profile, on the other hand, is quite flat, partly as a consequence of the assumed first-wall reffection coefficient of unity, but also because of the unisual flow pattern (see below) that results in a very weak net flow into the divertor.

Intense recycling (global recycling coefficient, $R=0.995$ ) is achieved at the divertor target based on the simple neutral-particle model in ODESSA, which, as discussed in 


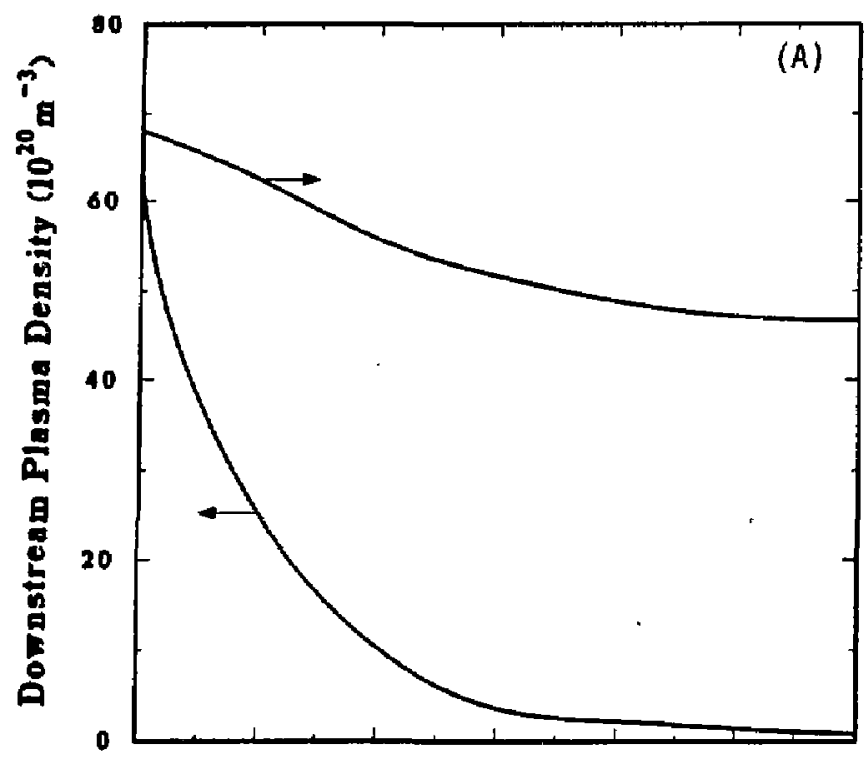

2.0

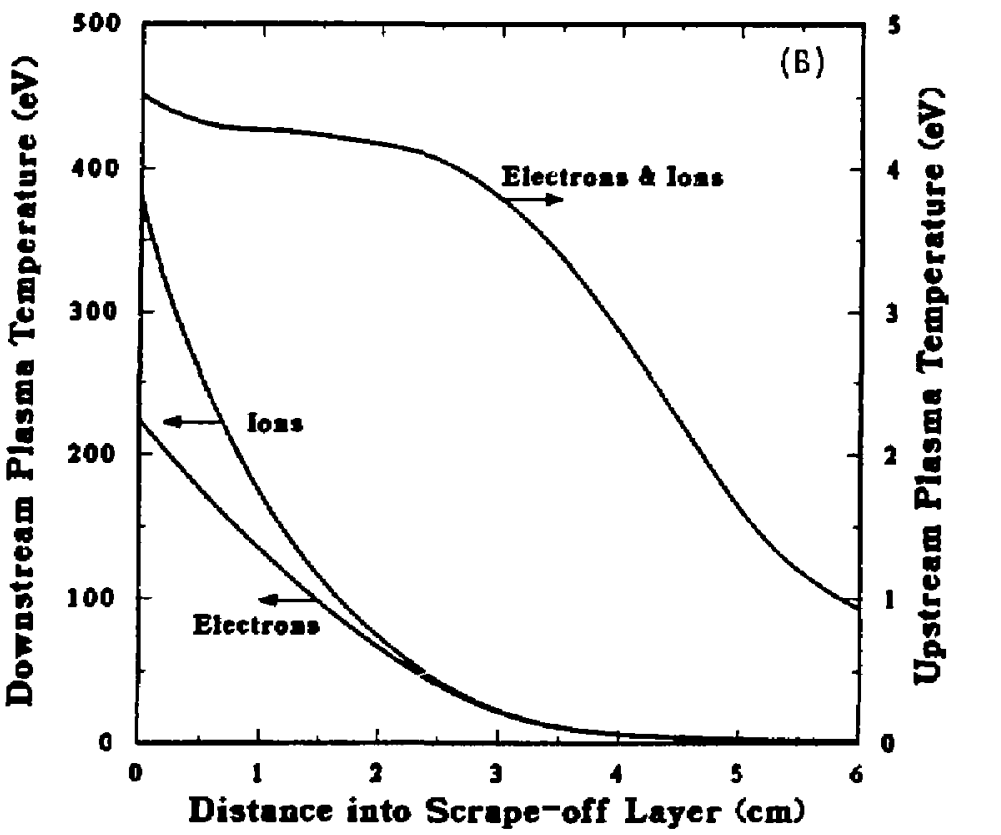

Figure 5.4-2. Radial profiles of plasma temperature (A) and density (B), in upstream (SOL) and downstream (divertor) zones. 
Section 5.5, is borne out by more detailed Monte Carlo simulations. This is characterized by high density $\left(\sim 10^{21} \mathrm{~m}^{-3}\right)$ and low temperature $\left(T_{\mathrm{e}} \simeq T_{\mathrm{i}} \simeq 5 \mathrm{eV}\right)$ in the divertor plasma. Because the cross section for electron-impact ionization is sensitive to the electron temperature, the ionization rate is greater near the separatrix than in the cooler regions near the first wall (most of the energy here is radiated upstream). As discussed in Section 5.5, the low temperature achieved is a significant factor in permitting a viable first wall and divertor design from an erosion viewpoint.

Figure 5.4-3 shows the radial profile of the upstreasn flow velocity towards the divertor. A striking observation is that the flow is reversed in the vicinity of the separatrix and accelerates to nearly sonic flow at the first wall. This complex flow pattern is a consequence of intense recycling in the divertor that causes the local recycling coefficient to exceed unity at certain locations. This in turn induces the axial pressure profile to invert, resulting in particles being driven out of the divertor there. A detailed explanation of this phenomenon, which describes the complex dependence on the $T_{E}$ profile, has been published elsewhere [25]. A diffusive/convective circulation is thus set up between the SOL and divertor. This effect occurs independently of the radiation in the edge, but it rules any attempt to localize all radiative cooling to the divertor plasma because impurities could not be confined exclusively to that region. For this reason, the decision was made to allow radiation uniformly in the plasma.

\subsubsection{Sensitivity Study}

In view of the considerable uncertainty in many of the assumptions used in the edgeplasma analysis, an extensive sensitivity study was undertaken in an effort to determine how the divertor design might change as the edge-plasma conditions change. For each different value used for an input parameter, the ODESSA code was rerun. Although it was too time-consuming to iterate with the core-plasma code for each case studied, the variations about the central values were sufficiently small that the results would not. be greatly different for a fully converged solution. Since every output parameter (e.g., plasma temperatures, densities, heat fluxes, etc.) is different for each variation, it would be impractical to examine the effect of changes on each parameter of interest. Because the divertor-target heat flux has been the critical issue for the TITAN divertor design, this factor has been emphasized in the sensitivity study. Further, to reduce the focus of attention to a single value, the heat flux has been averaged across the target, as adjustments in the shaping of the target could be made to smooth out sharp peaks in the loading. 


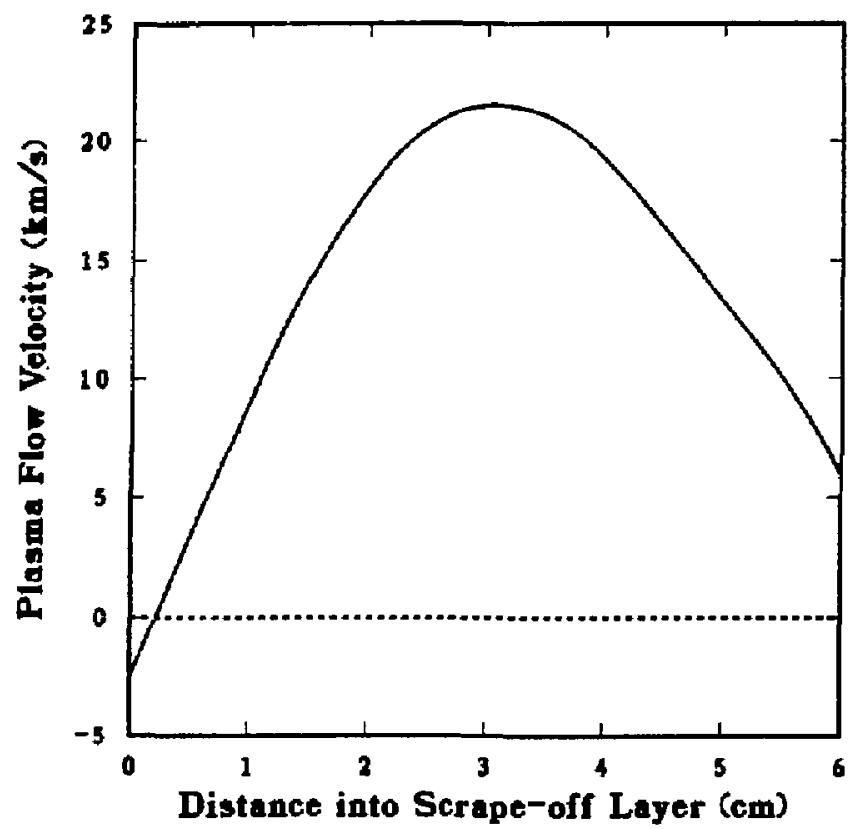

Figure 5.4-3. Radial profile of plasma flow velocity in upstream (SOL) zone.

Variations were considered in the following parameters: radial particle-diffusion coefficient, $D_{\perp}$; ratio of thermal-to-particle diffusivitjes, $\chi_{\perp} / D_{\perp}$; neutral-particle pumping time, $\tau_{\text {promp }}$ i constants in the impurity model, $L_{0}$ and $T_{0}$; field-line connection length, $L_{\| ;}$ acrape-off layet thickness, $\Delta_{S O L}$; core plasma-radiation fraction, $f_{R A D}^{f}$; and the number of divertors, $N_{D}$. The results of this study are given in Table 5.4-I, giving the average divertor-target heat flux, $q_{D}^{\prime \prime}$. Note that this heat flux is strictly for TITAN-I, but the trends in the data would exist for TITAN-II.

Fairly substantial changes (factor 2-3) in the transport coefficients and in the neutral pumping time lead to relatively small changes in the heat flux on the divertor target of less than $10 \%$ to $12 \%$. Note that the profile of heat flux in the plasma may vary quite noticeably, particularlv as the transport coefficients are adjusted, but the total power flow to the target (or, equivalently, the average heat flux) is less sensitive. The results are somewhat more sensitive to the parameters in the impurity radiation model, since $20 \%$ chenges in the input variables yield changes in the heat flux of up to $10 \%$. The connection length makes little difference to the heat flux (verifying that including a different vatue for each field line is not important), and there is also little sensitivity to the 
Table $\mathbf{6} .4-I$.

SENSITIVTTY STUDY FOR EDGE-PLASMA ANALYSIS

\begin{tabular}{ccccc} 
& $\begin{array}{c}\text { Reference } \\
\text { Value }\end{array}$ & New & $q_{D}^{\prime \prime}$ & Percent \\
Variable & Value & $\left(\mathrm{MW} / \mathrm{m}^{2}\right)$ & Change \\
\hline
\end{tabular}

Reference case

6.8

$D_{\perp}\left(\mathrm{m}^{3} / \mathrm{s}\right)$

1.0

0.5

$7.6+11.9$

$x_{\perp} / D_{\perp}$
$\tau_{\text {pump }}(\mathrm{ms})$

2.0

$6.1-11.3$

$L_{0}\left(10^{-12} \mathrm{eVm}^{3} / \mathrm{s}\right)$

4.0

2.0

7.3

$+7.2$

6.0

6.5

$-4.4$

(10)

0.3

0.1

7.2

$+5.9$

$T_{0}(\mathrm{eV})$

10.

1.0

6.6

$-3.2$

4.0

7.5

$+9.7$

6.0

6.4

$-6.6$

$L_{\|}(\mathrm{m})$

69.

8.0

7.3

$+7.2$

12.0

6.5

$-4.8$

$\Delta_{\text {SOL }}(\mathrm{cm})$

64.

7.0

$+3.1$

74.

6.7

$-2.5$

$f_{R A D}^{c}$

6.0

5.0

6.7

$-2.1$

7.0

7.0

$+2.1$

0.70

0.65

$7.9+16.0$

$N_{D}$

3

$5.0-27.0$

2

$8.0+16.9$

4

$6.2-9.2$ 
SOL thickness. The strongest sensitivity occurs with the core plasma-radiation fraction, where the average heat flux varies approximately as $\left(1-f_{\text {RAD }}^{e}\right)^{-1}$. This sensitivity could be utilized to adjust for any uncertainties in other parameters (especially the radiation model, which is probably the weakest aspect of the model) by varying the impurity density to ensure that the heat flux on the divertor is acceptable. Finally, the sensitivity of the heat flux to the number of divertors is weaker than the expected $1 / N_{D}$ dependence, which is explained by the following observations: For an increased number of divertors, the connection length is reduced, leading to less radial diffusion and a lower average density in the SOL. The impurity radiation is therefore reciuced and more power is deposited on the divertor target.

\subsection{NEUTRAL TRANSPORT AND EROSION}

\subsubsection{Neutral Transport Calculations}

This section describes the modeling of neutral particle transport that was performed for the TITAN divertor design. The actual geometry modeled was based on the TITAN-I divertor and vacuum-system designs, but the general trends of the results are aleo valid for TITAN-II. The reason for undertaking detailed neutral-transport simulations was to ensure that the simplified neutral model incorporated in the ODESSA edge-plesme modeling (Section 5.3) adequately predicted the recycling and particle removal in the divertor. Further, certain aspects of the modeling can only be carried out with a full neutral-particle model; for example, the question of whether the open divertor geometry used in TITAN, and the consequent proximity of the divertor target to the core plasma, leads to an unacceptable leakage of neutral particles from the divertor across the separatrix.

The detailed neutral-transport modeling was provided by DEGAS, a fully 3-D Monte Carlo transport code [24], which uses the most recent and thorough data base of cross sections and reflection coefficients.

The geometry of the TITAN-I toroidal-field divertor was modeled using an equatorialplane cross section of the divertor plasma and duct region. This planar section was then rotated about the plasma centerline to obtain the poloidally continuous divertor chamber. The divertor cross section actually varies continuously with poloidal angle, because of the variation of toroidal field with mejor radius, but the computational effort required for a nonsymmetric 3-D model was considered excessive. Therefore, both outboard and 
inboard geometries were examined (each with an assumed poloidal symmetry) in an effort to bound the result.

The duct leading from the divertor to the vacuum tank is not poloidally continuous and is located over the outboard $90^{\circ}$ in poloidal angle only. This feature is modeled by specifying this surface to be an absorbing wall over this angle, and to be solid over the remaining $270^{\circ}$.

The outboard cross-section geometry is shown in Figure 5.4-1 in relation to the divertor coils and blanket. In Figure 5.5-1, the grid representing this area in DEGAS is shown with a single particle trajectory. As this particular particle was launched from an inboard zone, the outermost boundary is a wall, rether than a pumping duct. In this case, the particle bounces off the walls in the duct for a long time until it either returns to the plasma and is re-ionized or it eventually finds its way around poloidally to the exit duet.

The plasme conditions that are required as an input to the code are taken from the edge-plasma code ODESSA. Since the axial (i.e., along the field line) variation of

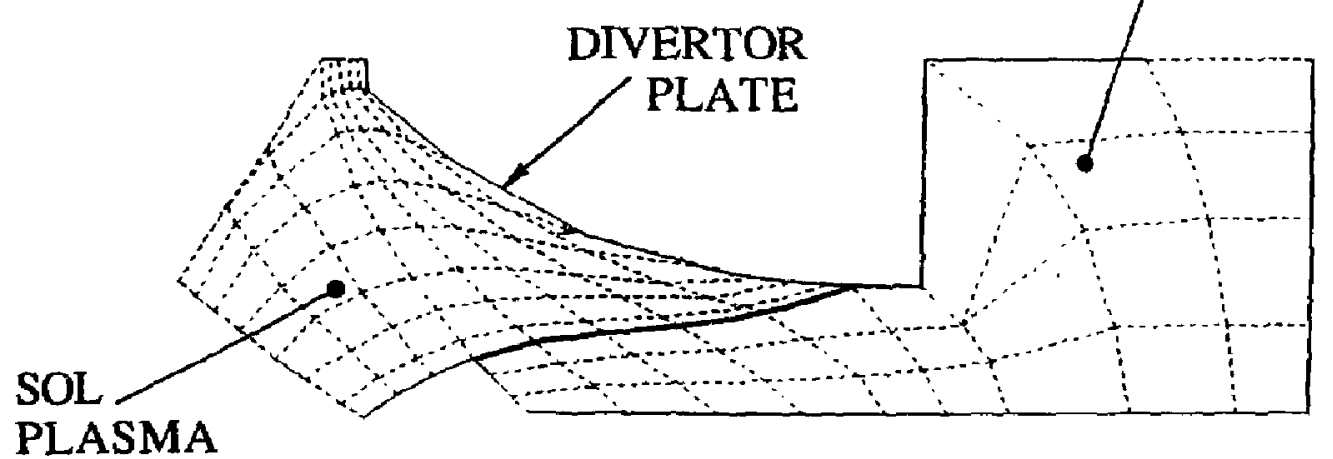

Figure 5.5-1. Mesh used for neutral particle modeling with DEGAS cade, showing a typical particle trajectory. 
the plasma parameters is needed but ODESSA provides only values at only two axial locations, a linear interpolation is made between the downstream values that apply at the divertor target, and the upstream values that are taken as applying at a set of radial points passing through the null point. Further upstream, the plasma parameters are assumed to be constant. Magnetic flux expansion at the target is also accounted for in computing the particle flux and neutral source at the target.

Figures 5.5-2 and 5.5-3 show the poloidally averaged neutral-particle density for, respectively, the species atomic $\mathrm{D}$ and molecular $\mathrm{DD}$, and for He for the outboard divertor geometry. Going upstream in the plasma away from the divertor target and in the SOL near the separatrix, the neutral densities fall very sharply so that the flux of neutral particles across the separatrix and into the core plasma is negligible. The lack of any significant leakage into the core plasma arises from the high plasma density $\left(\sim 10^{21} \mathrm{~m}^{-3}\right)$ near the divertor target, which leads to the neutrals emitted from the target having a short mean free path to ionization. Therefore, despite the closeness of the divertor target to the core plasma, the proposed configuration acts as a divertor rather than as a limiter.

Further upstream from the target, in the SOL area near the first wall, the neutral particle density falls off much more slowly giving rise to a neutral channeling effect between the first wall and the higher density and warmer (i.e., more ionizing) plasma nearer the separatrix.

The densities are high in the duct region and, for atomic $D$, fairly constant throughout the duct region, with pressures of about 15 to 75 intorr depending on the poloidal location; the poloidal asymmetry in the pressures is discussed later. These high pressure values pose a qualification on the assumption used in the neutral transport model that the neutrals are in the free streaming regime, in which case neutral-neutral collisions can be neglected. This assumption is poor at these high pressures.

It is noted that there is little margin for movement of the separatrix position with respect to the target, and the possible movement due to the proposed oscillating-field current drive or other sources of fluctuations is not known. An additional point is that the results apply to the full-power operating state and the transient conditions during start-up were not addressed with respect to this issue. Consideration must be given to these transient conditions so that a period of low plesma density does nc: coincide with a period of relatively high divertor power. This combination can give rise to an excessive interaction between the divertor target and the core plasma, as well as the possibility of high plasma temperatures at the divertor target and the resultant problem of sputtering damage. Fueling in the divertor may help to alleviate these undesirable conditions. 

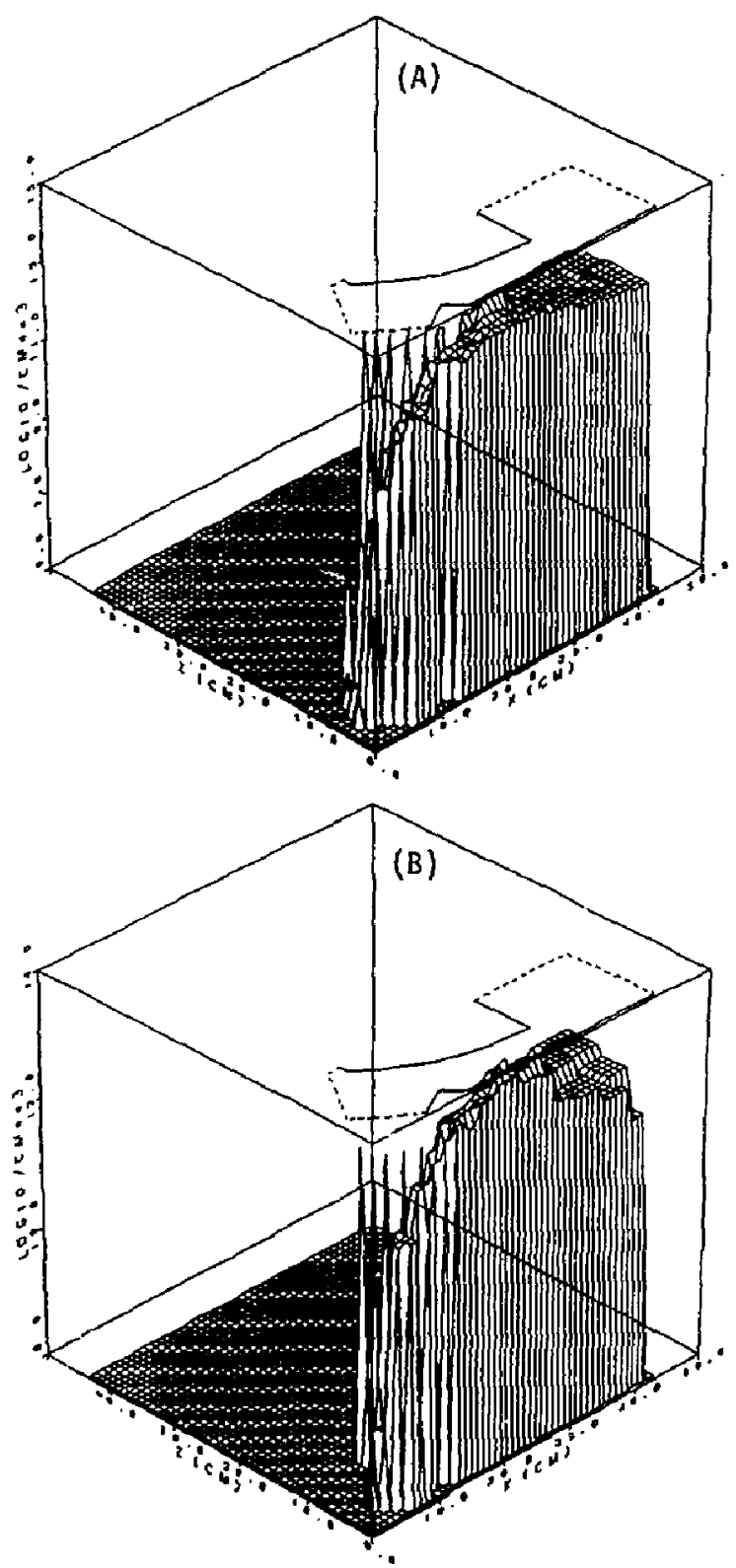

Figure 5.5-2. Poloidally averaged density of atomic D (A) and molecular DD (B) for TITAN-I divertor geometry. 


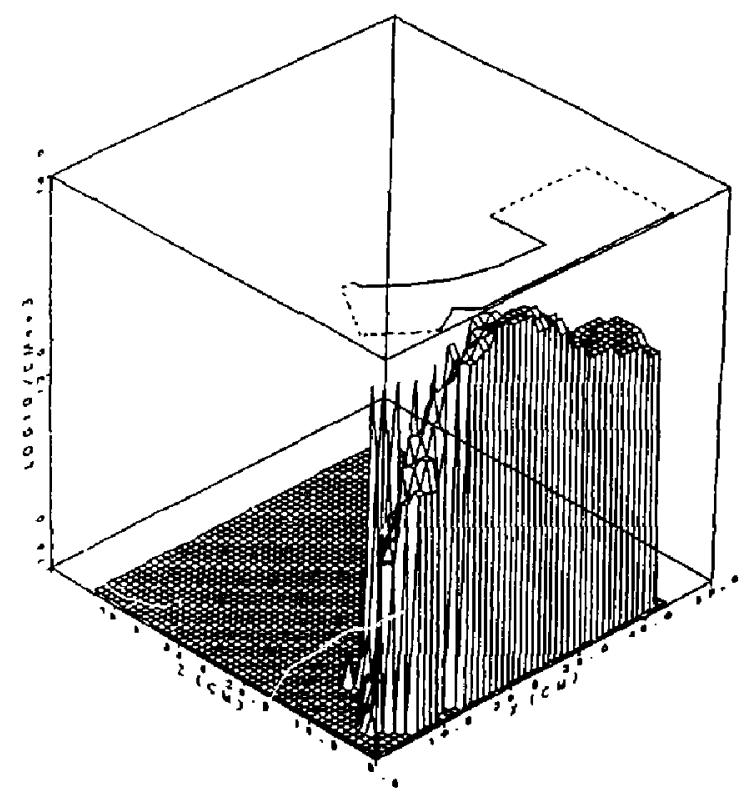

Figure 5.5-3. Poloidally averaged density of He for TITAN-I divertor geometry.

The neutral transport results show that the global recycling coefficient, $R$, lies in the range 0.95 to 0.98 , depending on the geometry and pusma specifics; the corresponding value predicted by the ODESSA edge-plasma cor $:$ is 0.995 . This slight mismatch in recycling coefficients exists because the codes have not been iterated to convergence. To achieve the higher recycling desirable for optimum divertor operation, it may be necessary to make minor adjustments to the divertor aitet geometry to increase the fraction of neutrals that are reflected back into the plasma and re-ionized.

The radial profile of plasma flow velocity upstream of the recycling zone can be evaluated by integrating the ionization source term along the field line away from the target, and ignoring cross-field transport. This analysis indicates that the flnw should reverse, as discussed in Section 5.3 and Reference [25]. However, the DEGAS results imply that the reversal will occur on field lines near the center of the SOL, in contrast to the ODESSA restitis where reversal occurs near the separatrix. Iteration between the two codes, particularly in the areas of radial neutral-particle profiles, would remove this discrepancy.

The fluxes of neutral particles escaping into the pumping duct were found to be significantly different from the plasma fluxes on the divertor target, specifically ihe neutral 
gas was enriched in $D$ and He relative to $T$. For the assumed case of equal target fluxes of D and $T$, and for an He plasma flux of 5\% that of DT, the neutral gas escaping was enriched in He relative to DT by a factor of $\sim 3$ and in D relative to $T$ by 8 factor of 3.7 .

The enrichment of $\mathrm{He}$ in the neutral exhaust, which has been observed in other simulations (e.g., Reference [26]), improves the ash pumping efficiency and this benefit has been included in the specifications for the vacuum system design. The enrichment arises from the smaller cross sections for charge exchange and for ionization of He relative to DT, which lead to a longe. mean frec path and an increased probability of escape from the plasma.

Enrichment $r$ f the pumped gas in $D$ relative to $T$ appears to be a new result ard is due to the mass difference between the two isotopes. The probability, $p$, of escape of a neutral from the divertor plasma can be approximated as $\exp (\Delta / \lambda)$, where $\Delta$ is the average path length of a neutral from the target through the plasma, and $\lambda$ is its mean free path. If $\Delta$ is taken as $0.1 \mathrm{~m}$, a reasonable estimate for the TITAN geometry, then for the average plasma density of $2 \times 10^{21} \mathrm{~m}^{-3}$ and a plasma temperature of $4 \mathrm{eV}$, the enrichment ratio of $D$ relative to $T$ is estimated to be about 3 , in reasonable agreement with the DEGAS results. Although a more extensive study of this effect is necestary, the apparent de-enrichment in tritium reduces the tritium throughput in the gan handling systems which is an important benefit.

The neutral particle pressure shows a marked asymmetry in poloidal angle because of the prescuce of the pumping duct only on the outboard side of the divertor. For atomic $D$, the pressure on the outboard side of $\sim 15$ mtorr is less than the inboard pressure by a factor of 4 to 5 , because the outboard duct acts as a particle sink. The asymmetry is even larger for DD where the inboard pressure is about 10 times more than that of the outboard. For this case, the source of DD is neutrals emitted from the chamber walls with the wall area being larger on the inboard side. Thus, the source, as well as the sink, is poloidally asymmetric and so the pressure differential is increased for DD over that for atomic $\mathrm{D}$.

The neutral particle density within the plasma region, especially that of atomic $D$, however, is largely constant with poloidal angle. There is an asymmetry (about a factor of 2 greater intoard than out) in the density of molecular DD in the low plasma-density region of the SOL, but the plasma density source is dominated by the atomic D species. These observations show that the assumption that the plasma conditions retain a poloidal symmetry while the neutral particle pressure in the duct is poloidally asymmetric is justified. 


\subsubsection{First Wall Erosion}

The DEGAS neutral-transport code was used to estimate erosion on the first wall of TITAN; the wall material chosen was vanadium (TITAN-I) but the results should be similar for the ferritic steel proposed for TITAN-II. Sputtering due to both ions and charge-exchange (C-X) neutrals was considered, using the upstream plasina protiles provided by ODESSA. The wall flux could not be taken directly from the results because a flat density profile was assumed as a wall boundary condition to conserve particles. Therefore, the DT ion flux on the wall was estimated at $2 \times 10^{21} \mathrm{~m}^{-2} \mathrm{~s}^{-1}$, with a $5 \%$ He ion fraction, from an approximate model of recycling at the first wall.

The reference case of the ODESSA model predicts a plasma temperature (ion and electron) of slightly less than $2 \mathrm{eV}$ at the first wall. This implies negligible ion erosion since the ion energies are below the threshold for sputtering. Further, the high plasma density near the first wall prevents the neutral particles released from the wall from penetrating to regions of high plasma temperature and then returning to the wall to pose a sputtering problem. However, the edge-plasma models contain a fairly large degree of uncertainty and, therefore, a sensitivity study was performed to estimate the erosion rate for different values of the plasma temperature at the first wall. Because it is not clear whether a sheath will form at the wall, cases with and without the presence of a negative sheath were considered. Table 5.5-I shows the results of this analysis in which redeposition was ignored.

As the engineering design of the first wall provides for a $0.25-\mathrm{mm}$ allowance for erosion, these results imply that a plasma temperature of $\sim 6 \mathrm{eV}$ is acceptable if a sheath exists at the wall, while the temperature is allowed to rise to $\sim 10-20 \mathrm{eV}$ if there is no sheath; the limits would be increased further if redeposition were taken into account. ODESSA predicts a temperature of $\leq 2 \mathrm{eV}$ so it appears that there is a large safety margin in the first wall design from considerations of erosion.

\subsubsection{Divertor Plate Erosion}

Erosion of the divertor target was calculated from the LT and He ion fluxes, allowing for acceleration through the plasma sheath. The location chosen for the calculation was a point close to where the separatrix strikes the divertor target, where the particle flux and temperature have their highest values. Allowing for magnetic flux expansinn and the inclination of the target to the field lines, tie DT ion flux is estimated to be $\sim 9 \times 10^{23} \mathrm{~m}^{-2} \mathrm{~s}^{-1}$; the He ion flux is taken as $5 \%$ of this value. The first material 
Table 5.5-I.

SENSITIVTTY STUDY FOR FIRST-WALL EROSION RATE(a)

\begin{tabular}{cllll}
$\begin{array}{c}\text { First Wall } \\
\text { Temperature (eV) }\end{array}$ & DT Ions & $\begin{array}{l}\text { DT C-X } \\
\text { Neutrals }\end{array}$ & He Ions & Total \\
\hline 1.7 & $0.00(0.00)$ & $0.01(0.01)$ & $0.00(0.00)$ & $0.01(0.01)$ \\
6.0 & $0.00(0.03)$ & $0.01(0.01)$ & $0.00(0.25)$ & $0.01(0.29)$ \\
10.0 & $0.02(1.02)$ & $0.02(0.02)$ & $0.02(0.78)$ & $0.06(1.82)$ \\
20.0 & 0.75 & 0.09 & 0.22 & 1.06 \\
30.0 & 2.55 & 0.21 & 0.54 & 3.30 \\
\hline
\end{tabular}

(a) Erosion rate in $\mathrm{mm} / \mathrm{y}$ without (with) a sheath.

considered was vanadium (TITAN-I first-wall material) but the sputtering due to He bombardment was found to be excessive $(\sim 5-10 \mathrm{~mm} / \mathrm{y})$ even at the low plasma temperature of $4 \mathrm{eV}$. It was concluded, therefore, that a high atomic number $(Z)$ armor would be required to protect the cooled divertor plate; tungsten (alloyed with rhenium to improve its ductility) became the final choice (Sections 11 and 17).

Helium and DT sputtering on tungsten are negligible for the TITAN divertor piasma conditions. Based on considerations of the expected concentration of xenon impurities in the divertor plasma, the total Xe flux onto the target was estimated at $\sim 10^{20} \mathrm{~m}^{-2} \mathrm{~s}^{-1}$. Many uncertainties exist regarding the behavior of the recycling Xe impurity, especially with respect to the flow speed and charge state when it strikes the target. There is a tendency for the impurity to be frictionally accelerated by the drifting background plasma such that it can be flowing at close to the sonic velocity of the DT ions. However, the mean free path for ionjzation of the neutral Xe atoms emitted from the target is short at these high plasma densities atid there is little distance for the impurity to be accelerated to a high velocity before returning to the target. As an example calculation, it was assumed that the recycling Xe has a flow speed of one-tenth that of the DT plasma ions, but that the primary $\mathrm{Xe}$ flux has the same flow speed as the background plasma. If these epecies have charge states of, respectively, 2 and 4, then the erosion rate is 
less than $2 \mathrm{~mm} / \mathrm{y}$ even if redeposition is ignored. However, strong recleposition would be expected at the high plasma density found near the divertor target and may dominate the net erosion rate, implying that higher charge states and flow speeds could be tolerated. Acknowledging the great uncertainties regarding erosion estimates for the divertor target, the specification for the divertor design was that a 2-mm-thick layer of tungsten should be provided to give a lifetime of one full-power year. Further investigations into the effects of heavy impurities on the erosion rate of divertor targets clearly are required.

Significant erosion can occur on the wall facing the divertor plate from chargeexchange neutrals originating from the divertor target. Orientation of this wall at a relatively oblique angle to the target can reduce this erosion but it may be necessary to protect this surface with a thin layer of an armor material such as tungsten. Since this region of the wall is shadowed from the core plasma and receives a small heat load, this protection should not lead to thermal problems in the structure.

\subsection{FUELING}

The TITAN-I externel fueling rate balances the combined DT exhaust and fusion burnup in steany state. The rate of generation and, hence, removal of helium ash by the fusion reactor is given by

$$
\phi_{H e}=\frac{P_{F}}{17.6 k_{B}},
$$

which for $P_{F}=2,300 \mathrm{MW}$ and $k_{B}=1.602 \times 10^{-19} \mathrm{~J} / \mathrm{eV}$ equals $\phi_{\text {He }}=8.16 \times 10^{20} \mathrm{He} / \mathrm{s}$ or $3.07 \mathrm{~Pa} \mathrm{~m} / \mathrm{s}$ (23.4 torr $1 / \mathrm{s}$ ). The TITAN-I tritium burnup is $0.353 \mathrm{~kg} / \mathrm{d}$ at a fractional burnup is 0.24 , such that the tritium throughput rate is $1.5 \mathrm{~kg} / \mathrm{d}$. The performance of TITAN-II is similar. Assuming that the confinement times of deuterium and tritium in the plasma are equal ( $\tau_{p i} \simeq 3.6 \mathrm{~s}$ ), the fueling stream can maintain the nominal composition of the plasma (including Xe impurities), subject to isotopic separation and removal of the alpha-particle ash.

Edge refueling is presumed to be inappropriate in TITAN because particles introduced at the first wall will tend to be swept out along the magnetic-divertor scrape-off (outside the separatrix) to the divertor plate and vacuum ducts and, therefore, will be unavailable to refuel the core plasma. Pellet fueling $[27,28]$ using cryogenic pellets $\left(\rho_{o} \simeq 250 \mathrm{~kg} / \mathrm{m}^{3}\right)$ is the fueling option of choice. The RFP plasma confinement is provided largely near the reversal surface $\left(r_{r} \simeq 0.55 \mathrm{~m}\right.$ for TITAN with $\left.r_{p}=0.60 \mathrm{~m}\right)$, with turbulent mixing 
of particles assumed to occur within the core plasma. It is assumed, therefore, that the pellet inventory needs to be deposited mostly just inside the reversal surface. Deep penetration to the central plasma is not required in an RFP, such that ultra-high pellet injection speeds $(>3 \mathrm{~km} / \mathrm{s})$ or advanced acceleration technologies [29] beyond the presently available pneumatic or centrifugal approaches [28], should not be required in the TITAN case.

The pellet ablation process has been considered by several authors [27,30-32]. The simple model applied here is the so-called "neutral-ablation-cloud" model [30]. Corrections for the perturbation of the local magnetic field by the pellet lead to somewhat (up to $\sim 50 \%$ ) reduced pellet-ablation rates and longer lifetimes [31]. The surface ablation (not including alpha-particle effects) of a spherical pellet with initial radius $a(\mathrm{~m})$ is represented by the following exptession [30] for the time-rate-of-change of the pellet radius:

$$
\frac{d a}{d t}=-1.0 \times 10^{-8} \frac{n_{e}^{1 / 3} T_{e}^{5 / 3}}{a^{2 / 3} m^{1 / 3}},
$$

where $m$ (amu) is the molecular weight of the fuel (e.g., $5 \mathrm{amu}$ for DT), $n_{c}\left(\mathrm{~m}^{-3}\right)$ is the target-plasma electron density, and $T_{e}(\mathrm{keV})$ is the target-plasma electron temperature. Integration of Equation 5.6-2 gives the pellet lifetime, $t_{p}(s)$, or the time after initial injection $(t=0)$ at which the pellet completely evaporates $(a \rightarrow 0)$. Assuming a constant pellet injection speed, $u(\mathrm{~m} / \mathrm{s})$, the maximum pellet-penetration distance, $d(\mathrm{~m})$, is given by $d=u t_{p}$. The integration of Equation 5.6-2 can be rewritten in the following form

$$
\frac{3}{5} a^{5 / 3}=\int_{.}^{r} \frac{1.0 \times 10^{-B} n_{e}^{1 / 3}(r) T_{e}^{5 / 3}(r)}{m_{i}^{1 / 3} \mu} d s,
$$

where $s=r_{p}-d$ is the penetration distance along a pernendicular injection trajectory aimed toward the plasma center $(r=0)$. Asymmetric pellet-surface ablation and consequent deflection of the pellet trajectory has peen observed on the ZT-40M experiment [33] but is ignored in this model. The TITAN radial profiles of electron temperature, $T_{e}(r)$, and density, $n_{e}(r)$, are illustrated in Figure 5.6-1, and represent the profiles obtained by the 1-D plasma simulations fitted by, respectively, Equations 5.3-10 and 5.3-11.

Ablation of the pellet while traversing the scrape-off region between the first wall and separatrix is typically small but included in the present calculation. Ablation of the pellet, as the pellet penetrates the plasma, contributes to the local plasma parricle inventory. The model assumes that the radial plasma-transport tirne $\left(\tau_{D} \simeq 20 \mathrm{~ms}\right)$ is much longer than the parallel-transport time ( $\tau_{\|} \simeq 240 \mu \mathrm{s}$ ), which in turn is much longer 


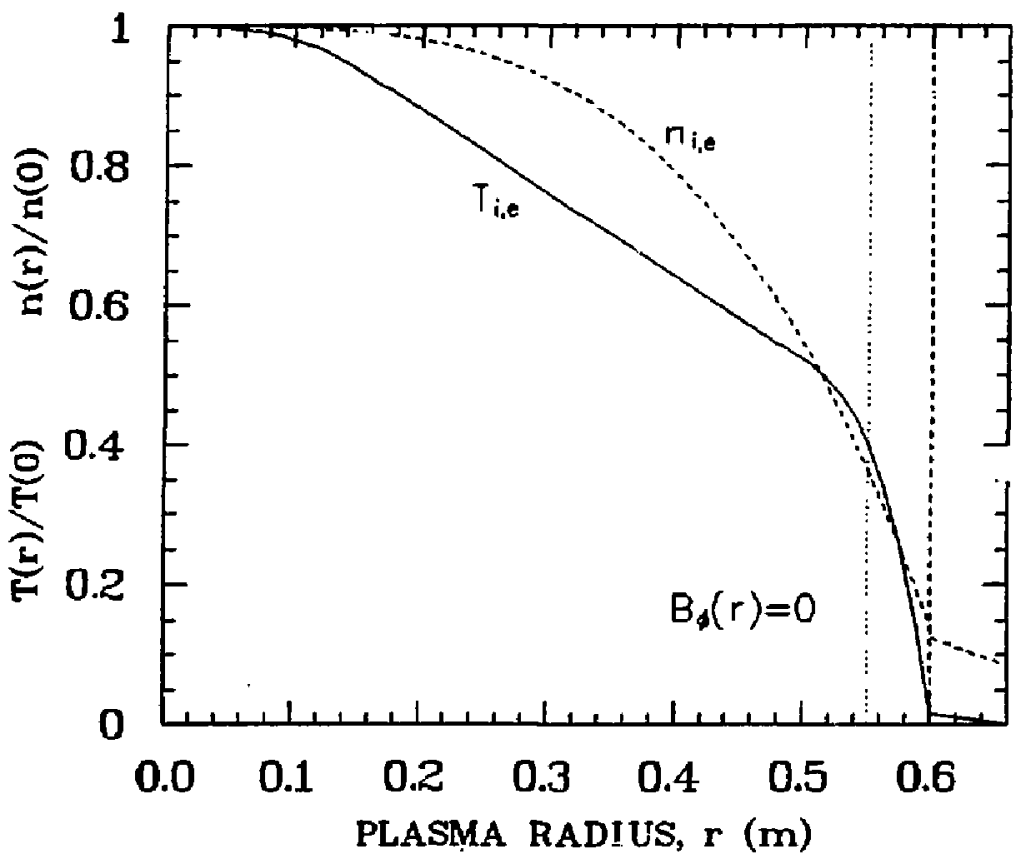

Figure 5.6-1. Radjal profiles of electron temperatures, $T_{e}(r)$, and density, $n_{e}(r)$, used in the calculation of pellet injection/ablation. The reversal surface $\left(B_{\phi}=0\right.$ at $\left.r_{\mathrm{r}}=0.55 \mathrm{~m}\right)$ and plasma separatrix $\left(r_{\mathrm{p}}=0.60 \mathrm{~m}\right)$ are indicated by, respectively, dotted and dashed vertical lines.

than the time for a pellet to cross the flux surface $\left(\tau_{\text {pel }} \simeq 5 \mu \mathrm{s}\right)$. The pellet, therefore, first ablates, then the particles spread out along the flux surface, and finally particles diffuse radially to readjust plasma profiles. Larger initial pellet radius or higher injection speed allows deeper penetration into the plasma. It is desirable to limit the individualpellet particle inventory to a smali fraction, $g$, of the nominal plasma particle inventory to reduce fluctuations in the plasma density and fusion power during refueling. Ablation caused by fusion-product alpha particles is ignorable for the shallow penetration case of interest to TITAN. If deep penetration is required, incremented injection speed must be provided to overcome the additional ablation from energetic alpha particles, although the magnitude of this effect is not well known and may, in fact, be negligib?. 
The time interval between pellet injections is given by

$$
\tau_{I}=\frac{\boldsymbol{M}_{p}}{\dot{M}_{p}},
$$

where $\dot{M}_{\mathrm{p}}=1.22 \times 10^{-4} \mathrm{~kg} / \mathrm{s}$ is the required mass flow rate and $M_{\mathrm{p}}$ is the peilet mass (with $\rho_{o}=250 \mathrm{~kg} / \mathrm{m}^{3}$ and $n_{o}=3.18 \times 10^{20} \mathrm{~m}^{-3}$ ). Pellet fueling parameters for several combinations of assumed ratios of initial pellet-to-plasme inventory, $g$, and normalized penetration distance, $d / r_{p}$, are summarized in Table 5.6-I. Results for the deep penetration options are included; advanced acceleration technologies would need to be developed in such cases, however. It may be desirable to synchronize the pellet injection time with the OFCD oscillation period (Section 7) to optimize profile control. As seen from the results presented in Table 5.6-I, a $g$-value just below 0.05 provides this benefit. An injection speed $u \simeq 2.1 \mathrm{~km} / \mathrm{s}$ results in a penetration depth $d / r_{p} \simeq 0.15$, consistent with the goal of pellet deposition iust inside of the reversal layer. This speed is consistent with that available from near-term acceleration technology [28].

A more advanced model [34] of pellet ablation has also been applied in the TITAN study. In this model, illustrated schematically in Figure 5.6-2, an energy balance is made at the plasma surface [34] to yield the following relationship:

$$
\lambda_{p}^{m} \dot{N}=-\lambda_{s}^{m} n_{r}^{m} 4 \pi a^{2} \dot{a}=4 \pi a^{2} Q_{p},
$$

where,

$$
\dot{a}=-\frac{Q_{p}}{\lambda_{\dot{q}}^{m} n_{\boldsymbol{q}}^{m}},
$$

$\lambda_{p}^{m}$ is the molecular heat of sublimation, $\dot{N}$ is the atomic evaporation rate at the pellet surface, $n_{p}^{m}$ is the molecular density of the solid, $a$ is the pellet radius, and $Q_{p}$ is the effective incident energy-flux averaged over the pellet surface. All energy reaching the pellet surface is assumed to result in evaporation rather than bulk heating of the pellet (i.e., energetic runoway electrons are ignored).

A detailed fit to a separate hydrodynamic solution $[34,35]$ of the expansion of the neutral-particle ablation cloud then gives the pellet surface ablation rate,

$$
\dot{a}=-\frac{1.25}{2 a n_{a}^{m}} \int_{a}^{r_{0}} n^{\alpha}\left[q^{\circ} a 0.5(\gamma-1)\right]^{1 / 3} d l,
$$

where,

$$
q^{\circ}=\frac{k_{B}\left(Q_{o}-Q_{p}\right)}{2 A_{p} m_{p} \int_{a}^{\tau_{0}} n^{\circ} d l}
$$


Table 5.6-I.

TITAN PELLET-FUELING PARAMETER VARIATIONS ${ }^{(a)}$

$\begin{array}{cccc}\text { Pellet inventory/plasma inventory } & 0.01 & 0.05 & 0.10 \\ \text { Pellet radius, a (mm) } & 0.97 & 1.71 & 2.15 \\ \text { Pellet injection interval, } \tau_{I}(\mathrm{~ms}) & 3.69 & 18.5 & 36.9^{(b)} \\ \text { Pellet mass, } M_{p}(\mathrm{~kg}) & 1.04 \times 10^{-7} & 5.20 \times 10^{-6} & 1.04 \times 10^{-0} \\ & & & \\ \text { Penetration depth, } d / r_{p} & & \text { Pellet Speed, } u(\mathrm{~km} / \mathrm{s}) \\ 0.05 & 0.48 & 0.19 & 0.13 \\ 0.10 & 2.26 & 0.93 & 0.63 \\ 0.15 & 5.16 & 2.11 & 1.43^{(c)} \\ 0.20 & 8.80 & 3.59 & 2.45 \\ 0.25 & 13.1 & 5.34 & 3.63 \\ 0.50 & 44.1 & 18.0 & 12.3 \\ 0.75 & 92.6 & 37.9 & 25.8 \\ 1.00 & 155.2 & 63.5 & 43.2\end{array}$

(a) Fixed parameters: $n_{e}=9.39 \times 10^{20} \mathrm{~m}^{-3}, T_{e}=9.5 \mathrm{keV}, r_{p}=0.60 \mathrm{~m}$, $\tau_{p i}=3.6 \mathrm{~s}$, and DT mass injection rate, $\dot{M}_{\mathrm{p}}=2.82 \times 10^{-5} \mathrm{~kg} / \mathrm{s}$.

(b) Synchronization with the oscillations of OFCD at $f=25 \mathrm{~Hz}$ would imply a pellet injection interval of $40 \mathrm{~ms}$ at a slightly higher value of $g$.

(c) Reference case consistent with near-term acceleration technology [28]. 


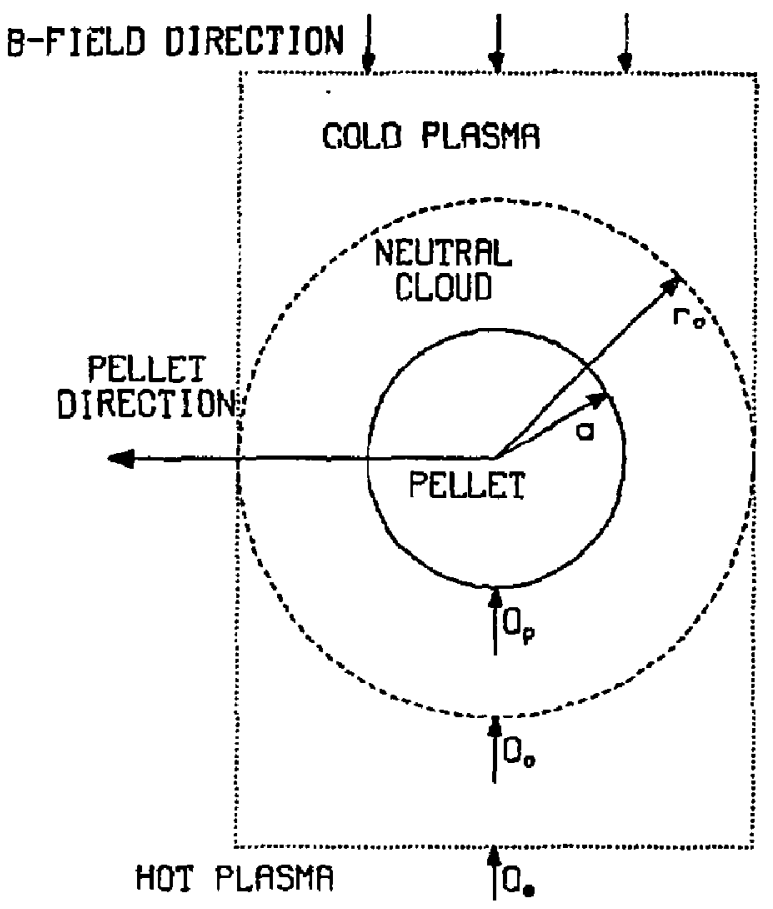

Figure 5.6-2. Schematic representation of the advanced (spherical) pellet-ablation model [34].

where $q^{\circ}$ is the net energy flux normalized to the thickness of the neutral cloud, $\gamma=7 / 5$ is the ratio of specific heats for hydrogenic gas, $m_{p}$ is the mass of a proton, $A_{p}$ is the atonic mass number of the peliet species, $Q^{\circ}$ is the incident energy-flux averaged over the surface of the neutral cloud, $n^{\circ}$ is the atomic density in the neutral cloud, $r^{\circ}$ is the radius of the neutral cloud. and the integral represents the thickness of the neutral cloud. Additional features of the model [34] include the following:

- A multiple-energy-group (number of energy groups, NEG =1 to 10) description of the Maxwellian electron-energy distribution at the cloud surface is used in calculating the heat flux penetrating to the pellet.

- A self-limiting-ablation model accounts for the depletion of electrons from energy groups within a flux surface.

- A cold-plasma-shielding model accounts for the cold plasma formed by jonization of the neutral particle cloud. 
Results of the simple and advanced models are compared in Figure 5.6-3. Agreement is satisfactory only for the shallow penetration case of interest to applications. For deep (central) penetration, the advanced model predicts significantly higher required pellet-injection speeds and implies the required development of advanced acceleration technologies.

The local perturbations of the TITAN plasma density and temperature profiles are calculated by the advanced model and illustrated in Figure 5.6-4. As expected, density increases locally where the pellet is ablated and the edge region temperature is suppressed prior to particle diffusion and energy equilibration. Results depend upon which features of the advanced model are invoked. Best agreement between the simple and advanced model occurs (for the shallow penetration regime) when multiple-energy-group and coldplasma-shielding effects are includea; self-limiting ablation is relatively unimportant for shallow penetration.

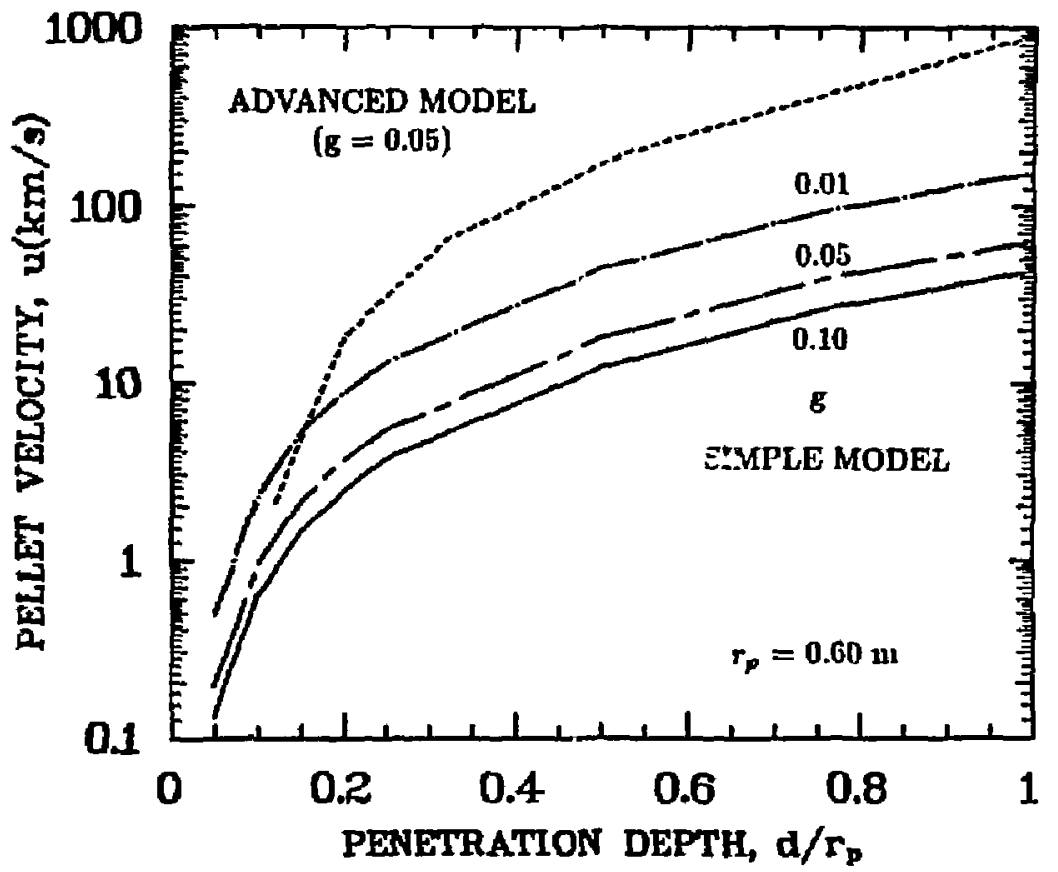

Figure 5.6-3. Comparison of pellet penetration results from the simple and advanced [34] models used in the TITAN study. Agreement is satisfactory only for the shallow penetration case of interest to TITAN. 

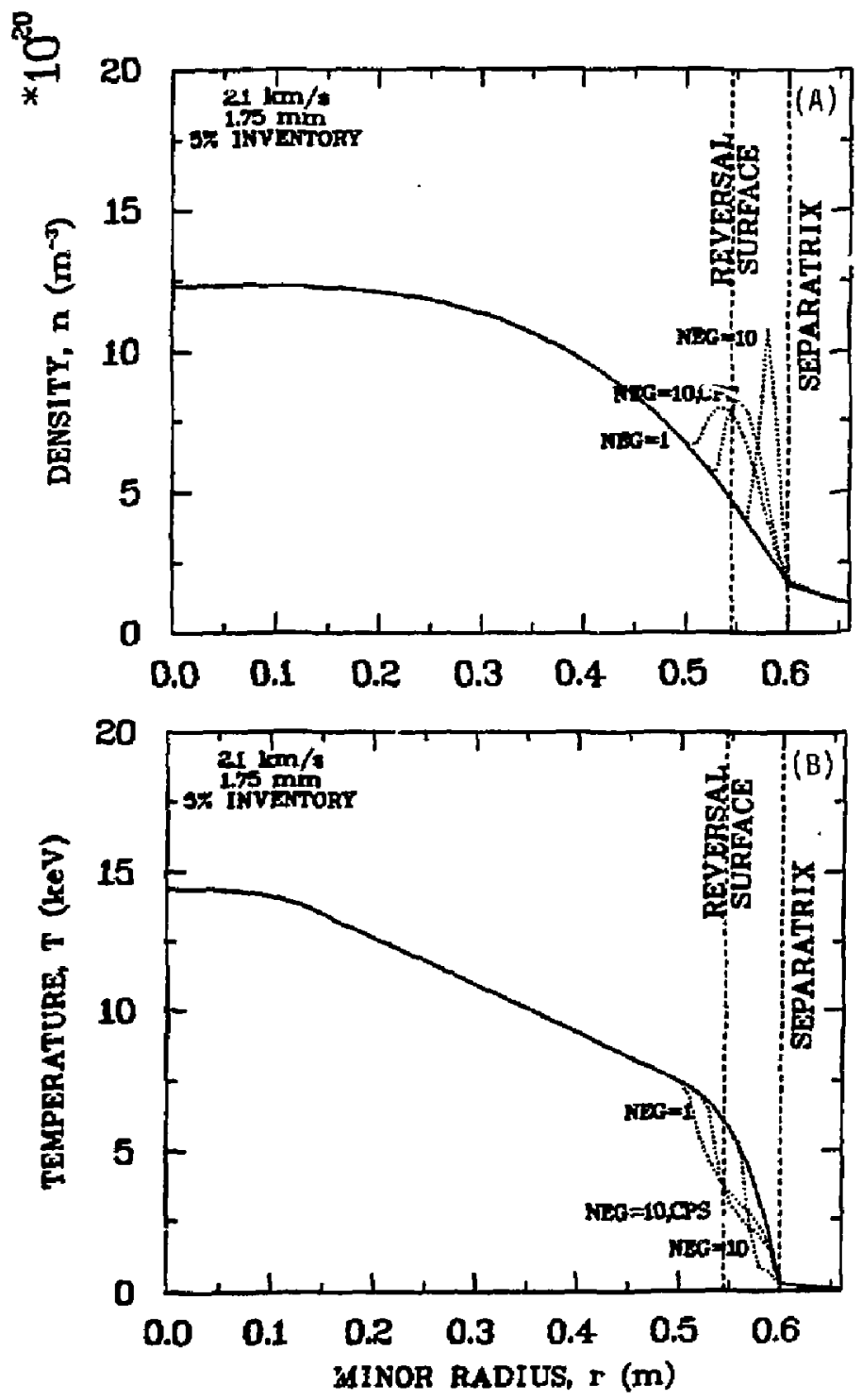

Figure 5.6-4. Local perturbation of the TITAN plasma density (A) and temperature (B) profiles caused by pellet ablation calculated by the advanced model [34]. Options invoked by the advanced model include cold plasma shielding and the number of energy groups, NEG $=1$ to 10 . 
In summary, fueling of the TITAN RFP reactor appears to be relatively straightforward, A pellet injector based on present technology can inject $2.1-\mathrm{km} / \mathrm{s}$ pellets past the reversal radius $\left(r_{\mathrm{r}} \simeq 0.55 \mathrm{~m}\right)$. Presently available injector frequencies $(\sim 6 \mathrm{~Hz})$ will bave to be increased to $\sim 25 \mathrm{~Hz}$ for TITAN applications. For fixed injection speed, pellets do not penetrate as far as the simple-nit model $(d=0.07 \mathrm{~m}$ versus $0.09 \mathrm{~m}$ at $u=21 \mathrm{~km} / \mathrm{s})$, particularly for deep penetration $(d=0.19 \mathrm{~m}$ versus $0.60 \mathrm{~m}$ at $u=64 \mathrm{~km} / \mathrm{s})$. A single large pellet penetrates as deeply as two sequential small pellets of equal total inventory. Pellets composed of TT penetrate slightly deeper than DD pellets $(d=0.13 \mathrm{~m}$ versus $0.12 \mathrm{~m}$ for $u=21 \mathrm{~km} / \mathrm{s} 1.75-\mathrm{mm}$ pellets), but the difference does noi justify DT isotopic separation. Future work should self-consistently incorporate the pellet-refueling source profiles into the temporal 1-D, core-plasma transport description to evaluate the profile effects on global plasma characteristics. Also, a model [33] of asymmetric pellet ablation (Section 2.3.3) remains to be applied to RFP reactors.

It is assumed that TITAN will be provided with two injection systems (5 M\$ each) for redundancy. The injectors themselves can be placed relatively remote from the TITAN FPC (e.g., outside the vacuum task for TITAN-I or well above the TITAN-II FPC to avoid penetrations through the water pool enclosure.) Each injector would occupy an essentially cubic box with a volume of approximately one cubic meter [28]. An evacuated drift tube would connect each injector to the FPC in the vicinity of the divertors to avoid penetrations through the blanket/shieid subsystems.

\subsection{SUMMARY AND CONCLUSIONS}

Extensive stvdies of the TITAN burning plasma have been performed in order to analyze the plasma behavior and performance (e.g., equilibrium, stability, and transport), and to study key plasme-support systems (e.g., current drive, fueling, impurity control, and particle exhaust). As a whole, these analyses provided detailed descriptions of the burning plasma for all engineering activities and design efforts. The results also provide information to the system analysis activity for improving parametric systems models that were then used to generate new cost-optimized design points for further conceptual design and analysis.

Although two separate fusion-power-core designs were studied for the TITAN reactor, lithium cooled for TITAN-I and an aqueous solution of a lithium sali as the coolant and breeder for TITAN-II, both designs had the same plasma parameters. Therefore, must of the burning-plasma simulation effort was not duplicated and, although there are certain 
references specifically to the TITAN-I design, only minor modifications would be required for TITAN-II.

The TITAN burning-plasma simulations include analyses of the equilibrium and stability of the TITAN plasma (Section 5.2). These equilibrium analysen were performed using a large-aspect-ratio approximation. Two-dimensional equilibrium analysis, however, was also performed to substantiate the accuracy of such an approximation for the TITAN effort. The most important equilibrium and stability issue is the need for a conducting shell. The existence and role of a close-fitting conducting shell that surrounds the RFP strongly impacts all physics and engineering aspect of the design.

Experimental observation oi the scaling of RFP plasma pressure with the toroidal current, $n T_{e}(0) \propto I_{\phi}^{2}$, is suggestive of operation near a $\beta$ limit. Under this condition, the intrinsic transport would adjust by MHD activity, radiation, or other mechanism to lose energy at a sufficient rate to maintain $\beta_{\theta}$ constant. Taking advantage of this RFP characteristic, the TITAN plasma operates with a highly radiative core plasma, deliberately doped with $\mathrm{Xe}$ impurities, to reduce the heat flux on the divertor plates. One-dimensional transport simulations of the TITAN core plasma were performed to study the behavior of highly radiative core plasmas (Section 5.3). It was found that high- $Z$ impurities are preferable since the required core-radiation fraction can be achieved with a smaller amount of impurity (and smaller $Z_{\text {eff }}$ ). Important physics issues remain that should be further explored by the RFP program. They include: (1) $\beta$-limited confinement scaling in the reactor plasma regime; (2) limit on the local beta; (3) the level and mechanism of the intrinsic transport; (4) the collapse of the temperature profile due to impurity injection; (5) the interaction of competing profile effects (e.g., refueling, alphs-particle transport, $T_{i}: T_{e}: n$ split); (6) the impact of the impurities on the edge plasma and the wall (e.g., sputtering, wall buildup, vacuum, and tritium systems); and (7) the effects of the impurities on the start-up transient.

The characteriatics of the edge plasma have been of major importance in driving the TITAN design, and extensive simulations of the edge plasma have been undertaken during the study. To obtain a self-consistent view of the behavior of the plasma and neutral purticles, this analysis has been coupled to the modeling of the cure plasma (Section 5.3) and the neutral particle transport (Section 5.5). The TITAN reactors use three toroidal-field divertors with an open geometry. Edge-plasma analysis suggests that the divertor design for the TITAN high-power-density RFP reactor is feasible, provided that operation in a radiation-dominated regime is possible, with only a sanall fraction of the steady-state plasma heating power deposited on the divertor target. An intensely recycling $(R=0.995)$, high-density $\left(\pi_{e} \sim 10^{21} \mathrm{~m}^{-3}\right)$, low-temperature $\left(\mathrm{T}_{e} \sim 5 \mathrm{eV}\right)$ stable 
divertor plasma is produced, with adequate ash removal. A high- $Z$ material is required for the divertor target in order to avoid excessive sputtering from the alpha particles even at the low plasma temperatures. This requirement has led to the inclusion of a 2-mmthick tungsten-rhenium armour on the divertor plate. Strong radiative cooling due to the xenon impurity results in low plasma temperature at the first wall $\left(T_{e} \sim 2 \mathrm{eV}\right)$ so that sputtering erosion is insignificant, even accounting for a possible negative sheath at the vanadium wall. Neutral-particle transport calculations with DEGAS indicate that a negligible leakage of particles from the target to the core plasrna should occur and that the neutral gas escaping to the pumps is entiched in He relative to DT by a factor $\sim 3$. Minimal atomic data exist for cross sections and cooling rates of high- $Z$ elements at low plasma temperatures $\left(T_{e} \leq 100 \mathrm{eV}\right)$. In as much as the high radiation regime appears essential for any high-power-density reactor, an experimental program to improve this data base would be of great value.

The TITAN burning-plasma simulations incorporate the latest understanding and models developed for reversed-field pinches (RFPs), as is summarized in Section 2; in several cases, new and improved models had to be developed for the TITAN study. Because of the relative lack of theoretical and experimental data bases for RFPs, the sensitivity of the design point to various physics assumptions has also been investigated (Section 3.4.2). A detailed description of the necessary R\&D areas for compact RFP reactors has also been produced and is reported in Section 8 . 


\section{REFERENCES}

[1] I. B. Bernstein, E. A. Frieman, M. D. Kruskal, and R. M. Kulsrud, "An Energy Principle for Hydromagnetic Stability Problems," Proc. Royal Soc. 244 (1958) 17.

[2] V. D. Shafranov, "Plasma Equilibrium in a Magnetic Field," Reviews of Plasma Physics 2, Consultant Bureau, New York (1966) 103.

[3] D. J. Strickler, J. B. Miller, K. E. Rothe, and Y-K. M. Peng, "Equilibrium Modeling of the TFCX Poloidal Field Coil System," Oak Ridge National Laboratory report ORNL/FEDC-83-10 (1984).

[4] D. C. Robinson, "High- $\beta$ Diffuse Pinch Configurations," Plasma Phys. 13 (1971) 439.

[5] D. C. Robinson, "Tearing-Mode-Stable Diffuse-Pinch Configurations," Nud. Fusion 18 (1978) 939.

[6] J. W. Connor and J. B. Taylor, "Resistive Fluid Turbulence and Energy Confinement," Phys. Fluids 27 (1984) 2676.

[7] V. Antoni, D. Merlin, S. Ortolani, and R. Paccagnella, "MHD Stability Analysis of Force-Free Reversed-Field Pinch Configurations," Nucl. Fusion 26 (1987) 1711.

[8] A. D. Merlin, S. Ortolani, and R. Paccagnella, "Free Boundary MHD Stability Configurations," in Proc. 1/th European Conf. on Cont. Fusion and Plasma Phys., Madrid, Spain (June 22-26, 1987).

[9] S. Ortolani, in Proc. Int. School of Plasma Phys. Course on Mirror-Based and FieldReversed Approaches to Magnetic Fusion, Varenna, Italy (1983).

[10] R. G. Wati and R. A. Nebel, "Sawteeth, Magnetic Disturbances, and Magnetic Flu: Regeneration in the Reversed-Field Pinch," Phys. Fluids 26 (1983) 1168.

[11] R. A. Nebel, "Quasi-Steady Operation of Reversed Field Pinches," Ph. D. Thesis, University of Mlinois (1980).

[12] K, A. Werley "Stable Alfvén-Wave Dynamo Action in the Reversed-Field Pinch," $\mathrm{Ph}$. D. Thesis, University of Illinois (1984).

[13] M. M. Pickrell, J. A. Phillips, C. J. Buchenauer, T. Cayton, J. N. Downing, A. Haberstich, et al, "Evidence for a Poloidal Beta Limit on ZT-40M," Bull. Am. Phys. Soc. 29 (1984) 1403. 
[14] J. P. Freiberg and D. W. Hewett, "Eigenmode Analysis of Resistive MHD Stability by Matrix Shooting," J. Plasma Phys. 26 (1981) 177.

[15] R. S. Massey, R. G. Watt, P. G. Weber, G. A. Wurden, D. A. Baker, C. J. Buchenauer, et al., "Status of the ZT.40M RFP Experimental Program," Fusion Technol. 8 (1) (1985) 1571.

[16] P. Thullen and K. Schoenberg (Eds.), "ZT-H Reversed Field Pinch Experiment Technical Proposal," Los Alamos National Laboratory report LA-UR-84-2602 (1984)

[17] F. Najmabadi, N. M. Ghoniem, R. W. Conn, et al., "The TITAN Reversed-FieldPinch Fusion Reactor Study: Scoping Phase Report," joint report of UCLA, General Atomics, Los Alamos National Laboratory, and Rensselaer Polytechnic Institute, UCLA-PPG-1100 (Januaty 1987).

[18] A. K. Prinja and R. W. Conn, "An Axjally-Averaged Radial Transport Model of Tokamak Eさge Plasmas," J. Nucl. Mater. 128-129, (1984) 135.

[19] A. K. Prinja, R. F. Schafer, R. W. Conn, and H. C. Howe, "Combined Cere/Boundary Layer Transport Simulations in Tokamaks," J. Nucl. Mater. 145147, (1987) 868 .

[20] A. K. Prinja, R. F. Schafer, and R. W. Conn, "Modeling of Plasma Boundary Conditions with Pump Limiters and Divertors," Bull. Am. Phys. Soc. 31 (1986) 1536 .

[21] M. F. A. Harrison, E. S. Hotston, and A. de Matteis, "Plasma Edge Physics for NET/INTOR," NET report EUR-FU/XII-361/86/50 (1985).

(22] D. E. Post, R. V. Jensen, C. B. Tarter, W. H. Grasberger, and W, A. Lokke, "SteadyState Radiative Cooling Rates for Low-Density High-Temperature Plasmas," Atomic and Nucl. Data Tables 20 (1977) 397.

[23] M. Petravic, D. Heifetz, and D. E. Post, "Modelling Analyses of Tokamaks with Divertors and Pumped Limiters," Proe. 10th Int. Canf. Plasma Phys. and Cont. Nucl. Fusion Res., London, U. K. (September 1984) IAEA, Vienna 2 (1985) 103.

[24] D. Heifetz, D. E. Post, M. Petravic, J. Weisheit, and G. Bateman, "A Monte-Carlo Method for Neutral-Particle Transport in Diverted Plasmas," J. Comp. Phys. 46 (1982) 309. 
[25] P. I. H. Cooke and A. K. Prinje, "An Analytic Model for Flow Reversal in Divertor Plasmes," Nucl. Fusion 27 (1987) 1165.

[26] A. K. Prinja and R. W. Conn, "Theory and Modelling of Helium Enrichment in Plasme Experiments with Pump Limiters," Nucl. Fusion 25 (1985) 1775.

[27] S. L. Milora, "Review of Pellet Fueling," J. Fusion Energy 1 (1981) 15.

[28] S. L. Milora, "Plamma Fueling Program," in Fusion Enengy Division Annual Progress Report, Oak Ridge National Laboratory report ORNL-6322 (October 1987).

[29] L. J. Perkins, S. K. Ho, and J. H. Hammer, "Deep Penetration Fueling of ReactorGrade Plesmas with Accelerated Compact Toroids," Lawrence Livermore National Laboratory preprint UCRL-96894 (June 1987), submitted to Nucl. Fusion.

[30] P. B. Parks and R. J. Turnbull, "Effect of Transonic Flow in the Ablation Cloud on the Lifetime of a Solid Hydrogen Pellet in a Plasma," Phys. Fluids 21 (1978) 1735.

[31] R. P. Gilliard and K. Kim, "Significance of Magnetic Shielding in the Ablation of a Solid Hydrogen Pellet in a Plasms," IEEE Thans. Plasma Sci. PS-8 (1980) 477.

[32] W. A. Houlberg, M. A. Iskra, H. C. Howe, and S. E. Attenberger, "Pellet-A Computer Routine for Modeling Pellet Fueling in Tokamak Plasme," Oak Ridge National Laboratory report ORNL/TM-6549 (January 1979).

[33] G. A. Wurden, P. G. Weber, R. G. Watt, C. P. Munson, J. C. Ingraham, R. B. Howell, T. E. Cayton, K. Buechl, and E. J. Nilles, "Pellet Refueling of the ZT-40M Reversed Field Pinch," Nucl. Fusion 27 (1987) 857.

[34] W. A. Houlberg, S. L. Milora, and S. E. Attenberger, "Neutral and Plasma Shielding Model for Pellet Ablation," Oak Ridge National Laboratory report ORNL/TM10556 (October 1987).

[35] S. L. Milora and C. A. Foster, "A Revised Neutral Gas Shielding Model for PelletPlasma Interactions," IEEE Trans. Plasma Sci. PS-6 (1978) 578. 


\section{PLASMA TRANSIENT OPERATIONS}

Farrokh Najmabadi Charles G. Bathke Robert A. Krakowski
Yuh-Yi Chu

Ronald L. Miller 


\section{Contents}

6.1. INTRODUCTION $\ldots \ldots \ldots \ldots \ldots \ldots \ldots \ldots \ldots \ldots . \ldots \ldots$

6.2. RFP START-UP DATA BASE $\ldots \ldots \ldots \ldots \ldots \ldots \ldots$

6.2.1. Breakdown and Formation . . . . . . . . . . . 6-4

6.2.2. Current Ramp-Up . . . . . . . . . . . . 6 6-12

6.3. FORMATION OF THE TITAN PLASMA . . . . . . . . . 6 6-14

6.4. IGNITION REQUIREMENTS $\ldots \ldots \ldots \ldots \ldots \ldots$. . . . . . . . . . .

6.4.1. Particle Balance . . . . . . . . . . . . . . . 6-20

6.4.2. Power Balance . . . . . . . . . . . . . 6-21

6.4.3. Energtic Alpha Particles . . . . . . . . . . . 6-23

6.4.4. Ignition Analysis $\ldots \ldots \ldots \ldots \ldots \ldots \ldots \ldots$

6.5. CURAENT RAMP-UP TO IGNITION AND BURN $\ldots \ldots \ldots \ldots$. . . . 6-29

6.5.1. Start-Up Sequence . . . . . . . . . . . . . . . 6-29

6.5.2. Circuit Model . . . . . . . . . . . . . . . 6-34

6.5.3. Plasma-Circuit Simulations $\ldots \ldots \ldots \ldots \ldots \ldots$. . . . . . .

6.6. SHUTDOWN \& TERMINATION OF TITAN PLASMA $\ldots \ldots \ldots \ldots$. . . . 65

6.6.1. Plasma Accidents . . . . . . . . . . . . . . . 6 6-56

6.6.2. Shutdown Frocedures $\ldots \ldots \ldots \ldots \ldots \ldots \ldots$. . . . . . . .

6.6.3. Termination Simulations . . . . . . . . . . . . . 6-57

6.7. SUMMARY AND CONCLUSIONS $\ldots \ldots \ldots \ldots \ldots \ldots$ 6-62

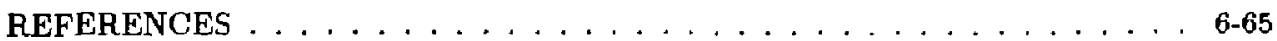




\section{PLASMA TRANSIENT OPERATIONS}

\subsection{INTRODUCTION}

A typical reversed-field-pinch (RFP) experimental discharge can be divided into four phases: (1) breakdown and formation, (2) current ramp-up, (3) sustainment, and (4) termination. A representative time history of an RFP dircharge, taken from ZT-40M experiments [1], is shown in Figure 6.1-1. Ignition and fusion burn in a reactor are achieved during the current ramp-up phase, and operation of the current-drive system is required during the sustainment phase. The brealrdown and formation phase encompasses the time from the start, which begins by establishing a toroidal magnetic field inside the discharge chamber in the absence of the plasma, to the formation of a "seed" RFP plasma. At the time of peak toroidal magnetic fieid, poloidal-field windings are activated to produce a flux chatige through the center of the torus and, consequently, a toroidal voltage around the discharge chamber. This voltage typically ionizes the gas in a few microseconds and the toroidal current is initiated in the resulting plasma. The toroidal plasma current and the toroidal magnetic field within the plasma increase while the toroidal magnetic field at the wall decreases, keeping the average toroidal field (and the toroidal flux) in the chamber almost constant. Eventually the toroidal magnetic field at the wall changes sign and is crowbarred in the reverse direction relative to the back-biased condition of the breakdown and formation phase.

The plasma current is then increased to the peak value during the curreit ramp-up phase. The poloidal-field (PF) coil system provides the poloidal flux and the majority of toroidal flux contained within the full-current plasma by the RFP dynamo. The duration of an RFP experiment is extended by using either a passive crowbar applied to the poloidal circuits, to give a decaying waveform, or an active (power) crowbar to produce a flat-top current waveform. For a steady-state device, the sustainment phase requires operation of the current-drive system (oscillating-field current drive for TITAN).

Reversed-fielkl-pinch discharges normally end abruptly and the plasma current decreases rapidly to zero. Accompanying this fast current "termination" is a positive pulse in the toroidal voltage at the liner, as is: also shown on Figure 6.1-1. This current termination is in contrast to the negative spike in toroidal voltage that accompanies the current disruption in a tokamak, indicating a difference in the flow of magnetic energy to or from the plasma during the respective events. Furthernore, the RFP current termi- 
0-2
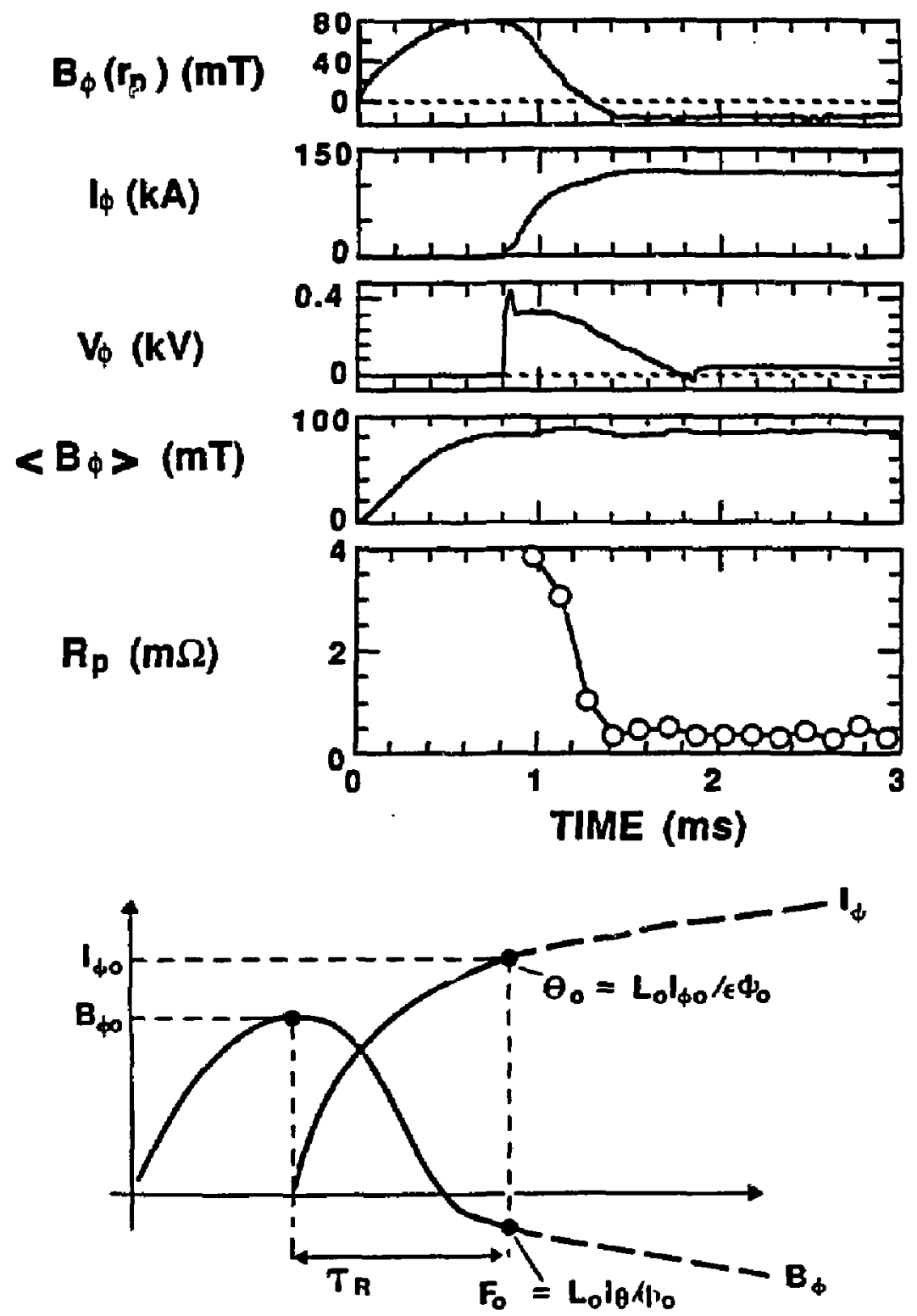

Figure 6.1-1. Typical matched-mode RFP formation for ZT-40M leading to the values of $\Theta_{a}, F_{o}$, and $I_{\phi o}[1]$ used as initial conditions for start-up, ignition, and burn simulations. 
nation can be influenced through the control of the density or toroidal magnetic field [2]; generally, the RFP terminates only when toroidal-field reversal is lost.

It became evident towards the end of the early RFP reactor studies [3] and during the scoping phase of the TITAN study [4] that the design limits for both the toroidalfield (TF) and PF coil systems would be determined more by the plasma breakdown, formation, and ramp-up transients than by the steady-state operational phase. The desire to use the RFP dynamo to generate internal totoidal flux (rather than injecting all the toroidal flux by the TF coils) and the constraints on bias stress and power strongly influence the TF and PF coil designs. Furthermore, the PF-coil configuration determines the coupling of ohmicheating (OH) coils with the plasma, the magnitude of the stray vertical field, and the degree of multipolarity of field nulls in the plasma chamber. These in turn influence the breakdown and RFP formation conditions through the amount of initial (vacuum) toroidal field, $B_{\phi \circ}$, and ultimately affect the TF-coil design.

Section 6.2 reviews the existing RFP experimental data base for RFP formation and start-up, which is then applied to the TITAN designs (Section 6.3). The ignition requirements for ohmically heated RFPs are studied (Section 6.4) in order to identify the optimum path for TITAN start-up. Using the TITAN seed RFP conditions, a 0-D, profileaveraged plasma-circuit code is used to simulate the evolution of the TITAN plasma through current ramp, ignition, and burn transients (Section 6.5). Current termination is a safety and economic concern because of large magnetic stored energy in the TITAN plasma. Techniques for control of current termination and plasma shutdown, leading to a "soft-landing," are discussed in Section 6.6. Summary and conclusions are given in Section 6.7.

\subsection{RFP START-UP DATA BASE}

A body of experimental evidence is beginning to accumulate that better defines the windows for RFP breakdown, formation, and current ramp-up. The status in each of these areas is summarized. Although much of this information is not fully understood theoretically and extrepolation from ZT-40M-class experiments to a reactor is uncertain, nevertheless this information and experience is assimilated for the first time and used as part of the TITAN study [4].

Three modes of operation are generally used for RFP formation in present-day experiments: self-reversal, matched, and aided-reversal. In the self-reversal mode, a conducting shell maintains and conserves the toroidal flux inside the chamber. The self-reversal mode 
of RFP formation is used on OHTE/RFP and often on HBTX1A. In the matched mode, the external circuits are programmed to conserve the toroidal flux inside the chamber by maintaining the poloidal electric field, $E_{\theta}$, near zero at the liner, thereby simulating the action of a conducting shell. This mode is usually used on ZT-40M. In the aided-reversal mode, the external circuits supplement the plasma self-reversal effect, as is typically used on ETA-BETA-II. Field control during the formation phase provides flexibility in varying the pinch parameter, $\Theta$, on which the configuration depends. The choice of the formation mode also affects the consumption of the poloidal flux during this phase of the RFP discharge. The final plasma parameters, however, are not particularly sensitive to the mode used for RFP formation.

In a conventional start-up sequence, the peak current is nearly reached at the time the toroidal field at the wall reverses (end of the formation phase). This start-up mode is undesirable in a large experiment or in a reactor because: (1) the RFP formation phase is power intensive until reversal is reached, and (2) large voltages are required. It is advantageous to set up the RFP configuration in a relatively short time at a low current and low stored energy and then slowly increase the current to the desired value while maintaining the RFP profiles. This mode of RFP start-up, called the ramped start-up, has been demonstrated on the ZT-40M experiment. Figure 6.1-1 shows such a ramped start-up, as well as the formation phase and key notation.

The ramped start-up scenario relies on the plasma relaxation process. During the current ramp, the toroidal flux must be increased proportionally to the current to maintain the RFP profiles while holding $F$ and $\Theta$ constant. This process requires the generation of toroidal flux by the RFP dynamo, since the toroidal field at the wall is negative while the average toroidal flux within the conducting shell is positive. The plasma must generate an equal and opposite amount of negative and positive flux to satisfy Faraday's law and then expel the negative flux from the plasma to generate a net positive flux increase. Indeed, the ramped discharges show that toroidal flux (Figure 6.1-1) continues to be generated, and negative flux is expelled from the plasma; this process occurs on a multi-millisecond time scale. Ramped start-up operation has also recently been reported for the HBTX1B experiment [5]. The TITAN reactor design also relies on a rapid formation of a "seed" RFP followed by a slow plasma-current ramp to the final value.

\subsubsection{Breakdown and Formation}

Plasma discharge and subsequent RFP formation generally occurs for values of the ratio of toroidal electric field to initial filling pressure, $E_{\phi} / P_{o}$, that are similar to tokamak 
values, but closer to electron runaway conditions. For example, for the JET experiment, a value of $E_{\phi} / P_{\sigma} \geq 0.66 \times 10^{4} \mathrm{~V} / \mathrm{m}$-torr is reported $[6]$, compared to 1 to $2 \times 10^{4} \mathrm{~V} / \mathrm{m}$-torr for ZT-40M [7], which is close to electron runaway conditions. Figure 6.2-1 gives typical breakdown and formation characteristics for a range of tokamaks [8] and for ZT-40M [9, 10]. Generally, breakdown and discharge formation are not problems for RFPs, but the degree of pre-ionization can greatly influence the discharge quality and poloidalflux consumption $[9,10]$. Since, to date, stable and reliable RFP formation appears to require a high $E_{\phi}$, the generally common $E_{\phi} / P_{o}$ values for both RFPs and tokamaks give significantly higher values of $P_{0}$ required to create a robust RFP. An initial electric field of $E_{\phi}=55 \mathrm{~V} / \mathrm{m}$ is indicated in Figure 6.1-1 for matched-mode operation.

A toroidal-field line of strength, $B_{\phi o}$, in the presence of a vertical field, $B_{V}$, will intersect the first wall and thereby prevent the formation of a continuous discharge if the ratio $B_{V} / B_{\phi o}$ is too large. The condition for the confinement of a single toroidal trajectory with a field null at a minor radial position, $r_{o}$, is given by

$$
\frac{B_{V}}{B_{\phi o}} \leq \frac{\epsilon}{2 \pi} \sqrt{1-\left(r_{o} / r_{p}\right)^{2}},
$$

where $\epsilon=r_{p} / R_{T}$ is the inverse plasma aspect ratio. For TITAN with $1 / \epsilon \simeq 6.5$ and setting $r_{a} \simeq r_{p} / 4$, Equation $6.2-1$ results in $B_{V} / B_{\phi_{o}} \leqq 0.02$.

In addition, a drift constraint, $E_{\phi} /\left(B_{V} / B_{\infty}\right) \geq 10^{3} \mathrm{~V} / \mathrm{m}$, has been suggested for JET [8]. This drift constraint together with Equation $6.2-1$ results in $E_{\phi} \geq 22 \mathrm{~V} / \mathrm{m}$, which is about a factor of 2 below experimental values derived from the ZT-40M experience. Therefore, breakdown voltage in the range 500 to $1,000 \mathrm{~V}$ may be required for the reactor. Careful designs that minimize field errors can possibly reduce these start-up voltages.

Therefore, in characterizing the TITAN initial conditions, the following possible constraints on the toroidal electric field were considered:

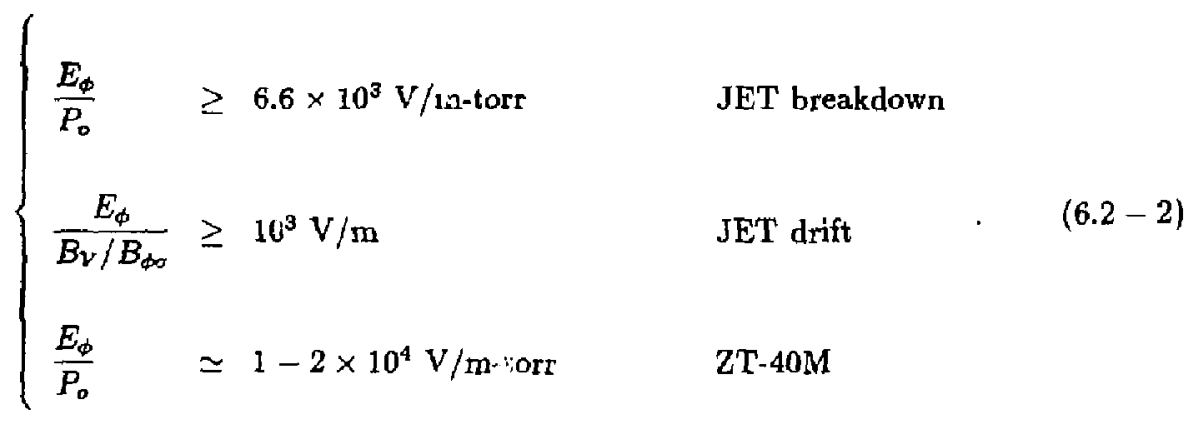



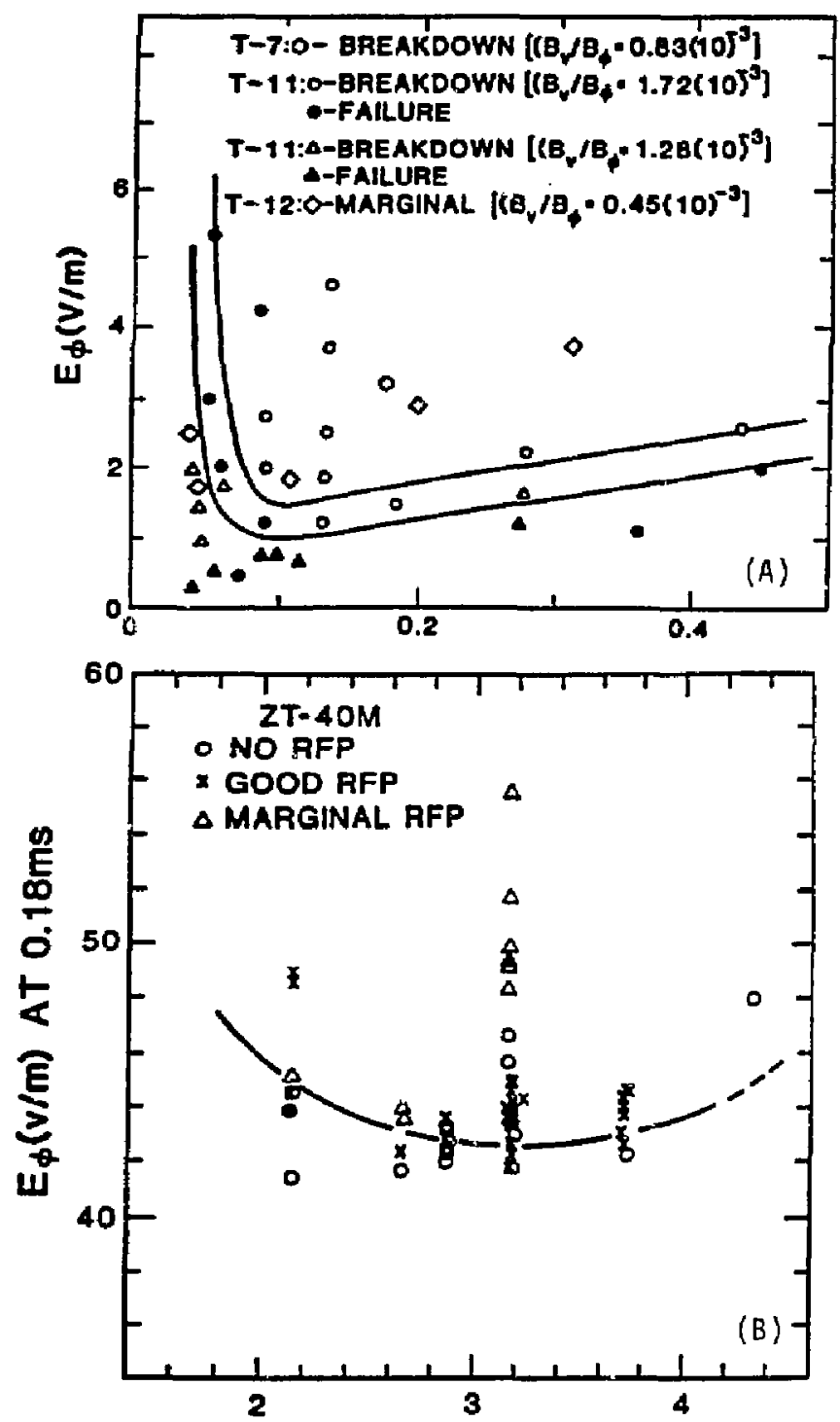

FILLING PRESSURE, $P_{0}$ (mTorr)

Figure 6.2-1. Typical breakdown curves for (A) tokamaik [8] and (B) RFP formation [9, 10]. 
In order to establish the parameters of the seed RFP, the relationship between $B_{\phi_{0}}$ and the average toroidal flux within the initial RFP, $\left\langle B_{\phi}\right\rangle$, must be determined (Figure 6.1-1). Generally, $\left\langle B_{\phi}\right\rangle \simeq B_{\phi 0}$ for RFP formation. Figure 6.2-2 shows the relationship between $B_{\phi o}$ and $\left\langle B_{\phi}\right\rangle$ for a range of ZT-40M discharges, illustrating the experimental basis for this assumption. For a given $\left\langle B_{\phi}\right\rangle$ and the initial pinch parameter, $\theta_{n}$, the initial (minimum) RFP current or current density is determined from

$$
I_{\phi 0}=5 \times 10^{6} r_{\mathrm{p}} \Theta_{\mathrm{o}}\left\langle B_{\phi}\right\rangle
$$

Hence, for $\left\langle B_{\phi}\right\rangle \simeq B_{\phi o}=0.05 \mathrm{~T}$ at formation, and TITAN plasr a conditions of $\Theta_{0} \simeq 1.5$ and $r_{p}=0.6 \mathrm{~m}$, the initial RFP current is $I_{\phi_{0}} \simeq 0.2 \mathrm{MA}$.

Although the specification of $\Theta_{0}$ and $\left\langle B_{\phi}\right\rangle$ at formation determines an initial current density (e.g., $j_{\phi o} \simeq 0.2 \mathrm{MA} / \mathrm{m}^{2}$ for above example for TITAN), other more dominant constraints may -xist. For example, the $2 \mathrm{~T}-40 \mathrm{M}$ experiment exhioits a minimum current-density limit which tronslates erupiricaliy to $j_{\phi o} \geq 0.4 \mathrm{MA} / \mathrm{m}^{2}$, below which RFP formation is difficult. This constraint is not well understood, but the application of such a coistraint to TITAN represents a conservative connection to experiment and for the above condition would requite a coubling of $B_{\psi_{0}} \simeq\left\langle B_{\phi}\right\rangle$. Secondly, a number of RFP experiments [11] have shown an impurity burn-through constraint, shown in Figure 6.2-3 for $2 \mathrm{~T}-40 \mathrm{M}$. For these conditions, burn-through requires that

$$
\frac{j_{\phi}}{n} \geq 10^{-14} \mathrm{Am}
$$

This constraint, however, when applied io th: $0.2-$ to $0.4-\mathrm{keV}$ plasmas expected for these formation conditions may place the plasnua sirongly in the electron runaway regime. If the runaway regime is to be avoided, which may or may not. be a requirement, higher densities will be required (e.g., for $\xi \equiv v_{D} / v_{T H e} \sim 0.0$., $n \geq 2 \times 10^{19} \mathrm{~m}^{-3}$ for $j_{b}=0.4 \mathrm{MA} / \mathrm{m}^{2}$ and $T \simeq 0.2 \mathrm{keV}$ ).

Generally, if the density pump-out is too great prior to toroidal-field reversal for a given initial filling pressure, $P_{o}$, unreliable RFP formation occurs [7], as is shown in Figure 6.2-4(A). S rularly, for a given $P_{0}$, a maximum initial bias field, $B_{\phi o}$, is found above which RFP formation does not occur [7], as is shown in Figure 6,2-4(B). Although RFPs form at lower values of $B_{\phi \infty}$, these RFPs have excessive poloidal-flux consumption, as is also shown in Figure $6.2-4(\mathrm{~B})$, for the $2 \mathrm{~T}-40 \mathrm{M}$ conditions examined. It should be noted that a variable and poorly controlled wall condition creates hystereses and related unpredictable effects in many of these data correlations. The constraints of Figures 6.2-4(A) and (B) have been combined in Figure 6.2-4(C) to eliminate the filling 


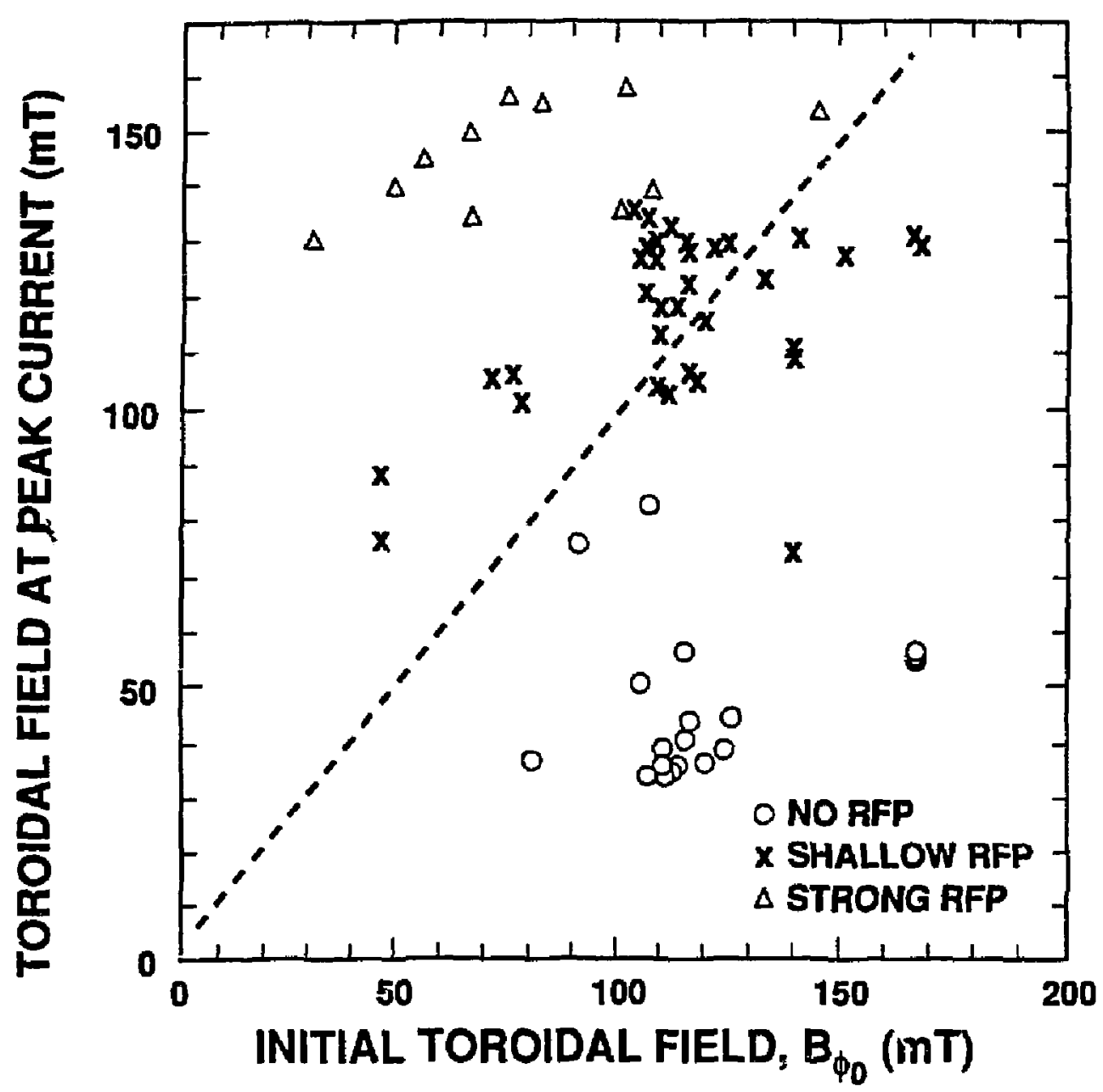

Figure 6.2-2, Relationship between $B_{\phi_{0}}$ and $\left\langle B_{\phi}\right\rangle$ for a range of $Z T$-40M discharges [7, 10) where robust RFP formation occurred, as well as no RFP formation and/or very shallow, spheromak-like RFPs were formed. 


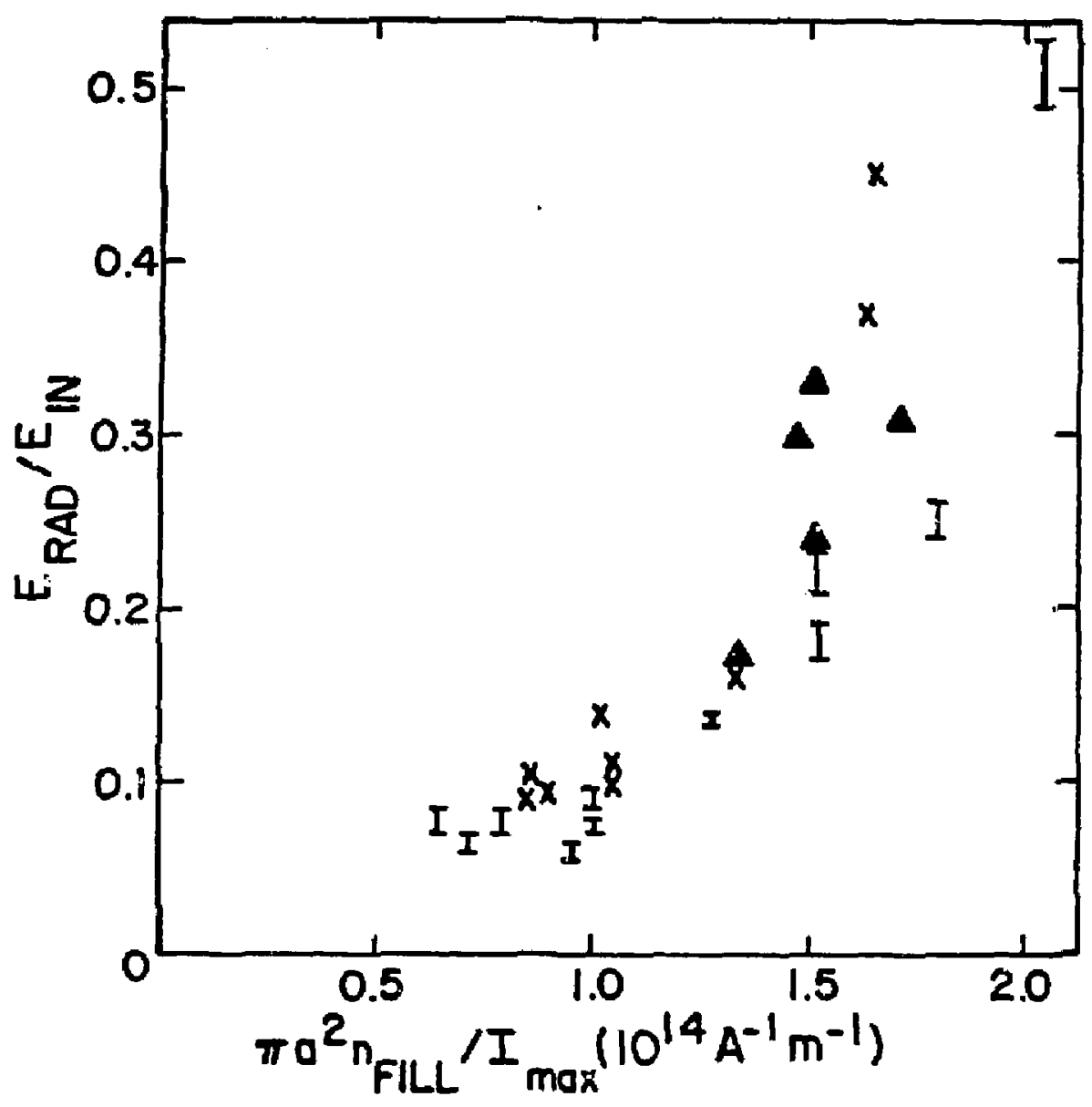

Figure 6.2-3. Typiral impurity burn-through constraint for $2 \mathrm{~T}-40 \mathrm{M}[7]$. 

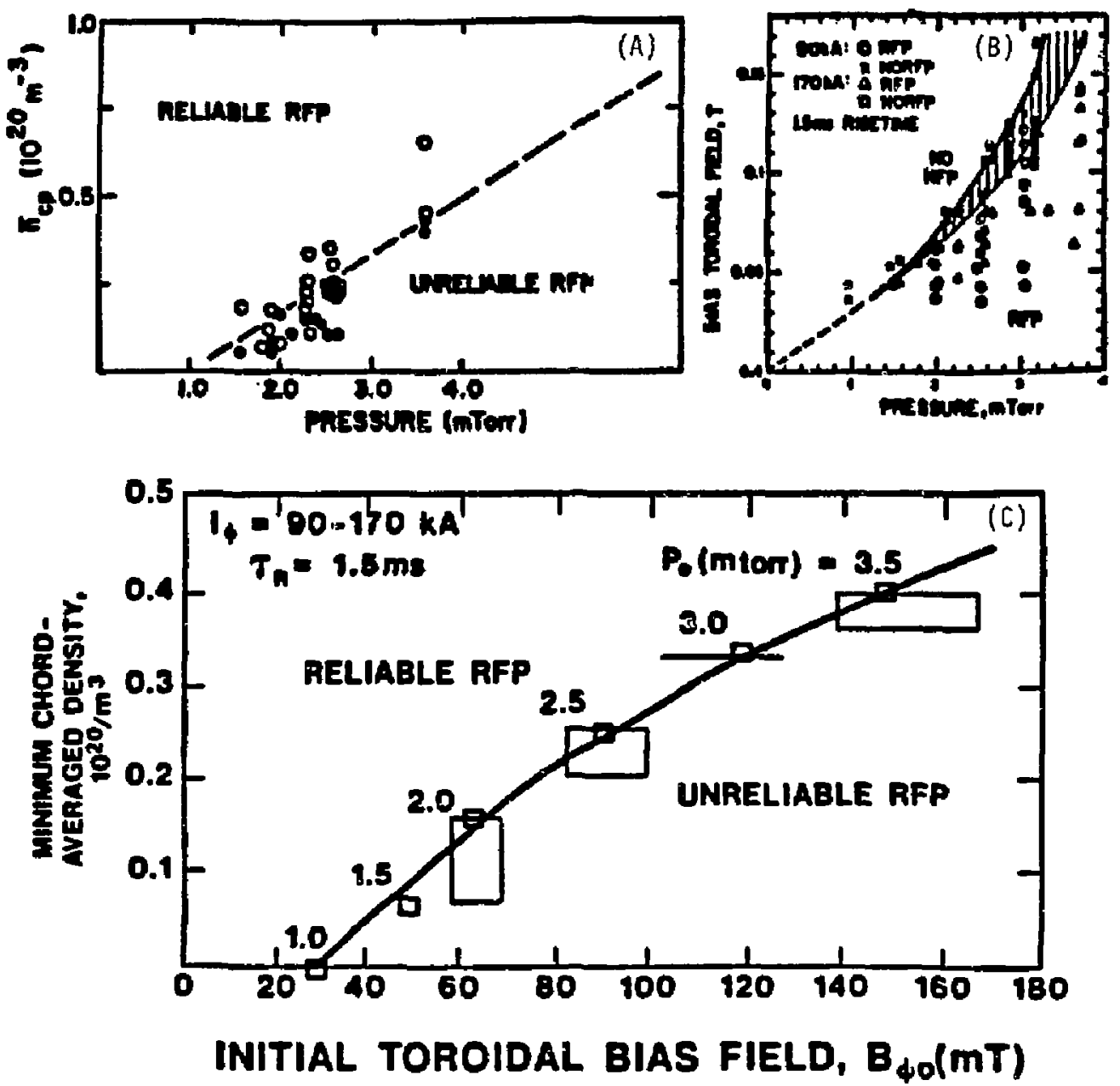

Figure 6.2-4. Typical RFP formation windows showing: (A) dependence on a critical plasma density, (B) magnitude of the initial toroidal bias field, and (C) a combination of the two constraints [ $\tilde{\imath}]$. 
pressure as a variable and perhaps to reduce the impact of these uaresolved wall effecto on these data. The result is a relationship between minimum plasma density and initial bias field that may be useful in assuring robust RFP formation:

$$
n \geq\left\{\begin{array}{ll}
10^{10} & \text { for } B_{\phi 0}<0.036 \mathrm{~T} \\
2.78 \times 10^{20} B_{\phi 0} & \text { for } B_{\phi 0}>0.036 \mathrm{~T}
\end{array} .\right.
$$

Most present-day RFPs experience a significant loss of density or pump-out upon formation, as is shown in Figure 6.2-5. The degree of density reduction between the initial filling pressure and the final RFP formation is not well understood but it depends strongly on wall preconditioning. Hence, the pump-out is expressed in terms of the ratio of initial filling density, $n_{0}$, to the final RFP plasma density, $n$, and the assumption must be made for the reactor that pump-out and $P_{0}$ can be minimized, thereby minimizing the $E_{\phi}$ required under start-up conditions.

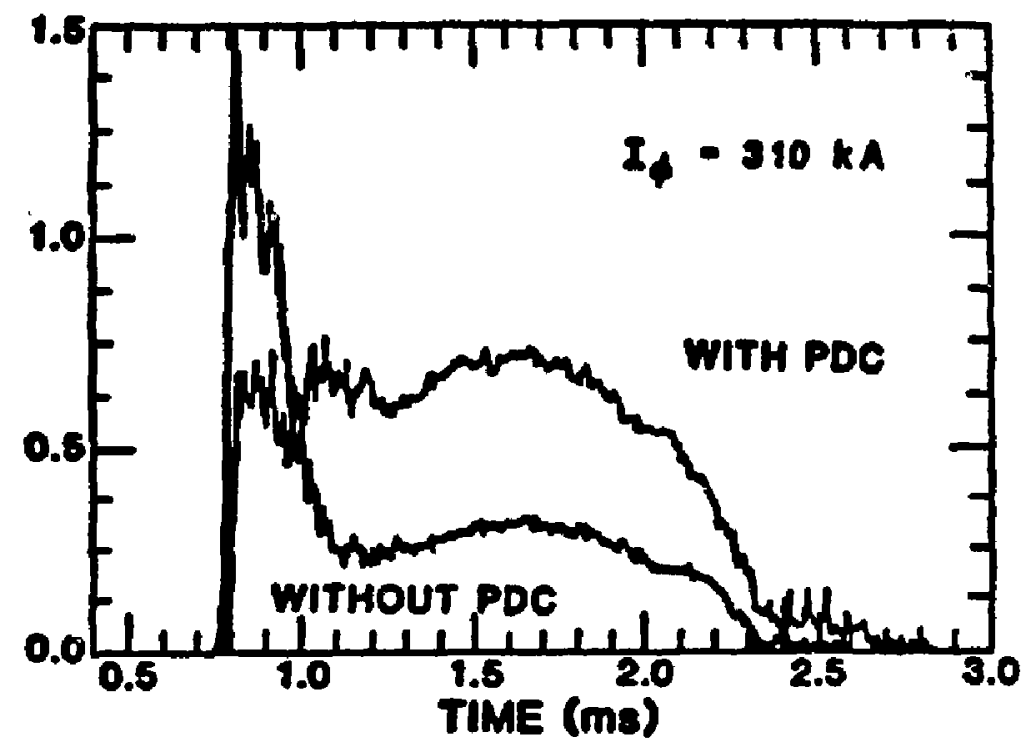

Figure 6.2-5. Loss of density or purmp-out for a typical ZT-40M discharge. Also shown is the impact of pellet fueling on reducing pump-out [12]. 


\subsubsection{Current Ramp-Up}

While the formation phase leading to the low-current $(\sim 0.2-\mathrm{MA})$ seed RFP is characterized by a rapid current rise (150 MA/s for the case shown in Figure 6.1-1), and the flux consumption can be a small fraction of the inductive flux delivered to the plasina, the subsequent current ramp to ohnic ignition and burn in the TITAN plasma $\left(I_{\phi}=18 \mathrm{MA}\right)$ represents a greater concern from the viewpoint of resistive flux consumption and the implication on OH-coil and related power-supply designs.

Figure 6.2-6 shows that slow current ramp rates $(9 \mathrm{MA} / \mathrm{s})$ have been achieved in ZT-40M. These current ramp rates are still large compared to those typical of tokamaks ( 1 to $2 \mathrm{MA} / \mathrm{s}$ ) and are possible in RFPs because of the anomalous penetration of magnetic flux. The slow current ramps of the kind shown are desirable for reactors since the bulk of the flux injection can be supplied directly from the electrical grid at relatively low power, rather than from an expensive on-site energy storage.

A significant decrease in plasma loop voltage is measured upon reversal of the toroidal field and formation of the RFP. This behavior is shown for ZT-40M in Figure 6.2-7, which also shows an optimal value of the pinch parameter from the viewpoint of loop voltage and
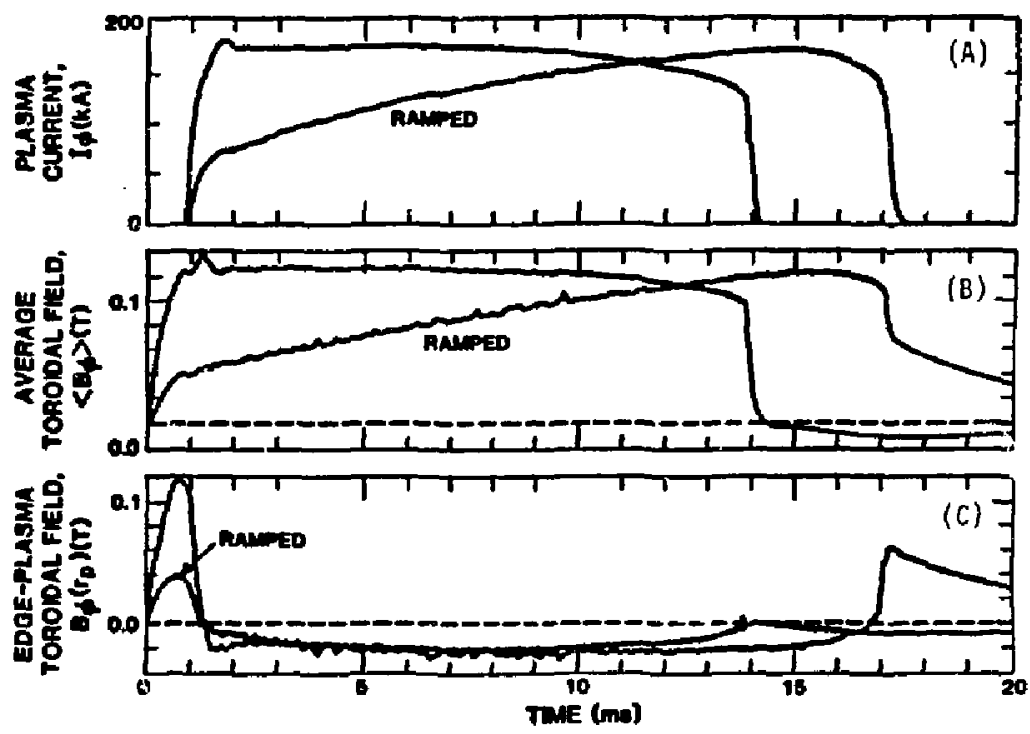

Figure 6.2-6. Slow and fast current ramps in $Z T-40 \mathrm{M}[7]$. 

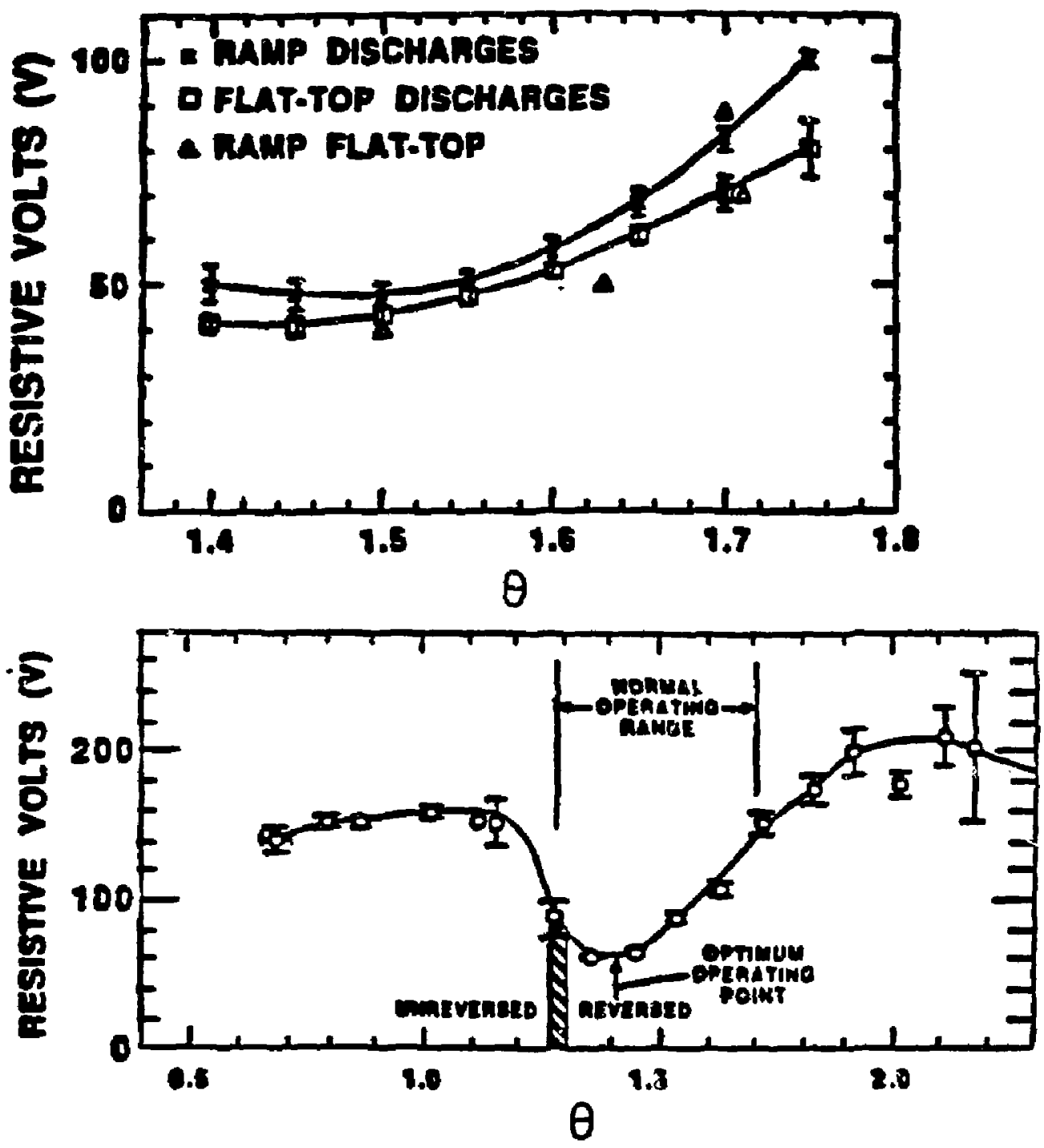

Figure 6.2-7. The dependence of the plasma loop voltage on the pinch parameler upon formation of RFP, showing an optimal value of $\Theta$ \{1]. 
resistive poloidal-flux consumption. The reduction in resistive flux consimption upon controlling $\theta$ at the optimal value is shown in Figure 6.2-8, which gives the resistive volt-second consumption for a range of conditions, including the volt-second efficient matched-mode operation. The Z'Г-40M data shown on Figure 6.2-8 indicate a constant (resistive) voltage scaling ( ${ }_{R E S} \simeq 32.5 \mathrm{~V}$ ), which implies that the plasma resistance is decreasing inversely with plasma current in this region. The implication of this scaling on the TITAN-I reactor design is examined in Section 8.5.3.

\subsection{FORMATION OF THE TITAN PLASMA}

In this section, the set of constraints and options for breakdown and RFP formation described in Section 6.2 are applied to the TITAN plasma formation. The notation used here follows that of the previous section in that a o subscript denotes the parameters of the seed RFP with the exceptions of $B_{\phi o}, n_{0}$, and $P_{0}$ which denote, respectively, the initial (bias) toroidal field, and the fill gas density and pressure. It is further assumed that

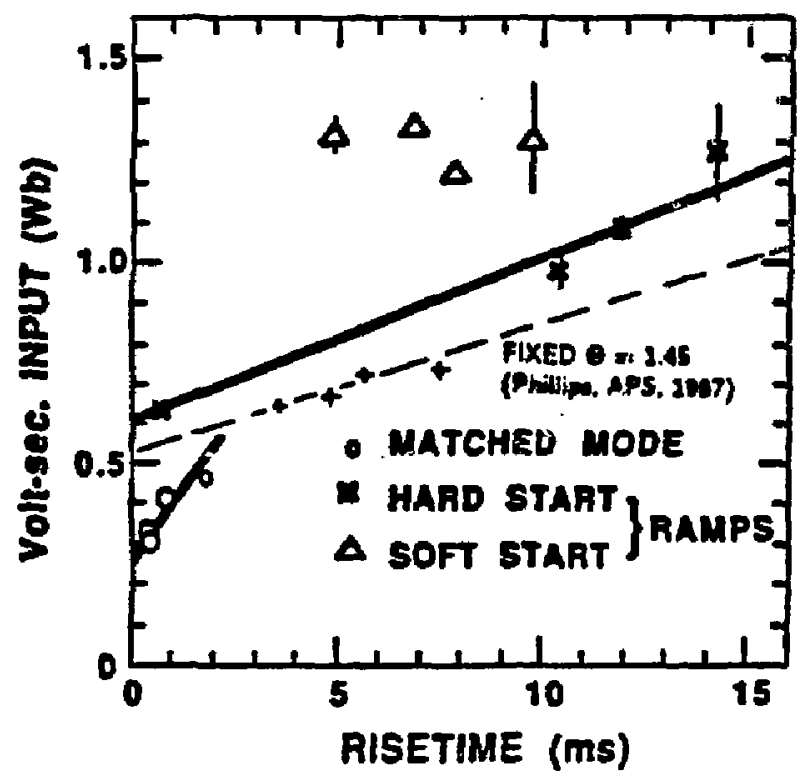

Figure 6.2-8. Dependence of flux input on current risetime for a range of ZT-40M operating conditions $[1,7]$. 
during the RFP formation phase, the toroidal flux inside the plasma chamber remains constant (i.e., $\left\langle B_{\phi}\right)=B_{\phi o}$ ) and the density pump-out is minimized (i.e., $n=n_{0}$ ).

Vertical-field constraint. Applying Equation 6.2-1 to the TITAN designs with $1 / \epsilon$ $=6.5$ and ${ }_{0} \leq \leq r_{p} / 4$ results in $B_{V} / B_{\phi o} \leq 0.024$. The stray vertical field for TITAN-I and TITAN-II designs are, respectively, 0.43 and $2.3 \mathrm{mT}$ (Section 4.5 ). Therefore, minimum initial bias fields are $B_{\phi o} \geq 18 \mathrm{mT}$ for TTTAN-I and $B_{\phi o} \geq 97 \mathrm{mT}$ for TITAN-II.

Density and current-density constraints. The impurity burn-through constraint, Equation 6.2-4, results in a minimum value for $j_{\phi} / n$. Assuming that $B_{\phi o}>0.036 \mathrm{~T}$ in Equation 6.2-5 and substituting for $\left\langle B_{\phi}\right\rangle=B_{\phi o}$ from Equation 6.2-3 results in a maximum value for $j_{\phi} / n$. For TITAN plasma conditions of $\Theta_{0} \simeq 1.5$ and $r_{p}=0.6 \mathrm{~m}$, then

$$
10^{-14}<\frac{j_{\phi}}{n}(\mathrm{~A} \mathrm{~m})<1.43 \times 10^{-14}
$$

Although the specification of $\Theta_{a}$ and $\left\langle B_{\phi}\right\rangle$ at formation determines an initial current density, other more dominant constraints may exist. First, the ZT-40M experiment exhibits a minimum current-density limit, which translates empirically to $j_{\phi_{0}} \geq 0.4 \mathrm{MA} / \mathrm{m}^{2}$, below which RFP formation is diffeult. This constraint is not well understood, but its application to TITAN represents a conservative connection to experiment. Second, the maximum value of the poloidal beta of the seed RFP, $\beta_{\theta 0}$, results in another constraint on the minium value of $j_{\phi}$ and/or the maximum value of $n$. The temperature of the seed RFP should also be reasonable for the chosen value of $j_{\phi}$. Third, high values of current density (and/or lor $n$ ) nay place the plasma strongly in the electron runaway regime. If the runaway regime is to be avoided, which may or not may be a requirement, the electron drift parameter should be limited to $\xi \lesssim 0.01$, where

$$
\xi=\frac{v_{D}}{v_{T H e}}=\frac{1.05 \times 10^{13}}{\sqrt{T_{e}}} \frac{j_{\phi}}{n},
$$

and SI units are used except for temperature which is in $\mathrm{eV}$.

Based on the above disiussion, the current density in the reference seed TITAN plasma is set at $j_{\phi o}=0.4 \mathrm{MA} / \mathrm{m}^{2}$ in order to provide a conservative connection to the ZT-40M experiment. This choice results in $I_{\phi o}=0.45 \mathrm{MA}$ and $B_{\phi o}=\left\langle B_{\phi}\right\rangle=100 \mathrm{mT}$ which satisfies the stray vertical-field constraint for both TITAN-I and TITAN-II designs. The temperature and density of the seed plasma are set, respectively, at $T_{e}=100 \mathrm{eV}$ and 
$n_{o} \simeq n=2.8 \times 10^{19} \mathrm{~m}^{-3}$. The corresponding gas $\left(\mathrm{D}_{2}\right)$ fill pressure is $\boldsymbol{P}_{o}=0.4 \mathrm{mtorr}$. This value of $n$ is the minimum density that satisfies Equation 6,3-1 and it is chosen to ensure $\beta_{\theta_{0}}=0.1$. The resulting drift parameter, however, is $\xi=0.015$.

The above set of parameters for the TITAN seed RFP is not unique and reflects a compromise among breakdown and formation constraints. For example, higher plesma temperatures can be obtained for higher values of $\beta_{\theta 0}$, which also reduce the value of $\xi\left(T_{e}=200 \mathrm{eV}\right.$ and $\xi=0.011$ for $\left.\beta_{\theta o}=0.2\right)$. Alternatively, one can increase $n$ to $4 \times 10^{18} \mathrm{~m}^{-3}$ (corresponding to $j_{\phi} / n=10^{-14} \mathrm{~A} \mathrm{~m}$ ) to have $\xi=0.011$ and $\beta_{\theta_{a}}=0.14$ for $T_{e}=100 \mathrm{eV}$. Finally, in order to reduce formation energy and flux consumption, $j_{\phi_{0}}$ can be reduced to $0.28 \mathrm{MA} / \mathrm{m}^{2}$, relaxing the $j_{\phi o}=0.4 \mathrm{MA} / \mathrm{m}^{2}$ connection to ZT-40M. Then, choosing $n_{\circ} \simeq n=2.8 \times 10^{19} \mathrm{~m}^{-3}\left(j_{\phi} / n=10^{-14} \mathrm{~A} \mathrm{~m}\right)$ results in $\xi=0.011$ a.1d $\beta_{80}=0.2$ for $T_{e}=100 \mathrm{eV}$. For this case, however, the initial bias field is $B_{\phi o}=\left\langle B_{\phi}\right\rangle=70 \mathrm{mT}$, which satisfies the stray vertical-field constraint for TITAN-I but violates this constraint for TITAN-II.

Toroidal electric-field constraint. Given the reference parameters of the TITAN seed RFP, the necessary toroidal electric field can be found from Equation 6.2-2 to be $E_{\phi o} \gtrsim 2.6 \mathrm{~V} / \mathrm{m}$ from the JET breakdown condition and $E_{\phi o} \gtrsim 4$ to $8 \mathrm{~V} / \mathrm{m}$ from the ZT-40M constraint. Note that the $E_{\phi \infty}$ value from the ZT-40M constraint is an order of magnitude lower than that actually used in the ZT-40M experiment $(\sim 40 \mathrm{~V} / \mathrm{m})$ because the gas fill pressure in TITAN is much lower. The JET drift condition results in $E_{\phi_{0}} \gtrsim 4.3 \mathrm{~V} / \mathrm{m}$ for TITAN-I and $E_{\phi o} \gtrsim 23 \mathrm{~V} / \mathrm{m}$ for TITAN-IL. This wide range of $E_{\phi_{0}}$ translates into a loop voltage, $V_{\phi} \equiv 2 \pi R_{T} E_{\delta}$, in the range of 100 to $500 \mathrm{~V}$.

Low values of loop voltage are desirable because they reduces the requirements on the power supplies and the insulation of the vessel. On the other hand, higher loop voltages reduce the RFP formation time and may result in reducing the necessary resistive energy and volt-seconds. In order to estimate the resistive energy consumed during the formation phase, we ignore the compressional and risistive voltages during the initial phase of the RFP formation, and approximate the current rise time by a sinusoidal, inductive waveform (capacitor power supply). The current rise time, $\tau_{R}$, is then:

$$
\frac{I_{\phi o}}{\tau_{R}}=\frac{2}{\pi} \frac{V_{\phi o}}{L_{p}},
$$

where $L_{\mathrm{p}}$ is the plasma internal inductance ( $L_{p} \approx 13.3 \mu \mathrm{H}$ for the TITAN plasma). For the ZT-40M experirent, $I_{\phi o} / \tau_{R} \sim 30$ to $40 \mathrm{MA} / \mathrm{s}$ while for TITAN, $I_{\phi o} / \tau_{R} \sim 4.5$ to $22 \mathrm{MA} / \mathrm{s}$ (for $E_{\phi \infty} \sim 4$ to $20 \mathrm{~V} / \mathrm{m}$ ). 
Poloidal flux consumption. In addition to the required inductive flux, $L_{p} I_{\phi \circ}$, the resistive flux consumption during the breakdown and formation phase can be estimated using the average resistivity of

$$
\eta=4.91 \times 10^{-2} I_{\phi 0}^{-0.64},
$$

which has been reported for the matched-mode start-up of the ZT-40M experiment [1]. It can be assumed that the tbove relationship can be directly applied to the TITAN plasma. Alternatively, one can assume that plasmas with similar current densities have similar resistivities. In the latter case, the resistivity of the TITAN plasma during formation is

$$
\eta=3.85 \times 10^{-1} r_{p}^{1.28} I_{\phi_{0}^{-04}}^{-0.44}
$$

which indicates a fivefold increase in the TITAN plasma resistance compared with estimates from Equation 6.3-4.

Assuming that the resistivity remajns constant during the formation phase, the resistive portion of the formation energy is

$$
\begin{aligned}
W_{R E S} & =\left(2 \pi R_{T}\right)\left(\pi r_{p}^{2}\right) \int_{0}^{\tau_{R}} \eta j_{\phi}^{2} d t \\
& =\frac{R_{T}}{\tau_{p}^{2}} \eta I_{\phi \infty}^{2} \tau_{R}
\end{aligned}
$$

and the resistive flux corisumption is

$$
\int_{0}^{T_{R}} V_{\phi} d t=\frac{2}{I_{\dot{~} \delta}} W_{R E S} .
$$

The parameters of the TITAN seed RFP are given in Table 6.3-I. The formation time, energy, and flux consumption are calculated for both estimates of the TITAN plasma resistivity (Equations 6.3-4 and 6.3-5). The formation parameters are reported for three values of toroidal electric field of 4,8 , and $20 \mathrm{~V} / \mathrm{m}$ to cover the range of required loop voltages discussed previously and also to study the resultant resistive flux consumption. Table 6.3-I shows that as $E_{\phi o}$ is increased from 4 to $20 \mathrm{~V} / \mathrm{m}$, the formation time is reduced from 100 to $20 \mathrm{~ms}$ and the resistive flux consumption and formation energy decrease by fivefold. For $E_{\phi o}=20 \mathrm{~V} / \mathrm{m}$ and plasma resistively scaling with current (Equation 6.3-3), the resistive components of flux consunption and formation energy become small compared to the total values, while for $E_{\phi o}=4 \mathrm{~V} / \mathrm{m}$, the resistive components dominate.

In summary, the corditions for plasma breaklown and subsequent RFP formation for the reactor are expected to differ little from the conditions in present and planned 
Table 6,3-I.

PARAMETERS OF REP FORMATION PHASE FOR TITAN

Plasma current, $I_{\phi o}$ (MA)

Plasma current density, $j_{\phi_{0}}\left(\mathrm{MA} / \mathrm{m}^{2}\right)$

Initial toroidal field, $B_{\phi o}(\mathrm{~T})$

Average toroidal field, $\left\langle B_{\phi}\right\rangle(\mathrm{T})$

Toroidal field at $r_{p}, B_{\phi}\left(r_{p}\right)(\mathrm{T})$

Poloidal field at $r_{p}, B_{\theta o}\left(r_{p}\right)(\mathrm{T})$

Pinch parameter, $\Theta_{\sigma}$

Reversal parameter, $F_{n}$

Poloidal beta, $\beta_{\theta 0}$

Drift parameter, $\xi$

Fill pressure, $P_{o}$ (mtorr)

Plasma density, $n\left(m^{-3}\right)$

Electron temperature, $T_{\mathrm{e}}(\mathrm{keV})$

Toroidal electric field, $E_{\downarrow_{\infty}}(\mathrm{V} / \mathrm{m})$

Loop voltage $V_{\phi o}(\mathrm{~V})$

Formation time, $T_{R}(\mathrm{~ms})$

Resistivity from Equation 6.3.3

Resistive flux consumption ( $\mathrm{Wb}$ )

Total flux consumption $(\mathbf{W b})^{(a)}$

Resistive formation energy (MJ)

Total formation energy $(\mathrm{MJ})^{(a)}$

Resistivity from Equation 6.3-4

Resistive flux consumption ( $\mathrm{Wb}$ )

Total flux consumption (Wb) ${ }^{(a)}$

Resistive formation energy (MJ)

Total formation energy (MJ) ${ }^{(a)}$
11.7

17.9

2.64

4.06

47.5

53.8

10.8

12.2
0.452

0.400

0.100

0.100

$-0.010$

0.151

1.5

$-0.1$

0.1

0.015

0.395

$2.80 \times 10^{19}$

0.100

8.0

20.

196.

490.

50.4

20.2

5.8

12.1

8.62

1.32

0.53

2.74

1.95

23.8

9.5

30.0

15.8

5.38

2.15

6.80

(a) Including $L_{p} I_{p}=6.29 \mathrm{~Wb}$ and $0.5 L_{p} I_{p}^{2}=1.42 \mathrm{MJ}$. 
RFP experiments [13], Likewise, the conditions of the seed RFP plasma required to start up the TITAN reactor, except for plasma size, are similar to present-day RFP parameters $\left(I_{\phi}=0.2\right.$ to $0.4 \mathrm{MA}, n=1$ to $3 \times 10^{18} \mathrm{~m}^{-3}, T=0.1$ to $\left.0.4 \mathrm{eV}\right)$. For the reference formation scenario, loop voltage in the range of 200 to $500 \mathrm{~V}$ is necessary to ensure short formation time and acceptable resistive flux consumption and formation energy. The ecaling of plasma resistivity during the formation phase is an important issue that may be resolved with data from larger RFP experiments. Better density and impurity controi during the breakdown and formation process may also be required.

Formation of a seed RFP with somewhat smaller $j_{\phi_{0}}$ (and lower $B_{\phi_{0}}$ and $n$ but higher $\beta_{\theta_{0}}$ ) would reduce the formation time and is desirable (but requires the relaxation of $j_{\phi_{0}}=0.4 \mathrm{MA} / \mathrm{m}^{2}$ connection to $2 \mathrm{~T}-40 \mathrm{M}$ ). For example, $j_{\phi_{0}}=0.28 \mathrm{MA} / \mathrm{m}^{2}$ and $n_{0} \simeq n=2.8 \times 10^{19} \mathrm{~m}^{-3}\left(j_{\phi} / n=10^{-14} \mathrm{~A} \mathrm{~m}\right)$ result in $\xi=0.011$ and $\beta_{\theta 0}=0.2$ for $T_{e}=100 \mathrm{eV}$. This $30 \%$ reduction in $j_{\phi_{0}}$ results in $30 \%$ reduction in $\tau_{R}, 40 \%$ reduction in resistive flux consumption, and $60 \%$ in cesistive formation energy for the same value of $E_{\phi o}$. For this case, however, the initial bias field is $B_{\phi_{0}}=\left\langle B_{\phi}\right\rangle=70 \mathrm{mT}$, which satisfies the stray vertical-field constraint for TITAN-I but violates this constraint for TITAN-II.

\subsection{IGNITION REQUIREMENTS}

The steady-state analysis of global plasma power balance provides useful information for the optimization of the plasma approach to ignition. Results of this type of analysis for auxiliary-heated fusion devices are usually presented in the form of required auxiliary power for power balance as a function of plasma density and temperature. This information is then used to identify the path to ignition that requires minimum auxiliary heating Fower. Similar analysis can be applied to compact RFP reactors in which the plasma is heated to ignition by ohmic heating alone (no auxiliary heating).

The plasma models used for this analyses are reported in this section and they are also used for the plasma-circuit interaction code of Section 6.5. The plasma parameters reported throughout this section are all volume-averaged quantities, that is

$$
n \equiv \frac{?}{r_{1}^{2}} \int_{0}^{r_{p}} n(r) r d r,
$$

except as otherwise noted. For example, the temperature is defined as:

$$
T \equiv \frac{2}{n r_{p}^{2}} \int_{0}^{r_{p}} n(r) T(r) r d r,
$$


which is density-weighted volume averaget! so that the plasma pressure represents a volume-averaged quantity.

The plasma profiles used in the transient analyses are based on the equilibrium analyses of Section 5.2. For the TITAN plasma analysis, two distinct sets of $\mu$ and $p$ profiles have been used. For start-up and transient calctiations of this section, a standard set of profiles,

$$
\begin{aligned}
& \mu(r)=\mu(0)\left[1-\left(r / r_{p}\right)^{8}\right], \\
& n(r)=n(0)\left[1-\left(r / r_{p}\right)^{2.5}\right], \\
& T(r)=T(0)\left[1-\left(r / r_{p}\right)^{4}\right],
\end{aligned}
$$

are used. At steady-state full-power operation, the TITAN plasma is deliberately doped with a trace amount of $\mathrm{Xe}$ impurity to enhance core-plasma radiation and to reduce the heat load on the divertor target plates. One-dimensional transport analysis (Section 5.3) has been performed and the following plasma profiles were obtained:

$$
\begin{aligned}
& \mu(r)=2.843\left[1-0.44\left(r / r_{p}\right)^{\theta}-0.56\left(r / r_{p}\right)^{8}\right], \\
& T_{e}(r)= \begin{cases}14.40-46.94\left(r / r_{p}\right)^{2.9504} & \left(0 . \quad<r / r_{p}<0.25\right) \\
16.07-10.29\left(r / r_{p}\right) & \left(0.25<r / r_{p}<0.833\right), \\
8.111-7.886\left(r / r_{p}\right)^{14.025} & \left(0.833<r / r_{p}<1\right)\end{cases} \\
& n(r)=1.23 \times 10^{21}\left[i-0.8577\left(r / r_{p}\right)^{3.44}\right] .
\end{aligned}
$$

These profiles have been used in the simulation of the steady-state, burning plasma of TITAN designs. The normalized profiles of plasma parameters $(n, T, P$, and $\mu$, magnetic field, current density, and safety factor, $q$, for both sets of profiles are shown in Figures 5.2-1 through 3.2-4.

\subsubsection{Particle Balance}

The particle balance equation for ion species " $\mathrm{j}$ " ( $\mathrm{j}=\mathrm{D}, \mathrm{T},{ }^{4} \mathrm{He}$, etc. $)$ is

$$
\frac{d n_{j}}{d t}=S_{j}-\frac{n_{i}}{\tau_{p j}}-R_{j} .
$$

Here $S_{j}$ is the particle source strength, $\tau_{p j}$ is the effective particle confinement of species " $\mathrm{j}$ " (including recycling), and $R_{j}$ is the low. rate of particle due to fusion reactions (if 
they fuse). The electron density is obtained from charge neutrality condition,

$$
n_{e}:=\sum_{j} Z_{j} n_{j}
$$

where $Z_{j}$ is the atomic number of ion species " $\mathrm{j}$ ". The effective plasma charge, $Z_{\text {eff }}$ is given by,

$$
Z_{e f f}=\frac{1}{n_{e}} \sum_{j} n_{j} Z_{i}^{2}
$$

In the study of TITAN start-up, ignition requirement analyses of Section 6.4.4 are used to find an "optimum" density evolution, $n(t)$, which results in the shortest and most effective approach to ignition. Equation 6.4-9 is then used to compute the correspcnding fueling rate, $S_{j}$.

\section{B.4.2. Power Balanze}

The power balance equation for ion species " $\mathrm{j}$ " ( $\mathrm{j}=\mathrm{D}, \mathrm{T},{ }^{4} \mathrm{He}$, etc. $)$ is

$$
\frac{3}{2} \frac{d}{d t}\left(n_{j} k T_{j}\right)=P_{\alpha j}-\frac{3}{2} \frac{n_{j} k T_{j}}{\tau_{E_{j}}}-\frac{3}{2} n_{j} k\left(\frac{T_{j}-T_{e}}{\tau_{j e}^{e q}}+\sum_{J} \frac{T_{j}-T_{j}}{\tau_{j J}^{e q}}\right) .
$$

Here $P_{a j}$ is the power deposited into ion species " $\mathrm{j}$ " by energetic $\alpha$ particles as they slow down (Section 6.4.3), $\tau_{E j}$ is the energy confinement time and summation on " $J$ " is carried over all ion species. The energy equilibration time between Maxwellian species " $j$ " and "s," $\tau^{e q}$, is given by

$$
\tau_{j s}^{e q}=\frac{3 \sqrt{2 \pi^{3}} \epsilon_{0}}{e^{4}} \frac{m_{j} m_{v}}{Z_{j}^{2} Z_{s}^{2} \lambda_{j s}}\left(\frac{k T_{j}}{m_{j}}+\frac{k T_{b}}{m_{b}}\right)^{3 / 2},
$$

where $\lambda_{j}$ is the coulc.nb logarithm, $Z_{j}$ and $m_{j}$ are, respectively, the atomic number and the mass of species " $\mathrm{j}$," $k$ is the Boltzmann constant, and $\epsilon_{0}$ is the permittivity of free space.

The electron power balance equation is

$$
\frac{3}{2} \frac{d}{d t}\left(n_{e} k T_{e}\right)=P_{a e}+P_{a H M}-P_{R A D}-\frac{3}{2} \frac{n_{e} k T_{e}}{\tau_{E e}}-\frac{3}{2} \sum_{J} n_{e} k\left(\frac{T_{e}-T_{J}}{\tau_{e}^{e q}}\right),
$$


where $P_{\text {RAD }}$ is the radiative power loss. The ohmic heating power into the plasma, $P_{\text {DHAf }}$ is

$$
P_{\text {oHM }}=\frac{2}{r_{p}^{2}} \int_{0}^{r_{p}} \eta_{\|}(r) J_{\|}^{2}(r) r \mathrm{~d} r,
$$

where $\eta_{\|}$, the classical resistivity of the plasme, is

$$
\begin{aligned}
\eta_{\|} & \therefore=0.51 \frac{4 \sqrt{2 \pi}}{3} \frac{m_{e}^{1 / 2}}{k^{3 / 2}}\left(\frac{e}{4 \pi \epsilon_{0}}\right)^{2} \frac{Z_{e f f}}{N\left(Z_{e f f}\right)} \lambda_{e i} T_{e}^{-3 / 2}(r), \\
N\left(Z_{s f f}\right) & \simeq 1+0.718\left(\frac{Z_{e f f}-1}{Z_{e j f}}\right)^{2} .
\end{aligned}
$$

The plasma-resistance composite-profile factor, $g_{O H M}$, which includes the effect of the temperature profile as well as the field-line screw-up ractor, was defined in Section 5.2 such that

$$
P_{\text {OHM }}=g_{\text {CHM }}\left\langle\eta_{\|}\right\rangle\left\langle J_{\|}\right\rangle^{2}
$$

where $\left(\eta_{\|}\right)$is the clossical resistivity computed by using the average electron temperature, $T_{\text {e }}$. The parameter $g_{O H M}$ includes all profile information, is infependent of plasma current and size, and only depends on the plasma profiles $(\mu, n$, and $T), \beta_{\theta}$, and $F$ or $\Theta$ values (Section 5.2). For $F=-0.1$ and power profiles (Equations 6.4-3 through 6.4-5), $g_{\text {он } M}$ ranges from 4.0 to 3.4 as $\beta_{\theta}$ is increased from 0 . to 0.2 .

The radiative power loss from plasma, $P_{R_{A D}}$, includes bremsstrahlung, synchrotron, and line radiation losses. For the TITAN plasma, synchrotron radiation losses are usually very small. The burning TITAN plasma is deliberately doped with xenon impurities to spread the plasma losses uniformly over the first wall, which reduces the heat load to the divertor plates. In this case, most of the plasma power is radiated in the form of line radiation caused by xenon impurities (Section 5.3). These impurities, however, are introduced into the plasma after the ignition phase in order to reduce the time and energy nefled to achieve ignition and steady-state burn. Therefore, !ne radiation is ignored during start-up transient simulations. The radiative power, therefors, is mostly by bremsstrahlung,

$$
P_{R A D} \simeq P_{b r e m}=4.23 \times 10^{-29} g_{b r e m} n_{e}^{2} Z_{e f f}\left(k T_{e}\right)^{1 / 2},
$$

where $g_{\text {trem }}$ is the bremsstrahlung profile fastor,

$$
\text { gtrem } \equiv \frac{2}{r_{p}^{2}} \int_{0}^{r_{p}} \hat{n}_{e}^{2}(r) \hat{T}_{e}^{1 / 2}(r) r d r
$$


The parameter, gbrem depends only on the profiles of electron density $\left[\hat{n}_{e} \equiv n_{e}(r) / n_{e}(0)\right]$ ard temperature $\left[\hat{T}_{e} \equiv T_{e}(r) / T_{e}(0)\right]$. For power profiles (Equations 6.4-3 through 6.4-5), $g_{\text {brem }}=1.329$ and for radiative profiles (Equations 6.4-6 through 6.4-8), $g_{b r e m}=1.172$.

The reactivity profile factors, $g_{D T}$, can be defined in the same manner,

$$
g_{D T}=\frac{2}{r_{p}^{2}} \int_{0}^{r_{p}} \hat{n}_{D}(r) \hat{n}_{T}(r) \frac{\langle\sigma v\rangle\left[T_{i}(r) j\right.}{\langle\sigma v\rangle\left(T_{i}\right)} r d r,
$$

where $\hat{n}$ is the normalized density profile and $\langle\sigma v\rangle\left(T_{i}\right)$ is DT reactivity computed for the plasma average temperature, $T_{i}$, while $\langle\sigma v\rangle\left[T_{i}(r)\right]$ is the local DT reactivity. Then, the $\alpha$-particle power, $P_{\alpha}$, is

$$
P_{\alpha}=k E_{\alpha} g_{D T}\left(T_{i}\right) n_{D} n_{T}(\sigma v)\left(T_{i}\right)
$$

where $E_{\alpha}=3.52 \mathrm{MeV}$ is the birth energy of $\alpha$ particles. Note that $g_{D T}$ is a function of average temperature, $T_{i}$.

Finally, the RFP electron energy-confinement time, $\tau_{E e}$, is assumed to scale from values obtained in present-day experiments (Section 2.3.5) according to

$$
\tau_{\mathrm{Ee}}=C_{\nu} I_{\phi}^{\nu} r_{p}^{2} f\left(\beta_{\theta}\right)
$$

with typical values of the current scaling exponent, $\nu$, and the cor.esponding numerical coefficient, $C_{\nu}$, summarized in Table 6.4-I and Figure 2.3-20 for $\tau_{\nu_{i}}=4 \tau_{E_{e}}$. The function $f\left(\beta_{\theta}\right)$ models the soft $\beta$ limit and is assumed to have a value of one for $\beta$ values below the $\beta$ limit, $\beta_{8 e}$, and to go to zero when the $\beta$ limit value is exceeded. For TITAN transient analyses, a value of $\nu=1.05$ is used and $f\left(\beta_{\theta}\right)$ is modeled by a Fermi-Dirac probability function,

$$
f\left(\beta_{\theta}\right)=\left[1+\exp \left(\alpha \frac{\beta_{\theta}-\beta_{\theta_{c}}}{\beta_{\theta c}}\right)\right]^{-1},
$$

with $\alpha=50$ and $\beta_{\theta_{\mathrm{c}}}=0.23$.

\subsubsection{Energtic Alpha Particles}

The distribution function of the fusion product " $\mathrm{a}$ " (e.g., $\alpha$ particles) in the plasma cen be found using the Fokker-Planck equation, which for isotropic distribution functions is given by

$$
\frac{\partial f_{a}}{\partial t}=\sum_{s} \frac{1}{v^{2}} \frac{\partial}{\partial v}\left[\mathcal{P}_{a s}(v) \frac{\partial f_{a}}{\partial v}+\mathcal{Q}_{a s}(v) f_{a}(v)\right]+S_{a}(v)-L_{a}(v)
$$


Table 6.4-I.

RFP ELECTRON ENERGY-CONFINEMENT

SCALING PARAMETERS ${ }^{(a)}$

\begin{tabular}{cc}
\hline$\nu$ & $C_{\nu}\left(\mathrm{s} / \mathrm{m}^{2}-\mathrm{M}\right.$ \\
\hline 1.50 & 0.1400 \\
1.40 & 0.1140 \\
1.25 & 0.0837 \\
1.20 & 0.0614 \\
1.10 & 0.0620 \\
1.05 & 0.0554 \\
1.00 & 0.0500 \\
0.90 & 0.0407 \\
0.80 & 0.0331
\end{tabular}

$$
\begin{aligned}
(a) \tau_{E} & \equiv 2\left(1 / \tau_{E e}+1 / \tau_{E i}\right)^{-1} \\
\tau_{E e} & =C_{\nu} I_{\phi}^{L} r_{p}^{2} ; \tau_{E i}=4 \tau_{E e}
\end{aligned}
$$

Here, the summation is carried out over all plasma species (i.e., electrons, ions, and fusion products), $S_{a}$ is the source of fast particles (e.g., fusion products), $L_{a}$ is the fast-particle loss term,

$$
L_{a}(v)=\frac{f_{a}(v)}{T_{p a}}
$$

$\tau_{p a}$ is the fast-particle confinement time, and $P$ and $\mathcal{Q}$ are given by

$$
\begin{aligned}
& \mathcal{P}_{a s}=\frac{4 \pi}{3} \Gamma_{a s}\left[\int_{0}^{v} f_{s}(u): u^{4} d u+v^{2} \int_{v}^{\infty} f_{s}(u) u d u\right], \\
& \mathcal{Q}_{a s}=4 \pi \frac{m_{a}}{m_{s}} \Gamma_{a s} \int_{0}^{v} f_{s}(u) u^{2} d u,
\end{aligned}
$$


where

$$
\Gamma_{a}=\frac{4}{\pi}\left(\frac{Z_{a} Z_{b} e^{2}}{4 \pi \epsilon_{0}}\right)^{2} \lambda_{a \mu}
$$

The density and pressure of fusion products, then, can be found from $f_{\mathrm{a}}(v)$ :

$$
\begin{aligned}
& n_{a}=4 \pi \int_{0}^{\infty} f_{a}(v) v^{2} d v \\
& p_{a}=\frac{4 \pi m_{a}}{3} \int_{0}^{\infty} f_{a}(v) v^{4} d v .
\end{aligned}
$$

The power transfer due to collisions from species " $\mathrm{a}$ " to species " $\mathrm{s}$ " is

$$
P_{a A}=4 \pi m_{a} \int_{0}^{\infty}\left[P_{a s}(v)+v \frac{d \mathcal{P}_{a s}}{d v}-v \mathcal{Q}_{a s}(v)\right] f_{a}(v) d v .
$$

If species "s" has a Maxwellian distribution, $f_{v}(v)=\left(n_{a} / \pi^{3 / 2} v_{a}^{3}\right) \exp \left(-v^{2} / v_{a}^{2}\right)$, $P_{a s}$ and $Q_{a}$ can be written as

$$
\begin{aligned}
\mathcal{P}_{a s}(v) & =\frac{2}{\sqrt{\pi}} n_{b} v_{a} \Gamma_{a}, G\left(v / v_{a}\right), \\
Q_{a s}(v) & =\frac{2}{\sqrt{\pi}} n_{a} v_{a} \Gamma_{a} \frac{2 m_{a}}{m_{a}} G\left(v / v_{a}\right), \\
\mathcal{P}_{a d}+v \frac{d \mathcal{P}_{a s}}{d v} & =\frac{2}{\sqrt{\pi}} n_{a} v_{a} \Gamma_{a s}\left(\frac{v}{v_{b}}\right)^{2} \exp \left(-\frac{v^{2}}{v_{d}^{2}}\right),
\end{aligned}
$$

where $v_{s}=\sqrt{2 k T_{\mathrm{a}} / m_{\mathrm{s}}}$ is the thermal speed of species "s,"

$$
G(x) \equiv \frac{\sqrt{\pi}}{4 x} \operatorname{erf}(x)-\frac{1}{2} \exp \left(-x^{2}\right)
$$

and $\operatorname{erf}(x)$ is the error function.

For the TITAN transient simulations, Equation 6.4-25 is solved using the numerical method of Reference [14], which is an extension of Chang and Cooper technique [15]. Equation 6.4-25 is nonlinear (functions $\mathcal{P}_{\mathrm{aa}}$ and $\mathcal{Q}_{a a}$ depend on $f_{a}$ ). Therefore, at each time step, an iteration scheme over the distribution function of fast species, $f_{a}$, were used: $\mathcal{P}$ and $Q$ functions are evaluated using the old values of $f_{a}$, new values for $f_{a}$ are calculated based on these $\mathcal{P}$ and $\mathcal{Q}$ functions, these new values of $f_{\mathrm{a}}$ are used to update $\mathcal{P}$ and $\mathcal{Q}$ functions, and $f_{a}$ values are again updated until convergence is achieved. The distribution of background plasma speries are assumed to be Maxwellian. Because the ion temperature is much smaller than the fusion $\alpha$-particle birth energy $\left(T_{i} \ll E_{\alpha}\right)$, the $\alpha$-particle source is asimed to be a delta function at energy $E_{\alpha}$. 


\subsubsection{Ignition Analysis}

The steady-state analysis of global plasma power balance provides useful information for the optimization of plasma approach to ignition. Results of this type of analysis for auxiliary-heated fusion devices are usually presented in the form of required auxiliary power for power balance as a function of plasma density and temperature. This information is then used to identify the path to ignition that requires minimum auxiliary heating power. Similar analysis can be applied to compact RFP reactors in which the plasma is heated to ignition by ohmic heating alone (no auxiliary heating).

Addition of the ion and electron power-balance equations (Equations 6.4-12 and 6.4-14) for steady state results in

$$
\frac{3}{2}\left(\frac{n_{e} T_{e}}{\tau_{E e}}+\sum_{j} \frac{n_{j} T_{j}}{\tau_{E_{j}}}\right)=P_{\alpha}+P_{O H M}-P_{R A D}
$$

where $P_{\text {OHM }}, \mathrm{P}_{R, D}$, and $P_{\alpha}$ are given, respectively, by Equations 6.4-18, 6.4-19, and 6.4-22. Equation 6.4-37 can been solved for the required plasma current for power balance, $I_{\phi}$, as a function of density and temperature for a given plasma size, ion mixture, plasma profiles $\left(n, T\right.$, and $\mu$ ), $F$ or $\Theta$ values, and the scaling of $\tau_{E}$ as given by Equation 6.4-23 and 6.4-24 (current exponent, $\nu$, and the soft $\beta$ limit, $\beta_{\theta_{\varepsilon}}$ ). Because of the high density of RFP plasmas, the electron/ion energy-equilibration time, $\tau_{e i}^{\text {eq }}$, is short resulting in $T_{e} \simeq T_{i} \simeq T$.

Results of this analysis for the TITAN plasma $\left(r_{p}=0.6 \mathrm{~m}\right)$ are shown in Figure 6.4-1 for a 50:50 DT mixture, $n_{\mathrm{e}} \simeq n_{i} \simeq n\left(Z_{\text {eff }}=1\right)$, plasma power profiles (Equations 6.4-3 through 6.4-5), and $F=-0.1$. A current exponent of $\nu=1.05$ and a soft $\beta$ limit of $\beta_{\theta_{c}}=0.23$ are used. Figure 6.4-1(A) shows a "ridge" in the $I_{\phi}-n-T$ space above which the path to ignition and burn should be located (similar to corresponding diagrams for auxiliary heated devices where i ridge for auxiliary heating exists). The optimum ignition scenario attempts to pass over the ridge at its lowest height (saddle point). This saddle point, which is denoted by a filled circle in the figure, is at $T \simeq 7 \mathrm{keV}$ and $n \simeq 3 \times 10^{20} \mathrm{~m}^{-3}$ with a current of. $I_{\phi} \simeq 16 \mathrm{MA}$. The TITAN start-up path through ignition and burn passes close to this saddle point (Section 6.5.3).

The corresponding contours of constant $I_{\phi}$ are shown in Figure 6.4-1(B). The chain dashed line in this figure is the contour of $\beta_{\theta}=0.2$ and the points above this line all have $\beta$ values of $\sim 0.2$ because of the assumption of the $\beta$-limited confinement. This point is emphasized in Figure 6.4-2, which illustrates the $\beta_{\theta}$ corresponding to $I_{\phi}$ values of Figure 6.4-1. The flat part of the $\beta_{\theta}$ surface at high $T$ and $n$ represent the operating 

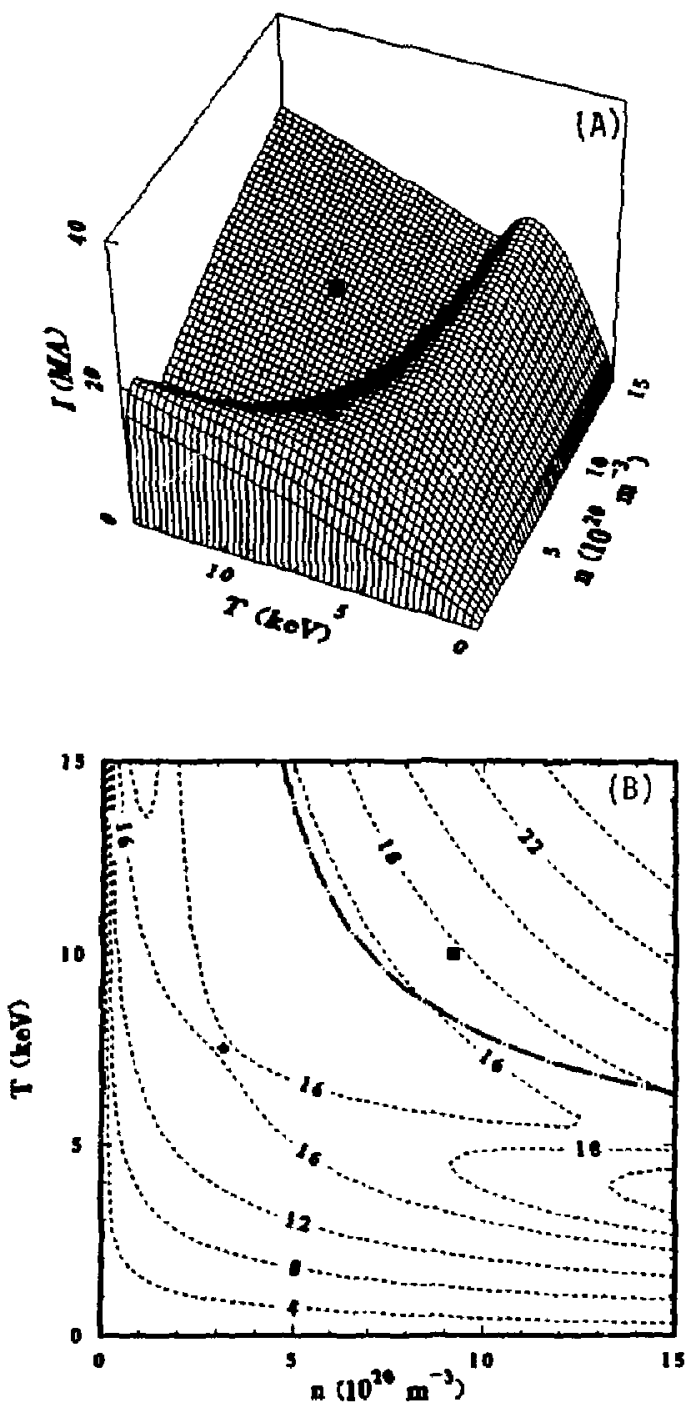

Figure 6.4-1. The required current for power balance as a function of TITAN plasme density and temperature (A) and the corresponding contour plot (B). The steady-state TITAN plasma is denoted by a filled square and the saddle point by a filled circle. The path to ignition and burn should be located above this surface. 

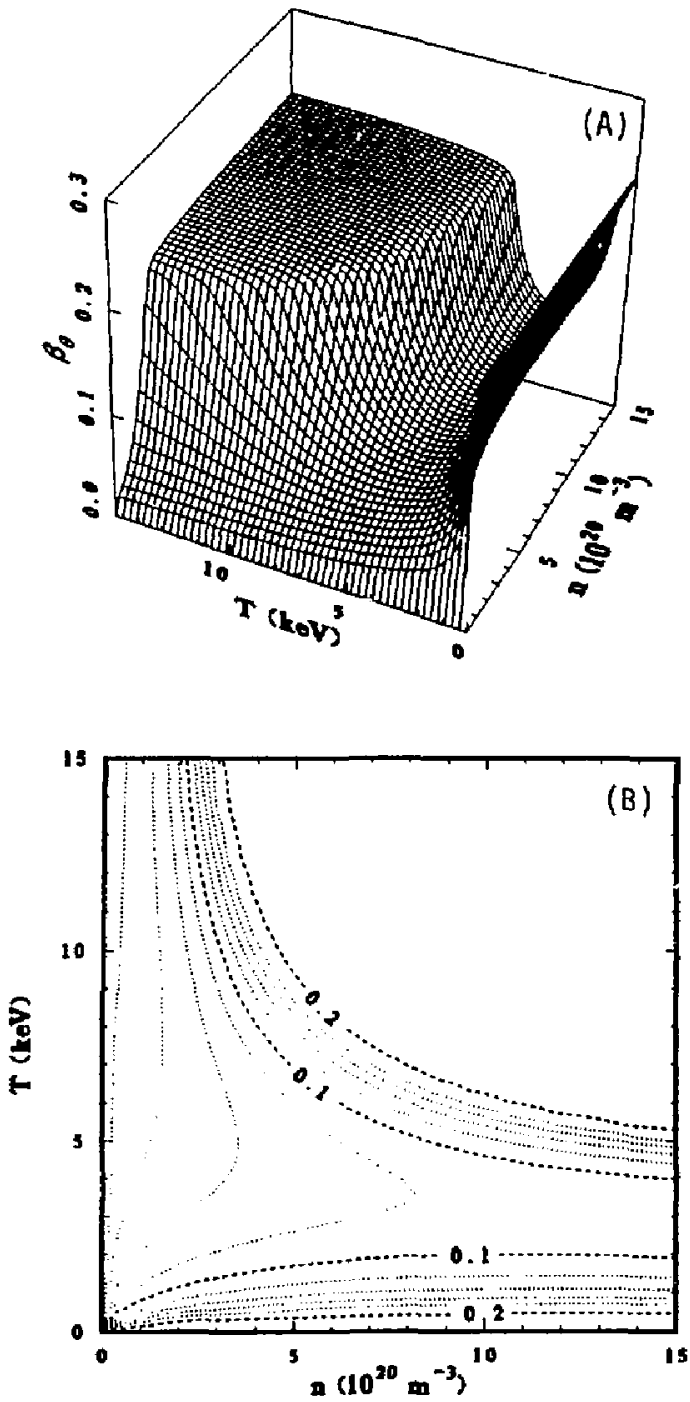

Figure 6.4-2. The plasma $\beta$ corresponding to the required current for power balance as a function of TITAN plasma density and temperature $(A)$ and the corresponding contour plot (B). The flat portiou of the $\beta_{\theta}$ surface is due to the $\beta$-limited confinement assumption. The path to ignition and burn should be located below this surface. 
point at the $\beta$ limit. Note that the path to ignition should stay below this $\beta_{\theta}$ surface since it should stay above the current surface of Figure 6.4-1. The $\beta_{\theta}$ value for the sadile point is about $7 \%$.

The sensitivity of the structure of the $I_{\phi}-n-T$ ignition surface to the various assumptions has been also studied. The $\beta$-limited confinement assumption does affect the ridge structure and only changes the current values above the chain-dashed line of Figure 6.4-1. If the confinement scaling is more favorable with current (higher $\nu$ values), the saddle point moves towards lower currents and slightly lower densities. For example, for $\nu=1.2$, the current is reduced to $12 \mathrm{MA}$. Increasing $Z_{\text {eff }}$ from 1.0 to 1.7 , increases the current by about $10 \%$ and electron density by $50 \%$ (note that line radiation is ignored).

\subsection{CURRENT RAMP-UP TO IGNITION AND BURN}

The TITAN start-up scenario inrough current ramp, ignition, and burn transients is shosen such that the start-up power is directly extracted from the power grid without requiring an on-site p.ower-storage system. This start-up scenario is described in Section 6.5.1 and its implications for the poloidal-field-coil system are reviewed. The evolution of the TITAN plasma through the start-up sequence is investigated by using a $0-D$, profile-averaged plasma-circuit code. The plasma models were described in Section 6.4. The circuit model for the plasma, the poloidal-field (PF) system, and the eddy currents in the FPC are reviewed in Section 6.5.2. A description of the plasma-circuit code as well as the simulation results are presented in Section 6.5.3.

\subsubsection{Start-Up Sequence}

The current in the TITAN plasma is initiated and then ramped to full value through induction by the PF-coil system. In addition to producing the required flux change, the PF system must also generate the necessary equilibrium-field distribution. The most efficient way to produce a net flux change is through bipolar operation of the coil system since the stored energy, magnetic-field strength, coil stress, and joule losses would be minimized. Plasma equilibrium depends on the magnitude of the plasma current and the equilibrium field produced by the PF coils should closely match the required vertical field for equilibrium.

For the TITAN designs, the PF-coil system is divided into two sets of coils: the ohmicheating $(\mathrm{OH})$ coil set, which produces most of the flux swing but does not produce any 
equilibrium (vertical) field; and the equilibrium-field (EF) coil set, which produces the necessary vertical field and may or may not contribute to the flux swing. This approach allows the $\mathrm{OH}$ coils to be operated independently of the magnitude of the plasma current. Futhermore, we will show that the magnetic properties of the OH- and EF-coil sets and the start-up switching sequence can be chosen such that the EF coil produces the required vertical field approximately, without any need for a highly controlled power supply for the EF coils.

The TITAN-I start-up scenario is chosen such that the start-up power is directly extracted from the power grid without requiring an on-site power-storage system (other than the coils themselves). The start-up switching sequence is shown in Figure 6.5-1. An illustration of the corresponding current and voltage waveforms are given in Figure 6.5-2. The TITAN start-up sequence uses a bipolar swing of the $\mathrm{OH}$-coil currents and begins with charging the $\mathrm{OH}$ coils to their full back-bias values. The $\mathrm{OH}$ coils are then discharged into a transfer resistor, while the EF coils are connected in parallel to the OH coils ("formation and fast discharge" phase in Figures 6.5-1 and 6.5-2). The value of the transfer resistor is set by the constraint on the maximum voltage across the superconducting $\mathrm{EF}$ coils. The fast-discharge phase lasts about $i$ to 2 seconds.

As the $\mathrm{OH}$ coils are discharging, the voltage across the circuit drops. When the voltage across the $\mathrm{OH}$ coils reaches that of the grid power supply, the transfer resistor is disconnected from the circuit and the grid power supply is directly applied to the $\mathrm{OH}$ and EF coils ("slow-ramp" phase). The $\mathrm{OH}$ coils are driven to their full, forward-bias current value and the EF coils and the plasma to their respective steady-state currents. The voltage of the grid power supply is usually a few kilovolts and its value is determined by the maximum power from the grid. The current-drive system begins operation during this phase and will be fully operational at the burn phase, maintaining the steadystate current in the plasma. After achieving the steady-state burn condition, the OFCD system is initiated while the $\mathrm{OH}$ coils are discharged slowly, from the full forward-bias current value to zero, in order to minimize the recirculating power and the coil-cooling requirements. Initiation of OFCD operation during the slow-ramp phase is advantageous.

With the proper magnetics design of the $O H$ and $E F$ coils, this start-up scenario will provide approximate plasma equilibrium throughout the start-up sequence. To find the magnetics design requirements, we consider the fast- and slow-ramp phases of the start-up sequence (Figure 6.5-1). Ignoring the trim-coil circuit and eddy currents that may be induced in the fusion power core (FPC), the circuit equations for the plasma and 

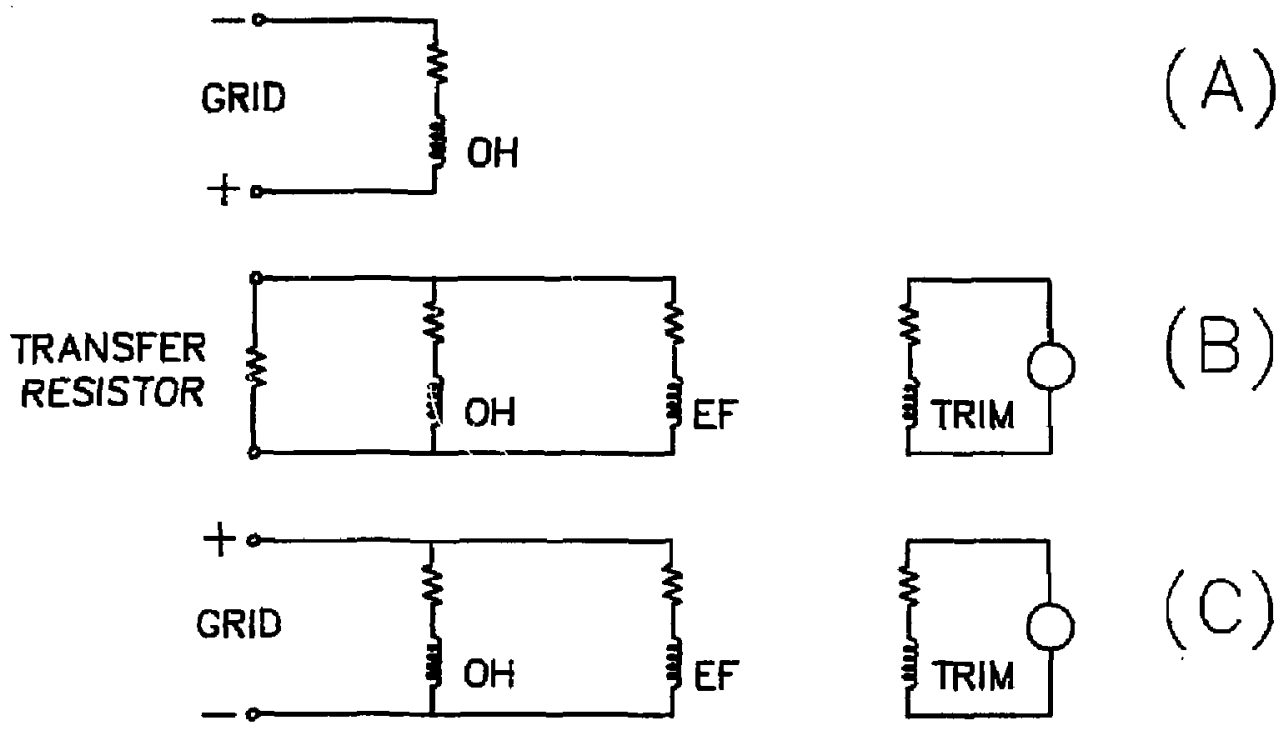

DISCHARGE

RESISTOR
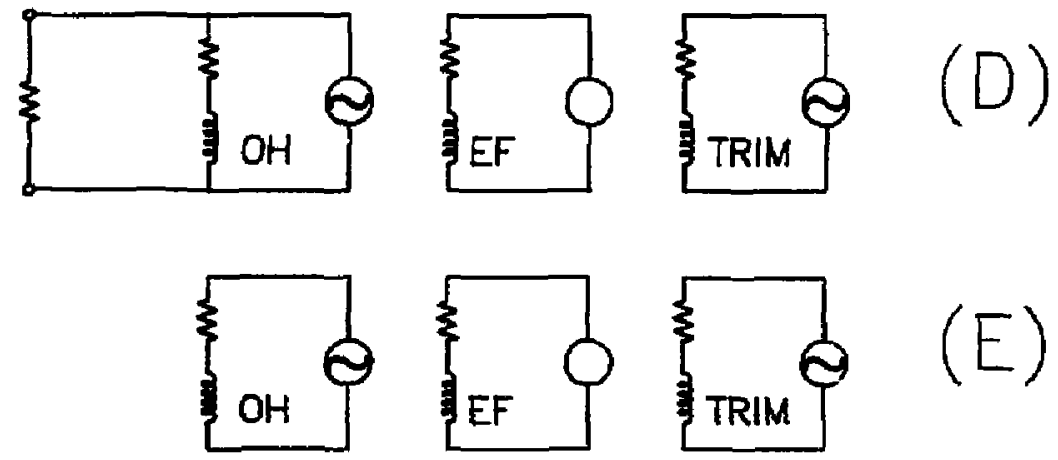

Figure 6.5-1. Start-up sequence for the TITAN reactors: (A) Charge-up - OH coils are charged to full back-bias value, (B) Formation and fast discharge - OH coils are discharged through a transfer resistor (EF coils are connected in parallel to the $\mathrm{OH}$ coils), (C) Slow ramp - grid power drives the $\mathrm{OH}$ coils to full forward biai current and the EF coils to their steady-state value, (D) Transition - the $\mathrm{OH}$ coils are slowly discharged while OFCD is initiated, and (E) Steady state - current-dtive system is fully operational. Initiation of OFCD operation during the slow-ramp phase is advantageous. 

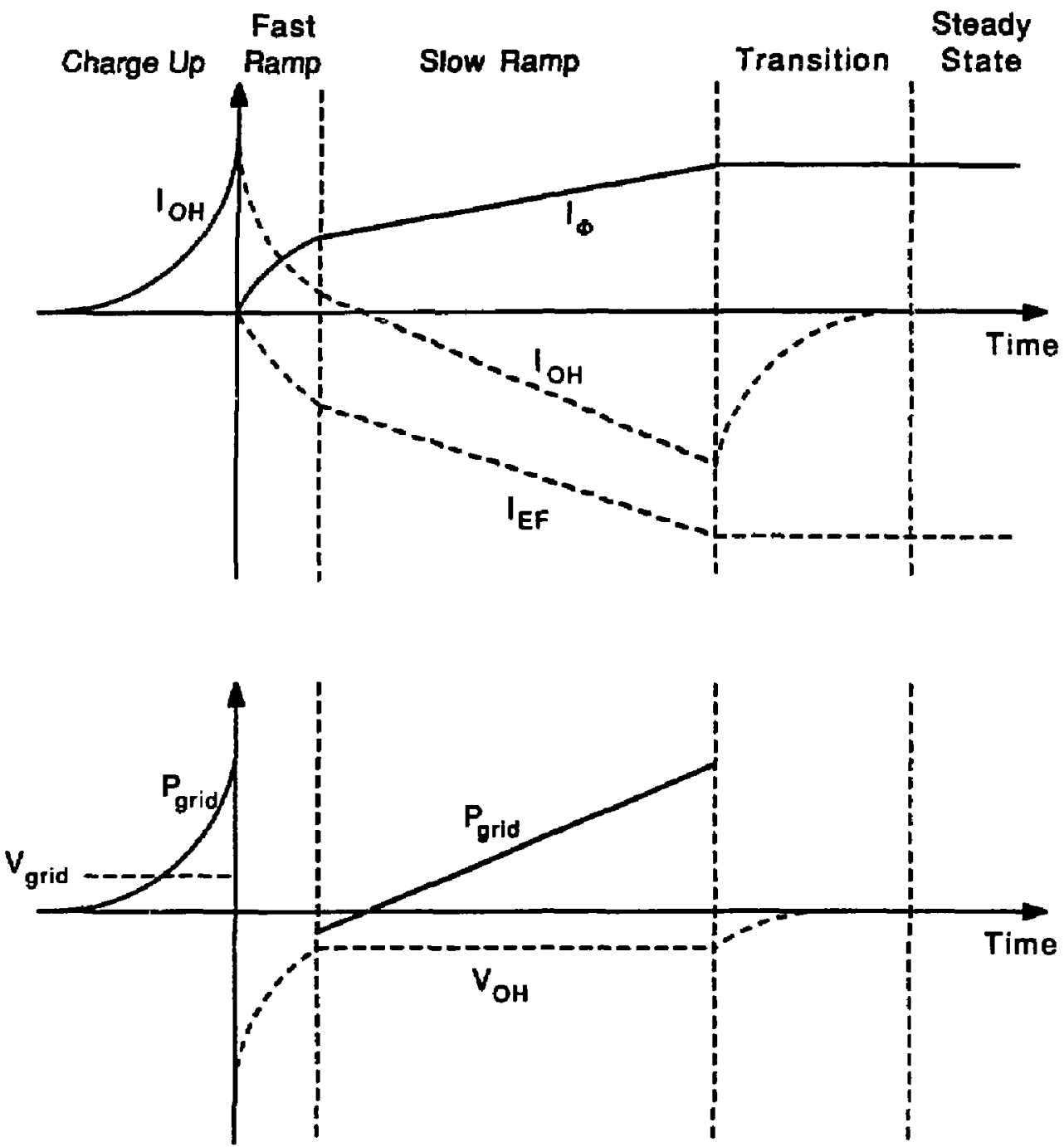

Figure 6.5-2. Schematic of coil currents and voltages during the TITAN start-up sequence corresponding to Figure 6.5-1: (A) Charge-up, (B) Formation and fast-discharge, (C) Slow-ramp, (D) Transition, and (E) Steady-state phases. Note that the time axis is not in scale. 
the $\mathrm{OH}$ and $\mathrm{EF}$ coils are, respectively,

$$
\begin{aligned}
L_{p} \frac{d I_{\phi}}{d t}+M_{O H, p} \frac{d I_{O H}}{d t}+M_{E F, p} \frac{d I_{E F}}{d t} & =-R_{P} I_{\phi}, \\
M_{O H, p} \frac{d I_{\phi}}{d t}+L_{O F} \frac{d I_{O H}}{d t}+M_{E F, O H} \frac{d I_{E F}}{d t} & =\frac{v_{O H}}{N_{O H}}-R_{O H} I_{O H}, \\
M_{E F, p} \frac{d I_{\phi}}{d t}+M_{E F, O H} \frac{d I_{O H}}{d t}+L_{E F} \frac{d I_{E F}}{d t} & =\frac{v_{E F}}{N_{E F}}-R_{E F} I_{E F},
\end{aligned}
$$

where the direction of the plasma current is taken to be the positive direction for the current in all circuits, $L$ and $M$ represent equivalent single-turn inductances, currents are given in Ampere-turn, and $N$ denotes the number of turns in each coil set. Eliminating $I_{O H}$ from Equations 6.5-1 through 6.5-3 and noting that $v_{O H}=v_{E F}$ during both fastand slow-ramp phases, one has

$$
\begin{aligned}
& {\left[\frac{N_{O H}}{N_{E F}}\left(M_{O H, p}^{2}-L_{O H} L_{p}\right)-\left(M_{O H_{1,}} M_{E F, p}-M_{O H, E F} L_{p}\right)\right] \frac{d I_{\phi}}{d t}+} \\
& {\left[\frac{N_{O H}}{N_{E F}}\left(M_{O H, p} M_{O H, E F}-L_{O H} M_{E F_{1, p}}\right)-\left(M_{O H, p} L_{E F}-M_{O H, E F} M_{E F, p}\right)\right] \frac{d I_{\partial F}}{d t} } \\
= & R_{P} I_{\phi}\left(\frac{N_{O H}}{N_{E F}} L_{O H}-M_{O H, E F}\right)-M_{O H, p}\left(\frac{N_{O H}}{N_{E F}} R_{O H} I_{O H}-R_{E} I_{E}\right) .
\end{aligned}
$$

If the resistances are ignored (circuit $L / R$ time is much longer than start-up duration), Equation 6.5-4 can be integrated subject to the initial condition of $I_{\phi}=I_{E F}=0$ at time $t=0$ to get

$$
-\frac{I_{E F}}{I_{\phi}}=\frac{\frac{N_{O H}}{N_{E F}}\left(M_{O H, p}^{2}-L_{O H} I_{p}\right)-\left(M_{O H, p} M_{E F, p}-M_{O H, E F} L_{p}\right)}{\frac{N_{O H}}{N_{E F}}\left(M_{O H, p} M_{O H, E F}-L_{O H} M_{E F, p}\right)-\left(M_{O H, p} L_{E F}-M_{O H, E F} M_{E F_{p}}\right)} .
$$

For a fixed PF-coil set, the vertical field produced by the EF coils directly scales with the EF current, while the vertical field necessary for plasma equilibrium scales directly with $I_{\phi}$. Hence, the needed ratio of the final EF-coil current to the final plasma current, $-I_{E F}^{+} / I_{\phi}^{+}=\left|I_{E F}^{+} / I_{\phi}^{+}\right|$, and the single-turn inductances are known. Equation 6.5-5 can then be used to find the value of $N_{O H} / N_{E F}$ so that the proper equilibrium is maintained during the discharge:

$$
\frac{N_{O H}}{N_{E F}}=\frac{M_{O H . E F}\left(C_{1}-C_{3}\right)}{L_{O H}\left(C_{1}-C_{2}\right)}
$$


where

$$
\begin{aligned}
& C_{1} \equiv \frac{1}{M_{O F, p}}\left(L_{p}-M_{E F, p}\left|\frac{I_{E F}^{+}}{I_{\phi}^{+}}\right|\right) \\
& C_{2} \equiv \frac{1}{L_{O H}}\left(M_{O H, p}-M_{O H, E F}\left|\frac{I_{E F}^{+}}{I_{\phi}^{+}}\right|\right) \\
& C_{3} \equiv \frac{1}{M_{O H, E F}}\left(M_{E F, p}-L_{E F}\left|\frac{I_{E F}^{+}}{I_{\phi}^{+}}\right|\right)
\end{aligned}
$$

Parameters $C_{1}$ through $C_{3}$ reflect the degree of coupling between various circuit elements.

Since the eddy current induced in the FPC and resistances in various circuits are ignored in arriving at Equation 6.5-5, the equilibrium provided by the EF coils during the start-up sequence is an approximate equilibrium. The TITAN PF-coil systerm, therefore, includes a pair of small, normal-conducijug trim coils to maintain exact equilibrim during the start-up sequence. Using this approach, only the power supplies for the EF trim coils have to be feedback controlled to ensure proper equilibrium. The EF trim coils are also used during the steady-state operation for plasma equilibrium control and OFCD cycles (Section 7).

The magnetics design of the PF-coil systems for the TITAN designs is given in Section 4.5 and magnet engineering issues are discussed in Section 10.5. The relevant circuit parameters for the TITAN PF coils are given in Table 6.5-I.

\subsubsection{Circuit Model}

The time-varying electromagnetic fields incurred during the plasma transients induce eddy currents in all the conducting materials surrounding the FPC (i.e., those of the first wall, liner/conducting shell, vacuum vessel, blanket, shield, and structures). These eddy currents retard and modify the plasma response to externally applied magnetic fields. Furthermore, these eddy currents give rise to magnetic fields affecting the plasma equilibrium, to electromagnetic forces on all conducting materials that carry the eddy currents, and to an additional energy drain from the external circuits to compensate for joule losses by eddy currents. Eddy-current modeling is, therefore, critical and it is usually the most difficult task in plasma-circuit interaction analysis. The approach to this problem, adopted for the TITAN study, is to divide the conducting material into small strips that simulate the actual eddy-current path and distribution. Each strip is modeled as an element of a complex circuit that also includes the external circuitiry 
Table 6.D-I.

\section{CIRCUIT PARAMETERS FOR TITAN PF-COIL DESIGNS ${ }^{(a)}$}

\begin{tabular}{|c|c|c|}
\hline & TITAN-I & TITAN-II \\
\hline \multicolumn{3}{|c|}{ Self inductances $(\mu \mathrm{H})$} \\
\hline$\cdot L_{p}$ & 13.29 & 13.29 \\
\hline - $L_{O H}$ & 2.74 & 3.68 \\
\hline$\cdot L_{E F}$ & 15.02 & 13.35 \\
\hline$\cdot L_{T r i m}$ & 18.36 & 19.35 \\
\hline \multicolumn{3}{|c|}{ Mintual inductances $(\mu \mathrm{H})$} \\
\hline - $M_{O H, \mathrm{p}}$ & 2.87 & 3.92 \\
\hline$\cdot M_{O H, E F}$ & 2.26 & 3.04 \\
\hline$\cdot M_{O H, T+i m}$ & 2.99 & 4.03 \\
\hline$\cdot M_{E F_{p p}}$ & 3.86 & 4.12 \\
\hline$M_{E F, T r i m}$ & 8.69 & 8.22 \\
\hline$\cdot M_{T r: n, p}$ & 5.60 & 6.15 \\
\hline \multicolumn{3}{|c|}{ Current levels (MA-turn) } \\
\hline$\cdot I_{\phi}$ & 17.75 & 17.82 \\
\hline$I_{E F}$ & 19.24 & 18.60 \\
\hline$\cdot \Delta I_{O H}$ & 55.80 & 40.82 \\
\hline \multicolumn{3}{|c|}{ Magnetic fluxes (Wb) } \\
\hline - Plasma & 236.0 & 236.9 \\
\hline . EF coil & 74.5 & 77.1 \\
\hline . $\mathrm{OH}$ coil & 161.5 & 159.8 \\
\hline
\end{tabular}

(a) Equivalent single-turn inductance values are given. 
and an equivalent circuit element for the plasma. The interaction of these elements is taken into account through the circuit-inductance matrix, $L$, containing self- and mutual inductances for all elements. The matrix circuit equation describing the evolution of currents in vitious circuit elements is

$$
\frac{d}{d t}(\mathbf{L} \cdot \mathbf{I})=\mathbf{V}-\mathbf{R} \cdot \mathbf{I},
$$

where $I$ and $V$ are column vectors of currents (Ampere-turn) and voltages (volt/turn) and $\mathbf{R}$ is the diagonal matrix of resistances. For a given time history of voltages and switch. 8 sequences, the above matrix equation can be solved to obtain the currents and, then, magnetic fields and power flow through the circuits. In principle, the accuracy of such a procedure should be improved by increasing the number of the equivalent eddycurrent circuit elements, which, in turn, increases the complexity of the overall circuit analysis as well as the computation time.

The starting point of this procedure is the division of the conducting material into equivalent eddy-current circuit elements. Such a division, however, requires a priori knowledge of eddy-current paths and distributions. Furthermore, introducing resistive breaks to suppress the magnitude of eddy currents and associated effects results in "saddle" current loops. Circuit simulations for the TITAN start-up transient analysis is limited to the equivalent circuits for the poloidal field. Each eddy-current path is approximated by a toroidal "conductor" model, whereas the effect of the resistive breaks is modelled only as a dramatic increase in the resistance of the eddy-current circuit. The poloidal component of the saddie current loops that impacts the toroidal-field system is ignored. Note that both poloidal and toroidal coinponents of saddle loop currents are included in the circuit simulation of OFCD of Section 7.

Special attention is also given to the EF-coil circuits. At all times, the current in the EF coils should provide the necessary vertical field to maintain the plasma equilibrium. The required value of this vertical field at the magnetic axis is given by Shafranov [16]:

$$
B_{V}=\frac{\mu_{0} I_{\phi}}{4 \pi R_{T}}\left[\ln \left(\frac{8 R_{T}}{r_{\mathrm{p}}}\right)+\beta_{\theta}+\frac{l_{i}}{2}-1.5\right],
$$

where $l_{i}$ is the plasma internal inductance per unit length. It has been shown [17] that the Shafranov for: tula is an accurate measure of the required vertical field for an RFP over a wide range of plasma conditions. In the presence of conducting material, the vertical field produced by the EF coils, together with contributions from other external coils and eddy currents, should be equal to the vertical field given by Equation 6.5-11:

$$
\sum_{j \neq p l a m a}^{-}\left\{B_{z}\right\}_{j}=B_{V},
$$


where $\left(B_{x}\right)_{j}$ is the vertical field produced by circuit " $j$ " and $B_{V}$ is the required vertical field. Since $\left(B_{x}\right)_{j} \propto I_{j}$ and $B_{V} \propto I_{p}$, Equation 6.5-12 can be written as

$$
\sum_{j \neq p l a m a} c_{j} I_{j}+c_{p} I_{p}=0
$$

where $c_{p} \equiv-B_{W} / I_{p}$ can be found from Equation 6.5-11 and constants $c_{j}$ depend only on coil or conductor geometry and location. Taking the time derivative of Equation 6.5-13, one finds

$$
\frac{d}{d t}\left(\sum_{j} c_{j} I_{j}\right)=0
$$

where the summation is over all circuits (including the plasma).

\subsubsection{Plasma-Circuit Simulations}

The evolution of the TITAN plasma through current ramp, ignition, and burn transients is investigated by using a $0-D$, profile-averaged plasma-circuit code. The plasma profiles ( $n, T$, and $\mu$ ) used in the transient analyses are the power profiles given by Equations 6.4-3 through 6.4-5. Equilibrium calculations of Section 5.2-are used to compute the piasma-resistance composite-profile factor, $g_{\text {us }}$, and the plasma internal inductance per unit length, $l_{i}$. It is shown in Section 5.2 that these two parameters are independent of plasma current and size, and only depend on the plasma profiles, $\beta_{\theta}$, and $F$ or $\Theta$ values. It is assumed that the value of $F$ is held constant during start-up transients by proper programining and/or feedback control of the TF coils at $F-0.1$. Therefore, $g_{O H M}$ and $l_{i}$ change only in response to change in $\beta_{\theta}$ during the start-up transients, as is shown in Figure 6.5-3. To save cimputational time, a table for valuez of $g_{O H M}$ and $l_{i}$ as functions of $\beta_{\theta}$ is made at the start of the calculations and the necessary values at each time are found through interpolation.

The plasma-circuit simulation code solves the ion and electron power-balance equation (Section 6.4.2) and the Folkker-Planck equation for fusion $\alpha$ particles (Section 6.4.3) using an implicit time-differencing scheme. The desired evolution of the plasma density, obtained from the ignition requirement analysis of Section 6.4.4, is used together with the particle balance equation (Equation 6.4-9) to determine the required fueling rate. Between each time step, a circuit-analysis package integrates the circuit equations using an adaptive Rung-Kutta method [18]. At the start of the analysis and for the given FPC geometry and external coil sets, the code divides the FPC into small sectors and 

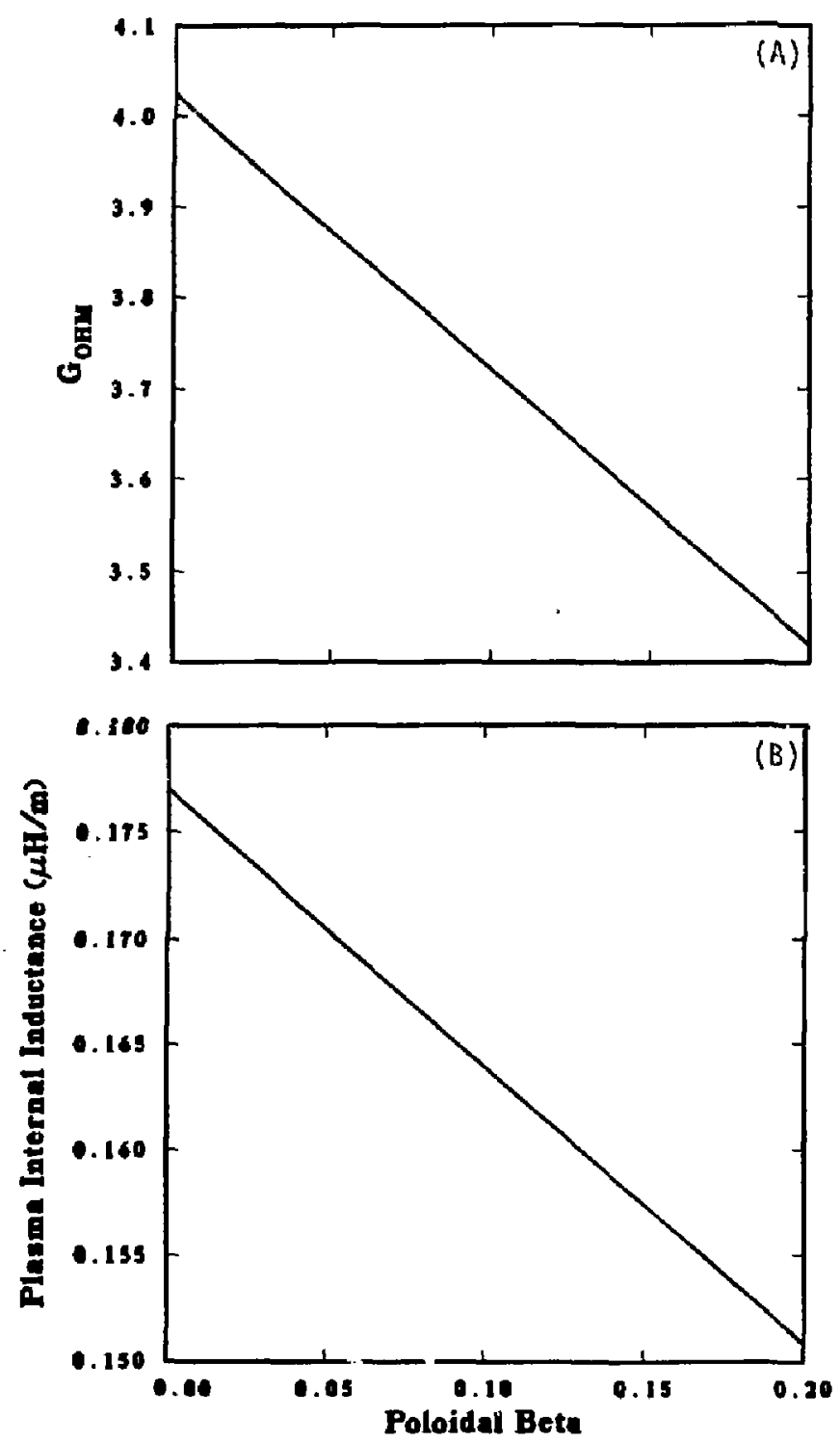

Figure 6.5-3. The plasma-resistance corr . $_{1}$ osite-profile factor $(A)$ and the plasma inter$\mathrm{nal}$ inductance per unit length $(B)$ as functions of $\beta_{\theta}$ for $F=-0.1$ and power profiles (Equations 6.4-3 through 6.4-5). 
models each as an eddy-current circuit element. The self- and mutual inductances of the various circuit elements are then calculated (as described in Section 4.5), and the overall inductance matrix is constructed.

Ignoring changes in the plasma position during the start-up transients, only the plasma inductance in the circuit inductance matrix changes in time since plasma intiernal inductance changes as $\beta_{0}$ evolves. Including the $\dot{L}_{\mathrm{p}}$ term in the plasma resistance, the circuit equation (Equation 6.5-11) can be written as

$$
\dot{\mathbf{I}}=\mathbf{L}^{-1} \cdot(\mathbf{V}-\mathbf{R} \cdot \mathbf{I}),
$$

where $\mathbf{L}^{-1}$ is the inverse of the inductance matrix. Because the plasma inductance changes, a new $\mathbf{L}^{-1}$ should be computed at each time step. Instead of inverting $\mathbf{L}$ at each time step, however, one can use the Sherman-Morrison formula [18] to find $L^{-1}$ since only one element of $L$ changes. A special case of this formula for matrices $L$ and $L^{*}$, which are identical except in element $L_{11}$, is

$$
L_{i j}^{*-1}=L_{i j}^{-k}-\frac{L_{i 1}^{-1} L_{1 j}^{-1}\left(L_{i 1}^{*}-L_{11}\right)}{1+L_{11}^{-1}\left(L_{i 1}^{*}-L_{11}\right)} .
$$

The TITAN PF-coil system includes a pair of EF trim coils to provide the exact equilibrium through the plasma transient operations. The code computes the necessary voltage that should be applied to the trim coils through the following procedure: Denoting $\mathrm{I}^{*}$ as the solution to Equation 6.5-15 for $v_{\text {Trim }}=0$, then

$$
\dot{I}_{i}=\dot{I}_{i}^{*}+L_{i, \text { Trim }}^{-1} \frac{v_{\text {Trim }}}{N_{\text {Trim }}} .
$$

Substituting the above equation in Equation 6.5-14 results in a first order equation in $v_{\text {Trim }}$ that can be solved to find the necessary voltage that should be applied to the EF trim circuit. The same procedure can also be applied when the main EF coils reach their steady-state value and are crowbarred $\left(\dot{I}_{E F}=0\right)$, and when the plasma current reaches its final value and the $\mathrm{OH}$ coils are crowbarred $\left(\dot{I}_{O H}=0\right)$.

In addition to the plasma size and the magnetics information, the input to the plasmacircuit code includes the final values of the plasma and EF-coil currents, the back-bias current for the $\mathrm{OH}$-coil set, the grid voltage, and the value of the transfer resistor (Figure 6.5-1). These latter circuit values determine the evolution of the plasma current during the start-up transients. An analytical estimate of the circuit behavior can be found by ignoring the EF trim-coil and eddy-current circuits and using Equations 6.5-1 through 6.5-3. 
Charge-up phase. During the charge-up phase [Figure 6.5-1(A)], the $O H$ coils are charged from the grid. Denoting the back-bias $\mathrm{OH}$-coil current by $\Gamma_{O H}^{-}$and the duration of the charge-up phase from $-t_{c u}$ to $t=0$, we have

$$
\begin{aligned}
t_{c u} & =-\tau_{c u} \ln \left(1-\frac{I_{O H}^{-}}{I_{G, C U}}\right), \\
I_{O H}(t) & =I_{G, C U}\left[1-\exp \left(-\frac{t+t_{c u}}{\tau_{c u}}\right)\right],
\end{aligned}
$$

where $\tau_{C U} \equiv L_{O H} / R_{O H}, v_{G, C H}$ is the voltage applied to $\mathrm{OH}$ coils during the charge-up phase, and $I_{G, c u} \equiv v_{G, E u} /\left(N_{O H} R_{O H}\right)$. Using the expression for $I_{O H}(t)$, the maximum power extracted from the grid, $P_{\mathrm{cu}, M a x}$ is

$$
P_{\mathrm{cu}, M a x}=\frac{v_{G, \mathrm{cu} S} I_{\hat{O} H}}{N_{O H}}=R_{O H} I_{G, \mathrm{cu}} I_{O H}^{-} \cdot
$$

The total energy extracted from the grid, $W_{G_{1}, a s}$, including both the magnetic energy stored in the $O H$ coils $\left(W_{M, \text { cu }}\right)$ and the joule losses during the charge-up phase $\left(W_{n, c u}\right)$ is

$$
W_{G, \mathrm{cu}}=I_{G, \mathrm{cu}}\left(v_{G, \mathrm{cu}} t_{\mathrm{cu}}-L_{O H} I_{O H}^{-}\right) \text {, }
$$

and a measure of the inefficiency of the charge-up phase is

$$
\frac{W_{\mathrm{\Omega}, \mathrm{cu}}}{W_{M, c u}}=-2\left(\frac{I_{G, \mathrm{GU}}}{I_{O H}^{-}}\right)^{2} \ln \left(1-\frac{I_{O H}^{-}}{I_{G, \mathrm{CU}}}\right)-1-\frac{2 I_{G, \mathrm{Cu}}}{I_{O H}^{-}} .
$$

Normalized values of $t_{e u} / \tau_{e u}$ and $W_{\Omega, e u} / W_{M, a u}$ are presented in Table 6.5-II for different values of $I_{G, a} / I_{O H}^{-}$. This table shows that for an efficient charge-up phase (relatively small joule losses in the $\mathrm{OH}$ coils), values of $I_{G, \mathrm{w}} / I_{O H}^{-}$of 3 or larger is desirable. Smaller values result in large joule losses that should be removed by the $\mathrm{OH}$-coil cooling circuit.

Fast-ramp phase. During both fast- and slow-ramp phases, the OH- and EF-coil circuits are in parallel, that is $v_{O H}=v_{E F}$. The circuit equations (Equations 6.5-1 through $6.5-3)$ then can be solved by using a perturbation expansion on the small parameter $R / L$ of each circuit. Assuming that the number of turns in $\mathrm{OH}$ and EF coils are set according to Equation 6.5-6, the circuit equations car be solved to get

$$
\begin{aligned}
\frac{d I_{\phi}}{d t} & =-\frac{1}{\left(C_{1}-C_{2}\right) L_{O H}} \frac{v_{O H}}{N_{O H}}, \\
I_{O H}(t) & =I_{O H}^{-}-C_{1} I_{\phi}(t), \\
I_{E F}(t) & =-\left|\frac{I_{E F}^{+}}{I_{\phi}^{+}}\right| I_{\phi}(t),
\end{aligned}
$$


Table 6.5-II.

INORMALIZED PARAMETERS OF THE CHARGE-UP PHASE

\begin{tabular}{ccc}
\hline $\begin{array}{c}\text { Charge-Up Voltage, } \\
I_{G, \mathrm{cu}} / I_{\text {OH }}^{-}=v_{G, \mathrm{cu}} /\left(R_{\text {OH }} N_{\text {OH }} I_{\text {OH }}^{-}\right)\end{array}$ & $\begin{array}{c}\text { Joule Losses, } \\
W_{\mathrm{n}, \mathrm{cu}} / W_{M, \mathrm{Gu}}\end{array}$ & $\begin{array}{c}\text { Charge-Up Time, } \\
-t_{\mathrm{cu}} / \tau_{\mathrm{cu}}{ }^{(a)}\end{array}$ \\
\hline 1.01 & 6.40 & 4.62 \\
1.1 & 2.60 & 2.40 \\
1.2 & 1.76 & 1.79 \\
1.5 & 0.94 & 1.10 \\
2.0 & 0.55 & 0.69 \\
3.0 & 0.30 & 0.41 \\
5.0 & 0.16 & 0.22 \\
10.0 & 0.07 & 0.11 \\
\hline
\end{tabular}

(a) For the TITAN-I design, $\tau_{c u}=20.7 \mathrm{~s}$.

and

$$
W_{M}(t)=\frac{1}{2} L_{O H} I_{O H}^{-2}-\left(C_{1}-C_{2}\right) L_{O H} I_{\phi}(t) I_{O H}^{-}+\frac{\left(C_{1}-C_{2}\right) L_{O H}}{2 C_{4}} I_{\phi}^{2}(t)
$$

where $W_{M}(t)$ is the total magnetic stored energy in the system (plasma and $\mathrm{OH}$ and EF coils), parameters $C_{1}$ through $C_{\mathrm{3}}$ are given by Equations 6.5-7 through 6.5-9, and

$$
C_{4} \equiv\left(C_{1}+\frac{N_{O H}}{N_{E F}}\left|\frac{I_{E F}^{+}}{I_{\phi}^{+}}\right|\right)^{-1}
$$

During the slow-ramp phase, the $\mathrm{OH}$ coils are discharged into a transfer resistor, $R_{t r}$, while the EF coils are connected in parallel to the $\mathrm{OH}$ coils [Figure 6.5-1(B)]. Substituting for $v_{O H}$ from

$$
v_{O H}=v_{E F}=-R_{t r}\left(\frac{I_{O H}}{N_{O H}}+\frac{I_{E F}}{N_{E F}}\right)
$$


in Equations 6.5-23 through 6.5-25 results in

$$
\begin{aligned}
v_{O H}(t) & =v_{M a x} \exp \left(-\frac{t}{\tau_{f_{r}}}\right), \\
I_{\phi}(t) & =C_{4} I_{O H}^{-}\left[1-\exp \left(-\frac{t}{\tau_{f^{*}}}\right)\right],
\end{aligned}
$$

and the evolution of $\mathrm{OH}$ - and EF-coil currents can be found by substituting for $I_{\phi}$ from the above equation in Equations 6.5-24 and 6.5-25. Also,

$$
\tau_{f r}=\frac{N_{O H}^{2} C_{4}\left(C_{1}-C_{2}\right) L_{O H}}{R_{\mathrm{tr}}}
$$

and

$$
v_{M a x}=\frac{R_{t r} I_{O H}}{N_{O H}}
$$

is the maximum voltage that appears on the $\mathrm{OH}$ and EF coils. The value of $R_{t r}$ is chosen such that $v_{M a x}$ satisfies the electrical-engincering design constraints of the coils.

The fast-ramp phase continues until the voltage across the $O H$ and EF coils becomes equal to the grid power-supply voltage, $v_{G}$. The duration of the fast-ramp phase, $t_{f r}$, is

$$
t_{f r}=\tau_{f r} \ln \left(\frac{v_{G}}{v_{M a x}}\right)
$$

and the plasma current at $t=t_{f r}$ is

$$
I_{\phi}\left(t_{f r}\right)=C_{4} I_{\mathcal{H}}^{-}\left(1-\frac{v_{G}}{v_{M a x}}\right) .
$$

The maximum joule heating in the transfer resistor is at the initiation of the fast-ramp phase $\left(R_{t r} I_{O H}^{-2} / N_{O H}^{2}\right)$ and the total joule losses in the transfer resistor, $W_{n, t r}$, are

$$
W_{n, t r}=\frac{C_{4}\left(C_{1}-C_{2}\right)}{2} L_{O H} I_{O H}^{-2}\left[1-\left(\frac{v_{G}}{v_{M a x}}\right)^{2}\right] .
$$

The joule losses in the $O H$ and EF coils can be found by using the appropriate expressions for the current evolution in each circuit.

Note that larger values of $v_{M a x}$ :esult in larger $R_{t r}$ and shorter duration for the fast-ramp phase. However, the plasma cur ent at the end of the fast ramp, as well as the energy loss in the transfer resistor, is insensitive to the value of $v_{M a x}$ since usually $v_{G} / v_{\text {Max }} \ll 1$. 
Slow-ramp phase. During the slow-rnmp phase, the grid power is directly applied to the $\mathrm{OH}$ and EF circuits, driving the $\mathrm{OH}$ current to zero and in the forward-bias direction [Figure 6.5-1(C)]. Substituting for $v_{O H}=v_{E F}=-v_{G}$ in Equations 6.5-23 through 6.5-25 results in

$$
I_{\phi}(t)=I_{\phi}\left(t_{f r}\right)+\frac{v_{G}}{N_{O H}\left(C_{1}-C_{2}\right) L_{O H}}\left(t-t_{f r}\right),
$$

and the evolution of $\mathrm{OH}$ - and EF-coil currents can be found by substituting for $I_{\phi}$ from the above equation in Equations 6.5-24 and 6.5-25. At the end of the slow-ramp phase, $t_{\text {or }}$, the plasma current has reached its final value. Therefore, the duration of the slowramp phase, $t_{\sigma r}-t_{f_{r}}$, is

$$
t_{\text {rr }}-t_{f r}=\frac{N_{O H}\left(C_{1}-C_{2}\right) L_{O H}}{v_{G}}\left[I_{\phi}^{+}-I_{\phi}\left(t_{f r}\right)\right] .
$$

The power extracted from the grid, $P_{G}$, is given by

$$
P_{G}(t)=\frac{v_{G}}{C_{4} N_{O H}}\left[I_{\phi}(t)-C_{4} I_{O H}^{-}\right] \text {. }
$$

The grid voltage, $v_{G}$, in the above equations is set based on the constraint of the maximum power extracted from the grid, $P_{G, M a x}$, which occurs at the end of slow-ramp phase:

$$
v_{G}=\frac{C_{1} N_{O H}}{I_{\phi}^{+}-C_{1} I_{O H}^{-}} P_{G, M a x}
$$

The total energy extracted from the grid during the slow-ramp phase is

$$
W_{G}=\frac{\left(C_{1}-C_{2}\right) L_{O H}}{2 C_{4}}\left[I_{\phi}^{+}-I_{\phi}\left(t_{f r}\right)\right]\left[I_{\phi}^{+}+I_{\phi}\left(t_{f r}\right)-2 C_{4} I_{O H}^{-}\right] \text {. }
$$

The above equations show that the plasma current and power extracted from the grid increase linearly in time during the slow-ramp phase. At the beginning of this phase, $P_{G}<0$ indicates that the $\mathrm{OH}$ coils are still discharging into the shunt resistors of the grid power supply, but this part $\left(P_{G}<0\right)$ is of short duration. Also, using Equation 6.5-36, one finds

$$
\Delta I_{O H}=I_{\mathrm{j} H}^{-}-I_{O H}^{+}=C_{1} I_{\phi}^{+}
$$

which shows that parameter $C_{1}$ directly reflects the degree of coupling between the $\mathrm{OH}$ and plasme circuits. 
Equations 6.5-18 through 6.5-41 provide estimates for the evolution of the plasma current during the current ramp-up phase. In addition to the coil geometry and values $I_{\phi}^{+}$and $I_{E F}^{+}$, the input data for these equations include the $O H$-coil back-bias current value, $I_{O H}^{-}$, grid voltage for the charge-up phase, $v_{G, u s}$ (or the maximum power extracted from the grid during the charge-up phase, $\left.P_{c u, M a x}\right)$, the maximum voltage allowed on the $\mathrm{OH}$ and EF coils, $v_{\text {mas }}$ (or the value of the transfer resistor, $\boldsymbol{R}_{t r}$ ), and the maximum power extracted from the grid during the slow-ramp phase, $P_{G, M a c}$ (or the grid voltage during the slow-ramp phase, $\left.v_{G}\right)$. As mentioned before, the value of the plasma current at the end of the fast-ramp phase is insensitive to the value of $v_{\max }$. A value of $v_{\max }=80 \mathrm{kV}$ is chosen for the TITAN analysis, which results in a maximum voltage of $40 \mathrm{kV}$ on each superconducting EF coil. It is further assumed that the same power supply is used during the charge-up and slow-ramp phases $\left(v_{G, e u}=v_{G}\right)$, and the maximum grid power is set at $500 \mathrm{MW}$. For these conditions, the solutions to Equations 6.5-18 through 6.5-4: are reported in Table 6.5-IlI for different values of $\mathrm{OH}$-coil back bias, ranging from $I_{O H}=28$ (symmetric swing for TITAN-I) to 50 MA-turn.

Table 6.5-III shows that as $I_{O H}^{-}$is increased, the voltage of the grid power supply also increases and, thus, $I_{G, \mathrm{Cu}}$ increases such that the ratio of $I_{G, \mathrm{cu}} / I_{O H}^{-}$assymptotes to about 1.4. As a result, the duration of the charge-up phase asymptotes to $\sim 25 \mathrm{~s}$ and the ratio of $W_{n, c u} / W_{M, c s}$ to $\sim 1$ (Table 6.5-II). Since the magnetic stored energy in the $\mathrm{OH}$ coil at the end of the charge-up phase increases as $I_{O H}^{-}{ }^{2}$, joule losses during this phase also increase as the square of the $\mathrm{OH}$ back-bias current.

For larger values of $I_{O H}^{-}$and for fixed values of $v_{M a x}$, the transfer resistor should be smaller (Equation 6.5-32), resulting in longer $\tau_{f r}$ and $t_{f r}$. The plasma current at the end of the fast-discharge phase is also larger and directly scales with $I_{O H}^{-}$. As a result, Equation 6.5-38 shows that for a fixed $P_{G, M a x}$, the voltage of the grid power supply is larger, as was mentioned before. Large values of $I_{O_{H}}$, together with the resultant larger values of $v_{G}$, increase the plasma-current ramp rate during the slow-ramp phase and decrease the duration of this phase. The $\mathrm{OH}$ forward-bias current will also be amaller, such that the total current swing in the $\mathrm{OH}$ coils remains the same. The total joule losses in the $\mathrm{OH}$ coils during both fast- and slow-ramp phases is approximately constant and independent of $I_{O H} \cdot$

Even though the duration of the current ramp-up phases becomes shorter as $I_{O H}^{-}$is increased, $\tau_{c u}$ also increases such that the total duration of start-up remains the same. The total energy extracted from the grid increases for larger values of $I_{O H}^{-}$- mainly because of increased joule losses in the $\mathrm{OH}$ coils during the charge-up phase but also partly because of larger losses in the transfer resistor. 
Table 6.5-III.

\section{ESTIMATES OF TITAN-I START-UP PARAMETERS ${ }^{(0)}$}

\begin{tabular}{|c|c|c|c|}
\hline Back-bias $O H$-coil current, $I_{O H}^{-}$(MA-turn) & 28. & 40. & 50. \\
\hline Grid voltage, $v_{G}=v_{G, \text { cu }}(\mathrm{kV})$ & 2.50 & 2.98 & 3 \\
\hline Transfer resistor, $R_{t r}(\Omega)$ & 1.06 & 0.74 & \\
\hline \multicolumn{4}{|l|}{ Charge-Up Phase } \\
\hline$I_{G, c u}(M A-t u r n)$ & 50.7 & 60.5 & \\
\hline Duration, $t_{c u}(\mathrm{~s})$ & 16.6 & 22.4 & \\
\hline Maximum power from grid, $P_{G, a u}(\mathrm{MW})$ & 188. & 321. & \\
\hline Joule losses in $\mathrm{OH}, \mathrm{L} / \mathrm{R}_{\mathrm{N}, \mathrm{S}}(\mathrm{MJ})$ & 695. & 2030. & 35 \\
\hline Energy from the grid (MJ) & 1770. & 4223. & 69 \\
\hline Magnetic stored enerizy, $W_{M}(t=0)(\mathrm{MJ})$ & 1075. & 2193. & 342 \\
\hline \multicolumn{4}{|l|}{ Fast-Ramp Phase } \\
\hline$\tau_{f r}(s)$ & 0.19 & 0.27 & \\
\hline Duration, $t_{f r}(s)$ & 0.65 & 0.88 & \\
\hline Plasma current, $I_{\phi}\left(t_{f r}\right)(\mathrm{MA})$ & 4.72 & 6.70 & 8. \\
\hline OH-coil current, $I_{O H}\left(t_{f^{\prime}}\right)$ (MA-turn) & 13.1 & 18.9 & \\
\hline Joule losses in $R_{\mathrm{tr}}(\mathrm{MJ})$ & 561. & 1144. & 178 \\
\hline Joule losses in OH (MW) & 32.4 & 91.2 & \\
\hline Magnetic stored energy, $W_{M}\left(t_{f+}\right)(M J)$ & 514. & 1049. & 164 \\
\hline \multicolumn{4}{|l|}{ Slow-Ramp Phase } \\
\hline Rate of increase in $I_{\phi}(\mathrm{MA} / \mathrm{s})$ & 0.82 & 0.97 & 1. \\
\hline Duration, $t_{g r}-t_{f r}(s)$ & 16.0 & 11.4 & \\
\hline OH-coil current, $I_{O H}\left(t_{a r}\right)$ (MA-turn) & -28.1 & -16.1 & -6.09 \\
\hline Joule losses in OH (MJ) & 299. & 242. & 19 \\
\hline Energy from the grid $(\mathrm{MJ})^{(b)}$ & 3955. & 2780. & 195 \\
\hline \multicolumn{4}{|l|}{ Start-Up Total } \\
\hline Current ramp-up duration, $t_{\text {or }}$ (s) & 16.7 & 12.3 & 9 \\
\hline Total duration, $t_{c u}+t_{s r}(s)$ & 33.2 & 34.6 & 33. \\
\hline Energy from the grid $(\mathrm{MJ})^{(\mathrm{b})}$ & 6056 & 7336. & 929 \\
\hline Total joule losses in $\mathrm{OH}(\mathrm{MJ})^{(\mathrm{c})}$ & 2101 & 2718. & \\
\hline
\end{tabular}

(a) For $v_{M a x}=80 \mathrm{kV}, P_{G, M a x}=500 \mathrm{MW}$, the TITAN-I magnetics parameters of Table 6.5-I $\left(C_{1}=3.16, C_{2}=0.154, C_{3}=-5.47, C_{4}=0.174\right)$, $N_{O H}=372, N_{E F}=156$, single-turn equivalent $R_{O H}=0.133 \mu \Omega$, and $R_{E F}=0$.

(b) Does not include joule losses in the plasma during the current ramp-up.

(c) Including joule heating during the transition phase (see text). 
As mentioned before, the maximum voltage on the coils, $v_{M a x}$, has only a small effect on the overall start-up scenario since $v_{G} / v_{M a s} \ll 1$ (Equations 6.5-34 and 6.5-35). For example, increasing $v_{\text {Max }}$ from 80 to $100 \mathrm{keV}$ for the case of $I_{O H}^{-}=40 \mathrm{MA}$-curn of Table 6.5-III reduces $t_{f r}$ to $0.75 \mathrm{~s}$ but has negligible effect on any other parameter.

Analysis of Table 6.5-III shows that the start-up scenario strongly depends on the values of $I_{O H}^{-}$and $P_{G, M a x}$. For a steady-state reactor where start-up is an infrequent event, the total energy absorbed from the grid is not an issue and the cost of the startup power supply is directly proportional to its capacity, $P_{G, M a x}$. The $\mathrm{OH}$-coil back-bias value, on the other hand, has important implications for the design and cost of the OHcoil system. Equation 6.5-41 and values from Table 6.5-III show that for a fixed final plasma current and coil geometry, the current swing in the $O H$ coil, $\Delta T_{O H}$, is constant (56 MA-turn for TITAN-I). Therefore, a symmetric swing $I_{O H}^{-}=28 \mathrm{MA}$-turn results in smallest peak stresses in the coils.

It is preferable for the $O H$-coil cooling circuit to only be designed to handle the steady-state heat generated in these coils by nuclear heating and by the oscillating-field current-drive (OFCD) system. For the TITAN-I design, the steady-state heat generation in the $\mathrm{OH}$ coils is about $14 \mathrm{MW}$ (Section 10.5.1.1), which is much lower than the heat generated during start-up. The start-up joule losses are absorbed by the thermal inertia of the coils, adiabatically raising the temperature of the conductor. Larger start-up joule losses are associated with larger values of $I_{\mathrm{OH}_{H}}^{-}$. On the other hand, smaller values of $I_{O H}^{-}$ result in larger values of $I_{O H}^{+}$and larger joule losses during the transition phase to steady state [Figure 6.5-1(D)] where the OH coils are discharged very slowly ( $L / R$ time). Since the smallest resistance possible is for short-circuited $\mathrm{OH}$ coils, the longest transition time is the same as $\tau_{c u}(21 \mathrm{~s}$ for TITAN-I). For this case, the magnet energy stored in the forward-biased $\mathrm{OH}$ coil appears as heat in the $\mathrm{OH}$ coils. The total $\mathrm{OH}$ joule losses for the start-up, listed in Table 6.5-III is based on this assumption and shows that the heat deposited in the $O H$ coils scales roughly as $I_{\text {or }}^{-}$(and not the square of the current).

The magnet engineering constraints point towards a symmetric $\mathrm{OH}$ current swing as the preferred mode of operation. However, it should be noted that during the transition phase, the current-drive system should work harder to oppose the electric field generated in the plesma by the discharging $\mathrm{OH}$ coils. Since the maximum decay tıme of the $\mathrm{OH}$ circuit is set by $t_{\mathrm{C}_{4}}$ and is independent of $I_{O H}^{-}$, smaller values of back bias (larger $I_{O H}^{+}$) will require higher excess capacity for the OFCD system. Finally, lower values of $I_{o}^{-}$ increase the duration of the slow-ramp phase such that the amount of energy supplied to the plasma during this phase may not be enough to balance the transport losses. In fact, for TITAN-I start-up simulations, ignition could only be achieved for $I_{O H}=50 \mathrm{MA}$-turn 
as is discusaed below. This is partly caused by the assumption of a constant-voltage, $v_{G}$, power supply for the grid. This assumption causes grid power used by the system to increase linearly in time (Equation 6.5-38), and the full capacity of the power supply is utilized only for a small portion of the start-up sequence. In principle, one can envision a scenario in which a large voltage is applied at the beginning of the slow-ramp phase (larger plasma-current ramp rate) and then slowly reduced, step by step, such that large power can be extracted from the grid at all times. This procedure reduces the duration of the slow-ramp phase by up to $50 \%$ and will allow for symmetric OH-coil current swings.

The above discussion shows that the optimum value of $I_{O_{H}}^{-}$can only be obtained through complex analyses of the detailed OH-coil design, the OFCD-aystem excesscapacity requirements, and the extrn cost that may be associated with a variable-voltage grid power supply. Because of the complexity of this optimization problem, and becaute the cost of the $\mathrm{OH}$ system and the start-up equipment represents a very small fraction of the cost of the TITAN reactors, such an optimization was not performed.

The plasma-circuit analysis of the TITAN-I design uses $I_{O H}=50 \mathrm{MA}$-turn and other relevant parameters that are similar to those listed in the footnote of Table 6.5-III. Figures 6.5-4 through 6.5-9 show the evolution of the TITAN-I plasma during the startup sequence, ignoring the eddy currents that may be flowing in the FPC. Figure 6.5-4 shows the time histories of the plasma and PF circuit currents. Because of the effect of plasma resistance, the EF-coil current reaches its final value before the plasma current and is crowbarred first. These figures also show the required current for the EF trin coil to achieve the exact equilibrium throughout the discharge. The vertical field provided by the main EF is shown in Figure 6.5-5(A) and is compared to the required value from the Shafranov formula (Equation 6.5-11). The vertical field provided by the EF trim coil to produce the exact equilibrium is also shown. The value of $N_{O F} / N_{E F}$ is slightly adjusted, as compared to the value from Equation 6.5-6(A), to minimize the power to the EF trim circuit. The valies of currents and the duration of the the discharge are comparable to the estimates of Table 6.5-III.

Figure 6.5-5(B) shows that total power extracted from the grid and the power delivered to the $\mathrm{OH}$ and $\mathrm{EF}$ coils during the start-up sequence. which, again, are in good agreement with the analytical estimates. At $t \simeq 11 \mathrm{~s}$, final EF-coil current is achieved and the EF coils are crowbarred resulting in a drop in EF-coil power (and grid power). Since the plasma and $\mathrm{OH}$-coil currents are still changing, power has to be supplied to the EF-coil in order to maintain the EF-coil current. Full plasma current is achieved at $t \simeq 13 \mathrm{~s}$ and $\mathrm{OH}$ coils are crowbarred, resulting in decreases in OH- and EF-coil powers. Between $t \simeq 13$ and $15 \mathrm{~s}, \beta_{\theta}$ and, therefore, plasma inductance, are changing rapidly. 

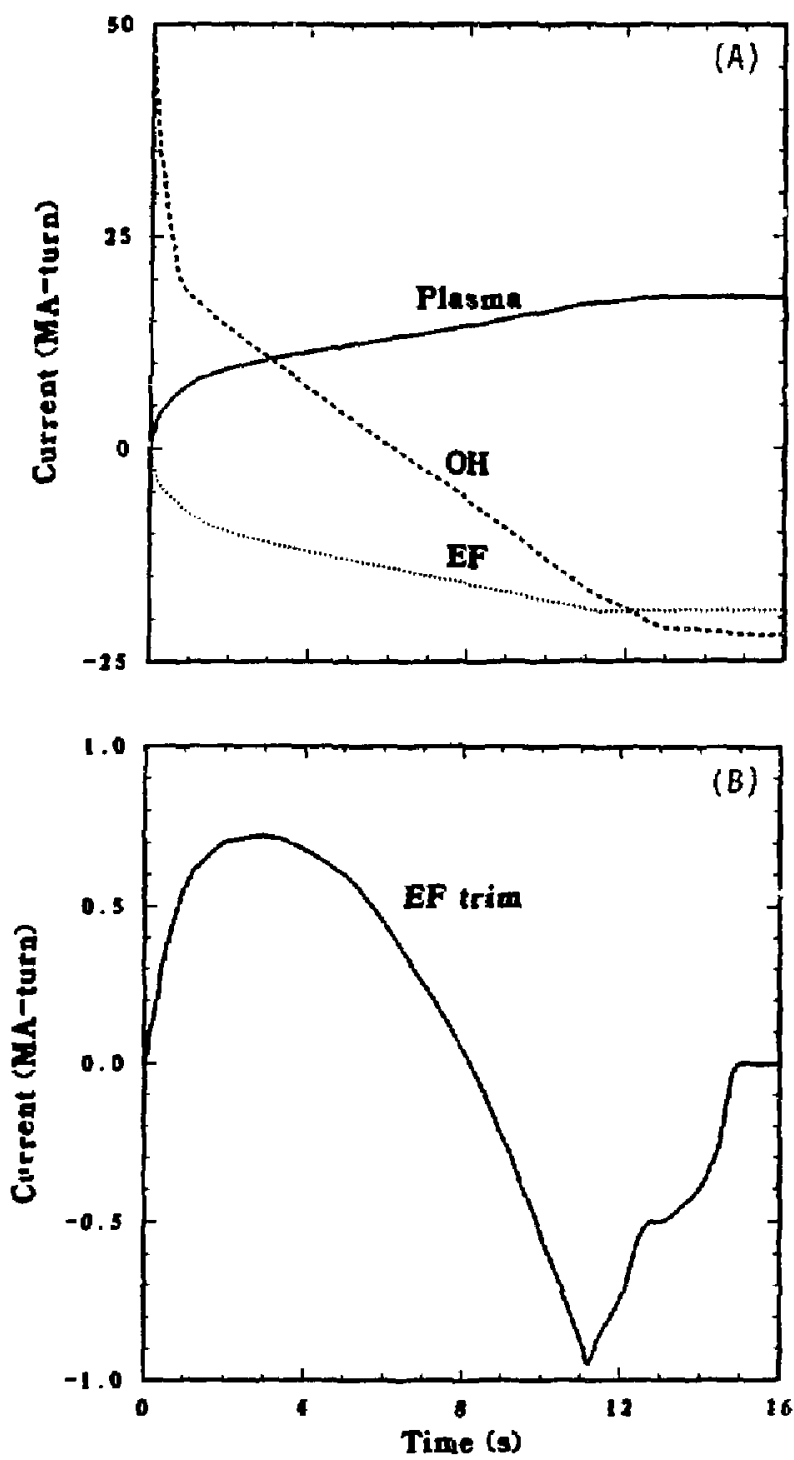

Figure 6.5-4. The TITAN-I start-up simulation results for the evolution of the current in (A) the plasma, superconducting EF coil, and $\mathrm{OH}$ coil; and (B) the EF trim coil. 

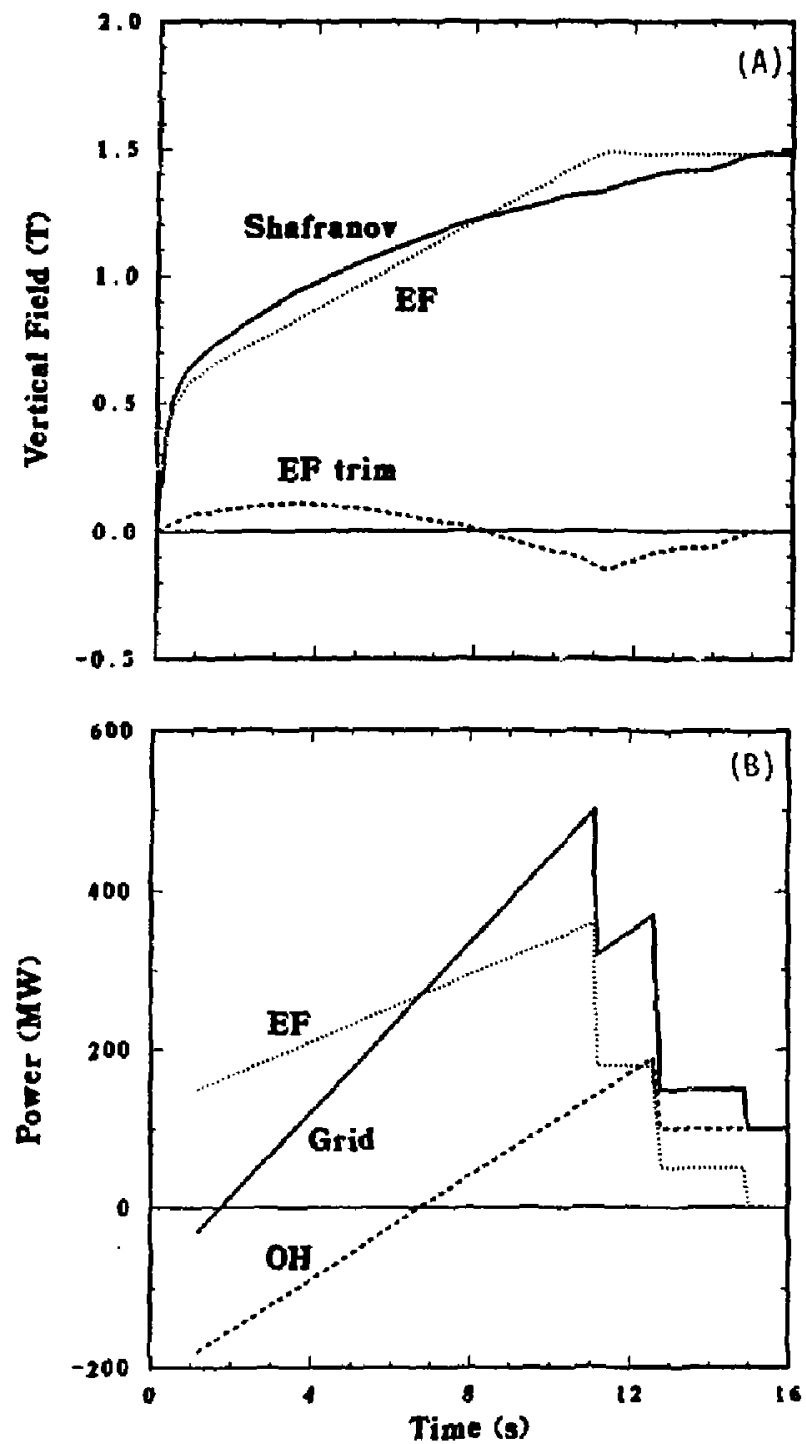

Figure 6.5-5. The TITAN-I start-up simulation results for the evolution of (A) vertical fields produced by the superconducting EF and EF trim coils and the required vertical field from the Shafranov formula; and (B) power deposited in the superconducting EF coil, OH coil, and the total power extracted from the grid. 

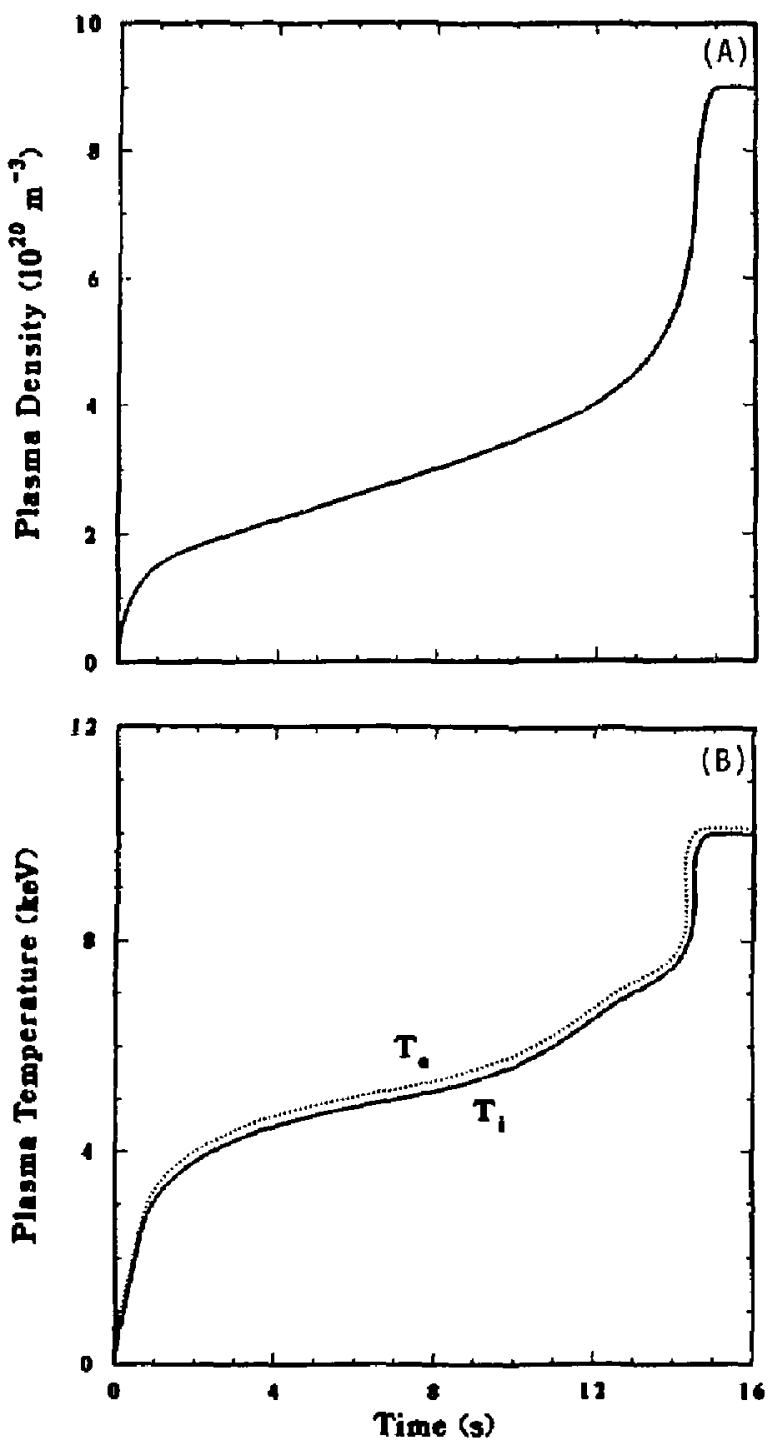

Figure 6.5-6. The TITAN-I start-up simulation results for the evolution of plasme density $(A)$ and temperature $(B)$. 


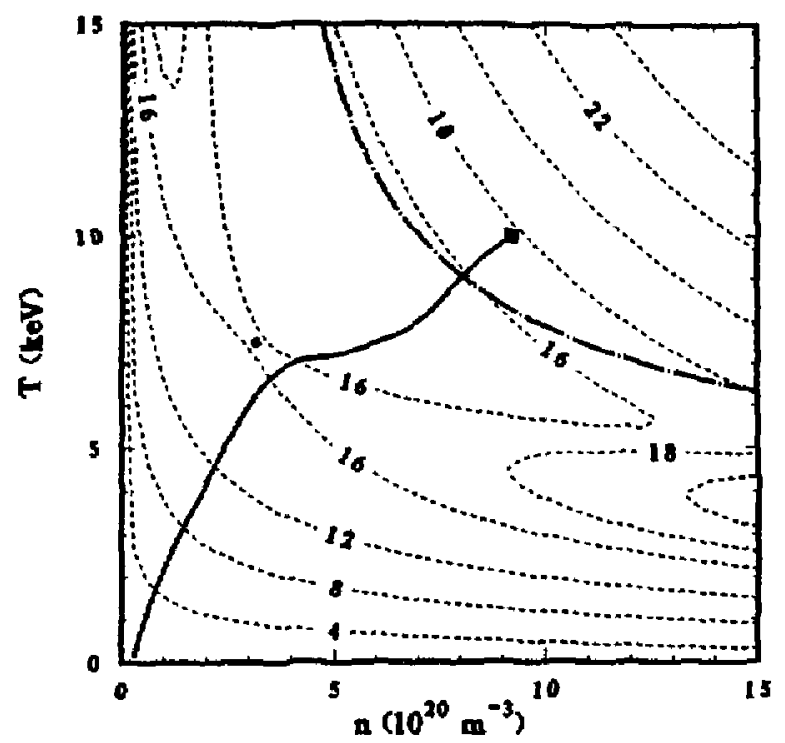

Figure 6.5-7. The TITAN-I start-up path projected in the $I_{\phi}-n-T$ space as found by the analysis of the ignition requirements (Figure 6.4-1). The start-up path (solid line) passes close to the saddle point.

During this period, the trim-coil curreat is also dropping to zero. Some power still has to be applied to the EF-coil to couriter the two effects. The OH-coil power after $t \simeq 13 \mathrm{~s}$ is mostly due to joule heating in the OH coils. It should be noted that the current, voltage, and power to the EF trim-coil circuit is calculated to keep the plasma in exact equilibrium (no shift in magnetic axis). Permitting small shifts in the plasma position will greatly reduce the power required for the EF trim circuit.

Figure 6.5-6 shows the evolution of plasma density and temperature during the startup sequence. The evolution of plasma density and temperature in the ignition $I_{\phi}-n-T$ space is shown in Figure 6.5-7. The ion density evolution is an input to the plasmacircuit code and was adjusted to ensure that the start-up path is located close to the ignition saddle point. The corresponding fueling rate is calculated from the particle balance equation and is shown in Figure 6.5-8(A). The evolution of the plasma density is also chosen such that the plasma streaming parameter remains small and the electron runaway condition is avoided [Figure 6.5.8(B)]. The evolution of $\beta_{\theta}$ and the volt-second 

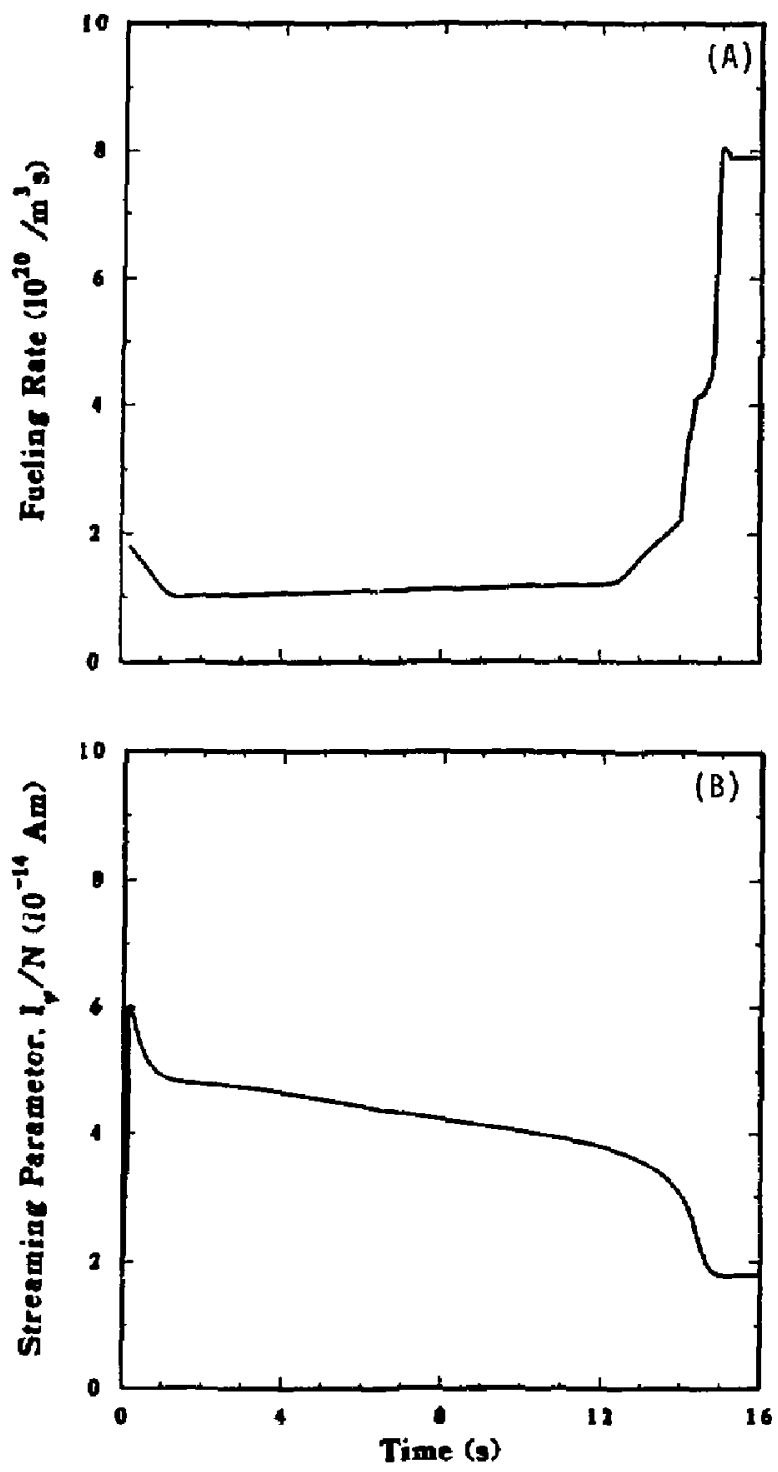

Figure 6.5-8. The TITAN-I start-up simulation results for the required fueling rate (A) and the resultant plasma streaming parameters, $I_{\phi} / N$ (B). 

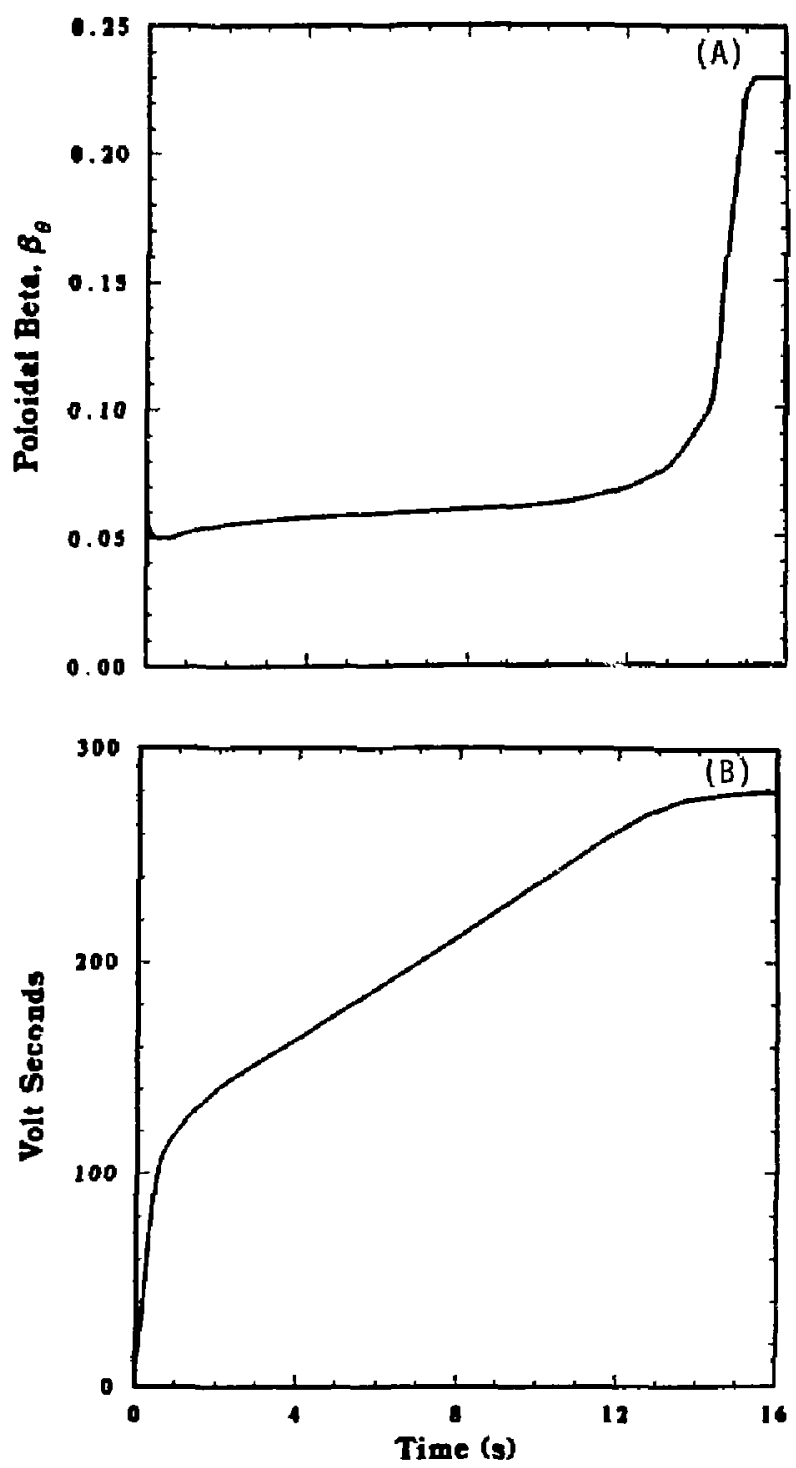

Figure 6.5-9. The TITAN-I start-up simulation results for the evolution of $\beta_{\theta}(A)$ and the consurned volt-seconds $(B)$. 
consumption during the start-up sequence are shown in Figure 6.5-9. It can be seen that plasma ignition is achieved at $\beta_{\theta}$ values of about $8 \%$ and that the final value of $\beta_{t}$ is achieved only after ignition.

The impact of the eddy currents on the start-up sequence has also been studied. The TITAN-I FPC is located in a thick vacuum tank with no resistive break. However, resistive breaks are used in the FPC itself. As a result, the eddy currents in the tanks produce the dominant effect on the start-up. Because the magnitudes of the eddy currents are small, the evolution of the plasma current is not affected. The vertical fields produced by the eddy currents, even though small, affect the plasma equilibrium during the fastdischarge phase (Figure 6.5-10) and programming voltage to the EF trim coils should be modified to keep the plasma in exact equilibrium. However, the power requirement for the EF trim power supplies is not increased by the presence of eddy currents.

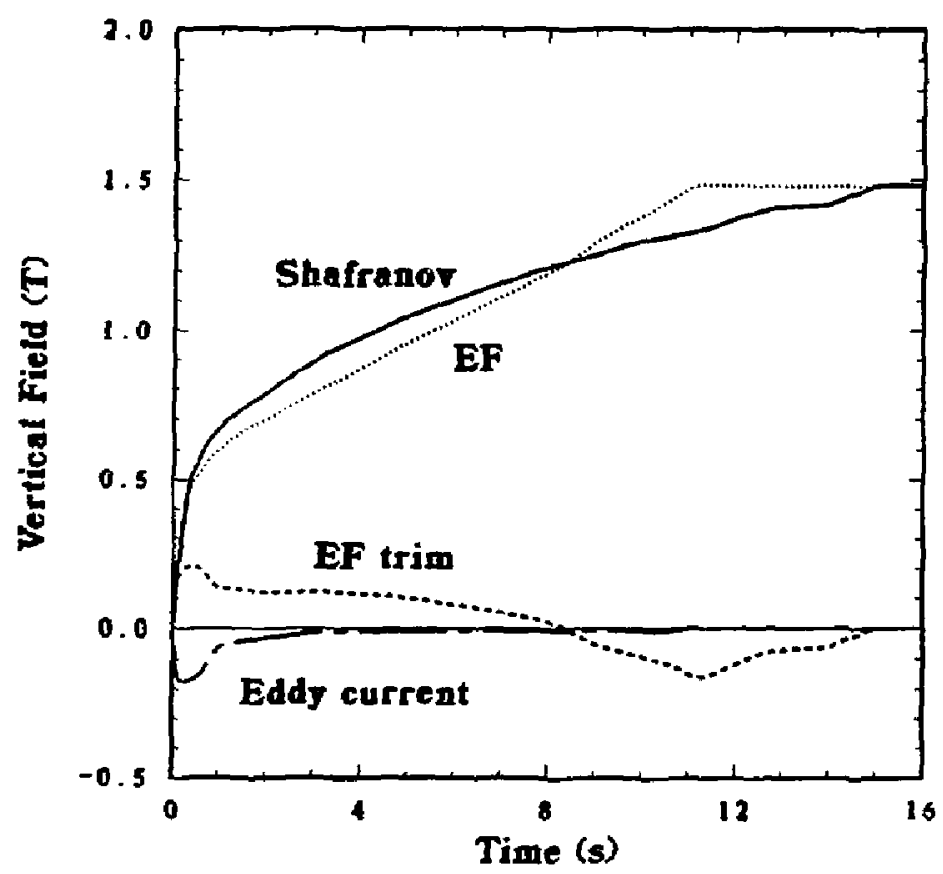

Figure 6.5-10. The TITAN-I start-up sinulation results for the evolution of vertical fields produced by the superconducting EF-coil, EF-trim coil, and eddy currents, and the required vertical field from the Shafranov formula. 


\subsection{SHUTDOWN \& TERMINATIOSं OF TITAN PLASMA}

In RFPs and tokamaks, in addition to the plasma thermal energy, a significant arnount of energy is stored in the poloidal magnetic field. At full operational conditions, the stored energy in TITAN-I plasma includes $0.1 \mathrm{GJ}$ of kinetic (thermal) energy and $W_{M} \sim 4.3 \mathrm{GJ}$ of magnetic energy ( $\sim 5.2 \mathrm{GJ}$ for $\mathrm{OH}$ coils with full forward-bias current). The magnetic energies internal to the plasma are $0.3 \mathrm{MJ}$ in the toroidal field and $0.4 \mathrm{GJ}$ in the poloidal field. The magnetic energies outside the plasma are $<2 \mathrm{MJ}$ in the toroidal field and $3.6 \mathrm{GJ}$ in the poloidal field. Any rapid release of these stored energies (e.g., similar to disruptions in tokamaks) may lead to severe consequences.

Operating RFP experiments tusually end with a "current termination" phase where the plasma current is rapidly reduced to approximately zero. Current termination is characterized by the loss of toroidal-field reversal and is accomparied by a positive voltage spike (as opposed to a negative voltage spike for tokmmak disruptions) and large density and magnetic-field fluctuations. A number of variables, such as plasma dersity, toroidal-field reversal, magnetic-field errors, and impurities appear to affect RFP terminations. A complete and satisfactory explanation of RFP current terminations is not yet available. Evidence, however, suggests that the onset of termination may be related to a loss of density, possibly leading to a streaming parameter that exceeds a critical value for runaway electrons. Since the value of the streaming parameter for the TITAN plasma is only a percent of the critical value for runaway electrons, a current termination is not expected during normal, steady-state operation of the TITAN reactor; rather only failure of plasma support technologies leading to an uncontrolled ramp-down of the plasma current will result in a current termination.

A method of controlled current ramp-down has been tested on the HBTX1B experiment in which the TF-coil circuit is controlled so that the pinch parameter (and the field reversal) is maintained at a given value as the current is decreased to a relatively low level [2]. Maintaining the field reversal in this way is found to delay termination, and the current can be reduced to between $10 \%$ and $20 \%$ of the maximum value (and the stored magnetic field energy reduced to $1 \%$ to $4 \%$ of the maximum value) before the termination occurs. Controlled ramp-downs of this kind forestall the loss of toroidal-field reversal as long as possible and are required for the reactor. 


\subsubsection{Plasma Accidents}

During normal, steady-state operation of the TITAN reactors, the following plesma support technologies are operational: (1) fueling, (2) current-drive, (3) toroidal-field, (4) divertor-field, and (5) equilibrium-field systems. Consequences of failure of each of these systems are discussed below.

Fueling system. The effective particle-confinement time in the TITAN plasma is very long (a few seconds) because of the operation with high-recycling divertors. The standard shutdown scenario envisioned for the TITAN plasma starts with the termination of plasma fueling and current divertor operations. Therefore, the loss of fueling system (and vacuum pumping) will not initiate an accident and the standard plasma shutdown scenario will be implemented.

Current-drive system. Once the current drive fails, the plasma current will resistively decay with time scales of $L_{p} / R_{p} \simeq 200$ to $400 \mathrm{~s}$. As for the case of failure of the fueling system, the standard plasma shutdown scenario can be implemented here which will prevent any accidents.

Toroidal-field system. The TF coils provide the reversed toroidal field at the plasma edge. If the TF-coil system (power supplies) fail, the RFP dynamo activity would increase to generate the necessary toroidal flux, resulting in a decay of the plasma current. An emergency plasma shutdown is recommended in this case even though the decay time for the plasma current is probably long enough that a standard shutdown would be possible.

Divertor-field system. The TITAN reactors operate with three toroidal-field divertors. If any of the divertor coils fail, the reactor can still be operated at reduced power levels. Failure of all three divertors will result in the plasma "riding" on the first wall in a limiter mode. Since the first wall is designed to handle about $95 \%$ of the plasma heat flux (if distributed uniformiy on the first wall), a standard shutdown process would probably suffice to avert any accidents.

Equilibrium-field system. The TITAN reactors use two sets of EF coils: a pair of stperconducting magnets provide the main equilibrium field, and a pair of small normalconducting "trim" coils provide the exact equilibrium (for feedback control of plasma 
position and for OFCD cycles). With a loss of control on plasma equilibrium and position, not only can the plasma energy be deposited locally, but also the plasma current will decrease rapidly, usually leading to a disruption or current termination. The failure of the equilibrium-field system, therefore, appears to be the most severe plasma-related accident for the TITAN reactor (and for any current-carrying toroidal system).

\subsubsection{Shutdown Procedures}

The TITAN plasma shutdown procedures are guided by the above observations to ensure (1) plasma current is reduced through a controlled ramp-down in order to forestall current termination, (2) plasma equilibrium is maintained during current ramp-down, (3) failure of the equilibrium-field system (i.e., quench of the superconducting EF coils) will automatically lead to an emergency shutdown, and (4) most of the magnetic energy stored in the plasma is removed during the shutdown.

The plasma shutdown scenarios envisioned for the TITAN plasma, therefore, start with terminating fueling and current-drive operations and simultaneously discharging the EF coils. For the standard shutdown procedure, the duration of the EF-coil discharge can be on the order of a few to tens of seconds. During the emergency shutdown procedure, however, the EF coils are discharged rapidly $(\sim 0.1 \mathrm{~s})$ through a resistor that can be combined with the quench protection system for the EF coils. Therefore, failure of the equilibrium-field system will automatically initiate the emergency shutdown procedure.

Because of the strong magnetic coupling between the plasma and EF and OH coils in TITAN, a fast discharge of the EF coils results in a rapid decrease in the plasma current; that is, the plasma stored magnetic energy is removed through the EF-coil circuit rather than appearing as heat on the first wall. The parameters of the EF- and OH-coil circuits, however, are chosen such that the plasme equilibrium is approximately maintained during this discharge without any need for an equilibrium control system. The large time constant of the IBC TF coils for field penetration is also utilized to ensure maintenance of the field reversal during the shutdown in a manner similar to the controlled current ramp-down [2].

\subsubsection{Termination Simulations}

A series of simulations with the plasma-circuit code has been performed to assess the effects of plasma termination and the effectiveness of the above procedures in reducing 
the impact of termination. In these simulations, full plasma parameters are assumed at time $t=0$. The effect of current termination is simulated by an exponential increase in the value of the plasma resistance:

$$
R_{p}(t)=R_{p}(0) \exp \left(\frac{t}{\tau_{M H D}}\right),
$$

where $\tau_{M H D}$ is the growth time of resistive MHD modes perceived to be responsible for loss of reversal and termination ( $\sim 10 \mathrm{~ms}$ for TITAN) and $R_{p}(0)$ is the classical plasma resistance at time $t$ which is computed based on plasma temperature at that time.

The first case simulates the situation where the EF coils remain at full current. The evolution of the plasma current is shown in Figure 6.6-1(A). Because the plasma resistance is very small at time $t=0$, the plasma current starts to decrease at about $80 \mathrm{~ms}$ and is reduced to zero in about $30 \mathrm{~ms}$. Figure 6.6-1(B) shows the joule heating in the plasma, which is assumed to be directly deposited on the first wall. The $\sim$ 4-GJ magnetic stored energy in the plasma appears as heat at a peak rate of $\sim 120 \mathrm{GW}$. If the $\mathrm{OH}$ coils are short-circuited during normal operation, a large current can be induced in the $\mathrm{OH}$ coils, reducing the heat deposited in the plasma to $\sim 1.6 \mathrm{GJ}$ and the peak heating to $\sim 60 \mathrm{GW}$. The loop voltage induced in the plasma is $\sim 20 \mathrm{kV}$ and large voltages also appear on superconducting EF coils. These results underline the severity of the problem.

For the second case, it is assumed that at the initiation of the accident the superconducting EF coils discharge rapidly in a resistor that can be combined with the quench protection system for the EF coils. It is further assumed that $O H$ coils are shorted out. The evolution of circuit currents and the corresponding heating power in the plasma are shown in Figure 6.6-2. It can be seen that the major part of the magnetic stored energy in the system is removed through the discharge of the EF coils. The total energy that appears as heat in the plasma (and, therefore, on the first wall) is $\sim 400 \mathrm{MJ}$, representing a factor of 10 reduction. The peak heating rate and the plesma loop voltage are also reduced tenfold. Because the TITAN PF-coil system is designed to provide approximate equilibrium during the start-up phase, approximate equilibrium is also maintained during the fast discharge of the EF coils as shown in Figure 6.6-3. The change in the plasma position, because of the mismatch between the vertical field and the required field from Shafranov formula, is small during most of the termination simulation. The eddy-current effects have also been investigated and, although small, found to improve the situation (i.e., smaller energy appears in the plasma and less mismatch occurs between the required vertical field and that produced by the EF crils).

These preliminary simulations of the TITAN emergency shutdown procedure appear to indicate that most of the stored magnetic energy is removed from the system and 

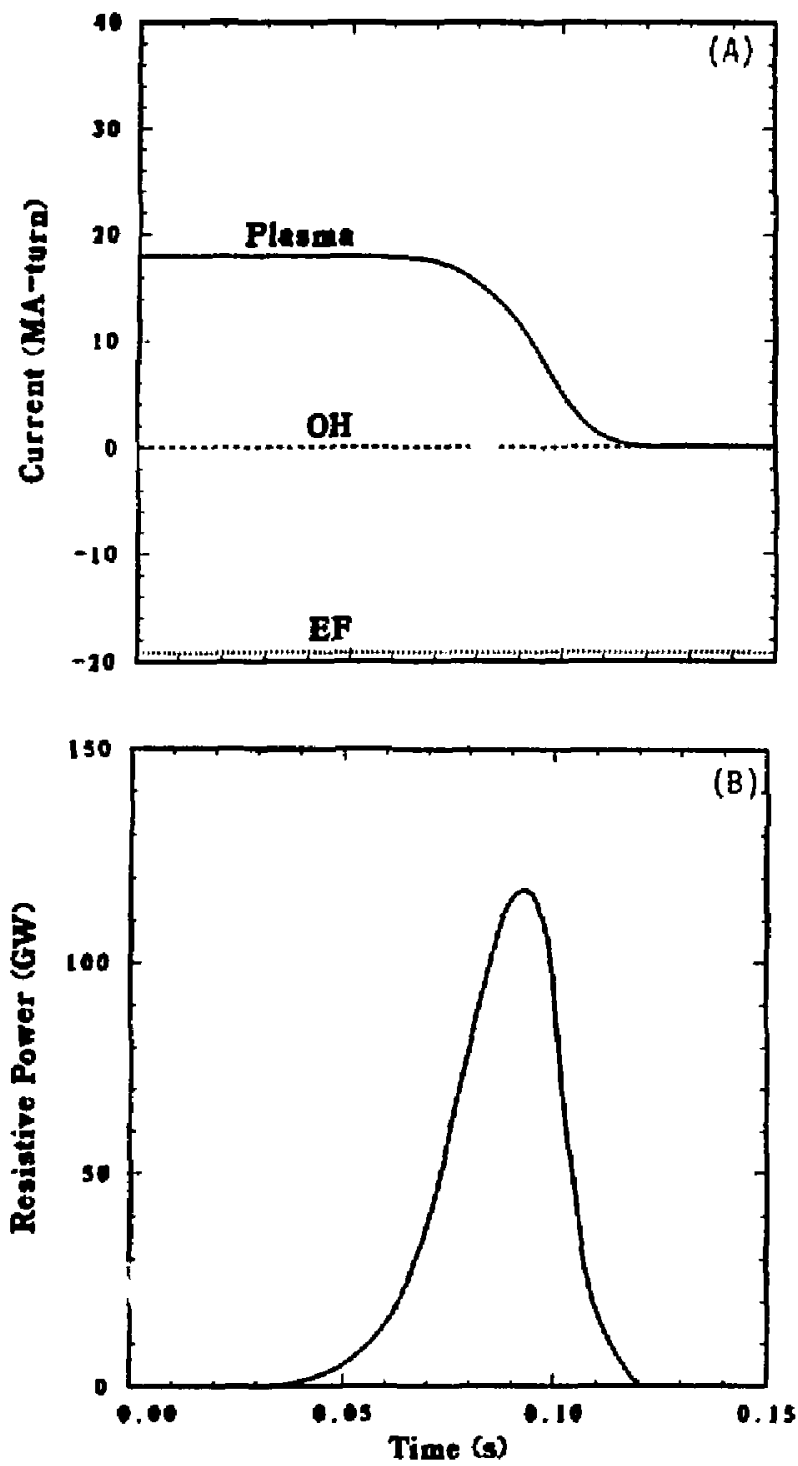

Figure 6.6-1. The TITAN-i termination simulation results for the evolution of $(A)$ the plasma, EF-coil, and $\mathrm{OH}$-coil currents; and (B) the heating power in the plasma. Note that the FF-coil current is kept at its full value and the $\mathrm{OH}$-coil is open circuit. 

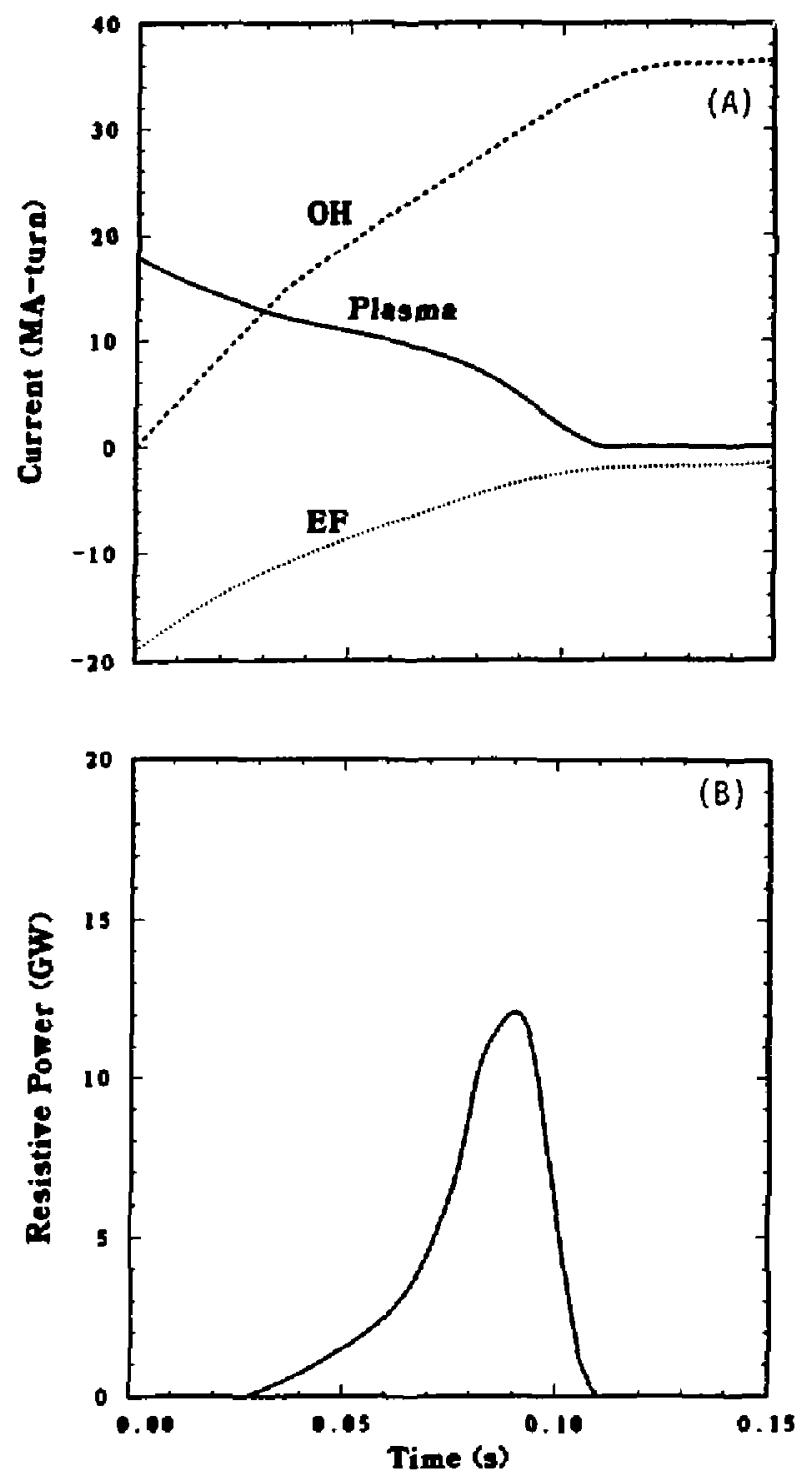

Figure 6.6-2. The TITAN-I termination simulation results for the evolution of $(A)$ the plasma, EF-coil, and OH-coil currents; and (B) the heating power in the plasma. Note that heating power is reduced tenfold in this case. 


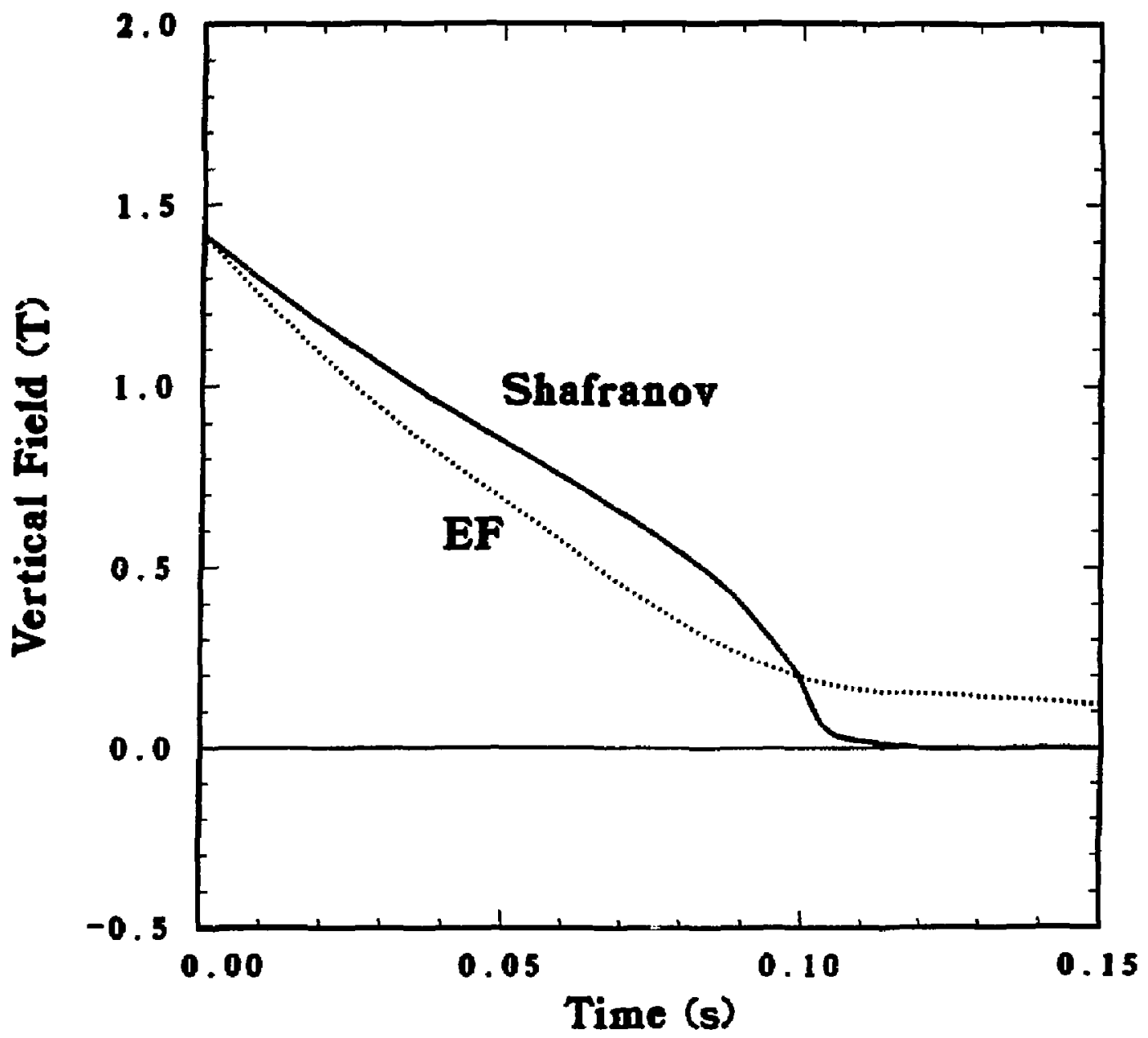

Figure 6.6-3. The TITAN-I termination simulation results for the evolution of vertical fields produced by the superconducting EF-coil current and the required vertical field from the Shafranov formula. 
dumped through the discharge resistor. Only about $200 \mathrm{MJ}$ of energy is transferred to the first wall in a time scale of 50 to $100 \mathrm{~ms}$, resulting in an average temperature rise in the first wall of about $300^{\circ} \mathrm{C}$; therefore, failure of the first wall is not expected.

Despite these favorable results, the RFP theoretical and experimental data base is not very extensive. In particular, no experimental data on high-current, high-temperature, diverted RFP plasmas exist. Furthermore, a complete and satisfactory explanation of current termination in RFPs is not yet available. The safety impact of plasme accidents, therefore, should be further investigated and the shutdown procedures, such as those envisioned for the TITAN plasma, should be experimentally explored.

\subsection{SUMMARY AND CONCLUSIONS}

A typical reversed-field-pinch (RFP) experimental discharge can be divided into four phases: (1) breakdown and formation, (2) current ramp-up, (3) sustainment, and (4) termination. Ignition and fusion burn in a reactor are achieved during the current ramp-up phase, and operation of the current-drive system is required during the sustainment phase. The breakdown and formation phase encompasses the time from the start, which begins by establishing a toroidal magnetic field inside the discharge chamber in the absence of the plasma, to the formation of a "seed" RFP plasma. At the time of peak toroidal magnetic field, poloidal-field windings are activated to produce a flux change through the center of the torus and, consequently, a toraidal voltage around the discharge chamber. This voltage typically ionizes the gas in a few microseconds and the toroidal current is initiated in the resulting plasma. The toroidal plasma current and the toroidal magnetic field within the plasna increase while the toroidal magnetic field at the wall decreases, keeping the average toroidal field (and the toroidal flux) in the chamber almost constant. Eventually the toroidal magnetic field at the wall changes sign and is crowbarred in the reverse direction relative to the buck-biased condition of the breakdown and formation phase.

The existing RFP experimental data base for RFP formation and start-up was reviewed in Section 6.2 and was then applied to the TITAN designs (Section 6.3). To summarize, the conditions for plasma breakdown and subsequent RFP formation for the reactor are expected to differ little from the conditions in present and planned RFP experiments [13]. Likewise, the conditions of the seed RFP plasma required to start up the TITAN reactor, except for plasma size, are similar to present-day RFP parameters $\left(I_{\phi}=0.2\right.$ to $0.4 \mathrm{MA}, n=1$ to $3 \times 10^{1 \mathrm{k}} \mathrm{m}^{-3}, T=0.1$ to $\left.0.4 \mathrm{eV}\right)$. For the reference 
formation scenario, loop voltage in the range of 200 to $500 \mathrm{~V}$ is necessary to ensure short formation time and acceptable resistive flux consumption and formation energy. The scaling of plasma resistivity during the formation phase is an important issue that may be resolved with data from larger RFP experiments. Better density and impurity control during the breakdown and formation process may also be required.

Formation of a seed RFP with somewhat smaller $j_{\phi o}$ (and lower $B_{\phi o}$ and $n$ but higher $\beta_{0_{0}}$ ) would reduce the formation time and is desirable (but requires the relaxation of the $j_{t_{0}}=0.4 \mathrm{MA} / \mathrm{m}^{2}$ connection to ZT-40M). For example, $j_{\phi_{0}}=0.28 \mathrm{MA} / \mathrm{m}^{2}$ and $n_{0} \simeq n=2.8 \times 10^{1 \theta} \mathrm{m}^{-3}\left(j_{\phi} / n=10^{-14} \mathrm{~A} \mathrm{~m}\right)$ result in $\xi=\bar{u} .011$ and $\beta_{\theta_{0}}=0.2$ for $T_{e}=100 \mathrm{eV}$. This $30 \%$ reduction in $j_{\phi o}$ results in reductions of $30 \%$ in $\tau_{R}, 40 \%$ in resistive flux consumption, and $60 \%$ in resistive formation energy for the same value of $E_{\phi a}$. For this case, however, the initial bias field is $B_{\phi o}=\left\langle B_{\phi}\right\rangle=70 \mathrm{mT}$, which satisfies the stray vertical-field constraint for TITAN-I but violates this constraint for TITAN-II.

Using these seed RFP conditions, a 0-D, profile-averaged plasma-circuit code is used to simulate the evolution oi the TITAN plasma through current ramp, ignition, and burn transients (Section 6.5). During the current ramp phase, the plasma current is increased to the peak value. The poloidal-field (PF) coil system provides the poloidal flux and the majority of toroidal flux contained within the full-current plasma by the RFP dynamo.

The steady-state analysis of global plasma power balance provides useful information for the optimization of the plasma approach to ignition. Results of this type of analysis for auxiliary-heated fusion devices are usually presented in the form of required auxiliary power for power balance as a function of plasma density and temperature. This information is then used to identify the path to ignition that requires minimum auxiliary-heating power. A similar analysis can be applied to compact RFP reactors in which the plasma is heated to ignition by ohn $c$ heating alone (no auxiliary heating). The ignition requirements for ohmically heated RFPs are studied (Section 6.4) in order to identify the optimu: 3 path for TITAN start-up. It was shown that the results of this analysis can be presented as the required current for power balance as a function of plasma density and temperature. Also, the resultant surface includes a "ridge" in the $I_{\phi}-n-T$ space above which the path to ignition and burn should be located (similar to corresponding diagrams for awiliary-heated devices where a ridge for auxiliary heating exists). The optimum ignition scenario attempts to pass over the ridge at its lowest height (saddle point). This saddle point for TITAN designs is located at $T \simeq 7 \mathrm{keV}$ and $n \simeq 3 \times 10^{20} \mathrm{~m}^{-3}$ with a current of $I_{\phi} \simeq 16 \mathrm{MA}$. The TITAN start-up path through ignition and burn passes close to this saddle point (Section 6.5.3). 
The TITAN start-up scenario through current ramp, ignition, and burn transients is chosen such that the start-up power is directly extracted from the power grid without requiring an on-site power-storage system. This start-up scenario is described in Section 6.5.1 and its implications for the PF.coil system are reviewed. The evolution of the TITAN plasma during the start-up sequence is investigated by using a 0-D, profileaveraged plasma-circuit code. The simulation results are presented in Section 6.5.3. Analytical estimates of the start-up sequence were also produced which agree well with simulation results. To summarize, the full plasma parameters can be achieved in $<128$ by utilizing grid power supplies; no on-site storage facility is necessary. The TITAN PF coils are designed such that the superconducting $E F$ coils provide an approximate equilibrium during the start-up sequence and a pair of small, normal-conducting EF trim coils maintain the exact equilibrium. Using this approach, only the power supplies for the EF trim coils have to be feedback controlled to ensure proper equilibrium.

Reversed-field-pinch discharges normally end abruptly and the plas:na current decreases rapidly to zero. Accompanying this fast current "termination" is a positive pulse in the toroidal voltage at the liner. This current termination is in contrast to the negative spike in toroidal voltage that accompanies the current disruption in a tokamak, indicating a difference in the flow of magnetic energy to or from the plasme during the respective events. Furthermore, the RFP current termination can be influenced through the control of the density or toroidal maznstic field [2]; generally, the RFP terminates only when toroidal-field reversal is lost.

Current termination is a safety and economic concern because of large magnetic stored energy in the TITAN plasma. Techniques for control of current termination and plasma shutdown, leading to $c$ "soft-landing," are discussed in Section 6.6. The preliminary simslations of the TITAN emergency shutriown procedure appear to indicate that by discharging the superconducting EF coils at the initiation of the accident, most (90\%) of the stored magnetic eniergy is removed from the system and dumped through the discharge resistor. Only about $200 \mathrm{MJ}$ of energy is transferred to the first wall in a time scale of 50 to $100 \mathrm{~ms}$, resulting in an average temperature rise in the first wall of about $300^{\circ} \mathrm{C}$; therefore, failire of the first wall is not expected.

Despite these favorable results, the RFP theoretical and experimental data base is not very extensive. In particular, no experimental data on high-current, high-temperature, diverted RFP plasmas exist. Furthermore, a complete and satisfactory explanation of curresit termination in RFPs is not yet available. The safety impact of plasma accidents, therefore, should be further investigated and the shutdown procedures, such as those envisiozed for the TITAN plasma, should $b$, explored experimentally. 


\section{REFERENCES}

[1] J. A. Phillips, D. A. Baker, R. F. Gribble, and C. Munson, "Start-up of ReversedField Pinches and Current Ramping Using Dynamo Action," submitted to Nucl. Fusion (1987).

[2] A. A. Newton and P. G. Noonan, "Controlled Termination of Reversed-Field-Pinch Discharges," Nucl. Instr. and Methods A245 (1986) 167.

[3] R. L. Hagenson, R. A. Krakowski, C. G. Bathke, R. L. Miller, M. J. Embrechts, N. M. Schnurr, et al., "Compact Reversed-Field Pinch Reactors (CRFPR): Preliminary Engineering Considerations," Los Alamos National Laboratory report LA-10200-MS (August 1984).

[4] F. Najmabadi, N. M. Ghoniem, R. W. Conn, et al., "The TITAN Reversed-FieldPinch Fusion Reactor Study: Scoping Phase Report," joint report of UCLA, General Atomics, Los Alamos National Laboratory and Rensselaer Polytechnic Institute, UCLA-PPG-1100 (January 1987).

[5] P. G. Noonan, C. G. Gimblett, and A. A. Noonan, "Plasma Behavior During Programmed Current Decay and Ramping in the Reversed-Field Pinch," in Proc. Ifth European Conf. on Cont. Fusion and Plasma Phys., Madrid, Spain (June 1987).

[6] A. Kellman, E. Lazzaro, P. Noll, F. C. Schveller, and P. Thomas, "Breakdown Conditions in JET," Bull. Am. Phys. Soc. 30 (1985) 1524.

[7] J. A. Phillips, L. C. Burkhardt, A. Haberstich, R. B. Howell, J. C. Ingrahan, E. M. Little, K. S. Thomas, and R. G. Watt, "ZT-40M Current Risetime Study," Los Alamos National Laboratory report LA-9717-MS (1983).

[8] F. Engelmann, N. Fujisawa, B. B. Kadomtsev, V. I. Pistunovich, J. A. Schmidt, and M. Sugihara, "Physics Design Basis," in International Tokamak Reactor, Phase One, IAEA, Vienna (1982) Chap. III, p. 127.

[9] R. G. Watt and J. N. Downing, "Pre-ionjzation Studies in ZT-40M," Los Alamos National Laboratory report LA-9163-MS (February 1982).

[10] J. A. Phillips, Los Alamos Natioral Laboratory, private communication (August 1986).

[11] H. A. Bodin, R. A. Krakowski, and O. Ortolani, "The Reversed-Field Pinch: from Experiment to Reactor," Fusion Technol. 10 (1986) 307. 
[12] G. A. Wurden, P. G. Weber, R. G. Watt, C. P. Munson, T. E. Cayton, and K. Buchl, "Rufueling and Density Control in the ZT-40M Reversed-Field Pinch," in Proc. Int. School of Plasma Phys. Course and Workshop on Physics of Mirrors, Reversed-Field Pinches, and Compact Tori, Varenna, Italy (September 1987).

[13] D. B. Thomson (Ed.), Proc. Int. Workshop on Engineering Design of Next Step Reverged Field Pinch Devices, Los Alamos National Laboratory (July 13-17, 1987).

[14] E. W. Larsen, C. D. Levermore, G. C. Pomraning, and J. G. Sanderson, "Discretization Method for One-Dimensional Fokker-Planck Operators," J. Comp. Phys. 61 (1985) 359.

[15] J. S. Chang and G. Cooper, J. Comp. Phys. 6 (1970) 1.

[16] V. D. Shafranov, "Plasma Equilibrium in a Magnetic Field," Reviews of Plasma Physics 2, Consultant Bureau, New York (1966) 103.

[17] D. A. Baker, L. W. Mann, and K. F. Schoenberg, Nucl. Fusion 23 (1983) 380.

[18] W. A. Press, B. P. Flannery, A. A. Teukolsky, W. A. Vetterling, Numerical Recipes, Cambridge University Press, Cambridge, United Kingdom (1986). 


\section{CURRENT DRIVE}

Charles C. Bathke Robert A. Krakowski

Tak-Kuen Mau 


\section{Contents}

7.1. INTRODUCTION . . . . . . . . . . . . 7-1

7.2. CURRENT-DRIVE OPTIONS $\ldots \ldots \ldots \ldots \ldots \ldots \ldots$ 7-2

7.2.1. Oscillating-Field Current Drive $\ldots \ldots \ldots \ldots \ldots \ldots . \ldots 7-2$

7.2.2. Bootstrap Current $\ldots \ldots \ldots \ldots \ldots \ldots \ldots \ldots$ 7-5

7.2.3. Fast-Wave Current Drive . . . . . . . . . . . 7-6

7.2.4. Relativistic Electron Beams . . . . . . . . . . 7-11

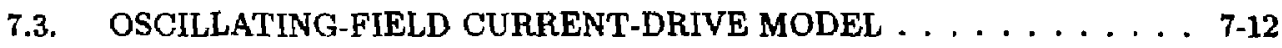

7.3.1. Plasma Model . . . . . . . . . . . . . . . . 7-12

7.3.2. Circuit Model . . . . . . . . . . . . . . . 7-22

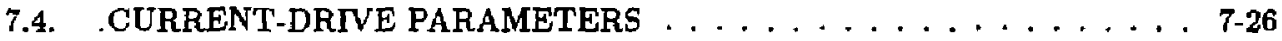

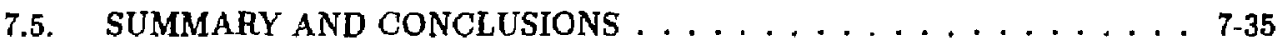

REFERENCES . . . . . . . . . . . . . . . 7-40 


\section{CURRENT DRIVE}

\subsection{INTRODUCTION}

At full plasme current of $18 \mathrm{MW} / \mathrm{m}^{2}$, the energy stored in the reference TITAN plasma includes $W_{M} \sim 5 \mathrm{GJ}$ of magnetic energy and $0.1 \mathrm{GJ}$ of kinetic energy. The magnetic stored energies internal to the plasma are $0.3 \mathrm{GJ}$ in the toroidal field and $0.4 \mathrm{GJ}$ in the poloidal field. The magnetic energies outside of the plasme are $<2 \mathrm{MJ}$ in the toroidal field aid $4 \mathrm{GJ}$ in the poloidal field for zero OH-coils current (6 GJ for OH-coils in full forward-bias current). Since, the toroidal magnetic stored energy internal to the plasma is supplied by the poloidal-field circuit during the start-up, the reference TITAN design requires a poloidal flux of $L_{p} I_{\phi} \simeq 250 \mathrm{~Wb}$ to achieve full plasma current. Because of the large plasma resistance in the TITAN designs, an inductively pulsed burn would be sustained for a pulse length of the order of $L_{p} / R_{p} \simeq 200$ to $400 \mathrm{~s}$. Therefore, steady-state operation is essential considering issues such as the total power balance, thermal cyclic fatigue in a high-power-density environment, as well as the costs of on-site energy storage (frequent grid-assisted start-up seems unlikely) and thermal storage. An inductively pulsed RFP reactor is a possibility [1]. The parameters of such a reactor, lowever, should be optimized to minimize the plasma resistance, which results in larger plasmas, lower power density, and possibly the use of superconducting coils throughout the FPC.

A number of current-drive options for the RFP have been considered (Section 7.2). Although the use of radio-frequency (RF) fast-wave current-drive scheme hes not been fully explored for the RFP, the high plasma density $\left(n \sim 9 \times 10^{20} \mathrm{~m}^{-3}\right.$ in TITAN) and currents relative to those for the tokamak indicate problems with the efficiency of RF current-drive schemes. On the other hand, because of the relaxation processes in RFPs, there is no need to drive the current at the plasma center and some of the issues related to wave penetration may be negated. Bootstrap current is also expected to be low, if such current mists at all in RFPs, since $\beta_{0}$ and $\epsilon=r_{p} / R_{T}$ are small relative to the tokamak.

The close coupling of poloidal and toroidal currents and magnetic fields that determine the near-minimum-energy states of the RFP offers the possibility of a current-drive method based on "magnetic helicity injection" because the resistive decay of plasma current can be viewed as a dissipation of magnetic helicity [2]. For the TITAN reactors, helicity injection by the oscillating-field current drive (OFCD) has been selected as the means to sustain the toroidal plasma current. From a theoretical viewpoint, the required 
physics processes for OFC:D in RFPs are identified [3,4], several numerical simulations are summarized in Reference [5], and some experimental data on OFCD [6] are also available (Section 7.2.1). An analogue circuit model for OFCD is presented in Section 7.3 and is used to investigate parametrically the characteristics of the OFCD system for the TITAN reactor. Design points for TITAN-I and TITAN-II are suggested in Section 7.4. Section 7.5 presents a summary and conclusions.

\subsection{CURRENT-DRTVE OPTIONS}

\subsubsection{Oscillating-Field Current Drive}

The strong coupling of poloidal asd toroidal magnetic fields that determine the nearmininum-energy states of the RFP [i] offers the possibility of a current-drive method based on "magnetic helicity injection" because the resistive decay of plasma current can be viewed as a dissipation of magnetic helicity [2]. Current drive through "electrostatic helicity injection" has been experimentally demonstrated in spheromaks [8], which are also relaxed-state systems like RFPs. Another helicity-injection teclnnique is the oscillating-field current drive (OFCD) $[2,9]$. In this scheme, audio-frequency oscillating voltages are applied to the toroidal and poloidal circuits in the appropriate phase $(\delta=\pi / 2)$ to drive a DC toroidal current in the plasma with the plasma, in effect, behaving as a nonlinear rectifier. As originally proposed [2], OFCD is based on the premise that maintenance of the RFP configuration simply ree(uires the supply of magnetic helicity at a rate equal to its dissipation. The helicity balance is given by $[2,10]$

$$
\frac{d K}{d t}=2 \phi V_{\phi}-2 \int \mathbf{E} \cdot \mathbf{B} d V_{p},
$$

where the integral gives the rate of helicity dissipation throughout the plasma volume and the remaining product of toroidal flux and voltage gives the rate of helicity injection or ejection through the plasma surface. Helicity is effectively injected into the plasma if $\Phi$ and $V_{\phi}$ are sinusoidal and are ostillated in phase with each other (e.g., $V_{\theta}=-\dot{\Phi}$ and $V_{\phi}$ are in quadrature), even though the time-averaged electric fields are zero. Hence, with t] $F-\Theta$ diagram providing the required connection between $V_{\theta}$ and $V_{\phi}$, a noninvasive and potentially efficient means to drive currents in high-density thermonuclear plasma is possible. Current drive by helicity injection has also been proposed for tokamaks $[11-13]$, provided that a similar, globally nondisruptive mechanism for profile relaxation exists.

Experimental data on OFCD in RFPs are reviewed in detail in Section 2.3.8 $[9,6]$ Low-power OFCD tests $\left(\sim 7 \mathrm{MVA}, I_{\phi} \simeq 60\right.$ to $\left.70 \mathrm{kA}\right)$, shown on Figure 7.2-1, were con- 

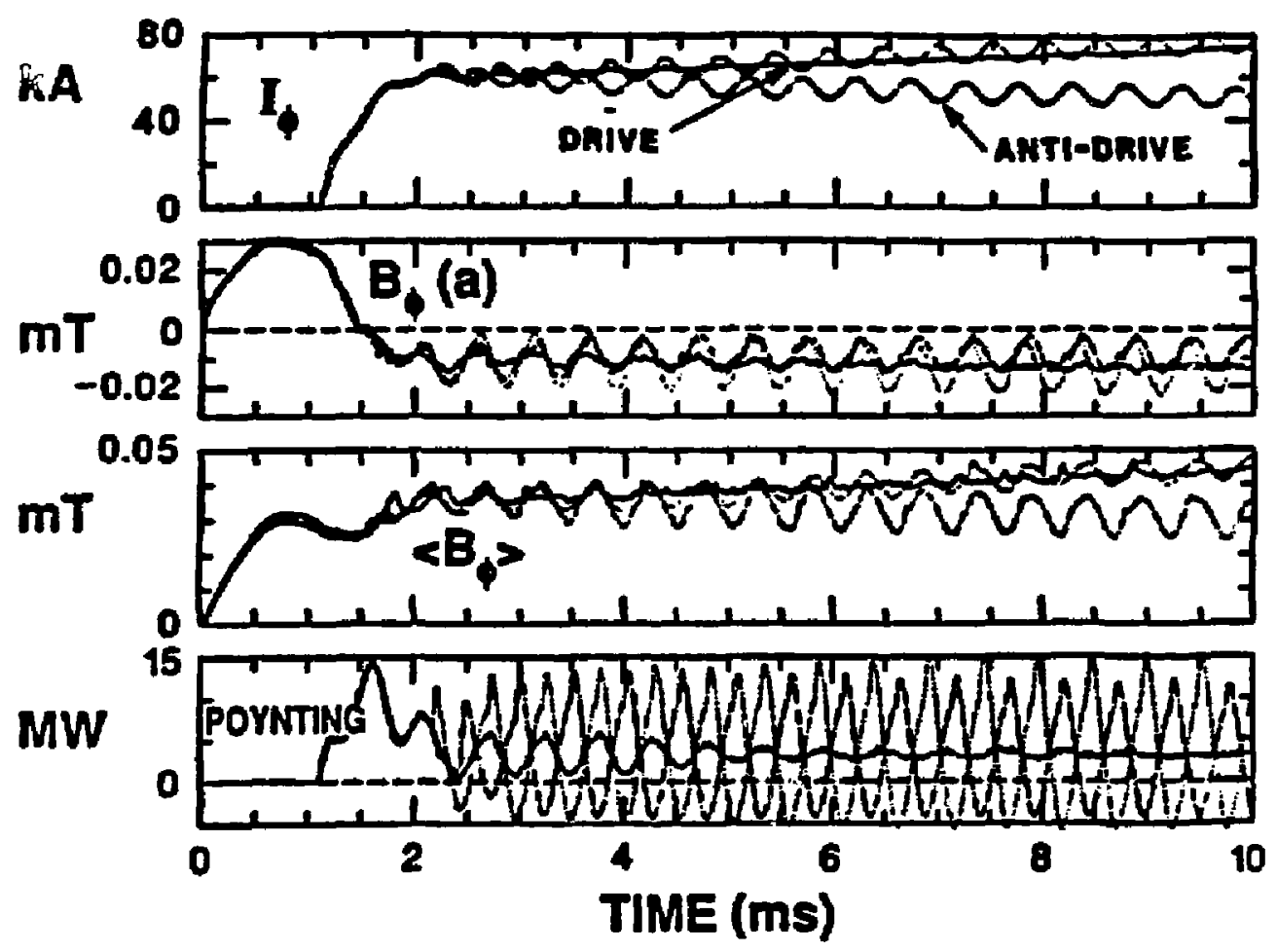

Figure 7.2-1. Low-power OFCD discharge results from Reference [6]. Shown are traces of current, edge toroidal field, toroidal flux, and Poynting vector for a standard discharge, a discharge with the optimal phase between toroidal and poloidal OFCD circuits for driving current, and a discharge with the optinal phase in the OFCD circuit for anti-drive. 
ducted on ZT-40M. These ramped discharges were at low temperature and, hence, a high plasma resistance. With optimal phasing $(\delta \simeq \pi / 2)$, an approximately $5 \%$ increase in poloidal flux was observed when OFCD was applied. While a clear demonstration of sulstantial current drive by OFCD nust await RFPs operating with hotter plasmas and reduced wall interaction [14], the strong dependence of the plasma response on $\delta$ and the spatial and temporal beliavior of the nean magnetic fields are in general agreement with magnetic helicity models and simulations.

Most of the analysis and design for the OFCD system is based on circuit-analogue models $[2,9]$. Such a model is described in Section 7.3 and is used to analyze the TITAN reactor current-drive system. A heuristic method for explaining OFCD in RFPs on the basis of MHD [5] considers the evolution of the q-profile for one OFCD cycle, as is shown in Figure 7.2-2. Initially the near-mininum-energy RFP state is claracterized by a value of $q=d \psi / d \phi$ that is less than unity on axis, with $q$ falling to zero near the plasma edge and reversing at the plasma surface as the toroidal field clanges direction. In order to affect OFCD in the RFP, the exterual toroidal- and poloidal-field circuits are oscillated about steady-state values, with the most efficient "pumping" (i.e., maximum current for mininum reactive power) occurring when the toroidal and poloidal voltages are approximately 90 degrees out of phase. A decrease in the external poloidal flux, $\psi$, and an increase in amplitude of the external toroidal flux, $\phi$, will result in a more negative $F$ value (i.є., deeper reversal) which appears as a compression phase in Figure 7.2-2. The plasuza under these condjtions is unstable to a series of resistive-MHD nodes with high toroidal mode numbers, $n$, all of which have a poloidal mode number $m=1$. These instabilities drive a Kadomtsev reconnection process (Figure 7.2-2). resulting in a flattesing of the central portion of the $q$ profile and increasing the poloidal flux $[3,4]$. The Kadomtsev reconnection process may also occur during the compression phase and need not be a distinct phase, as is shown in Figure 7.2-2. The plasma is then decompressed as the external circuits approach the end of a period when the external poloidal flux is increasing and the totoidal flux is decreasing. With the value of $q$ at the plasma edge reset to the initial value, the plasma then relaxes by means of a double reconnection [4] (i.e., dynamo effect) into the initial near-minimum-energy state, and the OFCD cycle is complete. As indicated previously, frequency of a few tens of $\mathrm{Hz}$ are generally required for reactor grade plasmas, with higher frequency $(\sim 5,000 \mathrm{~Hz})$ for the more resistive, present-day experiments. 

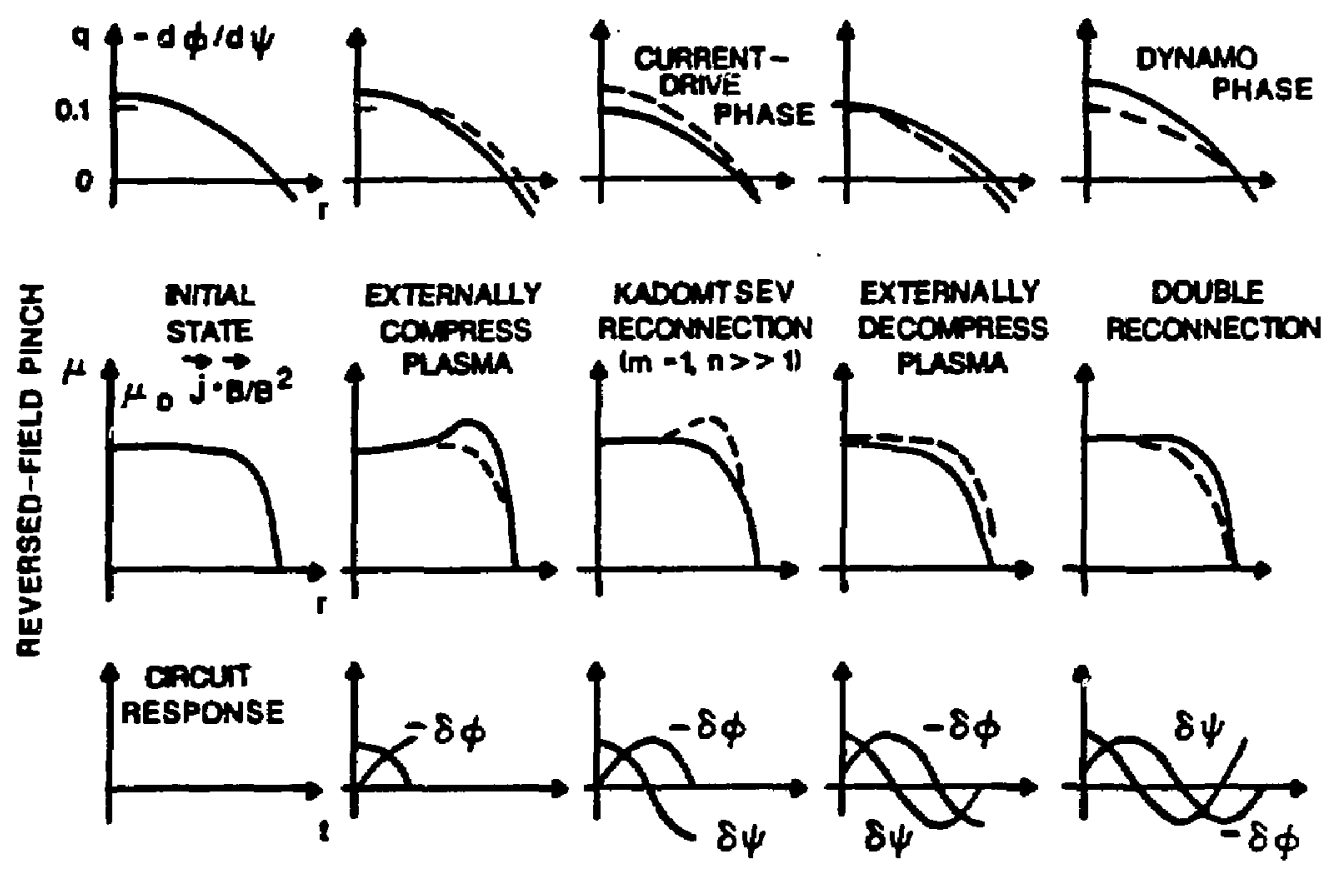

Figure 7.2-2. Schematic representation of OFCD in terms of a q-profile evaluation [5],

\subsubsection{Bootstrap Current}

Neoclassical theory predicts the existence of a bootstrap current caused by radial diffusion. An expression for this bootstrap current density is given by [15]

$$
j_{B c}=-\frac{\epsilon^{1 / 2}}{B_{\theta}}\left(\frac{\partial p}{\partial r}\right)
$$

where $\epsilon=r_{\mathrm{p}} / R_{T}$ is the inverse aspect ratio. The high $\beta_{\theta}$ that characterizes the RFP suggests a large bootstrap current, but the high aspect ratio will tend to reduce the effect. For example, if the pressure profile is given by $p / p_{o}=1-\left(r / r_{p}\right)^{\nu}$, then the contribution of boot:trap current to the overall current deusity is given by

$$
\frac{j_{B c}}{j_{\phi}}=\beta_{\theta} \epsilon^{1 / 2} \frac{\nu^{2}}{4(\nu+2)}
$$

which for $\nu \simeq 2, \beta_{\theta} \simeq 0.2$, and $\epsilon \simeq 1 / 6$ (TITAN conditions) gives $j_{B c} / j_{\phi} \simeq 0.02$. Significantly steeper pressure gradients and lower aspect ratios would be required to make the 
bootstrap curreut a significant contributor, if it exists at all in RFPs. Bootstrap current is not considered a possibility for TITAN at this time.

\subsubsection{Fast-Wave Current Drive}

A variety of current-drive methods using radio-frequency (RF) waves have been explored in the context of maintaining a steady-state tokamak reactor [16,17]. These waves are usually classified according to their frequency ranges, namely: electron cyclotron (ECH; $\sim 100 \mathrm{GHz}$ ), lower-hybrid ( $\mathrm{LH} ; \sim 1 \mathrm{GHz}$ ), ion cyclotron (ICRF; $\sim 100 \mathrm{MHz}$ ), and low-frequency Alfven ( $\mathrm{AW} ; \sim 1 \mathrm{MHz}$ ). Little experimental and theoretical work has been done for RF curtent drive in RFPs, particularly as applied to driving poloidal currents in the outer regions of the core plasma.

The most successful RF current-drive experiment to date has been with LH slow waves in a tokamak [18]. In this scheme, the wave energy is launched toroidally in one direction and is absorbed by electrons through the Landau damping process. In essence, the wave electric field accelerates those electrons with parallel speed $v_{\|}=\omega / k_{\|}$, where $\omega$ is the wave frequency and $k_{\|}$is the wave number parallel to the DC magnetic field. These resonant electrons that receive the wave momentum carry a plasma current, which is subsequently dissipated through collisions with the thernal particles. Consequently, accelerating higher energy electrons results in ligher current-drive efficiency. For a Maxwellian electron distribution, a higher temperature $\left(T_{e}\right)$ plasma naturally leads to more efficient current drive. Likewise, a denser plasma through collisional dissipation will result in less favorable current-drive efficiency. Disregarding accessibility issues temporarily, the current-drive efficiency, $I_{\phi} / P_{C D}$, scales as $T_{e} / n_{e}$. In fact, this conclusion can be extended to other RF schemes, which may drive current by creating a toroidally asymmetric resistivity (e.g., ECH waves) or by minority ion heating (e.g., ICRF waves).

In actuality, many physical processes in the reactor plasma can affect the RF currentdrive efficiency: (1) Because of the variation of the magnetic field along the field line, electrons can be trapped in a magnetic well and consequently become incapable of carrying currents. Any portion of the wave power absorbed by these trapped electrons, therefore, will not contribute to the current and will lower the efficiency: (2) Other conlpeting wave damping processes exist that do not result in a current. An example of these damping processes is absorption by the nonthermal alpha particles and fuel ions in the vicinity of their liarmonic cyclotron resonance surfaces. Damping by the alpha particles is particularly severe for waves in the LH range of frequencies [19], as will be shown later in thiss section. (3) In some instances, electron Landau danping may simply be so strong 
in the plasma periphery that none of the wave power penetrates to the core where it is most needed. This plienomenon is peculiar to the LH slow wave. (4) The bulk of the wave energy may be deposited in the thermal electrons, which are not efficient current carriers. This suggests that if a high-energy electron component can be created in the plasma, a synergistic effect may occur, in that these relatively collisionless electrons absorb most of the wave energy and carry the resultant current.

The fact that $I_{\phi} / P_{C D} \propto T_{e} / n_{e}$ inmediately suggests a rather low efficiency for RF current drive in the core of the TITAN plasma (relatively high density of $9 \times 10^{20} \mathrm{mi}^{-3}$ and moderate temperature of $\sim 10 \mathrm{keV}$ ). Moreover, absorption by the large population of alpha particles will also pose a serious accessibility problem for the incoining wave. For instance, the alpha-particle damping length in the TITAN core plasma is of the order of $0.1 \mathrm{~m}$ or less for the LH waves. However, in an RFP device, core current drive may not be crucial. The stable field configuration is maintained by the continuous relaxation of the plasma to a force-free, minimum-energy state, in which the current is dominantly toroidal in the plasma center and poloidal in the periphery. It is then plausible to drive a poloidal current in a narrow region just. inside the toroidal-field reversal layer ( $q=0$ surface) and allow the inherent turbulent relaxation process to redistribute the induced toroidal fux throughout the entire plasma. As a result, an incremental current is continuously generated to replenish the current lost through ohmic dissipation. The feasibility of this scheme of using waves in the LH range of frequencies in TITAN is examined below.

A 1-D coupled model of RF and plasma should be constructed for the region neas the toroidal-field reversal layer, where $\left|B_{\phi}\right| \ll B_{\theta}$. Neglecting $B_{\phi}$ and taking advantage of the approximate poloidal symmetry, therefore, results in a simple, yet sufficient, 1-D radial plasma model. Defining $N_{\|} \approx N_{\theta}$, and $N_{\perp} \approx N_{r}$, where $N=k c / \omega$ is the wave index of refraction, the wave dispersion relation can be written as follows:

$$
D(\omega, \mathbf{r}, \mathbf{N})=P_{6} N_{\perp}^{6}+P_{4} N_{\perp}^{4}+P_{2} N_{\perp}^{2}+P_{\mathrm{D}}=0,
$$

where the coefficients $P_{i}$ are functions of $N_{\|}, r$, and $\omega$. The electron Landau danping decrement, $\gamma_{e}$, is given by

$$
\gamma_{e}=-\frac{D_{I}^{(e)}}{\partial D / \partial k_{\perp}}
$$

with $D_{i}^{(e)}=2 \pi^{1 / 2}\left(\omega_{p e} / \omega\right)^{2} N_{\perp}^{2} N_{\|}^{2} w^{3} \exp \left(-w^{2}\right)$ and $w=\omega /\left(k_{\|} v_{e}\right)$. A similar expression exists for electron damping resulting from transit-time magnetic pumping (TTMP), which is more important for the fast wave. 
For the important alpha-particle damping decrenent, the unnagnetized-ion-orbit mortel $[19]$ is used because of the large energy of the alpha particles. This model gives the following expression for $\gamma_{\alpha}$ :

$$
\gamma_{\alpha}=\frac{\omega\left(\epsilon_{\|}-N_{\perp}^{2}\right) \epsilon_{\perp a}}{c \epsilon_{\perp} N_{\perp}},
$$

for the slow mode, and

$$
\gamma_{a}=\frac{\omega N_{\perp} \epsilon_{\perp a}}{c\left(N_{\|}^{2}-\epsilon_{\perp}+\epsilon_{x}^{2} / \epsilon_{\|}\right)},
$$

for the fast mode, where $\epsilon_{\perp \alpha}=0.2 \pi\left(\omega_{p o} / \omega\right)^{2}$ and $\epsilon_{\perp}, \epsilon_{\|}$, and $\epsilon_{x}$ are the plasma dielectric tensor elements.

The RF power flux, $P(r)$, along the radial direction is then calculated as

$$
P(r)=P\left(r_{p}\right) \exp \left(-\int_{r_{p}}^{r} \gamma d r^{\prime}\right),
$$

where $\gamma=\gamma_{e}+\gamma_{\alpha}$ and $r_{p}$ is the plasma radius. To calculate the driven curcent, the power absorbed by electrons over an incremental radius is determined and averaged over the flux surface to obtain the local absorbed power density, $P_{c}$. The local driven current, $j_{\|}^{-r}$, is then given by

$$
j_{\| f}^{r f}=\frac{19.2 \times 10^{18}}{\ln \Lambda} \frac{T_{e}}{n_{e}} \hat{\eta} P_{e}
$$

with $T_{e}$ in $\mathrm{keV}$ and the other variables in MKS units. In Equation 7.2-9, $\tilde{\eta}$ is the FischKarney nornalized current-drive efficiency [20] and is given by

$$
\hat{\eta}=\frac{8 \omega^{2}}{5+Z}+2+\frac{12(6+Z)}{(5+Z)(3+Z)}+\frac{2 C}{Z \nu},
$$

where $Z \equiv Z_{e f f}$ and $C$ is 3.76 for Landau damping and 8.09 for TTMP.

Using the nominal TITAN parameters listed in Table 7.2-I and the following density and temperature profiles:

$$
\begin{aligned}
& n_{e}(r)=\left[n_{e}(0)-n_{e}\left(r_{p}\right)\right]\left[1-\left(r / r_{p}\right)^{6.25}\right]+n_{e}\left(r_{p}\right) \\
& T_{e}(r)=\left[T_{e}(0)-T_{e}\left(r_{p}\right)\right]\left[1-\left(r / r_{p}\right)^{3.4}\right]+T_{e}\left(r_{p}\right)
\end{aligned}
$$

both the fast and slow waves have been examined as a means to drive current in the plasma periphery. For the slow mode, severe accessibility problems exist at the plasma 
PARAMETERS FOR TITAN RF CURRENT-DRIVE ANALYSIS

Plasma current, $I_{\phi}(\mathrm{MA})$

Major plasma radius, $R_{T}(\mathrm{~m})$

Minor plasnja radius, $r_{p}(\mathrm{~m})$

Reversal surface minor radius, $r_{r}(m)$

Poloidal field at radius $r, B_{\theta}(r),(\mathrm{T})$

Average electron density; $n_{e}\left(\mathrm{~m}^{-3}\right)$

Edge electron density, $n_{e}\left(r_{p}\right)\left(\mathrm{m}^{-3}\right)$

Density peaking ratio, $n_{e}(0) / n_{e}$

Average electron temperature, $T_{e}(\mathrm{keV})$

E dge electron temperature, $T_{e}\left(r_{p}\right)(\mathrm{keV})$

Tenperature peaking ratio, $T_{e}(0) / T_{e}$

A verage electron temperature, $T_{e}$
17.82

3.6

0.6

0.55

$17.3 J_{\mathrm{o}}(5.17 r)$

$8.9 \times 10^{20}$

$1.6 \times 10^{20}$

1.26

9.6

0.25

1.57

9.6

edge because of the high density. Mode conversion to the fast wave or to the ion plasma wave is dominant, with the latter process leading to undesirably. strong fuel ion and alpha-particle damping. For the fast wave in the LH regime, coupling to the slow mode also presents a severe problem, resulting in the wave energy being trapped in the plasma edge. This problem, however, can be overcor:ce by lowering the frequency to achieve conplete decoupling from the slow wave.

Fesults at a frequency of $\omega=1 \mathrm{GHz}$ are given in Figure 7.2-3, with $N_{\|}=1.2,1 \mathrm{f}$ 2.0, 2.5, and 3.0. It is clear that the wave power damps over a very short radial distance $(\leq 0.15 \mathrm{~m})$ inside the separatrix, as is shown in Figure $7.2-3\left(A_{2}\right)$. Also, the lower the value of $N_{\|}$, the weaker the absorption and the deeper the penetration. For the purpose $c:$ driving poloidal currents inside the $r=0.48-\mathrm{m}$ surface, only the $N_{\|}=1.2$ and 1.5 wave spectral components are desirable. As seen in Figure 7.2-3(B), the generated current profiles for these two components are relatively broart and low compared with those of 

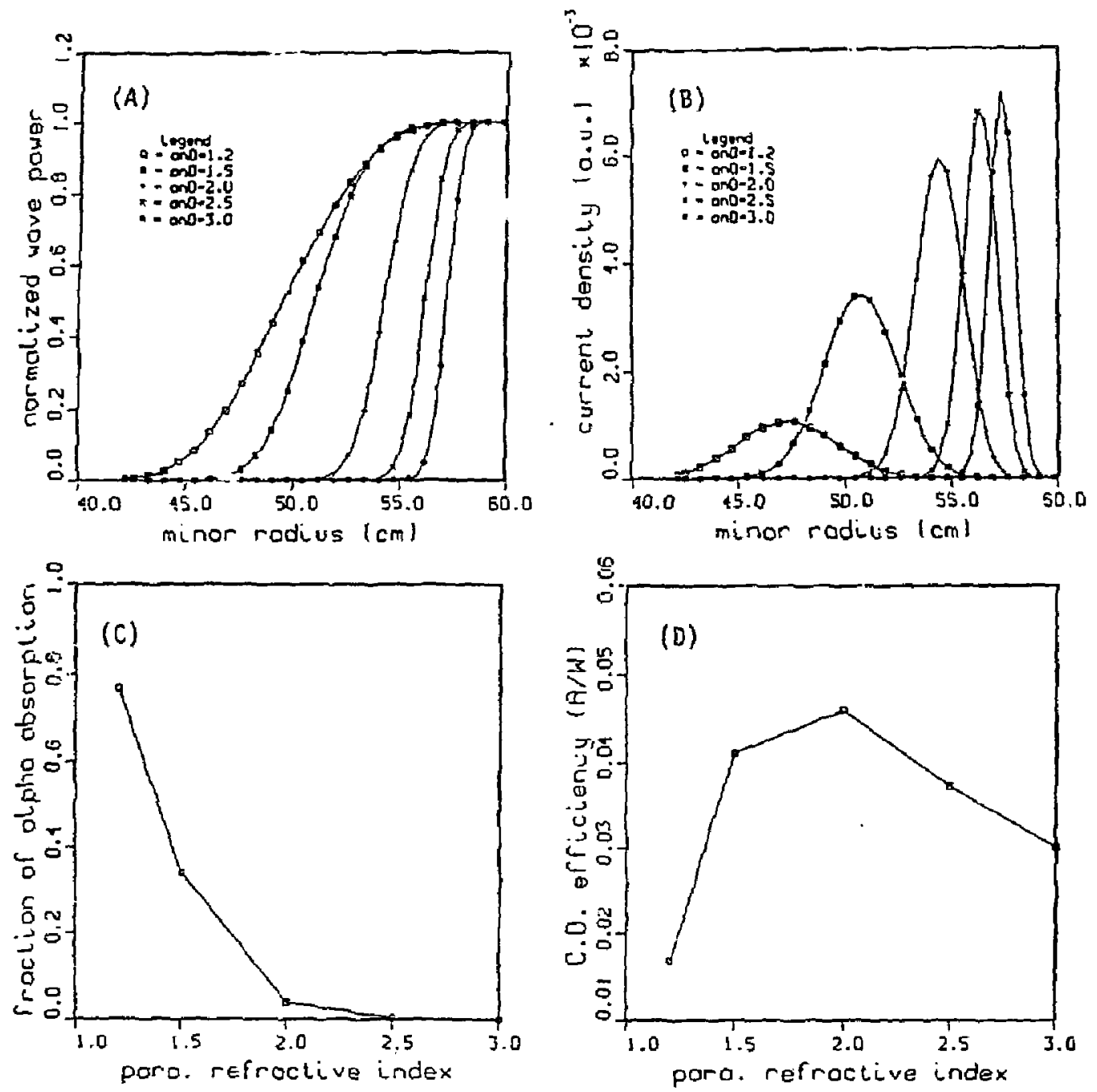

Figure 7.2-3. Profiles of the normalized wave power (A) and RF-driven currentdensity (B) along the plasma minot radius for a $\mathrm{LH}$ fast wave launched into TITAN, with $\omega=1 \mathrm{GHz}$ and $N_{\|}=1,2,1.5,2.0,2.5$ and 3.0 , tespecfively. Also shown are fraction of power ailsorbed by alpha partirles (C) and the resultant current-drive efficiency (D) as functions of $N_{\|}$. 
the higl $-N_{\|}$components. In Figure 7.2-3(C), it is found that a large portion of the wave energy is deposited in the alpha particles for the low- $N_{\|}$modes; likewise their current-drive efficiencies are low $(<0.05 \mathrm{~A} / \mathrm{W}$ ). The means by which this edge currentdrive efficiency will translate into an overall efficiency when the turbulent relaxation process is taken into account, however, is not clear. An assessment of this efficiency requires solving the radial flux-diffusion equation in the presence of a localized toroirlalflux generator inside the $q=0$ surface which is not within the scope of this work. Given the low current-drive efficiency, even at the plasma periphery, it is imlikely that the global efficiency will be found to be within the range desired.

Basically, the low current-drive efficiency found for the LH fast waves results from the high density and modest temperature found in the periphery of the TITAN plasma. This current-drive scheme relies on the external RF power to distort the electron velocity distribution asymmetrically along the direction of the DC magnetic field. Collisional scattering of the distorted velocity space with the background plasina tends to rapidly restore the distribution to a Maxwellian. In a high-density and low-temperature plasma, this restoring force becomes strong and substantial wave energy is required to maintain the non-Maxwellian distribution under these conditions. In fact, as mentioned earlier, all the conventional RF current-drive schemes use the same mechanism, whether it is ECH, ICRF, LH, or AW, and are not expected to yield a good current-drive efficiency under TITAN plasma conditions. Using synergistic effects by creating a higl-energy component in the plasma a priori and having the incoming wave damped on ihese electrons may improve somewhat the resultant current-drive efficiency. This particular method, lowever, has not been examined in this study.

\subsubsection{Relativistic Electron Beanus}

Reference [21] presents a detailed analysis and design for a relativistic electron-beam (REB) current-drive system for the FED-A fusion device. Those calculations indicate that the current-drive power for such a system can be taken approximately as

$$
P_{R E B}=2 I_{\phi}^{2} R_{p}
$$

where $R_{p}$ is the plasma resistance. Applying Equation 7.2-13 to the TITAN reactor with $I_{\phi} \simeq 18 \mathrm{MA}$ results in a current-drive system with $16 \mathrm{MW}$ of REB power, consistent with a. 1.6-MJ pulse energy and a period of $0.1 \mathrm{~s}$ between pulses. The REB current drive las been demonstrated experimentally on several tokamaks and has been used successfully for start-up without the use of $\mathrm{OH}$ coils. 
Although the low-power requirement makes REBs attractive, a number of obstacles to implementation can be identified: (1) The REB current-drive system involves the repeated firing of megavolt capacitor banks, giving rise to questions of reliability when applied to a commercial reactor. (2) Theory indicates that the REB current will only penetrate a few centimeters into the plasma, leading to a hollow current profile. Deeper penetrations can be achieved if very high voltages $(\sim 50 \mathrm{MV})$ are used. One hope in dealing with the hollow current profile is that the REB-created currents will diffuse rapidly into the plasme core through anomalous processes or be transported into the central plasma through $\mathrm{MHD}$ activities. A quantitative assessment of these ideas must await future studies.

\subsection{OSCILLATING-FIELD CURRENT-DRIVE MODEL}

This section develops a methodology [10] by which a power-flow analysis of OFCD, illustrated schematically in Figure 7.3-1, can proceed based on energy balance rather than helicity balance. In developing and evaluating a global picture of $O F C D$, the circuit parameters and definitions summarized in Table 7.3-I are used to arrive at the OFCD plasma equation which is then incorporated into an overall circuit model and used to obtain the parametric OFCD results reported in Section 7.4.

\subsubsection{Plasma Model}

The plasma is described in terms of the plasma magnetic helicity, $K$, toroidal $f$ lux, $\phi$, and magnetic energy, $W_{M}$. The relationship between these parameters and the circuit variables (i.e., resistances, inductances, currents, voltages) constitutes the overall currentdrive model. The time dependence of $K, \phi$, and $W_{M}$ results directly from the Maxwell equations:

$$
\begin{aligned}
\frac{d K}{d t} & =2 \phi V_{\phi}-2 \int \eta \mathbf{j} \cdot \mathbf{B} d V_{p}^{\prime} \\
\frac{d \phi}{d t} & =V_{\theta} \\
\frac{d W_{M}}{d t} & =I_{\phi} V_{\phi}-I_{\theta} V_{\theta}-\int \eta \mathbf{j} \cdot \mathbf{j} d V_{p} \\
E_{\phi} & =\eta j_{\phi}-\alpha B_{\phi}
\end{aligned}
$$




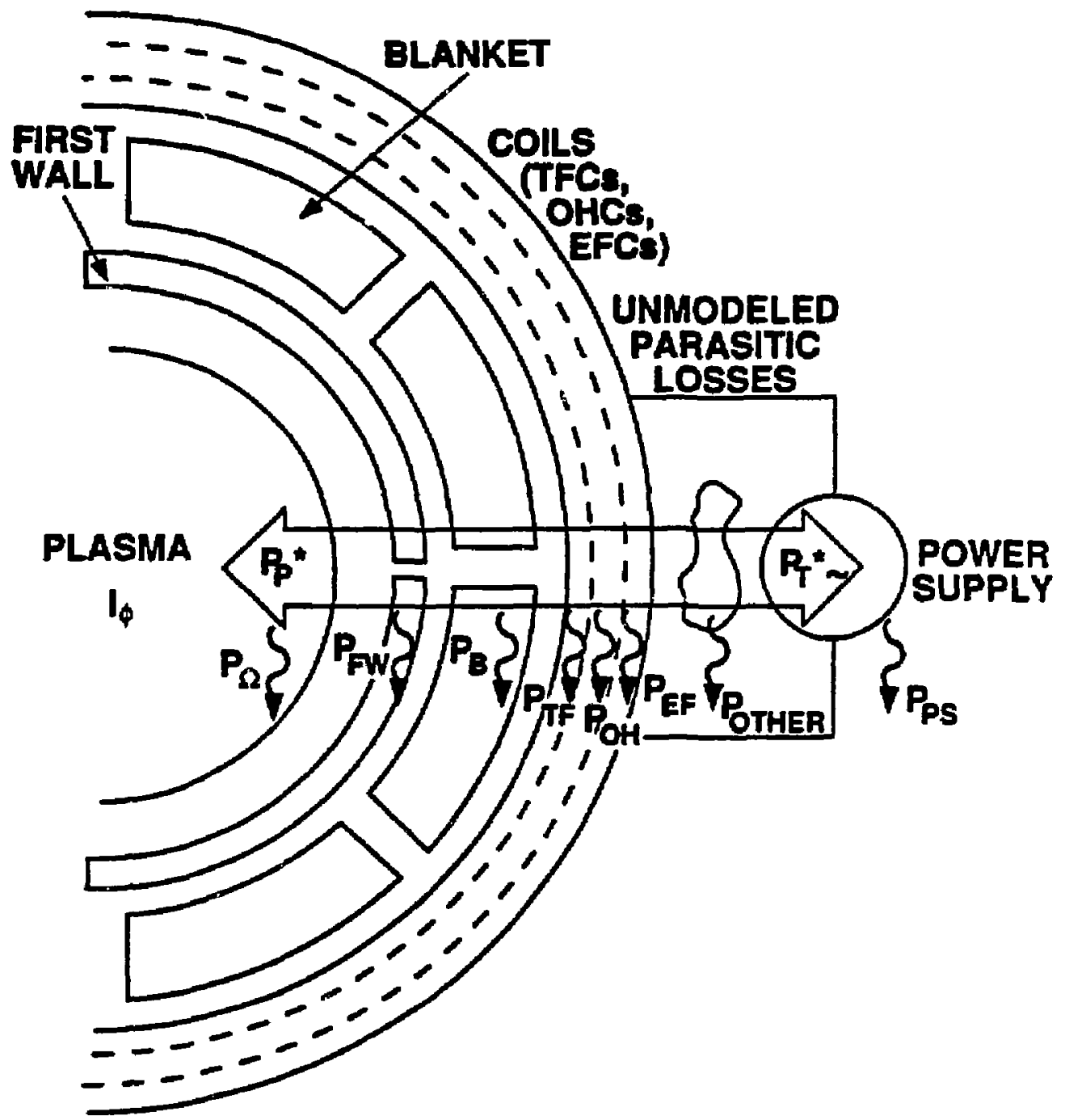

Figure 7.3-1. A simple power-flow diagram for the OFCD model. Shown are the plasma with a dissipation of $P_{\mathrm{n}}$, first wall $\left(P_{F W}\right)$, blanket $\left(P_{B}\right)$, toroidal-field coils $\left(P_{T F}\right)$, ohmic-heating coils $\left(P_{O H}\right)$, equilibrium-field coils $\left(P_{E F}\right)$, and power supply $\left(P_{P S}\right)$. 


\section{OFCD MODEL DEFINITIONS AND NOTATION}

Toroidal voltage on plasma

Toroidal current in plasma

Poloidal voltage at plasma surface

Poloidal current in external conductors

Plasna resistauce

Magnetic helicity

Toroidal flux

Total field energy within plasma

Vacuum toroidal inductance

Inverse aspect ratio

A verage toroidal field within plasma

Reversal parameter

Pincl parameter

$$
\begin{gathered}
V_{\phi} \\
I_{\phi} \\
V_{\theta}=\partial \phi / \partial t \equiv \dot{\phi} \\
I_{\theta} \\
R_{p} \\
K=\left(1 / \mu_{o}\right) \int \mathbf{A} \cdot \mathbf{B} d V_{p} \\
\phi=2 \pi \int_{0}^{r_{p}} B_{\phi}(r) r d r \\
W_{M}=\int_{0}^{r_{p}}\left(B^{2} / 2 \mu_{o}\right) d r \\
L_{o}=\mu_{o} r_{p}^{2} / 2 R_{T} \\
\epsilon=r_{p} / R_{T} \\
\left\langle B_{\phi}\right\rangle=\phi / \pi r_{p}^{2} \\
F=B_{\phi}\left(r_{w}\right) /\left(B_{\phi}\right)=L_{o} I_{\theta} / \phi \\
\Theta=B_{\theta}\left(r_{w}\right) /\left(B_{\phi}\right)=L_{o} I_{\phi} / \epsilon \phi
\end{gathered}
$$

where a positive Faraday's-law convention is adopted for the toroidal circuit to orient $\phi$ and $I_{\phi}$ in the same direction and the last, expression is the torojdal component of the Ohm's law, $\mathbf{E}=\boldsymbol{\eta} \mathbf{j}-\mathbf{v} \times \mathbf{B}$, corrected for the plasma dynamo effect.

With the plasma resistance defined in terms of a classical resistivity, $\eta[22,23]$, according to

$$
I_{\phi}^{2} R_{p}=\int \eta \mathbf{j} \cdot \mathbf{j} d V_{p}
$$

the plasma helicity and energy equations become

$$
\begin{aligned}
\frac{d K}{d t} & =2 \phi\left(V_{\phi}-I_{\phi} R_{H}\right), \\
\frac{d W_{M}}{d t} & =I_{\phi} V_{\phi}-I_{\theta} V_{\theta}-I_{\phi}^{2} \bar{F}_{p},
\end{aligned}
$$


where the helicity dissipation term of Equation 7.3-1 is written in terms of a "helicity resistance," $R_{H}$, defined by $2 \phi I_{\phi} R_{H} \equiv 2 \int \eta \mathbf{j} \cdot \mathbf{B} d \tau$. For the special case of $\beta=0$ with a constant $\mu$ profile (Bessel-function model, BFM), the helicity resistance is exactly equal to the plasina resistance [22]. Denoting time-averaged quantities by (\rangle , Equation 7.3-6 shows that for $\langle d K / d t\rangle$ to be equal to zero, then $2\left\langle\phi V_{\phi}\right\rangle$ must equal the dissipation term, which in turn implies that $\phi$ and $V_{\phi}$ should be nominally in phase. Hence, $V_{\theta}=d \phi / d t$ and $V_{\phi}$ should be out of pliase by $90^{\circ}$ for an optimal OFCD effect.

The plasma magnetic energy, $W_{M}$, can be written in terms of plasma and circuit parameters as follows:

$$
W_{M}=\frac{1}{2} L_{p} I_{\phi}^{2}+\frac{\phi^{2}}{2 L_{\mathrm{o}}}
$$

where $L_{p}$ is a plasma internal inductance that does not include vacuum toroidal flux and $L_{o}$ is the vacuum toroidal inductance defined in Table 7.3-I. Combining Equations 7.3-2, $7.3-\bar{\tau}$, and $7.3-8$ leads to the following expression for the toroidal voltage around the plasma, $V_{\phi}$ :

$$
V_{\phi}=I_{\phi} R_{p}+\left(L_{p}+\frac{\Theta}{2} \frac{d L_{p}}{d \Theta}\right) \dot{I}_{\phi}+\left(\frac{1-F}{\epsilon \Theta} \cdot \frac{\epsilon \Theta^{2}}{2 L_{o}} \frac{d L_{p}}{d \Theta}\right) V_{\theta},
$$

where $V_{\phi, \theta}$ are the toroidal and poloidal voltages applied to the plasna. If (1) the coupling of fields is sufficiently strong to make $L_{p}$ a function of $\Theta$, and (2) if a mechanism exists to allow the perturbation to the near-minimum-energy state to be relaxed to some point in $F-\Theta$ space on a time scale of relaxation, $\tau_{R}$, then oscillations of $V_{\phi, \theta}$ in proper plase at. frequency less than $\sim 2 \pi / \tau_{R}$ can give a net time-averaged current, $\left\langle I_{\phi}\right\rangle$, with $\left\langle V_{\phi}\right\rangle=0$ (i.e., no net flux change). Hence, a nonintrusive means to drive current using primarily the main confining coil system in a low-frequency, low-amplitude plasma-oscillation mode becomes possible.

In evaluating Equation 7.3-9 to determine the flux changes, field oscillations, and power flows, the relationship between $F$ and $\Theta$, as well as the dependence of field and current profiles on $\theta$ in order to determine $L_{p}$ and $R_{p}$, must. be determined. A 1-D MHD model described in Section 5 is used for these calculations. The MHD morlel is based on the following specification of the current density parallel and perpendicular to the magnetic field:

$$
\begin{aligned}
\mathbf{j}_{\|} & =\mu \mathbf{B}, \\
\mathbf{j}_{\perp} & =\frac{\nabla p \times \mathbf{B}}{B^{2}}, \\
\nabla \times \mathbf{B} & =\mu_{o} \mathbf{j}=\mu_{\circ}\left(\mathbf{j}_{\|}+\mathbf{j}_{\perp}\right),
\end{aligned}
$$




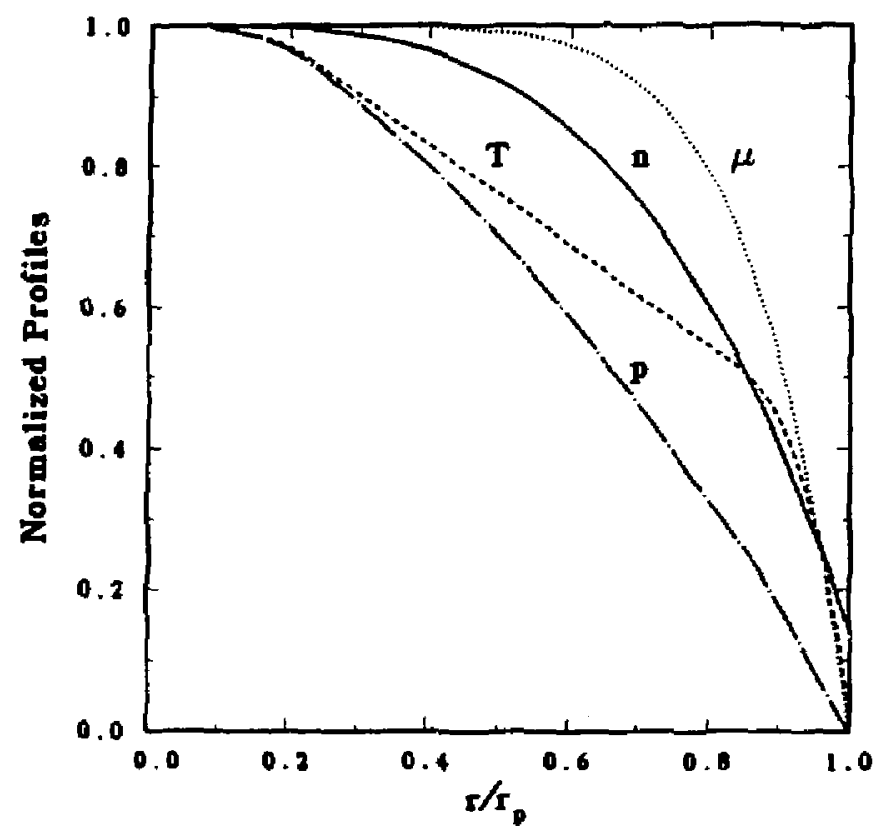

Figure 7.3-2. Normalized density, temperature, and $\mu$ profiles from Section 5.3 which are used in the 1-D MHD model.

where $\mu_{0}$ is the permeability of free space. The first equation invokes the Taylor minimumenergy state [7], the second equation gives the prescription for supporting a plasma pressure, $p$, in equilibrium, and the last equation is Ampere's law. The MHD model requires $\mu$ and pressure profiles as input. The pressur profile is derived from an assumed $\mu$ profile and from fits to density and temperature profiles calculated with the 1-D plasma simulations (Section 5.3) and shown in Figure 7.3-2.

Equations 7.3-10 th.ough 7.3-12 are solved for field and current-density profiles by specifying $\Theta$ subject to the following constraints of fixed $\beta_{\theta}$ and $I_{\phi}$ :

$$
\begin{aligned}
& \beta_{\theta}=\frac{4 \mu_{0}}{r_{p}^{2} B_{\theta}^{2}\left(r_{p}\right)} \int_{0}^{r_{p}} p r d r, \\
& I_{\phi}=2 \pi \int_{0}^{r_{p}} j_{\phi} r d r .
\end{aligned}
$$

Plasma magnetic-field and rurrent-density profiles corresponding to the mean steadystate plasma conditions for $\operatorname{TITAN}\left(i . e,(\Theta)=1.556,\left\langle\beta_{\theta}\right\rangle=0.22\right.$, and $\left\langle I_{\phi}\right\rangle=17.82 \mathrm{MA}$ ) 
are shown in Figure 7.3-3. Since the pressure profile (derived from the density and temperature profiles of Figure 7.3-2) has a non-zero first derivative at the plasma edge, the plasma has a surface current (i.e., a non-zero current density at the plasma edge) as is shown in Figure 7.3-3; this condition is inconsistent with a toroidal-field null. The surface currents are small, however, and should produce a negligible effect on the results based on this madel.

The $F-\Theta$ curve shown in Figure 7.3-4 is generated by the repeated application of the 1-D MHD model over a range of $\Theta$ values. To generate the $F-\Theta$ curve, the plasma current is assumed to scale linearly with $\Theta$. In order to obtain a finite current density at $\Theta \sim 0$ and a finite plasma resistance as predicted in the BFM [22], a $\beta_{\theta}(\Theta) \propto \Theta^{2}$ scaling must be used. This $\beta_{\theta}$ scaling gives rise to an inconsistency with the use of divertors. The plasma separatrix in TITAN is held stationary during OFCD to avoid periodically forcing the plasma into the first wall. This stationary plasma condition is achieved by oscillating the divertor coils in synchronization with the toroidal-field (TF) coils and should result in a plasma pressure invariant to changes in $\Theta$. The effect of using a pressure invariant scaling (i.e., $\beta_{\theta}(\Theta) \propto \Theta^{-2}$ ) produces only a $1 \%$ change in the current-drive efficiency reported in Section 7.4 conpared to values for $\beta_{\theta}(\Theta) \propto \Theta^{2}$ scaling. The plasma inductance and resistance are also calculated as functions of $\Theta$ and shown in Figure 7.3-5. These results are based on the plasma magnetic-field and current-density profiles obtained with the $\beta_{\theta}(\Theta) \propto \Theta^{2}$ scaling.

The poloidal- and toroidal-circuit equations, coupled through Equation 7.3-9, can be solved numerically for $I_{\phi}$ once driver functions, such as those given below, are selected for $\phi$ and $V_{\phi}$ :

$$
\begin{aligned}
\phi & =\phi_{0}+\delta \phi \cos \omega t, \\
V_{\theta} & =\dot{\phi}=\delta \phi \omega \sin \omega t \\
V_{\phi} & =\delta V_{\phi} \cos \omega t .
\end{aligned}
$$

The computational algorithm for solving Equation 7.3-9 is shown in Figure 7.3-6. This algorithm fixes the value of $\delta \phi / \phi_{o}$ and iterates on the value of $\delta V_{\phi} / V_{\phi o}$ until the plasmacurrent solvtion to Equation 7.3-9 becomes periodic, i.e., $I_{\phi}(t)=I_{\phi}(t+2 \pi / \omega)$. The plasma current is reset to the desired value at the beginning of each simulation period. In an outer loop the time scale is adjusted to ensure that the mean current during a period is the same as the current at the beginning of the period. Another loop that is not operated at present adjusts the $\mu$ and pressure profiles to achieve a value of $R_{H}$ which conserves helicity. 

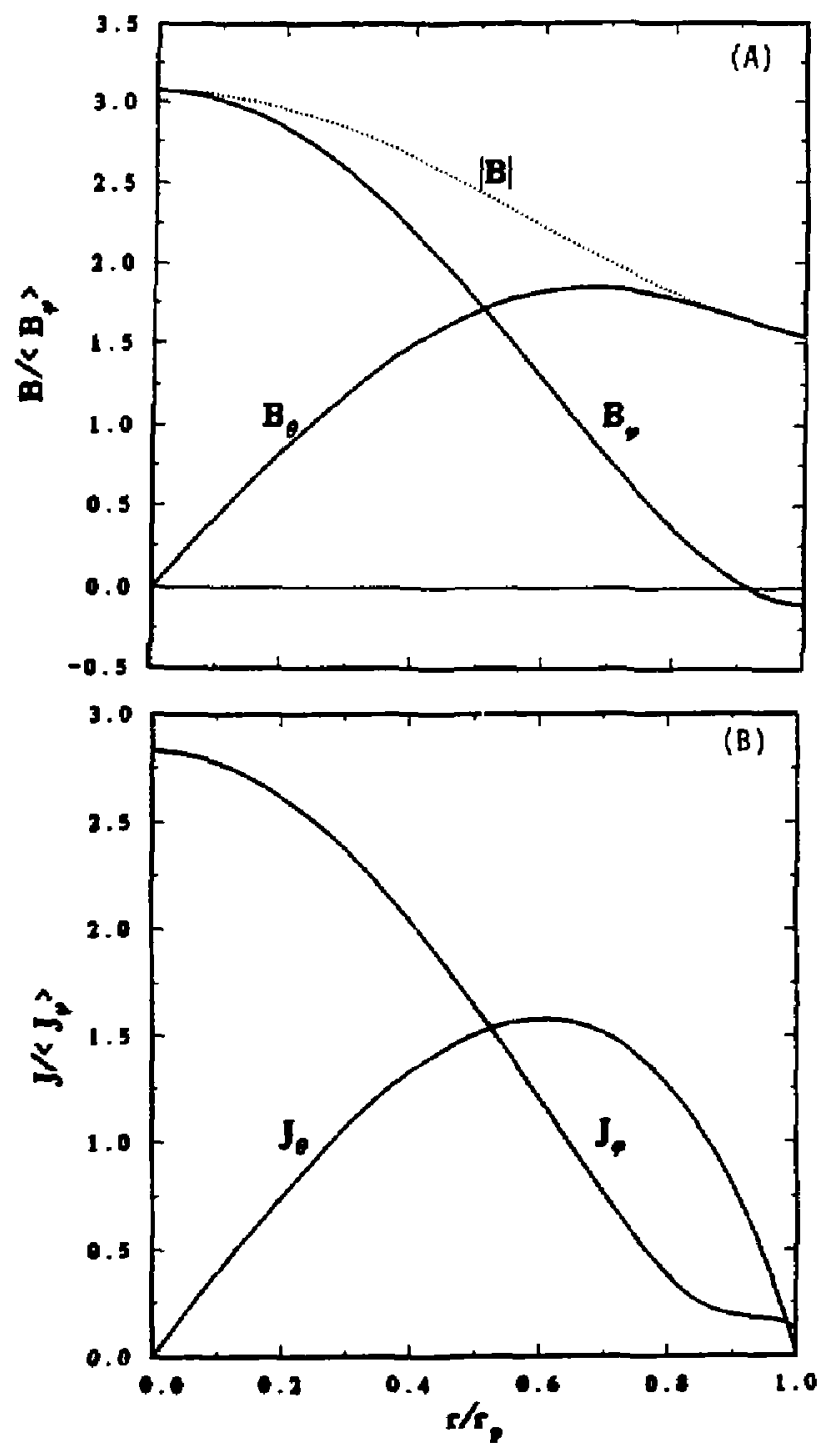

Figure 7.3-3. The profiles of the plasma magnetic field (A) and current density (B) calculated by the 1-D MHD model and used to determine the magnetic energy and plasma inductance for $\beta_{\theta}=0.22$ (includes energetic alphaparticle pressure) and $I_{\phi}=17.82 \mathrm{MA}$. 


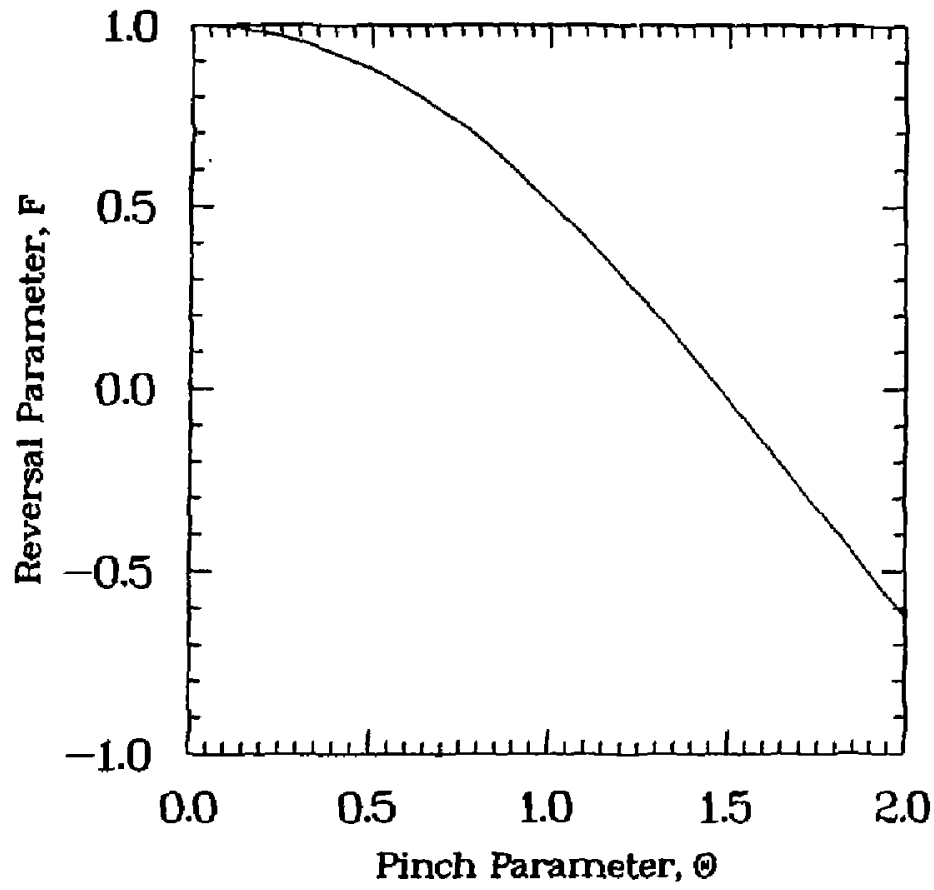

Figure 7.3-4. The F- $\Theta$ curve produced by the 1-D MHD model for the OFCD calculations.

The algorithm described in Figure 7.3-6 requires an initial guess for $\delta V_{\phi}$. The constraint that the time-averaged helicity is constant, $\langle d K / d t\rangle=0$, can be used to estimate the magnitude of the field oscillations required to sustain a given toroidal plasma current. If the ohmic dissipations for both the induced and driven cases are similar, and if the induced case is characterized by $\phi_{o}$ and $V_{\phi o}$, then $d K / d t$ for the driven case is given by Equation 7.3-6 with $V_{\phi o} \equiv I_{\phi} R_{H} \simeq I_{\phi} R_{p}$. Hence,

$$
\frac{d K}{d t} \simeq 2 \phi\left(V_{\phi}-V_{\phi \circ}\right) \text {. }
$$

Using the driver functions given by Equations 7.3-15 through 7.3-17, the tine average of $d K / d t$ for the driver case given above becomes

$$
-\left\langle\frac{d K}{d t}\right\rangle=\delta \phi \delta V_{\phi}+2 \phi_{o} V_{\phi \infty}
$$



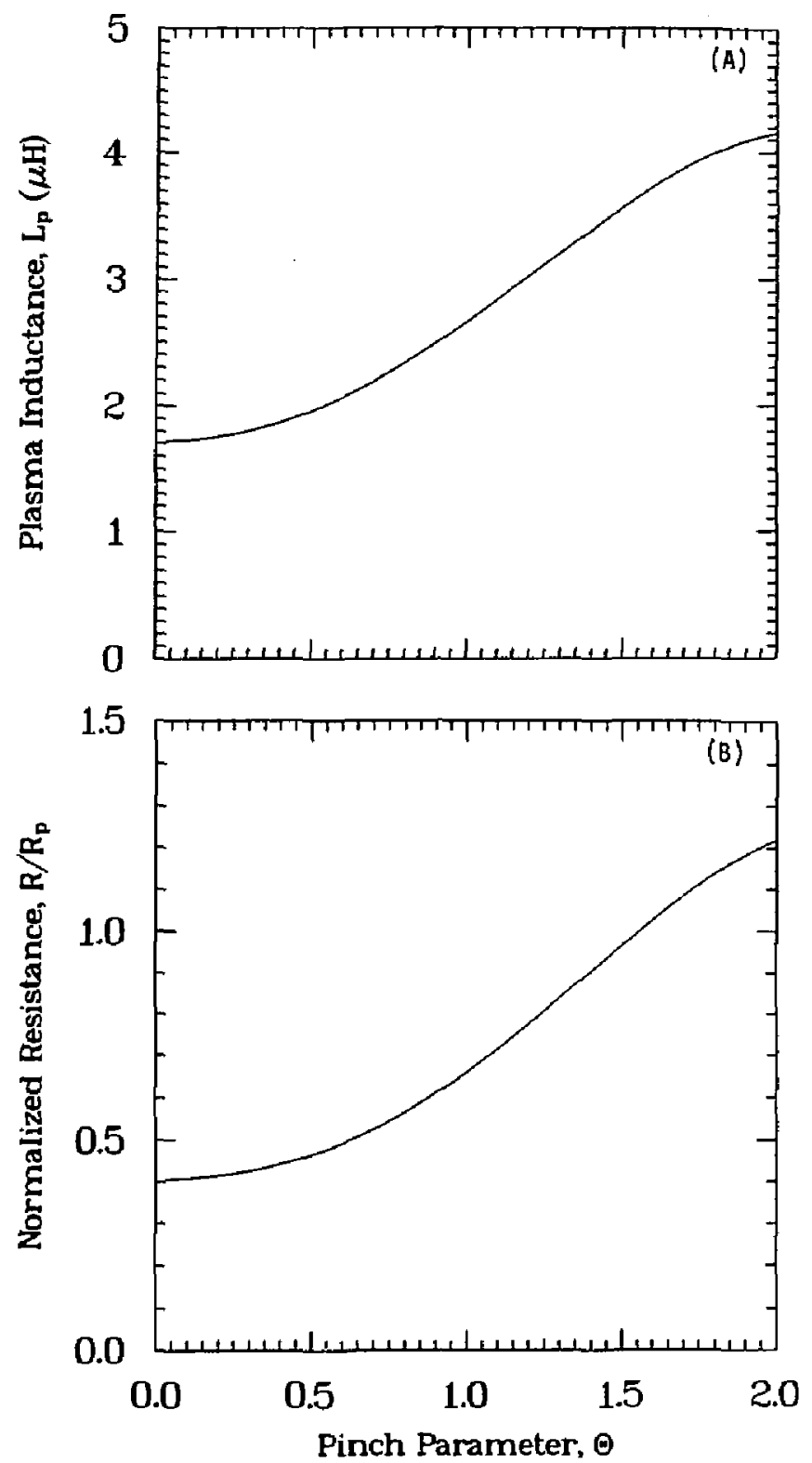

Figure 7.3-5. The dependence of the plasma inductance (A) and resistance (B) on $\Theta$ as determined by the 1-D MHD model. 


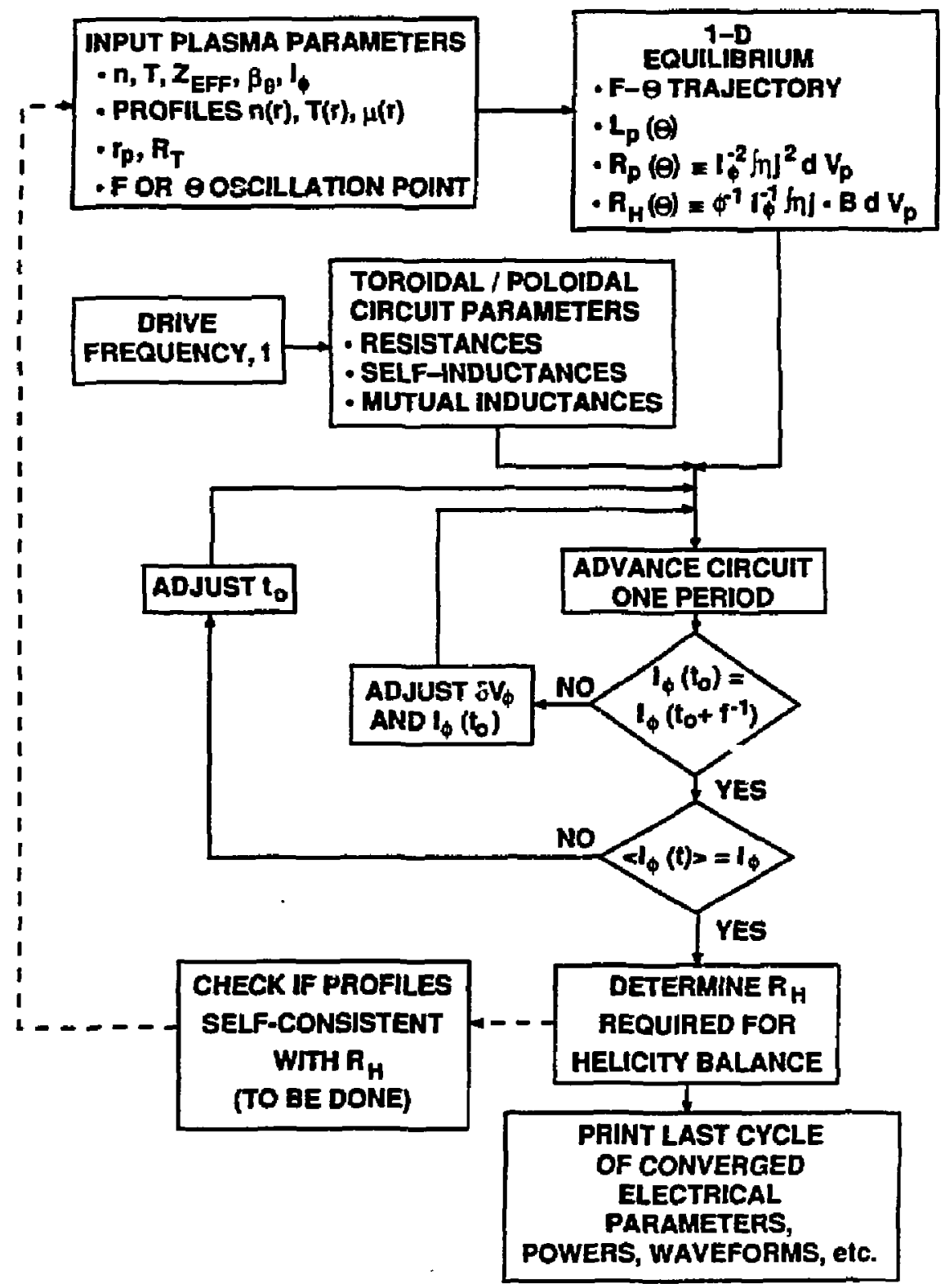

Figure 7.3-6. The algorithm used to solve OFCD plasma/circuit equations. 
For $\langle d K / d t\rangle \simeq 0$, the following condition on the amplitudes of the toroidal flux and voltage oscillation results:

$$
\left(\frac{\delta \phi}{\phi_{0}}\right)\left(\frac{\delta V_{\phi}}{V_{\phi a}}\right) \simeq-2 .
$$

Because toroidal-flux oscillations much above $\delta \phi / \phi_{\mathrm{o}} \simeq 0.05$ are expected to seriously impact the RFP configuration (i.e., loss of toroidal-field reversal), the $A C$ toroidal voltage needed to drive a $\mathrm{DC}$ toroidal current with $\langle d K / d t\rangle \simeq 0$ can be $\sim 40$ tiules greater than the voltage needed to sustain an inductively driven RFP.

\subsubsection{Circuit Model}

An assessnent of OFCD efficiency requires the modeling of the circuit elements external to the plasma in addition to the plasma itself, as indicated schematically in Figure 7.3-1. The governing matrix circuit equation is written as follows:

$$
\underline{\underline{\mathbf{L}}} \frac{d}{d t} \underline{\mathbf{I}}+\underline{\underline{\mathbf{R}}} \underline{\mathbf{I}}=\underline{\mathbf{V}}
$$

where $\underline{I}$ and $\underline{V}$ are column vectors representing the currents and voltages, respectively, $\underline{\underline{R}}$ is a diagonal matrix of resistances, $\underline{\underline{L}}$ is the inductance matrix, and the inductances are assumed invariant in tine. Separate matrix circuit equations are derived for poloidal, $\theta$, and toroidal, $\phi$, current paths and are labeled according to the current direction, $\theta$ and $\phi$, respectively.

A shell model is used to determine the juductances and the resistances used in the respective matrices. The self-inductances in the toroidal and poloidal direction for the ith element are given, respectively, by

$$
\begin{aligned}
L_{i, \phi}= & \mu_{o} R_{T}\left[\ln \left(\frac{R_{T}}{r_{c}}\right)-2\right], \\
L_{i, \theta}= & \mu_{a} R_{T}\left[1-\left(1-\gamma_{I}^{2}\right)^{1 / 2}-\frac{1}{3}\left(1-\gamma_{O}^{2}\right)^{1 / 2}\left(2+\gamma_{O}^{2}\right)\right. \\
& +\gamma_{O}\left(\gamma_{O}-\gamma_{I}\right)^{-2}\left(\sin ^{-1} \gamma_{I}-\sin ^{-1} \gamma_{O}\right) \\
& \left.+\frac{1}{3}\left(\gamma_{O}-\gamma_{I}\right)^{-2}\left(1-\gamma_{I}^{2}\right)^{1 / 2}\left(2+\gamma_{I}^{2}+3 \gamma_{O}^{2}-3 \gamma_{O} \gamma_{I}\right)\right],
\end{aligned}
$$

where $\gamma_{I}=r_{I} / R_{T}$ and $\gamma_{O}=r_{O} / R_{T}$, the major and minor radii of the shell are $R_{T}$ and $r_{c}$, and the inner and outer minor radii of the shell are $r_{I}$ and $r_{o}$, respectively. The 
internal inductance is ignored in Equation 7.3-22. The mutual inductance between the ith and $j$ th elements, $M_{i j}$, is the smaller of the ith aud $j$ th self-inductances in the shell model. The nutual inductance betweets two elements then is the self-inductance of the element with the smaller minor radius for poloidal currents and that of the element with the larger minor radius for toroidal currents. The resistance of the $i$ th element is

$$
R_{i}=\frac{2 \eta_{i} R_{T}}{r_{O}^{2}-r_{I}^{2}},
$$

where $\eta_{i}$ is the resistivity of the material in the conducting shell. The circuit elements simulated are the plasma, first wall (FW), the toroidal-field (TF) coils, a portion of the windings of the ohnic-heating $(\mathrm{OH})$ coils, a primary equilibriun-field (EF) coil set, a secondary EF-trim coil set, and the reflector and shield (R/S) for the TITAN-I and the blanket for the TITAN-II,

The current vector $\underline{I}$ in Equation 7.3-21 has components corresponding to each circuit element. The plasma current in the toroidal-circuit version of Equation 7.3-21 is the $I_{\phi}$ solution to Equation 7.3-9. The plasma current in the poloidal-circuit version of Equation 7.3-21 is a model artifact required for inductive transfer of magnetic-field energy to the plasma from the external elements and resembles a plasma skin current (physically, it is not a skin current).

The self-inductances and resistances used for constructing $\underline{\underline{L}}$ and $\underline{\underline{R}}$ matrixes in Equation 7.3-21 are presented in Table 7.3-II and Table 7.3-III, respectively, for TITAN-I and TITAN-II. The TF-coil set for TITAN-I has been separated into six individual elenents that physically correspond to the six radial rows of integrated-blanket-coil (IBC) tubes (Section 4.3.2). This configuration is used because the tube rows are connected electrically in parallel (Section 10.5) and the current penetration skin depth at the frequencies considered $(\sim 25 \mathrm{~Hz})$ is comparable to the tube dianeter. The plasma resistance is taken as zero in Equation 7.3-21, because the plasma resistance is included in Equations 7.3-7 and 7.3-9. The plasma inductances listed in Tables 7.3-II and 7.3-III are only the external inductances; the internal inductances appear in Equations $7.3-8$ and 7.3-9.

The voltage vector, $\underline{V}$, in Equation 7.3-21 contains time-varying applied voltages for the elements in which current is driven (i.e., the TF, OH, EF, and trim coil sets). The voltage on the TF coil is cletermined by requiring the toroidal field at the plasma surface be produced by all the elements with continuous poloidal current paths. The voltage of the $\mathrm{OH}$ coil is derived from knowing the solution for $I_{\phi}$ from Equation $7.3-9$. In the case of the EF coil, the voltage is maintained at a constant value corresponding 
to the mean equilibrimu fiehd. The EF trins-coil soltage is deternined by requiring the trim coil to track the oscillating equilibrium-field requirement of the plasma [d]. For 1.he passive elements, FW and R/S, the voltages are zero. The plasma voltage in the toroidal-circuit version of Equation $7,3-21$ is taken to be $-l_{\phi}$, because $l_{\phi}$ is a voltage drop in Equation 7.3-9. In the poloidal-circuit equation, the plasma voltage is takeu to be $V_{\theta}$, because of the decision to use a positive Faraday's law (Equation 7.3-2).

Table '.3-II.

CIRCUIT PAR AMIETERS FOR TITAN-I OFCD ANALYSIS

\begin{tabular}{|c|c|c|c|c|}
\hline \multirow[b]{2}{*}{ Circuit Element } & \multicolumn{2}{|c|}{ Inductance $(\mu \mathrm{H})$} & \multicolumn{2}{|c|}{ Resistance $(\mu \Omega)$} \\
\hline & Toroidal & Poloidal & Toroidal & Poloitlal \\
\hline Plasna & 9.56 & 0.0580 & 0. & 0. \\
\hline \multicolumn{5}{|l|}{ First wall: } \\
\hline without gaps & 9.06 & 0.07 & 760. & 6.95 \\
\hline with gaps & 0.0387 & 0.00114 & 3,040 & 27.8 \\
\hline \multicolumn{5}{|l|}{ TF coil: } \\
\hline 1st tube row & - & 0.0786 & - & 2.83 \\
\hline 2nd tube row & - & 0.0896 & - & 2.96 \\
\hline 3rd tube row & - & 0.101 & - & 3.09 \\
\hline 4th tube row & - & 0.114 & - & 3.22 \\
\hline 5th tube row & - & 0.127 & - & 3.35 \\
\hline 6th tube row & - & 0.141 & - & 3.48 \\
\hline $\mathrm{OH}$ coil & 5.15 & - & 1.51 & - \\
\hline Reflector \& shield & 1.65 & 0.161 & 25.2 & 2.33 \\
\hline EF coil & 0.752 & - & $\sim 0$. & - \\
\hline Trin coil & 3.18 & - & 2.93 & - \\
\hline
\end{tabular}


Table 7.3-III.

CIRCUIT PARAMETERS FOR TITAN-II OFCD ANALYSIS

\begin{tabular}{|c|c|c|c|c|}
\hline \multirow[b]{2}{*}{ Circuit Element } & \multicolumn{2}{|c|}{ Inductance $(\mu \mathrm{H})$} & \multicolumn{2}{|c|}{ Resistance $(\mu \Omega)$} \\
\hline & Toroidal & Poloidal & Toroidal & Polojdal \\
\hline Plasma & 9.56 & 0.0580 & 0. & 0. \\
\hline First wall & 0.0387 & 0.00114 & 3,040 . & 27.8 \\
\hline TF coil & - & 0.225 & - & 0.525 \\
\hline OH coil & 5.15 & - & 1.51 & - \\
\hline Blanket & 2.60 & 0.144 & 642,000 & 33,000 \\
\hline EF coil & 0.752 & - & $\sim 0$. & - \\
\hline Trim coil & 3.18 & - & 2.93 & - \\
\hline
\end{tabular}

Calculations with a continuous first wall (Section 7.4) indicate a need to mociel passive elements with resistjve breaks or gaps. The model derived here treats each passive element as consisting of an inner and outer current path, as is shown in Figure 7.3-7. The current is assumed to flow in the smaller of (1) half of the radial build of the passive element or (2) a current penetration skin depth. The self-inductance of the element with gaps is the difference of the self-inductances of the inner and outer current-path elements. The nutual inductance between an element with gaps and a continuous element is the difference of the minimum of the self-inductances of the inner current-path element and the continuous element and the minimum of the self-inductances of the outer currentpath element and the continuous path element. If the continuous element has a smaller self-inductance than eitiner the inner or outer current-path elements, then a zero mutual inductance rosults in the shell model. The resistance of the elenent with gaps is the sum of the resistances of the inner and outer current-path elements. This eddy-current model yields an accurate accounting of dissipated power when the oscillating magnetic fields are tangential to the passive elements, as is shown in Section 4.6.2.

The time-dependent current and voltage solutions to the toroidal and poloidal versions of Equation 7.3-21 are solved in conjunction with the $I_{\phi}(t)$ solution to Equation 7.3-9. 


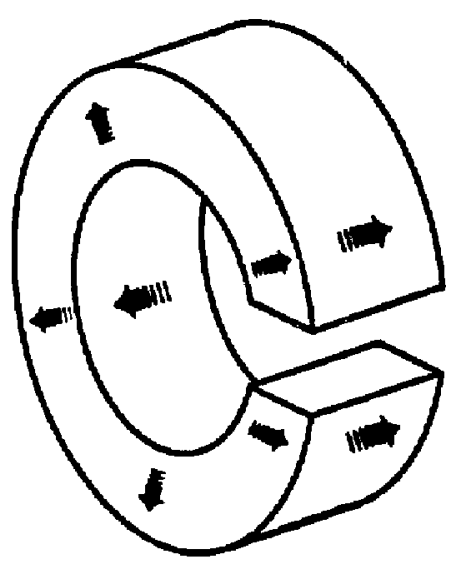

TOROIDAL

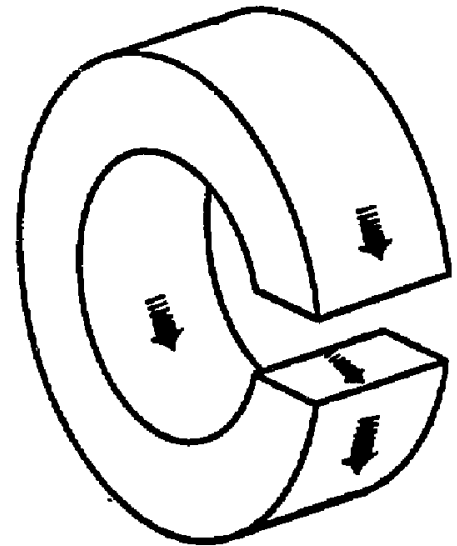

POLOIDAL

Figure 7.3-7. The current paths envisioned for the model of the passive circuit elements with resistive breaks orthogonal to the corresponding continuous current direction.

The electrical time constants of the external circuits are sufficiently short so that periodic solutions to Equations 7.3-9 and 7.3-21 are obtained simultaneously. The dissipated powers and peak reactive powers of the entire system, schematically represented in Figure 7.3-1, are derived fiom the calculated current and voltage waveforms.

\subsection{CURRENT-DRIVE PARAMETERS}

The first application of the algorithms described in Section 7.3 was to the TITAN-I design shown in Figure 7.4-1. This design has a continuous first wall, but the reflector and shield, superconducting EF coils, and the EF trim-coil circuit elements were not included in the sinulations. The results of this simulation are reported in Table 7.4-I. The most prominent result of this reduced circuit simulation is that $\leadsto 120 \mathrm{MW}$ of power is dissipated in the first wall. Efforts to reduce the dissipated power initially focused on varying the toroidal-flux swing, $\delta \phi / \phi_{o}$, and the drive frequency, $f$. The results are shown in Figure 7.4-2(A). The operating window for $\delta \phi / \phi_{o}$ is bounded above and below for both frequencies because of a loss of field reversal. The upper bound is the result 


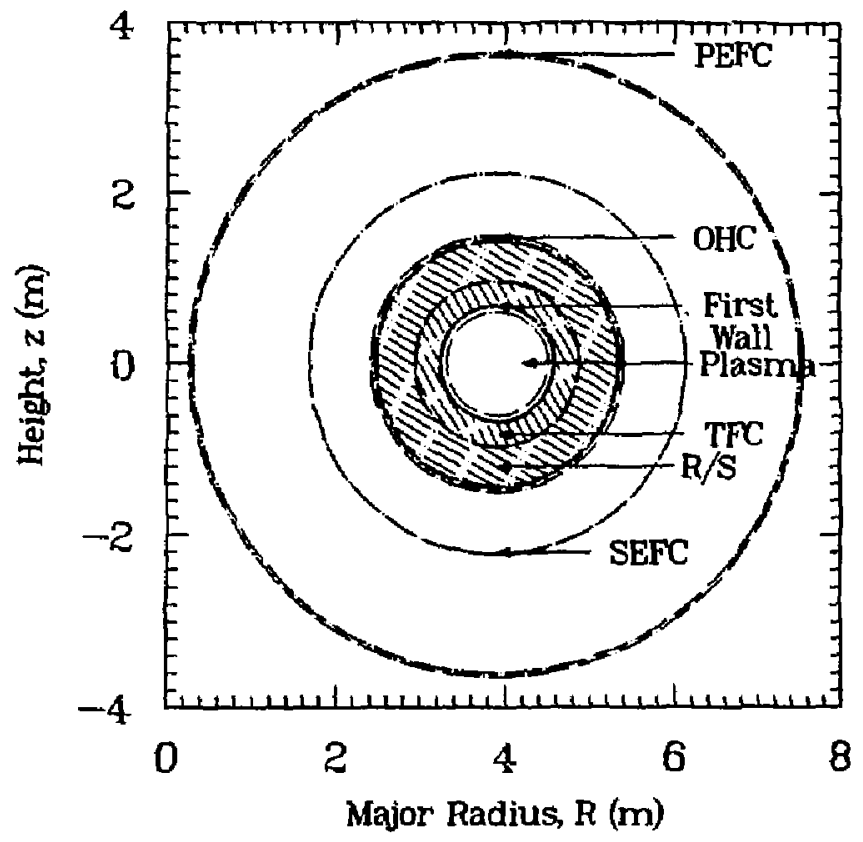

Figure 7.4-1. A cross-sectional view of the TITAN-I shell model used for OFCD calculations. The circuit elements simulated are the plasma, first wall, IBC TF coils (TFC), reflector and shield (R/S), OH coils (OHO), the trim coils (SEFC), and the superconducting EF coils (PEFC).

of too large oscillations in $\phi$ at a shallow reversal $(F=-0.1)$. The lower bound is the result of too large oscillations in $I_{\phi}(\geq 5 \%)$ and, hence, $\Theta$. This lower bound causes a loss of field reversal because adherence to an $F-\Theta$ curve is strictly enforced. The $\delta \phi / \phi_{0}$ operating window shrinks with lower frequencies until completely disappearing at frequencies between 5 and $10 \mathrm{~Hz}$. A drive frequency of $25 \mathrm{~Hz}$ was selected for the TITAN study because the $\delta \phi / \phi_{c}$ operating window is relatively unrestricted in this region, power supplies at $25 \mathrm{~Hz}$ are commercially available, and the effect of frequency on dissipated power has nearly saturated at $25 \mathrm{~Hz}$. A flux swing of $\delta \phi / \phi_{o}=0.035$ was selected because it is in the middle of the $\delta \phi / \phi_{0}$ operating window.

The effect of changing just the first-wall resistance at a $25-\mathrm{Hz}$ drive frequency is shown in Figure 7.4-2(B). The magnetically induced voltage on the first wall, $V_{F W}$, is independent of the first-wall resistance, so the first-wall dissipated power scales as 
Table 7.4-I.

PRELIMINARY OFCD RESULTS FOR TITAN-I DESIGN ${ }^{(a)}$

\begin{tabular}{|c|c|c|}
\hline & Without Grap & With Gap \\
\hline Average plasma current, $I_{\phi}$ (MA) & 17.82 & 17.82 \\
\hline Drive frequency, $f(\mathrm{~Hz})$ & 25. & 25. \\
\hline Toroidal flux swing, $\delta \phi / \phi_{0}$ & 0.035 & 0.035 \\
\hline$\Theta$ variation & $1.499-1.616$ & $1.499-1.616$ \\
\hline$F$ variation & $-0.032--0.173$ & $-0.032--0.173$ \\
\hline \multicolumn{3}{|c|}{ Power flow in toroidal (poloidal) circuits (MW): } \\
\hline Plasma Poynting power, $P_{P}^{*}$ & $3,959.99(247.31)$ & $3,959.99(247.31)$ \\
\hline Terminal reactive power, $P_{H}$ & $325.44(790.31)$ & $191.20(504.08)$ \\
\hline Plasma dissipation, $P_{\Omega}$ & $28.55(0)$. & $28.55(0)$. \\
\hline First-wall dissipation, $P_{F W}$ & $39.47(80.73)$ & $0.00(0.01)$ \\
\hline Coil dissipation, $P_{H}$ & $0.78(75.77)$ & $0.50(47.41)$ \\
\hline Total dissipation, $P_{D}^{\prime}$ & $68.80(156.5)$ & 29.05 ( 47.42 ? \\
\hline Real (lost) terminal power, $P_{T}$ & $42.20(183.11)$ & $2.44(74.03)$ \\
\hline Power-supply dissipated power, $P_{P S}{ }^{(b)}$ & $3.25(7.90)$ & $1.91(5.04)$ \\
\hline TF-coil DC power, $P S S$ (MW) & 29.15 & 29.15 \\
\hline Dissipated power, $P_{D}(\mathrm{MW})$ & 236.46 & 83.43 \\
\hline Current-drive power, $P_{C D}(\mathrm{MW})$ & 207.31 & 54.28 \\
\hline Current-drive efficiency, $I_{\phi} / P_{C D}(\mathrm{~A} / \mathrm{W})$ & 0.09 & 0.33 \\
\hline
\end{tabular}

(a) Excluding the reflector \& shield, EF coils, and trim-coils circuit elements.

(b) Assuming the power supplies are $99 \%$ efficient $\left(Q_{P S}=100\right)$. 

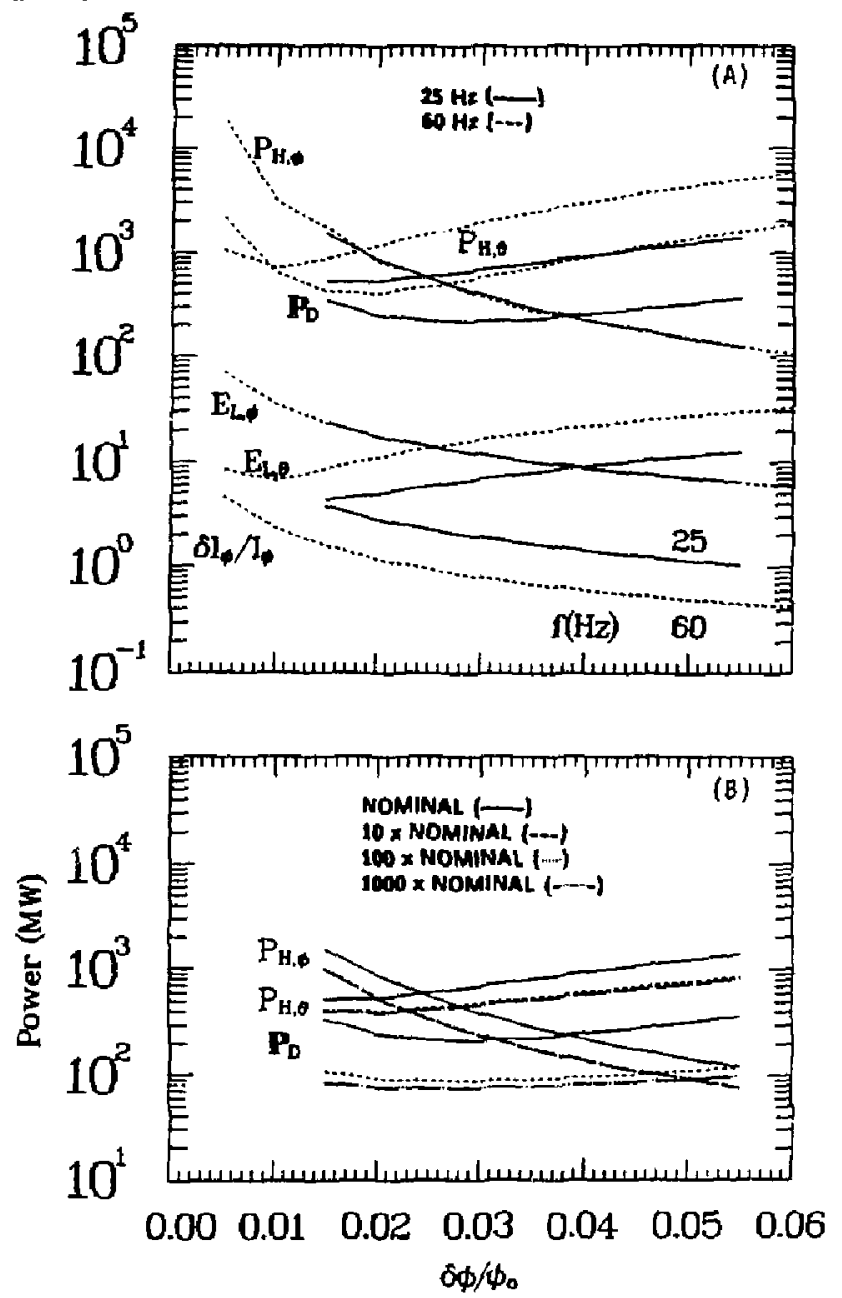

Figure 7.4-2. Power flow in TITAN-1 OFCD system as a function of the amplitude of the toroidal-flux swing normalized to the mean toroidal flux (excluding the $\mathrm{R} / \mathrm{S}, \mathrm{EF}$, and trim circuit elements). (A) Results at frecuencies of 25 and $60 \mathrm{~Hz}$ for nominal first-wall resistance. (B) Results at $25 \mathrm{~Hz}$ for the nominal, $10 \times$ nominal, $100 \times$ nominal, and $1000 \times$ nominal first-wall resistance. Terminal reactive powers for the toroidal $\left(P_{H_{\phi} \phi}\right)$ and poloidal $\left(P_{K, \theta}\right)$ circuits, the dissipated power $\left(P_{D}^{\prime}\right)$ in the plasma, first wall, TF, and $\mathrm{OH}$ coils, as well as electric fields at the first wall in the torsidal $\left(E_{L, \phi}\right)$ and poloidal $\left(E_{L, \theta}\right)$ directions and the amplitude of the plasma current oscillations normalized to the mean curreut $\left(\delta I_{\phi} / I_{\phi}\right)$ are show11. 
$P_{F W} \propto V_{F W}^{2} / R_{F W}$. The nominal first-wall design (and hence, resistance) is determined by thermal-hydraulics considerations (Section 10.4). To achieve the two-order-of-magnitude increase in first-wall resistance that is required to reduce the first-wall dissipated power to negligible levels, a new first-wall material that is 100 times more resistive is required. In addition, the nominal first wall provides a vertical-field penetration time of $3 \mathrm{~ms}$, which falls within the 1.8-57.9-ms requirement for plasma-wall stabilization projected for TITAN (Section 5.2). This latter constraint would be difficult to meet for a nuch more resistive first wall. The only way to emulate an increased first-wall resistance while maintaining wall stabilization is by using gaps or insulating breaks.

The gap model described in Section 7.3 was applied to the same reduced circuit morlel of the TITAN-I design and is reported in Table 7.4-I in parallel with the results for a continuous first wall. The effect of the first-wall gap is primarily to reduce the dissipated power in the first wall and secondarily to reduce the coil powers. The net effect of gaps is to increase the current-drive efficiency by a factor of 3.7 over that for a continuous (without gaps) first wall.

The full capability of the circuit model was then exercised on the TITAN-I and TITAN-II designs shown in Figures $7.4-1$ and 7.4-3, respectively. Initially the trim coils were disabled, which resulted in an $\sim 7$-GW reactive power in the EF coils. The trim coils, subsequently, were enabled to reduce the EF-coil reactive power because the power supplies are costed at $\sim 10 \mathrm{M} \$ / \mathrm{GW}$ of reactive power (Section 3.3). The results of the full simulation of TITAN-I and TITAN-II for the various powers of interest as a function of $\delta \phi / \phi_{0}$ are shown in Figure 7.4-4. The two designs perform similarly with the exception of the TF coils. The TITAN-II TF coils are further from the plasma than in TITAN-I and, therefore, require a larger reactive power. The TF-coil dissipated power, however, is smaller in TITAN-II, even though the TITAN I and TITAN-II DC powers are nearly equal. The TITAN-iI TF coils are series wound and have a uniform current density, whereas the TITAN-I TF coils are comnected in parallel with an overall radial build greater than the current-penetration skin depth with most of the current concentrating within a skin depth, as is discussed later. The gaps in both TITAN-I and TITAN-II first. wa'is must hold off $\sim 2 \mathrm{~V}$ in order to maintain the electrical-break effect; this condition could be met by using (1) an $\sim 1-\mathrm{nm}$ wide vacuum gap or (2) even thinner anount of electrical insulator. The small-amplitucle $(<2 \%)$ plasma-current oscillations should not. arlversely affect the plasma stability or transport.

The TITAN-I and TITAN-II OFCD design points summarized in Table T.4-lI were selected from the midcle of the $\delta \phi / \phi_{0}$-operating window to provicle a maximum safety margin against the accidental loss of field reversal. The power clissipated in the first. 


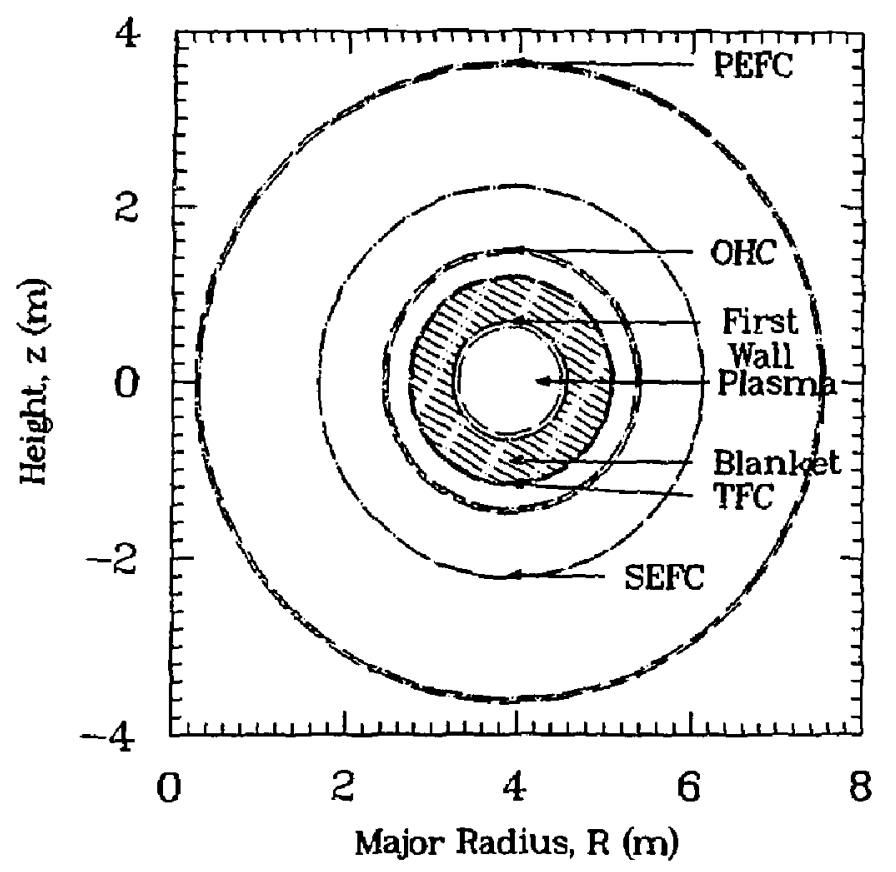

Figure 7.4-3. A cross-sectional view of the TiTAN-II shell model used for OFCD calculations. The circuit elements simulated are the plasma, first wall, blanket, TF coils ( $T F C$ ), $\mathrm{OH}$ coils ( $\mathrm{OHC}$ ), trim coils ( $\mathrm{SEFC}$ ), and superconducting EF coils (PEFC).

wall of each design (with breaks) is the same because the first walls are physically the same. The power dissipated in the TITAN-1 R/S is larger than in the TITAN-II blanket primarily because of a lower $R / S$ resistance. Because the TITAN-1 $R / S$ is positioned outside of the IBC TF coils, none of the poloidal-circuit elements couple to the $R / S$ and no power is dissipated in the $\mathrm{R} / \mathrm{S}$ from that circuit. The dissipated and reactive powers in the coils of the toroidal circuit (i.e., OH, superconducting EF, and trim coils) are slightly larger for TITAN-II because the toroidal-circuit blanket inductance is larger for TITAN-II (i.e., the blanket is less transparent to the power flowing through its surfaces).

The voltage waveforms for the TITAN-I and TITAN-II OFCD design points are shown in Figure 7.4-5. These waveforms indicate that the much larger TITAN-11 blanket resistance causes a large $(\sim 100 \mathrm{~V})$ induced voltage, which is not found in the TITAN-I design. The TITAN-II voltage waveforms more prominently display a phenomena com. 

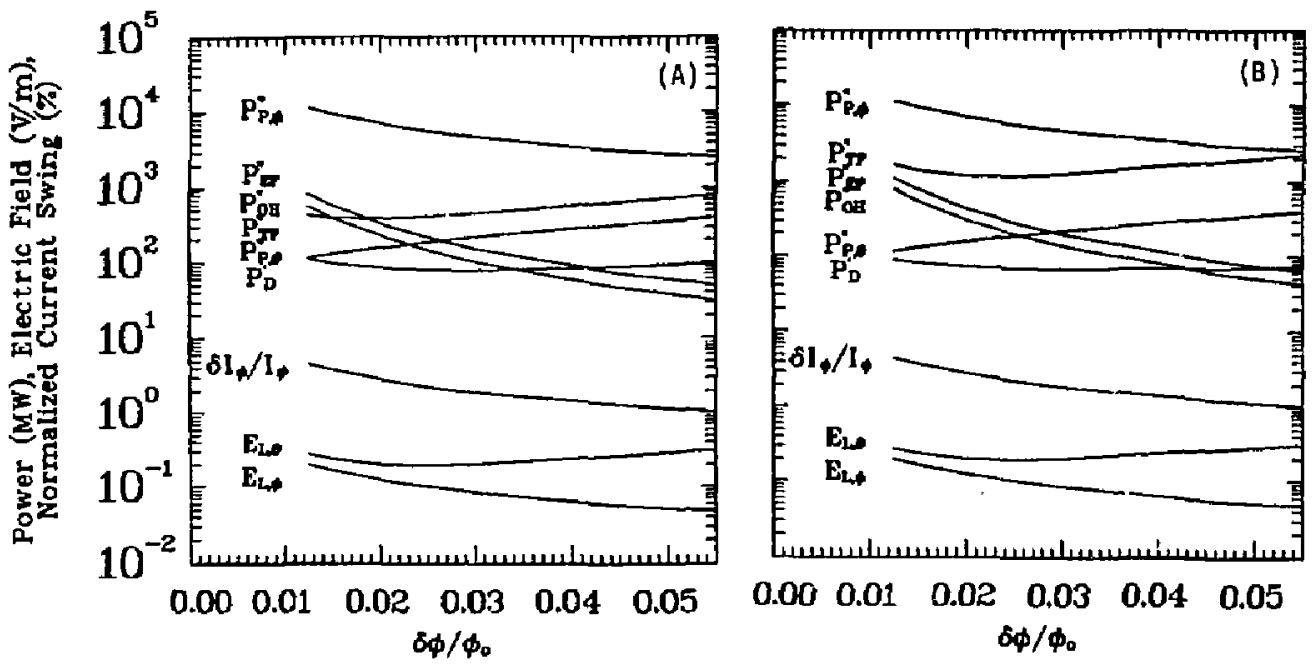

Figure 7.4-4. Power flow in TITAN-I (A) and TITAN-II (B) OFCD systems as a function of the amplitude of the toroidal-flux swing normalized to the niean toroidal flux at $25 \mathrm{~Hz}$. Terminal reactive powers for the $\mathrm{TF}$ coils $\left(P_{\bar{T} F}\right)$, trim coils $\left(P_{E F}^{*}\right), \mathrm{OH}$ coils $\left(P_{O H}^{*}\right)$, ihe plasma Poynting power in the toroidal $\left(P_{P_{, \phi}}^{*}\right)$ and poloidal $\left(P_{P_{, \theta}}^{*}\right)$ circuits, and the dissipated power $\left(P_{D}^{\prime}\right)$ in the plasma, FPC components, and TF, trim, and $\mathrm{OH}$ coils, as well as the electric fields at the first wall in the toroidal $\left(E_{L, \phi}\right)$ and poloidal $\left(E_{L, \theta}\right)$ directions and the amplitnde of the plasma current oscillations normalized to the mean current $\left(\delta I_{\phi} / I_{\phi}\right)$. 
Table 7.4-II.

COMPARISON OF OFCD IN TITAN DESIGNS

\begin{tabular}{|c|c|c|c|}
\hline & \multicolumn{3}{|c|}{ Interim } \\
\hline & TITAN-I & TITAN-II & TITAN-II \\
\hline A verage plasma current, $I_{\phi}$ (MA) & 17.82 & 17.82 & 17.82 \\
\hline Drive frequency, $f(\mathrm{~Hz})$ & 25. & 25. & 25. \\
\hline Toroidal-flux swing, $\delta \phi / \phi_{o}$ & 0.035 & 0.035 & 0.035 \\
\hline$\Theta$ varjation & $1.499-1.616$ & $1.499-1.616$ & $1.499-1.616$ \\
\hline F variation & $-0.032--0.173$ & $-0.032--0.173$ & $-0.032--0.173$ \\
\hline \multicolumn{4}{|l|}{ Toroidal (poloidal) circuit power (MW): } \\
\hline Plasma Poynting power, $P_{P}^{*}$ & $3959.99(247.31)$ & $3959.99(247.31)$ & $3959.99(247.31)$ \\
\hline Plasma dissipation, $P_{n}$ & $28.55(0 . \quad)$ & $28.55(0)$. & $28.55(0)$. \\
\hline First-wall dissipation, $P_{F W}$ & $0.00(0.01)$ & $0.00(0.01)$ & $0.00(0.01)$ \\
\hline Blauket dissipation, $P_{B}$ & $1.04(0)$. & $0.01(0.19)$ & $0.01(0.17)$ \\
\hline \multicolumn{4}{|l|}{ Terminal reactive power, $P_{i}^{*}$ (MW): } \\
\hline TF coils & 503.88 & 1413.75 & 1413.77 \\
\hline $\mathrm{OH}$ coils & 74.92 & 101.99 & 101.99 \\
\hline EF coils & $\sim 0$. & $\sim 0$. & $\sim 0$. \\
\hline Trim coils & 113.44 & 147.16 & 147.16 \\
\hline \multicolumn{4}{|l|}{ Coll dissipation, $P_{H}(\mathrm{MW})$} \\
\hline TF coils & 47.38 & 35.69 & 11.44 \\
\hline $\mathrm{OH}$ coils & 0.13 & 0.17 & 0.17 \\
\hline EF coils & $\sim 0$. & $\sim 0$. & $\approx 0$ \\
\hline Trim coils & 1.95 & 2.49 & 2.49 \\
\hline \multicolumn{4}{|l|}{ Real (lozt) terminal power, $P_{T}$ (MW): } \\
\hline TF coils & 74.00 & 62.50 & 38.23 \\
\hline $\mathrm{OH}$ coils & 1.62 & 1.15 & 1.15 \\
\hline EF coils & $\sim 0$ & $\sim 0$. & $\sim 0$ \\
\hline Trim coils & 3.44 & 3.46 & 3.46 \\
\hline TF-coil DC power, $P_{H, \theta}^{S S}(\mathrm{MW})$ & 29.15 & 29.13 & 9.34 \\
\hline Power-supply dissipation, $P_{P S}(\mathrm{MWV})^{(a)}$ & 6.92 & 16.62 & 15.34 \\
\hline Total dissipation, $P_{D}(\mathrm{MW})$ & 85.93 & 83.74 & 58.19 \\
\hline Current-drive power, $P_{C D}$ (MW) & 56.83 & 54.61 & 48.85 \\
\hline Efficiency, $I_{\phi} / P_{C D}(\mathrm{~A} / \mathrm{W})^{(b)}$ & 0.33 & 0.33 & 0.36 \\
\hline
\end{tabular}

(a) Assuming the power supplies are $99 \%$ efficient $\left(Q_{P S}=100\right)$.

(b) Based on the total power consumed including driver efficienry and transmission losses. 

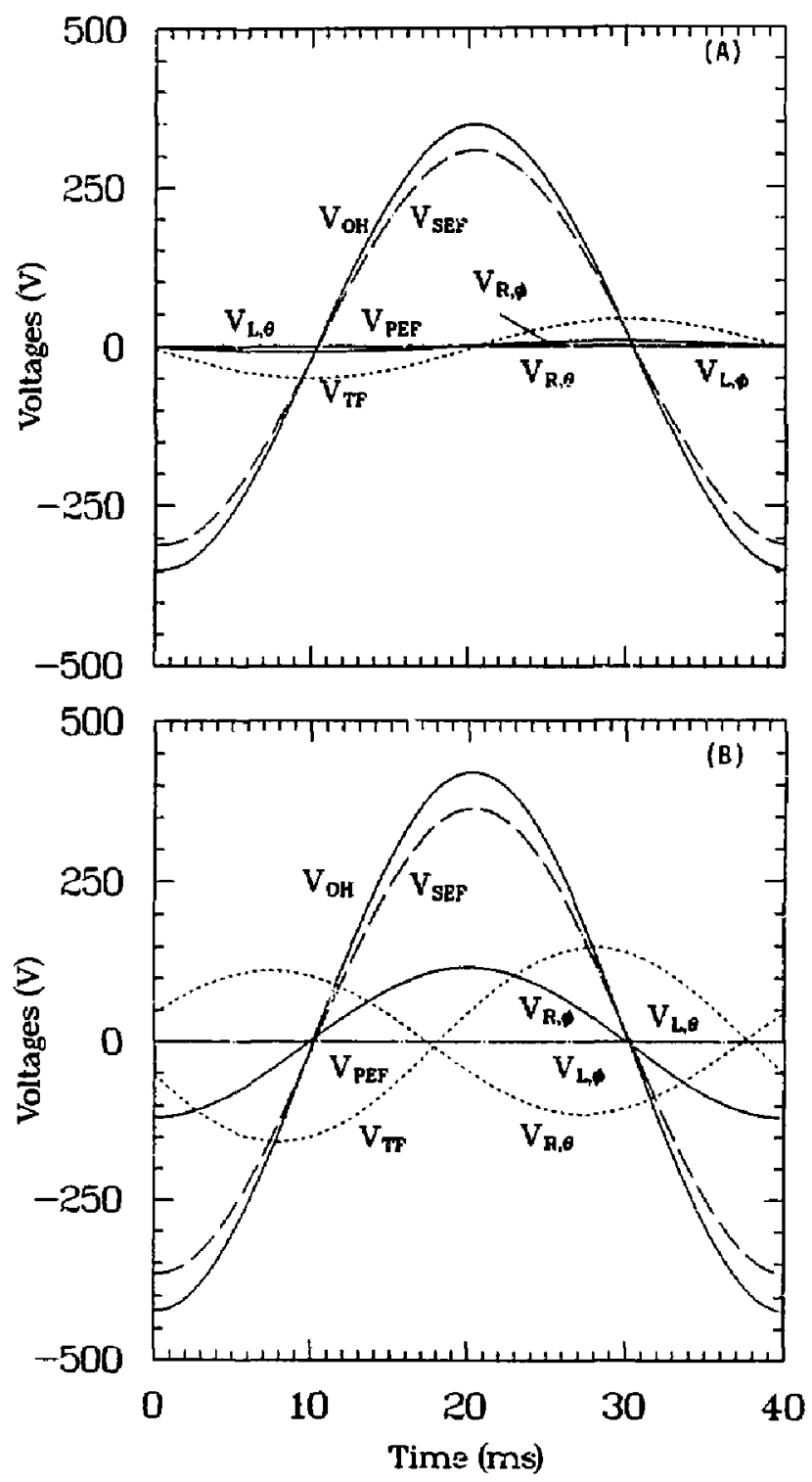

Figure 7.4-5. The voltage waveforms for TITAN-I (A) and TITAN-II (B) design for one OFC:D period. The voltages across $O H$ coils $\left(V_{O H}\right)$, trim coils $\left(V_{S E F}\right)$, TF coils $\left(V_{T F}\right)$, first wall $\left(V_{L, \phi}\right.$ and $\left.V_{L, \theta}\right)$, and across TITAN-I reflector and shield or TITAN-II blanket $\left(V_{R, \phi}\right.$ and $\left.V_{R, C}\right)$ are shown. 
mon to both designs: the $90^{\circ}$ phase shift between the toroidal $\left(V_{\phi}\right)$ and poloidal $\left(V_{\theta}\right)$ plasma voltages, which yields the optimal current-drive efficiency, is generated by a different phase shift between the toroidal ( $V_{O H}$ and $V_{S E F}$ ) and poloidal $\left(V_{T F}\right)$ coil vaitages. The phase shift between the coil voltages is dependent upon $\delta \phi / \phi_{o}$ in addition to the TF-coil position.

The current waveforns for the TITAN-I and TITAN.II OFCD dasign points are shown in Figure 7.4-6. Even though the superconducting EF coils are maintained at a constant (albeit negligible) voltage, the current in these coils oscillates vith an amplitude of $\leq 2 \mathrm{MA}$. Furthermore, the EF-coil current oscillations are out of plase with the trin- and $O H$-coil oscillations. The TITAN.I and TITAN-II TF-coil current wareforms are nearly the same, even though the voltage waveforms are quite different because the plasmacurrent and, heice, the toroidal-field waveforins are required to be identical and because the toroidal field is determined primarily by the TF coils (the toroidal field is affected only slightly by the first wall and blanket). The maximum, mininumu, and average values of the voltage and current waveforms are presented in Table 7.4-1Il.

Whereas the TiTAN-II TF-coil current density is uniform, the TITAN-I TF-coil current density has a radial variation shovin in Figure 7.4.\%. The expected exponential decay of the current radially through the IBC TF-coil tube bank occurs because the currentpenetration skin dept $h$ is greater than the coil radial build. In addition the current from radial row to radial cow incurs a phase shift. This radial non-uniformity of the $I B C$ TF-coil current gives rise to the direrences in the TITEN.I and TITAN-1I TF-coil dissipated powers. The TiTAN-II design ultimately dissipates less power in the first wall, blanket, and coils than TITAN-I, but has a larger terminal reactive power because of the different TF-coil designs. When the efficiency of the power supplies $(Q=100$ assumed $)$ is included in the current-drive efficiency, both designs operate at comparable efficiencies of $\sim 0.35 \mathrm{~A} / \mathrm{W}$. This frequency is based on the total power supplied to the system, inrluding driver efficiency and transmission losses.

\subsection{SUMMARY AND CONCLUSIONS}

Because of the large plasma resistance in the TITAN designs, an inciuctively pulsed burn would be sustained for a pulse length of the order of $L_{p} / R_{p} \simeq 200-400$ s. Therefor , steady-state operation is essential considering issues such as the total power balance, thernal cyclic fatigue in a high-power-deusity enviconment, as well as the costs of on-site energy storage (frequent grid-assisted start-up seenls unlikely) and thermal storage. An 

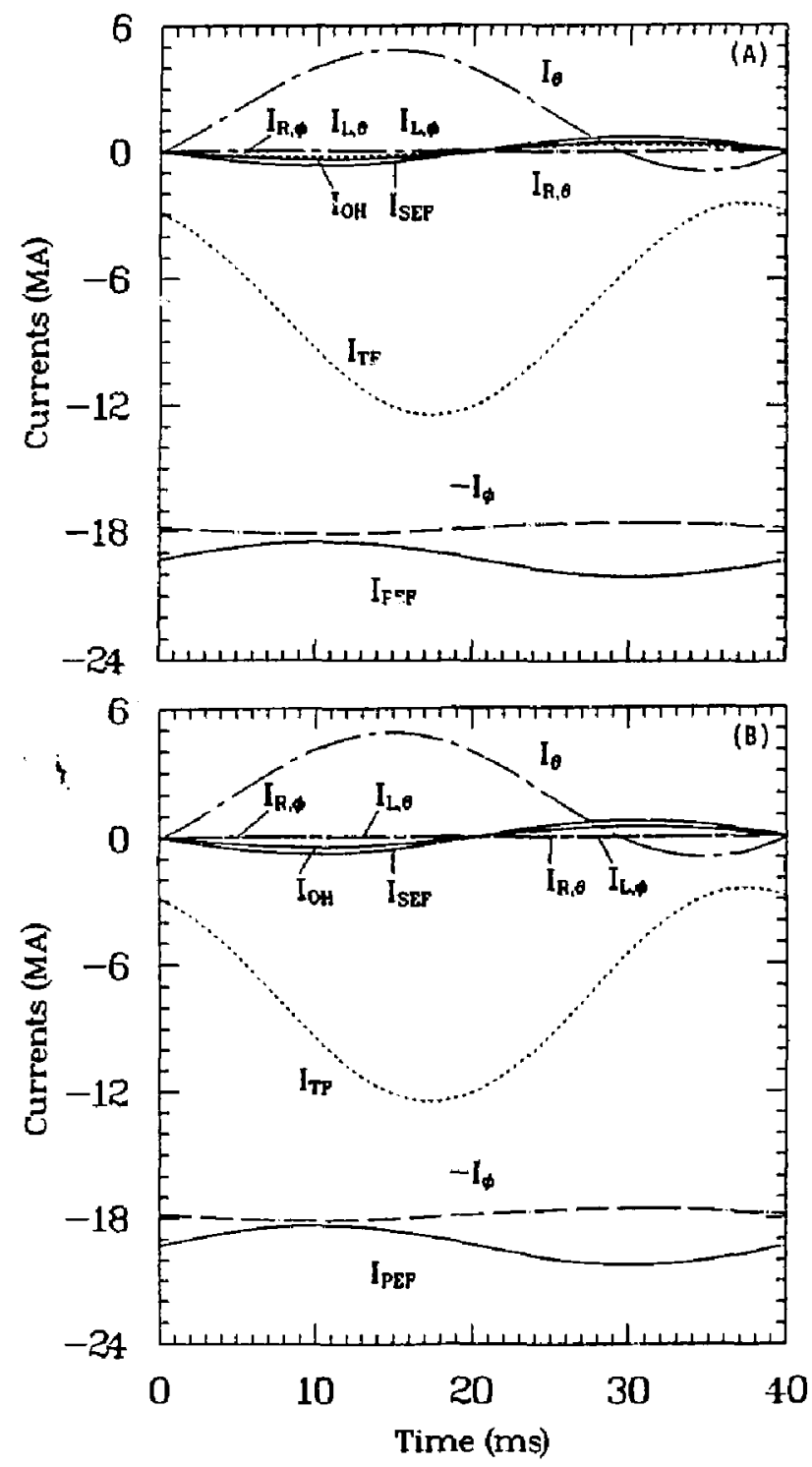

Figure 7.4-6. The current waveforms for TITAN-I (A) and TITAN-II (B) design for one OFCD period. The currents in the OH coils $\left(J_{O H}\right)$, trim coils $\left(J_{S E F}\right)$, superconducting EF coils $\left(I_{P E F}\right)$, TF coils $\left(I_{T F}\right)$, first wall $\left(I_{L, \phi}\right.$ and $\left.V_{l_{\theta}}\right)$, and in TITAN-I reflector and shield or TITAN-II blanket. $\left(I_{R, \phi}\right.$ and $\left.I_{R, \theta}\right)$ are shown. 
Table 7.4-III.

OFCD SINGLE-TURN VOLTAGES AND CURRENTS

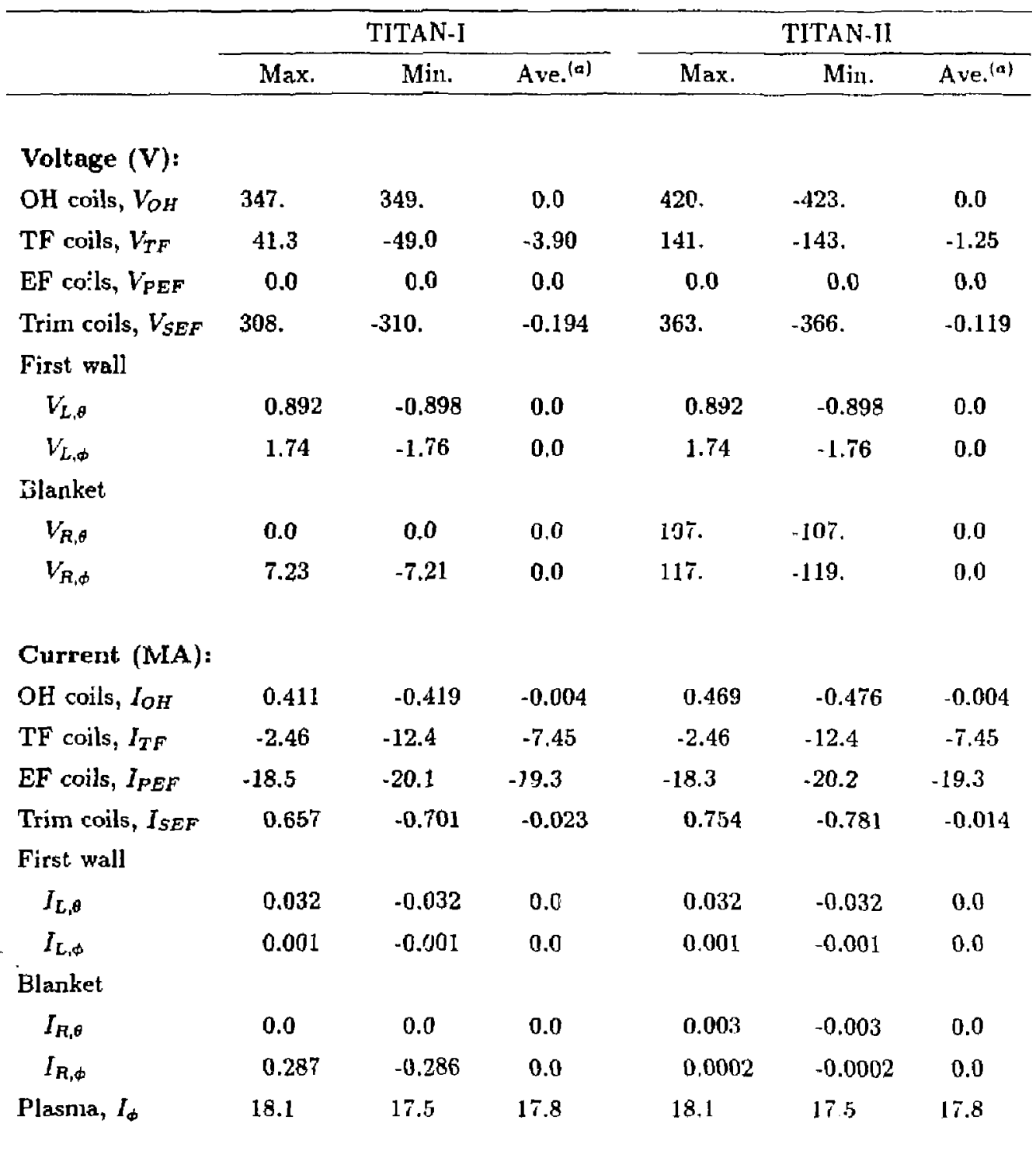

(a) Average value of $x$ is $\langle x\rangle \equiv \tau^{-1} \int_{0}^{r} x(t) d t$, where $\tau$ is one OTCD period. 


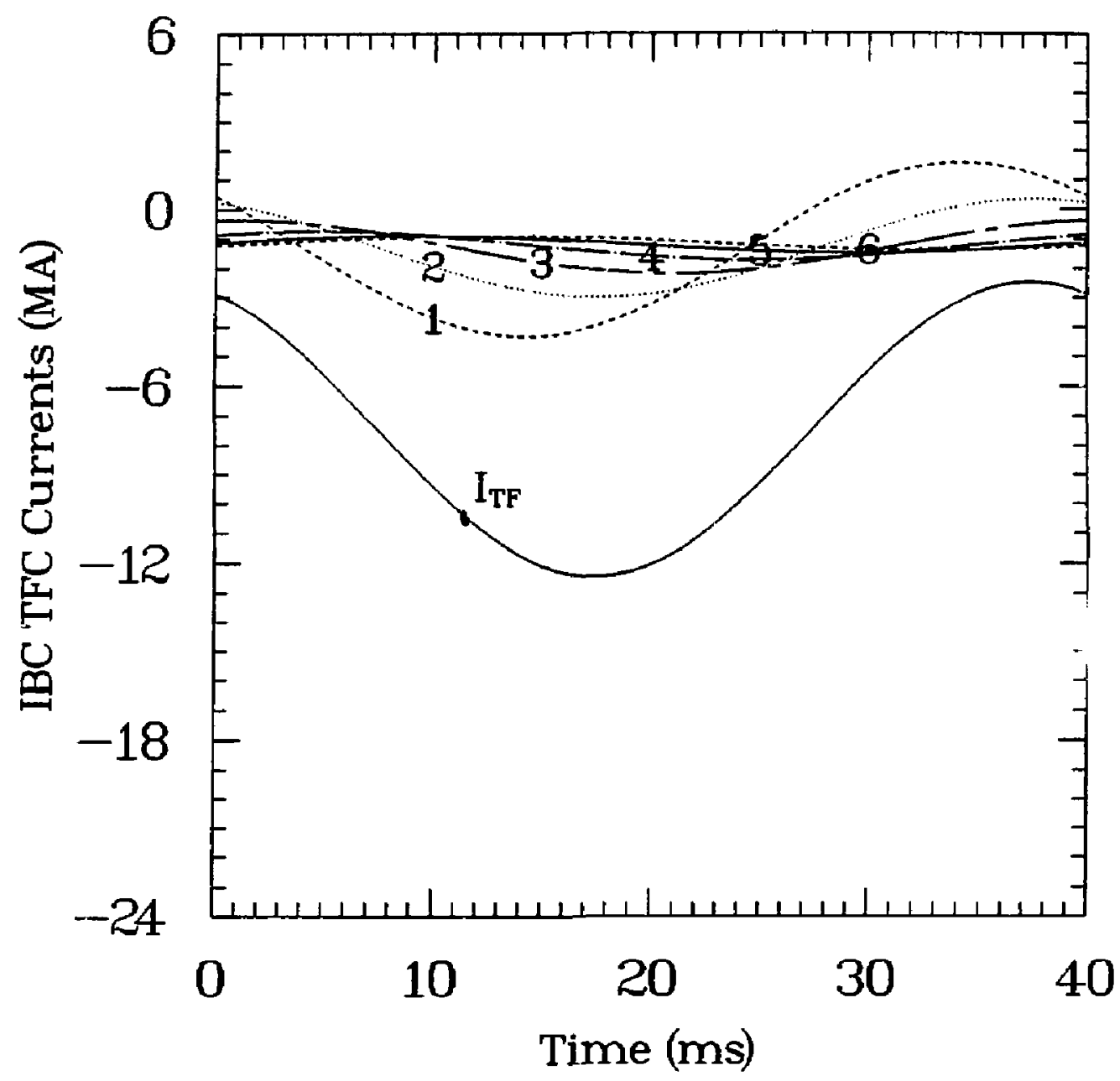

Figure 7.4-7. TITAN-I TF-coil current waveform $\left(I_{T F}\right)$ resolved into the current waveform in each of the six radial rows of $I B C$ tubes. Curve 1 corresponds to the inner most row of tubes, with increasing number indicating consecutively the outer rows. 
inductively pulsed RFP reactor is a possibility [1]. The parameters of such a reactor, however, should be optimized to mimimize the plasma resistance, which results in larger plasmas, lower power density, and possibly the use of superconducting coils throughout the FPC:

A number of current-drive options for the RFP have been considered (Section 7.2 . Althongh the use of fast-wave current-drive schemes has not been fully explored for the RFP, the high plasma density $\left(n \sim 9 \times 10^{20} \mathrm{~m}^{-3}\right.$ in TITAN) and currents relative to those for the tokamak indicate problems with the efficiency of radio-frecuency (RF) currentdrive schemes. On the other hand, because of the relaxation processes in RFPs, there is no need to drive the current at the plasma center and some of the issues related to wave penetration nlay be negated. Bootstrap current is also expected to be low, if such current exists at all in RFPs, since $\beta_{\theta}$ and $\epsilon=r_{p} / R_{T}$ are small relative to the tokamak.

The close coupling of poloidal and toroirlal currents and magnelic fields that determine the near-minimum-energy states of the RFP offers the possibility of a current-drive method based on "magnetic helicity injection" because the resistive decay of plasma current can be viewed as a dissipation of magnetic helicity [2]. For the TITAN reactors, helicity injection by the oscillating-field current drive (OFCD) has been selected as the means to sustain the toroidal plasma current.

A circuit model was developed that simulates the major elements associated with OFCD in order to determine the jnjected and/or dissipated powers. The model was used to quantify the need for toroidal and poloidal gaps or insulating breaks in structures such as the first wall, which will have currents induced by the OFCD. It is concluded that these breaks are needed to achieve acceptable current-drive efficiencies $(\sim 0.35 \mathrm{~A} / \mathrm{W})$. Detajled analysis of the TITAN-I and TITAN-II designs revealed a preference for the following: (1) series winding of all OFC:D coils; (2) the positioning of these coils as close to the plasina as possible; and (3) in the case of coil sets with small amplitude oscitlations about. large average currents, the splitting of the coil set into a set devoted to the oscillation and another set to produce the mean current. Future work should focus on effects of field errors introriuced by gaps during curreut oscillations, a better modeling of helicity balance and profile changes, and the generation of a better engineering understanding of the interaction of the OFCD system with other major FPC subsystems $(\epsilon, g$. equilibriun control, impurity control, and startup and shutdown systems). 


\section{REFERENCES}

[1] R. Hancox, R. A. Krakowski, and W. R. Spears, "The Reversed-Field Pinch Reactor Concept," Nucl. Eng. and Res. 63 (1981) 251.

[2] M. K. Bevir and J. W. Gray, "Relaxation, Flux Consumption and Quasi Steady State Pinches," Proc. of RFP Theory Workshop, Los Alamos, NM (1980), Los Alamos National Laboratory report LA-8944-C (1982) 176.

[3] D. D. Schnack, E. J. Caramana, and R. A. Nebel, "Three-Dimensional Magnetohydrodynamic Studies of the Reversed-Field Pinch," Phys. Flutds 28 (1985) 321.

[4] E. J. Caramana, R. A. Nebel, and D. D. Schnack, "Nonlinear, Single-Helicity Magnetic Reconnection in the Reversed-Field Pincl,," Phys. Fluids 26 (1983) 1305.

[5] R. A. Scardovelli, R. A. Nebel, and K. A. V'erley, "Transport Simulation of the Oscillating Field Current Drive Experiment in the ZT-4OM," Los Alamos National Laboratory report LA.JR-2802.

[6] K. F. Schroenberg, J. C. Ingraliam, C. P. Munson, P. G. Weber, D. A. Baker, R. F. Gribble, et al., "Oscillating-Field Curr ant Drive Experiment in a Reversed Field Pinch," Phys. Fluids 31 (1988) 2285.

[7] J. B. Taylor, "Relaxation and Magnetic Reconnection in Plasma," Rev. Mod. Phys. 58 (1986) 741; also "Relaxation of Torojdal Plasma and Generation of Reversed Magnetic Fields," Phys. Rev. Lett. 33 (1974) 1139; and "Relaxation of Toroidal Discharges," Proc. 3rd Topical Conf, on Pulsed High-Beta Plasmas, A bingdon (Sept. 1975), Pergamon Press, London (1976) 59.

[8] C. W. Barnes, J. C. Fernandez, I. Henins, H. W. Hoida, T. R. Jarboe, S. O. Knox, G. J. Marklin, and K. F. McKenna, "Experimental Determination of the Conservation of Magnetic Helicity from the Balance Between Source and Spheromak," Phys. Fluids 29 (1986) 3415.

[9] K. F. Schoenberg, C. J. Buchenaner, R. S. Massey, J. G. Meiton, R. W. Moses, R. A. Nebel, and J. A. Phillips, " $F-\Theta$ Pumping and Field Modulation Experiments on a Reversed Field Pinch Discharge," Phys. Fluids 27 (1984) 548.

[10] K. F. Schoenberg, R. F. Gribble, and D. A. Baker, "Oscillating Field Current Drive for Reversed Field Pinch Discliarges," J. Appl. Phys. 56 (1984) 2519. 
[11] J. T. Hogan, Bull. Am. Phys. Soc. 31 (1986) 1548.

[12] J. M. Finu and T. M Autonsen, Jr., "Anomalous Current Penetration and Osciliating Curcent Drive in Tokamaks," Phys. Fhids 30 (1987) 2450.

[13] M. Ono, G. J. Greene, D. Dajrrow, C. Forest, H. Park, and T. H. Stix, "Stesdy-State Tokamak Discharge via Helicity Injection," Phys. Rev. Lett. 59 (1987) 2165.

[14] Proc. Int. Workshop on Engineering Design of Next Step Reversed Field Pinch Devices, D. B. Thomson (Ed.), Los Alamos National Laboratory (July 13-17, 1987).

[15] R. Gross, Fusion Energy, Wiley and Sons, New York (1984) p. 118.

[16] N. J. Fisch, "Theory of Current Drive in Plasmas," Rev. Mod. Phys. 59 (1987) 175.

[17] M. Abdow, C. Baker, J. Brooks, D. Defreece, D. Ehst, et al., "A Demonstration Tokamak Power Plant Study-Interim Report," Argonne Iational Laboratory report. ANL/FPP/TM-154 (1982) Chapter 2.

[18] M. Porkolab, B. Blackwell, P. Bonoli, D. Griffin, S. Kr.owlton, et al., "Lower Hybrid Heating, Current Drive and Ion Gyclotron Heating Experiments on Alcator $\mathrm{C}$ and Versator II Tokamaks," Proc. 10th Int. Conf. Plasma Phys. and Cont. Nuc. Fusion Res., London (September 1984) 463.

[19] K. L. Woug and M. Ono, "Effects of Ion Cyclotron Harmonic Damping on Current Drive in the Inwer Hybrid Frequency Range," Nucl. Fusion 24 (1984) 615.

[20] N. J. Fiscls and C. F. F. Karney, "Current Generation with Low Frequency Waves," Phys. Fluids 24 (1981) 27.

[21] D. A. Ehst, "Pelativistic Electron Beam Current Drive," in FED-A, An cidvanced Performance FED Based on Low Safety Factor and Current Drive, Oak Ridge National Laboratory report ORNL/FEDC-83/1 (August 1983).

[22] R. L. Hagenson, R. A. Krakowski, C. G. Bathke, R. L. Miller, M. J. Embrechts, et al., "Compact Reversed-Field Pinch Reaciors (CRFPR): Preliminary Engineering Considerations," Los Alamos National Laboratory report LA-10200-MS (1984).

[23] S. I. Braginskii, "Transport Processes in a Plasma," in Revieus of Plasma Physics, M. A. Leontovich (Ed.), 1, Consultants Bureau, New York (1965). 


\title{
8. PHYSICS ISSUES FOR COMPACT REVERSED-FIELD-PINCH REACTORS
}

\author{
Roijert A. Krakowski
}

Farroklı Najuabadi 


\section{Contents}

8.1. INTRODUCTION .................... 8-1

8.2. CONFINEMENT $\ldots \ldots \ldots \ldots \ldots \ldots \ldots \ldots \ldots$ 8-8

8.2.1. Reactor Coudtitious . . . . . . . . . . . . . . 8-8

8.2.2. Physics Data Base $\ldots \ldots \ldots \ldots \ldots \ldots \ldots$. . . . . . . . . .

8.2.3. Reactor Implications . . . . . . . . . . . . . 8-18

8.3. CURRENT DRIVE . . . . . . . . . . . . . . . 8-21

8.3.1. Reactor Conditions . . . . . . . . . . . . . 8.22

8.3.2. Plysics Data Base . . . . . . . . . . . . . . 8-22

8.3 3. Reactor Inplications . . . . . . . . . . . . 8-25

8.4. IMPURITY CONTROL . . . . . . . . . . . . . . 8-28

8.4.1. Reactor Conditions . . . . . . . . . . . . . 8-28

8.4.2. Physics Data Base . . . . . . . . . . . . 8-30

8.4.3. Reactor Implications . . . . . . . . . . . . 8-32

8.5. FORM

8.5.1. Reactor Conditions . . . . . . . . . . . 8.36

8.5.2. Physics Data Base . . . . . . . . . . . . . 8-37

8.5.3. Feactor Implications . . . . . . . . . . . . . 8.47

8.6. SUMMARY AND RECOMMENDATIONS . . . . . . . . . . . 8-51

REFERENCES . . . . . . . . . . . . . . . 8.53 


\section{PHYSICS ISSUES FOR COMPACT REVERSED-FIELD-PINCH REACTORS}

\subsection{INTRODUCTION}

The TITAN research program is a multi-institutional [1] effort to determine the potential of the reversed-field-pinch (RFP) magnetic fusion concept as a compact, highpower-density, and "attractive" fusion energy system from economics (cost of electricity, COE), safety, environmental, and operational viewpoints.

In recent reactor studies, the compact reactor option [2-5] has been identified as one approach toward a more affordable and competitive fusion reactor. The main feature of a compact reactor is a fusion power core (FPC) with a mass power density in excess of 100 to $200 \mathrm{kWe} /$ tonne. Mass power density (MPD) is defined [2] as the ratio of the net electric power to the mass of the FPC, which includes the plasma chamber, first. wall, blanket, shield, magnets, and related structure. The increase in MPD is achieved by increasing the plasma power density and neutron wall loading, by reducing the size and mass of the FPC through decreasing the blanket and shield thicknesses and using resistive magnet coils, as well as by increasing the blanket energy multiplication. A compact reactor, therefore, strives toward a system with an FPC comparable in mass and volume to the heat sources of alternative fission power plants, with MPDs ranging from 500 to $1000 \mathrm{kWe} /$ tonne and competitive cost of energy.

Other potential benefits for compact systems can be envisaged in addition to iunproved economics. The FPC cost in a compact reactor is a small portion of the plant cost and, therefore, the economics of the reactor will be less sensitive to changes in the unit cost of FPC components or the plasma performance. Moreover, since a high-MPD FPC: is smaller and cheaper, a rapid development progrann at lower cost should be possible, changes in the FPC design will not. introduce large cost perlalties, and the economics of learning curves can be readily exploited throughout the plant life

The RFP has inherent characteristics which allow it to operate at very high mass power densities. This potential is available because the main confining field in an RFP is the poloidal field, which is generated by the large toondal current flowing in the plasma. This feature results in a low field at the external magnet coils, a high plasma beta, and a very high engineering beta (defined as the ratio of the plasma pressure to the square 
of the magnetic field strength at the coils) as compared to other confinement schemes. Furthermore, sufficiently low magnetic field at the external coils pernits the use of normal coils while joule losses remain a small fraction of the plant output. This option allows a thimner blanket and shield. In addition, the high current density in the plasma allows nlunic heatiug to ignition, elininating the need for anxiliary heating extupnetut. Also, the RFF concept promises the possibility of efficient current-drive systems baserl on low-frequency oscillations of poloidal and toroidal fluxes and the theory of HFP relaxed states. The RFP confinement concept allows arbitrary aspect ratios, and the circular cross section of plasma eliminates the need for plasma shaping coils. Lastly, the higher plasma densities particularly at the edge, together with operalion with a highly racliative RFP plasma, significantly reduce the divertor heal flux and erosion problems.

These inherent characteristics of the RFP [6] allow it to meet, and actually far exceed, the economic threshold MPD value of $100 \mathrm{kWe} /$ tonne. As a result, the TITAN study also seeks to find potentially significant benefits and to illnminate main drawbacks of operating well above the MPD thresiold of $100 \mathrm{kWe} /$ tonne. The prograin, therefore, has chosen a minimum cost, high neutron wall loading of $18 \mathrm{MW} / \mathrm{m}^{2}$ as the reference case in order to quantify the issue of engineering practicality of operating at high mass powe: densities. The TITAN study has also put strong emphesis on safety and environmental features in order to deternine if high-power-density reartors can be designed with a high level of safety assurance and with low-activation material to qu- lify for Class-C: waste disposal.

An important potential lenefit of operating at a very high MPD is that the smal] physical size and mass of a compact reactor permits the clesign to be made of only a few pieces and a single-piece maintenance approach will be feasible $[7,8]$. Single-piece maintenance refers to a procedure in which all of components that must be changed during the scheriuled maintenance are replaced as a single unit, although the actual maintenance procedure may involve the movenent, storage, and reinstallation of some other reactor components. In TITAN designs, the entire reactor torus is replaced as a single unit during the annual scheduled maintenance. The single-piece maintenance procedure is expected to result in the shortest period of downtine during the scheduled maintenance period because: (1) the number of connects and disconnects needed to replace componemts will be minimized; and (2) the installation time is much shorter because the replaced components are pretested and aligned as a single unit before committment to service. Furthermore, recovery from unscheduled events will be nore standard and rapid because complete components will be replaced and the reactor brought back on line. The tepair work will then be performed outside the reactor vault. 
To demonstrate the possibility of multiple engineering appioaches to the high-powerdensity option, the TITAN study has developed two FPC embodiments: TITAN-I, a selfcooled liquid-lithium-metal loop design with vanadium-alloy structure, and TITAN-II, a self-cooled aqueous-lithium-salt pool design with ferritic-steel structure.

The operating space of a compact RFP reactor has been examined using a compre. hensive parametric systems model which includes the evolving state of knowledge of the physics of RFP confinement and embodies the TITAN-I and TITAN-II enginering approaches (Section 3). Two key figures of merit, the cost of electricity (COE) and mass power density (MPD), are monitored by the parametric systems model and are displayed in Figure 8.1-1 as functions of the neutron wall loading. Figure 8.1-1 shows that the COE is relatively insensitive to wall loadings in the range of 10 to $20 \mathrm{MW} / \mathrm{m}^{2}$, with a shallow minimum at about $19 \mathrm{MW} / \mathrm{m}^{2}$. The MPL is found to increase monotonically with the

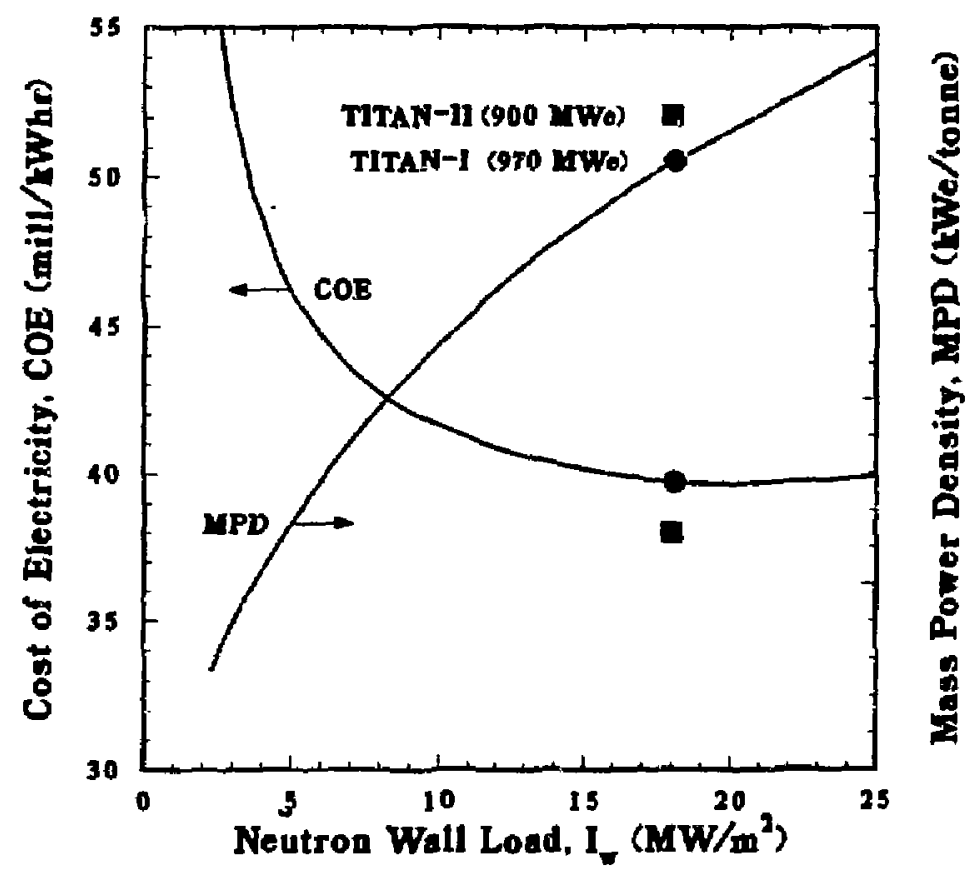

Figure 8.1-1. The COE and MPD as functions of neutron wall loading for the TITANclass RFP reactors. TITAN-I (filled circle) and TITAN-II (filled squares) reference design points are also shown. 
wall load. For designs with a neution wall load larger than about $10 \mathrm{MW} / \mathrm{m}^{2}$, the FPC: is physically small enough such that single-piece FPC! maintenance is feasible. These considerations point to a design window for compart RFP reartors with neutron wall loading in the range of 10 to $20 \mathrm{MW} / \mathrm{m}^{2}$. The TITAN-class RFP reactors in this clesign window bave an MPD in excess of $500 \mathrm{kWe} /$ tomne, and an FPC engineering powet density in the range of 5 to $15 \mathrm{MWt} / \mathrm{m}^{3}$; these values represent improvements by fartors of 10 to 30 compared with earlier fusion reactor designs. The FPC $\cos t$ is a smaller portion of the total plant cost (typically about $12 \%$ ) compared with $25 \%$ to $30 \%$ for earlier RFP designs $[4,5]$. Therefore, the unit direct sost (UUC:) is less sensitive to related physics and techuology uncertainties.

Near-minimum-COE TITAN-I and TITAN-II design points, incorporating distinct blanket thermal-hydraulic options, materials cloices, and neul.ronics performances have been identified in Figure 8.1-1. The major parameters of the TITAN reartors are summarized in Table 8.1-1. The engineering analyses of TITAN-I and TITAN-II FP('s are presented, respectively, in Sections 9 through 14 and Sections 15 through 20 . The parameters of the TITAN reference design points, based on detailed subsystem design, are included in Appendices $\mathrm{A}$ and $\mathrm{B}$ and follow the DOE/OFE standard reporting format. Appendices also include cost tables and parametric systems code predicions of subsystem parameters for comparison with DOE/OFE tables.

In order to permit a comparison, the TITAN reference design points have similar plasma parametets and wall loadings allowing for certain plasma engineering analyses to be common between the two designs. Major pliysics features of the TITAN designs are summarized in Table 8.1-II.

One of the major ohjectives of the TITAN study is to identify and assess the physics requirements of high-MPD RFP reactors. The experimental and theoretical bases for RFPs have grown rapidly during the last few years [6], but a large degree of extrapolation] to I'ITAN-class reactors is still required (Table 8.1-III). The degree of extrapolation is one to two orders of magnitude in plasma current and temperature and two to three orders of unagnitude in energy confinement time. However, the TITAN plasna density, poloidal beta, and plasma current density all are close to present-day experimental achievenents. The next generation of RFP experiments $[9,10]$ with hotter plasmas will extend the data base toward reactor-relevant regimes of operation. The TITAN study has brought out and illuminated a number of key physics issues, sone of which require greater attention from the RFP physics community.

The physics of confinement scaling, plasma transuort, RFP plasma formation and start-up, and the role of the conducting shell are already major efforts in RFP research. 
Table 8.1-1.

\section{OPERATING PARAMETERS OF TITAN FUSION POWER CORES}

\section{TITAN.I}

3.9

0.60

0.66

17.8

0.30

0.23

18

4.6

Radiation heat flux on first wall (MW/ $/ \mathrm{m}^{2}$ )

Primary coolant.

Structural materia]

Breerler material

Neutron multiplier

Coolant inlet temperature $\left({ }^{\circ} \mathrm{C}\right)$

First-wall-coolant exit temperature $\left({ }^{\circ} \mathrm{C}\right)$

Blanket-coolant exit temperature $\left({ }^{\circ} \mathrm{C}\right)$

Coolant punping power (MW)

Fusion power (MW)

Total thermal power (MW)

Net electric power ( $M W$ )

Gross efficiency

Net efficiency

Mass power density, MPD ( $\mathrm{kWe} /$ tome)

Cost of electricity, COE (mill/kWh)
Liquid lithium V-3Ti-1Si

Liquid lithium

none

320

440

700

48

2301

2935

970

$44 \%$

$33 \%$

$75 \pi$

39.7
TITAN-II

3.9

0.60

0.66

17.8

0.36

0.23

18

4.6

Aqueous solution Ferritic steel 9.C!

$\mathrm{LiNO}_{3}$ Be 298 330 330 49 2290 3027 900 $35 \%$

$30 \%$

806

38.0 
Table 8.1-II.

\section{PHYSICS FEATURES OF TITAN REACTORS}

- Confinement scaling: $\tau_{E,} \propto J_{\phi}^{\prime} r_{p}^{2} f\left(\beta_{\theta}\right)$ with a soft. beta limil.;

- Poloidal bet.a: $\beta_{\theta} \simeq 0.22$. including energetic alpha particles;

- Radiation-dominated plasma: $f_{R A D} \simeq 0.96$ for the combined core and edge plasmai

- Poloidally symmetric, toroidal-field divertors for impurity control and particle exltaust:

- Current drive 'ising oscillating-fie!d helicity injection;

- Shell stabilization for $\leq 10 \mathrm{~ms}$;

- Relaxation-assisted start-up and sustainment;

- Deusity and field control of current terminations;

- Open poloislal-field-coil configuration with superconducting eguilibrium-field coils;

- Bipolar start-up with low back-bias toroiclal field.

However, the TITAN stuig points to three other major issues. First, operafing highpower-density fusion reactors with intensely radiating plasmas is crucial. C'onfirning that the global energy confinement time remains relatively unaffected while core-plasma radiation increases (a possible unicjue feature of RFP) is extremely important. Second, the TITAN study has aclopted the use of three "open-geometry" toroidal divertors as the impurity-control and particle-exhaust system. Fven with an intensely radiative plasma, using an array of polvidal pump-limiters as the impurity-contcol system would suffer from the serious etosion of the liniter blades (and possibly the first wall). The physirs of toroidal-field divertors in RFPs must be examined, and the impact of the nuagnetic separatrix on RFP corfinement nust be studied. If toroidal divertors are comsistent. with confinenent and stability in RFPs, then high-recycling divertors and the predicted 
Talsle 8.1-III.

PARAMETERS OF MAJOR RFP DEVICES

\begin{tabular}{|c|c|c|c|c|c|c|c|}
\hline Device & $\begin{array}{c}\text { Major } \\
\text { Rarlius } \\
(\mathrm{m})\end{array}$ & $\begin{array}{c}\text { Minor } \\
\text { Radius } \\
\text { (in) }\end{array}$ & $\begin{array}{c}\text { Plasuna } \\
\text { Current } \\
\text { (MA) }\end{array}$ & $\begin{array}{c}\text { Current } \\
\text { Density } \\
\left(\mathrm{MA} / \mathrm{m}^{2}\right)\end{array}$ & $\begin{array}{c}\text { Electron } \\
\text { Temperature } \\
(\mathrm{keV}) \\
\end{array}$ & $\begin{array}{c}\text { Average } \\
\text { Desssity } \\
\left(10^{20} \mathrm{~m}^{-3}\right) \\
\end{array}$ & $\begin{array}{c}\begin{array}{c}\text { Poloidal } \\
\text { Beta }\end{array} \\
\end{array}$ \\
\hline TPE-1RM ${ }^{(n)}$ & 0.50 & 0.09 & 0.13 & 5.1 & 0.60 & 0.3 & 0.1 \\
\hline ETA-BETA-II $I^{(b)}$ & 0.65 & 0.125 & 0.15 & 3.0 & 0.08 & 1.0 & 0.1 \\
\hline $\mathrm{HBTX}_{1} \mathrm{~A}^{(c)}$ & 0.80 & 0.26 & 0.32 & 1.5 & 0.10 & 0.2 & 0.05 \\
\hline OHTE/RFP(d) & 1.24 & 0.20 & 0.50 & 4.5 & $0.4-0.6$ & $0.5-3.0$ & $0.1-0.2$ \\
\hline$Z \mathrm{~T}-40 \mathrm{M}^{(e)}$ & 1.14 & 0.20 & 0.44 & 3.5 & $0.3-0.5$ & $0.4 \cdot 0.9$ & $0.1-0.2$ \\
\hline $\operatorname{RFX}(f)$ & 2.00 & 0.48 & 2.0 & $2.8^{\circ}$ & $0.5-2.0$ & $0.3-2.0$ & 0.10 \\
\hline CPRF/ZTH ${ }^{(9)}$ & 2.40 & 0.40 & 4.0 & 8.0 & $0.5-5.0$ & $0.3-5.0$ & 0.10 \\
\hline $\mathrm{FTF} / \mathrm{RFP} \mathrm{P}^{(h)}$ & 1.80 & 0.30 & 10.4 & $3 \pi$. & 10. -20 & $6.0-9.0$ & $0.1-0.2$ \\
\hline TITAN $\{i\}$ & 3.80 & 0.60 & 18.2 & 16. & 10. -20 . & 9.t & 0.2 \\
\hline
\end{tabular}

(a) Existing experiment at ETL, Japan [11].

(b) Existing experiment at Padova, Italy [12].

(c) Existing experiment at. Culham, U. K. [13].

(d) Existing experiment at General 4tunics, U. S. A. [14].

(f) Existing experiment at Los Alamos National Laboratory, U. S. A. [15].

(f) Planned experiment at Parlova, Italy $[10,16]$.

(g) Planned experiment at Los Alamos National Laboratory, U. S. A. $[9,15]$.

(h) Conceptual neutron source, a Los Alamos National Laboratory study, U. S. A. [17].

(i) Conceptuai reactor design, a UCLA-led multi-institutional study, U. S A. 
high-density, low-temperaf ure scrape-oft layer must. be also comfirmed. Third, enrly work in the TITAN stuty convinced the tean that high-MPD, compact RPP reactors must. operate at steady state. Cinrrent drive by magnetic helicity injection atilizing the nat ural relaxation process in RFP plasma is predicted to be efficient $[18,19\}$ lut experingents on oscillating-field current drive (OFCD) are inconclusive. Testiug OF(!) in higher temperat.me plasmas must awajt the next generation of RFP experiments. namely ZIII $[9,16]$ and $\operatorname{RFX}(10,16]$.

The key physics issues of confinement, impurity control, current drive, and RPP formation and start-up are reviewed in detail in Sections 8.2 through 8.5 , respectively. These issues are to varying degrees interrelated and, therefore, interfactes and connertions are identified when appropriated. For each physics issue, the TYTAN reactor requirements and the present physics data base are reviewed in order to ithentify those high-leverage areas that require further experinental and theoretical work. The scusitivity of the reactor performance to these physics assumptions and extrapolation liave also been st.utied using the parannetric systems code.

\subsection{CONFINEMENT}

The physies issues related to plasma confinement include: (1) energy aud parlicle confinement in a plasma with dynano-related turluslence, (2) the magnitude and scaling of the normalized plasma pressure, $\beta_{\theta}$, and (3) the deglee to whicf the plasua can shed emergy by radiation as opposed to intrinsic 1 ransport channels without impacting the global confinement. These confinement issues should be addressed for both olumic-heated and alpha-particle-heated discharges.

\subsubsection{Reactor Conditions}

As for most fusion reactor designs, the sjecifications of the thet-electic power (i.e., tolal fusjon power, $P_{F}$ ), plasma power balance (i.e., $n \tau_{E} T$ ), and neut.on wall loarling, $I_{w}$, give the global energy confomement. time, $\tau_{E} \times\left(P_{F} / A\right)^{1 / 4}\left(n \tau_{E} T\right) / I_{1, "}^{3 / 4}$. The value of neutron wall loading is deternined by the minimization of ('OE in TITAN, therefore, the above value of $\tau_{E}$ represent an "economic" confinement time, $\tau_{E}(E C O N)$, which must be compared with experimental predictions, $\tau_{E}$ (PHYS), The economic confinement required to achieve the minimum-COE design point for 'Tl'IAN (Figure 8.1-1) is $\tau_{E}(E C O N)=0.22 \mathrm{~s}$, or $x_{E}=0.32 \mathrm{~nm}^{2} / \mathrm{s}$. For TITAN, this level of global energy confinement must be attained in a highly radiating core plasma (with a radiation fraction, 
$f_{R A D}^{\subset} \simeq 0.7$ ) with a poloidal beta, $\beta_{\theta}=0.22$ (including a beta increment of $\sim 0.02$ for energetic alpha particles).

A sinuplified energy balance for a non-radiating, ohmically heated plasma gives

$$
\tau_{B}=\frac{3 \mu_{0}}{16} \frac{\beta_{\theta} \tau_{p}^{2}}{\eta}
$$

where $\eta$ is the effective plasma resistivity. Under conditions of constant $\beta_{\theta}$ and $j_{\phi} / n$, pressure balance gives $T \propto I_{\phi}$, and for a classical scaling of resistivity with temperature $\left(\eta \propto T^{-3 / 2}\right)$ results in $\tau_{\mathrm{Be}} \propto I_{\phi}^{\nu} r_{\mathrm{p}}^{2}$ with $\nu=1.5$. Any variations from these assumptions as well as variations in profile factors, impurity concentration, and $Z_{e s f}$, will alter the dependence of $\tau_{s}$ on $I_{\phi}$ and $r_{p}$. Until experinent data on the plasnia profiles and $Z_{\text {ef } j}$ become available, the expression $\tau_{\varepsilon e} \propto C_{\nu} I_{\phi}^{u} r_{p}^{2}$ is used to paranetrically fit the existing experinental data.

The TITAN systems code, as well as the plasina engineering effort, has used a physics scaling of the form $\tau_{E}$ (PIYYS) $=2\left(1 / \tau_{E_{e}}+1 / \tau_{E i}\right)^{-1}$ with $\tau_{E_{e}}=C_{\nu} I_{\phi}^{\nu} r_{p}^{2} f\left(\beta_{\theta}\right)$ and $\tau_{E_{1}} \simeq 4 \tau_{B_{e}}$. The current exponent $\nu=0.8$ to 1.5 represents a range of fits to the global confinement observed in experiments after corrections for rarliative loss liave been applied. The function $f\left(\beta_{\theta}\right)$ reflects an attempt to model a beta limit that may exist in RFPs with $f\left(\beta_{\theta}\right)=1$ for low values of $\beta_{\theta}$ and $f\left(\beta_{\theta}\right) \rightarrow 0$ when $\beta_{\theta}$ exceeds a critical value of beta, thereby giving considerable thermal stability to the burn.

Several important points should be noted: (1) The ahove empirical scaling is derived from experiments with limited variations in both $I_{\phi}$ and $r_{p}$. (2) In the present. chmically leated discharges, $\beta_{\theta}$ and $\tau_{B}$ (or $\chi_{z}$ ) are inexorably coupled and it is not clear that the above empirical scaling of confinement will be applicable to fusion discharges where alpha-particle heating is dominant. (3) Zero-dimensional simulation of plasma startup for TITAN reactors with the above scaling shows that the ignition is achieved at $\beta_{\theta} \simeq 0.05-0.1\left(\tau_{E} \simeq 0.4 \mathrm{~s}\right)$ and the value of $\beta_{\theta} \simeq 0.22$ is only reacied at the stearty-state burn condition. Since the above scaling is derived for the flat-top portion of experimental discharges with $\beta_{\theta}$ being close to its linnit, applicability of this scaling to TITAN start-up sinulations is questionable.

The level of field error, particularly as related to the size and formation of magnetic islands, must be sufficiently low to not adversely impact plasma confinement. The toroidalfield (TF) and divertor coils of TITAN reactors are designed such that the toroidal-field ripple is constrained to $\Delta B_{R} / B_{\theta} \leq 0.03$. As a result, the overall island widths are less than a quarter of the distance between the reversal surface and plasma (separatrix) surface; this annular region is assumed to be crucial for RFP confinement. 
Table 8.2-I.

\section{IMPURITY CONCENTRATION AND RADIATION FRACTIONS IN THE TITAN PLASMA}

\begin{tabular}{lccc}
\hline Location & $n_{X c}\left(10^{18} \mathrm{mi}^{-3}\right)$ & $n_{i}\left(10^{20} \mathrm{~m}^{-3}\right)$ & $f_{R A D}=P_{R A D} / P_{T O T}^{(a)}$ \\
\hline Core & 0.289 & 8.9 & 0.695 \\
Edge & 0.289 & 1.7 & 0.232 \\
Divertor & 10.50 & 62.0 & 0.039
\end{tabular}

(a) $P_{T O T}=P_{O H M}+P_{\alpha}=552 \mathrm{MW}$.

One-dimensional (radial) plasma simulations were performed to deter:nine achievable values of $f_{R A D}^{c}$ for a given kind and quantity of injected impurity (Section 5.3). For these calculations, the local beta limit described above in terms of $f\left(\beta_{\theta}\right)$ is imposed. This limit enhances the local electrou perpendicular thermal conductivity and the particle diffusivity by large factors aluove classical values, especially in the central plasma region when the on-axis beta exceeds a critical limit. For ohmically heated plasmas with small radiation losses, these assumptions lead to a global scaling of the form given above with $\nu \simeq 1.5$.

The 1-D plasma simulations were performed assuming a uniform impurity concentration and a coronal equilibrium model. The values of the core-plasma radiation fraction $\left(f_{R A D}^{c}\right)$ and the resultant $Z_{e f f}$ are estinated as functions of the impurity concentration for several impurity species and the results are given in Figure 8.2-1. High- $Z$ impurities are preferred because a high value of $f_{\mathrm{RAD}}^{c}$ can be achieved for ninimun $Z_{\mathrm{c} f f}$ (to minimize $V_{\phi}$ and the OFCD requir ments). (Combined with similar estimates of the edge-plasma and divertor radiation fractions, a total radiation fraction of $f_{R A D}^{T O T}=0.97$ is possible for the Xe impurity concentrations given on Table 8.2-1.

\subsubsection{Plysics Data Base}

The essential elements of the RFP experinental data base in the confinement area are: (1) scaling of plasma temperature and/or pressure with current (i,e., confining 

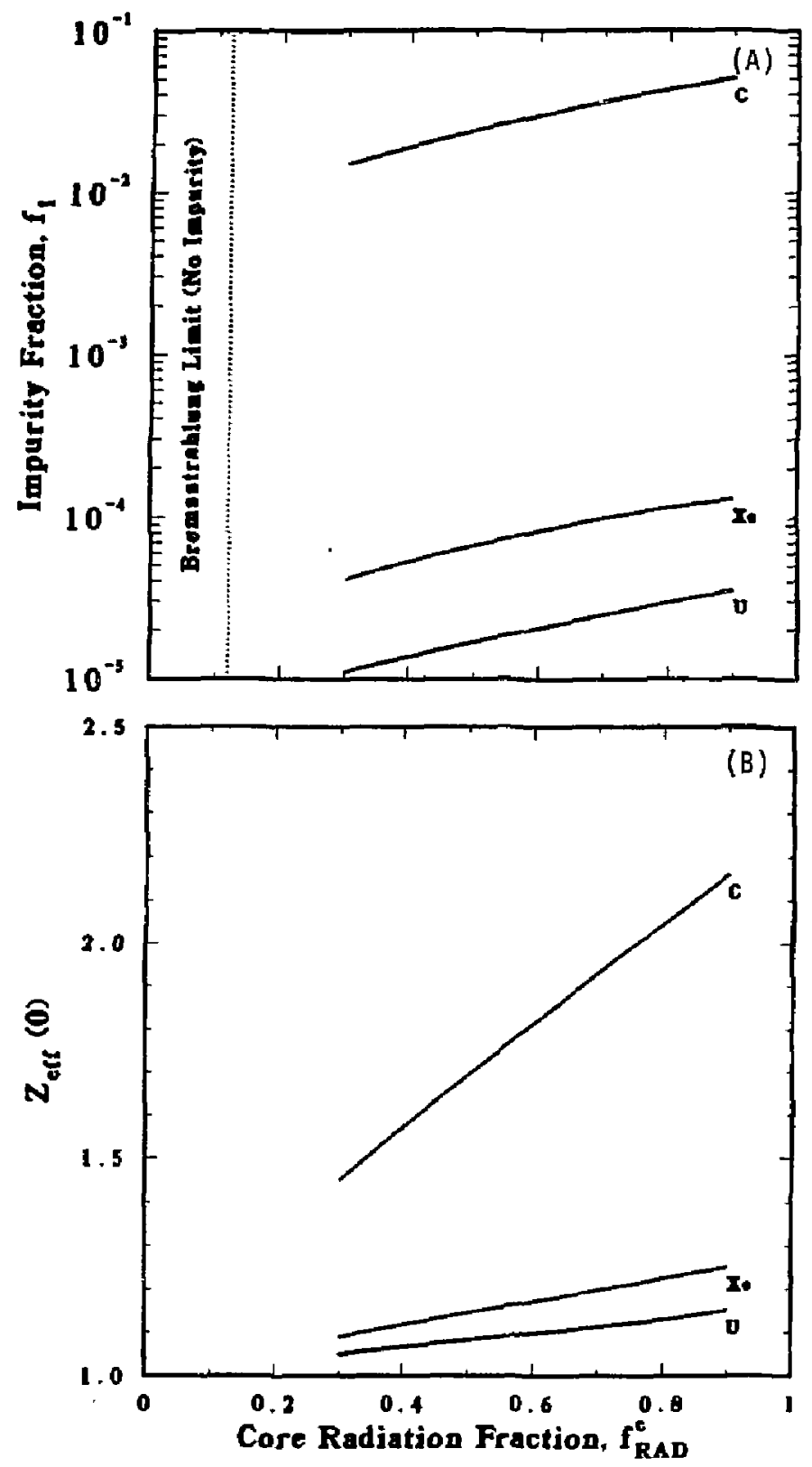

Figure 8.2-1. The required inpurity fraction (A) and the resultant $Z_{\text {efs }}(\mathrm{B})$ as functions of the core radiation fraction, $f_{h A D}^{c}$, for different impurities. 
magnetic field); (2) scaling of plasma energy confinement with plasma current and size; and (3) partition of plasma energy loss between radiative and intrinsic (conduction and convection) chamels. A more extensive review of the present clata base is given in Section 2.3; only examples typical of desirable beharior are repeated here.

\subsubsection{Temperature, density, and pressure scalings}

Extensive measurements of the dependence of the plasmn temperature on cursent for a range of RFPs indicare that the on-axis electron temperattue increases with plasma current raised to a power in the range of 0.5-1.0. Temperature increases on the order of $1 \mathrm{eV} / \mathrm{kA}$ have been observed. Figures 8.2-2 and 8.2-3 show this behavior for the OHTE/RFP [14] and the ZT-40M [20] experiments. T'he ZT-40M data are for a range of conditions and show $T_{e}(0) \propto I_{\phi}^{1.2}$ and $n_{e} T_{e}(0) \propto I_{\phi}^{2}$ (i.c., approximately constant $\beta_{\beta}$ if $T_{e}(0) / T_{e}$ is constant and $\left.T_{i} \simeq T_{\varepsilon}\right)$. In other experiments on $\mathrm{Z}^{\prime} \mathrm{T}^{\prime}-40 \mathrm{M}$ with current flattop operation and longer pulses, it was found that $T_{e}(0) \propto I_{\phi}^{0.7}$, but in these conditions $n \propto l_{\phi}^{1.3}$, again resulting in $n_{e} T_{e}(0) \propto l_{\phi}^{2}$ and a constant-beta scaling.

More recent results from HBTX1A [12] and ZT-40M [21] suggest that the temperalurecurrent scaling might be better described by postulating a constant beta, with a slope determined by $I_{\phi} / N$. Evidence from a number of experiments indicates that the maximum value of $\beta_{\theta}$ varies relatively little over a range of conditions and from one thachine to another. Some variation of $\beta_{\theta}$ with $I_{\phi} / N$ has been reported, with $\beta_{\theta}$ increasing somewhat as $I_{\phi} / N$ is reduced. It should also be noted that the range over which favorable scaling is obtained appears to be extended by improved wall-conditioning methods and by reduction in field erross [13].

\subsubsection{Confinement scaling}

Only linited confinement-scaling information for RFPs is available, and the variation of the main parameters, particularly geometry, is limited. Furthermore, all RFPs to date are ondy ofmusical]y beated. 'The first recent estimates of energy confinement in RFPs lave been made possible by the relatively stationary conditions achieved in flat-top current discharges. With the global energy confinement time, $r_{E}$, defined as plasma energy divided by the ohmic power and defining an effective global diffusivity as $\chi_{E}=(3 / 16) r_{p}^{2} / \tau_{E}$, it follows that in general $\chi_{E}=\eta \beta_{\theta} / \mu_{0}$. Depending on assumptions made with respect to the constancy of $\beta_{\theta}, j_{\phi} / n, Z_{e f f}$, profiles, etc., and the scaling of $\eta$ with tenperature 

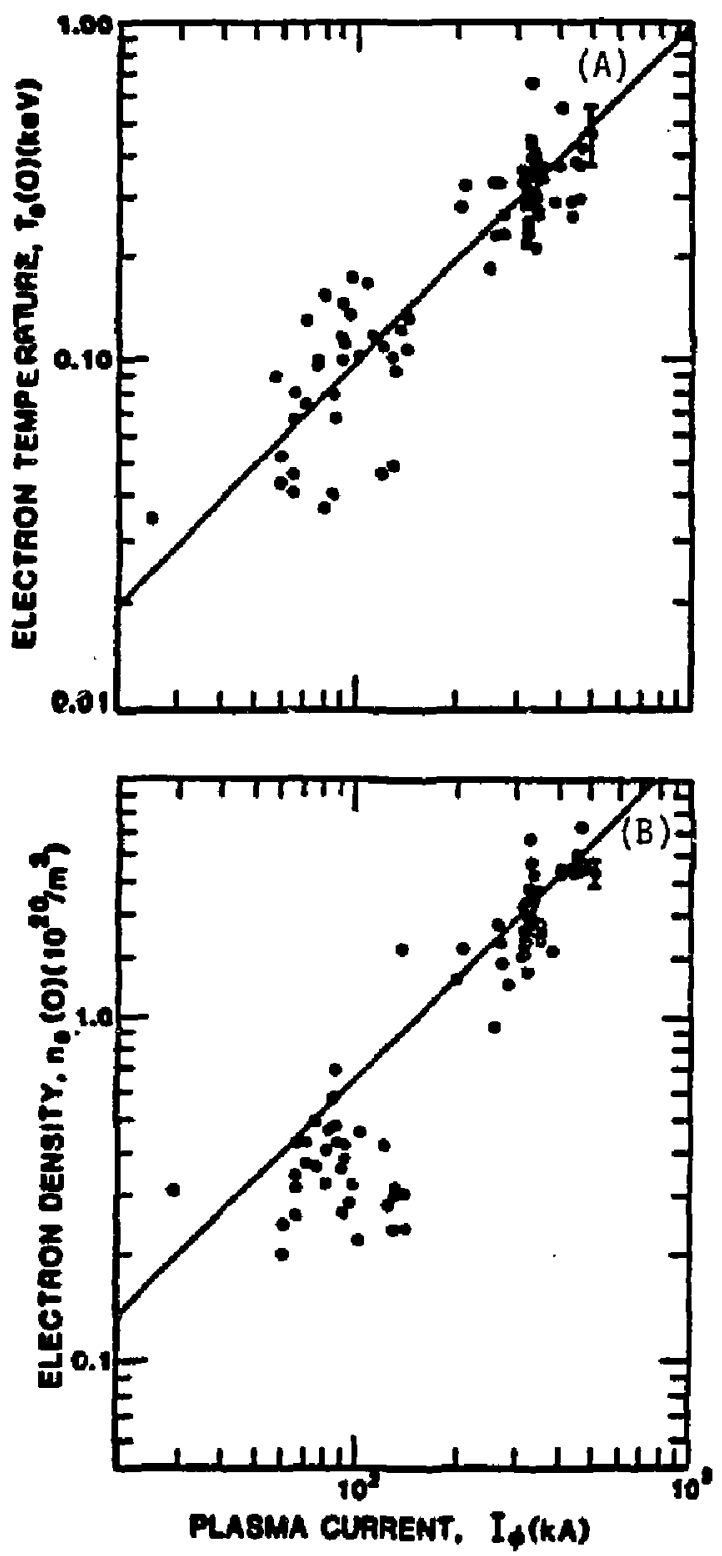

Figure 8.2-2. Variation of central electron tenperature (A) and central electron density (B) with the plasma current for the OHTE/RFP experinent [14]. 

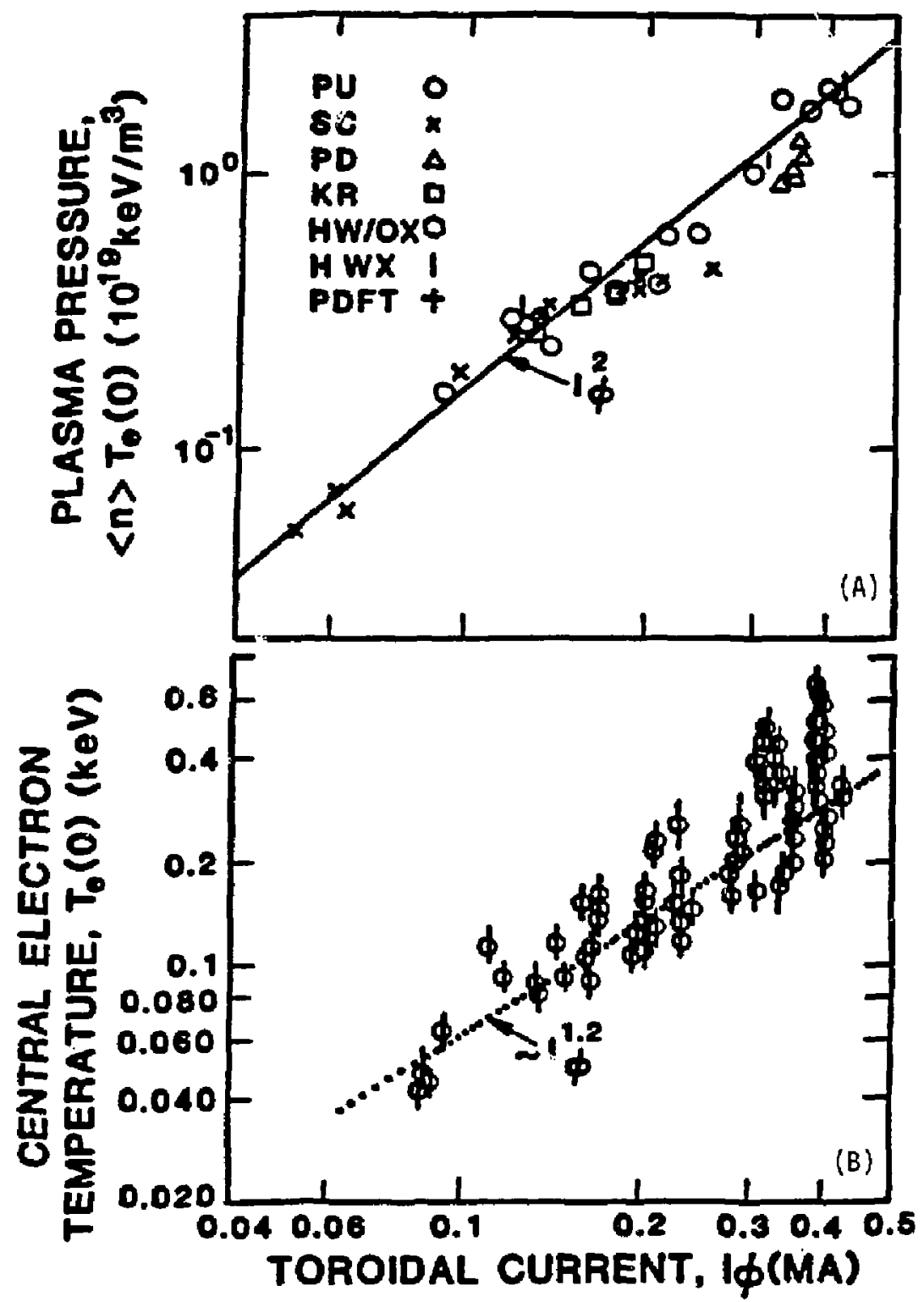

Figure 8.2-3. Variation of the product of central electron temperature with the average electron density (A) and the central electron lemperature (B) with the plasma current for the ZT-40M experiment [20]. 
or current, a range of scaling of $\chi_{s}$ wit.l, current, beta, and geonetry can be derived. Assuming classical scaling for $\eta$, the definitions of $\beta_{\theta}$ and $x_{E}$ result in

$$
1 / X_{E} \propto\left(j_{\phi} / \pi\right)^{3 / 2} \beta_{\theta}^{5 / 2} I_{\phi}^{3 / 2} / Z_{e f f}
$$

which for coustant $\beta_{\rho}$ and $j_{\phi} / n$ predicts $1 / \gamma_{s} \propto T_{\phi}^{3 / 2} / Z_{\text {eff }}$. The inverse global diffusivity (colrected for radiative losses) is pistted in Figure 8.2-4 for a number of RFP experiments. Also shown on Figure 8.2-5 is the dependence of $n \tau_{E}$ on $I_{\phi}$ for ZT-40M [20], with the assumptions of constant $\beta_{\theta}$ and $j_{\phi} / n$ predicting $n \tau_{E} \propto \beta_{\theta}^{2} I_{\phi}^{5 / 2}$.

Figures 8.2-4 and 8.2-5 represents the early RFP data base, an extension of which has been summarized in Section 2.3.5. As noted in that section, even more recent extensions of this clata hase indicate that the experinenta: beta (hased on average density, but central electron temperature) nay ise clecreasing somewhat with increasing curent. This apparest decrease could be attributalble to flattening temperature profiles or $T_{i}>T_{e}$ in regions where $I_{\phi} / N$ is increasing. Furthermore, $\tau_{\varepsilon}$ for a given clevice (again measured using average density and central electron temperat nle) may be increasing with current less rapidly than predicted by the simplified scaling relationships for $\tau_{E}$ or $x_{E}$ given aloove. These recent extensions of the RPP scaling clata base generaily represent increases in statistical breadth, with an emphasis placed on the lower-current $(\sim 50-70 \mathrm{kA})$ regime. Increases in $Z_{\text {ef } f}$ and broadening of temperature profiles can explain these more recent. projections. A clear resolution of these issues, however, must await higher-curcent experiments $\left(-0.5 \mathrm{MA}\right.$; that use improved $Z_{\text {eff }}$ and profile diagnostics as well as stricter edge-plasma and density control. Until these issues are resolved by experiments presently being designed and built, an : anpirical scaling of the kind snggested above $\left(\tau_{E} \propto I_{\phi}^{t} r_{p}^{2}\right)$ is parametrically applied to quantitatively exjress the main reactor requirements. These issues are addressed in more detail in Section 2.3 .

\subsubsection{Highly 1.adiative RFP plasısa}

The scaling of plasina pressure with current observenl in Figures 8.2-2 and 8.2-3 singgests a beta limit wherein intrinsic transport would adjust through changing MHD activity if other loss channels (c.g., radiation) becone available. Inder this hypothesis, as the fraction of the total energy luss carried through the racliation clanuel, $f_{R A D}^{c}=P_{R A D} / P_{O H M}$, is increased, the non-radiative (i.c., intrinsic) confinement time, $\tau_{E}^{N R}$, is expected to jucrease according to $\tau_{\mathrm{E}} /\left(1-f_{R A D}^{c}\right)$.

A preliminary test of this hypothesis that energy loss cliannels can be adjusted to maintain a constant plasma energy content was performed on ZT-40M by adding trace 


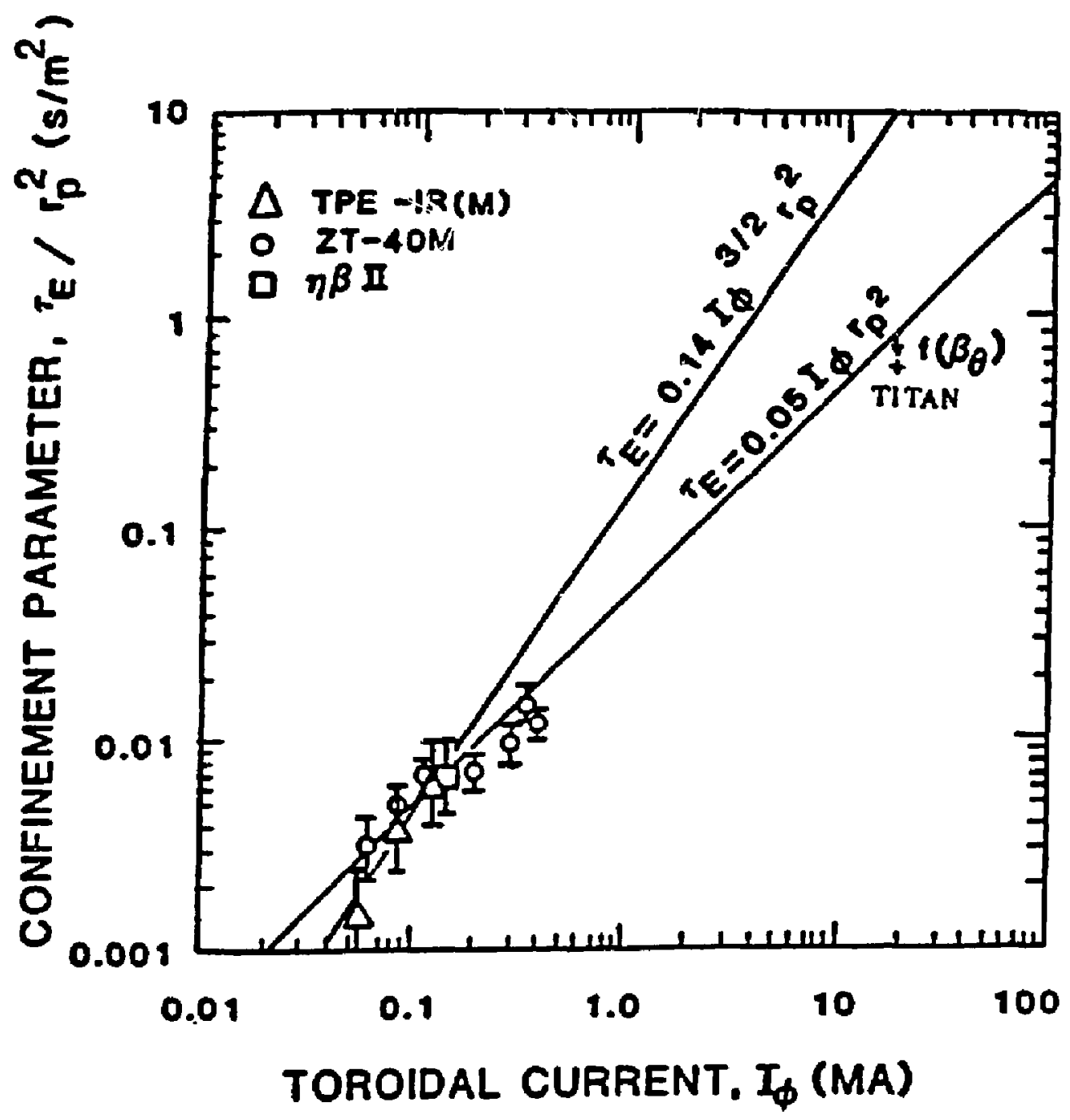

Figure 8.2-4. Variation of the confinement parameter $\left(1 / \lambda_{E} \propto \tau_{E} / r_{p}^{2}\right)$ with plasma current with data from several experinents $[20]$. 


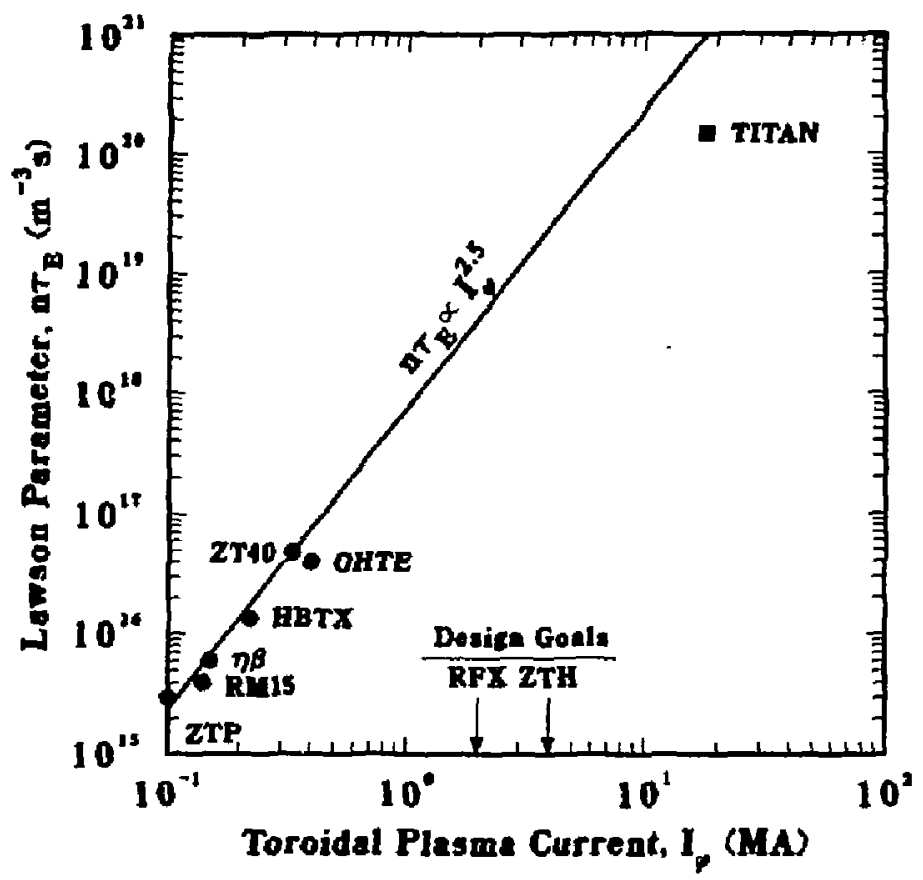

Figure 8.2-5. Variation of the Lawson parameter, $n \tau_{s}$, with the plasma current with data from several experiments [20].

quantities of krypton impurities to the plasma [22]. The radiation loss, $P_{R A D}$, increased, the beta remained selatively constant, and the ohmic input power, $P_{O H M}$, increased only slightly. The data for the krypton-injection experinent shown on Figure 8.2-6 are in quantitative agreement with this prediction and offer the potential for the beta-limited RFP to radiate (more-or-less uniformly) a large fraction of its energy without significantly reducing the global energy confinement time.

This potential for highly radiative plasmas is important for the compact, high-MPD reactor embodiment in order to control $n$ nd distribute the expected high heat fluxes unifornly over the first wall. Generally, the highly radiative plasma regime without degreing overall confinement is not available to the tokamak plasma; radiation loss is added to intrinsic losses and degrades the overall confinement. 


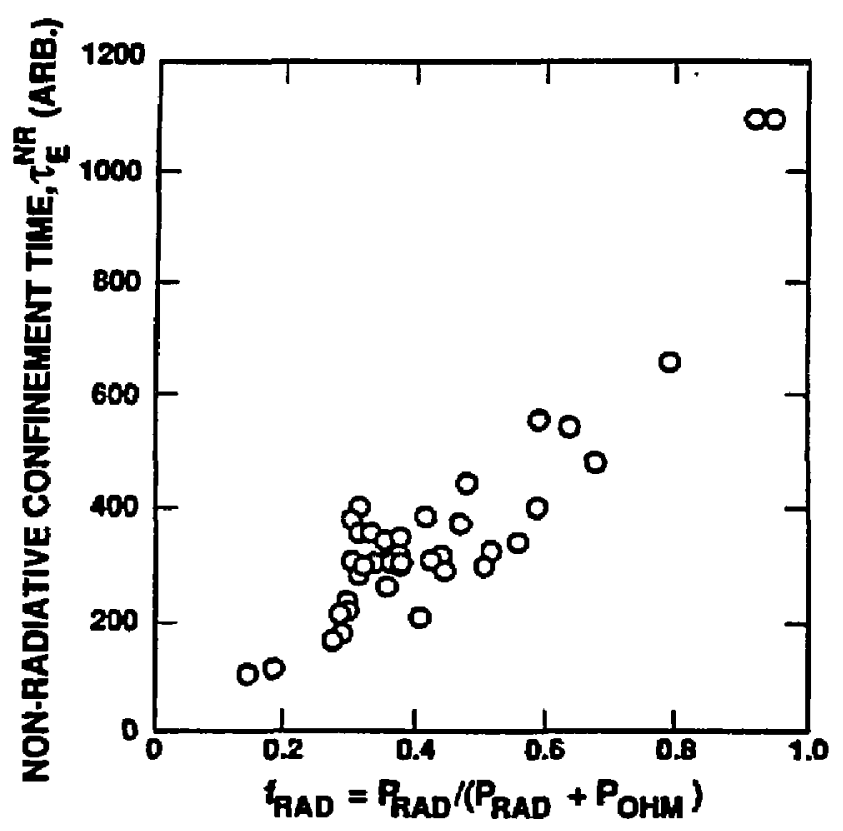

Figure 8.2-6. Scaling of the non-radiative energy confinement time with the fractional radiative power loss in the ZT-40M experiment [22].

\subsubsection{Reactor Implications}

The TITAN parametric systems model (Section 3) was used to investigate the impact of the RFP confinement (e.g., $\beta_{\theta}, \tau_{E}, f_{R A D}^{c}$ ) on the reactor cost. The COE increases as $\beta_{\theta}$ is decreased, as is shown in Figure 8.2-7, which is caused primarily by the need to establish and drive more plasma current, as is reflected in increased OFCD power consumption and increased coil mass (reduced MPD). Values of $\beta_{\theta}$ much below $\sim 0.1$ result in substantial increases in $\mathrm{COE}$.

Using the experimental scaling of the confinement time, $\tau_{e} \propto I_{\phi}^{\nu} r_{p}^{2} f\left(\beta_{\theta}\right)$, the impact of the plasma current scaling exponent, $\nu$, on achieving the minimum-COE TITAN-I design is illustrated in Figure 8.2-8. For each respective constant $\nu$ curve, the condition $\tau_{E}(\mathrm{ECON}) \leq \tau_{E}(\mathrm{PHYS}) \equiv 2\left(1 / \tau_{E e}+1 / \tau_{E i}\right)^{-1}$ with $\tau_{E i} \simeq 4 \tau_{E_{e}}$ is met to the right (i.e., higher $r_{p}$ ). The accessibility to minimum-COE designs depends on the value of $v$. In addition, for $\nu$ values much below $\sim 0.8$, the demands on the $\mathrm{OH}$-coil system during the ohmic-heating transient to ignition and burn can be serious. Also, it should be noted 


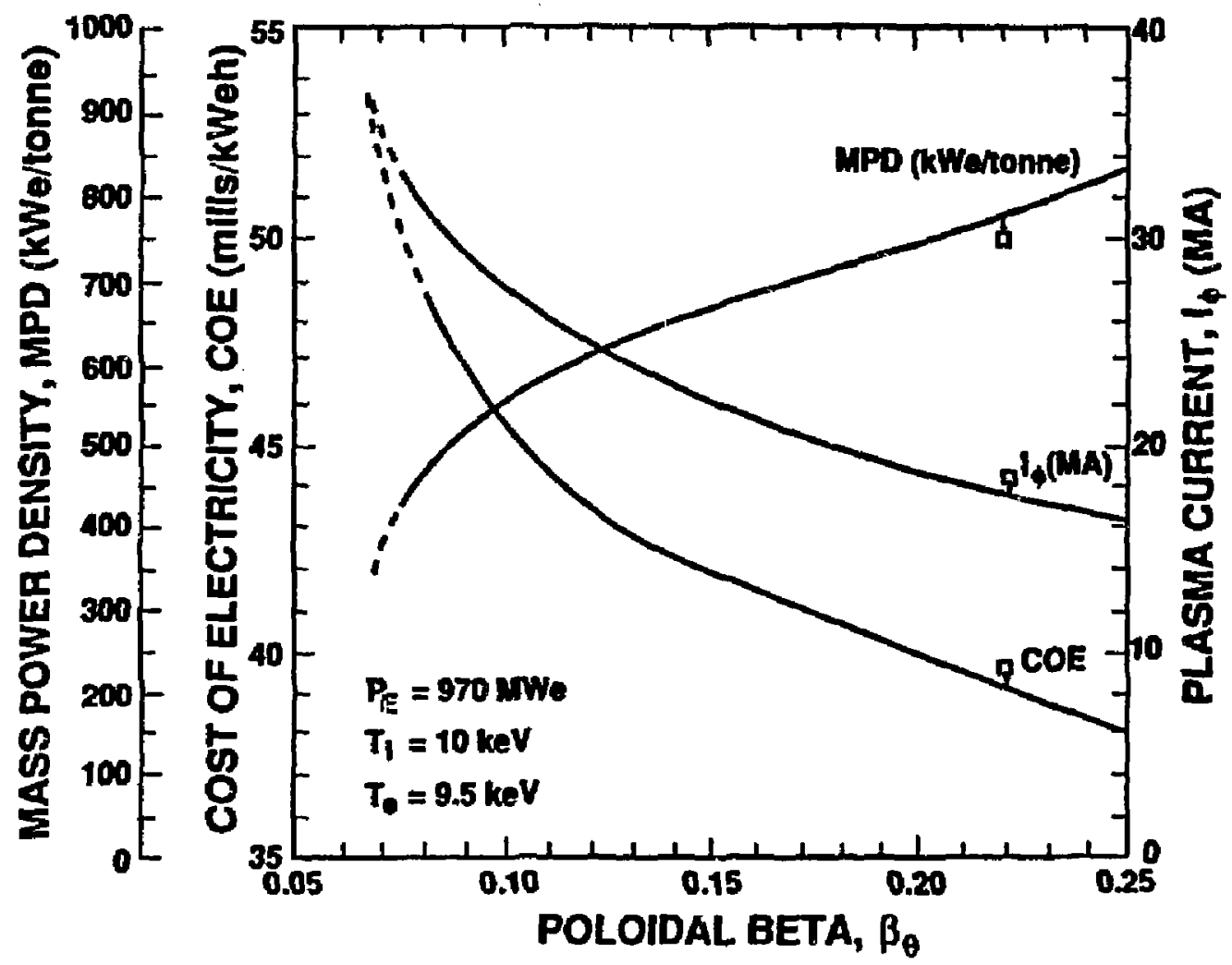

Figure 8.2-7. Dependence of minimum-COE TITAN-I design values on poloidal beta; the near-nunimum-COE TITAN-I reference design values are also shown. 


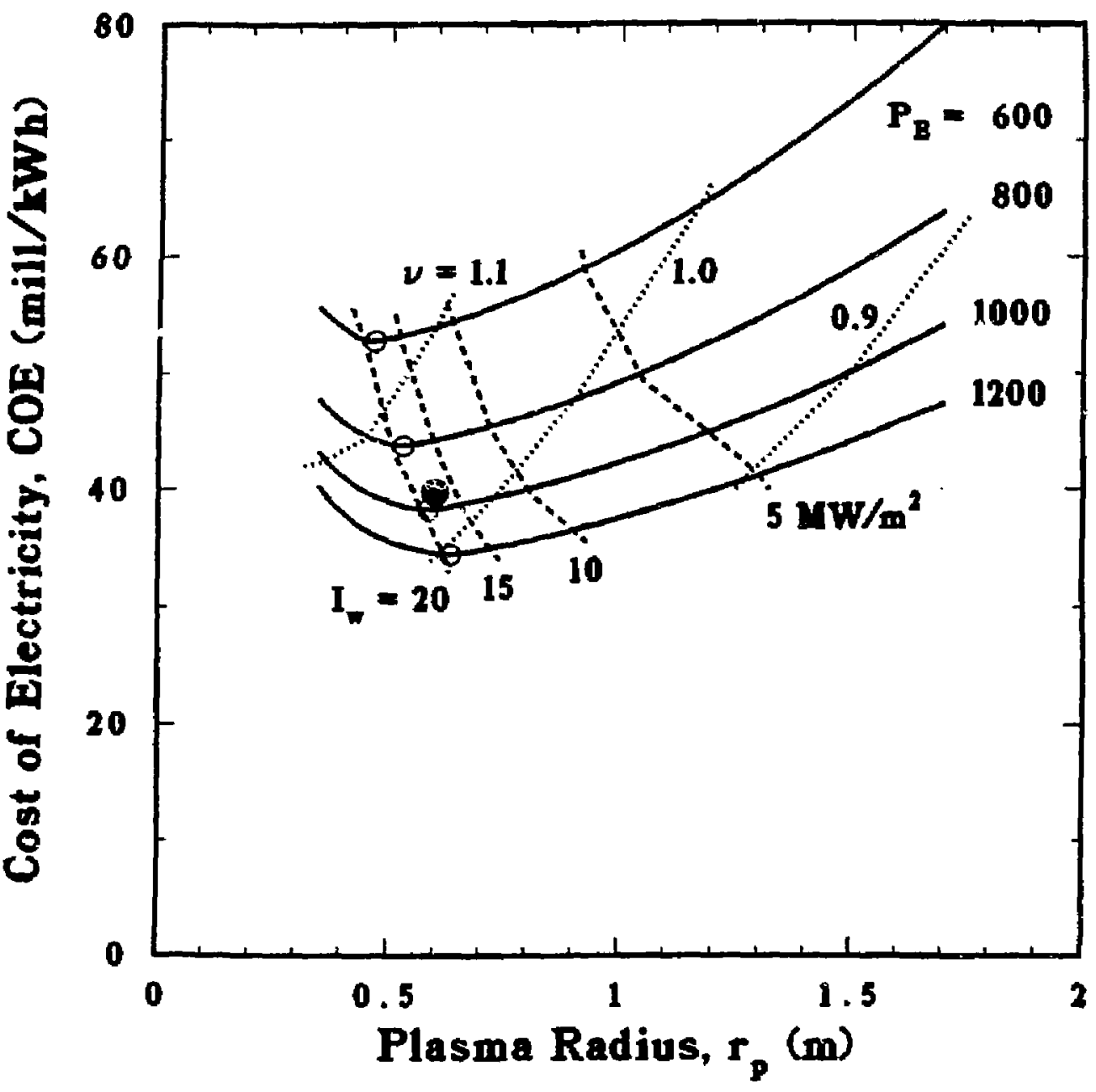

Figure 8.2-8. Parametric trade-off of TITAN-I cost with level of confinement required $\left(\tau_{E_{e}}=C_{\nu} I_{\alpha^{2}}^{2},{ }_{p}^{2} f\left(\beta_{\theta}\right), \tau_{E_{i}}=4 \tau_{E_{e}}\right)$, expressed as the magnitude of exponent $\nu$ required for $\tau_{E}(E C O N) \leq \tau_{E}($ PHYS $)=2\left(1 / \tau_{E_{7}}+1 / \tau_{E}\right)^{-1}$. Accessible design points are to the right of curves of constant $\nu$. Minimum-COE design points with $\tau_{E}(O P T)=\tau_{E}(E C O N)$ for fixed net power output $P_{E}$ are indicated by open circles. The near-minimum-COE TITAN-I ieforence design at $P_{E}=970 \mathrm{MWe}$ and $I_{w}=18.1 \mathrm{MW} / \mathrm{m}^{2}$ is denoted by a filled circle. 
that the flexibility of operation of TITAN resctors at lower than nominal power (for load following or checkout) requires better intrinsic plasma confinemer: (i.e., higher $\nu$ ).

It stould be re-emphasized that the scaling given on Figures $8.2-2$ and $8.2-3, r i$ hough reproducible and representative of a statistically large number of discharges, does not occur for all machine conditions. Operating conditions have been obaerved where $T_{e}(0)$ scales leas than linearly with plasma current, and the product $n_{e} T_{e}(0)$ scales less strongly than the square of the plasme current (i.e., beta under certain conditions appear to de$r$. ase somewhat with increasing current). These operating conditions may result from inadequate control of $I_{\phi} / N$ (e.g., pump out) and field errors, leading to increased $Z_{\text {eff }}$ (a parameter not yet measured experimentally in RFPs), cooling of electrons and heating of ions caused by current-driven instabilities, changing edge-plasma conditions and plasma profiles, and other unresolved effects. Hence, a range of plasma current exponents in the scaling of $T_{*}(0), n_{*}, T_{*}(0) n_{e}$, and $\beta_{0}$ exists, ultimately leading to the runge of $\tau_{*}$ (PHYS) scalings suggested in Figure 8.2-4 as $\nu$ is varied. Better control of experimental discharges carried out over a wider range of quasi-stationary, flat-top-current conditions is needed and represents a main goal of the generation of RFP experiments presently under construction [16].

\subsection{CURRENT DRIVE}

At full plasma cirrent of $18 \mathrm{MW} / \mathrm{m}^{2}$, the energy stored in the reference TITAN plesma includes $W_{M} \sim 5$ GJ of magnetic energy and 0.1 GJ of kinetic energy. The magnetic stored energies internal to the plasma are $0.3 \mathrm{GJ}$ in the toroidal field and $0.4 \mathrm{GJ}$ in the poloidal field. The magnetic energies outside of the plasma are $<2 \mathrm{MJ}$ in the toroidal field and $4 \mathrm{GJ}$ in the poloidal field for zero OH-coils current 6 (6 GJ for OH-coils in full forward-bias current). Since, the toroidal maguetic stored energy internal to the plasma is supplied by the poloidal-field circuit during the start-up, the reference TITAN design requires a poloidal flux of $L_{p} I_{\phi} \simeq 250 \mathrm{~Wb}$ to achieve full plesma cursent. Because of the large plasma resistance in the TITAN designs, an inductively pulsed burn would be sustained for a pulse length of the order of $L_{p} / R_{p} \simeq 200$ to $400 \mathrm{~s}$. Therefore, steady-state operation is essential considering issues such as the total power balance, thermal cyclic fatigue in a high-power-density environment, as well as the costs of c-i-site energy storage (frequent grid-assisted start-up seems unlikely) and thermal storege. An inductively pulsed RFP reactor is a possibility [23]. The parameters of such a reactor, however, should be optimized to minimize the plasma resistance, which results in larger plasmas, lower power density, and possibly the use of superconducting coils throughout the FPC. 


\subsubsection{Reactor Condifions}

A number of current-drive options for the RFP have been considered (Section 8.2). For the TITAN reactors, helicity injection by the oscillating-fielcl current drive (OPCD) las been selected as the mean to sustain the toroidal plasma current.

The major parameters of TITAN-I OFCD system are presented in Table 8.3-1. Al1hough oscillations in the plasura current and toroidal flux are small $\left(\delta I_{\phi} / I_{\phi}=0.02\right.$ and $\left.\delta \phi / \phi_{0}=0.035\right)$, the reactive power flow across the plasma surface and at the OFCD power supply terninals can be large, requiring careful and poswibly expensive energy management. Figure 8.3-1 gives the variation of the main power flows for the TJTAN-I OFCID system which uses the main confining magnets for the OFC:D.

A circuit nodel was developed that simulates the major elements associated with OFCD in order to deternine the injected and/or dissipated powers (Section 7 ). The model was used to quantify the need for toroidal and poloidal gaps or insulating breaks in structures such as the first wall, which will have currents induced by the OFCD. It. is concluded that these breaks are needed to achieve acceptable current-drive efficiencies $(\sim 0.35 \mathrm{~A} / \mathrm{W})$. Detailed analysis of the TITAN-1 and TITAN-1I designs revealed a jreference for the following: (1) series winding of all OFCD coils; (2) the positioning of these coils as close to the plasma as possible; and (3) in the case of coil sets with small anplitucle oscillations about. large average currents, the splitting of the coil set into a set devoted to the oscillation and another set to produce the mean current. Future work should focus on effects of field errors introduced by gaps during current oscillations, a better modeling of lielicity balance and profile clianges, and the generation of a bet. ter engineering understanding of the interaction of the OFCD system with of.'pr major FPC: subsystems $(e, g$. equilibrium control, impurity control, and startup and slutdown systems).

\subsubsection{Pliysics Data Base}

Tests of OFCD on the relatively resistive ZT-40M experiment proved encouraging but inconclusive [24]. The key experimental parameters, as for the reactor study, are the frequency, the F- $\Theta$ oscillation point, and the phase angle between poloidal and toroidal drive voltages ( $\pi / 2$ at the plasma surface giving theoretically the st rongest OFCD effect unter ideal conditions). Tests of OFCD on ZT-40M examined modulations of 180 to 200-kA discharges (40 MVA) and 60 to $70-\mathrm{kA}$ discharges (7 MVA). T'he lat.ter lower power tests were pursued in an effori to minimize nodulation-driven plasma/wall interactions. 
Table 8.3-I.

COMPARISON OF OFCD IN TITAN DESIGNS

\begin{tabular}{|c|c|c|}
\hline & TITAN-I & TITAN-II \\
\hline A verage plasma current, $I_{\phi}$ (MA) & 17.82 & 17.82 \\
\hline Drive frequency, $f(\mathrm{~Hz}\}$ & 25. & 25. \\
\hline Toroidal-flux swing, $\delta \phi / \phi_{\mathrm{o}}$ & 0.035 & 0.035 \\
\hline$\Theta$ variation & $1.499-1.616$ & $1.499-1.616$ \\
\hline$F$ variation & $-0.032--0.173$ & $-0.032--0.173$ \\
\hline \multicolumn{3}{|l|}{ Toroidal (poloidal) circuit power (MW): } \\
\hline Plasma Poynting power, $P_{P}^{*}$ & $3959.99(247.31)$ & $3959.99(247.31)$ \\
\hline Plasma dissipation, $P_{\Omega}$ & $28.55(0 . \quad)$ & $28.55(0)$. \\
\hline First-wall dissipation, $P_{F W}$ & $0.00(0.01)$ & $0.00(0.01)$ \\
\hline Blanket dissipation, $P_{B}$ & $1.04(0)$. & $0.01(0.17)$ \\
\hline \multicolumn{3}{|l|}{ Terminal reactive power, $P_{i}^{*}(\mathrm{MW})$ : } \\
\hline TF coils & 503.88 & 1113.77 \\
\hline OH coils & 74.92 & 101.99 \\
\hline EF coils & $\sim 0$ & $\sim 0$ \\
\hline Trim coils & 113.44 & $14 i, 16$ \\
\hline \multicolumn{3}{|l|}{ Coil dissipation, $P_{H}(M W)$ : } \\
\hline TF coils & 47.38 & 11.44 \\
\hline OH coils & 0.13 & 0.17 \\
\hline EF coils & $\sim 0$ & $\sim 0$ \\
\hline Trim coils & 1.95 & 2.49 \\
\hline \multicolumn{3}{|l|}{ Real (lost) termunal power, $P_{T}(\mathrm{MW})$ : } \\
\hline TF coils & 74.00 & 38.23 \\
\hline $\mathrm{OH}$ coils & 1.62 & 1.15 \\
\hline EF coils & $\sim 0$ & $\sim 0$ \\
\hline Trim coils & 3.44 & 3.46 \\
\hline TF-coil DC power, $P S S$ (MW) & 29.15 & 9.34 \\
\hline Power-supply rissjpation, $P_{P S}(M W\}^{(a)}$ & 6.92 & 15.34 \\
\hline Total dissipation, $P_{D}(\mathrm{MW})$ & 85.93 & 58.19 \\
\hline Current-drive power, $P_{C D}(\mathrm{MW})$ & 56.83 & 48.85 \\
\hline Efficiency, $I_{\phi} / P_{C D}(\mathrm{~A} / W)^{(b)}$ & 0.33 & 0.36 \\
\hline
\end{tabular}

(a) Assuning the power supplies are $99 \%$ efficient $\left(Q_{P S}=100\right)$.

(b) Based on total power consumed including clriver efficiency and transunission losses. 

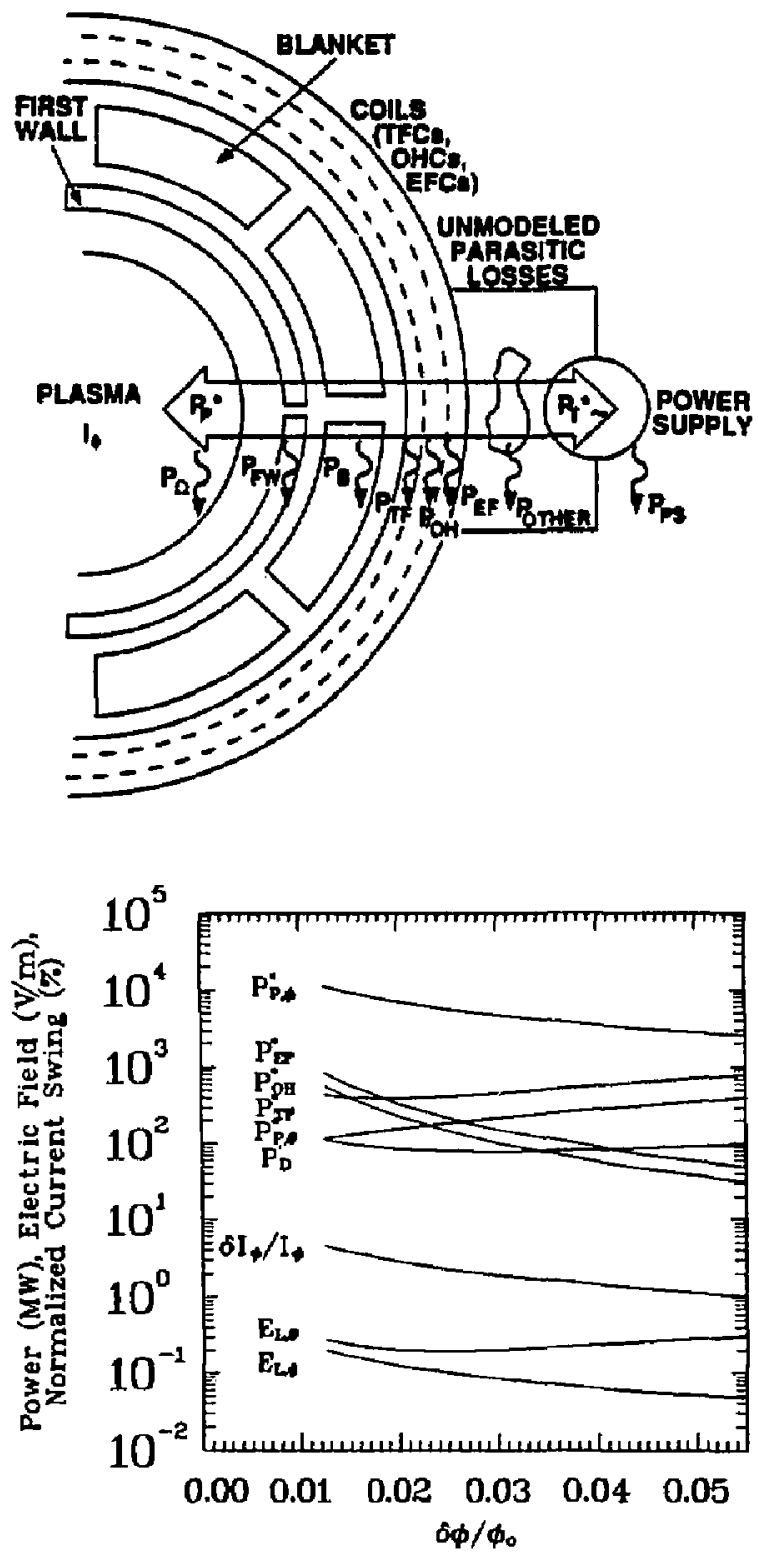

Figure 8.3-1. Variation of the main power flows, current swings, and driver voltages as a function of toroidal flux swing for the TITAN-I OFCD system. 
Figure 8.3-2 gives the time dependence of plasma current, reversed toroidal ficld $\left(B_{\phi R}\right)$, average toroidal field ( $\mathrm{Aux}$ ), and reactive powers on the ZT-40M experiments for (1) no drive, (2) drive (phase shift of $\pi / 2$ ), and (3) anti-drive (phase shif of $-\pi / 2$ ). As predicted by theory, $I_{\phi}$ is reduced in the anti-drive mode, but failure to drive significant. current in the drive mode possibly resulted from modulation-driven plasma-wall interactions. Attempts to drive current in lower-current discharges requiring less modulation of a coller, higler-resistance plasma also proved inconclusive. In arldition to agreeing with theoretical predictions of the impact of the modulation phase, measured spatial ausd temporal behavior of the mean toroidal field within the plassua during modulations also agree with OFCD theory. Future tests of the OFCD principle must await high-temperature, lower-resistance plasmas that require less modulation of $F_{-} \Theta$, lower frequency, reduced plasma-wall interaction, and ideally use an RFP with a fixed separatrix in order to better control the equilibrium and the plasma/wall interaction.

\subsubsection{Reactor Inplications}

Steady-state operation of the high-power-density TI'TAN-I reactor is mandatory, and some form of current drive will be required. The TITAN plasma is seeded with impmrities and is highly radiative in order to spread the lseat load unjormly over the first wali and to reduce the divertor-plate heat load to manageable levels. Seeting of the plasna with impurities, however, increases the current-drive requirements through increased $Z_{\text {ef } f}$ and plasma current dissipation. Attempls to reduce the plasma ohmic loss and rate of lelicity injection through OFCD by increasing the plasma temperature also requires higher impurity concentrations and $Z_{\text {ef } j}$ to maintain the design value of $f_{R A D}$. It was found that the TITAN OFCD requirements under this $f_{A A D}$ constraint actually increased as the average plasma temperature was increased above the $10-\mathrm{keV}$ design value.

Assuming that the large ( $\sim 4 \mathrm{GW}$ ) Poynting flux to and from the plasma under the OFCD conditions can be efficjently managed and the field errors introduced by gaps in the FPC components during OFCD oscillations can be controlled, the management of 500 to 1,500-MW reactive powers at the TF-coil termijals with high-Q efficiency (Table 8.3-I) at reasonable cost $(10-15 \$ / \mathrm{kVA})$ represents the main engineering concern. The impact of the circuit $Q$-value on $\mathrm{COE}$ and other reactor parameters is illustrated in Figure 8.3-3. It can be seen that $Q$-values much below $\sim 50$ will begin to have economic inpact on the TITAN-I design.

Piasna parameters, particularly the $F$ - $\Theta$ oscillating point, will also have serious cost and design impacts. Deeper reversals (i.e., more negative $F$-value) are desirable since 


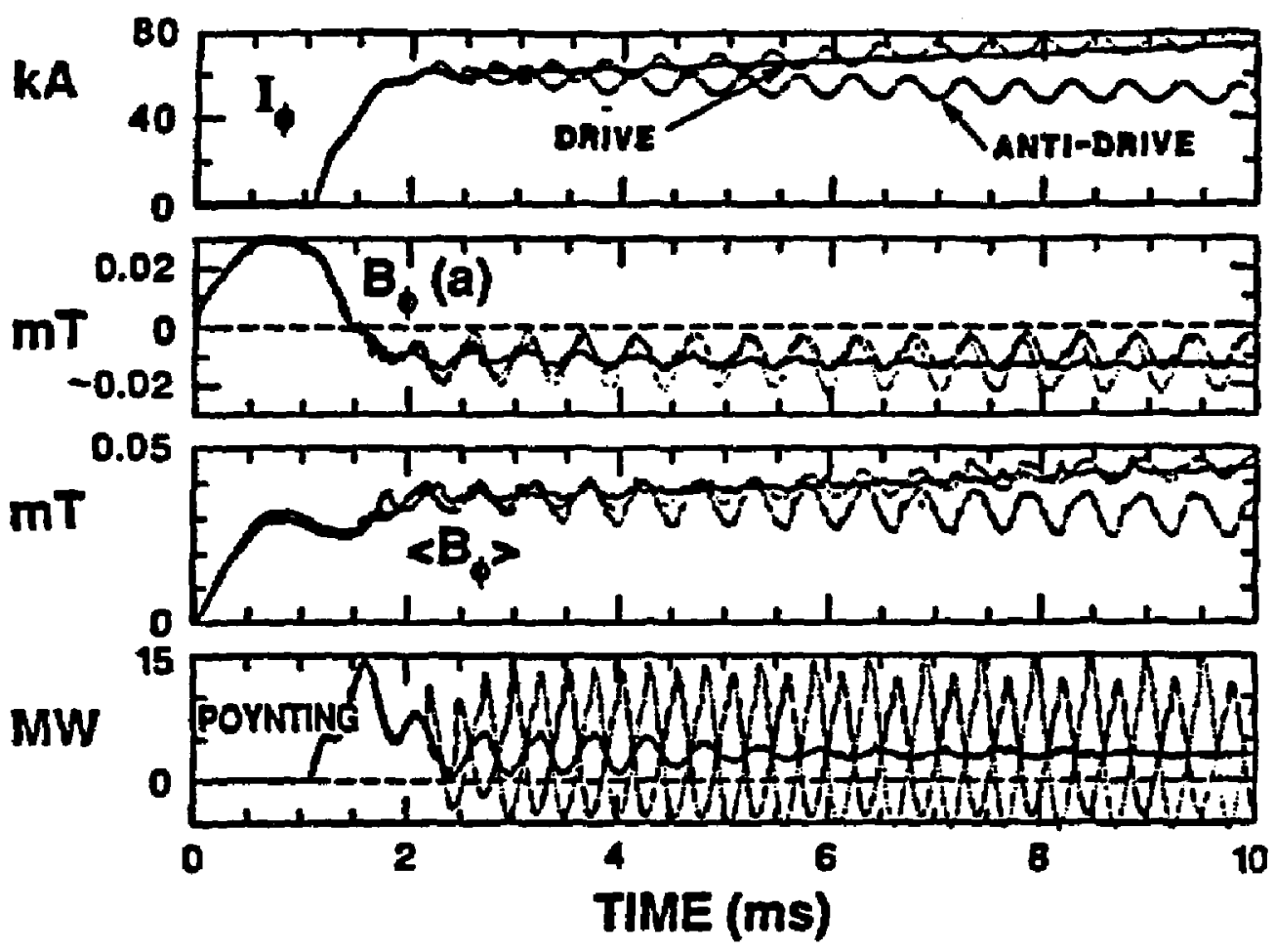

Figure 8.3-2. Low-power OFCD discharge results from Reference [24]. Shown are traces of current, edge toroidal field, toroidal flux, and Poynting vector for a standard discharge, a discharge with the optimal phase between toroidal and poloidal OFCD circuits for driving current, and a discharge with the optimal phase in the OFCD circuit for anti-drive. 

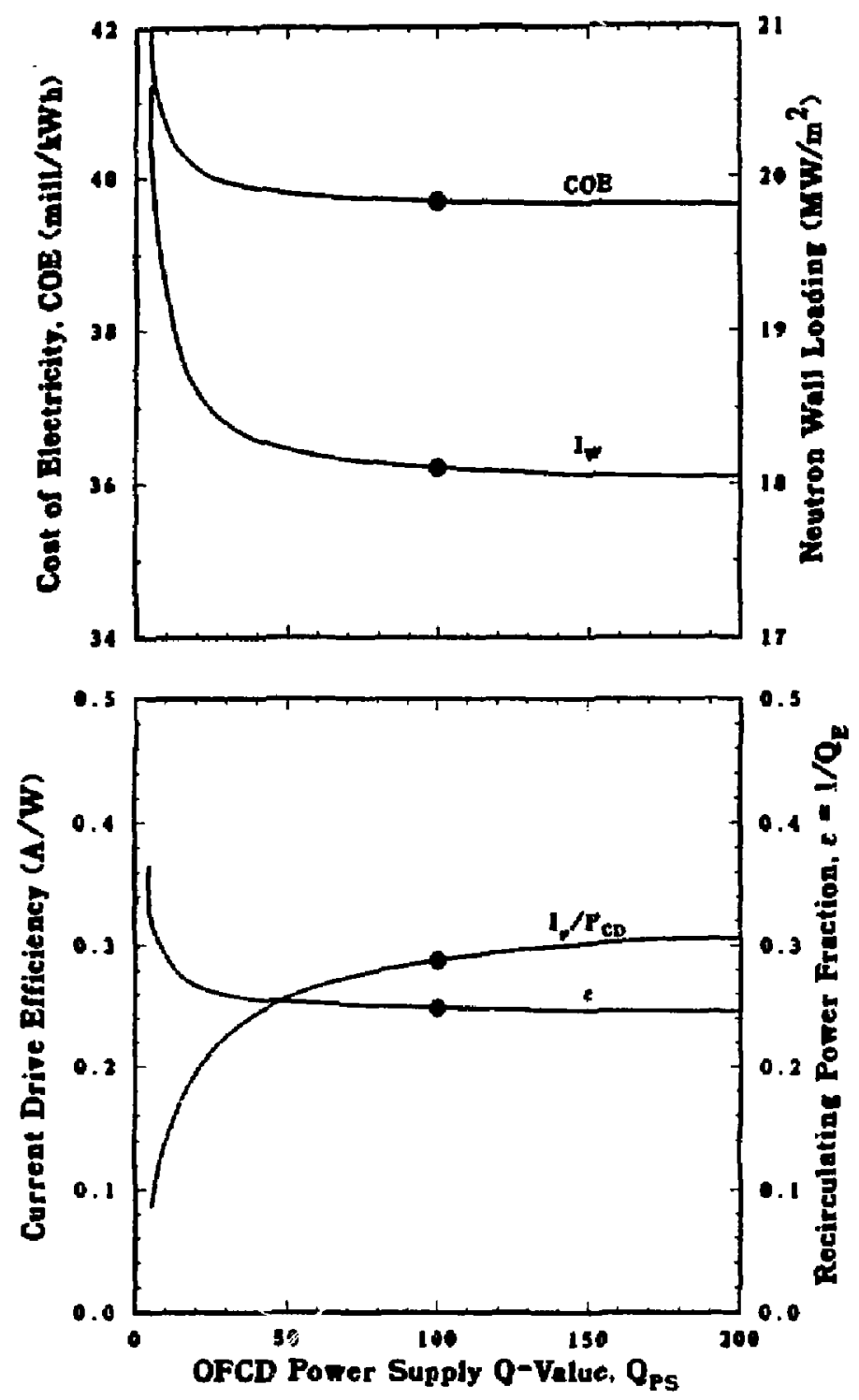

Figure B.3-3. Impact of OFCD power supply Q-value on TITAN-I operating parameters. The TITAN-I reference case is denoted by a filled circle at $Q_{P S}=100$. 
the design reported in Table 8.3-1 oscillates close to loss of reversal. Deeper reversals, however, will increase an already large TF-coil circuit reactive power, as well as increasing steady-state losses in the TF and divertor coils. Figure 8.3-4 illustrates the impact of the $F-\Theta$ operating point on the overal] TITAN-l design point.

Lastly, the impact of OFCD frequency and modulation amplitude on the plasma resistance and overall confinement represents areas of future conceru. Other means to deliver magnet helicity to RFPs also warrant future examination (c.g., DC helicity jnjection perhaps using the divertor plates as electrodes [25] or RF-driven poloidal current in the lower-density edge plasma).

\subsection{IMPURITY CONTROL}

The design of in-vessel components (divertor plate, limiter, first wall) is a critical issue for all fusion reactors. The key problem is to remove the steady-state plasma power (alpha-particle and ohmic) while maintaining acceptable heat fluxes and erosion rates on all components. In the scoping phase of the TITAN study [1], a simulation of the edge plasma without impurity radiation indicated plasma temperatures in the range of 50 to $100 \mathrm{eV}$ at the first wall and very high heat fluxes on the divertor target. As a result the TITAN reactors operate with a highly radiative plasma with balanced radiation from core, edge: and divertor plasmas. The plasma is deliberately doped with a trace annount of high- $Z$ xenon impurities to create strong radiative cooling and spreading the heat load uniformly over the largest possible area (first wall). This higl-radiation regime of operation, which appears to be an essential ingredient for a high-power-density reactor, may be more easily achieved in RFPs than in tokamaks because experiment al evidence suggests that RFPs operate with a soft- $\beta$ limit. (Section 8.3 ).

\subsubsection{Reactor Conditions}

The use of either poloidal pump limiters or magnetic divertors in the context of a ligh-power-density RFP requires that the majority of the plasma self-heating power be radiated uniformly to the first wall and other in-vessel components. Further analysis of the array of 24 poloidal limiter blades suggested in early studies [5] indicated possible problems with gross erosion of those surfaces in contact with the hot edge plasma. The TITAN designs, therefore, are based on toroidal-field divertors to minimize the perturbation to the global magnetic configuration (toroital field is the ninority field in RFPs) and to minimize the coil currents and inagnet.ic forces [26]. 

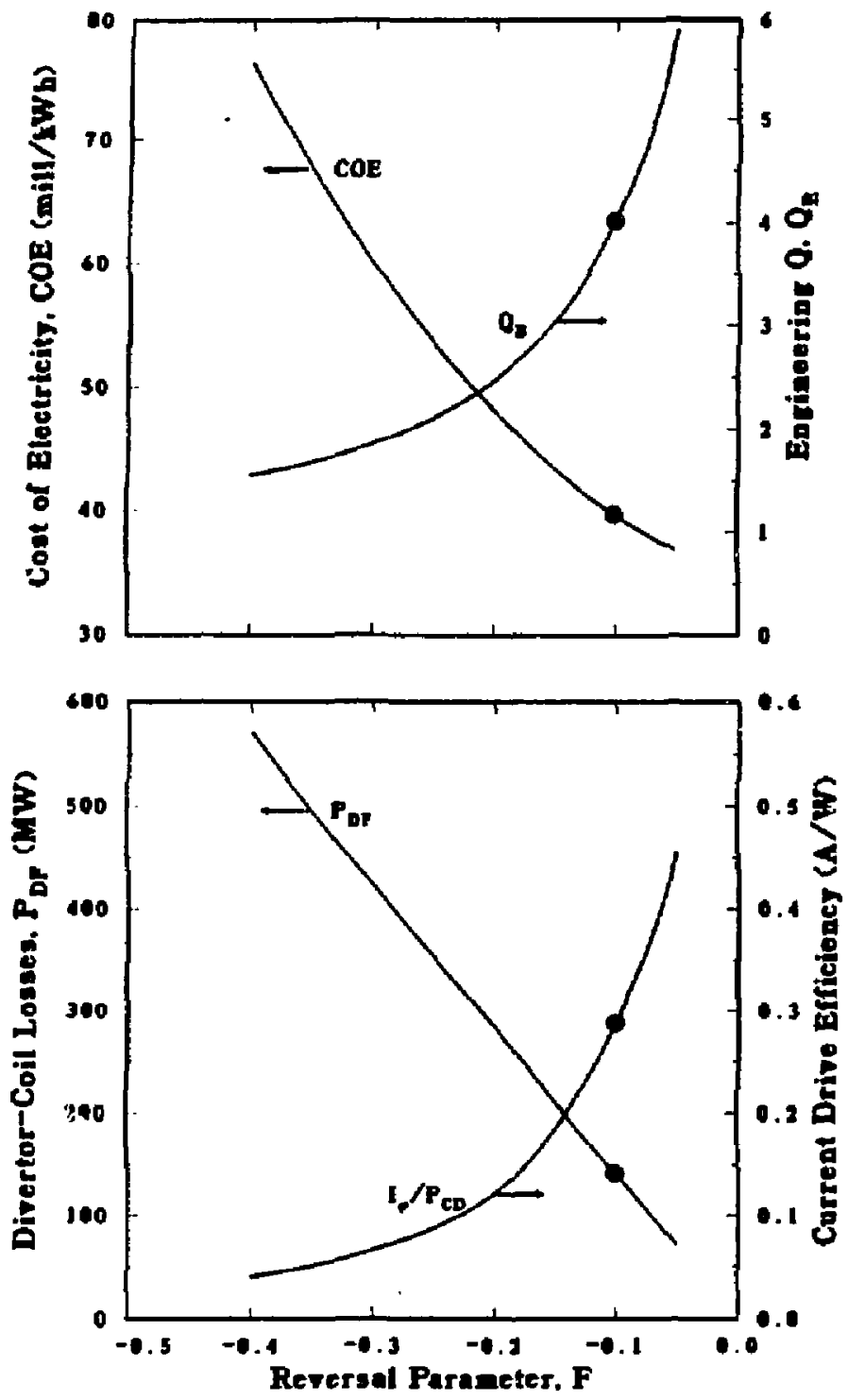

Figure 8.3-4. Impact of the reversal parameter on the overall TITAN-I design and cost. The TITAN-I reference design at $F=-0.1$ is denoted by a filled circle. 
The final TITAN divertor designs represent the result of extensive iterations between erlge-plasma analysis, magnetic design, thermal-hydraulic and structural analyses, and neutronics. The TITAN divertors uses an "open" configuration, in which the clivertor target is located close to the null point and faces the plasma, rather than in a separate chamber (Figure 8.4-1). This positioning takes advantage of the increased flux expansion in this region which tends to recluce the heat loading on the divertor plate. The high plasma density in front of the divertor plate eusures that the neutral particles emitted from the surface have a short mean free path and only a negligible fraction of these neutral particles enter the plasma (Section 5.5).

The final magnetic design includes three divertor modules, located $120^{\circ}$ apart in t.oroidal direction. The local field ripple produced by these poloidally symmetric toroidalfielal divertors anounts to $\Delta B_{R} / B_{\theta}=0.01-0.02$ with a conmection length in the range to to $75 \mathrm{~m}$, flux expansion factors in the range 2 to 4 , and scrape-off-layer thicknesses of $\sim 60 \mathrm{~mm}$. Table 8.4-I compares the impact of the divertor-induced ripple on the magnetic-island width, $\Delta r$, with the case where divertors are not used. The magneticisiand widtlıs is estimated from

$$
\frac{\Delta r}{r_{r}}=\left[16 \frac{\Delta B_{R} / B_{\theta}}{N r_{r}(d q / d r)}\right]^{1 / 2},
$$

where the poloidal field at the plasma surface is $B_{\theta}=5.9 \mathrm{~T}$, the shear at the reversal surface is $r_{T}(d q / d r)_{r_{r}}=-0.165$, and the mode number $N=2 \pi R_{T} / l_{T F}$, with $R_{T}=3.9 \mathrm{n}$ being the major radius and $l_{T F}$ being the toroidal extent of the toroidal-field perturbation.

The radial profile of edge-plasma density and temperature, as predicted by the edgeplasma transport code OUESSA [2T], is given in Figure 8.4-2. The resultant heat flux ols the divertor target from an integrated thermal-hydraulics/divertor-plate positioning code (Section 11) are shown in Figure 8.4-3. For the estimated density and temperature profiles in the scrape-off layer, sputter-erosion rates at the first-wall and divertor-plate surface are estimated to be below $0.1 \mathrm{~mm} / \mathrm{y}$. These low erosion rates for the high-powerdensity RFP are possible because of the high-density (low-temperature) first-wall and divertor-plate conditions.

\subsubsection{Physics Data Base}

Experience operating RFPs with a magnetic separatrix is nonexistent. Althouglı plans exist for shell-less HFP operation with an ortapole-like (poloidal-fielul) separatrix $[16,28]$, this configuration is not relevant to the configuration proposed for the TITAN-I. 
I-NVLIL Ioj uo!

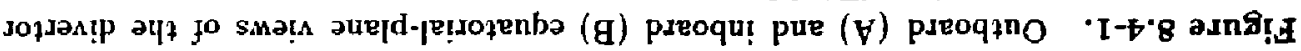
(u) $k$

$\begin{array}{ccccccc}90 & 50 & b^{\circ} 0 & \varepsilon^{\circ} 0 & 20 & 10 & 00\end{array}$

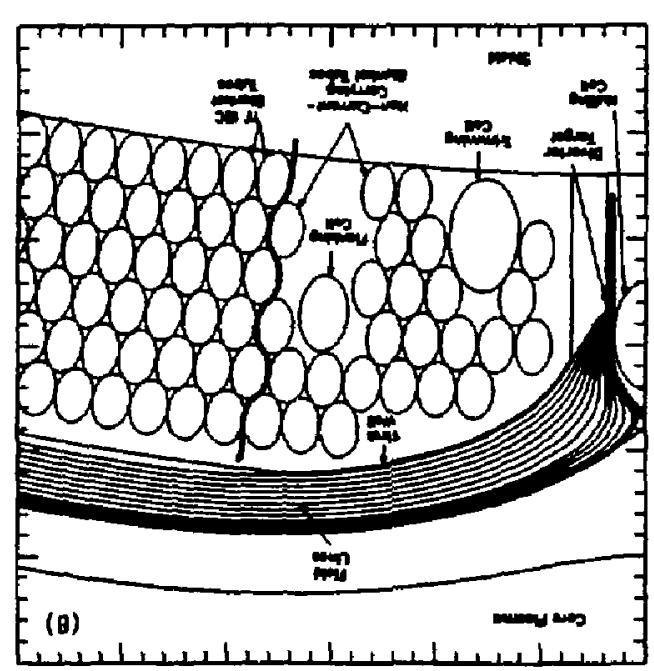

82

$6 Z$

$0 \%$

L8

Ž

$\varepsilon \varepsilon$

t'E

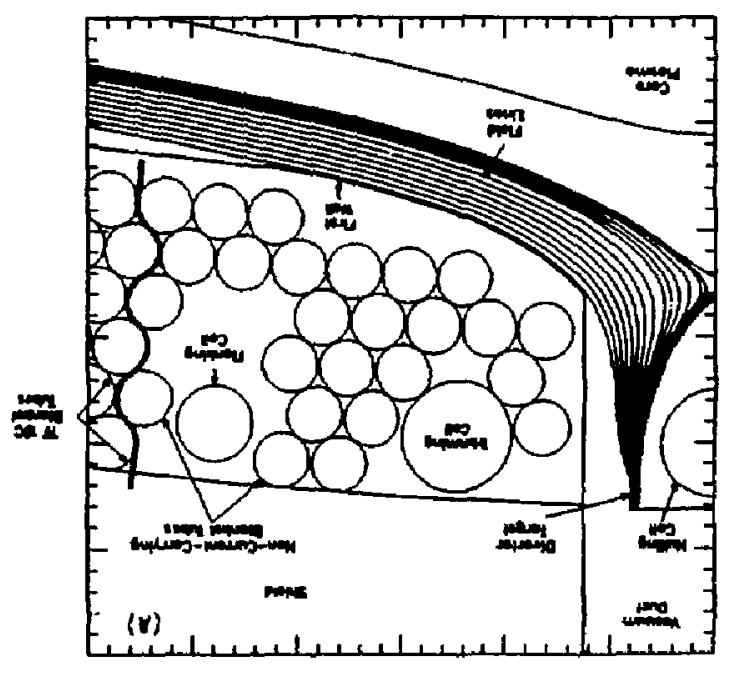

$\forall+t$

$\varsigma^{\prime \prime}$

9

$L \stackrel{\times}{3}$

$8 ' t$

$6 \circ$

0.5 
Table 8.4-I.

\section{COMPARISON OF FIELD RIPPLE WITH AND WITHOUT TOROIDAL-FIELD DIVERTORS}

\begin{tabular}{lccc}
\hline & With Divertors & Withoust Divertors(a) \\
\hline Location & $r_{\mathrm{r}}=0.55 \mathrm{~m}$ & $r_{\mathrm{p}}=0.60 \mathrm{~m}$ & $r_{\mathrm{p}}=0.60 \mathrm{~m}$ \\
Radial field, $\Delta B_{R}(\mathrm{mT})^{(b)}$ & $84 / 76$ & $136 / 103$ & $\sim 10^{-3}(2 / 14)$ \\
Ripple parameter, $\Delta B_{R} / B_{\theta}{ }^{(b)}$ & $0.014 / 0.013$ & $0.023 / 0.017$ & $\sim 0(\sim 0 / 0.002)$ \\
Effective mode number, $N$ & $>300$ & $>300$ & $\sim 1,000(30)$ \\
Magnetic-island width, $\Delta r / r_{r}$ & $\leq 0.067 / 0.065$ & $\leq 0.086 / 0.074$ & $\sim 0(\sim 0 / 0.025)$ \\
\hline
\end{tabular}

(a) Values for TITAN-1 TF-IBC coils.

Values for TITAN-1I copper TF coils are given in parenthesis.

(b) Maximum values at inboard/outboard locations.

\subsubsection{Reactor Implications}

The high-recycling toroidal-field divertor appears to provide the most viable impuritycontrol scheme for the high-power-density RFP reactor. Although the local ripple induced by the divertor is large, the size of the associated nugnetic islands can be held to a smalt fraction of the distance between the reversal and plasma (separatrix) sufaces. The divertor should be positioned as close as is possible to the plasma in order to minimize local perturbation in the magnetic topology and also to minimize coil dissipation.

The impact of the distance between the chivertor coils and the plasma on the power consumption by the divertor coils is shown in Figure 8.4-4 for the TITAN-1, wherein acided shielding could conceivably be required if the insulator racliation lifetimes fall below the estimated design values (Section 10.2). The distance plotted on Figure 8.4-4 is defined by the toroidal-field null and the mulling-coil inner surface. These results were obtained by varying the divertor-coil radial localion and currents for a fixed current. density of $38 \mathrm{MA} / \mathrm{m}^{2}$ and a fixed angle of $3.5^{\circ}$ between the nulling-and fanking-coil current centers. The lower bound on $\Delta s / r_{p}$ in Figure $8.4-4$ is set by the maximum 

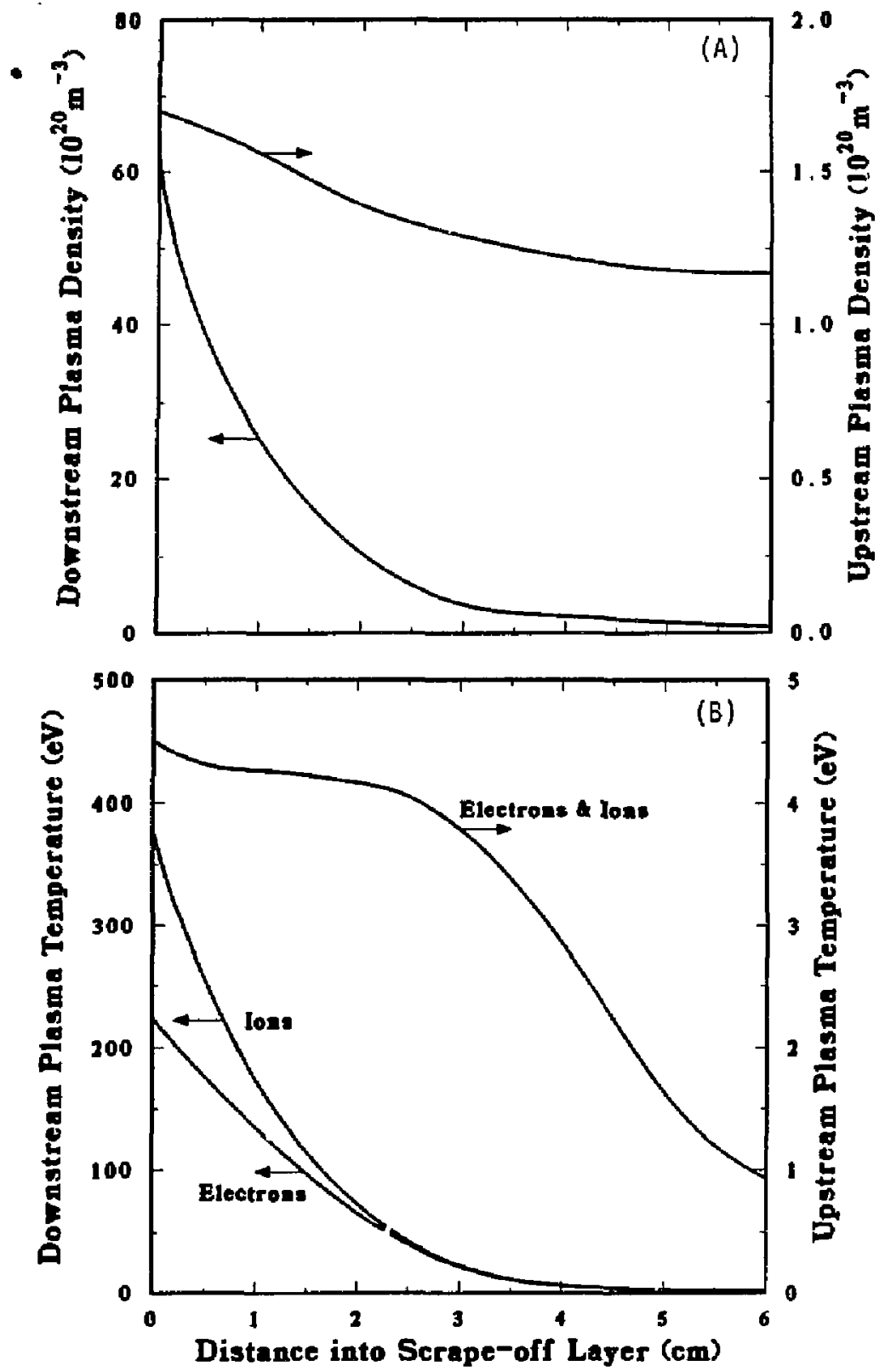

Figure 8.4-2. Radial profile of edge-plasma density (A) and temperature $(B)$ in the scrape-off layer for upstrean and downstream axial positions in TITAN-I. 

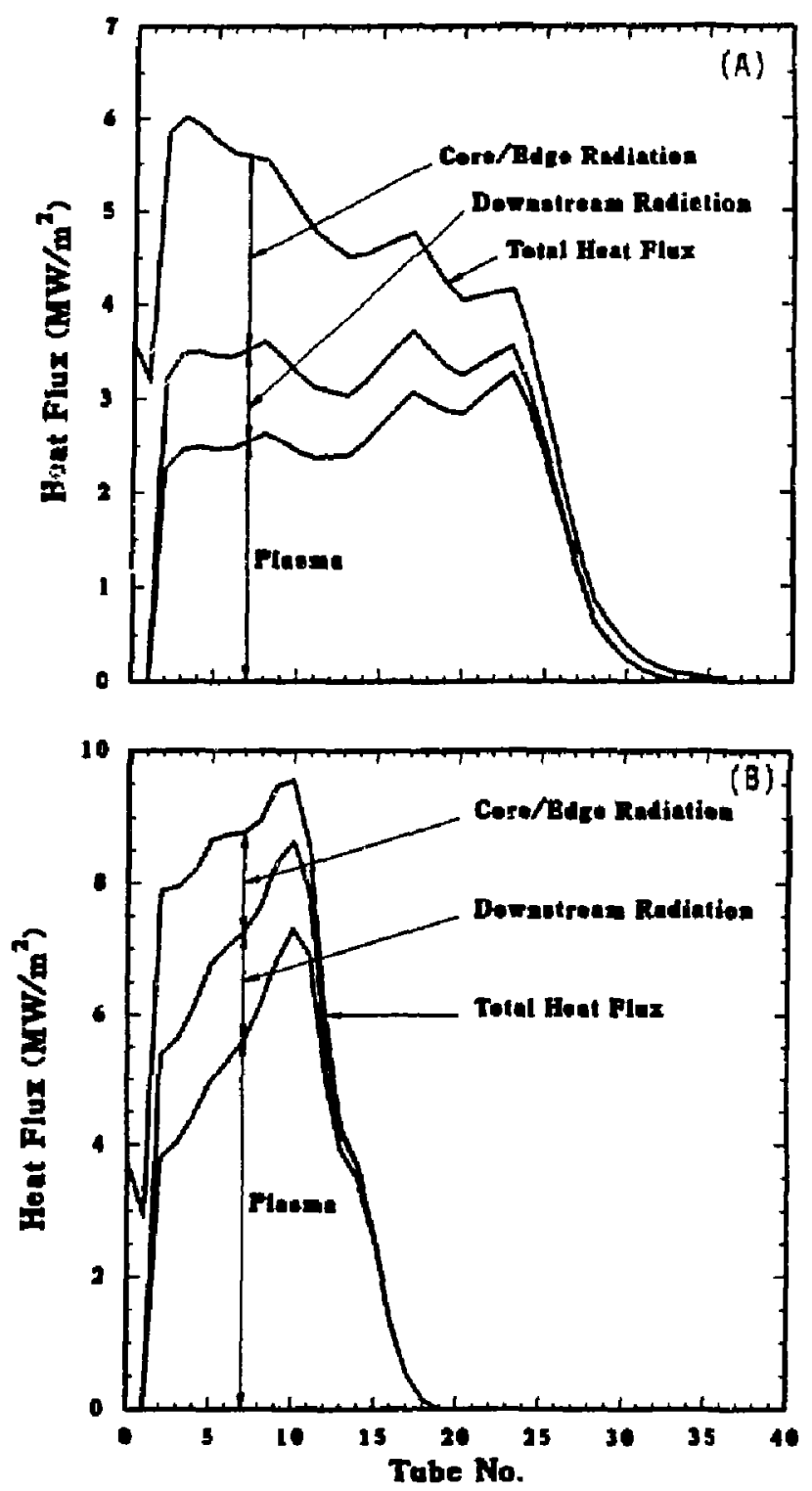

Figure 8,4-3. Heat flux distribution on the outboard (A) and inboard (B) sections of the TITAN-I divertor target. Coolant tubes are numbered from the apex or symmetry point of the target between the nulling coil and the core. plosma. 
heat flux (i.e., size of divertor-field plume) and is $\geq 0.0 \%$ and $\geq 0.12$ for TITAN-I and TITAN-II, respectively. Recirculated power considerations set upper bounds on $\Delta s / r_{p}$.

The operation of the TITAN divertors is very sensitive to precise positioning of the separatrix and the edge-plasma field lines with respect to the divertor target. Control of the divertor-coil current as well as the overall pliasing of the divertor operation with the start-up and subsequent OFCD transients present other concerns. Generally, operation of OFCD with a fixed separatrix location and a hot plasma at the separatrix should reduce both the plasma resistance, plasma wall interertion, and hence, the OFCD requirements, provided the scrape-off region does not promote additional helicity dissipation [29].

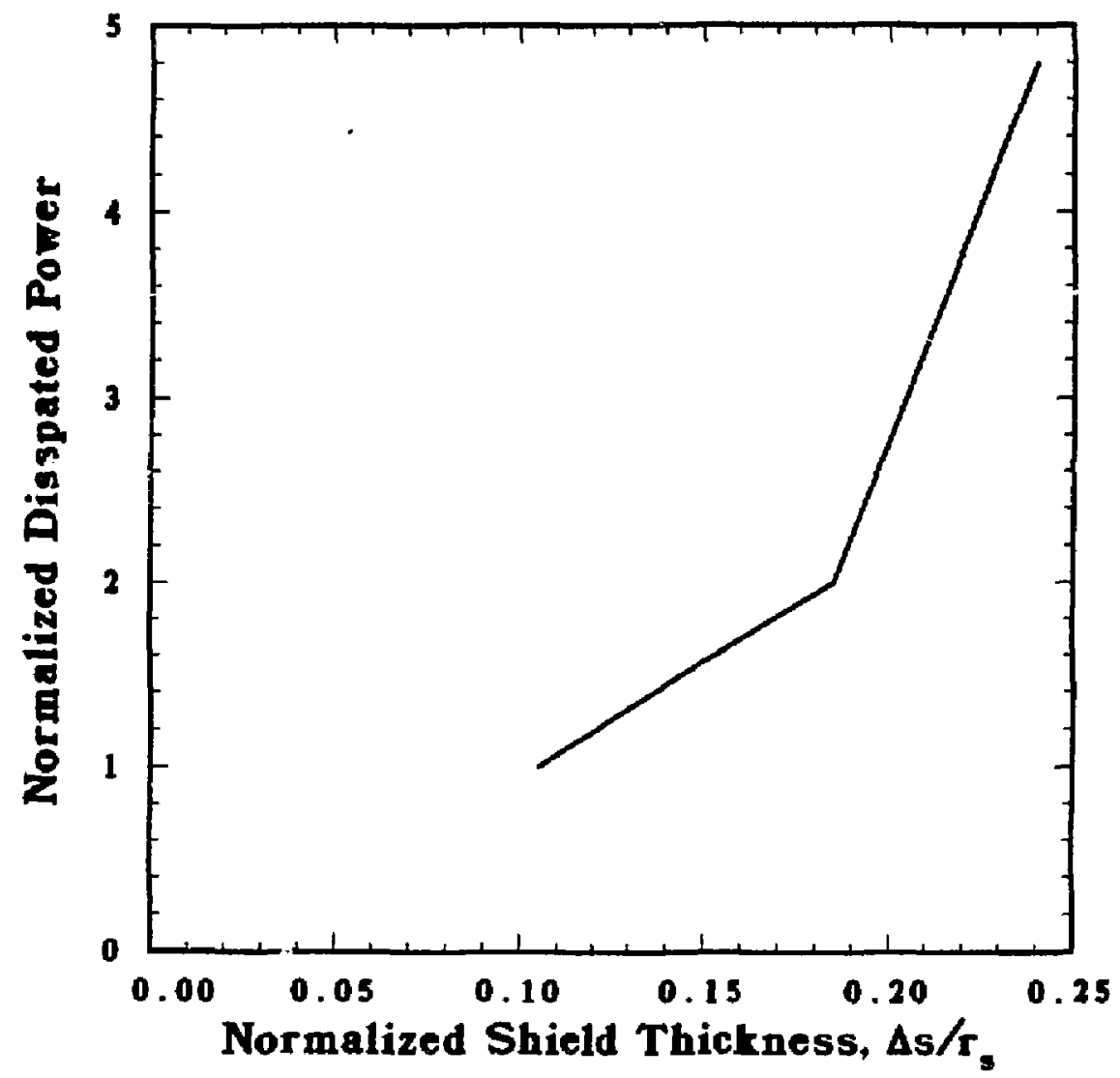

Figure 8.4-4. Sensitivity of TITAN-I divertor-coil power consumption to normalized shield thickness. 


\subsection{FORMATION AND START-UP}

\subsubsection{Reactor Conditions}

The four main pliases of the TITAN start-up towards achieving olunic ignition and ultimately a steady-state, $I_{\phi}=18$-MA burn are illustrated in Figure 8.5-1. The proposed start-up scenario includes: (1) a 1 to 10 -ms formation phase $(0.1$ to $0.2 \mathrm{MA})$, (2) a fast current ramp ( 2 to $3 \mathrm{~s}, 0.1 \rightarrow 10 \mathrm{MA}$ ), (3) a slow current ramp ( 8 to $20 \mathrm{~s}, 10 \rightarrow 18 \mathrm{MA}$ ), and (4) steady-state buru. Throughout this current ramp-up phase, active equilibrium control (by main equilibrium-field and trim coils) will be required. Impurity control should probably start during the fast current-ramp pliase and the separatrix position held fixed during the start-up transients. The OFCD systen may starts operation during the slow-ramp phase to assist in achieving the full plasma current. Initially, about 0.2 to $0.3 \mathrm{~Wb}$ of toroidal flux would be injected by the $\mathrm{TF}$ coil. The majority of the $\sim 5 \mathrm{~Wb}$ of toroidal flux contained within the final burning plasma, however, would be generated through the poloidal-field (PF) coil system by the RFP dynano and, hence, presents an added, but small, flux requirement for the ohmic-heating (OH) coils.

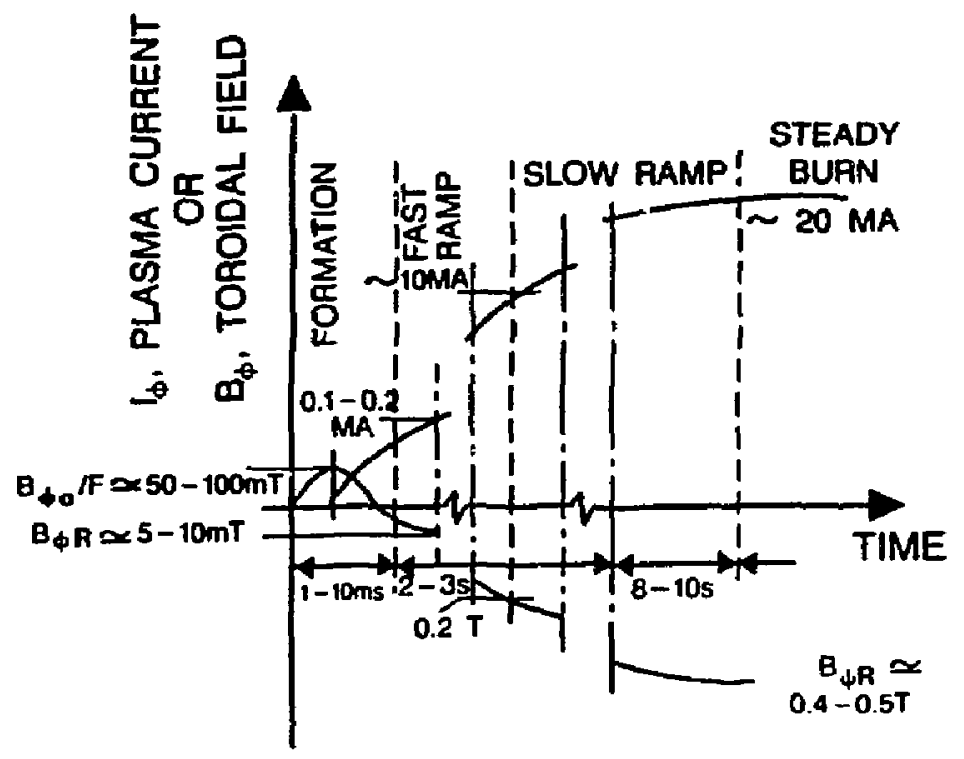

Figure 8.5-1. Schematic of TITAN start-up and burn cycle. 


\subsubsection{Physics Data Base}

A body of experimental evidence is beginning to accumulate that better defines the windows for RFP breakdown, formation, and ramp-up. The status in each of these areas is summarized. Because of the present position of the RFP physics program, a majority of the RFP data base pertains to the formation/current ramp and, to a lesser extent, to the confinement areas.

\subsubsection{Breakdown}

Plasma discharge and subsequent RFP formation generally occurs for values of the ratio of toroidal electric field to initial filling pressure, $E_{\phi} / P_{o}$, that are dimilar to tokamak values, but closer to electron runaway conditions. For example, for the JET experiment, a value of $E_{\phi} / P_{0} \geq 0.66 \times 10^{4} \mathrm{~V} / \mathrm{m}$-torr is reported [30], compared to 1 to $2 \times 10^{4} \mathrm{~V} / \mathrm{m}$-torr for 2T-40M [31], which is close to electron runaway conditions. Figure 8.5-2 gives typical breakdown and formation characteristics for a range of tokamaks and for ZT-40M. Generally, breakdown and discharge formation are not prohlems for RFPs, but the degree of pre-ionization can greatly influence the discharge quality and poloidal-flux consumption $[33,34]$. Since, to date, stable and reliable RFP formation appears to require a high $E_{\phi}$, the generally common $E_{\phi} / P_{\phi}$ values for both RFPs and tolearnaks give significantly higher values of $P_{0}$ required to create a robust RFP. An initial electric field of $E_{\phi}=55 \mathrm{~V} / \mathrm{m}$ is indicated in Figure 8.5-3 for matched-mode operation.

A toroidal-field line of strength, $B_{\phi o}$, in the presence of a vertical field, $B_{V}$, will intersect the first wall and thereby prevent the formation of a continuous discharge if the ratio $B_{V} / B_{\phi_{0}}$ is too large. The condition for the confinement of a single toroidal trajectory with a field null at a minor radial position, $r_{o}$, is given by

$$
\frac{B_{V}}{B_{\phi o}} \leq \frac{\epsilon}{2 \pi} \sqrt{1-\left(r_{o} / r_{p}\right)^{2}},
$$

where $\epsilon=r_{p} / R_{T}$ is the inverse plasma aspect ratio. For TITAN with $1 / \epsilon \simeq 6.3$ and setting $r_{o} \simeq 0.5 r_{F}$ result in $B_{V} / B_{\phi_{0}} \leq 0.02$.

In addition, a drift constraint, $E_{\phi} /\left(B_{V} / B_{\phi_{0}}\right) \geq 10^{3} \mathrm{~V} / \mathrm{m}$, has been suggested for JET [32]. This drift constraint together with Equatios 8.5-1 results in $E_{\phi} \geq 22 \mathrm{~V} / \mathrm{m}$, which is about a factor of 2 below experimental values terived from the ZT-40M experience. Therefore, breakdown voltage in the range 500 to $1,000 \mathrm{~V}$ may be required for the reactor. Careful designs that minimize field errors can possibly reduce these start-up voltages. 

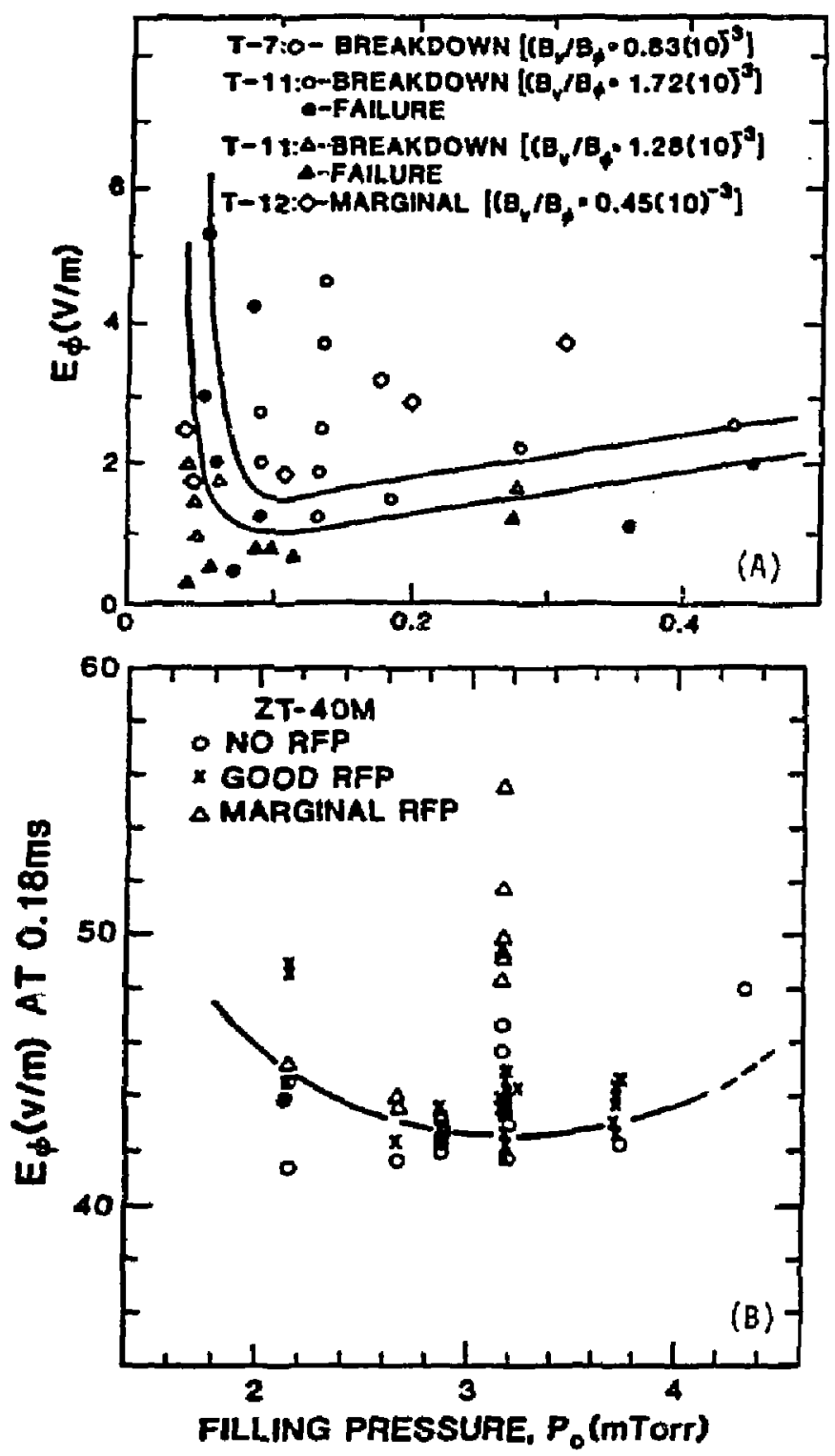

Figure 8,5-2. Typical breakdown curves for (A) tokamak [32] and $(B)$ RFP formation $[33,34]$. 

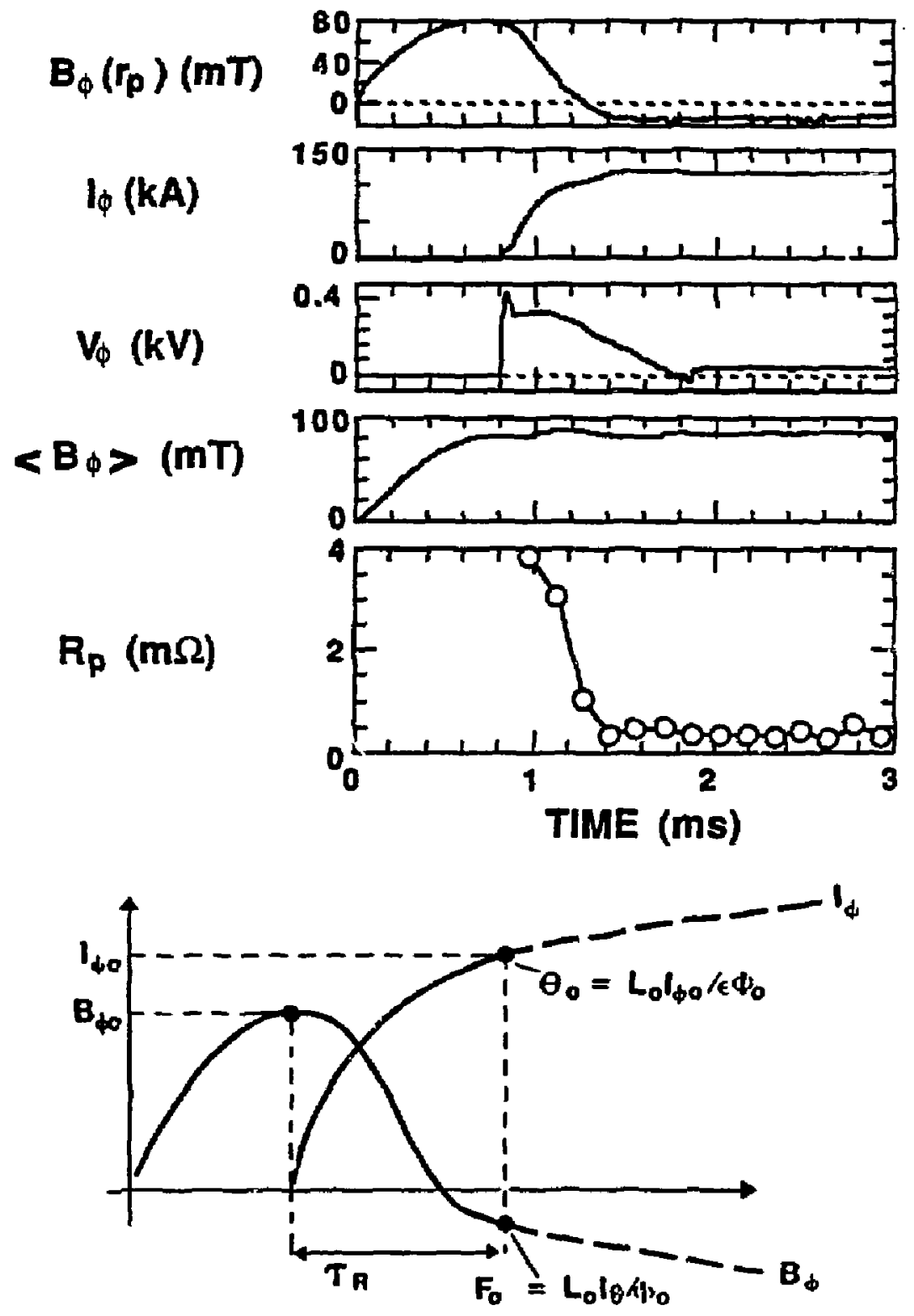

Figure 8.5-3. Typical matched-mode RFP formation for ZT-40M leading to the values of $\Theta_{o}, F_{o}$, and $I_{\phi \infty}[35]$ used as initial conditions for start-up, ignition, and burn simulations. 


\subsubsection{RFP formation}

In order to establish the parameters of the "seed" RFP, the relationship between $B_{\phi o}$ and the average toroidal flux within the initial RFP, $\left\langle B_{\phi}\right\rangle$, must be determined (Figure 8.5-3). Generally, $\left\langle B_{\phi}\right\rangle \simeq B_{\phi 0}$ for RFP formation. Figure 8.5-4 shows the relationship between $B_{\phi_{0}}$ and $\left\langle B_{\phi}\right\rangle$ for a range of ZT-40M discharges. illustrating the experimental basis for this assumption. For a given $\left\langle B_{\phi}\right\}$ and the initial pinch parameter, $\Theta_{a}$, the initial (minimum) RFP current or current density is determined from

$$
I_{\phi \infty}=5 r_{p} \Theta_{o}\left\langle B_{\phi}\right\rangle .
$$

Hence, for $\left\langle B_{\phi}\right\rangle \simeq B_{\phi o}=0.05 \mathrm{~T}$ at formation, and TITAN plasma conditions of $\Theta_{0} \simeq 1.5$ and $r_{p}=0.6 \mathrm{~m}$, the initial RFP current is $I_{\phi o} \simeq 0.2 \mathrm{MA}$.

Although the specification of $\Theta_{0}$ and $\left\langle B_{\phi}\right\rangle$ at formation determines an initial current density (e.g., $j_{\phi_{0}} \simeq 0.2 \mathrm{MA} / \mathrm{m}^{2}$ for TITAN), other more dominant constraints may exist. For exarnple, the ZT-40M experiment exhibits a minimum current-density limit which translates empirically to $j_{\phi_{0}} \geq 0.4 \mathrm{MA} / \mathrm{m}^{2}$, below which RFP formation is difficult. This constraint is not well understood, but the application of such a constraint to TITAN represents a conservative connection to experiment and for the above condition would require a doubling of $B_{\phi o} \simeq\left\langle B_{\phi}\right\rangle$. Secondly, a number of RFP experiments [6] have shown an impurity burn-through constraint, shown in Figure 8.5-5 for ZT-40M. For these conditions, burn-through requires that $j_{\phi} / n \geq 10^{-20} \mathrm{MA} \mathrm{m}$. This constraint, however, when applied to the $0.2-$ to $0.4-\mathrm{keV}$ plasmas expected for these formation conditions places the plasma st:ongly in the electron runaway regime. If the runawray regime is to be avoided, which may or may not be a requirement, higher densities will be required (e.g., for $\xi \equiv v_{D} / v_{T H e} \leq 0.01, n \geq 2 \times 10^{1 \theta} \mathrm{m}^{-3}$ for $j_{\phi}=0.4 \mathrm{MA} / \mathrm{m}^{2}$ and $T \approx 0.2 \mathrm{keV}$ ).

Generally, if the density pump-out is too great prior to toroidal-field reversal for a given initial filling pressure, $P_{o}$, unreliable RFP formation occurs [31], as is shown in Figure 8.5-6(A). Similarly, for a given $P_{\mathrm{o}}$ a maximum initial bias field, $B_{\not \circ}$, is found above which RFP formation does not occur [31], as is also shown in Figure 8.5-6(B). Although RFPs form at lower values of $B_{\phi 0}$, these RFPs have excessive poloidal-flux consumption, as is shown in Figure 8.5-6(B), for the $2 \mathrm{~T}-40 \mathrm{M}$ conditions examined. It should be noted that a variable and poorly controlled wall condition creates hystereses and related unpredictable effects in many of these data correlations. The constraints of Figures 8.5-6(A) and (B) have been combined in Figure 8.5-6(C) to eliminate the filling pressure as a variable and perhaps to reduce the impact of these unresolved wall effects on these data. The result is a relationship between average plasma density and initial 


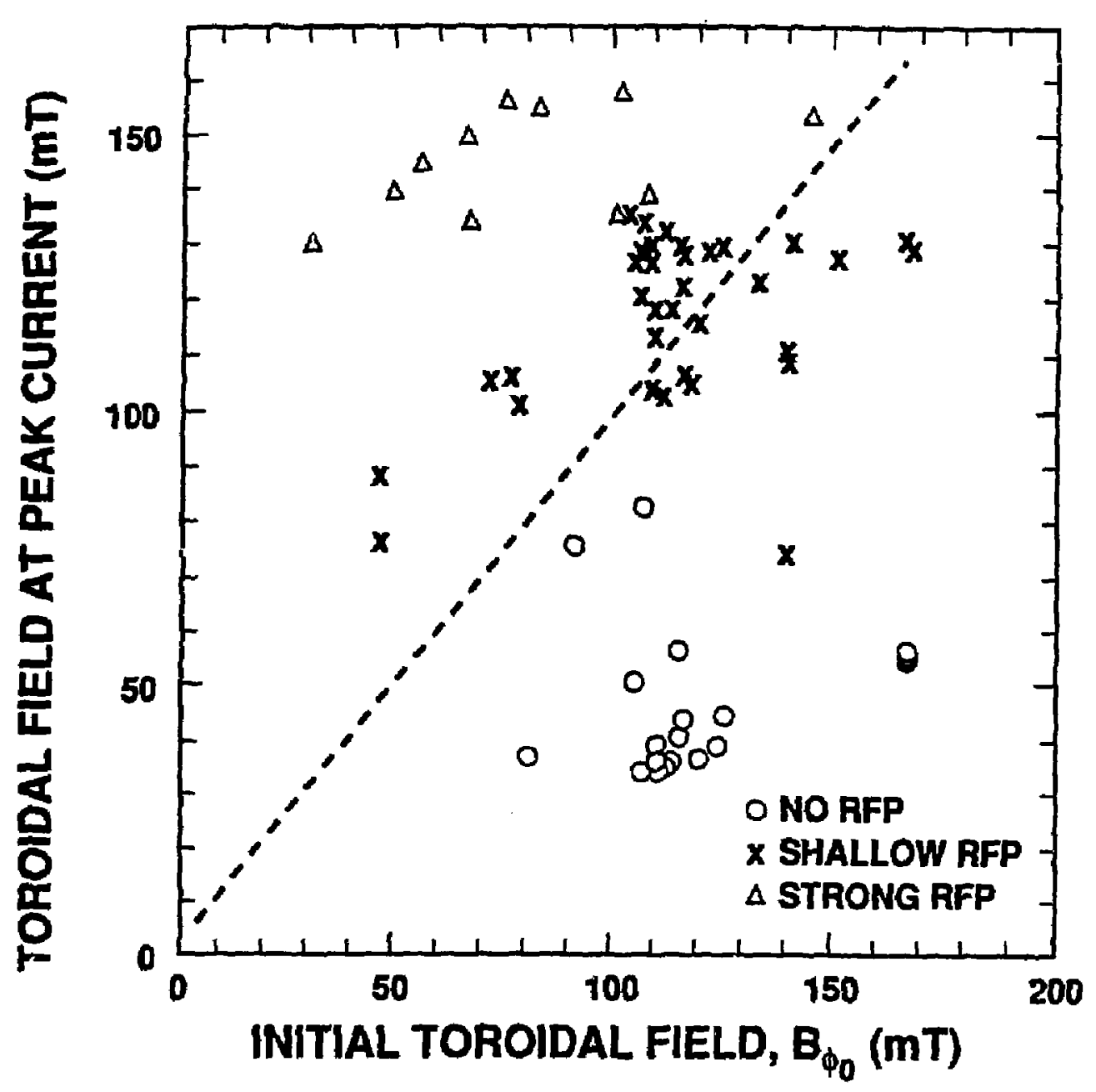

Figure 8.5-4. Relationship between $B_{\phi \circ}$ and $\left\langle B_{\phi}\right)$ for a range of ZT-40M discharges [ 31 , 34] where robust RFP formation occurred, as well as no RFP formation and/or very shallow, spherornak-like RFPs were formed. 


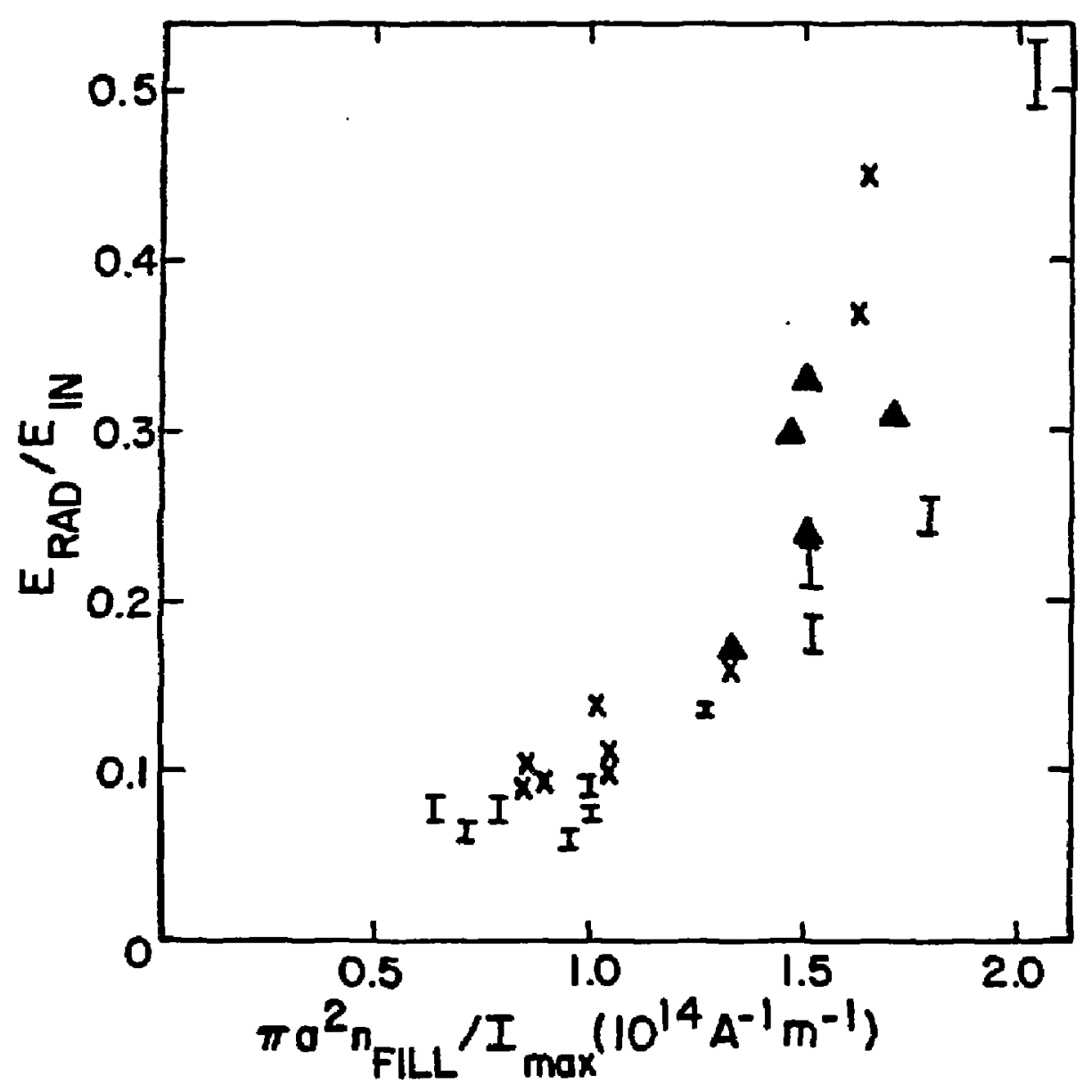

Figure 8.5-5. Typical impurity burn-through constraint for ZT-40M [31]. 

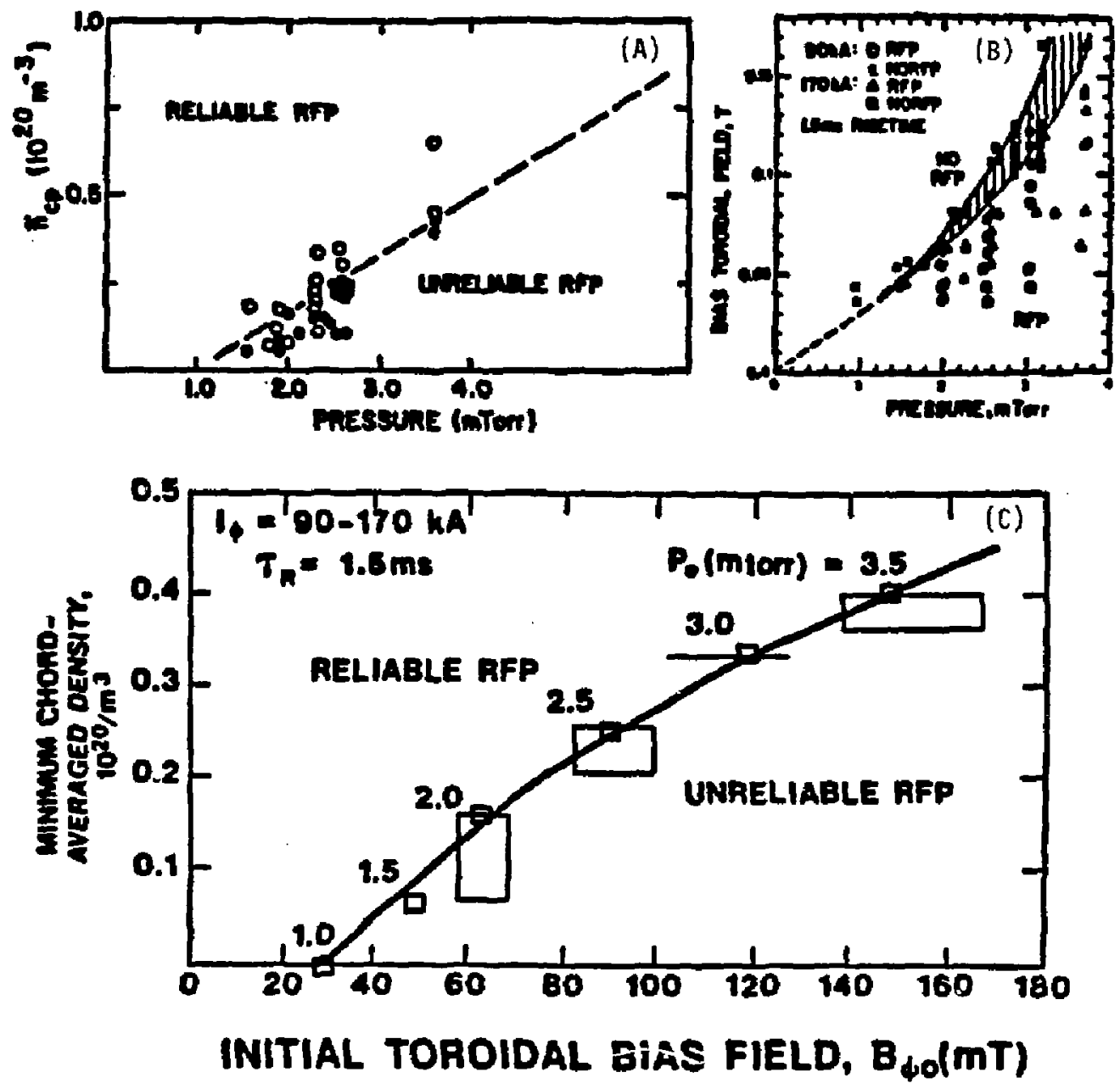

Figure 8.5-6. Typical RFP formation windows showing: (A) dependence on a critical plasma density, (B) magnitude of the initial toroidal bias field, and (C) a combination of the two constraints $\{31\}$. 


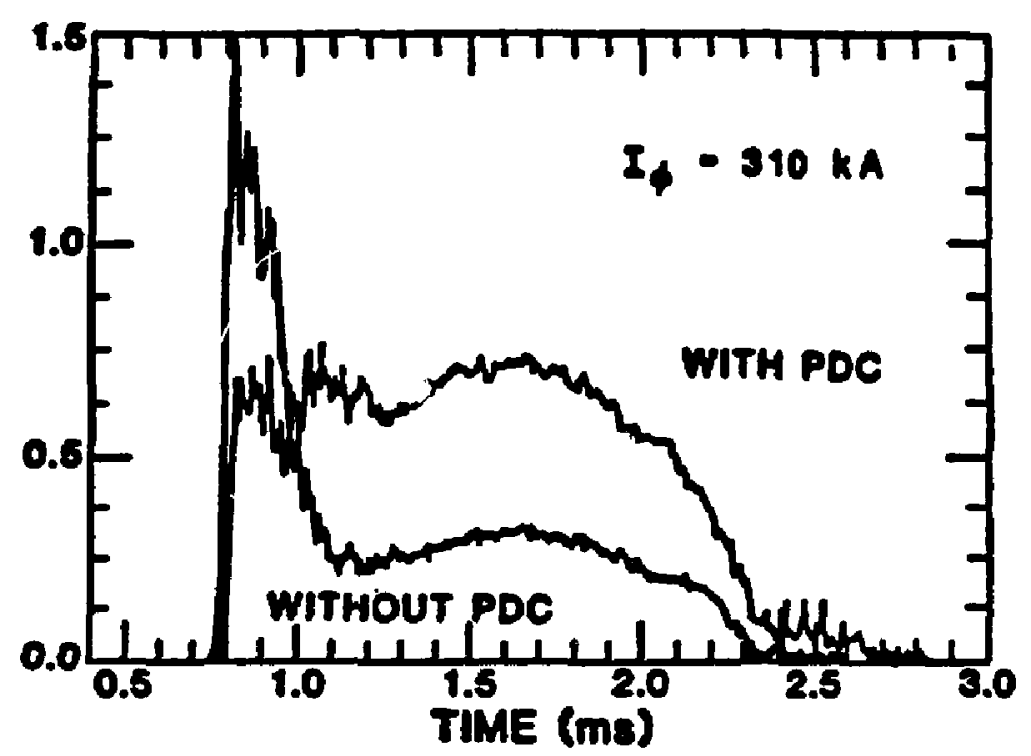

Figure 8.5-7. Loss of density or pump-out for a typical ZT-40M discharge. Also shown is the impact of pellet fueling on reducing pump-out [36].

bias field that may be useful in assuring robust RFP formation. Typically, for values of $B_{\phi o}$ being considered, the minimum critical density for reliable RFP formation is above that needed to assure a reasonable safety margin against electron runaway upon RFP formation.

Most present-day RFPs experience a significant loss of density or pump-out upon formation, as is shown in Figure 8.5-7. The degree of density reduction between the initial filling pressure and the fingl RFP formation is not well understcod but it depends strongly on wall preconditioning. Hence, the pump-out is expressed in terms of the ratio of initial filling density, $n_{o}$, to the final RFP plasma density, $n>n_{e}$, and the assumption must be made for the reactor that pump-out and $P_{o}$ can be minimized, thereby minimizing the $E_{\phi}$ required under start-up conditions.

\subsubsection{Current ramp-up}

While the formation phase leading to the low-current ( 0.2-MA) "seed" RFP is characterized by a rapid current rise (150 MA/s for the case shown in Figure 8.5-3), 

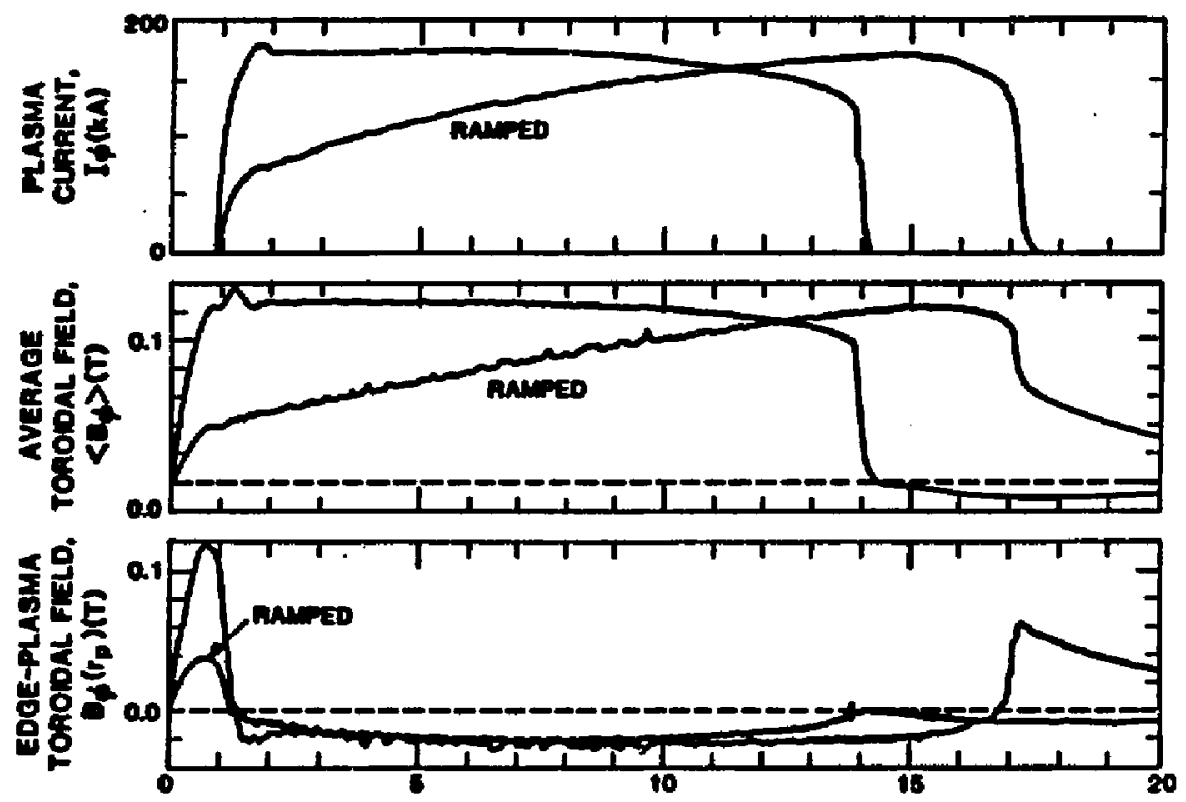

THE (n)

Figure 8.5-8. Slow and fast current ramps in ZT-40M [31].

and the flux consumption can be a small fraction of the inductive flux delivered to the plasma, the subsequent current ramp to ohmic ignition and burn ( $\left.I_{\phi}=18 \mathrm{MA}\right)$ represents a greater concern from the viewpoint of resistive flux consumption and the implication on $\mathrm{OH}$-coil and related power-supply designs. Figure 8.5-8 shows that slow current ramp rates $(9 \mathrm{MA} / \mathrm{s})$ have been achieved in $\mathrm{ZT}-40 \mathrm{M}$. These current ramp rates are still large compared to those typical of tokamaks ( 1 to $2 \mathrm{MA} / \mathrm{s}$ ) and are possible in RFPs because of the anomalous penetration of magnetic flux. The slow current ramps of the kind shown are desirable for reactors since the bulk of the flux injection can be supplied directly from the electrical grid at relatively low power, rather than from an expensive on-site energy storage.

A significant decrease in plasma loop voltage is measured upon reversal of the toroidal field and formation of the RFP. This behavior is shown for ZT-40M in Figure 8.5-9, which also shows an optimal value of the pinch parameter from the viewpoint of loop voltage and resistive poloidal-flux consunption. The reduction in resistive flux consumption upon controlling $\Theta$ at the optimal value is shown in Figure 8.5-10, which gives the resistive 

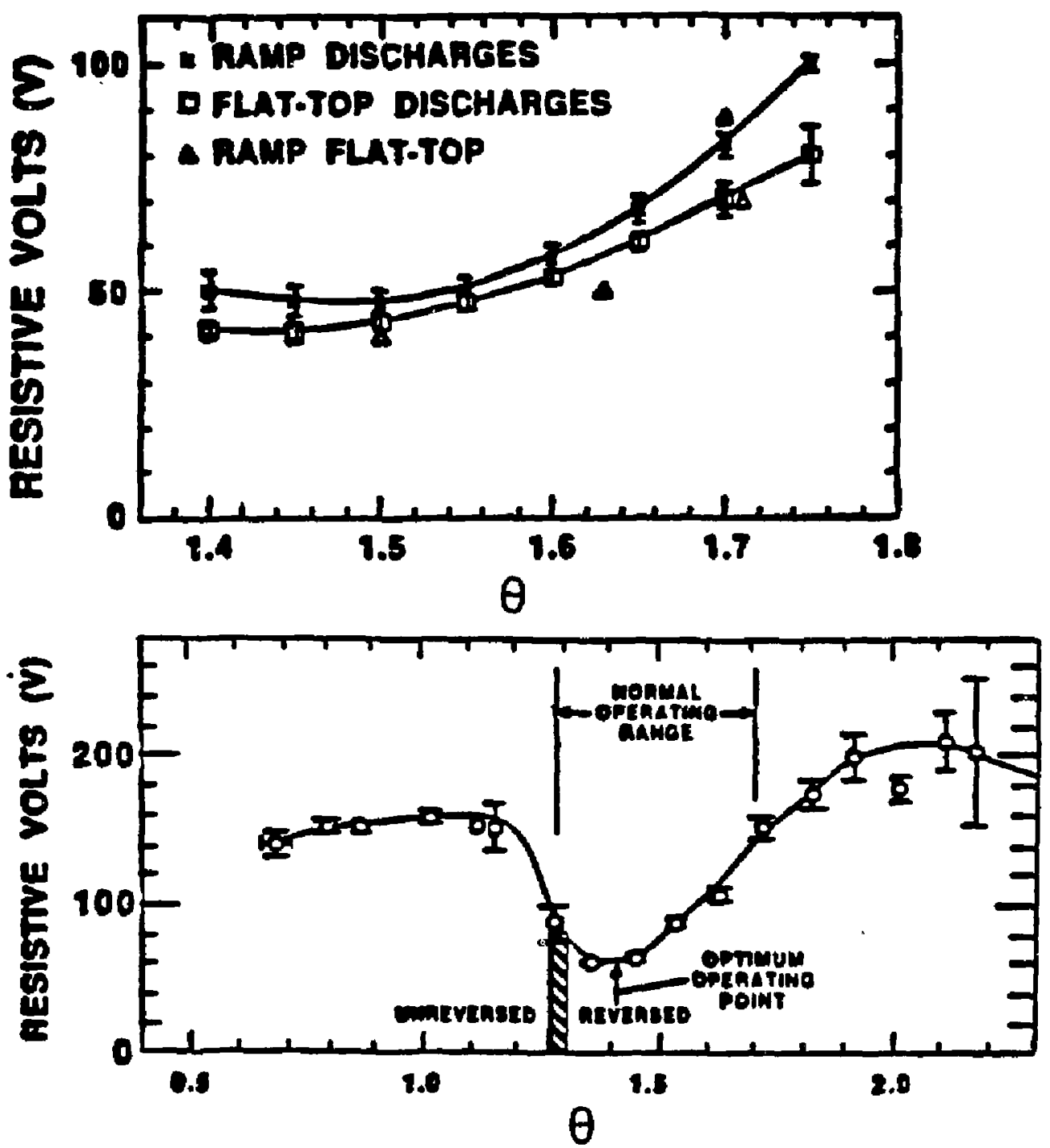

Figure 8.5-9. The dependence of the plasma loop voltage on the pincl paraneter upon formation of RFP, showing an optinal value of $\Theta$ [35]. 


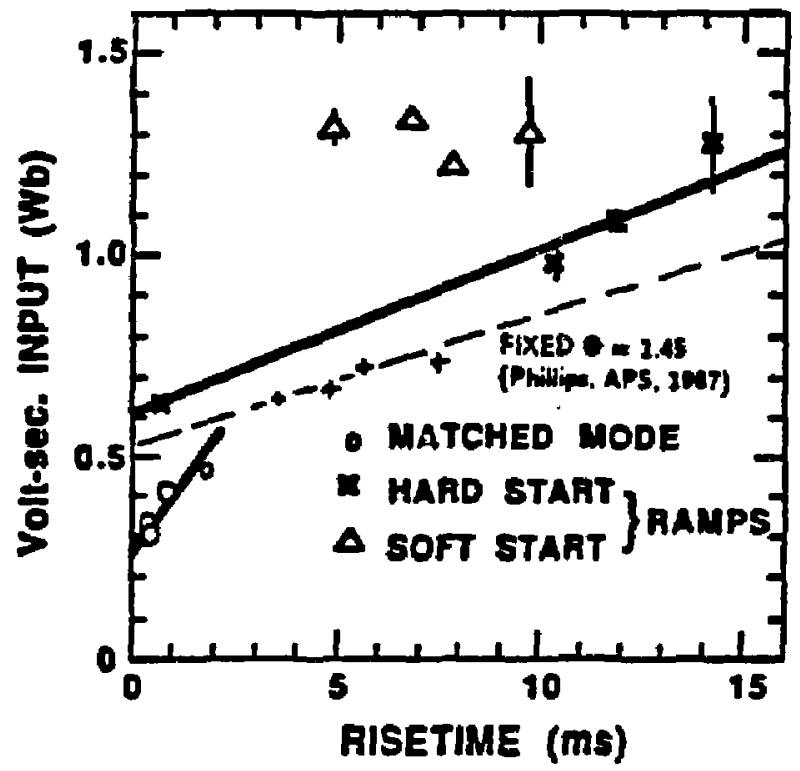

Figure 8.5-10. Dependence of flux input on current risetime for ange of 2T-40M operating conditions $[31,35]$.

volt-second consumption for a range of conditions, including the volt-second efficient matched-mode operation. The ZT-40M data shown on Figure 8.5-10 indicate a constant (revistive) voltage scaling ( $V_{R E S} \simeq 32.5 \mathrm{~V}$ ), which implies that the plasma resistance is decreasing inversely with plasma current in this region. The implication in this scaling on the TITAN-I reactor design is examined in the folloring section.

\subsubsection{Reactor Implications}

The conditions for plasma breakdown and subsequent RFP formation for the reactor is expected to differ little from the conditions in present and plantied RFP experiments [16]. The conditions of the seed-RFP plasma required to start up the TITAN reactor, except for plasma size, are similar to present-day RFP parameters $\left(I_{\phi}=0.2-0.4 \mathrm{MA}\right.$, $\left.n=1-2 \times 10^{10} \mathrm{~m}^{-3}, T=0.2-0.4 \mathrm{eV}\right)$. Somewhat lower values of $j_{\phi} / n$, however, are desirable for the reactor starting plasma, and better density and impurity control during the $\sim 10$-ms breakdown and formation process may be required.

Assuming that the breakdown and formation windows defined for most operating RFP experiments remain unchanged and a seed-RFP plasma of the above-mentioned 
characteristics can be provided in larger sizes and longer times, the main physics issue for start-up reduces to one of resistive poloidal-flux consumption cluring the current ramp-u\}).

The TITAN stari-up scenalio includes a fast current caup to $t_{\phi} \simeq 10 \mathrm{MA}$ (at 3 to $5 \mathrm{MA} / \mathrm{s}$ ) to rapidly achieve a plasma of reduced resistance. The fast ramp is followed by a slower ramp to ignition and burn, $I_{\phi}=18 \mathrm{MA}$ (at $\sim 1 \mathrm{MA} / \mathrm{s}$ ). During the current. ramp phases, toroidal flux within the TF coil increases from $0.3 \mathrm{~Wb}$ to $5.3 \mathrm{~Wb}$ (gross) (4.5 Wb, net). This toroidal flux will be supplied by the RPP dynamo llasough the PF. coil system since the direct injection of the entire toroidal fux at. formation would require considerable upgrading of the TF coils and /or considerably increased current ramp rates and is viewed as impractical for the reactor.

The fact that the final plasnia state has nore toroidal fhux than ihe TF coils alone are apable of supporting, however, must be consjdered during the current rundown phase of the reactor. For example, if the initial toroidal bias field is $B_{\text {dro }}=0.1 \mathrm{~T}$, the initial toroidal fux injected into the reactor torus for a nominal coil ractius of $r_{c \phi}=1.0 \mathrm{~m}$ is $0.3 \mathrm{~Wb}\left(\dot{\phi} \simeq 0.3-1.0 \mathrm{kV}\right.$-turns for $\left.\tau_{\mathrm{Ro}_{0}} \simeq 10 \mathrm{nus}\right)$. The reversed and plasma toroidal fields at $1:$ in conditions are, respectively, $B_{\phi R} \simeq-0.4 \mathrm{~T}$ and $\left\langle B_{\phi}\right\rangle=-B_{\phi R} / F \simeq 4.0 \mathrm{~T}$, which correspond to the respective toroidal fluxes of $\phi^{-} \simeq \pi\left(r_{c \phi}^{2}-r_{p}^{2}\right) B_{\phi R}=-0.8 \mathrm{~Wb}$ and $\phi^{+} \simeq \pi r_{p}^{2}\left\langle B_{\phi}\right\rangle=4.5 \mathrm{~Wb}$. Wence, the average toroirtal field that would result if the plasma suddenly "disappeared" would be $\left(\phi^{+}+\phi^{-}\right) / \pi r_{c \phi}^{2}=1.2 \mathrm{~T}$ or 10 times the initial tomidal field. Furthermore, the net flux change amounts to a field of $\left(\phi^{+}-\phi^{-}\right) / \pi r_{\text {eq }}^{2}=1.7 \mathrm{~T}$, which, thougl, not large by tokamak standiards, would nevertheless requive a structural redesign of the TF coil, and the 'TF IBC: used in the TITAN-I design would also recpuire a reassessment of the start-up sower consumption. Lastly, almost all of the funal touncial f ${ }^{*} x$ within the plasma, $\phi^{+} \simeq 4.5 \mathrm{~Wb}$, must be supplied eluring the current ranp-up by the $O H$ coil through the dymamo. Therefore, if the conversion of poloidal flux to toroidal flux occurs wit] an efficiency of $\sim 50 \%$, an aclditional $\sim 10 \mathrm{~Wb}$ must be resigned into the $\mathrm{OH}$-coil set $($ an $\sim 4 \%$ effect $)$.

Th- impact of resistive flux consumption for the reactor during the ramp-up of plasma current is summarized in Table 8.5-I, which compares the experimenta] (ZT-40M) [35] and TITA N-I ranp-up conditions. The TITAN-I ramp-up conditions recuire a bipolar $\mathrm{OH}$ coil designed for $\sim 500-\mathrm{MWe}$ maxinum power from the grid that can supply $24 \mathrm{~T}$ Wb of inductive poloidal flux and $\sim 26 \mathrm{Wl}$ of resistive flux dissipation over the current rampup period. The resistive dissipation inclucles only that deposited in a plasma described by a Spitzer resisijivity $\left(\eta \propto T^{-3 / 2}\right.$ ), an appropriate profile factor ( $g_{\text {OHM }}=2.92$ as defined by Equetion 3.2-8), and a temperature scaling linearly witl current. 
Table 8.5-I.

IMPACT OF RESISTIVE FLUX CONSUMPTION DURING RAMP-UP

Typical Experimental and Reactor Parameters

Start-up mode

Pinch parameter, $\Theta$

Plasina current, $I_{\phi}$ (MA)

Ramp time, $\tau_{R}(s)$

Incluclive flux, $\phi_{\text {ind }}(\mathrm{Wb})$

Plastna resistive $\mathrm{Alux}_{\mathrm{u}} \phi_{\mathrm{rea}}^{\mathrm{P}}$ (Wb)

Major radius, $R_{T}(\mathrm{~m})$

Minor radius, $r_{p}(\mathrm{~m})$

Geometry iactor, $R_{T} / r_{p}^{2}(1 / \mathrm{m})$

Final plasma temperature, $T(\mathrm{keV})$

Final loop voltage, $V_{\phi}(\mathrm{V})$

Plasma ohnic power, POHM $_{\text {(MW) }}$

$\begin{array}{cc}\text { ramp } & \text { ramp } \\ 1.45^{(a)} & 1.5-1.6 \\ 0.05-0.2 & 18 . \\ 0.015 & 8.0^{(b)} \\ \sim 0.5 & 247 .\end{array}$

$32.5 \tau_{R}$

$26.4^{(c)}$

1.12

3.9

0.2

0.6

28.0

10.6

$0.05-0.20$

10.0

32.5

1.9

6.5

33.6

Resistive Flux Consumption for ZT-40M Scaling (Wb)

Direct application 260.

Corrected for geonetry 98.4

Corrected for temperature ${ }^{(d)}$ 54.6

Corrected for temperature and geometry 20.1

(a) Optimal (minimum fux consumption) for ZT-40M.

(b) Time to maximum current, almost equilibrium plasma conditions.

(c) Spitzer resistivity, $Z_{\text {eff }}=1.0 \rightarrow 1.05$, gOHM $=2.92$.

(d) $R_{p} \propto r_{-\phi}^{-3 / 2}$. 
The direct applicalion of the "scaling" suggested in Figure 8.5-10 (i. $\left.\epsilon_{+}, R_{p} \approx 32.5 / I_{\phi}\right)$ would give rise to a plasma resistive fiux consumption of $260 \mathrm{~Wb}$ (7able 8.5-I), almost doubling the volt-second requirement of the OH-coil set from TITAN-I. The TITAN-I design uncer these conditious would re-optimize at a faster current rise, more massive coils, increased peak (back-biased) power requirement, and possibly higher aspect ratio (to reduce $\mathrm{OH}$-coil stresses in the back-biased condition). Direct application of a geometry correction (i.e., $R_{T} / r_{p}^{2}$ ) to the ZT-40M resistive volt-second scaling reduces $\phi_{\text {res }}^{\text {p from }}$ $260 \mathrm{~Wb}$ to $98 \mathrm{~Wb}$. The assumption of an $R_{p} \propto I_{\phi}^{-3 / 2}$ (i.e., $T \propto I_{\phi}$ ) scaling of the ZT-40M data gives $\phi_{\mathrm{res}}^{\mathrm{p}}=55 \mathrm{~Wb}$. Application of both geometry and temperature scalings to the ZT-40M data combine to reduce $\phi_{\text {res }}^{\text {pes }}$ to $20 \mathrm{~Wb}$, which is close to the TI'lAN-1 design value of $26 \mathrm{~Wb}$.

In summary, the experimental data base for breakdown and formation is encouraging for direst application to TITAN-I conditions. Extension of the current ramp-up to higher currents and plasmas and to somewhat slower rann rates ( 1 to $2 \mathrm{MA} / \mathrm{s})$ is required, and the demonstration of more classical-like scaling of plasma resistance with plasma geometry $\left(R_{p} \propto R_{T} / r_{p}^{2}\right)$ and temperature $\left(R_{p} \propto T_{p}^{-3 / 2}\right)$ is highly desirable. Additionally, the data base for breakdown, formation, and ramp-up should be extended to higher currents and densities, but at lower values of $\xi=v_{D} / v_{\text {THe. }}$. Active control of density, $Z_{e f f}$, and equilibrium during start-up is highly desirable.

A broader definition of the RFP formation window is needed as expressed in terns of (1) the minimum $B_{\phi_{o}}$ as a function of the critical plasma density and/or filling pressure for a given vertical-fiek error, (2) a minimum $E_{\phi_{0}}$ for breakdown and impurity burn-throush, and (3) a better resolved relationship between $B_{\phi \infty}, E_{\phi o}, P_{o}$, and degree of purnp-out. Lastly, the ZT-40M scaled loop voltage for TITAN parameters is $\sim 17$ times that of the estimates using classica!-like scaling. Hence, significant reductions are reçuired from the viewpoint of current drive and ohnic power losses (i.e., a factor of 17 increase in $P_{O H M}$ would make ohmic heating comparable to the alpha-particle heating at steady-state bum conditions).

A number of siart-up issues are not expected to be addressed by the next generation of RFP devices, but are of sufficient importance to warrant further analysis. These issues deal with the combined need during the current ramp-up to concurrently initiate equilibrium (including separatrix formation), feedback control, active inpurity control, operation of high-heat-flux components, impurity injection to create a highly racliating plasma, pellet injection and density control, and the accommodaion of as yet unresolved 2.unges in the heating profiles when the plasma transcends from a predoninantly resistively heated entity to one that is heated primarily by clarged-particle reaction products. 
While these issues cannot be addressed by the RFX and ZTH devices presently under const.ruction, further understanding of these integrated transient effects and the respective plasma responses and associated timing of system responses (e.g., EF-coil control, divertor, impurity injection, fueling, surface cooling) can be developed by additional analytic studies.

\subsection{SUMMARY AND RECOMMENDATIONS}

The performance projecter for the TITAN reactor designs etepends crucially on the physics areas described in Sections 8.2 through 8.5 . Given below are the key plyysics issues for the high-power-clensity RFP reactors and recommendations to the $R F P$ experimental physics program.

Confinement: Determine spatially resolved profiles in non-transitory RFPs in order to resolve the scaling of intrinsic energy confinement time with plasma geometry, current or current density, and $F-\Theta$ value in hot plasmas with low values of $\xi=v_{D} / v_{T H e}$ and $Z_{\text {eff }}$. Special emplasis should be placed on investigating radiation-dominated operation and the limits of beta and intrinsic (non-radiative) confinement.

Current drive: Investigate both $D C$ and $A C$ technirues for helicity injection in hot RFPs with separatrices and minimal modulation-induced plasma-wall interaction. The inupact and management of high levels of reactive power require better resolution. Other means of current drive sliould also be investigated.

Inpurity control: Emphasize plasna operation with a toroidal-field separatrix in order to to minimize plasma-wall interaction in a lighly radiating plasma, eventually leading to high-recycling divertor operation and an easing of OFCD with a fixed separatrix position.

Formation and start-up: Clearly resolve the scaling of plasina resistance witl current ranp rate, current, geomet.ry, and $F-\Theta$ values in plasmas with low values of electron streasming parameters, and minimal and/or controllable plasma-wall interactions.

Clearly, these key issues and the conditions needed and/or assumed for the compact RFP reactor designs are interrelaterl and symbiotic. The existence and role of a closefitting electrically conducting shell that surrounds the RFP has strong impact on these 
physics issues. Hence, as a fifth, but highly integrating recommendation: resolve the need for and characteristics of a conducting shell with electricai breaks on the formation and start-up, confinement, current drive, and inpurity control which represent at present. an inadequately mapped issue for the RFP. This is an issue that the TITAN study circummavigated numerous times in its overall guest to tesolve physics and engineeringsystems issues for cost-effective and technologically attractive, higlt-power-density fusion reactors.

Finally, current termination is a safety and economics concern because of the large magnetic stored energy in the TITAN plasma. Experimental techniques for control of current ternination and plasma shut down, leading to a "soft-landing," especially by passive means, are essential for achieving a ligh degree of safety and envisonusental attractiveness for RFP reactors. 


\section{REFERENCES}

[1] F. Najmabadi, N. M. Ghoniem, R. W. Conn, et al, "The TITAN Reversed-Field Pincl Reactor Study, Scoping Phase Report," joint report of University of Califormia Los Angeles, General Atonics, Los Alamos National Laboratory, and Rensselaer Polytechnic Institute, UCLA-PPG-1100 (1987).

[2] R. W. Conn (clıaiman and editor), "Magnetic Fusion Advisory Committee Panel X Report on High Power Density Fusion Systems" (May 8, 1985).

[3] J. Sheffield, R. A. Dory, S. M. Cohn, J. G. Delene, L. F. Parsley, tt al., "Clost. Assessment of a Generic Magnetic l'usion Reactor," Oak Ridge National Laboratory report ORNL/TM-9311 (1986) 103.

[4] R. A. Krakowski, R. L. Miller, and R. L. Hagenson. "The Need and Prospect for Improved Fusion Reactors," J. Fission Energy 5 (1986) 213.

[5] R. A. Krakowski, R. L. Hagenson, N. M. Schnurr, C. Copenhaver, C. G. Bathke, R. L. Miller, and M. J. Embrechts, "Compact. Reversed-Field Pinch Reactors (CRFPR)," Nucl. Eng. Design/Fusion 4 (1986) 75.

[6] H. A. Bodin, R. A. Krakowski, and O. Ortolani, "The Reversed-Field Pinch: from Experiment to Reactor," Fusion Technol. 10 (1986) 307.

[7] R. L. Hagenson, R. A. Krakowski, G. G. Batlike, R. L. Miller, M. J. Embrechts, et al., "Compact Reversed-Field Pinch Reactors (CRFPR): Prelininary Eugineering Considerations," Los Alamos National Laboratory report LA-10200-MS (1984).

[8] C. Copenhaver, R. A. Krakowski, N. M. Schunrr, R. L. Miller, C. G. Bathke, et al., "Compact Reversed-Field Pinch Reactors (CRFPR)," Los Alamos National Laboratory report LA-10500-MS (1985).

[9] P. Thullen and K. Schoenberg (Eds.), "ZT-H Reversed-Field Piuch Experiment. Technical Proposal," Los Alamos National Laboratory report LA-UR-84-2602 (1984) 26.

[10] G. Malesani and G. Rostagni, "The RFX Experiment," Proc. I4th Symp. Fusion. Technology, Avignon (1986) 173.

[11] Y. Hirano, T. Shimada, Y. Maejimz, and K. Ogawa, "Improved Stability Period in High-Current-Density Operation of Reversed-Field Pinch of ETL-TPE-1R(M)." Nucl. Fusion 22 (1982) 1613. 
[12] B. Alper et al., "RFP Comfinement Studies in EPA-BETA-11," Proc. 12th Europran Conf. on Cont. Fusion and Plasma Phys., Budapest., Hungary (Septemler 1985), European Phys. Soc. 1 (1985) 578.

[13] H. A. B. Bodin, "New Results from the IIB'TX Experiment," Bull. Am. Phys. Soc. 32 (1987) 1784.

[14] T. Tamano, W. D. Bard, T. N. Carlstrom, C. Chu, B. Curwe, R. K. Fisher, et al., "Higlı C'urrent, High Beta Toroidal Pinch Experiment in OHT'E," in Proc. 10th Int. Conf. on Plasma Phys. and Cont. Nucl. Fusion Res., London, U. K. (September 1984), LAEA, Vienna (1985).

[15] D. A. Baker, C. J. Buchenauer, L. C. Burkliarclt, E. J. Caramana, J. N. DiMarco, J. N. Downing, et al., "Experiunental and Theoretical S1 udies of the ZT-40M Reversed-Field Pinch," jn Proc. 10th Int. Conf. on Plasma Phys. and Cont. Nucl. Fusion Res., London, U. K. (September 1984), IAEA, Vienna (1985).

[16] D. B. Thounson (Ecl.), Proc. Int. Workshop on Engineering Design of Ncxt Step Reversed Field Pinch Devices, Los Alamos National Laboratory (July 13-1T, 1987).

[17] C. Ci. Bathke, R. A. Krakowski, R. A. Krakowski, and R. L. Miller, "A DT Neutron Source Based on the Reversed Field Pinch," Proc. 12th IEEE Symp. on Ftusion Eng., Monterey, C:A (October 1987) 829.

[18] M. K. Bevir and J. W. Gray, "Relaxation, Flux Consumption and Quasi Steady State Pinclies," Proc. of RFP Theory Workshop, Los Alamos, NM, U. S. A. (1980), Los Alamos National Laboratory report LA-8944-C! (1982) 176.

[19] K. F. Schoenherg, J. C. Ingraham, C. P. Munson, ef al., "Oscillating-Field CurtentDrive Experinents in a Reversed-Field Pincl)," Phys. Fluids 8 (1989) 2285; also R. A. Scardovelli, R. A. Nebel, and K. A. Werley, "lranspont Sinmlation of the Oscillating Field Current Drive Experiment in the Z-40M," Los Alamos Nationa] Laboratory report LA-UR-2802 (1988).

[20] R. S. Massey, R. G. Watt, P. G. Weber, C. A. Wurden, D. A. Baker, C. J. Buchenauer, et al., "Status of the ZT-40M RFP Experimental Progran," Fusion Technol. 8 (1985) 1571.

[21] P. G. Weber ot al., "Results from the Los Alamos RFP Experiments," Proc. 12th European Conf. on Cont. Fusion and Plasm- Phys., Budlapest, Hungary (September 1985), European Phys. Soc. 1 (1985) 570. 
[22] M. M. Pickrell, J. A. Phillips, C. J. Buchenaler, T. Cayton, J. N. Downing, A. Haberstich, et al., "Evidence for a Poloidal Beta Lisit on ZT-40M," Bull. Am. Phys. Soc. 20 (1984) 1403.

[23] R. Hancox, R. A. Krakowski, and W. R. Spears, "The Reversed-Field Pinch Reactor Concept," Nucl. Eng. and Res. 63 (1981) 251.

[24] K. F. Schoenberg, J. C. Ingrahain, C. P. Munson, P. G. Weber, D. A. Baker, R. F. Gribble, et al., "Oscillat.ing-Field Current Drive Experiment in ZT-40M Reversed Field Pinch," Los Alamos Nalional Laboratory report LA-UR-88-122, submitted to Phys. Fluids (1988).

[25] R. L. Hagenson and R. A. Krakowski, "The Spheromak as a Compact Fusion Reactor," Los Alamos National Laboratory report. LA-10908-MS (March 1987).

[26] C. G. Batlike and R. A. Krakowski, "A Comparison Study of Toroidal-Field and Bundle Divertors for a Conupact Reversed-Field Pinch Reactor," Fusion Technol., 8 (1985) 1616.

[27] A. K. Prinja and R. W. Conn, "An Axially-Averaged Madial Transport Model of Tokamak Edge Plasmas," J. Nucl. Mater. 128-129, (1984) 135.

[28] A. F. Almagri, et al., "Reversed Field Pinch Experiments with Various Boundary" Conditjons," in Proc. Int. School of Plasma Phys. Course and Workshop on Physics of Mirrors, Reversed-Field Dinches, and Compact Tori, Vareme, Italy (September 1987 ).

[29] T. R. Jarboe and B. Alper, "A Model for Loop Voltage of a Reversed-Field Piuch," Phys. Flucids 30 (1987) 1177.

[30] A. Kellman, E. Lazzaro, P. Noll, F. C. Schveller, and P. Thomas, "Breakdown Conditions in JET," Bull. Am. Phys. Sac. 30 (1985) 1524.

[31] J. A. Phillips, L. C. Burkhardt, A. Haberstich, R. B. Howell, 3. C. Ingraham, E. M. Little, K. S. Thomas, and R. G. Watt, "ZT-40M Ciurrent Risetine Study," Los Alamos National Lalıoratory report. LA-9717-MS (1983).

[32] F. Enge]mann, N. Fujisawa, B. B. Karlonitsev, V. I. Pistunovicl, J. A. Sclunidt, and M. Sugihara, "Pliysics Design Basis," in International Tokamak Reactor, Phase One, IAEA, Vienna (1982) (liap. III, p. 127. 
[33] R. G. Watt and J. N. Downing, "Pre-ionization Studies in ZT-40M," Los Alanos National Laboratory report LA-9163-MS (February 1982).

[34] J. A. Phillips, Los Alamos National Laboratory, private communicaticn (August 1986).

[35] J. A. Phillips, D. A. Baker, R. F. Gribble, and C. Munson, "Start-up of ReversedField Pincles and Current Ramping Using Dynamo Action," submitted to Nucl. Fusion (1987).

[36] G. A. Wurden, P. G. Weber, R. G. Watt, C. P. Munson, T. E. Cayton, and K. Buchl, "Refueling and Density Control in the ZT-40M Reversed-Field Pinch," in Proc. Int. School of Plasma Phys. Course and Forkshop on Physics of Mimors, Reversed-Field Pinches, and Compact Tori, Varenua, Italy (September 1987). 